HN-190

AEC RESEARCH \&

DEVELOPMENT REPORT

UC-80, Reactors-General

TID-4500 Distribution

\title{
RELIABILITY ANALYSIS
}

OF

\section{NUCLEAR POWER PLANT PROTECTIVE SYSTEMS}

\author{
By \\ B. J. Garrick, Study Director \\ W. C. Gekler, Study Leader \\ L. Goldfisher \\ R. H. Karcher \\ B. Shimizu \\ J. H. Wilson \\ Prepared Under \\ Subcontract No. C-275 \\ PHILLIPS PETROLEUM COMPANY \\ Atomic Energy Division \\ Idaho Falls, Idaho
}

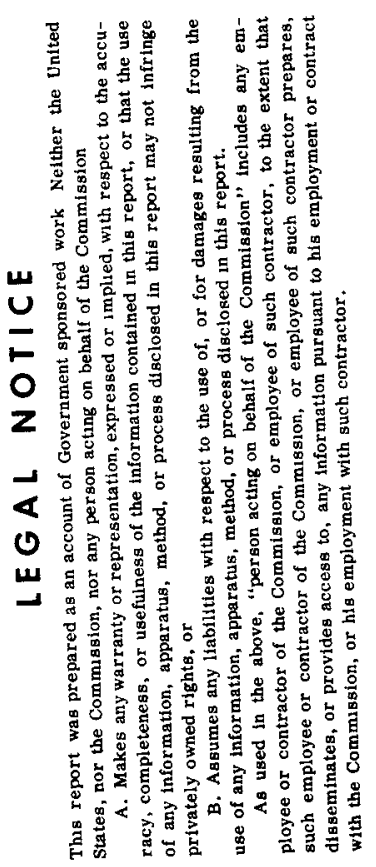

U. S. ATOMIC ENERGY COMMISSION

Contract No. AT(10-1)-205

Idaho Operations Office

May 1967

\section{HOLMES \& NARVER, INC. NUCLEAR DIVISION}




\section{DISCLAIMER}

This report was prepared as an account of work sponsored by an agency of the United States Government. Neither the United States Government nor any agency Thereof, nor any of their employees, makes any warranty, express or implied, or assumes any legal liability or responsibility for the accuracy, completeness, or usefulness of any information, apparatus, product, or process disclosed, or represents that its use would not infringe privately owned rights. Reference herein to any specific commercial product, process, or service by trade name, trademark, manufacturer, or otherwise does not necessarily constitute or imply its endorsement, recommendation, or favoring by the United States Government or any agency thereof. The views and opinions of authors expressed herein do not necessarily state or reflect those of the United States Government or any agency thereof. 


\section{DISCLAIMER}

Portions of this document may be illegible in electronic image products. Images are produced from the best available original document. 
$$
\text { . }
$$ 


\section{ACKNOW LEDGEMENTS}

Preparation of this report has involved the cooperation and advice of several individuals and organizations. We wish to thank the management and employees of the General Electric Company, the Westinghouse Electric Corporation, North American Aviaiion, Inc., and the Phillips Petroleum Company. We wish to especially thank Messrs. N. K. Sowards and E. O. Meals of the Phillips Petroleum Company for their constructive comments during the course of the study.

Special appreciation is extended to Mr. D. E. Brimley for consultation on data management and reliability analysis, to Messrs. R. C. Erdmann and $O$. C. Baldonado of Holmes \& Narver, Inc. for investigations of selected analytical techniques connected with reliability methods, and to Mr. H. P. Pomrehn, formerly of Holmes \& Narver, for contributions during early phases of the study. 


\section{PREFACE}

Much has been said about the safety of nuclear reactors compared to other facilities of moder $n$ industry. In fact, the safety record of the nuclear industry is without parallel. But we dare not be complacent for the fact remains that a large nuclear plant contains within itself an immensely significant amount of radioactive material. Thus, we must not relax; rather, we must continue to press for better understanding, better control of safety. Perhaps we can go so far as to quantify the notion of safety. That is, perhaps we can learn to provide definitions and $r$ ules by which one can arrive at a numerical figure of merit indicating the level of safety of a particular plant. Or, perhaps it will be a small cluster of figures of merit which collectively tell the story. Any quantification of this sort will be an aid so that people (designers, operators, regulators) can make better judgments of merit and risk, so that they can more rationally decide for or against various designs, sites, and operating procedures.

How then does one go about making precise and quantifying so vague a concept as that of level of safety? Clearly, the concept involves knowledge of the origins, likelihoods, and consequences of accidents. And clearly, it involves knowledge of routine and expected, though random, wearing out and failure of equipment and personnel. Such knowledge in turn derives from study and understanding of the basic physical phenomena and of the particular system in question. It derives also from study of the experience record of systems and facilities already in being. Assuming the concept of level of safety can be defined in crisp mathematical terms, we will still need the input, the basic physical data, the probabilities of component failure, and so on, Thus, we need two things: clarification of concept and collection of actual operating data. 
In the present study and those preceding it, ${ }^{(1,2,3)}$ we have taken steps to satisfy both these needs. We have gathered information, and we have identified and experimented with methods of interpreting and synthesizing the information into a quantified measure of safety. We have made detailed recommendations for a reliability monitoring program - for better methods of collecting and inter preting pertinent safety, reliability, and availability information.

The task has been a tedious one involving sifting, searching, and analyzing hundreds of in-plant records including instrumentation and equipment logs, maintenance work orders, and all manner of control room logs. It icicied lengthy discussions with utility executives, plant superintendents, plant engireers, operators, maintenance personnel, government employees, and design personnel. It involved many different plants, manweeks of effort to reconstruct plant systems in diagram form, data compiling and analyzing, and writing, writing, and writing. It was approached in this manner because all the easy ways seemed to lead to nothing. Like good design, good safety is based on details . It is based on knowing the day-to-day problems of plant operation. It is based on observing first-hand the recording and handling of data. Only this provides a solid foundation for a more quantitative basis for safety evaluations.

Finally, we must ask ourselves how the results of greater quantification will be revealed in the day-to-day practice of nuclear safety. Obviously, there will be direct benefits. Much needed reliability data will be generated. This data will serve as the grist for many

(1) B. J. Garrick, W. J. Costley, and W. C. Gekler, "A Study of Test Reactor Operating and Safety Experience, "HN-172 (Vols. I and II), U. S. Atomic Energy Commission, May 1963.

(2) B. J. Garrick, W. C. Gekler, J. M. Duncan, R. H. Karcher, and B. Shimizu, "A Study of Research Reactor Operating and Safety Experience, "HN-180, U. S. Atomic Energy Commission, June 1964.

(3) B. J. Garrick, W. C. Gekler, and H. P. Pomrehn, "An Analysis of Nuclear Power Plant Operating and Safety Experience, " HN-185 (Vols. 1 and 2), U. S. Atomic Energy Commission, December 1966 . 
other activities. Statistical bases will arise to more clearly define the performance of equipment. Industry norms will be established for comparing equipment and isolating unsatisfactory reliability performance. Test frequencies will be related to statistical expectations and we can approach ever closer to recognition of the underlying statistical distributions which give rise to the observed behavior.

We should also look for indirect benefits, not the least of which will be the enhancement of reliability analysis as a safety tool. Reliability data will permit this technique to be used in quantifying system reliability and establishing the relative importance of various components to system reliability. This knowledge can, in turn, provide a valuable lever for improving and controlling safety. Systems offered for the same safety function will be compared on the basis of expectation as well as capability. Physical modifications and procedural changes which improve the reliability of a system will be identified. Ultimately we will arrive at the goal of a figure of merit to quantify safety.

It is our hope that this work will be used to realize these and other benefits and thereby further increase our understanding of safety.

B. John Garrick

Study Director 


\section{TABLE OF CONTENTS}

$\underline{\text { PAGE }}$

ABSTRACT

xvi

INTRODUC TION

SUMMARY AND CONCLUSIONS

Data Management

Analytical Techniques

Application

Recommendations

CHAPTER 1 - RELIABILITY DATA MANAGEMENT SYSTEM 1-1

Introduction

Data Classification

Data Collection

CHAPTER 2 - RELIABILITY ANALYSIS TECHNIQUES 2-1

Automatic Reliability Mathematical Model (ARMM) Program 2-3

Description

Use of ARMM

Illustration of ARMM Application

Systems Analysis by Fault Tree Evaluation, SAFTE-1

SAF TE - 1 Program Description

Importance Sampling

Sample Calculation: Dresden-3 Emergency A-C Power System

Conclusions and Recommendations

References

CHAPTER 3 - EXAMPLE APPLICATIONS OF RELIABILITY ANALYSIS

Dresden Unit 3 Primary Containment

System Description

Reliability Considerations
$3-1$

$3-3$

3-3

2-3

2- 18

2-29

2-36

2-37

2- 42

$2-47$

2-50

2-51

3-14 
TABLE OF CONTENTS (continued)

$\begin{array}{lc} & \text { PAGE } \\ \text { Reliability Evaluation } & 3-15 \\ \text { Results of Analysis } & 3-19 \\ \text { Connecticut Yankee Safety Injection System } & 3-22 \\ \text { System Description } & 3-23 \\ \text { Reliability Considerations } & 3-27 \\ \text { Reliability Evaluation } & 3-28 \\ \text { Results of Analysis } & 3-29 \\ \text { Connecticut Yankee Containment Cooling } & 3-31 \\ \text { System Description } & 3-31 \\ \text { Reliability Considerations } & 3-33 \\ \text { Reliability Evaluation } & 3-33 \\ \text { San Onofre Safety Injection System } & 3-40 \\ \text { System Description } & 3-41 \\ \text { Reliability Evaluation } & 3-46 \\ \text { Results of Analysis } & 3-49\end{array}$

APPENDIX A - PRESENT DATA COLLECTION PRACTICES

IN OPERATING NUCLEAR POWER PLANTS A-1

APPENDIX B - DATA MANAGEMENT PRACTICES IN

RELATED AREAS B-1

APPENDIX C - EXAMPLE OF LOADSHEET PREPARATION AND DATA REDUCTION C-1

APPENDIX D - SELECTED FAILURE RATE DATA D-1

APPENDIX E - SAFTE-1 SOURCE PROGRAM LISTING E-1

APPENDIX F - TENTATIVE GUIDELINES FOR RELIABILITY ANALYSIS OF CONTAINMENT ISOLATION VALVE SYSTEMS F-1

APPENDIX G - GLOSSARY G-1

APPENDIX H - RELIABILITY ESTIMATES WITH CONFIDENCE LEVELS H-1 
TABLE OF CONTENTS (continued)

TABLES

PAGE

2. 1 Possible Failure Combinations Considered by ARMM of System in Figure 2. 1 (No Dependency) $2-52$

2. 2 Example Situations

$2-52$

2. 3 Modes Available, Modes Required, and Weighting Factors

$2-53$

2. 4 Failure Combinations and the Use of Depth Control 2-54

2. 5 Order of Magnitude Integration Error by Using

Simpson's Rule $\quad 2-55$

2.6 Time Intervals of Failure Modes 2-56

2. 7 Function and Component Data 2-57

2. 8 Time Profile 2-58

2. 9 Logical "Or" and "And" Gates Appearing in

Dresden-3 Emergency AC Power System Fault Tree 2-59

2. 10 Component Failure and Repair Data for Dresden-3

Emergency AC Power System

$2-61$

3. 1 Principal Design Parameters for Dresden-3

Primary Containment $\quad 3-50$

3. 2 Principal Penetrations of Primary Containment and

Associated Is olation Valves 3-5l

3. 3 Dresden-3 Standby Diesel Generator Loading

Requirements

3.4 Blowdown Accident Summary 3-53

3.5 Time Sequence for Blowdown Accident 3-54

3.6 Failure Rate Assumptions 3-55

3. 7 Summary of Results for Dresden-3 Primary

Containment Reliability Evaluation 3-56

3. 8 Major Contributors to Primary Containment Failure 3-57

3. 9 Component Grouping by Percentage Contribution to System Failure $\quad 3-58$

3. 10 Loss of Coolant Incident and Safety Injection System Summary $3-59$

3.11 Ruptured Pipe Sizes and Safety Injection System

Operation

$3-60$

$\begin{array}{lll}3.12 & \text { Failure Rate Summary } & 3-61 \\ 3.13 & \text { Summary of System }\end{array}$

3. 13 Summary of System Unreliability 3-62

3. 14 Major Contributors to System Unreliability 3-63

3.15 Connecticut Yankee Containment Cooling System
Component Failure Rates 3-64

3. 16 Component Failure Combinations Leading to Power System Failure $\quad 3-65$

3.17A Component Contributions to System Unreliability 3-66 
TABLE OF CONTENTS (continued)

TABLES (continued)

$\underline{\text { PAGE }}$

3. 17B Component Combinations Contributing to System Failure (Hand Estimate)

$3-67$

3. 18 Components - Mechanical Features 3-68

3.19 Recirculation Heat Exchanger $3-70$

3.20 Components - Electrical Parameters 3-71

3.21 Interval Component Contributes to System

Unreliability $\quad 3-72$

3.22 Failure Rate Data Used for San Onofre Safety Injection System

$3-75$

3.23 Major Contributors to Unreliability of San Onofre Safety Injection System During Three Time Intervals

B. 1 UKAEA Fault Classification System B-20

B. 2 Partial List of Plant Types B-21

B. 3 Partial List of Component Types B-22

B. 4 Fault Importance B-23

B. 5 Partial List of Fault Types B-24

B. 6 Effects $\quad$ B-25

B. 7 Miscellaneous Column Allocations B-26

B. 8 PM-1 Malfunction Packet Card No. 2 B-27

B. 9 Malfunction Packet Card No. $3 \quad$ B-28

B. 10 Partial List of Subsystem and Component Codes B-29

B. 11 Malfunction, Safety, and Shutdown Codes B-30

B. 12 Failure Codes B-31

B. 13 Levels of MARAD Classification B-32

B. 14 Systems and Subsystems B-33

B. 15 Component and Assembly List B-34

B.16 Component Type List (Index 2) B-35

B. 17 EEI - Type of Outage B-36

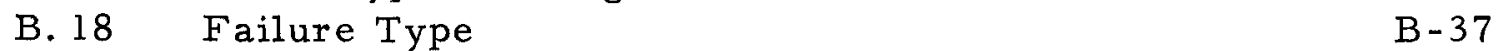

B. 19 Example of AEC-FPC Account Number Correlation B-39

B. 20 AEC Classification of Construction Accounts

Nuclear Power Plants B-40

B. 21 Example of Detailed Account Listing B-43

C. 1 List of Components in a Simple Pumping System C-5

D. 1 Failure Rate Data on Selected Electronic, Electrical, and Mechanical Equipment D-3

D. 2 Reference Sources for Failure Rate Data D-7

F. 1 Penetration Classification F-3 
TABLE OF CONTENTS (continued)

\begin{tabular}{|c|c|c|}
\hline 1. 1 & Comparison of Data Management Information & $1-23$ \\
\hline 1.2 & Proposed Data Management System & $1-24$ \\
\hline 1.3 & List of Existing Classifications & $1-25$ \\
\hline 1.4 & $\begin{array}{l}\text { Numbers of Levels, Groups, and Digits of Existing } \\
\text { and Proposed Classification Codes }\end{array}$ & $1-26$ \\
\hline 1.5 & $\begin{array}{l}\text { Comparison of Plant/System/Component Classifi- } \\
\text { cation/Multiple Component Identification Numbers }\end{array}$ & $1-27$ \\
\hline 1.6 & Nuclear Power Plant Classification & $1-28$ \\
\hline 1.7 & Reactor Plant Classification System & $1-29$ \\
\hline 1.8 & $\begin{array}{l}\text { Reactor Auxiliary Cooling and Heating System and } \\
\text { Emergency Shutdown Cooling System Classification }\end{array}$ & $1-33$ \\
\hline 1.9 & Partial List of Common Component Classification & $1-34$ \\
\hline 1.10 & Common Subclassification & $1-37$ \\
\hline 1.11 & Sample Classification of Safety Injection System & $1-38$ \\
\hline 1.12 & $\begin{array}{l}\text { Sample Classification of Neutron Monitor System } \\
\text { Source Range Channel }\end{array}$ & $1-39$ \\
\hline 1.13 & Failure Mode Classification & $1-40$ \\
\hline 1.14 & Failure Cause Classification & $1-41$ \\
\hline 1.15 & Failure Effect Classification & $1-42$ \\
\hline 1.16 & Use Event Classification & $1-42$ \\
\hline 1.17 & Reliability Input Data Flow Chart & $1-43$ \\
\hline 1. 18 & Required Reliability Information & $1-44$ \\
\hline 1.19 & $\begin{array}{l}\text { Typical Operational Testing Summary for Nuclear } \\
\text { Safeguards Systems }\end{array}$ & $1-45$ \\
\hline 1.20 & Comparison of Required Information & $1-46$ \\
\hline 1.21 & $\begin{array}{l}\text { Failure Report and Maintenance Request form with } \\
\text { Required Reliability Information }\end{array}$ & $1-47$ \\
\hline 1.22 & Modified Maintenance Request Form & $1-48$ \\
\hline 1.23 & Vendor's Reliability Input Data Form & $1-49$ \\
\hline 1.24 & Failure Rate Conversion Table & $1-50$ \\
\hline 2. 1 & Example System & $2-63$ \\
\hline 2.2 & Function-Component Summary Sheet & $2-64$ \\
\hline 2.3 & Input Form A & $2-65$ \\
\hline 2.4 & Input Form $B$ & $2-66$ \\
\hline 2. 5 & Input Form C & $2-67$ \\
\hline 2.6 & Input Form D & $2-68$ \\
\hline 2.7 & Input Form $\mathrm{E}$ & $2-69$ \\
\hline 2. 8 & Input Form G & $2-70$ \\
\hline 2.9 & Input Form $\mathrm{H}$ & $2-71$ \\
\hline 2.10 & ARMM Input Deck General Arrangement & $2-72$ \\
\hline
\end{tabular}




\section{TABLE OF CONTENTS (continued)}

FIGURES (continued)

PAGE

2. 11 Series System 2-73

\begin{tabular}{ll} 
2. 12 Parallel System 2-74 \\
\hline 2. 13 & 2-75
\end{tabular}

2. 13 Composite System 2-75

2. 14 Electrical System 2-76

2. 15 Change from Nonredundant to Redundant Components by Partial Data $\quad 2-77$

$\begin{array}{ll}\text { 2. } 16 \text { Hydraulic System } & 2-78\end{array}$

2. 17 Hydraulic System Flow Diagram 2-79

2. 18 Electrohydraulic System Block Diagram 2-80

2. 19 ARMM Input Loadsheets 2-81

2. 20 Completed Computer Output - Problem $1 \quad 2-88$

2. 21 Computer Output Summary

2. $22 P(\lambda T)$ as a Function of $\lambda T$ for Several Values of $A$.

$2-114$

The Model is a Two-Out-of-Three System with

Identical Components and No Repair

2. 23 Schematic of Dresden-3 Emergency A-C Power System

2. 24 Fault Tree for Dresden-3 Emergency A-C Power System

2. 25 Input Data Card Listing for Sample Calculation

(Analog)

2. 26A SAFTE-I Sample Calculation Output

2. 26B SAFTE-I Sample Calculation Output

2.26C SAFTE-I Sample Calculation Output

2. 26D SAFTE-I Sample Calculation Output

2. 26E SAFTE-I Sample Calculation Output

2. 26F SAFTE-I Sample Calculation Output

2. $27 \quad P(T)$ as a Function of Operating Time (Weeks) for the Dresden-3 Emergency A-C Power System

$2-125$

$2-126$

$2-127$

$2-128$

3.1

Schematic Cross-Section of Dresden-3 Containment

$3-78$

3. 2

Functional Diagram - Primary Containment

$3-79$

3.3

Functional Diagram - Drywell Assembly

$3-80$

3.4

Functional Diagram - Pressure Suppression Chamber

$3-81$

3. 5

Dresden-3 Primary Containment Penetrations

$3-82$

Containment Spray Cooling System

$3-83$

3.7

Schematic of Emergency A-C Power Supply for

Core Spray and Containment Cooling

$3-84$

3. 8 Containment Pressure for Various Available Engineered Safeguards

$3-85$

3.9

Example of Failure Mode and Effects Analysis

3-86 


\section{TABLE OF CONTENTS (continued)}

3. 10 Reliability Block Diagram Containment Isolation and Cooling Interval 1

3. 11 Reliability Block Diagram Core Spray - Interval 1 (Redundant Operation)

3. 12 Reliability Block Diagram Containment Cooling Interval 2

3. 13 Reliability Block Diagram Core Spray - Interval 2 (Redundant Operation)

3. 14 Reliability Block Diagram Core Spray - Interval 1 (Both Loops Required)

3. 15 Reliability Block Diagram Core Spray - Interval 2 (Both Loops Required)

3. 16 Automatic Reliability Mathematic Model

Dresden-3 Primary Containment - Case 2

3. 17 Process Flow Diagram - Safety Injection System Connecticut Yankee

3. 18 Schematic Flow Diagram Residual Heat Removal Secondary Water System Connecticut Yankee

3. $19115 \mathrm{KV} / 4160$ Volt Schematic Diagram for Safety Injection System

3. 20480 Volt Schematic Diagram for Safety Injection System

$3-114$

3. 21 Schematic Diagram

$3-115$

3. 22 Loss-of-Coolant Incident 3-Inch Charging Line Break

$3-116$

3.23

Failure Mode and Effect Analyses

$3-117$

3. 24 Reliability Block Diagram Safety Injection System Connecticut Yankee

3. 25 Reliability Block Diagram Emergency Power for Core Deluge System

ARMM Input Loadsheets - Case 1

$3-128$

3. 27

ARMM Input Loadsheets - Case 2

$3-141$

3. 28

Component Contributions to Unreliability

$3-145$

3.29

Function Contributions to Unreliability

$3-154$

3.30

Air Recirculation Fan - Cooling Coil Unit

Connecticut Yankee

$3-157$

3.31A Reliability Block Diagram Connecticut Yankee Air Recirculation Unit

3.31B Reliability Block Diagram Connecticut Yankee Containment Spray System

3.31C Reliability Block Diagram Connecticut Yankee Power Supply and Service Water System Pumps 


\section{TABLE OF CONTENTS (continued)}

FIGURES (continued)

PAGE

3. 31D Containment Cooling System Reliability Block Diagram - Connecticut Yankee

$3-161$

3. 32 Residual Heat Removal System in Containment Spray Mode Connecticut Yankee 3-162

3.33 Connecticut Yankee Containment Pressure Transient 3-163

3. 34A Failure Mode and Effect Analysis Connecticut

Yankee - Containment Spray System

$3-164$

3.34B Failure Mode and Effect Analysis Connecticut

Yankee - Air Recirculation Unit

$3-165$

$3-167$

3. 35 Schematic of San Onofre Safety Injection System

3. 36 Electrical Power to Safety Injection System San Onofre

$3-168$

3.37 Failure Mode and Effect Analysis San Onofre Safety Injection System

$3-169$

3. 38 Reliability Block Diagram Safety Injection System

$3-174$

A. 1 Maintenance Request Flow A-11

A. 2 Maintenance Request Flow A-12

A. 3 Maintenance Request Form A-13

A. 4 Maintenance Memorandum A-14

A. 5 Maintenance Memorandum Flow for Electrical and Mechanical Work

A. 6 Test Bureau Maintenance Memorandum Flow A-16

A. 7 Work Request (Form 23) Flow A-17

A. 8 Equipment Maintenance Record Form A-18

A. 9 Motor Maintenance Record Form A-19

A. 10 Maintenance Record Forms A-20

B. 1 Sample Safety Report B-44

B. 2 Sample Failure, Shutdown, and Availability Report B-45

B. 3 Sample Shipboard Data Collection Form B-47

B. 4 Sample Shipboard Data Collection Form B-48

B. 5 MARAD Maintenance and Reliability Program B-49

B. 6 Analysis of Component Forced Outages for the

Year 1964 Conventional Drum Type Units Only B-50

B. 7 Report Summary Sheet B-51

B. 8 FARADA Information Flow Chart B-52

B. 9 Sample of Completed "Background Information on

Failure Rate Data" Form B-53

B. 10 Sample of Completed "Tabular Failure Rate Data

Summary" Form

B -55 


\section{TABLE OF CONTENTS (continued)}

B. 11 Sample of Completed "Failure Mode Distributions" Form

B. 12 Typical Page of FARADA Computer Print-Out from Volume $1 \mathrm{~A}$

B. 13 Typical Page of FARADA Computer Print-Out from Volume $1 \mathrm{~B}$

B -58

B. 14 Typical Page of FARADA Failure Mode Distributions

B- 59

B. 15 Partial List of Major Classifications of the FARADA Part/Component Generic Code

B -60

B. 16 FARADA Common Subclassification

B -61

B. 17 Example of FARADA Part/Component Classification Code

B -62

B. 18 Sample Deficiency Report

B -63

B. 19 Deficiency Report Flow Chart

B -64

C. 1 Simple Pumping Station

C -6

C. 2 Operational History of a Simple Pumping System

C -7

C. 3 Input Loadsheet

C -8

C. 4

Type 2 Output Data Sheet Past Summary of Failure Rate Data

C -9

C. 5 Type 1 Output Data Sheet Current Listing of Component Failures

C -10

C. 6 Type 2 Output Data Sheet Current Summary of Failure Rate Data

C -11

H. 1 Lifetime Estimate for Standby Components at 75 Percent Confidence Level

$\mathrm{H}-10$

H. 2 Lifetime Estimate for Operating Components at 75 Percent Confidence Level 
ABSTRACT

Data and analytical requirements for a reliability monitoring program in nuclear safety have been identified. A data management system is defined incorporating a method for equipment and event classification and a plan for data collection. Equipment classification is accomplished by use of a 9-digit generic code which identifies equipment type, environment, and operating load. The event classification provides coded identification of failure mode, effect, and cause, as well as an index of accumulated experience.

The data collection plan uses a two-step recording process to enable use of existing plant practices and operating staffs. The first step entails in-plant recording of failure or repair data and operating and test data. The second step requires transferral of operating and failure data to coded input for a centralized data bank. Subsequently, data bank processing will convert and report accumulating experience as failure rate and repair data.

The accuracy and flexibility of the Automatic Reliability Mathematical Model (ARMM) technique has been increased for evaluation of the reliability of engineered safety systems. A computer program, Systems Analysis by Fault Tree Evaluation (SAFTE-1), has been developed to implement the fault tree concept.

Applications of both techniques to sample problems using estimated or available reliability data demonstrate that they provide useful reliability estimates. Results of these applications show that the quality of these estimates is determined more by the analyst's skill than the limitations of the techniques. It is revealed that the questioning and documentation process required in preparation for quantitative reliability estimates is of value in identifying potential trouble spots and suggesting procedural or design changes which can eliminate or reduce the adverse effects of component failures on safety.

It is concluded that reliability analysis can contribute to a more quantitative, systems-oriented measure of safety; techniques adequate for safety analysis of nuclear power plants now exist; the data necessary 
to support these analyses can be obtained through the suggested data management scheme; and the resulting reliability monitoring program can be executed without need for expanding nuclear power plant operating staffs. It is recommended that the reliability monitoring program be instituted in operating nuclear power plants at the earliest possible date to enable the accumulation of data of quality appropriate to reliability analysis of engineered safety systems. 


\section{INTRODUCTION}

In an earlier study ${ }^{(1)}$ it was noted that the usefulness of reactor operating experience could be greatly increased if this data were collected in a form suitable for reliability analysis. An investigation has been made of data and analysis requirements for a reliability monitoring program in power reactor safety.

One objective has been the definition of a system for the collection and analysis of operating, maintenance, inspection, and testing data on components of engineered safety systems $(2)$. This data management system will provide currently unavailable input data for assessing engineered safety system reliability. A second objective has been the investigation of techniques for the evaluation of reliability and the application of these techniques to engineered safety systems typical of water-cooled and-moderated power reactors. The applications provide information about the form and type of data which would be required in an operating reliability monitoring program. They also serve to illustrate the expected results.

In this report, the material has been arranged in the order of consideration expected in the reliability monitoring program. That is, the requirements for a reliability data management system are outlined in Chapter 1. Methods for using data to assess reliability are discussed in Chapter 2. Finally, Chapter 3 illustrates steps required for the application of anticipated data to reliability models of selected engineered safety systems in recently designed water-cooled and-moderated reactors. While specific reliability analysis methods and engineered safety systems have been cited in this study, they are not intended to exclude other methods or systems which can be used in a given reliability analysis. Rather, these examples are meant to illustrate the requirements for establishing a program for evaluating and monitoring reliability in nuclear safety. A glossary is provided in Appendix $G$ to indicate the meaning of selected terms used in this report.

(1) Garrick, B. J., W. C. Gekler, and H. P. Pomrehn, "An Analysis of Nuclear Power Plant Operating and Safety Experience, "HN-185, U. S. Atomic Energy Commission, December 15, 1966.

(2) Engineered safety systems is now the preferred terminology for systems previously referred to as engineered safeguard systems; i. e., systems provided to prevent or mitigate various possible reactor accidents. 


\section{SUMMARY AND CONCLUSIONS}

Reliability analysis can contribute to a more quantitative systemsoriented measure of safety. The techniques for analysis exist, having been tailored to meet the needs peculiar to safety analysis of nuclear power plants. The data necessary to support these analyses can be obtained through implementation of the data management scheme detailed in this report. The resulting reliability monitoring program can be executed without the need for expanding nuclear power plant operating staffs.

As noted in the Preface, a reliability monitoring program can benefit nuclear safety and the nuclear industry in many ways. Realization of these benefits is dependent on the effectiveness of the reliability monitoring program. Program effectiveness is, in turn, contingent on three factors: (1) the availability of suitable data; (2) analytical techniques which can describe system behavior; and (3) proper application of these data and techniques.

The requirements and techniques associated with proper treatment of each of these factors have been investigated and the results of this work are presented in this report in the form of a data management system, the review and development of two analytical techniques suitable to reliability analysis, and step-wise examples of application of these techniques and anticipated data to engineered safety systems.

\section{DATA MANAGEMENT}

In Chapter 1, a data management system has been defined for collection and evaluation of reliability data from operating plants and equipment manufacturers or vendors. This system consists of a method of classification and a plan for data collection which were derived from consideration of generally available data management systems in both nuclear and nonnuclear industry. The data classification concepts are based primarily on nonnuclear-oriented reliability programs while the data collection plan is strongly slanted to conformance with recording and reporting practices now used in nuclear power plants. The data classification system provides access to reliability data at the plant, system, or component level. It allows selective grouping for statistical searches or investigations of failure rate data as well as standardized format for reporting reliability data by system and generic component. Among 
data requirements considered in the classification system are the mode, cause, and effect of failure and the repair time associated with each failure. To convert specific occurrences of failure into failure rate information, a use event classification is described which allows correlation of failure to accumulated use or age.

With respect to data collection, it has been found that current practices in operating power reactors should lend themselves readily to orderly collection and processing of reliability data. Furthermore, the effort associated with collecting this data should result in little or no increase in operating staff workload. Much of the required data is already called for in existing practices. Some modification of existing records and recording procedures may be required but the basic ingredient for success will be the establishment and adherence to good record keeping practices, many of which already exist in these facilities. Encouragement of such practices can be enhanced by illustrating the potential value of reliability data to those involved in its collection and by procedures which assure that resulting data will be handled in a manner that objectively and fairly serves the interest of the designer, manufacturer, operator, and the public. An information flow scheme which would assure proper use and dissemination of data from the reliability monitoring program is shown in Figure 1.

As proposed, the data collection plan entails a two-step recording practice with subsequent processing by a data reduction agency. The first step involves completion of failure or maintenance report forms only slightly different from those currently in use by the plant personnel normally reporting and recording maintenance. The second step involves transferral of the failure information on these forms as well as use data from operating and outage summary reports to reliability data loadsheets. This transferral can be accomplished by in-plant personnel after a brief training period or by a representative of the data collection agency during periodic plant visits. Subsequently, the loadsheet data would be reduced and reported as failure rate and repair data summary information. Such information would be directly applicable to reliability analyses of engineered safety systems. It also would be of some use to any nonsafety pursuit of improved plant or system availability.

\section{ANALYTICAL TECHNIQUES}

A number of mathematical techniques or machine codes have been investigated which can be used in evaluating engineered safety system 
reliability. Two promising techniques utilized in aerospace applications have been examined in detail. They are the Automatic Reliability Mathematical Model (ARMM) and fault tree analysis. These techniques have been tailored to permit application to engineered safety systems.

The ARMM program possesses many attributes desirable in evaluating engineered safety system reliability. Among its capabilities are the simulation of redundancies, interdependence and mutually exclusive failure modes, and the operation of standby equipment. Results of an ARMM analysis include: (1) the probability of successful system operation; (2) each component's contribution to the probability of system failure; and (3) the failed component combinations which are major contributors to system unreliability. In the course of understanding and using $A R M M$, modifications have been made in program subroutines to better suit it to engineered safety system applications. These modifications are described in Chapter 2 along with identification of several other changes which, if made, would further improve the program. Perhaps the most important result of working with ARMM is recognition that its effectiveness is limited by the user's skill, especially in the initial applications.

Fault tree analysis is particularly appealing because it parallels reactor safety analysis practices. Fault tree analysis generates a logical statement of events which, in combination or separately, can lead to an undesired event, i. e., nuclear incident. The approach is to state the undesired event in real, measurable terms and subsequently identify the event sequences necessary and sufficient to cause the undesired event. The result is a gated logic (AND and OR gates) which identifies human errors, equipment malfunctions, and operating conditions necessary to the occurrence of an event. To enable use of fault tree analys is in nuclear safety, a computer program has been developed and written having the code name SAFTE-1 (Systems Analys is by Fault Tree Evaluation). SAFTE-1 is a computerized probabilistic model of the logic which allows simulation of system operation through a large number of trials ix. a short period of time to identify the likelihood of the undesired event and the relative contributions of various initiating component failures to this event. Application of SAFTE-1 to an engineered safety system has shown that the program yields reliability estimates. However, continued research is required to realize the full and highly desirable benefits of the program.

Regardless of format, any nathematical method for making quantitative reliability estimates is a simulation of system behavior. As the simulation 
becomes more realistic, its input requirements increase. The two methods previously referred to are believed to be near the upper bounds of practical simulation for reliability evaluation of engineered safety systems. They can allow changes in operating mode and configuration; they can accommodate repair and testing practices; they can recognize dependencies and redundancies; and they can incorporate human error either as a combined input with component failure rate or as a separate input.

\section{$\underline{\text { APPLICATION }}$}

In reliability analysis the methods for operating, maintaining, and testing a system are described to lay a basis for careful, step-by-step evaluation of the cause and effect of each failure mode of each critical component. These failure mode and effect analyses, sometimes called failure analyses, identify critical components in terms of failure effect and provide formalized documentation of actions which can be taken to eliminate the cause of failure or reduce the effect of failure. The components identified in a failure analysis are then introduced into reliability models or logical models of system operation. When properly developed, these models graphically display the relationship between components including such concepts as interdependence and redundancy.

As pointed out in Chapters 2 and 3, the primary factor in the usefulness and credibility of a reliability analysis for an engineered safety system, or for that matter, for any system is the analyst's skill and experience. Similar conditions hold for other engineering analyses in nuclear systems. Without proper background the analyst is confronted with what appears to be a rather complex bookkeeping task. With adequate background he is able to identify the equipment and operating patterns which most adversely affect system reliability and then concentrate his efforts in these areas.

The steps involved in applying data and mathematical models to a reliability analysis have been illustrated in Chapter 3 by example applications to four engineered safety systems from pressurized and boiling water reactors. These steps include:

1. System description.

2. Operating and testing procedure review.

3. Failure analysis. 
4. Reliability model development.

5. Reliability data selection.

6. Reliability analysis.

7. Evaluation of results.

In developing these applications, it has been found that the questioning and documentation required in preparation for quantitative reliability estimates are as valuable as the estimates themselves. These preparatory functions identify many of the trouble spots which are subsequently confirmed by reliability estimates. They also direct attention to procedural or design changes which can eliminate or reduce the adverse effects on safety of component failures.

The investigative processes involved in preparing for a reliability analysis also occur in the course of discussions between operator and designer, and between regulator and operator. Such discussions result from a need to make reliability judgment. It is believed that when properly applied, reliability analyses can readily provide orderly documented answers and permit a more detailed evaluation of systems important to reactor safety.

The example applications presented in Chapter 3 also illustrate the quantitative results of a reliability analysis and indicate ways in which these results may be used to benefit safety.

Specific examples of these results include:

1. Components or design features to which reliability improvement efforts may be most effectively applied.

2. Components contributing to system failure at a given probability level.

3. The effect of adding or removing redundancy on the reliability of the system.

4. Variations in system reliability which result from altered testing frequencies or procedures.

5. The sensitivity of system reliability to errors in component failure rate and repair data.

In conclusion, the reliability monitoring program described in this study provides a means for generating much needed statistical reliability data 
as well as guidelines for application of the data to quantitative reliability estimates. When initiated, it is believed that the program will prove useful in every step of the activity associated with design, manufacture, evaluation, and operation of engineered safety systems. Among the specific benefits are reliability data for use in evaluating new engineered safety system designs; a more quantitative basis for comparison of new and existing designs; evaluation of the significance of preoperational and operational testing practices; and a feedback mechanism for improvement of equipment and procedures in new and existing systems by identification of conditions adversely affecting reliability.

\section{RECOMMENDATIONS}

Analytical techniques and mathematical models necessary to perform a reliability analysis of typical commercial power reactor safety systems are now available. This study has also developed a data management system designed and tailored to collect and reduce experience data to reliability data for components of power reactor protective systems. What remains to be done is implementation of an active reliability monitoring program.

In view of the fact that approximately 17 water-cooled and-moderated power reactors with output greater than 50 Mwe are expected to be in operation by December 1969 and an additional 24 with outputs greater than 400 Mwe by December 1972, it is recommended that steps be taken now to initiate operation of the reliability monitoring program in operating power reactors.

From the standpoint of urgency, the most important aspect of the program is the collection and reduction of experience into useful reliability data. There is some demand for this data now and it can be expected to increase rapidly. Furthermore, there is strong justification to initiate data collection now in order to minimize the loss of currently accumulating experience. It is exceedingly difficult and expensive to attempt recovery of this experience in a reliability data sense using existing documentation practices.

While data collection is the most urgent aspect of the reliability monitoring program, there is also a need to verify the integrated concept of the program in actual practice. That is, a pilot test should be made to demonstrate the collection and reduction of data and the application of this data to the reliability analysis of well defined operating systems. 
Finally, this pilot test could provide a means of experimenting with other activities concerned with data collection problems. $(1,2)$

(1) Computer Handling of Reactor Data for Safety, CHORDS, under development by the Nuclear Safety Information Center.

(2) Griffin, C. W., Outline Plan for Collection, Evaluation and Dissemination of LMFBR Facilities Incident Report Information, Liquid Metal Engineering Center, March 15, 1967. 


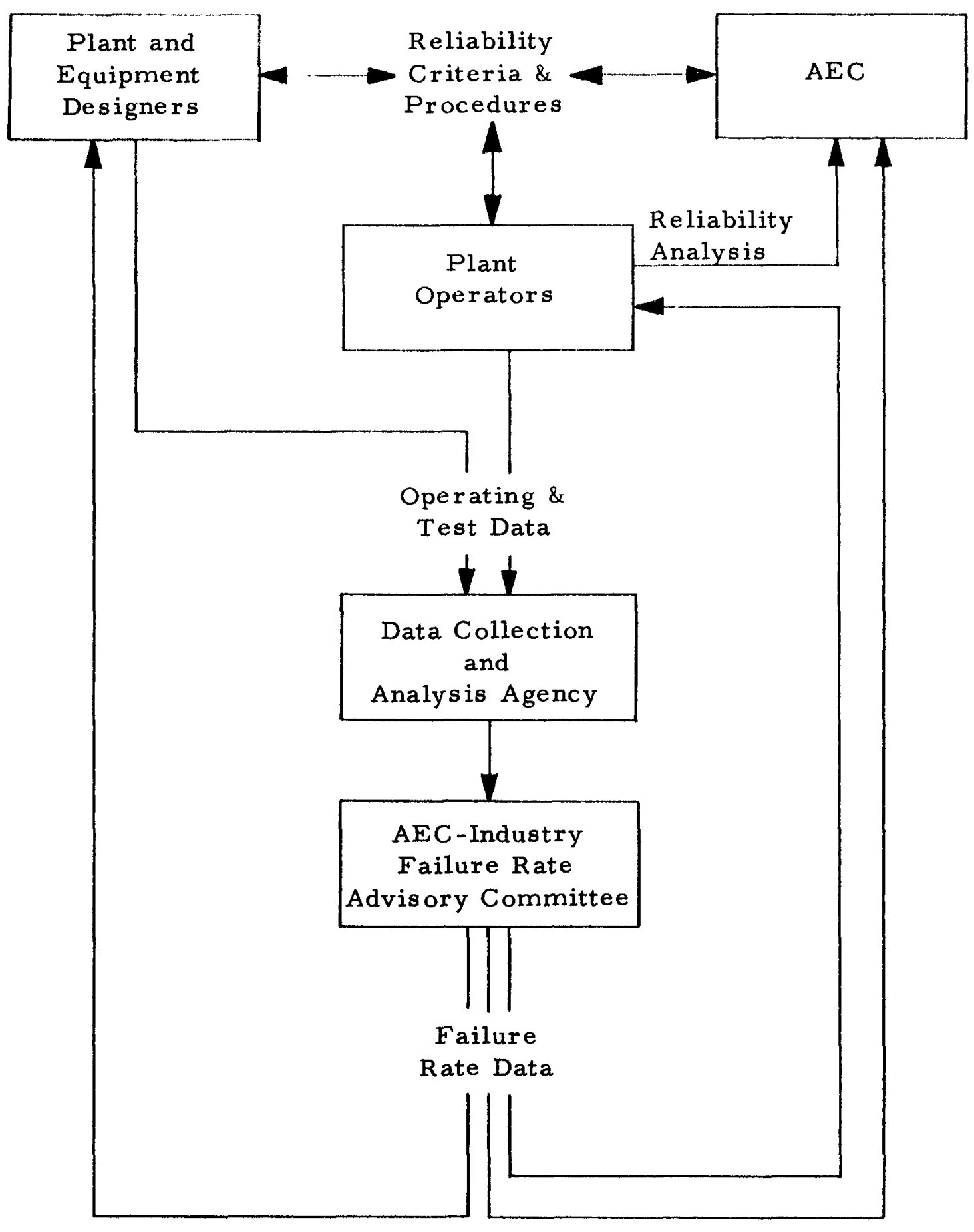

FIGURE 1

RELIABILITY MONITORING PROGRAM INFORMATION FLOW CHART 


\section{CHAPTER 1}

RELIABILITY DATA MANAGEMENT SYSTEM 


\section{CHAPTER 1}

\section{RELIABILITY DATA MANAGEMENT SYSTEM}

\section{INTRODUCTION}

A primary objective of this study has been the identification of a data management system for the collection and analysis of operating, maintenance, inspection, and testing data on the components of power reactor protective systems which will provide the necessary input for assessing protective system reliabilities. To be successful, a reliability data management system for reactor safety must be practical and flexible. The program must also recognize a variety of data sources, the most important of these sources being operating reactors. Within these general constraints, a data management system has been defined. The system concentrates primarily on the collection of useful reliability data from operating facilities. It also recognizes the very important source of reliability data represented by test programs in manufacturer's facilities and national laboratories. The approach used is based on the fact that significant amounts of information necessary to development of reliability data, particularly failure rate data, is being generated in a gross form via other administrative activities requiring data collection. What is now required is a method of selectively culling this existing information to arrive at a useful set of reliability data. This requirement can be accomplished by setting forth procedures and formats for collecting and classifying.reliability data from available data. Listed in Figure 1. 1 are the system objectives and the type of required information with which these objectives can be fulfilled.

To arrive at a selected data management system, a number of similar systems either proposed or in operation have been reviewed. These sys tems represent both nuclear and nonnuclear applications. Most of them are scoped to develop failure rates on the basis (1) that the collected failure data derive from chance failures and exclude failures due to wearout, or (2) that all failures are chance failures. The system outlined in this report has been developed to provide a systematic means of collecting component failure data and to permit the analys is of failure modes, and the identification of failure distribution functions associated with the various failure modes, wherever possible.

Reliability data management can be broadly divided into (1) data collection efforts which encompass collection, reduction and evaluation of in-plant or field data and manufacturer's or test data; and (2) classification of 
the collected data by equipment and event types in order to facilitate evaluation and correlation of data. Both activities must be predicated on the end use of the data. A breakdown of functions required in data collection and classification is illustrated in Figure 1.2. Since data classification identifies many of the format requirements for data collection, it will be discussed first.

\section{DATA :LASSIEICATION}

\section{Present Practices}

In considering the requirements for data classification, seven classification systems have been reviewed, along with current practices in several commercial nuclear power plants operating in the United States. Current practices in several U.S. power plants are summarized in Appendix A. Since there currently is no formalized reliability program in U.S. nuclear power plants, the data classification activities in these facilities is largely dictated by other management and maintenance goals, e. g. , cost control, spare parts inventory, and general availability measurement. Of the seven classification systems reviewed, three were related to nuclear plants here and abroad. The remainder were nonnuclear in orientation. These classification systems which are summarized in Appendix $B$ are:

1. United Kingdom Atomic Energy Authority (UKAEA), Reactor Fault Reporting System.

2. Computer Program for Military Nuclear Power Plant (PM-1) Data.

3. Uniform Subject Index for Nuclear Power Demonstration (NPD) Project.

4. Maritime Administration (MARAD) Classification of Merchant Ship Systems.

5. Edison Electric Institute (EEI) Outage Classification Code.

6. Interservice Data Exchange Program (IDEP), Guided Missile Data Exchange Program (GMDEP), and Failure Rate Data Program (FARADA).

7. AEC Uniform System of Accounts. 
While the majority of these systems deal with the subject of failure rate data, many of them also included other missions, as shown in Figure 1.3, which makes it difficult to compare directly all parameters between different classification systems. For example, the PM-l systems classification is understandably simple because of the simplicity of the installation and the single unit application. Simplicity of the Canadian (NPD) classification also derives to some extent from its single installation application. The UKAEA classification is relatively more.complicated since it includes not only light-water reactors but also heavy-water, gas-cooled, and liquid-metal reactors. In any case, it is obvious that there is more than one good classification system.

To demonstrate the relative complexity of these classification systems, the number of levels, groups, and digits used, and some typical examples of combined plant/system/component classification are shown in Figures 1.4 and 1.5 respectively. Disadvantages of these classifications are that (1) systems classifications are hardware rather than function-oriented; (2) component classifications are not in generic codes and thus by component classifications alone, similar components cannot be placed into common groups; and (3) multiple components of the identical kind cannot be distinguished.

The NPD and MARAD classifications provide functional system breakdowns, the FARADA classification provides component generic codes, and the PM-1 classification provides multiple component identification numbering. All of these are considered desirable qualities for a good data classification system.

A classification system which deserves special attention is the AEC Uniform System of Accounts which was developed primarily for cost accounting and estimating purposes. The functional breakdown provided in this system, particularly for the reactor plant, is rather appealing. It should be noted that the NPD classification system which is used to collect reliability data as well as to assist scheduling and accounting activities, was developed from the same source as the AEC system, namely the Federal Power Commission (FPC) Uniform System of Accounts.

Another important task in selecting a classification system is determining the number and detail of classification categories for describing failure events. These categories must be practical for use of field data and at the same time permit statistical examination. The tendency is to construct an extensive and detailed event classification system and identify field data collection procedures and forms which permit use of 
the classification system. The UKAEA and PM- 1 fault classifications with 200 and 65 fault categories, respectively, show the extent of detail which may be required. Such an approach can prove difficult to implement without adding extra burden to the workload of operating personnel. A checklist form such as that used in the MARAD program illustrates the type of field data requirement which may result. While more detailed failure event classification codes yield better failure data, it is considered desirable to match these codes as closely as possible to the capability of existing in-plant data recording and reporting practices.

The data classification system recommended in this study is effectively a composite of concepts derived from the systems summarized in Appendix B. The recommended system has been scoped for use in reliability data generation on engineered safety system components. It also reflects consideration of broader potential application to all nuclear systems and the possibility of eventual incorporation into data management systems concerned with all components in nuclear power plants. The immediate concern, however, has been reliability data for engineered safety systems. While the classification system presented here may be adaptable to broader use, it must be recognized that there are others which may be equally applicable. The point is there is a definite need for early standardization of nuclear power plant data management systems to avoid unnecessary duplication of data management activities while achieving useful data.

Recommended Plan

Data classification is divided into (1) systems classification which establishes the hierarchy of hardware or equipment in nuclear plants, and (2) event classification which identifies the how and why of failure as well as other events.

Systems Classification - It is desirable that the classification code be adaptable to all plant systems and at the same time be suitable for acceptance of vendor data. Such an approach is to identify individual components by the system to which they belong and by the function which they perform. The systems classification code outlined in this section is considered to satisfactorily accomplish the preceding goals. While complete, detailed development of the classification code can be performed by using the classifications described in Appendix $B$ as guides, it is believed that such an effort should be preceded by a coordinated evaluation by all potential users at the outline stage in order that the detailed classifications are of maximum utility.

As presented, the systems classification permits identification of data from a specific component in a system for a particular plant; it allows 
collecting data on a specific system in more than one plant; and it permits grouping of data on generically similar components in a single plant or all plants. The method of classification as discussed in the following sections includes plant identification, functional system identification, and in-plant and generic component identification.

Plant Identification - Identification of a nuclear power plant should be unique. Thus, classification of a particular plant has been made by use of a portion of the docket number associated with its utilization facility license. Serialized portions of docket numbers are preceded by "0" for those licensed under Part 50* and by " 5 " for those under Part 115*. Thus, a reactor with Docket No. 50-206 can be identified by 0206, while that with Docket No. 115-4 can be identified by 5004. Note that in order to match the column allocation, 0 's are used when the serialized portion of the docket number is less than three digits.

Functional System Identification - After reviewing the nuclear and nonnuclear equipment classification systems summarized in Appendix $B$, it is concluded that an adaptation of the approach used in the AEC Classification of Construction Accounts will give the best basis for formalizing functional systems for reliability purposes. This classification system is derived from the Federal Power Commission's Uniform System of Accounts, which applies to government records on all types of power generation and $t r a n s m i s s i o n$ facilities.

Another reason for using the AEC system as a basis for classification is that it already covers both pressurized and boiling water reactors and, in addition, provides for coverage of organic moderated, sodium graphite, and fast breeder reactors.

In adapting the $A E C$ code to the reliability monitoring program, the first digit of the AEC code (the number 2) has been dropped, since this digit indicates a nuclear power plant which is the only type of facility presently under consideration. The resulting functional classification system uses a numerical designation, generally consisting of five digits. A first-level breakdown of the system is shown in Figure 1.6. The first digit is a functional classification within the power plant. As can be seen, most of the equipment classes of interest will fall in the " 2 " or reactor plant group.

The first level is in turn provided with up to 9 major system classes by a second digit, also shown in Figure 1.6. The third, fourth, and fifth digits provide further subsystem breakdowns, as required. Figure 1.7 gives the numerical designations presently identified at the third and fourth levels. The primary difference between this breakdown and that contained in the AEC system is inclusion of the reactor containment (system class 21.9) as part of the reactor plant rather than under structures and improvements. This move is considered desirable because of the close

*Code of Federal Regulations, Title 10 
relationship between containment operability and reactor safety. An example of a further breakdown to the fifth digit is shown in Figure 1. 8 . Of course, some systems do not require four or five digit breakdowns.

Component Identification - Classification of a component has been made (1) by its plant identification number which is familiar to all first-line operating personnel and also distinguishes it among multiple components in any given system; and (2) by its generic code which allows grouping of data from similar components and comparison of vendor data to in-plant data for similar components.

1. Plant Identification - The plant identification number for a component can be most expeditiously established on a plantby-plant basis by adapting, for example, equipment numbers shown on the system diagrams, those appearing on the equipment history cards, or any other administrative numbering system that is used throughout the plant. After adopting the appropriate scheme, component identification numbers can be cross-indexed with the generic code for further utilization.

2. Generic Classification - A three-digit index number followed by three two-digit subclass groups has been used to provide generic component identification. The three-digit number is assigned from one of two groups of numbers, 001 to 199 or 200 to 999, depending upon the class of component involved. $(000$ is used if there is no heading necessary at the component level.) The first group of numbers (001 to 199) is for components which are unique to a given plant or reactor design, and for which a generic code has not been established. These unique components are assigned consecutive numbers within each facility from the 001 to 199 group and are associated with the systems classification for identification, until such time as a new generic code is established.

The second group of numbers (200 to 999) are for components which are common to many facilities and for which failure data can be accumulated from both in-plant and equipment vendor programs. For these components the numbers are preassigned. The suggested generic code is shown in partial development in Figure 1. 9.

Following the three-digit component index number are several subclass index groups of two-digit numbers which identify service, material, size, type, function, operation, etc. Several subclasses, e. g., service, material, and size, are 
applicable to a number of components. In these cases, common subclassifications are used as detailed in Figure 1.10. In all other cases, appropriate subclasses are created to suit the particular component. $(00$ is used if the re is no heading at any subclass level.)

While the preceding generic classification is similar in format to that used in the FARADA system, the specific code for components is different. This is considered desirable to assure that users apply generic data derived from equipment of the type found in nuclear power plants. Some FARADA data is applicable to nuclear systems but the user should be aware of the FARADA subclassifications and avoid using inapplicable failure rates. Other reasons for avoiding blanket use of the FARADA classification include (a) many FARADA components are not used at all in reactors (88 of 463 components in an available list have no counterpart in reactor systems), thus many component classification numbers (first three digits) are used for inapplicable data; and (b) some subclassifications (two digit numbers) pertain to variable ranges not encountered in reactor systems, e.g., motors over $1 \mathrm{hp}$ are lumped into a single subclass and operating media include exotic materials such as rocket fuels.

Complete system-component classifications of two representative systems in a typical PWR-type nuclear power plant are shown in Figures 1.11 and 1. 12. In-plant component identification numbers were taken from system diagrams.

Event Classification - The primary need in preparing reliability data is to relate failuces to accumulated use under specific operating conditions. Both failures and use are considered to be events in this classification system. In this context, failure events identify the how and why of failure; and use events provide a basis for correlating failures to accumulated use.

Failure Events - To evaluate failure data and allow proper use of this data for reliability analyses, it is necessary to classify individual failure events by (1) functional manifestation of the failure, i.e., failure mode; (2) physical manifestation or failure cause; and (3) effect of failure on system performance. The first two items are important because if we know how and why a failure occurred, we can learn to prevent it. The third item, failure effect, allows the most efficient use of failure analysis efforts to eliminate impor tance failure causes and/or failure modes.

1. Failure Modes - (The way in which a component fails) - To generate a listing of all types of functional failures for every possible component can lead to an almost endless list of 
failure modes. However, a very useful listing can be formulated by considering failure modes to be characterized as: (1) failure to open; (2) failure to close; (3) failure to start; (4) failure to stop; (5) failure to continue operation; (6) spurious failure, i. e., premature operation of a component when not called for;

(7) degradation; (8) erratic operations; (9) scheduled service; and (10) scheduled replacement.

The se functional failures are classified as shown in Figure 1.13. Applying a reasonable interpretation it can be shown that any conceivable failure will involve one of these functional manifestations. Being functional phenomena, the se failure modes are potentially detectable when they occur. Also, they have physical precursors (causes) which can be identified; hence, component failures become always theoretically predictable and preventable. However, they are not predictable until the cause is present.

2. Failure Causes - (What made the component fail the way it did) A generalized classification of failure causes is difficult, but not because of the outward appearance of many causes; rather, it results from the fact that, if pursued far enough, the root cause of every failure involves human action or inaction. Tentatively, however, it is believed that cause classifications should be oriented to stages in a component's life--a use-oriented classification. This method of classification is considered useoriented because in analysis of cause-effect relationships the desire is to eliminate or counteract those causes giving rise to undesirable effects. This goal can best be attained by identifying that part of a component's life wherein the causes of failure most frequently are generated. Put another way, it is desirable to know if the cause resulted from weak or improper design, poor fabrication, or improper use in operation. On the basis of the preceding discussion, three major causal categories are defined. They are design, fabrication, and operation. Detailed definition of the se different categories is shown in Figure 1.14.

Design causes reflect those failure causes resulting from improper or incomplete recognition of actual use conditions and environments; e.g., no anticipation of high humidity or incomplete heat transfer analysis. These causes may also result from lack of or misdirected developmental testing which, in turn, allows fabrication of weak components.

Fabrication causes are broken down into abnormalities in compcnent configuration and abnormalities in material composition. Configuration abnormalities include the commonly 
referred to failure causes resulting from poor assembly practices. Design and fabrication causes are more likely to be found in vendor's data.

Operation causes, with few exceptions, are generally unpredictable, since the causes are external to the component in question and lie in the future unless the misuse is chronic and has already resulted in wear or some othe $r$ detectable degradation. Operationcauses probably should dominate most of the in-plant failures.

3. Failure Effects - (What happened to system when component failed the way it did) - Even when performance levels are fairly well established, evaluation of failure effects will require some good engineering judgment. For ease of judgment, failure effects have been classified with respect to their importance to system performance. They are specifically divided into (1) no loss of performance, (2) partial loss of performance, and (3) total loss of performance. Use of many more levels of judgment has been shown to lead to inconsistency in repeated evaluation of marginal cases. The effect classification code is shown in Figure 1.15.

The effect of failure events on reactor performance is indicated by the use classification code which encompasses both failure and nonfailure or use data.

Use Events - The recommended classification code for use events is shown in Figure 1.16. This code provides a basis for characterizing or establishing the accumulated use on pieces of equipment. This objective is achieved by using changes in plant operating status and the occurrence of system tests outside the normal schedule as indices. The desire here is to relate each bit of reliability data to the plant status during the time in which the data was developed. Thus, a failure event will include a use classification which defines the plant status during the time that the data was accumulated. As indicated in Figure 1.16, the use codes are defined in such a way that they can be readily identified by operating or reviewing personnel.

\section{DATA COLLECTION}

Present practices in operating nuclear power plants include collection of (1) use data as a part of periodic management reports, (2) failure and repair data as an adjunct to performing maintenance operations, and (3) additional experience data in conjunction with special reports and studies. After reviewing these existing data sources, a recommended plan for data collection has been formulated using the previously defined classification system. 
However, before outlining the recommended data collection practices, it is desirable to briefly review existing practices in nuclear power plants in the United States and in other nonnuclear and foreign nuclear activities.

Present Practices

U. S. Nuclear Power Plants - As indicated in Appendix A, it is customary for commercial nuclear power plants in the U. S. to prepare periodic management reports in which plant operations and outages are summarized. In most cases, these reports provide adequate use data but little reliability information. These reports are derived from more basic records accumu.. lated in the power plants for purposes of guiding maintenance and providing a historical record of operations. The basic records include documents such as the reactor log, control room log, supervisor's log, and shift foreman's log. None of the se records possesses a format which includes consideration of reliability data generation, although they provide much of the basic input for reliability data.

Probably the single greatest source of failure data in commercial power plants is the documentation generated to direct and control maintenance. The se records may be called maintenance memorandums, maintenance requests, work item cards, or deficiency reports. Several examples of the formats for existing records are given in Appendix A along with descriptions of the records and their respective routing procedures. Here again the formats in use are not completely compatible to reliability data development; however, it is believed that a collection mechanism for failure data useful in reliability analysis can be developed from existing maintenance records with very little added effort.

Other Facilities - In the reliability data management programs that have been reviewed, data recording has been accomplished by either the checklist or narrative approach. In the checklist approach, plant personnel make an initial record of all data by filling blanks with quantitative data and checking boxes opposite applicable statements. Narrative statements are necessary only to describe unusual events not evaluable within the scope of available data requirements. The data on these forms are transferred directly to punch cards or other reliability evaluation forms which are used to derive summary data such as failure rates, repair time, repair costs, and availability. This approach has the advantage of eliminating or reducing to a minimum data transferral activities between data collection groups (operating and maintenance staffs) and data analysis and evaluation groups. On the other hand, it requires additional effort and knowledge on the part of the initial recorder to fill out a fairly elaborate form. An example of such a form, recommended for use by the Maritime Administration, is shown in Appendix B, Figure B.3. Of course, this form was developed to provide more data than required 
strictly for reliability information. Another possible drawback to checklist forms is the rather formidable appearance they present to personnel whose primary mission is to maintain and operate a plant in a safe and efficient manner.

In the narrative approach, a memorandum type work request form requiring a minimal amount of specific data is used as the primary source of failure and repair data. The form is reasonably simple and does not require familiarity with a set of terminology peculiar to subsequent uses of the raw data. The major disadvantage of this approach is that a reliability engineer or a person familiar with nuclear power plant systems and data analysis must translate the raw information into reliability data and transfer the data to records or input forms suitable for reliability evaluation. Such an approach is currently in use by the Ontario Hydroelectric Power Commission (HEPC) at its Nuclear Power Demonstration (NPD) Reactor. The basic record used at the NPD reactor is a deficiency report (DR) form. A sample of this form is shown in Appendix $B$, Figure B. 18. The primary use of the DR form is to initiate and control maintenance. Its contents and method of handling are also scoped to assure that periodic review by a reliability engineer (from the HEPC central offices) can quickly identify all failures and repairs associated with each component in the systems of interest. The DR review system is completely manual. Experience with this system is believed to show that virtually all of the reportable component failures are identified. It also has been reported that the reliability engineer requires one to two weeks per system per year in reviewing and reporting on the accumulated DR's. The use of a reliability engineer review is considered necessary to properly evaluate the significance of the data.

Typical narrative type work request forms, used in the U. S. power reactors, are shown in Appendix A, Figures A.3, A. 4, and A. 10. Comparing these examples with the Canadian deficiency report form suggests that current work requests might be used as failure report forms with little modification. Success of such an approach in the accumulation of failure data is contingent upon strong interest in implementation by plant supervisory personnel (as would be true of any recording system), and the provision of a person knowledgeable in reliability data require ments to translate work request data into input for data collection and evaluation.

\section{Recommended Plan}

Based on the preceding discussions, a narrative type approach to data collection is recommended. It is believed that this approach will provide adequate information and at the same time be most compatible to use in 
existing activities such as maintenance, control, and documentation. A plan whereby necessary data for reliability analysis can be collected is shown schematically in Figure 1.17. Because of the different degree of data reduction performed in operating plants and in manufacturing test facilities, entirely separate approaches will be made in collecting reliability data from each of these sources.

In-Plant Data - There are two sources of in-plant data that are of primary importance for reliability analysis. One is that generated by the operating group. It includes records of all use data for tests and normal operations. The second source is that resulting from failure events observed by the operating group and subsequently corrected by the maintenance group. These failure events are generally recorded on failure report and maintenance request ( $F R / M R$ ) forms. In essence, use data are mainly concerned with length in time, while failure events are related to point in time. The recommended plan for collection and correlation of these data follows.

1. Failure Data - When a failure has been reported, some type of preliminary investigation becomes necessary to determine the basic failure cause and effect. Otherwise, only a superficial description of abnormal symptoms will result. Usefulness of raw data collected for reliability analysis depends largely on the information required of the investigator.

Minimum information expected from the investigator for reliability purposes includes:

a. Component identification by name and system to which it belongs.

b. Component failure description including cause, mode, effect on system performance and plant operation, and time at which failure occurred or was discovered.

c. Component repair description including whether repair or replacement was made, length of time for repair, and time at which repair was completed.

In Figure 1.18 a list is presented for specific information re quired of in-plant personnel for eventual use in reliability analysis. Identification of the individual component is necessary in order to be able to calculate the length of time between failures for specific components as well as for identical or similar components in similar service. This is particularly 
important if it is desired to perform failure distribution analyses. Date and time of component failure and repair completion provide basic input to the calculation of failure rates and, if desired, the evaluation of failure distribution assumptions. Total repair time is the total elapsed time from the occurrence of a failure event to the completion of repair including time in which no active maintenance occurs. If a failed component is replaced by another new or repaired component, completion of repair occurs at the time installation of the new component is completed. Repair time is needed to compute actual use time and allows attainment of maximum effectiveness in reliability evaluation techniques such as fault-tree analysis. An example of a fault-tree type of technique is given in Chapter 2.

Mode of failure allows apportionment of the overall component failure rate among various critical failure modes. Mode and cause of failure are also important in evaluating failures and failure rates. For an unusual type of failure or a failure occurring at an unusually high rate, mode and cause data provide a means for retrospective review of failure data to permit detection and correction of gross deficiencies. Knowledge of failure effect on system performance and plant operation is necessary to link failure data to use data, i.e., effect data provides a means of identifying components put in operation or shutdown by a failure event. Effect data also provides a source of information for use in corroborating failure mode and effect assumptions in system reliability analyses.

Description of repair is required to the extent that it identifies the extent of repair, and particularly, whether failed items were replaced by completely new or repaired components, repaired in place with some part replacement, or adjusted or calibrated without any new parts. Component replacements must be identified in any analysis of failure distribution functions, especially when these replacements reflect component wearout.

2. Use Data - Use data is defined as the time element or "use time" by which successful operation of the plant or a system is measured. Individual entries from which use data can be derived are recorded chronologically in various log books; however, the most convenient sources of this information are periodic Operating and Outage Summary (O/OS) reports used in conjunction with the failure data.

In any system whose operation is continuous and directly measurable by length in time, the use time can be measured by one of the following time elements: 
a. Calendar Time - e.g., a radiation monitor which operates continuously with no relation to plant operation.

b. Plant Operating Time - e.g., reactor operating time and/or turbine-generator $(T / G)$ operating time.

There are systems whose use times are not exactly the same as those listed above but are proportional to them. For these systems, use time can be modified by a use fraction, $K$, and computed as follows:

$$
\begin{aligned}
\text { Use time }= & \mathrm{K} \times \text { Calendar time, or } \\
& \mathrm{K} \times \text { Reactor operating time, or } \\
& \mathrm{K} \times \mathrm{T} / \mathrm{G} \text { operating time. }
\end{aligned}
$$

Any component operation which is continuous in nature can be measured by one of the above use times. In order to facilitate the bookkeeping for various use times, the following rules are sugges ted:

Rule 1: Unless a system failure is reported, the use time of any system will correspond to that of the plant operation (reactor or $T / G$ ), appropriately modified by a use fraction.

Rule 2: Unless a component failure is reported, the use time of any component will correspond to that of the system to which it belongs.

This type of recording practice, namely "data management by exception, "where only exceptions to normal operation are recorded already exists in preparing O/OS reports. By extending the same technique, the paper work involved in recording use data can be simplified to initial identification of use fractions for components or systems in a plant. Subsequently, use for a component is computed by the product of the use fraction and the appropriate operating time less component down time recorded in failure event data.

Use time for any test operation which is cyclic in nature and not directly dependent on the length in time cannot be measured by the calendar time or plant operating time. Test intervals of routine operational tests required by the technical specifications* and operating procedures will provide a useful guide in

*Code of Federal Regulations, Title 10, Part 50. 
predicting the actual number of tests performed on any given system or component. In general, the number of successful tests of any component will correspond to that of the system to which it belongs; however, operational test procedures must be carefully studied to verify that individual components are indeed operationally tested when testing the entire system operation. For example, when an emergency core cooling system is periodically tested during power operation, not all components may be tested operationally with each test.

Typical intervals for operational tests are summarized in the technical specifications issued for a power plant, as shown in Figure 1.19. From this listing, the follcwing "usefractions" can be obtained:
a. Number of tests/calendar time.
b. Number of tests/reactor operating time.
c. Number of tests/T/G operating time.

By multiplying the appropriate use fraction by the calendar time or the appropriate plant operating time, the cyclic use of protection system components can be estimated.

In addition to components whose use times can be defined as above, there are some components which are also operated with each reactor startup and shutdown; e.g., reactor protection circuits. For components in this category, it becomes necessary to identify all plant startups and shutdowns, including reactor scrams, and to include these occurrences as part of the overall use.

Data Recording and Reporting - In-plant data recording and reporting will be accomplished in a two-step approach. The first step will be performed by operating and maintenance personnel using FR/MR forms to record all failure and repair data, and by periodic $O / O S$ reports to record all use data. This data recording activity already exists in nuclear power plants and can be upgraded to a level suitable for use in reliability evaluation by minor modification of the existing forms and reports, and provision of simple procedures to assure that all pertinent data are recorded on them. Of course, any changes in these forms should not detract from their original purposes.

Much of the failure and repair information is already available in existing $\mathrm{FR} / \mathrm{MR}$ forms. In order to ascertain what additional information will be required from operating personnel, the required information, shown in Figure 1.18, has been compared with the information asked for on some 
of the existing work order formats in Appendix A, Figures A. 3, A. 4, and A. 10, and in Appendix B, Figure B. 18. This comparison is shown in Figure 1.20.

Based on the comparison given by Figure 1.20, a suggested FR/MR format with necessary reliability information is shown in Figure 1.21. Although the suggested format differs in some respects from any in current use, these differences are small. In most cases existing forms could be adapted easily to the reliability oriented format by use of a rubber stamp form on the front or back of the existing form. An example of such a modification is given in Figure 1.22.

The next step in in-plant data collection requires periodic translation and transferral of raw data generated by the operating personnel to data forms suitable for data handling, indexing, storing, and retrieving with the possible use of computerized data handling systems. This step requires an engineer or technician familiar with both the subject plant systems and the reliability data classification requirements.

The use data reported in O/OS reports and failure data reported on $F R / M K$ forms would be codified onto an 80-column loadsheet using appropriate field allocations as shown in an example in Appendix C, Figure C. 3. These data inputs may be described as follows:

1. Date of Report - Date will be reported as Day $(01$ to 31), Month (01 to 12), and the last two digits of the calendar year.

2. Data Source - Identification number of the data source, i.e., plant I. D.

3. System Code - Refer to the classification of functional systems.

4. Component I. D. Number - Plant assigned I. D. No, of the failed component. Generic component identification is provided during data reduction.

5. New component I. D. Number - Enter only when repair is accomplished by replacement with a new component of different design.

6. New Component Manufacturer's Code - Enter only when repair is accomplished by replacement with a new component from a different manufacturer.

7. Time of Event - Time of the day when event occurred in Hours $(00$ to 24$)$ and Minutes (00 to 60). If information on time is not available, 0800 will be used. This is used to identify multiple failures or startups and shutdowns within the same day.

8. Total Repair Time - Enter to the nearest hour. 
9. Date of Recovery - Date will be reported in the same manner as for date of reporting in item (1).

10. Failure Mode - Refer to failure classification, Figure 1.13.

11. Failure Cause - Refer to failure classification, Figure 1.14.

12. Failure Effect - Refer to failure classification, Figure 1.15.

13. Use - Refer to use classification, Figure 1.16.

14. Description of Event - Enter in 20 letters or less, and in case of failure event it should identify the failed parts and indicate whether they were replaced, repaired, adjusted, or calibrated.

Two basically different line entries are made in the input loadsheet to assure proper correlation of use and failure data in generating failure rates and repair data. They are (1) entries which define the beginning of plant startups, and scheduled shutdowns; and (2) entries which define each failure event.

Using these line entries, including the failure effect and use codes, the operational history of each component can be reproduced either by hand calculation or a computer program. The proposed method of data reduction is explained in a subsequent section, and salient points in loadsheet preparation are demonstrated in Appendix C, with the use of a hypothetical system consisting of several components operating continuously, as well as cyclically.

Vendor Data - Qualification tests, production tests, and other tests are routinely conducted by various equipment vendors; and the reports summarizing these tests represent an additional source of reliability data. Consideration is given here to the requirements for collection of reliability data from equipment vendors in a form compatible with that from in-plant sources. The objective is to allow eventual accumulation of all the se data into one data bank.

To assure collection of vendor data in a form consistent with the in-plant data, the vendor's reliability input data format shown in Figure 1.23 is recommended for use in the reliability data program. In this format component identification can be made by model number or part number; but in any case it should also be identified by the generic code previously developed for in-plant data. The test condition entry called out in item 5 of Figure 1.23 may be divided into the following categories.

Test Condition

Life Test

Proof Test

Start-up Test
Description

Component operating in long-term test.

Component operating in short-term test, e.g., production test, qualification test, etc.

Component operating in-plant startup. 
Items $6,7,8$, and 9 are the basic statistical data necessary in evaluating failure rate. If known, the confidence level and assumed failure distribution should be noted as indicated in item 9. Functional modes of failure are the same as those defined for in-plant data. Another important data entry in reporting component failure rate is the derating factor. Headings are given in item 11 for the more commonly used derating parameters. Other environmental stress factors should be noted in item 12, if known. Finally, to avoid submission of the same data from different sources, it is necessary to reference the test document from which the test data were derived.

It should be noted that the format in Figure 1.23 suggests the necessary data from vendor sources. If existing vendor reports include the information indicated in Figure 1.23 then the se reports may be used as the first level input to the generalized data collection form. Reduction of both in-plant and vendor data is discussed in the following section.

Data Reduction - Reliability data collected from in-plant sources and equipment vendors should be compiled in a manner that allows easy retrieval and updating of all data. The formats illustrated in Appendix C are considered to satisfy this need. The output provided in this recommended format will yield the following information for reliability analysis:

1. Failure rates by a single component.

2. Failure rates by components of the same generic code.

3. Failure rates by failure mode for each component.

4. Mean repair time.

5. Standard deviation of failure rates or mean time between failure.

6. Standard deviation of mean repair time.

7. Time between failure of a single component which will enable detailed examination of failure distribution functions with accumulation of sufficient failure events.

As suggested in Appendix C, Figures C.4, C. 5, and C.6, the formats can be arranged as computer output sheets. The se formats also can be readily converted to calculation forms suitable for manual computation. The recommended formats are as follows:

Type 1 Output Data Sheet - Listing of Component Failures

Column 1 Date of Report - Date of report will be reported in the same manner as in the input loadsheet. 
Column 2 Component I. D. No. - Plant or manufacturer's I. D. No. for component, e.g., see Appendix C, Figure C. 3 .

Column 3 Manufacturer's Code - A unique three-digit code number assigned to the manufacturer of the equipment item on which data is to be given.

Column 4 Failure Mode - Same as shown on the input loadsheet (see Appendix C, Figure C. 3).

Column 5 Hours or Cycles - To distinguish the unit of use associated with the operation of the component.

Column 6 Time to Failure in Million Hcurs or Cycles - Time from the initial use until the failure of a single component or the time since the last failure of the component.

Column 7 Accumulated Time in Million Hours or Cycles The sum of Times to Failure in Million Hours or Cycles from the initial operation to the end of reporting period for a specific component.

Column 8 Repair Time - Same as the input loadsheet (see Appendix C, Figure C.3).

Column 9 Description of Repair - Same as the input loadsheet (see Appendix C, Figure C. 3).

Type 2 Output Data Sheet - Summary of Failure Rate Data

Column 1 Component I. D. No. - Plant or manufacturer's I. D. No. for component on which data is being given (see Column 2 of Type 1 Output Data Sheet).

Column 2 No. of Components - Number of components identified with the component I. D. No, given in Column 1 .

Column 3 Failure Mode - Same as Column 4 on Type 1 Output Data Sheet.

Column $4 \quad$ No. of Failures - Number of component failures identified on each component and for all components of a generic type from initial operation to end of reporting period.

Column 5 Hours or Cycles - Same as Column 5 on Type 1 Output Data Sheet. 
Column 6

Column 7

Column 8
Accumulated Time in Million Hours or Cycles The sum of Times to Failure in Million Hours or Cycles from initial operation to the end of reporting period.

Failures per Million Hours or Cycles - This is the quotient of No. of Failures divided by Accumulated Time in Million Hours or Cycles. The use of failure rates implies assumption that the observed failures relate to the chance or exponential failure distribution. With this assumption, the failure rate for nonfailed components may be reported as the inverse of Accumulated Time with 63 percent confidence. Failure rates, hence reliabilities, for both failed and nonfailed components may be estimated at othe $r$ confidence levels using the methods outlined in Appendix $\mathrm{H}$ or statistical charts such as provided in Appendix 4 of HN- $185 .^{* *}$

In vendor's input data, failure rates may be provided with either the number of failures and accumulated time or the estimated confidence level. The first form is the more desirable since it is better suited to estimating failure rates at other confidence levels. Another variation which may occur in reporting failure rates is the use of different units. For convenience, a conversion table is provided in Figure 1.24 to allow conversion to the suggested units of failures per million hours or cycles.

Failure Rate Standard Deviation - Standard deviation of the failure rate for generically similar components. This number may be calculated when the number of components is equal to or greater than three.

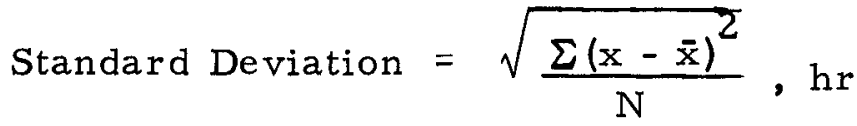

where $\quad x=$ Mean time between successive failures for a specific component in hours or cycles,

*Garrick, B. J., Gekler, W. C., and Pomrehn, H. P., "An Analysis

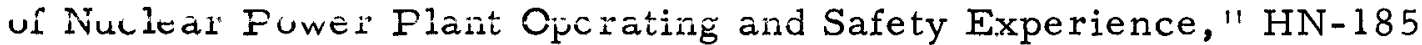
December 15, 1966. 
$\overline{\mathbf{x}}=$ Average mean time between failures for all generically similar components in hours or cycles, and

$\mathrm{N}=$ No. of components.

Note that a similar test can be applied to mean times between failure for a single component when more than three failures have occurred.

Column 9 Accumulated Repair Time - The sum of all Repair Time from the beginning to the reporting date.

Column 10 Mean Repair Time - The quotient of Accumulated Repair Time divided by No. of Failures or repairs.

Column 11 Repair Time Standard Deviation - Standard deviation may be calculated when No. of Repairs is equal to or greater than three.

$$
\begin{aligned}
& \text { Standard Deviation }=\sqrt{\frac{\sum(\mathrm{r}-\overline{\mathrm{r}})^{2}}{\mathrm{~N}}} \text {, hr } \\
& \text { where } \quad \begin{aligned}
\mathrm{r} & =\begin{array}{l}
\text { Repair time for individual failure } \\
\text { in hours, }
\end{array} \\
\overline{\mathrm{r}} & =\text { Mean repair time in hours, and } \\
\mathrm{N} & =\text { No. of failures }
\end{aligned}
\end{aligned}
$$

Many of the items noted above are direct inputs from the input loadsheet. However, it should be noted that Type 1 Output Data Sheet has line entries for failed components only while the Type 2 Output Data Sheet tabulates all components whether they failed or not during a particular reporting period.

To bridge the gap and provide information on successful (nonfailed) components, the principle of "data management by exception" mentioned earlier under the heading of Use Data must be used. Proper interpretation of this principle for data reduction computations may be accomplished by use of the following rules:

Rule 1: If no component failure is reported in the input loadsheet, the use time of any components will correspond 
to that of the plant operation, modified by the appropriate use fraction.

Rule 2: If a component failure accompanied by no loss in system performance is reported, the use time of other components in the same system will correspond to that of the system to which they belong in the same calendar period.

Rule 3: If a component failure accompanied by system loss is reported, the use time of other components in the same system will be decreased by the repair time identified for the failed component.

The key to use of the foregoing rules is proper identification of the failed component and the effect and use codes entered in the input loadsheet. One should be familiar enough with the systems operation or the computer subroutine should be written so that the status of all nonfailed components in the same system can be identified with the component failure in the system, or in case of no failures all components within the same system can be identified. Specific examples of the se mathematical operations are given in Appendix C. 


\begin{tabular}{|c|c|c|c|}
\hline \multirow[b]{2}{*}{ Program Objective } & \multicolumn{3}{|c|}{ Data Management Information } \\
\hline & Available & Minimal & Proposed \\
\hline $\begin{array}{l}\text { System/Component } \\
\text { Classification }\end{array}$ & By Name & $\begin{array}{l}\text { By Generic } \\
\text { Description }\end{array}$ & By Generic Code \\
\hline $\begin{array}{l}\text { Component Failure } \\
\text { Rate }\end{array}$ & $\begin{array}{l}\text { No. of Failures } \\
\text { Total Operating } \\
\text { Time }\end{array}$ & $\begin{array}{l}\text { No. of Failures } \\
\text { Total Operating } \\
\text { Time }\end{array}$ & $\begin{array}{l}\text { No. of Failures } \\
\text { Total Operating } \\
\text { Time }\end{array}$ \\
\hline $\begin{array}{l}\text { Failure Distribution } \\
\text { Function }\end{array}$ & None & $\begin{array}{l}\text { Identification of } \\
\text { Wearout or Chance } \\
\text { Failures }\end{array}$ & $\begin{array}{l}\text { Length of Time } \\
\text { or No. of Cycles } \\
\text { to Failure }\end{array}$ \\
\hline $\begin{array}{l}\text { Failure Mode and } \\
\text { Effect Analysis }\end{array}$ & $\begin{array}{l}\text { Safety Analysis } \\
\text { Reports }\end{array}$ & $\begin{array}{l}\text { Final Safety } \\
\text { Analysis Report; } \\
\text { Complete Descrip- } \\
\text { tions of Systems, } \\
\text { System Functions } \\
\text { and Operating and } \\
\text { Testing Procedures }\end{array}$ & $\begin{array}{l}\text { Failure Mode, } \\
\text { Cause and Effect } \\
\text { Analyses }\end{array}$ \\
\hline Repair Data & Repair Description & $\begin{array}{l}\text { Repair Description } \\
\text { Repair Time }\end{array}$ & $\begin{array}{l}\text { Repair Description } \\
\text { Repair Time }\end{array}$ \\
\hline
\end{tabular}




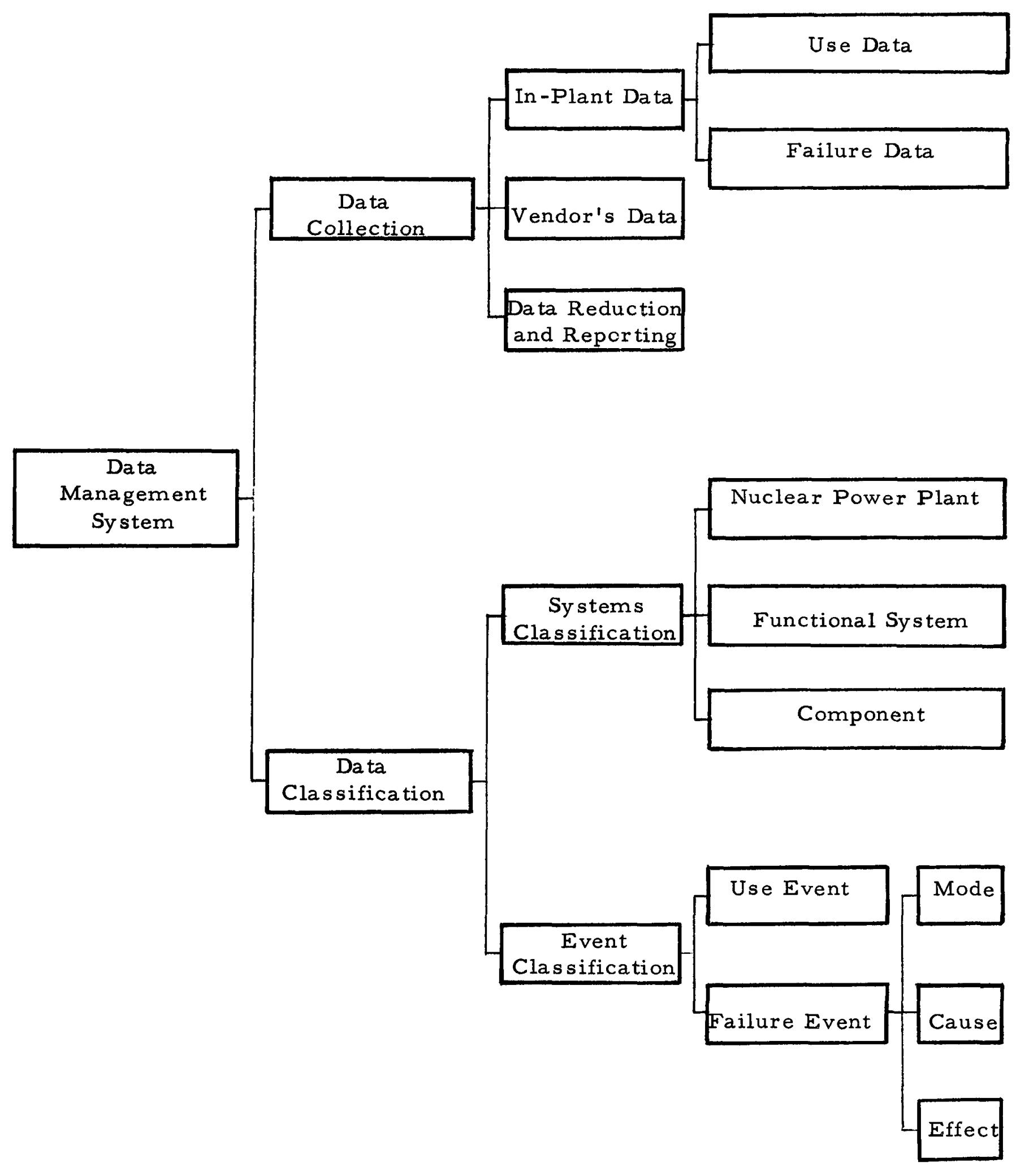

FIGURE 1.2 


\begin{tabular}{|c|c|c|}
\hline Classification & Organization & Purpose \\
\hline UKAEA & $\begin{array}{l}\text { United Kingdom Atomic } \\
\text { Energy Authority }\end{array}$ & $\begin{array}{l}\text { 1. Fault and incidence report. } \\
\text { 2. Safety assessment. } \\
\text { 3. Failure rate data. }\end{array}$ \\
\hline PM - 1 & U. S. Air Force & $\begin{array}{l}\text { 1. Plant performance and economics report. } \\
\text { 2. Safety assessment. } \\
\text { 3. Availability report. } \\
\text { 4. Failure rate data. }\end{array}$ \\
\hline NPD & $\begin{array}{l}\text { Nuclear Power Demonstration, } \\
\text { Ontario Hydro-Electric Power } \\
\text { Commission }\end{array}$ & $\begin{array}{l}\text { 1. Safety assessment. } \\
\text { 2. Failure rate data. } \\
\text { 3. Scheduling. } \\
\text { 4. Cost Accounting. }\end{array}$ \\
\hline MARAD & $\begin{array}{l}\text { Maritime Administration, } \\
\text { Department of Commerce }\end{array}$ & $\begin{array}{l}\text { 1. Failure rate data. } \\
\text { 2. Maintenance cost minimization. } \\
\text { 3. Inspection frequency optimization. }\end{array}$ \\
\hline EEI & Edison Electric Institute & $\begin{array}{l}\text { 1. Outage data. } \\
\text { 2. Equipment availability report. }\end{array}$ \\
\hline IDEP-FARADA & $\begin{array}{l}\text { Interservice Data Exchange } \\
\text { Program and Failure Rate Data } \\
\text { Program, DOD and NASA }\end{array}$ & $\begin{array}{l}\text { 1. Failure rate data. } \\
\text { 2. Part/component test data. }\end{array}$ \\
\hline $\begin{array}{l}\text { AEC Uniform } \\
\text { System of } \\
\text { Accounts }\end{array}$ & U. S. A tomic Energy Commission & $\begin{array}{l}\text { 1. Cost accounting. } \\
\text { 2. Cost estimating. }\end{array}$ \\
\hline
\end{tabular}

FIGURE 1.3 


\begin{tabular}{|c|c|c|c|c|c|c|c|c|}
\hline \multirow{2}{*}{ CLASSIFICATION } & \multicolumn{2}{|c|}{ S YSTEM CODE } & \multicolumn{2}{|c|}{ COMPONENT CODE } & \multicolumn{2}{|c|}{ FAULT CODE } & \multicolumn{2}{|c|}{ USE CODE } \\
\hline & Level & Digit & Level & Digit & Group & Digit & Group & Digit \\
\hline UKAEA & 8 & 8 & 6 & 6 & 3 & $2,4, \& 5$ & 2 & 2 \\
\hline PM-1 & 1 & 3 & 1 & 5 & 2 & 2 & 2 & 2 \\
\hline NPD & 3 & 3 & 2 & 2 & 2 & (2) & 1 & (2) \\
\hline MARAD & 3 & 3 & 4 & 8 & 4 & (3) & 1 & (3) \\
\hline EEI & 1 & 1 & 1 & 3 & (1) & & & \\
\hline IDEP / F ARADA & & & 7 & 9 & (4) & & & \\
\hline $\begin{array}{l}\text { AEC Uniform } \\
\text { System of Accounts }\end{array}$ & 6 & 6 & (5) & & & & & \\
\hline Proposed System & 6 & 6 & 7 & 9 & 3 & 1 & 1 & 1 \\
\hline
\end{tabular}

Notes

(1) Fault identified with component.

(2) Yes or No only.

(3) Checklist form.

(4) Number of fault groups varies with components.

(5) Component identified only to extent necessary for cost accounting.

\section{FIGURE 1.4}

NUMBERS OF LEVELS, GROUPS, AND DIGITS OF EXISTING

AND PROPOSED CLASSIFICA TION CODES 
PROPOSED

$\begin{array}{llllllllllllllllllllllllllll}0 & 2 & 0 & 6 & / & 2 & 1 & 4 & 1 & 0 & / & 2 & 0 & 2 & 5 & 6 & 5 & 4 & 5 & 2 & / & \mathrm{M} & 0 & \mathrm{~V} & 8 & 5 & 1 & \mathrm{~A}\end{array}$

San Onofre Unit \#1

Reactor Plant

Reactor

Reactor Auxiliary Cooling and Heating Systems

Safety Injection System

Valve

Service, water

Material, stainless steel

Size, over 12 inches

Type, gate

Function, shut off

Operation, motor

UKAEA

Multiple component number

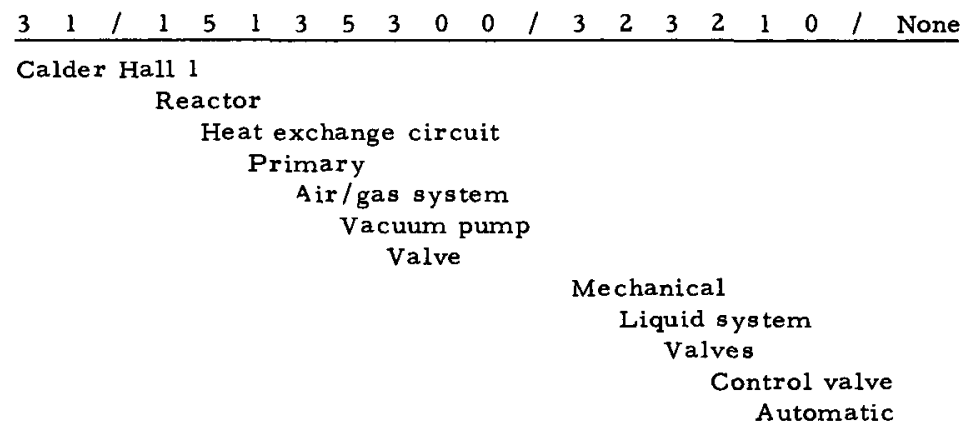

WESTINGHOUSE

$\begin{array}{llllllllllllll}P & M & I & / & P & I & A & / & \mathrm{R} & \mathrm{Y} & \mathrm{A} & / & 3 & 6\end{array}$

Air Force Sundance Reactor

Annunciator and temperature scan systems

Relay alarm

Location number

CANADIAN

None / 7 1 $2 / 2 / 200 /$ None

NPD

Common processes and services

Water supply system

Circulating water system

Pumps

MARAD

\begin{tabular}{llllllllllllllllllll}
2 & 6 & 4 & 7 & 0 & 4 & $/$ & $\mathrm{P}$ & $\mathrm{B}$ & $\mathrm{B}$ & $/$ & 0 & 0 & 1 & $\mathrm{D}$ & 0 & 2 & $/$ & 0 & 1 \\
\hline
\end{tabular}

S. S. President Arthur

Power and propulsion system

Steam subsystem

Steam Supply Unit

Boiler

Bent tube type

Steam drum

Starboard

FARADA / IDEP-II CODES

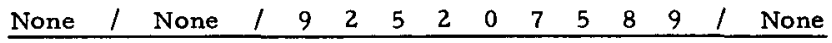

Valves

Fuel, exotic

Pressure, 200 psig

Function, shutoff

Type, globe

Activation, solenoid

FIGURE 1.5

COMPARISON OF PLANT/SYSTEM/COMPONENT CLASSIFICATION/ MULTIPLE COMPONENT IDENTIFICATION NUMBERS 

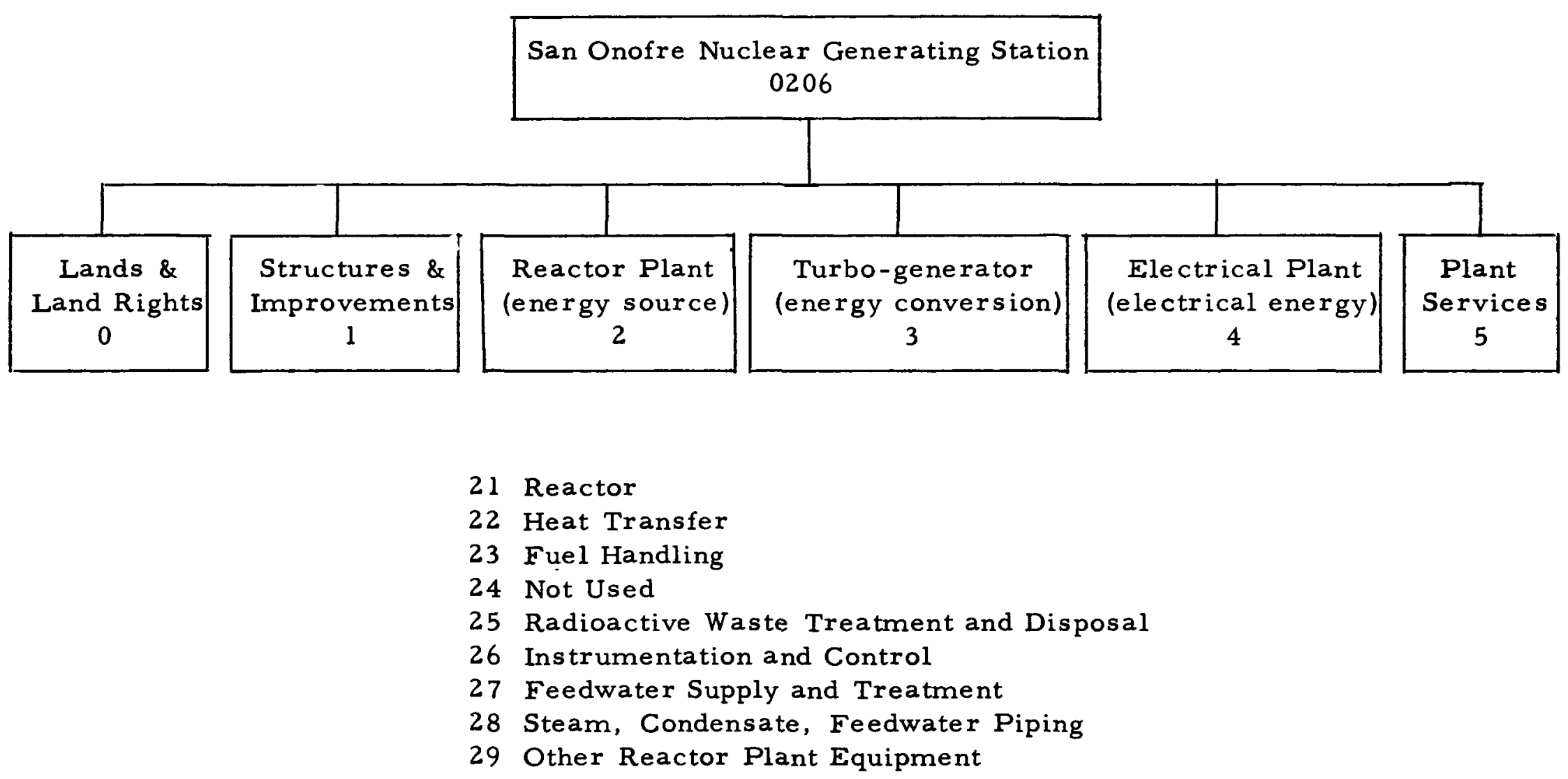

FIGURE 1.6 


\section{Reactor Equipment}

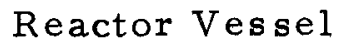

\section{FIGURE 1.7}

REACTOR PLANT CLASSIFICATION SYSTEM 
21.94

21.95

21.96

21.97

21. 98

21.99

22

22,1

22. 2

22. 23

22.3

22.31

22. 32

22.33

22. 34

22.35

22.4

22.41

22.42

22.43

22.44

22.45

22.5

22.51

22.52

22.7

22.71

22. 72

22.73

22.74

22.75

22.76

23

23. 1

23.2

23.21

23.22
Superstructure

Lighting and Electrical System

Plumbing and Drainage

Temperature Control System (heating and cooling)

Purge System

Fire Protection

Heat Transfer Systems

Reactor Coolant System

Intermediate Cooling System

Intermediate Heat Exchanger and Supports

Steam Generators, Superheaters, Steam Drums, and Steam Separators

Steam Generators and Supports

Superheaters

Steam Drums and Internais

Steam Separators

Reheaters

Reactor Coolant Receiving, Supply and Treatment

(and intermediate, if any)

Charge, Volume Control, Pressuriring and

Relief System

Purification System

Chemical Treatment, Blowdown and Sampling

Systems

Coolant Receiving, Storage and Makeup

Inert Gas Supply and Blanketing System

Reactor Moderator Auxiliary Systems

Gas Systems, Supply and Circulation

Cooling and Purification System for Gas and Liquids

Conventional Fossil Fuel Fired Superheaters or Boilers

Superheaters or Boilers

Firing Equipment

Coolant Systems

Fuel Handling and Storage Equipment

Blowdown Equipment

Ash Handling and Storage

Nuclear Fuel Handling and Storage Equipment

Cranes and Hoisting Equipment (fuel handling)

Special Tools and Service Equipment

Reactor Vessel Servicing Equipment

Fuel Removal, Assembly and Disassembly

Equipment

FIGURE 1.7 (cont.) 
23.23

23. 24

23. 25

23.3

23. 31

23.32

23.33

23. 34

23. 4

25

25. 1

25. 11

25. 12

25. 2

25. 3

25. 4

25.5

26

26. 1

26. 11

26. 12

26. 13

26. 14

26.2

26. 21

26.22

26.23

26. 24

26.25

26.3

26. 31

26. 32

26.4

26.41

26.42

26.43

26.5

26. 51
Control Rod Handling

Television, Other Viewing and Special Lighting

Containers and Racks

Spent Fuel Storage, Cooling, Cleaning and Inspection

Equipment

Spent Fuel Cooling and Cleaning

Spent Fuel Washing (organic coolant)

Spent Fuel Washing (sodium coolant)

Inspection Equipment

Shipping Casks and Cars

Radioactive Waste Treatment and Disposal

Liquid Waste

Aqueous

Organic

Gaseous Waste

Solid Wastes

Drain and Vent System, Reactor Coolant

Drain and Vent System, Intermediate Coolant

Instrumentation and Control

Reactor Plant Control System

Reactor

Reactor Shield

Reactor Auxiliary Systems

Vapor Containers

Heat Transfer System

Reactor Coolant System

Intermediate Coolant System

Steam Generators and Superheaters

Coolant Supply and Treatment

Coolant Receiving and Storage

Service to Fuel Handling and Storage

Spent Fuel Storage Cooling System

Fuel Wash System

Service to Radioactive Waste Treatment

and Disposal

Liquid Waste

Gaseous Waste

Solid Waste

Radiation Monitoring

Process

FIGURE 1.7 (cont.) 
26.52

26.53

26.6

27

27. 1

27.2

27.3

27.4

29

29. 1

29. 2

29.21

29.22

29.23
Health Physics

Off-site Environs

Steam Generator

Feedwater Supply and Treatment System

Raw Water Supply

Make-Up Water Treatment

Steam Generator Feedwater Purification

Feedwater Heaters

Other Reactor Plant Equipment

Chemical Decontamination System

Reactor Plant Maintenance Equipment

Remotely Controlled Tools

Radioactive Maintenance Facilities

Portable Shielding

FIGURE 1.7 (cont.) 
21.4 Reactor Auxiliary Cooling and Heating Systems

21.41 Safety injection system

21.42 Fmergency shutdown cooling system

21. 43 Decay heat removal system

21.44 Component cooling system

21.45 (not used)

21. 46 Post incident cooling system

21. 47 Pressure suppression system

$21.42 \quad$ Emergency Shutdown Cooling System

21.421 Turbine steam bypass system

21. 422 Emergency condenser system

21.423 Emergency feedwater system

21.424 Steam dump system

FIGURE 1.8

REACTOR AUXILIARY COOLING AND HEATING SYSTEM AND EMERGENCY SHUTDOWN COOLING SYSTEM CLASSIFICA TION 
200 Piping

201 Pipes and Fittings

201.00 Service and Material (common)

202 Valves

202.00 Service and Material (common)

202.00.00 Size (common) and Type

$\overline{01} \overline{\mathrm{Ba} 11}$

.02 Butterfly

.03 Flapper

.04 Gate

.05 Globe

.06 Needle

.07 Plug

.08 Poppet

202.00.00.00 Function

. 10 Check

.20 Multifunction

.30 Relief

.40 Servo

.50 Shutoff

.603 -Way Selector

.70 4-Way Selector

202.00.00.00 Operation

$.0 \overline{1}$ Manual

.02 Motor

.03 Pilot (remote manual)

.04 Pressure

.05 Solenoid

203 Filters and Strainers (nonelectrical)

203. 00 Service (common) and Filtering Material

.$\overline{01}$ Cloth

.02 Ceramic

.03 Earth

.04 Fiberglas

.05 Paper

.06 Resin

.07 Sintered Metal

.08 Spaced Plates

.09 Wire Mesh

FIGURE 1.9

PARTIAL LIST OF COMMON COMPONENT CLASSIFICATION 
203.00.00 Pressure (common)

$$
\begin{aligned}
\text { 203.00.00.00 } & \text { Operation } \\
.01 & \text { Chemical } \\
.02 & \text { Centrifugal } \\
.03 & \text { Electrostatic } \\
.04 & \text { Gravitational } \\
.05 & \text { Mechanical }
\end{aligned}
$$

220 Pump

221 Pump

221.00 Service and Material (common)

$$
\begin{aligned}
& 221.00 .00 \text { Capacity } \\
& \text {. } 10 \text { Under } 10 \text { GPM } \\
& .2010 \text { up to } 100 \mathrm{GPM} \\
& .30100 \text { up to } 1000 \mathrm{GPM} \\
& .40 \text { Over } 1000 \text { GPM }
\end{aligned}
$$

$221.00 .00 \frac{T}{.01} \frac{\text { Type }}{\text { Axial }}$

.02 Centrifugal

.03 Diaphragm

.04 Electromagnetic

.05 Gear

.06 Reciprocating

.07 Rotary

240 Heat Exchangers

240.00 Service and Material, primary side (common)

240.00. 00 Service and Material, secondary side (common)

240.00.00.00 Pressure (common)

400 Tanks

400. 00 Service and Material (common)

400.00 .00 Capacity

. io Under 1 gallon

.201 up to 10 gallons

.3010 up to 100 gallons

.40100 up to 1000 gallons

.50 Over 1000 gallons

400.00.00 Type

$.0 \overline{1} \frac{\text { Drain }}{.02}$

.02 Holdup

.03 Relief

.04 Storage

.05 Sump

.06 Surge

.07 Waste

400.00.00.00 Pressure (common)

FIGURE 1.9 (continued) 
500 Industrial Instrument

500.00 Service, Media Measured (common)

$$
\begin{aligned}
& 500.00 . \frac{00}{10} \frac{\text { Type }}{\text { Controller }} \\
& .20 \text { Indicator } \\
& .30 \text { Meter } \\
& .40 \text { Switch } \\
& .50 \text { Testing } \\
& .60 \text { Transmitter }
\end{aligned}
$$

500.00 .00 .00 Readout

$$
\begin{aligned}
& . \overline{10} \text { Audio } \\
& .20 \text { Dial } \\
& .30 \text { Digital } \\
& .40 \text { Plotting } \\
& .50 \text { Recording } \\
& .60 \text { Video }
\end{aligned}
$$

800 Nuclear Equipment

801 Flux Detector

$$
\begin{aligned}
801.00 & \text { Type } \\
.01 & \text { Ion Chamber } \\
.02 & \text { Fission Chamber } \\
.03 & \text { BF3 Counter } \\
.04 & \text { G-M Tube } \\
.05 & \text { Scintillator } \\
.06 & \text { Semi-Conductor } \\
.07 & \text { Detector Lead }
\end{aligned}
$$

802 Preamplifier

803 Amplifier

$$
\begin{aligned}
803.00 & \text { Type } \\
.01 & \text { Linear } \\
.02 & \text { Log } \\
.03 & \text { Magnetic } \\
.04 & \text { Pulse } \\
.05 & \text { Period } \\
.06 & \text { Bistable }
\end{aligned}
$$

804 Power Supply

$$
\begin{aligned}
& \text { 804. } \frac{00}{10} \frac{\text { Service }}{\text { Low Voltage }} \\
& .20 \text { High Voltage }
\end{aligned}
$$

804.00.00 Voltage Rating (common)

FIGURE 1.9 (continued)

$$
1-36
$$


Service, Media Handled

$\begin{array}{ll}.1 & \text { Air } \\ .2 & \text { Gas } \\ .3 & \text { Hydraulic } \\ .4 & \text { Liquid Metal } \\ .5 & \text { Water } \\ .6 & \text { Heavy Water }\end{array}$

$\underline{\text { Size }}$

.1 Under 1 inch

.21 up to 3 inches

.33 up to 6 inches

. 46 up to 12 inches

.5 Over 12 inches

Voltage Rating

.1 Under $1 \mathrm{mV}$

.21 up to $10 \mathrm{mV}$

$.310 \mathrm{mV}$ up to. $1 \mathrm{~V}$

.41 to $100 \mathrm{~V}$

.5100 to $1000 \mathrm{~V}$

.61 to $10 \mathrm{KV}$

.7 Over $10 \mathrm{KV}$

Material

. 1 Aluminum

.2 Brass/Bronze

.3 Copper

.4 Steel, Carbon

. 5 Steel, Galvanized

. 6 Steel, Corrosion Resistant

.7 Nonmetallic

.8 Multimetallic

.9 Composite-Metallic and Nonmetallic
Pressure Range

.1 Vacuum

.2 Ambient

.30 up to 100 psig

.4100 up to 1000 psig

.5 Over 1000 psig

Service, Media Measured

$.01 \quad$ Flow

.02 Level

.03 Pressure

.04 Temperature

.05 Vacuum

.20 Conductivity

$.21 \quad \mathrm{pH}$

.22 Hydrogen Concentration

.23 Hydrazine Concentration

.24 Oxygen Concentration

$.25 \mathrm{CO}_{2}$ Concentration

.30 Current

.31 Power

.32 Resistance

.33 Voltage

.40 Count Rate

.41 Dose Rate

.42 Flux

.43 Period

.50 Position

.51 Revolution

.52 Torque

.53 Speed

.54 Vibration

.60 Time

.70 Light

FIGURE 1.10

COMMON SUBCLASSIFICATION 


\begin{tabular}{|c|c|c|c|}
\hline \multirow[b]{2}{*}{$\begin{array}{c}\text { System } \\
\text { Code }\end{array}$} & \multirow[b]{2}{*}{$\begin{array}{c}\text { Generic } \\
\text { Code }\end{array}$} & \multicolumn{2}{|r|}{ COMPONENT } \\
\hline & & $\begin{array}{c}\text { Identification } \\
\text { Number } \\
\end{array}$ & Name \\
\hline 21.410 & 221.46 .42 & G50A & Safety Injection Pump \\
\hline 21.410 & 221.46 .42 & G50B & Safety Injection Pump \\
\hline 27.500 & 221.46 .42 & G3A & Feedwater Pump * \\
\hline 27.500 & 221.46 .42 & G3B & Feedwater Pump * \\
\hline 21.410 & 400.56 .54 .20 & & Refueling Water Storage Tank \\
\hline 21.410 & 202.56 .54 .52 & MOV853A & Feedwater Inlet Valve \\
\hline 21.410 & 202.56 .54 .52 & MOV853B & Feedwater Inlet Valve \\
\hline 27.370 & 202.56 .35 .55 & CV36 & Condenser Hotwell Valve \\
\hline 27.370 & 202.56 .35 .55 & CV37 & Condenser Hotwell Valve \\
\hline 21.410 & 202.56 .43 .14 & $867 \mathrm{~A} 06 \mathrm{C} 58$ & Check Valve \\
\hline 21.410 & 202.56 .43 .14 & $867 \mathrm{~B} 06 \mathrm{C} 58$ & Check Valve \\
\hline 21.410 & 202.56 .43 .14 & $867 \mathrm{C} 06 \mathrm{C} 58$ & Check Valve \\
\hline 26.132 & 500.01 .20 .60 & FI912 & Flow Indicator \\
\hline 26.132 & 500.01 .20 .60 & FI913 & Flow Indicator \\
\hline 26.132 & 500.01 .20 .60 & FI914 & Flow Indicator \\
\hline 26.132 & 500.01 .60 & F T912 & Flow Transmitter \\
\hline 26.132 & 500.01 .60 & F T914 & Flow Transmitter \\
\hline 26.132 & 500.01 .60 & F T914 & Flow Transmitter \\
\hline
\end{tabular}

(*) The se components normally functioning in different systems.

FIGURE 1.11 


\begin{tabular}{|c|c|c|c|}
\hline \multirow[b]{2}{*}{$\begin{array}{l}\text { System } \\
\text { Code }\end{array}$} & \multirow[b]{2}{*}{$\begin{array}{c}\text { Generic } \\
\text { Code }\end{array}$} & \multicolumn{2}{|r|}{ COMPONENT } \\
\hline & & $\begin{array}{c}\text { Identification } \\
\text { Number }\end{array}$ & Name \\
\hline $\begin{array}{l}26.111 \mathrm{~A} \\
26.111 \mathrm{~A} \\
26.111 \mathrm{~A} \\
26.111 \mathrm{~A} \\
26.111 \mathrm{~A} \\
26.111 \mathrm{~A} \\
26.111 \mathrm{~A} \\
26.111 \mathrm{~A} \\
26.111 \mathrm{~A} \\
26.111 \mathrm{~A} \\
26.111 \mathrm{~A} \\
26.111 \mathrm{~A} \\
26.111 \mathrm{~A} \\
26.111 \mathrm{~A} \\
26.111 \mathrm{~A} \\
26.111 \mathrm{~A} \\
26.111 \mathrm{~A} \\
26.111 \mathrm{~A} \\
26.111 \mathrm{~A} \\
26.111 \mathrm{~A} \\
26.111 \mathrm{~A} \\
26.111 \mathrm{~A}\end{array}$ & $\begin{array}{l}801.03 \\
801.03 \\
802.00 \\
802.00 \\
803.02 \\
803.02 \\
001 \\
001 \\
002 \\
002 \\
500.04 .20 .60 \\
500.04 \cdot 20.60 \\
500.04 .40 \\
500.04 .40 \\
500.40 .20 .60 \\
500.40 .20 .60 \\
500.40 .20 .60 \\
500.40 .20 .60 \\
500.40 .30 .20 \\
500.40 .30 .20 \\
500.40 .20 .50 \\
500.40 .20 .50\end{array}$ & $\begin{array}{l}\text { NE1201 } \\
\text { NE1202 } \\
\text { NM1201.4 } \\
\text { NM1202.4 } \\
\text { NM1201.1 } \\
\text { NM1202.1 } \\
\text { ND1201 } \\
\text { ND1202 } \\
\text { NT1201 } \\
\text { NT1202 } \\
\text { TA1200.1 } \\
\text { TA1200.2 } \\
\text { TE1201 } \\
\text { TE1202 } \\
\text { NFA1201.5 } \\
\text { NFA1202.5 } \\
\text { NLA1201.1 } \\
\text { NLA1202.1 } \\
\text { NLI1201 } \\
\text { NLI1202 } \\
\text { NRL1201 } \\
\text { NRL1202 }\end{array}$ & $\begin{array}{l}\mathrm{BF}_{3} \text { Detector } \\
\mathrm{BF}_{3} \text { Detector } \\
\text { Prea:nplifier } \\
\text { Preamplifier } \\
\text { Log Count Rate Amplifier } \\
\text { Log Count Rate Amplifier } \\
\text { Power Supply Cutoff Assembly } \\
\text { Power Supply Cutoff Assembly } \\
\text { Test and Calibration Assembly } \\
\text { Test and Calibration Assembly } \\
\text { Thimble Temperature Indicator } \\
\text { Thimble Temperature Indicator } \\
\text { Thimble Temperature Detector } \\
\text { Thimble Temperature Detector } \\
\text { Log Count Rate Alarm } \\
\text { Log Count Rate Alarm } \\
\text { Log Count Rate Alarm } \\
\text { Log Count Rate Alarm } \\
\text { Log Count Rate Meter } \\
\text { Log Count Rate Meter } \\
\text { Log Count Rate Recorder } \\
\text { Log Count Rate Recorder }\end{array}$ \\
\hline
\end{tabular}

FIGURE 1.12 


\begin{tabular}{|l|l|}
\hline Mode Code & \multicolumn{1}{|c|}{ Functional Failure Mode } \\
\hline 1 & Failure to close \\
2 & Failure to open \\
3 & Failure to start \\
4 & Failure to stop \\
5 & Failure to continue operation \\
6 & Spurious failure - premature \\
7 & operation \\
8 & Degradation \\
9 & Erratic operation \\
0 & Scheduled service \\
\hline
\end{tabular}

FIGURE 1.13

FAILURE MODE CLASSIFICATION 
Cause

Code

10 DESIGN

11 Not suitable to normal operating conditions.

12 Not designed to withstand design environment.

13 Not suitable for simultaneous application of all design stresses.

14 Useful life too short for design application.

15 Marginal design leading to reduced performance of several parts under stress.

20 FABRICATION

21 Configuration Abnormality

a) Breaks or cracks.

b) Distortion or deformation.

c) Part left out.

d) Presence of foreign objects, parts, or material.

e) Improper size or shape.

f) Depletion.

g) Wear or abrasion.

h) Mechanical or electrical maladjustment, including loose parts and faulty positioning.

22 Composition Abnormality

a) Wrong material used.

b) Excessive impurities in component material.

c) Change in composition or grain structure.

30 OPERATION

31 Actual environmental stresses exceed normal stresses called for in operation.

32 Actual operating conditions exceed those called for.

33 Maladjustment.

34. Operating error as a direct cause of failure.

35 Operation beyond stipulated design life.

00 UNSPECIFIED

FIGURE 1.14

FAILURE CAUSE CLASSIFICATION 


\begin{tabular}{|c|c|}
\hline Effect Code & Failure Effect on System Performance \\
\hline & \\
1 & No loss of performance \\
3 & Partial los of performance \\
& Total loss of performance \\
\hline
\end{tabular}

FIGURE 1.15

FAILURE EFFECT CLASSIFICATION

\begin{tabular}{|c|l|}
\hline Use Code & \multicolumn{1}{|c|}{ Type of Use Event } \\
\hline 0 & No Operation (Plant Remains Shutdown) \\
1 & Startup Operation \\
2 & Continue Operation \\
3 & Shutdown, Normal \\
4 & Shutdown, Scram \\
5 & System Test \\
\hline
\end{tabular}

FIGURE 1.16

USE EVENT CLASSIFICATION 


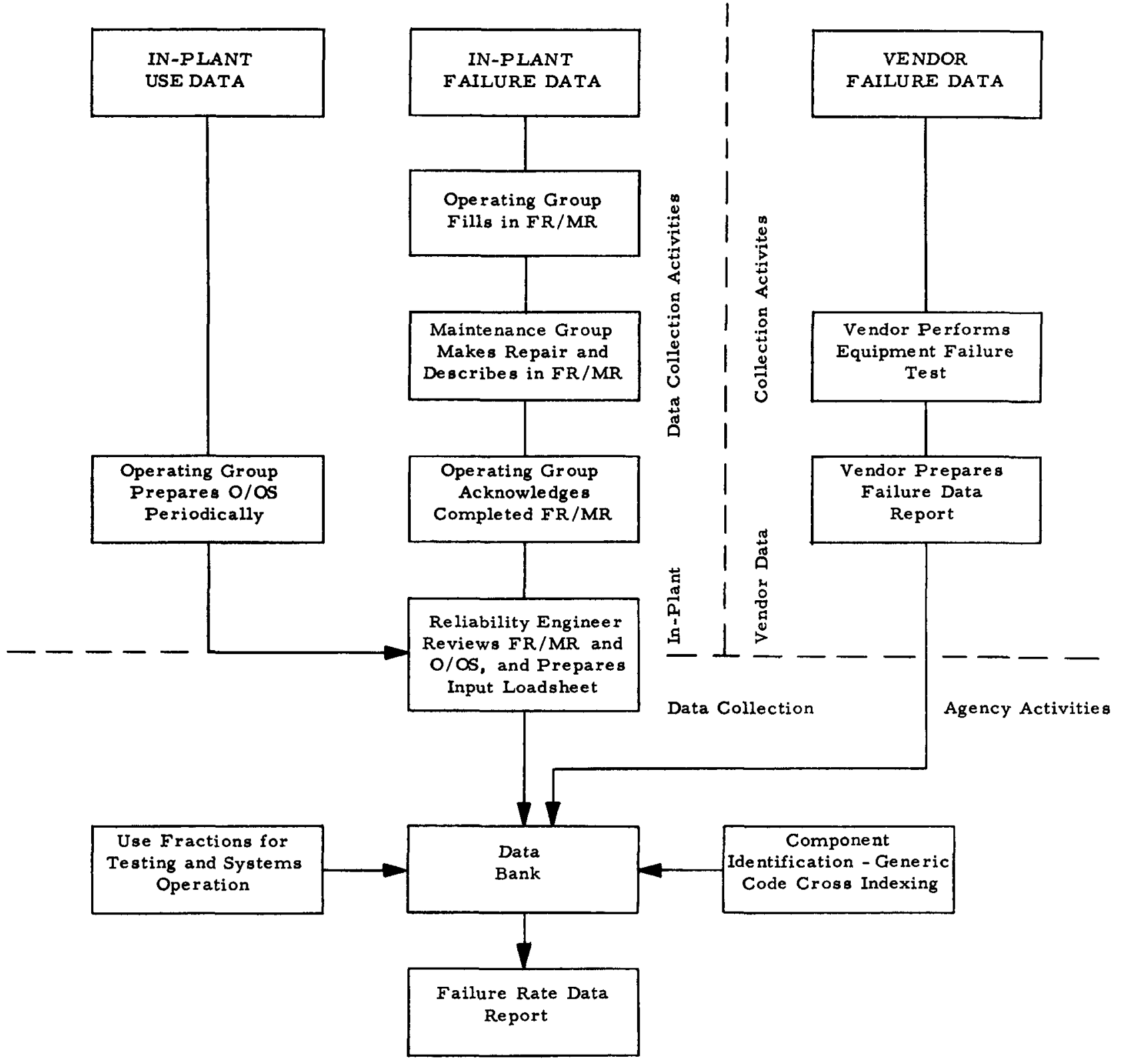

FR/MR denotes Failure Report and Maintenance Request $O / O S$ denotes Operating and Outage Summary

FIGURE 1.17

RELIABILITY INPUT DATA FLOW CHART 


\begin{tabular}{|c|c|c|}
\hline & Item & Required Information \\
\hline 1. & Component/System & $\begin{array}{l}\text { Name of component and system to which } \\
\text { it belongs. Where two or more identical } \\
\text { components exist in one system, indi- } \\
\text { vidual component must be identifiable. }\end{array}$ \\
\hline 2. & Date/Time of Failure & $\begin{array}{l}\text { Occurrence or discovery of failure in } \\
\text { day, month, year, hours, and minutes. }\end{array}$ \\
\hline 3. & Total Repair Time & $\begin{array}{l}\text { Total repair time, including off-hours, } \\
\text { recorded to the nearest hours and } \\
\text { maintenance man-hours. }\end{array}$ \\
\hline 4. & Completion Time of Repair & $\begin{array}{l}\text { Time when repair was completed and } \\
\text { system became operationa!. }\end{array}$ \\
\hline 5. & Functional Mode of Failure & $\begin{array}{l}\text { The way in which a component failed, } \\
\text { i. e., to close, open, start, stop, or } \\
\text { continue operation; premature, } \\
\text { degrading, or erratic operation; or } \\
\text { scheduled service or replacement. }\end{array}$ \\
\hline 6. & Cause of Failure & $\begin{array}{l}\text { What made the component fail the } \\
\text { way it did? }\end{array}$ \\
\hline 7. & $\begin{array}{l}\text { Effect of Failure on System } \\
\text { Performance }\end{array}$ & $\begin{array}{l}\text { The degree to which system perform- } \\
\text { ance was affected, i. e., no loss, } \\
\text { partial loss, or total loss of perform- } \\
\text { ance. }\end{array}$ \\
\hline & $\begin{array}{l}\text { Result of Failure on Reactor } \\
\text { Operation }\end{array}$ & $\begin{array}{l}\text { The way in which reactor operation } \\
\text { was affected, i.e., none, shutdown } \\
\text { or scram. }\end{array}$ \\
\hline 9. & Description of Repair & $\begin{array}{l}\text { Description of failed parts and } \\
\text { whether they were replaced, repaired } \\
\text { adjusted, calibrated etc.; and } \\
\text { name of manufacturer if replaced } \\
\text { with unit of different make. }\end{array}$ \\
\hline
\end{tabular}

FIGURE 1.18

REQUIRED RELIABILITY INFORMATION 


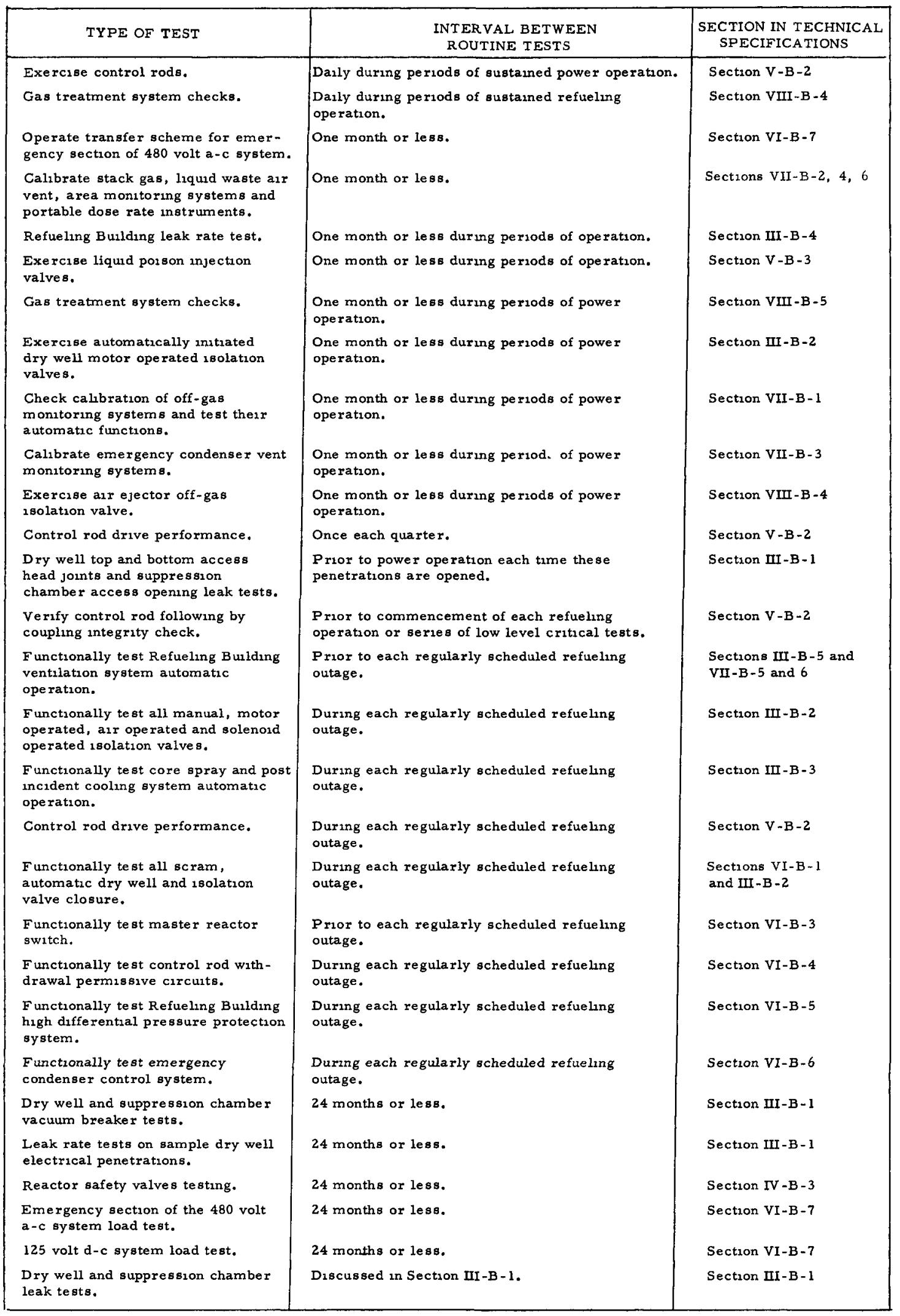

FIGURE 1.19 


\begin{tabular}{|c|c|c|c|c|c|}
\hline & Items & Yankee & $\begin{array}{l}\text { Indian } \\
\text { Point }\end{array}$ & $\begin{array}{l}\text { Shipping - } \\
\text { port }\end{array}$ & NPD \\
\hline (1) & Component/System & Yes & Yes & Yes & Yes \\
\hline (2) & Time of Failure & Yes & No & Yes & Yes \\
\hline (3) & Repair Time & No & No & Yes & No \\
\hline (4) & $\begin{array}{l}\text { Completion Time } \\
\text { of Repair }\end{array}$ & Yes & No & Yes & Yes \\
\hline (5) & $\begin{array}{l}\text { Functional Mode of } \\
\text { Failure }\end{array}$ & No & No & No & No \\
\hline (6) & Cause of Failure & No & No & No & No \\
\hline (7) & $\begin{array}{l}\text { Effect of Failure on } \\
\text { System Performance }\end{array}$ & No & No & No & Yes \\
\hline (8) & $\begin{array}{l}\text { Result of Failure on } \\
\text { Reactor Operation }\end{array}$ & No & No & Yes & Yes \\
\hline (9) & Description of Repair & Yes & Yes & Yes & Yes \\
\hline
\end{tabular}

FIGURE 1.20

COMPARISON OF REQUIRED INFORMATION 
1. Component/System

2. Date/Time of Failure 1

3. Functional Mode of Failure (circle one)

Failed to (a) close (b) open (c) start (d) stop or $(e)$ continue operation (f) premature operation (g) degradation (h) erratic operation (i) scheduled service (j) scheduled replacement

4. Cause of Failure (if known or required)

5. Effect of Failure on System Performance (circle one)

(a) no loss (b) partial loss or (c) total loss of performance

6. Effect of Failure on Plant Operation (circle one)

(a) none (b) continue operation (c) shutdown or (d) scram

7. Work to be Performed

8. Reported by

9. Work Assigned to

10. Date of Completion

11. Total Repair Time hours

12. Defective/Replaced Parts

13. Work Performed

14. Name of Manufacturer (if replaced with unit of different make)

FIGURE 1.21

FAILURE REPORT AND MAINTENANCE REQUEST FORM WITH REQUIRED RELIABILITY INFORMATION 


\begin{tabular}{|l|l|l|}
\hline $\begin{array}{l}\text { DATE AND TIME } \\
\text { OF FAILURE }\end{array}$ & $\begin{array}{l}\text { SHUTDOWN } \\
\text { REQUIRED }\end{array}$ & $\begin{array}{l}\text { REPAIR } \\
\text { TIME }\end{array}$ \\
\hline $\begin{array}{l}\text { FAILUAE } \\
\text { MODE }\end{array}$ & $\begin{array}{l}\text { FAILURE } \\
\text { EAUSE }\end{array}$ & $\begin{array}{l}\text { FAILURE } \\
\text { EFFECT }\end{array}$ \\
\hline
\end{tabular}

\section{MAINTENANCE MEMORANDUM No 81913}

\begin{tabular}{c|c}
\hline APPARATUS & WORK \\
\hline & \\
\hline & \\
\hline & \\
\hline & \\
\hline & \\
\hline
\end{tabular}

REPORTED BY

WATCH POREMAM

FIGURE 1.22

MODIFIED MAINTENANCE REQUEST FORM 
1. Vendor's Name

2. Component Name

3. Component Identification/Generic Code

4. Test Date or Duration

5. Test Condition (circle one or describe)
(a) Life Test
(b) Proof Test
(c) Startup Test
(d) Others

6. Total No. of Components Tested

7. Total No. of Failures

8. Total Operating Time million comp - $\underline{\text { r }}$. or cy.

9. Estimate of Failure Rate per million $\underline{\mathrm{hr}}$. or cy.

(a) Confidence Level $\%$

(b) Assumed Failure Distribution

10. Functional Mode of Failure by Percent of Total Failures

Failed to (a) close

(b) open

(c) start

(d) stop or (e) continue operation

(f) Premature operation

(g) Degradation

11. Derating Factor by Percent of Rated

(a) Voltage

(b) Current

(c) Power

(d) Frequency

(e) Pressure

(f) Temperature

(g) Others

12. Other Environmental Stress Factors

13. Reference

FIGURE 1.23

VENDOR'S RELIABILITY INPUT DATA FORM 


\begin{tabular}{|l|c|c|c|c|}
\hline & $\begin{array}{c}\text { Bits (\% } \\
\text { failures per } \\
\text { million hours) }\end{array}$ & $\begin{array}{c}\text { \% Failures } \\
\text { per } \\
1000 \text { hours }\end{array}$ & $\begin{array}{c}\text { Failures } \\
\text { per } \\
\text { million hours }\end{array}$ & $\begin{array}{c}\text { Failures } \\
\text { per } \\
\text { hour }\end{array}$ \\
\hline $\begin{array}{l}\text { Bits (\% } \\
\text { failures per } \\
\text { million hours) }\end{array}$ & 1 & $10^{3}$ & $10^{2}$ & $10^{8}$ \\
$\begin{array}{l}\text { \% failures per } \\
\text { loo0 hours }\end{array}$ & $10^{-3}$ & 1 & $10^{-1}$ & $10^{5}$ \\
$\begin{array}{l}\text { Failures per } \\
\text { million hours }\end{array}$ & $10^{-2}$ & $10^{1}$ & 1 & $10^{6}$ \\
$\begin{array}{l}\text { Failures } \\
\text { per hour }\end{array}$ & $10^{-8}$ & $10^{-5}$ & $10^{-6}$ & 1 \\
\hline
\end{tabular}

FIGURE 1.24

FAILURE RATE CONVERSION TABLE 


\section{CHAPTER 2}

\section{RELIABILITY ANALYSIS TECHNIQUES}




\section{CHAPTER 2}

RELIABILITY ANALYSIS TECHNIQUES

An objective of this study has been to define requirements and methods of application for data collected in a reliability monitoring program. Basically, what is needed is an examination of the types of reliability analys is techniques which might be used in such a program. The range of candidate techniques either in existence or currently conceivable is large. It includes part-count models requiring all components to function, simple block-diagram models allcwing credit for redundancy, more complex block-diagram models accounting for various component interdependencies and standby operation, and logical system simulation models which include human error. As the complexity of technique increases the data requirements increase and the results of the analysis technique can generally be expected to provide more realistic evaluations of the relative contributions to unreliability of the various parts of a system design. As the application of reliability analysis techniques to nuclear safety increases, it can be expected that the techniques will increase in sophistication and complexity.

Various reliability analysis methods currently in use have been investigated in the course of this study. Out of this review two methods have been selected whose data requirements are believed to represent the upper limit which might be encountered in a reliability monitoring program for reactor safeguard systems. These methods are the Automatic Reliability Mathematical Model (ARMM) and fault tree analysis. ARMM is a working, computerized reliability analysis program. Fault tree is a logical analys is concept developed during the Minuteman missile program. A preliminary fault tree computer program, SAFTE-1, has been developed during this study. Both of these methods are described in this chapter. An appreciation for the data requirements and method of data application which would be encountered in a reliability monitoring program is gained from a discussion of ARMM and fault tree. From this discussion, as well as the examples given in Chapter 3 , it will be seen that either method is capable of a comprehensive reliability examination of the system, given sufficient information regarding the equipment, the design, and the methods of operation and testing of the system. Items of information which are particularly important in either method include:

1. Equipment relationships determined by both functional and physical design. 
2. Significant failure modes for each item of equipment.

3. Failure rate data for each significant failure mode.

4. Time to detect and repair failed equipment.

5. Testing or inspection frequencies which reveal ability to perform or conversely failure to perform on each item of critical equipment which is not continuously monitored.

6. Normal or expected use patterns for aging of each item of equipment.

Finally, it should be noted that in either of these methods a primary limitation on the quality of results is the experience of the user. As repeated applications are made, the user will become aware of the fact that many apparently impossible configurations or sequences can be appropriately simulated. Such an observation applies equally to other promising reliability analys is techniques which were not included here. 


\section{AUTOMATIC RELIABILITY MATHEMATICAL}

MODEL (AR MM) PROGR AM

$\operatorname{ARMM}(1,2)$ is a general purpose computer program for deriving and solving a mathematical model of the reliability of complex systems. It is based on a sequential application of the conditional probability theorem to the probability of system failure. The program selects those combinations of component failures which cause a system failure, and derives and solves the reliability mathematical model for computing failure probabilities. The output includes (1) the probability of successful system operation; (2) the probability of occurrence of each failure combination; (3) the probability of function failure, its percentage of system failure, and rank of each function of the system; (4) each component's contribution to the probability of system failure, i.e., its percentage of the total probability and rank among the components; and (5) the component combinations which are the major contributors to system unreliability. Other useful aspects of the program are a built-in capability to handle dependent components and mutually exclusive failure modes, and the use of input data requirements which are simplified for engineers not familiar with programming methods.

ARMM was developed by North American Aviation, Inc, under contract to the U. S. Air Force. Subsequently, it has been revised and expanded to allow treatment of larger and more complex reliability models. It has been used by North American to evaluate reliability and reliability achievement in aircraft system design from the conceptual design phase through the detailed design phase. With the availability of test data it also has been found capable of calculating a system's demonstrated reliability; specific examples include analyses of systems in experimental or advanced design aircraft.

It will be noted that ARMM has limitations which can be of importance in some safeguard system reliability analyses; however, these limitations are not believed to be critical to the use of ARMM in nuclear applications. Each of the limitations and suggested modifications are discussed in the description and discussion of use which follow.

\section{DESCR IPTION}

Logical Concept

ARMM considers a system to be made up of functions which in turn are made up of components. When a function fails, the system fails if there 
are no other operating or standby functions which are alternate to the failed function. The failure of a function is determined by the relative values of the "modes available" and the "modes required" which are assigned to that function. A component can support more than one function, and for each of the functions a component supports it is assigned a "weighting factor." When the component fails, the corresponding weighting factor is subtracted from the modes available of each function it supports. When the modes available of a function become (by this subtraction at each component failure) less than the modes required, the function has failed.

A component is the smallest piece of equipr.ent the reliability analyst wants to consider and for which he has failure rate data. If a piece of equipment has several modes of failure, each mode can be considered as a unique component if there is failure rate data for the different modes. If the failure modes of a piece of equipment are mutually exclusive, i.e., the failure in one mode excludes the possibility of failure in another (e.g., a valve failing open cannot fail closed at the same time), this can be described to the program by making one modecomponent a "must not fail" dependent component on the other modecomponent. If two components are physically dependent on each other, e.g., a pump and its motor, but not mutually exclusive, this dependency is input to the program as a "must fail" dependency. This latter type of dependency can also be used between components which, though not physically dependent, are dependent in such a way that the failure of one makes consideration of failure in the other superfluous. Use of "must fail" dependency is especially helpful in reducing computer time.

\section{Mathematical Bases}

Application of the Conditional Probability Theorem to Reliability - What follows is a determination of the probability of system failure by the application of the conditional probability theorem. The system to be used throughout this discussion is shown in Figure 2. 1. It consists of five components arranged on three "success paths." So long as the components on at least one success path are operating, the system has not failed.

According to the conditional probability theorem, the probability of system failure can be written as:

$$
P(S)=P(S / A) P(A)+P(S / \bar{A}) P(\bar{A})
$$


where

$$
\begin{aligned}
S= & \text { system failure, } \\
A= & \text { component } A \text { is working (not failed), } \\
\bar{A}= & \text { component } A \text { has failed (the bar over the } \\
& \text { component name signifies the component } \\
\text { has failed), } & \text { probability of system failure, } \\
P(S)= & \text { probability of system failure given that } \\
P(S / A)= & \text { component A has not failed, } \\
P(S / \bar{A})= & \text { component A has failed, } \\
P(A)= & \text { probability that component A has not failed, and } \\
P(\bar{A})= & \text { probability that component A has failed. }
\end{aligned}
$$

The probability that component $A$ has or has not failed is determined by the failure density function for the component. Evaluated at time $t$ the probability of failure is

$$
P(\bar{A})=\int_{0}^{t} f_{A}(t) d t
$$

and the probability of no failure is

$$
P(A)=1-P(\bar{A})
$$

where

$$
\begin{aligned}
f_{A}(t)= & \text { failure density function for component } A, \\
f_{A}(t)= & \beta \alpha t^{\beta-1} e^{-\alpha t^{\beta}}, \\
\beta= & \text { Weibull shape parameter. If } \beta=1, f_{A}(t) \\
& \text { becomes the exponential failure density function, } \\
& \text { and } \\
\alpha= & \begin{array}{l}
\text { Weibull scaling parameter. If } \beta=1, \alpha \text { becomes } \\
\text { the failure rate, } \lambda .
\end{array}
\end{aligned}
$$


Given Equations (2) and (3), the probability of system failure $P(S)$ requires that $P(S / \bar{A})$ and $P(S / A)$ also be evaluated. These terms can be defined by a stepwise application of the conditional probability theorem to the system shown in Figure 2.1. If $A$ has not failed, the system can fail only if components $B, C$, and $D$ or $D$ or $E$ also fail. The following equations state this in the notation of Equation (1):

$$
P(S / A)=P(S / A, B) P(B)+P(S / A, \bar{B}) P(\bar{B}) \text {. }
$$

Since the system will not fail if both $A$ and $B$ are operating then

$$
P(S / A, B)=0
$$

and Equation (4) reduces to

$$
P(S / A)=P(S / A, \bar{B}) P(\bar{B}) \text {. }
$$

The probability of system failure given that $B$ has failed is evaluated as:

$$
P(S / A, \bar{B})=P(S / A, \bar{B}, C) P(C)+P(S / A, \bar{B}, \bar{C}) P(\bar{C}) \text {. }
$$

The first term on the right is zero since the system cannot fail if $C$ has not failed. Equation (5) becomes

$$
P(S / A, \bar{B})=P(S / A, \bar{B}, \bar{C}) P(\bar{C})
$$

The probability of system failure given that $C$ has failed is evaluated as :

$$
P(S / A, \bar{B}, \bar{C})=P(S / A, \bar{B}, \bar{C}, D) P(D)+P(S / A, \bar{B}, \bar{C}, \bar{D}) P(\bar{D})
$$

Since Equations ( $4 a$ ) and (5a) say that $B$ and $C$ have failed, then if $D$ fails the system fails (i. e., $P(S / A, \bar{B}, \bar{C}, \bar{D})=1$ ). All that remains to be evaluated so that Equation (4a) can be expressed in terms of unconditional component probabilities is the factor $P(S / A, \bar{B}, \bar{C}, D)$. It is evaluated by

$$
P(S / A, \bar{B}, \bar{C}, D)=P(S / A, \bar{B}, \bar{C}, D, E) P(E)+P(S / A, \bar{B}, \bar{C}, D, \bar{E}) P(\bar{E})(7)
$$


In Equation (7) $D$ has not failed, so $P(S / A, \bar{B}, \bar{C}, D, E)=0$ for if $D$ and $E$ have not failed, there remains an unfailed success path. But if $E$ fails then the system fails so $P(S / A, \bar{B}, \bar{C}, D, \bar{E})=1$. It is now possible to rewrite Equation (4a) as:

$$
P(S / A)=[1-P(E) P(D)][1-P(C)][1-P(B)]
$$

The other unknown in Equation (1), $P(S / \bar{A})$, can be evaluated in a manner similar to Equations (4) through (8)

$$
P(S / \bar{A})=P(S / \bar{A}, B) P(B)+P(S / \bar{A}, \bar{B}) P(\bar{B}) \text {. }
$$

Since A has failed

$$
\mathrm{P}(\mathrm{S} / \overline{\mathrm{A}}, \mathrm{B})=\mathrm{P}(\mathrm{S} / \overline{\mathrm{A}}, \overline{\mathrm{B}})
$$

which has been evaluated by Equations (5a), (6), and (7). So Equation (9) can be rewritten as:

$$
P(S / \bar{A})=[1-P(E) P(D)][1-P(C)] \text {. }
$$

If Equations (8) and (11) are substituted into Equation (1), the probability of system failure is:

$$
P(S)=[1-P(E) P(D)][1-P(C)][1-P(A) P(B)] \text {. }
$$

The probability of system failure for the simple system of Figure 2.1 could have been obtained by recognizing that for this system, with redundant success paths, the probability of failure is the product of the probabilities of failure of each of the success paths. In more complex systems, solution by inspection is difficult if not impossible and the value of this approach is apparent.

ARMM's Use of the Conditional Probability Theorem - ARMM's analysis of the system in Figure 2.1 is, of course, that of a computer. Therefore, it is a sequential application of the conditional probability theorem rather than an "it is clear that..." approach. ARMM proceeds down the list of components searching for combinations of failed components which cause the system to fail. The user specifies the maximum number of failed components ARMM needs to consider in a combination. Table 2. 1 lists, in the proper order, the combinations ARMM would consider for the system in Figure 2.1 if the maximum number of failed components allowed per combination were four. 
Note that after the system has failed, it is returned to operation by turning on the last failed component. This is first met by ARMM with the combination $\bar{A} \bar{B} \bar{C} \bar{D}$. Component $D$ is turned on and for the next combination $E$ is failed.

If no system failure occurs with the failure of four components as with the combination $\bar{A} \bar{B} C \bar{D} \bar{E}$ then as with a system failure the last failed component is turned back on. However, in this example the last failed component is the last component on the list. What ARMM does here is turn on $B$ and $D$ as well and then fail $C$.

If the user had specified that component B was a "must fail" dependent component to component $A$ then ARMM would not consider any combination with both $A$ and $B$ failed. So fewer combinations are considered by the program which means a savings in time.

Without the dependency and as shown in Table 2. 1, there are six failure combinations. They are: $\bar{A} \bar{B} \bar{C} \bar{D}, \bar{A} \bar{B} \bar{C} D \bar{E}, \bar{A} B \bar{C} \bar{D}, \bar{A} B \bar{C} D \bar{E}, A \bar{B} \bar{C} \bar{D}$, and $A \bar{B} \bar{C} D \bar{E}$. With the dependency there are four: $\bar{A} \bar{C} \bar{D}, \bar{A} \bar{C} D \bar{E}, A \bar{B} \bar{C} \bar{D}$, and $A \bar{B} \bar{C} D \bar{E}$. Note that since $P(B)+P(\bar{B})=1$, these two lists are the same. The first list required more computer time. If only three components were allowed to be failed at a time, and a look at Figure 2. 1 shows that really that is all that is necessary, then the two lists would not be the same and there would be an error in the results of the run without the dependency.

The probability of system failure is the sum of the probability of occurrence of each of the failure combinations. For the failure combination $A \bar{B} \bar{C} D \bar{E}$, the probability of its occurrence is calculated by $A R M M$ as:

$$
P(A \bar{B} \bar{C} D \bar{E})=P(\bar{B}) P(\bar{C}) P(\bar{E})[1-P(\bar{A}+\bar{D})]
$$

where

$$
\begin{aligned}
& P(\bar{A}+\bar{D})=\text { probability that } A \text { or } D \text { or both fail, } \\
& P(\bar{A}+\bar{D})=P(\bar{A})+P(\bar{D})-P(\bar{A}) P(\bar{D})=1-P(A) P(D), \\
& P(A)=1-P(\bar{A}), \text { and } \\
& P(D)=1-P(\bar{D}) .
\end{aligned}
$$

The factor in brackets in Equation (13) is the probability that neither A nor $D$ fail. The probability of system failure, SF, during each time 
interval, is obtained by summing the probability of occurrence of each of the $\mathrm{N}$ failure combinations

$$
S F_{i}=\sum_{k=1}^{N} P_{k} \text { (failure combination } k \text { in time }
$$

This sum is the probability of system failure during time interval $i$.

The probability that the system has failed by the start of time interval $i$ is given by the relation:

$$
\mathrm{TSF}_{i}=\mathrm{TSF}_{i-1}+\left(1-\mathrm{TSF}_{i-1}\right) \mathrm{SF}_{i-1}
$$

where by convention

$$
\mathrm{TSF}_{0}=0
$$

and, therefore,

$$
\begin{aligned}
& \mathrm{TSF}_{1}=0 \\
& \mathrm{TSF}_{2}=S F_{1} .
\end{aligned}
$$

For example, the probability that the system has failed by the start of the third interval $i=3$ is:

$$
\mathrm{TSF}_{3}=\mathrm{SF} F_{1}+\left(1-\mathrm{SF}{ }_{1}\right) \mathrm{SF} \mathrm{F}_{2}
$$

Equation (16) can be read as the probability that the system failed in interval one plus the product of the probability that it failed in interval two and the probability that it did not fail in interval one. This product suggests the use of the "good as new" approximation for all components at the beginning of each time interval.

Originally, ARMM evaluated the probability of failure of each component for the time interval $i$ from $t_{i}$ to $t_{i+1}$ by Equation (17)

$$
P(\bar{B})=\int_{t_{i}}^{t} f_{B}(t) d t
$$


This does not incorporate the "good as new" approximation. This equation has been changed so that the limits of integration are now from 0 to $\left(t_{i+1}-t_{i}\right)$ which does reflect the "good as new" approximation.

A comparison of the different integration limits can be made as follows. For the exponential density function

$$
P^{(1)}\left(t_{i+1}-t_{i}, 0\right)=P^{(1)}\left(t_{i+1}, t_{i}\right) e^{\lambda t_{i}}
$$

where the superscript in parenthesis specifies the value of $\beta$.

Here the factors in the argument of $P$ are the upper and lower limits to the integral, respectively. Equation (18) shows that the error in using the limits of Equation (14) is small if $\lambda t_{l}$ is small. When Weibull distributions are used, the following relation obtains:

$$
\begin{aligned}
& P^{(\beta)}\left(t_{i+1}-t_{i}, 0\right)=P^{(\beta)}\left(t_{i+1}, t_{i}\right) e^{\alpha t_{i}^{\beta}}\left[\frac{P^{(1)}\left(\left[t_{i+1}-t_{i}\right], 0\right)}{P^{(1)}\left(t_{i+1}^{\beta}-t_{i}^{\beta}, 0\right)}\right] . \\
& P^{(1)} \rightarrow P^{(\beta)}, \beta=1 .
\end{aligned}
$$

Hence the values of $\alpha$ and $\beta$ used will determine the difference involved in using the two sets of limits.

The probability that the unfailed components in the failure combination of Equation (13) do not fail is calculated as:

$$
[1-P(\bar{A}+\bar{D})]=1-\int_{0}^{t} \int_{i+1}^{-t}\left[\left(\lambda_{A}+\lambda_{D}\right) e^{-\left(\lambda_{A}+\lambda_{D}\right) t}\right] d t .
$$

Equations (17) and (20) use the exponential failure density function. ARMM in its present form also is able to use the Weibull failure density function in Equation (17) but not in Equation (20). Equation (20) was generalized in this study to include a Weibull failure density function. This yields 


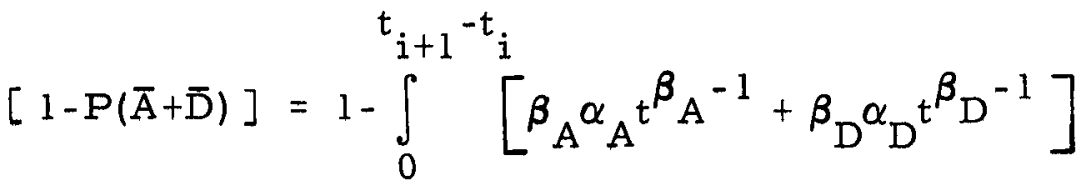

$$
\begin{aligned}
& \left.e^{-\left[\alpha_{A} t^{\beta} A+\alpha\right.} D^{t^{\beta} D}\right] d t \text {. }
\end{aligned}
$$

The result obtained using Equation (12), and the result obtained by ARMM, Equation (14), are identical for the simple system of Figure 2. 1. So for simple systems ARMM gives the same result for system failure during an interval as the conditional probability theorem. For complex systems, since ARMM limits the number of components failed in a failure combination, the results will not be identical to those of the conditional probability theorem, However, Equation (13) shows that the probability of system failure is proportional to the product of the probabilities of failure of each failed component, so for a failure combination with many failed components its contribution to the probability of system failure will be small. However, significant errors can result for simple systems when not enough failures are allowed.

System Probability - Bayes' the orem states:

$$
P(\bar{A} / S)=\frac{P(S / \bar{A}) P(\bar{A})}{P(S)}
$$

where $P(\bar{A} / S)$ is the probability, that given the system has failed, it was caused by the failure of component $A$. In other words, it is $A^{\prime} s$ contribution to system failure (or to system unreliability).

ARMM calculates the contribution of each of the components, $j$, to system unreliability in time interval i by the equation:

$$
P_{i}(j / S)=\frac{C_{i j} S F_{i}\left(1-T S F_{i}\right)}{a_{i}}
$$

where

$$
C_{i j}=\sum_{k=1}^{n} \frac{\lambda_{j}}{\lambda_{f c, k}}\left[\prod_{l=1}^{m} P_{l}\right]_{k}
$$




$$
\begin{aligned}
& \lambda_{f c, k}=\sum_{l=1}^{m} \lambda_{l, k} \\
& \begin{aligned}
\lambda_{l, k}= & \text { failure rate of component } l \text { which was involved } \\
& \text { in failure combination } k,
\end{aligned} \\
& \mathrm{~m}=\text { number of failed components in a given failure } \\
& \text { combination, } \\
& P_{l}=\text { probability of failure } \mathrm{cf} \text { component } l, \\
& \mathrm{n} \quad=\text { number of failure combinations in which component } \mathrm{j} \\
& \text { was involved, } \\
& a_{i}=\sum_{j=1}^{N} C_{i j} \text {, and } \\
& \mathrm{N}=\text { number of "on" components during interval i. }
\end{aligned}
$$

The factor $\mathrm{C}_{i j}$ is the sum of the contributions component $j$ makes to each failure combination of which it is a failed component. Its contribution is determined by the ratio of failure rates. For example, for component $A$ in the failure combinations $\bar{A} \bar{C} \bar{D}$ and $\bar{A} \bar{C} D \bar{E}$

$$
\begin{aligned}
c_{i, A}= & {\left[\frac{\lambda_{A}}{\lambda_{A}+\lambda_{C}+\lambda_{D}}\right] P(\bar{A}) P(\bar{C}) P(\bar{D}) } \\
& +\left[\frac{\lambda_{A}}{\lambda_{A}+\lambda_{C}+\lambda_{E}}\right] P(\bar{A}) P(\bar{C}) P(\bar{E}) .
\end{aligned}
$$

The contribution of component $j$ to system unreliability $P_{i}(\bar{j} / S)$ is included in the ARMM output for each time interval that component $j$ 
has not been turned off by the user. It is also part of the summary output which is the sum of system performance for all the time intervals. The contribution of component $j$ to system unreliability for the entire time, $P_{T}(j / S)$, is given by:

$$
P_{T}(\bar{j} / S)=\sum_{i=1}^{N} P_{i}(\bar{j} / S)
$$

for $\mathrm{N}$ time intervals.

If component $j$ was turned off during an interval, the $P_{i}(\bar{j} / S)$ for that interval is zero.

Standby Functions - ARMM can also handle standby functions. These are functions which do not operate until the function to which it is standby fails.

In Figure 2.1, for example, the function which is composed of components $D$ and $E$ could be standby to the function which is composed of component $C$.

The probability of occurrence of a failure combination which includes a failed standby component is calculated by an equation which is a modification of Equation 13. Since component $E$ is, for this consideration, a standby component which, along with component $D$; was brought into operation by the failure of component $C$, the product $P(\bar{C}) P(\bar{E})$ in Equation 13 is replaced by:

$$
\begin{aligned}
& t_{i+1}{ }^{-t} \\
& P(\bar{C} \bar{E})=\int_{0} \frac{\lambda_{C} \lambda_{E}}{\left(\lambda_{C}-\lambda_{E}\right)}\left[e^{-\lambda_{E} t}-e^{-\lambda_{C} t}\right] d t
\end{aligned}
$$

if $\lambda_{\mathrm{C}}=\lambda_{\mathrm{E}}=\lambda$ then:

$$
P(\bar{C} \bar{E})=\int_{0}^{t_{i+1}-t} \lambda^{2} t e^{-\lambda t} d t
$$


The general form for Equation 29, as used in ARMM, is

$P(n$ failures $)=\int_{0}^{t_{i+1}{ }^{-t}{ }_{i}} \frac{\prod_{j=1}^{n} \lambda_{j}}{\lambda_{1}-\sum_{j=2}^{n} \lambda_{j}}\left[\exp \left(-\sum_{j=2}^{n} \lambda_{j} t\right)-\exp \left(-\lambda_{1} t\right)\right] d t$

where

$\lambda_{1}=$ failure rate of the operating component whose failure "turns on" all $n-1$ standby components, and

$P(n$ failures $)=$ probability that the operating component and all of its standby components fail.

Equation 31 is for an operating component, and $n-1$ standby components all of which are brought into operation at the time the operating component fails. In the system in Figure 2.1, D and $E$ are standby to $C$ and both are turned on when $C$ fails; however, the system fails with eithe $r$ component $D$ or $E$ failed, so $n$ equals 2 for this system.

Equation 31 was derived by making the assumption that when the standby components were turned on, the probability of system failure could be written as the product of the probabilities of failure of the individual components. This yields Equation 31 which does not contain the limiting values of one and zero for large and small times, respectively.

Consider a simple standby model with three components $A, B$, and $C$, each having exponential failure densities. Component $A$ operates and when it fails both $B$ and $C$ are turned on. System failure occurs when all three components fail. The correct expression for system failure probability in this case is

$$
\begin{aligned}
P(t, 0)=1- & {\left[\frac{\lambda_{A} e^{-\lambda_{B}{ }^{t}-\lambda_{B} e^{-\lambda_{A} t}}}{\lambda_{A}-\lambda_{B}}+\frac{\lambda_{A} e^{-\lambda_{C} t}-\lambda_{C} e^{-\lambda} A^{t}}{\lambda_{A}-\lambda_{C}}\right.} \\
& \left.\frac{\lambda_{A} e^{-\left(\lambda_{B}+\lambda_{C}\right) t}-\left(\lambda_{B}+\lambda_{C}\right) e^{-\lambda_{A}^{t}}}{\lambda_{A}-\left(\lambda_{B}+\lambda_{C}\right)}\right] .
\end{aligned}
$$


The expression for $P(t, 0)$, based on the assumption of product probabilities, is from Equation 31

$P(t, 0)=\frac{\lambda_{B} \lambda_{C}}{\lambda_{B}+\lambda_{C}}-\frac{\lambda_{A} \lambda_{B} \lambda_{C}}{\lambda_{A}-\left(\lambda_{B}+\lambda_{C}\right)}\left[\frac{e^{-\left(\lambda_{B}+\lambda_{C}\right) t}}{\left(\lambda_{B}+\lambda_{C}\right)}-\frac{e^{-\lambda_{A} t}}{\lambda_{A}}\right]$.

An estimate of the difference between the two expressions has not been made. Equation 31 is exact for $n=2$.

ARMM, in its present form, does not allow for sequential activation of standby functions. That is, it does not consider a system wherein there is a standby function which turns on when another standby function fails. An expression for this configuration was obtained using a three component system model which covers most practical cases. The result is

$$
\begin{aligned}
P(t, 0)= & 1-\left[\frac{\lambda_{B} \lambda_{C} e^{-\lambda_{A}^{t}}}{\left(\lambda_{B}-\lambda_{A}\right)\left(\lambda_{C}-\lambda_{A}\right)}+\frac{\lambda_{A} \lambda_{C} e^{-\lambda_{B}}}{\left(\lambda_{A}-\lambda_{B}\right)\left(\lambda_{C}-\lambda_{B}\right)}\right. \\
& \left.+\frac{\lambda_{A} \lambda_{B} e^{-\lambda_{C} t}}{\left(\lambda_{A}-\lambda_{C}\right)\left(\lambda_{B}-\lambda_{C}\right)}\right] .
\end{aligned}
$$

Also, ARMM does not consider Weibull failure density functions for standby functions; it considers only the exponential. In extending this analysis to include Weibull densities, one obtains expressions which are not integrable in closed form. Hence, double integrals will appear and numerical procedures will have to be developed to handle them.

Function Contribution to Unreliability - Included in the ARMM output is the function contribution to unreliability for each function. The one exception of this is when a function has a standby function, then the 
contribution of the functions is assigned to its standby. The function contribution for function $(\mathrm{m})$ in time interval (i) is calculated by:

$$
\left.P_{i} \text { (function } m / S\right)=\frac{\left(1-T S F_{i}\right) S F_{i}\left[\sum_{k=1}^{a}\left(\begin{array}{ll}
n & \left.P_{l=1}\right) \\
\sum_{m=1}^{N}\left[\sum_{k=1}^{a}\left(\begin{array}{ll}
n \\
l=l
\end{array} P_{l}\right)\right.
\end{array}\right] m\right.}{m}
$$

whe re

$$
\begin{aligned}
& \mathrm{n}=\begin{array}{l}
\text { number of failed components in a given failure } \\
\text { combination whose failure is attributed to function } \\
\mathrm{m},
\end{array} \\
& \mathrm{a}=\begin{array}{l}
\text { number of failure combinations whose failure is } \\
\text { attributed to function } \mathrm{m} \text {, and }
\end{array} \\
& \mathrm{N}=\text { number of failure combinations in time interval i. }
\end{aligned}
$$

A failure combination is attributed to the function supported by the last failed component in that combination. For this reason, it is possible to have two parallel or redundant functions with the same type of components and yet different contributions to unreliability.

The contribution for a function for the entire time is given by the sum of its contribution for each inte rval it is on.

Serial Probability - The serial probability is the contribution each "on" component makes to system unreliability if all the "on" components in the system are in series. It is calculated by the equation:

$$
S P_{i j}=\frac{\lambda_{j}}{\lambda_{T}}\left(1-x_{i-1}\right) P_{i}
$$

where

$$
P_{i}=\int_{0}^{t_{i+1}{ }^{-t}} \lambda_{T} e^{-\lambda} T^{t} d t
$$




$$
\begin{aligned}
& \lambda_{T}=\sum_{j=1}^{n} \lambda_{j}, \\
& \lambda_{j}=\text { the failure rate of component } j, \\
& n=\text { number of "on" components, } \\
& x_{i}=x_{i-1}+\left(1-x_{i-1}\right) P_{i} \text {, and } \\
& x_{0}=0 .
\end{aligned}
$$

The factor, $\left(1-\alpha_{i-1}\right)$, is the probability that there has been no component failure up to the start of interval i.

The serial probability for the entire time is calculated by

$$
S P_{j T}=\frac{\lambda_{j} \Delta T_{j}}{\sum_{j=1}^{m} \lambda_{j} \Delta T_{j}} x_{N}
$$

where

$$
\begin{aligned}
\Delta \mathrm{T}_{j}= & \text { total operating time of the } j \text { th component during } \\
& \text { the } \mathrm{N} \text { time intervals. } \\
x_{N}= & \text { the probability of at least one component failure } \\
& \text { in the total operating time } t=0 \text { to } t=t_{N+1}
\end{aligned}
$$

The value of the serial probability is of little significance in systems possessing extensive amounts of redundancy. It does allow a conservative estimate of component contribution to unreliability in complex systems containing extensive cross linking. 
USE OF ARMM

As previously indicated, ARMM has been designed for use by engineers who do not have computer experience. To use ARMM to analyze the reliability of a system one is required to (1) prepare a reliability diagram of the system, and (2) identify the functions and their supporting components. Subsequently the information presented in the diagram is translated to numeric and alphanumeric input data.

The function and component summary sheet shown in Figure 2.2 is an aid in organizing the input data. The components are assigned a weight for each function they support. Also, the components are assigned failure rates and the functions are assigned "modes available" and "modes required." The relationship between modes available, modes required, and component weight is developed in detail in subsequent discussions of fields $4 \mathrm{~B}$ and $5 \mathrm{~B}$.

Each of the seven input forms shown in Figures 2.3 through 2.9 has been subdivided into data fields for appropriate input information. The fields are marked with vertical lines and have appropriate headings. Each line or row on a form corresponds to a single punch card of information. In each row columns 73 to 80 are used for assigning card identification or card sequencing numbers which assure correct arrangement of a complete input data deck.

It also should be noted that some of the input data must be given in fixed point numbers, e.g., 386, while other portions of the data must be in floating point numbers, e.g., 386.0. Fixed point numbers must be right adjusted, i.e., placed in the right hand columns within the field. The data fields that require a floating point number have a notation to that effect in the heading. The other fields use only fixed point numbers.

A review of the requirements for filling out each input form follows:

Form A-General Information - Rows 1 through 7

Field 1A - Columns 1 through 3 (Number of Components) - This field instracts the program about the number of components for which it will expect to read input information. This number must agree with the actual number of components for which input information has been provided or the program will reject the problem. The program will handle a maximum of 500 components. 
Field 2A - Columns 4 and 5 (Number of Time Intervals) - Every problem must consider 1 or more time intervals. The program is capable of considering a maximum of 20 . If the number in this column is not the same as the number of time intervals for which information is provided in Form $G$ entries, the program will not accept the problem.

Field 3A - Columns 6 through 10 (Operating Time) - The operating time is the length of time in hours from the beginning of the first time interval to the end of the last time interval. A floating point number should be used.

Field 4A - Columns 11 through 13 (Maximum Number of Components Failed at a Time) - This field is used to regulate the amount of detail that is to be used in the analysis, which in turn helps regulate the machine time required by the problem. As the maximum number of failed components is increased the machine time increases at a $r$ ate greater than that indicated by the linear rate of change in this number. Therefore, the maximum number of failed components considered must be judiciously selected to preserve sensitivity and yet limit computer run time.

Although the value of this number may be 8 or less, it is suggested that as a rule 3 or 4 will give an adequate level of sensitivity without excessive machine time for systems with limited redundancy. For highly redundant systems excessive run times may result if this number is greater than 2 or 3. In this case the depth control feature can then be used to consider failure combinations of a higher number for specific components. As discussed in Field 13C, the depth control allows specific adjustment of the maximum number of failed components considered with any given component. Thus, internal sensitivity control is provided elsewhere in the data, and the overall input level in Field $4 \mathrm{~A}$ may be set lower than that considered for specific components.

Field 5A - Columns 14 through 16 (Number of Functions) - This input information tells the program the number of functions for which it must read input information. The program will handle a maximum of 300 functions.

Field 6A - Columns 17 and 18 (Number of Integration Intervals) - ARMM calculates probability of failure by integrating a failure density function using Simpson's Rule. Simpson's Rule requires a division of the integration interval into subintervals. The input information gives the program the number of subintervals to use in the integration. The program can accept a maximum of 24 subintervals. As a rule 10 intervals should be sufficient. 
Field 7A - Column 19 (Print Equation) - This field should always have a 1.

Field 8A - Column 20 - This field is not used.

Field 9A-Columns 21 through 23 - This field is not used.

Field 10A - Column 24 (Insert Partial Data) - A zero (0) in this column instructs the program to erase all previous data and prepare to read all input data required for this problem. A one (1) in this colunn instructs the program to keep the input data from the previous problem and prepare to replace portions of the data. There are only certain portions of the data in this program which can be changed without: reading all of the input data. These portions are:

1. The number of modes available for a function. Field 4, Columns 25 through 28 of Form B.

2. The number of modes required for a function. Field 5, Columns 29 through 32 of Form B.

3. A component's name, failure rate, beta value for the Weibull distribution, and depth control. These are in Fields 3, 5, 6, 7, 12, and 13 of Form C.

4. Cumulative time to the start of this time interval. Field 3 of Form G. Probability of failure for this time interval. Fields 5 and 6 of Form G.

Field 11A - Columns 25 through 27 (Number of Major Contributors to System Unreliability)- The program can list from 1 to 100 combinations of failed components which cause system failure. Also, it will rank them and print the major combinations. This input governs the number of failure combinations which the program will list. Of course, if there are only $\mathrm{X}$ combinations it will only print X combinations if that is less than this input. As a rule it is not necessary to specify a value in this field.

Field 12A - Columns 30 through 72 (Problem Name) - These 42 spaces are provided for the problem name. Any FORTRAN symbol may be used. This name will be printed on each page of the output.

Form A - Descriptive Information - Rows 8 through 27 - Columns 3 through 68 - This space is reserved for any descriptive purpose to which the engineer may wish to apply it. It will accept and reproduce in the output any FORTRAN character. Examples of the use of this capability are a problem description and assumptions made in the analysis. 
Each card used for this purpose must be identified with a four (4) in Column 2. A maximum of 55 cards may be used for this pur pose.

Form B - Function Data

This form organizes entries for input information describing the system in terms of functions. Each card will contain the information for one function.

Field 1B - Columns 1 and 2 (Function Input Card Identification) - A 1 in Column 2 will identify this information as function information to the program. If a 1 is not in Column 2 of each function card, the program will give an error statement and proceed to the next card or job. In either case, the analysis will not be done.

Field 2B - Columns 3 through 6 (Function ID Number) - The program will accept any integer of 3 digits or less. They do not need to be in any order or have a numbering scheme. The program uses internal index numbers, but will always print the ID numbers. Standby functions are identified by a $(-)$ in Column 3.

Field 3B - Columns 7 through 24 (Function Name) - The program will accept an 18 letter name for each function. It will print this name in the input data printout and again in the output. This feature aids in the analysis of the problem printout.

Field 4B - Columns 25 through 28 (Number of Modes Available) - The number to be placed in this field is a numerical value which is given to a function when all components which support the function are in operating condition. As components supporting the function fail, they detract from the operability of the function and when a sufficient number of supporting components have failed the function will be failed. To reflect this change in function operability each component is given a "weighting factor" in each function that it supports. When a component fails, the program subtracts the component weighting factor from the "number of modes available" assigned to each function supported by the component.

A floating point number should be used in this field.

Field 5B - Columns 29 through 32 (Number of Modes Required) - The number to be placed in this field is a floating point number which is used by the program to determine when sufficient component failures have occurred to cause function failure. The program recognizes a function failure when it finds the number of modes available for the function has been reduced to less than the number of modes required. 
Field 6B - Columns 33 through 36 (Number of Alternate Functions) - An alternate function is a function which is capable of doing the same job. This input information states the number of function ID's which are alternate to a given function. There is a maximum number of 1100 alternate functions for the problem.

Field 7B through 14B - Columns 37 through 72 in Width (Alte rnate Functions) - Use only the ID number of the appropriate alternate function. Standby functions are considered to be alternate functions. However, when a standby function is listed as an alternate function, the minus sign should not be used here. Alter nate functions should be listed in increasing numerical ID numbers, one function for each 4 column field. The function numbers should be right adjusted and should not have more than 3 digits. If the function has as an alternate a series of functions all of which must work, e.g., function 10 has as alternate functions 20,30 , and 40 and they all must work, then an "A" must be put in the first column of the field of each of the alternate functions in the series except the first alternate function of the series. The "A" signifies the word "and" and indicates to the program that all $\mathrm{A}$ connected functions in the series must $w$ ork to provide an effective alternate function. In the example above, the alternate functions would be input as _-20A_30A_40. From the standpoint of program capacity, each function in a series counts as an alternate function. If more than 9 alternate functions are needed, the additional functions should be entered on a following function card with a 1 in Column 2 and alternate function number entries beginning in the field defined by Columns 37 through 40 .

Component Information

Forms $C, D$, and $E$ are used to input information about a single component. When assembling the cards for a specific component, the card from the entry on Form $C$ must be followed by cards from entries on Forms $D$ and $E$ which pertain to the same component if there are numerical entries in Fields 8 and 9 of Form C. There will always be a positive entry in Field 8.

Form C - Component General Information

Field 1C-Columns 1 and 2 - A 2 in Column 2 identifies this card as one which contains component information. Therefore, a 2 must appear in Column 2 of every row used on this page.

Field 2C - Columns 3 through 9 (Component ID Number) - These seven spaces are available for component identification. They provide space for a 3 -digit component ID number, a decimal point, and a 2 -digit subsystem 
identification number which may be used by the programmer for reference information; e.g., the particular failure mode being considered. The subsystem number and the decimal point need not be used; however, in either case, the component ID number should be right adjusted in Field $2 \mathrm{C}$.

Field 3C-Columns 10 through 27 (Component Name) - These 18 spaces are to be used for an alphanumeric component name. They are placed in temporary storage and printed beside the component ID numbers when the input and output data is printed.

Field 4C-Columns 28 through 32 - This field is not used.

Field 5C - Columns 33 through 39 (Cyclic Failure Rate) - This field is to be used only if the component operates cyclically and has a cyclic failure rate. If the component operates continuously, then this field should be left blank. When it is used, the failure rate must be expressed as failures per million cycles. A floating point number must be used.

Field 6C - Columns 40 through 46 (Average Number of Cycles per Hour) This field must be used if and only if Field $5 \mathrm{C}$ is used. A floating point number must be used.

Field 7C - Columns 47 through 54 (Continuous Failure Rate) - If a component operates continuously throughout the time intervals under consideration, then its failure rate must be listed here. The failure rate must be expressed as the number of failures per million hours. A floating point number must be used. If the Weibull function is used for this component, then the input in this field is the parameter $\alpha$.

Field 8C - Columns 55 through 57 (Number of Functions Supported) - The effect on the system of the failure of components is determined by the effect on the functions that the component supports. The number input in this field is the number of functions in which the component is involved. Since a component may support every function, it can support a maximum of 300 functions. The sum of the numbers placed in this section for every component in the problem must not exceed 1200. If a 0 is placed in this column, the program will not read a card from Form $E$. If this number is greater than 10 , then the program will expect 2 cards from Form $E$ to follow the component card. For each additional 10, the program will expect another card from Form E. A fixed point number must be used and it must be written as far to the $r$ ight of the field as possible, i. e., it must be right adjusted.

Field 9C - Columns 58 through 60 (Number of Dependent Components) - A component can have 3 kinds of dependent components. They are (1) the "must fail" type of dependent component, (2) the "must not fail" type of dependent component, and (3) the series dependent component. Each will be identified on Form $D$. 
A "must fail" type of dependent component is one which ceases to operate upon failure of the component upon which it is dependent. An example would be a pump which is dependent on a motor because when the motor fails, the pump ceases to perform its function. Clearly, it cannot subsequently fail, so it is taken out of consideration by the program.

A "must not fail" type of dependent component is one which cannot fail when the component on which it is dependent fails. It can be used to represent mutually exclusive failure modes of a single physical com ponent; e.g., a flip-flop switch which if it fails open in one circuit must fail closed in another circuit. Another example is a valve which can fail either open or closed. These modes of failure can have different effects on the system. Once the valve is considered failed open, it cannot be considered failed closed. Each of these modes of failure is dependent on the other. To consider different failure modes of a single physical com ponent, the physical component must be read into the program as several components.

When components are arranged in a series configuration in a function, some of the components may cease operation upon the failure of an earlier component in the series, while others may not. Those that cease operation have already been mentioned as "must fail" components. Subsequent failures of those components in the series, which are not of this type, have no effect on the system failure, since a preceding failed component has already caused function failure. Hence, these operating components can be taken out of consideration without altering the result and thereby reduce the number of combinations to be considered by the program. So, a component which is in series with and fails after another component whose failure makes its consideration superfluous is considered to be series dependent and may be listed as a "must fail" dependent component.

Components of the first and third type are handled alike. They are listed on Form $D$ with a minus preceding their ID number. Their information must be listed after the information for the component upon which they are dependent.

Components of the second type are listed on Form D without a sign preceding their ID numbers. As an example, consider a switch which may fail open or closed. Let Compon;nts 1 and 2 be the switch failing open and closed, respectively. A (2) must be listed on Form D so that it appears in a proper place in the information about Component 1 , and a (1) must be listed on Form D so that it appears in the information about Component 2. 
If a $(0)$ is placed in Field 9, the program will not look for a card from Form D. For each 18 dependent components, or part thereof, a card from Form D must be coded to follow the component card.

A single component may have any number of dependent components, but the total for all components must not exceed 500 .

Field 10C-Column 61 - This field is not used.

Field 11C-Column 62 - This field is not used.

Field 12C-Columns 63-67 (Weibull Shape Parameter) - The program has been coded to use a value of 1.0 for the shape parameter of a component if no number or 0.0 is put in this field. If other values are desired, then they must be put into this field as a floating point number.

Field 13C - Column 68 (Depth Control) - This number controls the maximum number of components which can fail in a failure combination headed by the failure of this component. For this component only it overrides the value of Field $4 \mathrm{~A}$ of the general information card. Depth control can be used to avoid superfluous consideration of failure combinations, in which a component failure makes no contribution to system failure. Conversely, it allows consideration of failure combinations for a specific component involving more failed components than allowed by the number of Field $4 \mathrm{~A}$.

Form D - Dependent Component Information

If Field $9 \mathrm{C}$ of the component card is empty or has a zero $(0)$, then no information is required on this form. If Field $9 \mathrm{C}$ of the component card has a positive integer, then the fields on Form $D$ must be coded with right adjusted, component ID numbers until the number of fields filled equals that number on the component card. Do not skip any fields. Each field is 4 columns wide.

If a component is a "must fail" type of component, then it must be coded with a minus sign preceding its ID number on this card. Place the minus sign in the first column of the section. If the component is a "must not fail" type, then the first column should be left blank.

The program's analysis will not be correct if a component's "must fail" type of dependent components are listed in the input data before it. For example, if Component 20 is dependent on 10 , then the cards for 10 must precede those for 20. The program has been coded to print an error statement and to refuse to execute if it finds any "must fail" type of dependent component earlier in the on-component array than the component upon which it is dependent. 
Form E - Functions Supported Information-10 Per Card-Each Field has 7 Columns

This form has ten 7-column data fields on each line. The first three columns in each field are for the ID of the function which is supported by a component. Do not use any minus signs to differentiate between operating functions and standby functions here. Use only the function ID number.

The last 4 columns in each data field are reserved for the component weight or weighting factor in the respective supported functions. The corresponding component weighting factor will be subtracted from the "modes available" of each of the supported functions upon failure of the component. Thus the weighting factor represents the effect of a component on the operation of a function. This number can be an integer if it is right adjusted. It also can be any nonnegative decimal number which appropriately represents the effect of the component on the function. The number of weighting factors assigned to each component must be equiva lent to the number of functions supported as listed in Field $8 \mathrm{C}$. If they are not equal, the program will print an error statement and refuse to execute the problem. If a component supports more than 10 functions, then an additional card should be used from Form $E$ for each additional 10 functions.

The weighting factor for a component in support of a given function must be greater than the difference between modes available (MA) and modes required (MR) for the function, if failure of the component will cause function failure. If two components must fail to cause function failure, then the sum of the failed component weighting factors must be greater than the difference given by MA-MR. Similar analogies are used in larger component failure combinations.

Form G - Time Profile Information

Field 1G - Columns 1-2 Input Card Type 3 - The program will recognize this information as time profile information if it finds a "3" in Column 2 on every card concerning the time profile. Every row coded on this page must have a "3" listed in Column 2. If there are $\mathrm{N}$ time intervals, then there should be $N+1$ row entries or cards filled out on Form $G$.

Field 2G - Columns 3-4 (Time Interval) - In order to define a time interval, its beginning and end must be specified. This implies that $\mathrm{N}+1$ times must be specified to define $\mathrm{N}$ time intervals. The time intervals should be numbered as their beginning. The card which enters the time for the end of the last time interval should have this field blank. 
The number of intervals listed here must agree with the value coded in Field $2 \mathrm{~A}$ on Form $\mathrm{A}$, or an error statement will be printed and the problem will be rejected. Twenty time intervals is the maximum that the program will handle.

Field 3G - Columns 5-13 (Cumulative Time to the Start of this Interval) This field should have a floating point number equal to the accumulated time at the start of an interval. Thus, the entry on the first card will be 0.0 , and the entry in this field on the last card for time profile will be the accumulative time to the end of the last interval.

Field 4G-Columns 14-18-This field is not used.

Field 5G - Columns 19-32 (Probability of System Success for this Time Interval) - If this probability is known from a previous $r$ un, then it may be listed here just as it appears on the print-out of the previous run. When this field is not blank, then the program accepts the value and skips the analysis for this time interval, and begins to consider the next time interval.

Field 6G-Columns 33-48-This field is not used.

Field 7G - Columns 47-49 (Number of Functions Requiring a State Change) Functions may be turned on or off at the beginning of a time interval. Components which support only those functions which have been turned off for this interval will not be considered until those functions are turned on again. Components may support both operating and nonoperating functions during a time interval, but the program will not check nonoperating functions for a system failure.

Standby functions are in the on-function array only after the failure of an associated function is in a nonfailed condition. Since they are brought into consideration in this manner, they are not turned "on" or "off" in this field.

In this field, place the sum of the number of functions to be turned either on or off. Place the ID's of the first five functions in the fields immediately following this field on this card, each proceded by a + or - sign, as described next.

Fields 8G, 10G, 12G, 14G, 16G - (-) Off / (t) On - If a function is to be turned off, then a minus should be placed in this single column field and the function ID should follow in the next section. If a function is to be turned on, then this column may be left blank or have a plus sign. 
The program will consider all functions, except standby functions, "on" at the beginning of the first time interval. If a function is not to be considered in the first time interval, then it will have to be turned off.

Fields 9G, 11G, 13G, 15G, 17G - Function ID - These fields must contain the ID of the function whose state is changed by the immediately-preceding function change of state field. If a function is to be turned off, then a minus must be in the preceding field.

If more than 5 functions are to change state at the beginning of a time interval, then the remainder must be listed on following cards, using the format of Form $H$. In Form $H$ they must be listed in the same pattern, but beginning in Column 3. A (3) must be placed in Column 2 of these cards also.

\section{Data Arrangement}

These instructions have been arranged in the order of card type; that is, card type (1) signified function information type, (2) component information and type, and (3) time profile information. The actual deck should be arranged as shown in Figure 2.10.

\section{End of Data}

The program must be able to recognize the end of the data for a problem. This is done by a ( 1 ) in Column 1 of the last card that contains an input card type entry; i.e., the last card in the time profile information. Several problems can be worked in succession by the program because it will read data until it reads a (1) in Column 1 of a proper card, work the problem, and then read the next set of data. When inserting partial data, the end of the data must be signified in the same manner. 


\section{ILLUSTRATION OF ARMM APPLICATION}

This section shows how ARMM handles a number of situations encountered in reliability analysis. The design detail has been kept at a minimum to avoid an overly complex system. Example applications of ARMM to real systems are given in Chapter 3. Table 2. 2 lists the situations illustrated by examples.

\section{Examples}

Reliability Block Diagrams - One of the simplest reliability block diagrams consists of several components connected in series and performing one or more functions. In Figure 2.11 such a series system is shown performing both as a single function and as two functions. The order in which the components are placed in the block diagram does not have to be the same as in the flow diagram. The simple system in Figure 2.11 has been divided into functions in two ways. First the three components are grouped in the same function; this is the simplest way. However, both the tank and pump perform continuously and may be required over several time intervals, but the valve is cyclic and its operation may be required in only one interval. To remove the valve from consideration during intervals when it is not needed it must be put in a separate function which will then be turned off in those intervals. This is shown as the second case in Figure 2. 11 and requires a rearrangement of the components (in the diagram).

Dependent Components - In Figure 2.11B, Case 1, Components V and P are dependent components of Component $\mathrm{T}$ since once the tank has failed the system has failed, and it is no longer necessary to consider the additional failure of Components V and P. Similarly, Component $\mathrm{P}$ is a dependent component on Component $\mathrm{V}$.

Redundancy - In ARMM, redundancy is achieved by either the use of the proper modes available, modes required, and component weighting factors within one function, or the use of alternate operating and standby functions. Consider the three valves in Figure 2.12A as one function. The various redundancy requirements which may be imposed on these valves are none, 2 out of 3 or 1 out of 3 . These redundancy requirements are shown in block diagram form in Figure 2.12B. The use of alternate functions is illustrated in Figure 2.13. Here a system of two units of redundant components is described in three different ways by grouping the components in different functions. In the first case each unit is a function and would have to be handled in the same way as the 
function in Figure 2.12. In the second case the system is divided into two parallel functions (numbered 3 and 4 ) each an alternate of the other. In the third case, six parallel functions are used (numbered 5, 6, 7, 8, 9, and 10). Functions $6,7,8,9$, and 10 are alternate to Function 5; Functions $5,7,8,9$, and 10 are alternate to Function 6; and similarly for Functions $7,8,9$, and 10 . The modes available and required and the component weighting factors for Functions $1,2,3$, and 5 are given in Table 2.3 .

If one of the pumps is in standby then a description of the system similar to Case 1 should be used, except each pump is now a function; the standby pump is a standby function. The other two cases could be used with the functions containing the standby pump input as standby functions.

An interesting situation involving a standby component arises in the placement of bus ties in a power system. This situation and how it can be described in the ARMM input is shown in Figure 2.14. The bus tie is a standby function and is alternate to the two transformers in Function 1. This arrangement assures that when either of the transformers fail the bus tie is turned on.

Partial Data Change - An interesting use of this option occurs in the investigation of the effect of different degrees of redundancy on system reliability. The ARMM program can handle a change between runs, in the number of modes available and modes required for a function, but it cannot handle a change in the number of components which support the function. The sample case illustrated in Figure 2.15 uses a scheme which allows ARMM to calculate the effect of redundancy without having to change the number of components in a function. Case 1 represents a single valve and Case 2 a two valve system. To convert from Case 1 to Case 2 using the partial data option, Case 1 is represented by the two valves of Case 1 a. Each of these valves has a fictitious failure rate. The value of these failure rates is found as follows.

If

$$
\begin{aligned}
\mathrm{P}(\mathrm{V})= & \text { probability of failure of valve } \mathrm{V}(\text { Case } \mathrm{l}), \\
\mathrm{P}(\mathrm{b})= & \text { probability of failure of Case } \mathrm{Ib}, \\
\lambda= & \text { failure rate of the real valve } \mathrm{V} \text {, and the two } \\
& \text { valves of Case } 2, \\
\lambda_{1}, \lambda_{2}= & \text { failure rate of the two fictitious valves } \mathrm{V}-1 \text { and } \\
& \mathrm{V}-2, \text { respe-tively, }
\end{aligned}
$$


and it is desired that

$$
P(V)=P(b) \text {, }
$$

since

$$
P(V)=1-e^{-\lambda t}
$$

and

$$
\begin{aligned}
P(b) & =1-\left[1-P\left(V_{1}\right)\right]\left[1-P\left(V_{2}\right)\right]=P\left(V_{1}\right)+P\left(V_{2}\right)-P\left(V_{1}\right) P\left(V_{2}\right) \\
& =1-e^{-\left(\lambda_{1}+\lambda_{2}\right) t},
\end{aligned}
$$

then it follows that for Case 1 and Case la to give similar results

$$
\lambda=\lambda_{1}+\lambda_{2}
$$

It is convenient to make $\lambda_{1}=\lambda_{2}=1 / 2 \lambda$.

After running the problem as Case la, the effect of redundancy can be investigated in the second run by changing the values of $\lambda_{1}$ and $\lambda_{2}$ to $\lambda$ and by changing the modes available from one to two.

Maximum Number of Components Failed at a Time and Depth Control The value, measured in savings in computer time, of a proper choice of either the maximum number of components failed at a time or the depth control on any of the components can be appreciated by considering the way ARMM goes down a list of components in its search for failure combinations. Table 2.4 lists the failure combinations considered by ARMM for the system in Figure 2.13. A value of two has been assumed for the maximum number of components failed at one time. The program considers the failure of components in the order they were read into the program. So the failure of Component $\mathrm{P}-1$ is considered with the four remaining components, while for P-2 only three are considered. Also note that a three component failure combination, which could not have been picked up by the program, would be considered 
if a depth control of three had been applied to Component $\mathrm{V}-1$. In arranging components in a block diagram, it saves computer time to place those components with the higher numbered depth control towards the end. This minimizes program consideration of failed component combinations which do not cause system failure.

Similarly, it is advisable to keep the value for the maximum number of components failed and/or depth control at a low level, since some failed component combinations causing system failure do not contribute significantly to the total probability of system failure. To illustrate this point, consider a system composed of components which have a probability of failure of $1 \times 10^{-2}$ for the particular syster. operation. The probability of any double failure occurring is $1 \times 10^{-4}$. In comparison, the probability of any quadruple component failure is $1 \times 10^{-8}$. Since ten thous and of these quadruple combinations are equivalent to one double component failure combination, the contribution to system failure from failure combinations containing four or more components is negligible.

Number of Integration Intervals - Simpson's rules calculate the area under a curve passing through a given set of ordinates spaced at two or three equal intervals, by approximating the curve with a second or third order polynomial, respectively. For two intervals, the method is referred to as the Simpson's first rule and for three intervals as the second rule. In $A R M M$ when the number of integration intervals is even, the first $r$ ule is applied half as many times as there are number of intervals. When the number is odd, the second rule is applied on the first three intervals and the first rule on the remaining intervals. As shown in Table 2.5, an estimate was made of the order of magnitude error introduced by use of Simpson's rules in integrating the exponential failure density function. Since ARMM performs numerical integration to eight significant figures, it can be seen that significant integration errors can occur in the ARMM output only when $\lambda T=1$ and the number of intervals is less than approximately 10. Table 2.5 may also be used to select the proper number of intervals to avoid integration error when $\lambda T$ is less than one. In this case, the integral of the exponential failure density function for time interval 0 to $T$ is of the order of $\lambda T$. Thus, from Table 2.5 the number of integration intervals can be chosen such that the integration error introduced by using Simpson's rules is at least eight to ten orders of magnitude smaller than $\lambda \mathrm{T}$. In choosing $\lambda \mathrm{T}$ look for the component which has the highest failure rate $\lambda$. Generally, $\lambda T$ is less than one so the use of 10 integ:ation intervals should yield results without significant error. 
Configuration Changes Between Time Intervals - The ability to turn functions on and off at the start of each time interval allows the use $r$ to change the configuration of the system with time. An illustration of this is given in Figure 2.16. Pumps in a parallel system are designed with check valves on the downstream side to prevent reverse flow through the failed pump. The system is considered for three time intervals. During the first interval the pumps are to start. During the second and third intervals they are to continue operating. In the first two intervals the failure of any one component fails the system; whereas, in the third interval only one pumping train is required. The system's various configurations are represented by the seven functions indicated in Figure 2.16. The time intervals these functions are on and the components which support them are listed in Table 2.6.

Mutually Exclusive Failure Modes - In Table 2.6 the check values are given separate designations to signify two different failure modes, i.e., blockage and leakage. These are referred to as mutually exclusive failure modes, since once the check valve has failed by blockage it no longer can fail by leakage. Also note that the second failure mode (leakage) must be in a standby function to the pump, since this failure mode cannot be considered until pump failure has occurred.

Miscellaneous - Additional ways in which computer time can be minimized include (1) the grouping of all the components which are in a long series and which do not have dependencies outside of the group into one representative component, (2) the utilization of only one time interval for preliminary studies, and (3) the placement of several small problems behind one another by use of the partial data option.

Sample Problem

The application of the ARMM program will be demonstrated by combining the examples shown in Figures 2.14 and 2.17 into the single system shown in Figure 2.18. Some minor variations are made to illustrate the use of the partial data option. The reliability of the basic block diagram as shown will be calculated as Problem 1 , and that of a modified system as Problem 2.

Connected to the tank is a valve, V, represented by two fictitious valves, $\mathrm{V}-1$ and $\mathrm{V}-2$, each assigned one half of the true failure rate. This enables the valves to as sume one out of two redundancy in Problem 2 . Following the valves is the block diagram of the electrical system. Next, the diagram splits into two branches. The first branch consists of the two operating pumping trains represented by Functions 30 through 75 . 
The other branch consists of a standby pumping train represented by Functions 80, 85, and 90. Pertinent data for the functions and components is shown in Table 2.7. Partial data changes to be applied in Problem 2 are noted by asterisks.

Information about the time profile is shown in Table 2.8. During time interval $l$ the system is considered to be in a state of readiness. In order to maximize the effect on system unreliability, this interval is the time between periodic testing. In Problem 1 the test interval is assumed to be one month, while in Problem 2 the test interval is reduced to one week. During time intervals 1 and 2, two pumps are required to be operational, while during interval 3 only ore pump is required for the system success. The loadsheets for the sample calculations are shown in Figure 2.19. The sequencing card numbers are in Columns 78 to 80 . The cards numbered from 1 to 300 are for Problem 1 and those in the $500^{\prime}$ s are the changes made for Problem 2. The final data deck is arranged as shown in Figure 2. 10.

Each of the example situations listed in Table 2.2 has been considered in this sample problem. They are discussed here in the order in which they appear in the loadsheets.

1. The maximum number of failed components in a failure combination (field $4 \mathrm{~A}$ ) is set at two, since nearly all conceivable failure combinations involve two or less components. This setting is modified on two components by use of depth control.

2. The number of integration intervals (field 6A) is chosen as 2 from Table 2.5 since the larges values of $\lambda \mathrm{T}$ are on the order of $1 \times 10^{-2}$.

3. The partial data change is indicated for Problem 2 by a "l" in field $10 \mathrm{~A}$.

4. The number of modes available (field 4B) and modes required (field 5B) are taken from Table 2. 7.

5. The standby functions (field $2 \mathrm{~B}$ ) are identified as listed in Table 2.7.

6. Alternate functions (fields $7 \mathrm{~B}$ to $14 \mathrm{~B}$ ) are selected by reviewing all possible success paths at function level including those of standby functions in the block diagram of Figure 2. 18. Many alternate paths can be eliminated 
by tracing only those functions that are operating during each time interval. Note that for Function 90 there are three alternate paths and each path contains four alternate functions. Also note that path 50-60-70-75 cannot exist in a real system.

7. A depth control (field 13C) of three is applied to components 140 and 200 , in order to include all potentially significant failure combinations. For example, for function 75 to fail all three valves in that function must fail. If a depth control of three is put on the card of the first valve (Component 200), then ARMM is able to consider this failure combination. Also note that for Component 200, the depth control has been reduced to 2 as a partial data change in Problem 2 .

8. Dependent components are identified on Form D. For example, Card No. 165 for Check Valve C-1 is followed by Card No. 166 listing Component 170 as a mutually exclusive dependent component and Component 180 as a "must fail" dependent component.

9. The weighting factors of and functions supported by the components are taken from Table 2.7 and listed on Form E. An example of the placement of these cards in the deck is Card No, 167 which follows Card No. 166 cited above.

10. Configuration change with time interval is taken from Table 2. 8.

A complete set of computer output for Problem 1 is presented in Figure 2.20; the summary of Problem 2 is presented in Figure 2.21. 
SYSTEMS ANALYSIS

BY FAULT TREE EVALUATION, SAFTE-1

Fault tree analysis ${ }^{(3)}$ is a technique which provides a concise and orderly description of the various combinations of possible occurrences within a system that can result in a predefined "undesired event." Equally as important, it makes available a means with which to measure the level of safety inherent in any particular configuration. The result is an engineering capability to not only identify potential problem areas but also evaluate their overall system impact.

The concept of fault tree analysis was developed by Bell Telephone Laboratories as a technique with which to perform a safety analys is of the Minuteman launch control system. Bell engineers discovered that the method used to describe the flow of "correct" logic in data processing equipment could also be used for analyzing the "false" logic which results from component failures. Further, such a technique was ideally suited to the application of probability theory in order to numerically define critical fault modes. The Minuteman safety study was successfully completed using the new technique and provided convincing arguments for the incorporation of a number of equipment and procedure modifications.

The Boeing Company ${ }^{(4,5)}$ subsequently developed fault tree analys is to the stage of mathematical simulation (Monte Carlo), utilizing hybrid* data processing systems and time decrementing numerical procedures.

In the present study, the simulation of fault tree networks by digital means exclusively has been investigated as has the elimination of costly time decrementing procedures. In connection with this effort, a Monte Carlo fault tree simulation program (SAFTE-1) has been developed. The program, written in FORTRAN IV, assumes exponential failure and normal (Gaussian) repair at the component level. Only single phase operation is permitted. A generalized importance sampling technique has been incorporated into the program and has been tested in a number of cases. Importance sampling is necessary to increase the frequency of unlikely events in the system and thereby accomplish the following results:

1. Make possible the estimation of system failure probability for operating times which are very short compared to the mean life of the components in the system.

*Coupled digital-analog system. 
2. Significantly shorten the computation time required to achieve a given level of convergence.

The following sections describe the general features of SAFTE-1, including subroutines, input and output, and importance sampling. The results of sample calculations for an idealized two-out-of-three system and for the Dresden-3 emergency a-c power system are summarized. A listing of the source program is given in Appendix E.

\section{SAFTE-1 PROGRAM DESCRIPTION}

The SAFTE-l program is a Monte Carlo procedure for fault tree simulation. It views the system or fault tree to be analyzed as a statistical assembly of components, each characterized by an exponential failure distribution and a normal repair distribution. The system is simulated mathematically by assigning a randomely determined time to failure (TTF) and time to repair (TTR) to each component. It is then tested to determine the time at which failure occurs at the system level. Estimates of system reliability are the $n$ obtained by testing a sufficiently large population of systems in this manner.

The mechanics of the procedure can be described as follows. Initial values of TTF and TTR are computed for each component. The component TTF's are arranged in computer memory according to component I. D. number and order in which failure occurs. The computer examines the list of $\mathrm{TTF}^{\prime} \mathrm{s}$ in order of failure palssing after each failure to determine if a failed state at the system level has been achieved. If at any step a previously failed component has been repaired, a new TTF and TTR are computed for that component based on the good-as-new assumption; and it is placed back in service at the time repair is completed. The new TTF is inserted in proper sequence in computer memory. This procedure continues until a system failure occurs or a step in time is reached which exceeds a specified maximum time of interest.

The advantages of this approach relative to earlier procedures are:

1. Elimination of the need for stepping-off small time increments and examining the list of TTF's and TTR's after each step.

2. Elimination of misinformation resulting from round-off of TTF's and TTR's to integer multiples of the time increment used.

3. Elimination of the requirement for a hybrid computer. 
The major disadvantage is the limitation on problem size due to the requirement for two-dimensional ( $\mathrm{n} \times \mathrm{n}$ ) array sequencing of component failure times, where $\mathrm{n}$ is the number of components in the system. Current $32 \mathrm{~K}$ computers will handie systems of about 100 to 150 components. The larger computers which are beginning to appear should ease this limitation significantly.

Subroutines

Eight subroutines are used in SAFTE-1. The function of each of these is as follows:

1. MAIN - This routine calls the various subroutines in the proper sequence to execute the program logic. The following subroutines are called: RANDIN, EXPRN, FLTRN, SETLOG, GAUS, SEQNCE, LOGIC, SUM, and EDIT. Input data is read and printed, and the estimator arrays are zeroed. Component TTF's and TTR's are computed, and the logical operations associated with estimator weight correction and undesired event detection are performed.

2. RANDIN - This subroutine is part of a random number package utilized by SAFTE-1, which also includes EXPRN and FLTRN. RANDIN is called by MAIN at the beginning of each case with the argument DUMMY $=0$ to initialize the random number generator. EXPRN selects random numbers with an exponential distribution. FLTRN selects random numbers uniformly distributed on the unit interval. This is a machine language routine.

3. SETLOG - This subroutine sets all the logical variables false. SETLOG is called by the main program at the beginning of each trial and whatever a component is repaired and placed back into service.

4. GAUS - This subroutine, written in machine language, generates random numbers with a normal distribution.

5. SEQNCE - This subroutine arranges the current list of component TTF's in a two-dimensional array, $\operatorname{ARRAY}(I, J)$. Dimension I corresponds to the component identification number, and dimention $\mathrm{J}$ corresponds to the order of failure (e.g., $J=1$ signifies the component with the smallest TTF). 
6. LOGIC - This subroutine contains the logical arithmetic needed to describe a given fault tree. A subroutine LOGIC must be written for each different fault tree to be analyzed. Subroutine LOGIC is called after the occurrence of each component failure to determine if a failed state for the system has been achieved. If the test is positive (signifying failure), the trial is terminated and the appropriate bookkeeping is performed. Given a good fault tree diagram, subroutine LOGIC is usually simple to prepare. An example of subroutine LOGIC is given in Appendix $\mathrm{E}$ for the Dresden-3 emergency a-c power system.

7. SUM - When system failure occurs in a given trial, this subroutine is called to perform the bookkeeping operations essential to the calculation.

8. EDIT - When the specified number of trials have been completed, this subroutine operates on the data stored by SUM to compute the failure frequency function and cumulative failure distribution for the system. Several other optional outputs are available also. This includes a detailed statistical error analysis for selected operating times less than or equal to TMAX and an estimate of the probability of a given component failure resulting in the undesired event before TMAX for each component in the system. The error analysis logic has not yet been debugged.

A complete listing of the SAFTE-1 source program is given in Appendix E, including subroutine LOGIC developed for the Dresden-3 emergency a-c power system.

Input Description

A minimum of seven input data cards are required per case. These cards contain the following information and format.

Card A: FORMAT $(\stackrel{a}{\mathrm{I}} 5, \stackrel{\mathrm{b}}{\mathrm{I}} \mathrm{5}, \underset{\mathrm{I}}{\mathrm{c}}, \stackrel{\mathrm{d}}{\mathrm{I}} \mathrm{5}, \stackrel{\mathrm{e}}{\mathrm{I}} 5)$

a. NTRIAL: The number of trials.

b. IMX: The number of components (maximum 90). Must be consistent with subroutine LOGIC.

c. NOINT: The number of time intervals (maximum 250).

d. NCONS: A dummy parameter currently not used. Set NCONS $=1$.

e. NPTH: A dummy parameter currently not used. Set $\mathrm{NPTH}=1$. 


$$
\text { a b } \quad c \quad d
$$

Card B: FORMAT (E12.5, E12.5, El2.5, E12.5)

a. TMAX: Maximum time of interest (hours). The trial is terminated if a point in time is reached in the calculation exceeding TMAX before the system achieves a failed sta te.

b. AA: Biasing parameter for TTF calculation. Values of AA greater than 1.0 cause short TTF's to be emphasized.

c. BB: Biasing parameter for TTR calculations. Values of BB greater than 1.0 cause short TTR's to be deemphasized.

d. CC: Biasing parameter for TTR calculations. Values of CC greater than 1.0 cause long TTR's to be emphasized.

The direct analog calculation (i.e., unbiased) is performed by specifying a value of 1.0 for each biasing parameter.

\section{a}

Card C: FORMAT (6E12.5)

a. XMTTF(I), I = 1, IMX: The mean time to failure (hours) for each component in the system. The list is sequenced in the same order as the component I. D.'s (i.e., XMTTF (1) corresponds to component No. 1). These parameters are punched six to a card. As many cards $C$ are used as are necessary to specify IMX values of mean time to failure.

a

Card D: FORMAT (6E12.5)

a. XMTTR(I), I = 1, IMX: The mean time to repair (hours) for each component in the system. The same instructions apply as for Card C.

Card E: FORMAT (6E12.5)

a. SIG(I), I = 1, IMX: The standard deviation (hours) associated with the repair distribution of each component in the system. The same instructions apply as for Card C.

a

Card F: FORMAT (6E12.5)

a. CONS(I), I = l, NCONS: Dummy variable of which NCONS values must be entered. 
Card G: FORMAT (5I5)

a. $\mathrm{Kl}(\mathrm{I}), \mathrm{I}=1,3$ : The time interval numbers selected for statistical error analysis. Since this part of the program is not debugged, it is recommended that a single value of $K l=0$ be entered. Error analys is is then omitted.

Successive cases can be performed by inserting the additional case cards A through $G$ necessary to completely specify a problem. A sample data deck listing is given in the section dealing with the Dresden-3 emergency power system calculations.

\section{Output Description}

The input to SAFTE-1 is printed out after it is read, allowing an easy verification of the input and providing a written record of the run. In addition, the case results are printed. These include:

1. The probability of system failure before TMAX, P (TMAX).

2. The failure density function of the system failure (the probability per unit time that the system fails at time $t$ ), $p(t)$, as a function of time for the time grid specified by the input. This function is in units of hours ${ }^{-1}$. NOINT values are printed 5 to a line in order of increasing time. The interval size is TMAX/NOINT.

3. The cumulative distribution function for system failure (probability of system failure before time $T$ ),

$$
P(T)=\int_{0}^{T} p(t) d t,
$$

as a function of time for the same grid as described under 2 . It should be noted that the system reliability at time $T$ is given by

$$
R(T)=1-P(T)
$$

4. A summary table of the contribution from each component $P_{i}(T M A X)$ to $P(T M A X)$, where

$$
P(T M A X)=\sum_{i=1}^{I M X} P_{i}(\text { TMAX }) .
$$


Physically, $P_{i}$ (TMAX) represents the probability that

component $i$ is the final failure in a sequence of component failures leading to the undesired event before TMAX. $P_{i}$ (TMAX) then, is a direct measure of the sensitivity of system reliability to the reliability of component $i$.

A sample case output is given in the section dealing with the Dresden-3 emergency power system.

\section{IMPOR TANCE SAMP LING}

The fault tree integral for an " $n$ " component system without repair is

$$
P(T)=\int_{S} W\left(t_{1}, t_{2}, \ldots t_{n}\right) \prod_{i=1}^{n} f\left(t_{i}\right) d t_{i}
$$

where $f\left(t_{i}\right) d t_{i}$ is the probability that component $i$ fails between $t_{i}$ and $t_{i}+d t_{i}$. In the SAFTE-1 program, where exponential component failure is assumed, $f\left(t_{i}\right)=\lambda_{i} e^{-\lambda_{i} t_{i}}$. S represents $n$ dimensional phase space of which there exists a subset $F$ consisting of all possible system failures before time $T$. The function $W$ is defined as follows:

$$
w\left(t_{1}, t_{2}, \ldots t_{n}\right)=\left\{\begin{array}{l}
1,\left(t_{1}, t_{2}, \ldots t_{n}\right) \in F \\
0,\left(t_{1}, t_{2}, \ldots t_{n}\right) \notin F
\end{array}\right.
$$

Thus, $W=1$ if $t_{1} \ldots t_{n}$ is a point contained in $F$, otherwise $W=0$.

Fault tree simulation is performed by generating $n$ random numbers $R_{l}$, $R_{2}, \ldots R_{n}$, and computing a random time to failure $t_{i}$ for each component from

$$
R_{i}=\int_{0}^{\lambda_{1} t_{i}} f\left(x_{i}\right) d x_{i}
$$

where $0 \leq R_{i} \leq 1$. 
If each simulation is thought of as a trial, an estimate of Equation (1) is given by

$$
\widetilde{P} \cong \frac{1}{N} \sum_{j=1}^{N} w_{j},
$$

where $\mathrm{N}$ is the total number of trials and $\mathrm{W}_{j}$, as defined by Equation (2), is the Monte Carlo estimator for trial $j$. This in essence is fault tree simulation by means of analog Monte Carlo.

Analog Monte Carlo is a powerful tool for analyzing system reliability, $R(T)=1-P(T)$, for values of $P(T)>10^{-4}$. The required number of trials and hence computer costs become prohibitive when estimates of $P(T)$ much below this level are desired. For example, $4 \times 10^{4}$ trials are required in order to obtain 50 percent confidence limits of approximately an order of magnitude in the estimate of $P(T)=10^{-4}$. As $P(T)$ gets smaller, the required number of trials increases. Small $P(T)$ generally occurs when $T<<1 / \bar{\lambda}$, where $\bar{\lambda}$ is the average failure rate for system components. Inadequate sampling is then obtained in the subset $F$ of phase space to provide adequate statistics.

This inefficiency in the analog Monte Carlo procedure can usually be overcome by what is referred to as importance sampling. To permit importance sampling, Equation (1) is rewritten in the following modified form

$$
P(T)=\int_{S}\left[\frac{W\left(t_{1}, t_{2}, \ldots t_{n}\right) \prod_{i=1}^{n} f\left(t_{i}\right)}{\prod_{i=1}^{n} f^{*}\left(t_{i}\right)}\right] \prod_{i=1}^{n} f^{* k}\left(t_{i}\right) d t_{i}
$$

where $\prod_{i=1}^{n} f^{*}\left(t_{i}\right)$ is the importance function from which $t_{i}$ is now selected, a rd the quantity in brackets is the new Monte Carlo estimator $w_{j}^{*}$. The optimum importance function is

$$
\frac{W\left(t_{1}, t_{2}, \ldots t_{n}\right) \prod_{i=1}^{n} f\left(t_{i}\right)}{\int_{S} W\left(t_{1}, T_{2}, \ldots t_{n}\right) \prod_{i=1}^{n} f\left(t_{i}\right) d t_{i}},
$$

and would yield the correct answer in a single trial. 
However, for complex systems it is impossible to construct the optimum importance function since an a priori knowledge of the answer to be calculated is required. In simple systems where exact analytical solutions are obtainable, the question of an optimum importance function becomes trivial. In practice, the refore, we try to construct an $\prod_{i=1} f^{*}\left(t_{i}\right)$ which will mimic $\prod_{i=1}^{n} f\left(t_{i}\right)$ to some extent, but which will sample the subset $F$ more frequently. The condition must also be satisified

$$
\prod_{i=1}^{n} \int_{0}^{\infty} f^{*}\left(t_{i}\right) d t_{i}=1 \text {. }
$$

The current version of SAFTE-1 permits component failure sampling of the following form

$$
\prod_{i=1}^{n} f^{*}\left(t_{i}\right)=A^{n} \prod_{i=1}^{n} \lambda_{i} e^{-A \lambda_{i} t_{i}},
$$

where $A$ is a biasing parameter and corresponds to $A A$ in the program input description.

For values of $A>1.0$, Equation (7) effectively shortens component mean time to failure. To offset this distortion and preserve expectation values, the Monte Carlo estimator of Equation (4), W, becomes

$$
W^{*}\left(t_{1}, t_{2}, \ldots t_{n}\right)=W\left(t_{1}, t_{2}, \ldots t_{n}\right) \frac{1}{A^{n}} \prod_{i=1}^{n} e^{(A-1) \lambda_{i} t_{i}} .
$$

The above procedure has been exercised for an idealized two-out-of-three system without repair in order to study its general applicability for fault tree analysis. The exact solution for the two-out-of-three system with identical components is

$$
P(\lambda T)=3\left(1-e^{-\lambda T}\right)^{2} e^{-\lambda T}+\left(1-e^{-\lambda T}\right)^{3} \text {, }
$$

and for $\lambda T<<1.0$

$$
P(\lambda T) \sim 3(\lambda T)^{2}
$$


Figure 2.22 shows the results of several calculations for values of $A=1,2.5$, 5, 10. Since identical components are assumed, it is convenient to express the independent variable in Figure 2.22 as the dimensionless parameter $\lambda T$. Each calculation represents 5,000 trials. An independent sequence of pseudo-random numbers ${ }^{*}$ was used in each case. The exact solution for the two-out-of-three system is shown by the solid line. Good agreement with the exact solution is obtained using analog Monte Carlo $(A=1)$ for values of $P(\lambda T) \geq 4 \times 10^{-4}$. This corresponds to $\lambda \mathrm{T} \geq 1.2 \times 10^{-2}$. When $A=2.5$, the Monte Carlo agreement is within 30 percent for values of $\lambda T \geq 7 \times 10^{-3}$. Samples were obtained for $\lambda T<7 \times 10^{-3}$ in this case; however, these estimates exhibit extremely large statistical variance, defined as

$$
\sigma^{2}=\frac{1}{N} \sum_{j=1}^{N} w_{j}^{*} 2-\left\{\frac{1}{N} \sum_{j=1}^{N} w_{j}^{*}\right\}^{2}
$$

For the case of $A=5$, the statistical variance is improved somewhat in the range $7 \times 10^{-3} \leq \lambda \mathrm{T} \leq 1.25 \times 10^{-2}$. When $A=10$, the solution in the range $\lambda \mathrm{T} \geq 6 \times 10^{-3}$ is consistently low by about a factor of two. However. for $\lambda T<6 \times 10^{-3}$, a significant improvement over previous solutions is obtained.

The calculations of Figure 2.22 show that good estimates of $P(\lambda T)$ of order $10^{-4}$ are practical using analog Monte Carlo. It is also noted that considerable improvement in estimates of smaller values of $P(\lambda T)$ can be obtained using importance sampling. The degree of importance sampling necessary (i.e., the size of $A$ ) depends on the value of $P(\lambda T)$ being estimated as well as the desired accuracy of the result. At present, the value of $A$ appropriate to a given calculation is best determined by experimentation.

SAFTE-1 also contains a provision for importance sampling component repair distributions. The probability per unit time that component $i$ is repaired at time $x_{i}$ is given by the normal or Gaussian distribution

*Pseudo-random sequence of numbers is a sequence calculated one number at a time as needed from a completely specified prescription so devised that reasonable statistical tests will detect no significant departure from randomness. 
The parameters $\mathrm{B}$ and $\mathrm{C}$ correspond to $\mathrm{BB}$ and $\mathrm{CC}$ respectively in the input description.

For $B>1.0$, the above procedure deemphasizes short repair times which result from sampling the left side of the normal distribution (i.e., $\alpha=-1$ ), and for $\mathrm{C}>1.0$ it emphasizes long repair times which occur in sampling the right side of the distribution (i.e., $\alpha=1$ ). As in the case of the parameter $A$, values of $B$ and $C$ appropriate to a given calculation must be determined through experimentation at the present time.

In order to preserve expectation values when repair distributions are importance sampled, the Monte Carlo estimator (Equation 8) is multiplied by the additional factor

$$
\prod_{i=1}^{n} \frac{r\left(\tau_{i}\right)}{r^{*}\left(\tau_{i}\right)}=\prod_{i=1}^{n} \frac{\sigma_{i}^{\prime}}{\sigma_{i}} e^{-\tau_{i}^{2} / 2\left(1 / \sigma_{i}^{2}-1 / \sigma_{i}^{\prime 2}\right)} \text {. }
$$

The repair logic of SAFTE-1 has only been used in the case of very large component mean time to repair (MTTR) and small standard deviations to simulate systems without repair. Calculations for systems with repair have not yet been performed; consequently, this area of program logic has not been verified.

\section{SAMP LE CALCULA TION: DRESDEN-3 EMERGENCY A-C POWER SYSTEM}

A fault tree was developed for the Dresden-3 emergency a-c power system as depicted in the schematic $(6)$ of Figure 2.23 , and several sample calculations were performed. The fault tree development (Figure 2.24), which was carried to a level of detail commensurate with the schematic, describes the various combinations of possible occurrences within the system that can result in the predefined "undesired event." The undesired event in the system is Loss of Emergency A-C Power. The symbols appearing in Figure 2. 24 are defined as follows:

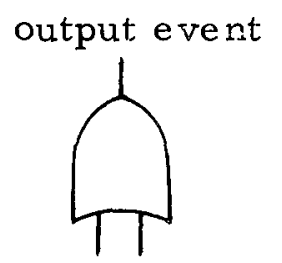

input events
The logical "OR" gate. This gate defines the logical operation whereby one or more input events are required to produce the output event. 


$$
r\left(x_{i}\right)=\frac{1}{\sigma_{i}} \frac{1}{\sqrt{2 \pi}} e^{-x_{i}^{2} / 2 \sigma_{i}^{2}}
$$

where $\sigma_{i}$ is the standard deviation for component $i$, and $x_{i}$ is displacement from the mean time to repair $\mu_{i}$. The elapsed time of repair, $d_{i}$, is then given by

$$
\mathrm{d}_{\mathrm{i}}=\mu_{\mathrm{i}}+\alpha \tau_{\mathrm{i}}
$$

where $\alpha_{T_{i}}$ is a randomly determined variation about $\mu_{1}$.

The value of $\tau_{i}$ is computed from the half-Gaussian

$$
R_{1}=\int_{0}^{\tau_{i}} r\left(x_{i}\right) d x_{i}=\frac{1}{\sigma_{i}} \sqrt{\frac{2}{\pi}} \int_{0}^{\tau_{i}} e^{-x_{i}^{2} / 2 \sigma_{i}^{2} d x_{i}},
$$

and $\alpha$ is a dummy parameter satisfying the conditions

$$
\begin{aligned}
\alpha & =1, \mathrm{R}_{2} \geq 0.5, \\
\alpha & =-1, \mathrm{R}_{2}<0.5,
\end{aligned}
$$

where $R_{1}$ and $R_{2}$ are independent, uniformly distributed random numbers. Importance sampling is performed by computing $\tau_{i}$ from the modified form of Equation (13),

$$
R_{1}=\int_{0}^{\tau_{i}} r^{*}\left(x_{i}\right) d x_{i}=\frac{1}{\sigma_{i}{ }^{\prime}} \sqrt{\frac{2}{\pi}} \int_{0}^{\tau_{i}} e^{-x_{i}^{2} / 2 \sigma_{i}{ }^{2}} d x_{i}
$$

where the following definitions apply

$$
\begin{aligned}
& \sigma_{i}^{\prime}=\sigma_{i} / \mathrm{B}, \alpha=-1, \\
& \sigma_{i}^{\prime}=\sigma_{i} \text { C, } \alpha=1 .
\end{aligned}
$$




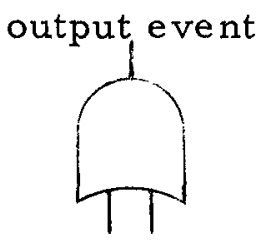

input events
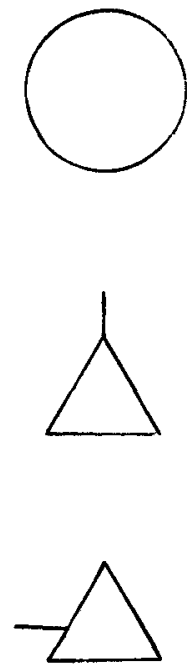

The logical "AND" gate. This gate describes the logical operation whereby the coexistence of all input events is required to produce the output event.

The circle defines a basic system component, characterized by a mé.n time to failure (MTTF) and a MrTR.

The triangle symbolizes transfer. A line from the apex of the triangle denotes transfer - in, and a line from the side denotes transfer - out.

The logical "OR" and "AND" gates appearing in Figure 2.24 are summarized in Table 2.9 with their respective consequences. Subroutine LOGIC for the Dresden-3 emergency a-c power system appears in Appendix E with the SAFTE-l program listing.

The Dresden-3 emergency a-c power system as defined in Figure 2.24 contains 57 components. The se are listed in Table 2.10 with values for MTTF and MTTR. The standard deviations assumed for the repair distributions also are given in the MTTR column. The values listed in Table 2. 10 are based on data contained in Appendix D and engineering judgment.

Several calculations were performed for various values of $A(i . e,, A=1$, $1.2,1.5)$ in which repair was not considered. Repair was deleted from the model by specifying a fictitious set of values for component MTTR's which were large compared to TMAX. Small values for standard deviation SIG were also specified. This assures that the probability of compone nt repair before TMAX will be zero. 
Figure 2.25 shows a listing of the input data card images as they appear in the analog calculation. In each calculation 3,000 trials were performed. The maximum time interval TMAX is $4.2 \times 10^{4}$ hours or 250 weeks. Using NOINT equal to 250 then requires the calculated results to be reported out with a grid size equal to 168 hours or one week. This was a convenient scale to adopt in the present calculations. A value of MTTR $=1 \times 10^{5}$ hours and SIG $=1 \times 10^{-1}$ hours was assigned to each component.

Figures 2.26A through $2.26 \mathrm{~F}$ show the most significant elements of computer output for the analog calculation. The calculated results for $P(T)$ have been plotted as a function of time in Figure 2.27 for the three cases. Identical random number sequences were used in each case. The abscissa shows operating time in weeks. As expected, fairly well converged (i.e., low variance) estimates were obtained for $P(T)$ at long operating times (i.e., $\mathrm{T} \geqq 40$ weeks) using the analog procedure. This corresponds to values of $P(T) \geqq 10^{-3}$.

When $A=1.2$, estimates for $P(T)$ are obtained at shorter operating times ( $\sim 24$ weeks); however, this appears to be accompanied by poorer convergence in the overall solution. We see this effect become even more pronounced for $A=1.5$ where the tail of the solution looks to be in error by an order of magnitude. The vertical bars shown at the 19 week data point, representing the 50 percent confidence limits for this particular point, encompass the estimated exact solution (solid curve). It is believed that significant improvements in convergence can be achieved by increasing the number of trials $N$. The extent to which $N$ must be increased in order to obtain satisfactory performance has not been determined.

Figure $2.26 \mathrm{~F}$ gives a tabulation of the individual contribution $\mathrm{P}_{\mathrm{i}}$ (TMAX) from each of the system components in the analog case. $P_{i}$ (TMAX) represents the probability that component $i$ is the final failure in a sequence of component failures which result in the undesired event previous to TMAX. Figure $2.26 \mathrm{~F}$ indicates that component X23, the diesel generator, is the largest contributor to P(TMAX). Components $\mathrm{X} 9$ and $\mathrm{X} 8$, the secondary and primary windings respectively of transformer, $T-3$, are the next largest contributors. The latter two components should contribute equally to P(TMAX); however, the number of trials performed was inadequate to show this.

Execution time for the analog calculation was approximately 81 minutes on the IC 6000, a 7094 simulator with a disadvantage factor of approximately 3 or 4 to 1 . The biased calculations required approximately 100 minutes on the same system. Although an equal number of trials were performed in each case, an increase in execution time for the 
two biased cases results from the larger average number of component failures which are considered per trial.

\section{CONCLUSIONS AND RECOMMENDATIONS}

The feasibility of fault tree simulation by digital means and without recourse to small time incrementing has been demonstrated. The Monte Carlo procedures outlined and the existing computer program (SAFTE-1) are believed to be useful tools for the mathematical analysis of fault trees. Additional work is necessary to develop these tools to their most efficient state.

It should be possible in most applications to obtain estimates of $P(T)$ as low as $10^{-3}$ or $10^{-4}$ using the analog method. Some form of importance sampling will generally be required to improve computational efficiency when smaller values of $P(T)$ are to be estimated. The procedures outlined for this purpose are relatively simple to apply and with further development. should prove very useful in this respect.

The principal factors influencing computational costs are the problem size (number of components) and the number of trials to be performed. Computational costs are directly proportional to the se factors.

Several areas exist in which additional Monte Carlo methods development and programming effort appear to be justified. These include:

1. Further investigation of importance sampling techniques.

2. Extension to multiphase operation.

3. More general failure and repair distributions.

4. Investigations of repair situations.

5. Human error.

6. More efficient random number generation.

7. Possible elimination of two-dimensional arrays.

8. Check out of error analysis portion of code. 


\section{REFERENCES}

1. McKnight, C. W., W. H. Hatton, L. J. Modiest, N. E. Schmidt, S. A. Stoneberger, and M. G. Singleton, "Automatic Reliability Mathematical Model," NA 66-838, North American Aviation, Inc.

2. McKnight, C. W., L. J. Modiest, and N. E. Schmidt, "An Automatic Reliability Mathematical Model, "Proceedings on the 1 th National Symposium on Reliability and Quality Control, Miami Beach, Florida, January 12-14, 1965.

3. Mearns, A. B., "Fault Tree Analysis: The Study of Unlikely Events in Complex Systems," System Safety Symposium Sponsored by the University of Washington and the Boeing Company, Seattle, Washington, June 1965.

4. Haasl, D. F., "Advanced Concepts in Fault Tree Analysis," System Safety Symposium sponsored by the University of Washington and the Boeing Company, Seattle, Washington, June 1965.

5. Nagel, P. M., "A Monte Carlo Method to Compute Fault Tree Probabilities, "System Safety Symposium sponsored by the University of Washington and the Boeing Company, Seattle, Washington, June 1965.

6. Dresden Nuclear Power Station, "Unit 3-Plant Design and Analysis Report," Commonwealth Edison Company, February 1966. 
TABLE 2.1

POSSIBLE FAILURE COMBINATIONS CONSIDERED BY ARMM

OF SYSTEM IN FIGURE 2.1 (NO DEPENDENCY)

\begin{tabular}{|c|c|c|}
\hline \multirow{2}{*}{$\begin{array}{c}\text { Possible Failure } \\
\text { Combinations }\end{array}$} & \multicolumn{2}{|c|}{ System } \\
\hline & Not Failed & Failed \\
\hline 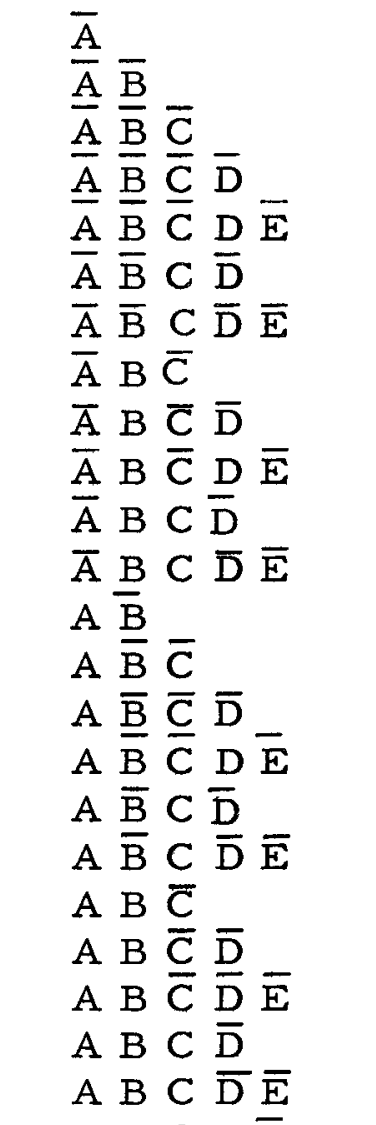 & $\begin{array}{l}\mathrm{X} \\
\mathrm{X} \\
\mathrm{X} \\
\mathrm{X} \\
\\
\mathrm{X} \\
\mathrm{X} \\
\mathrm{X} \\
\mathrm{X} \\
\mathrm{X} \\
\mathrm{X} \\
\mathrm{X}\end{array}$ & $\begin{array}{l}X \\
X\end{array}$ \\
\hline A B C D $\bar{E}$ & $\mathrm{X}$ & \\
\hline
\end{tabular}

TABLE 2.2

EXAMPLE SITUATIONS

\begin{tabular}{|l|}
\hline Reliability Block Diagrams \\
Dependent Components \\
Redundancy \\
Partial Data Change \\
Maximum Number of Components Failed at a \\
$\quad$ Time and Depth Control \\
Number of Integration Intervals \\
Configuration Changes Between Time Intervals \\
Mutually Exclusive Failure Modes \\
Miscellaneous \\
Sample Problem
\end{tabular}


TABLE 2.3

MODES AVAILABLE, MODES REQUIRED, AND WEIGHTING FACTORS

\begin{tabular}{|c|c|c|c|c|c|c|c|c|}
\hline & & & & CTIONS & & & & \\
\hline \multirow{4}{*}{ COMPONENT } & \multicolumn{2}{|c|}{1} & \multicolumn{2}{|c|}{2} & \multicolumn{2}{|c|}{3} & \multicolumn{2}{|c|}{4} \\
\hline & $\begin{array}{l}\text { Modes } \\
\text { Avail. }\end{array}$ & $\begin{array}{l}\text { Modes } \\
\text { Req'd. }\end{array}$ & $\begin{array}{l}\text { Modes } \\
\text { Avail. }\end{array}$ & $\begin{array}{l}\text { Modes } \\
\text { Req'd. }\end{array}$ & $\begin{array}{l}\text { Modes } \\
\text { Avail. }\end{array}$ & $\begin{array}{l}\text { Modes } \\
\text { Req'd. }\end{array}$ & $\begin{array}{l}\text { Modes } \\
\text { Avail. }\end{array}$ & $\begin{array}{l}\text { Modes } \\
\text { Req'd. }\end{array}$ \\
\hline & 2 & 1 & 3 & 1 & 3 & 1 & 1 & 1 \\
\hline & \multicolumn{2}{|c|}{$\begin{array}{c}\text { Component } \\
\text { Weighting } \\
\text { Factors }\end{array}$} & \multicolumn{2}{|c|}{$\begin{array}{c}\text { Component } \\
\text { Weighting } \\
\text { Factors }\end{array}$} & \multicolumn{2}{|c|}{$\begin{array}{c}\text { Component } \\
\text { Weighting } \\
\text { Factors }\end{array}$} & \multicolumn{2}{|c|}{$\begin{array}{c}\text { Component } \\
\text { Weighting } \\
\text { Factors }\end{array}$} \\
\hline $\mathrm{P}-1$ & \multicolumn{2}{|c|}{1} & & & \multicolumn{2}{|c|}{3} & \multicolumn{2}{|c|}{1} \\
\hline $\mathrm{P}-2$ & \multicolumn{2}{|c|}{1} & & & & & \\
\hline $\mathrm{V}-1$ & & & \multicolumn{2}{|c|}{1} & \multicolumn{2}{|c|}{1} & \multicolumn{2}{|c|}{1} \\
\hline
\end{tabular}


TABLE 2.4

FAILURE COMBINATIONS AND THE USE OF DEPTH CONTROL

\begin{tabular}{|c|c|c|}
\hline \multirow{2}{*}{ First Component Failed } & \multicolumn{2}{|c|}{ Combinations Considered } \\
\hline & No Depth Control & Depth Control of 3 on V- \\
\hline$P-1$ & $\begin{array}{l}P-1 \\
(P-1, P-2) \\
P-1, V-1 \\
P-1, V-2 \\
P-1, V-3\end{array}$ & $\begin{array}{l}P-1 \\
(P-1, P-2) \\
P-1, V-1 \\
P-1, V-2 \\
P-1, V-3\end{array}$ \\
\hline$P-2$ & $\begin{array}{l}P-2 \\
P-2, V-1 \\
P-2, \quad V-2 \\
P-2, \quad V-3\end{array}$ & $\begin{array}{ll}P-2 & \\
P-2, & V-1 \\
P-2, & V-2 \\
P-2, & V-3\end{array}$ \\
\hline$V-1$ & $\begin{array}{ll}\mathrm{V}-1 \\
\mathrm{~V}-1, & \mathrm{~V}-2 \\
\mathrm{~V}-1, & \mathrm{~V}-3\end{array}$ & $\begin{array}{l}\mathrm{V}-1 \\
\mathrm{~V}-1, \mathrm{~V}-2 \\
(\mathrm{~V}-1, \mathrm{~V}-2, \mathrm{~V}-3)\end{array}$ \\
\hline $\mathrm{V}-2$ & $\begin{array}{l}V-2 \\
V-2, \quad V-3\end{array}$ & $\begin{array}{l}\mathrm{V}-2 \\
\mathrm{~V}-2, \quad \mathrm{~V}-3\end{array}$ \\
\hline $\mathrm{V}-3$ & $V-3$ & $V-3$ \\
\hline
\end{tabular}

Notes: 1. Maximum number of components failed at a time $=2$.

2. Parenthesis indicates a failure combination. 


\begin{tabular}{|c|c|c|c|c|}
\cline { 2 - 5 } & \multicolumn{4}{|c|}{ Integration Error } \\
\hline$\lambda \mathrm{T}$ & 2 & 4 & 10 & 74 \\
\hline 1 & $10^{-5}$ & $10^{-6}$ & $10^{-7}$ & $10^{-11}$ \\
$10^{-1}$ & $10^{-10}$ & $10^{-11}$ & $10^{-12}$ & $10^{-16}$ \\
$10^{-2}$ & $10^{-15}$ & $10^{-16}$ & $10^{-17}$ & $10^{-21}$ \\
$10^{-3}$ & $10^{-20}$ & $10^{-21}$ & $10^{-22}$ & $10^{-26}$ \\
$10^{-4}$ & $10^{-25}$ & $10^{-26}$ & $10^{-27}$ & $10^{-31}$ \\
\hline
\end{tabular}

$\mathrm{N}$ : Number of integration intervals

$\lambda$ : Failure rate

T: Total time

TABLE 2.5

ORDER OF MAGNITUDE INTEGRATION ERROR BY USING SIMPSON'S RULE 


\begin{tabular}{|c|c|c|c|}
\hline Time Interval & Function Number & Supporting Components & Failure Mode \\
\hline 1 & 1 & $\begin{array}{l}\text { Pump P-1 } \\
\text { Pump P-2 } \\
\text { Check Valve C-1 } \\
\text { Check Valve C-1 }\end{array}$ & $\begin{array}{l}\text { Fail to start } \\
\text { Fail to start } \\
\text { Blockage } \\
\text { Blockage }\end{array}$ \\
\hline 2 & 2 & $\begin{array}{l}\text { Pump } P^{\prime}-1 \\
\text { Pump } P^{\prime}-2 \\
\text { Check Valve C-1 } \\
\text { Check Valve C-2 }\end{array}$ & $\begin{array}{l}\text { Fail to continue running } \\
\text { Fail to continue running } \\
\text { Blockage } \\
\text { Blockage }\end{array}$ \\
\hline \multirow[t]{5}{*}{3} & 3 & (same as Function 2) & \\
\hline & 4 & $\begin{array}{l}\text { Pump } P^{\prime}-1 \\
\text { Check Valve }\end{array}$ & $\begin{array}{l}\text { Fail to continue running } \\
\text { Blockage }\end{array}$ \\
\hline & (atandhy) & Check Valve & Leakage \\
\hline & 6 & $\begin{array}{l}\text { Pump } P^{\prime}-2 \\
\text { Check Valve C-2 }\end{array}$ & $\begin{array}{l}\text { Fail to continue running } \\
\text { Blockage }\end{array}$ \\
\hline & $\begin{array}{c}7 \\
\text { (standby) }\end{array}$ & Check Valve $\mathrm{C}^{\prime}-2$ & Leakage \\
\hline
\end{tabular}

TABLE 2.6

TIME INTER VALS OF F AILURE MODES 
TABLE 2.7

FUNCTION AND COMPONENT DATA

\begin{tabular}{|c|c|c|c|c|c|c|}
\hline $\begin{array}{l}\text { Function } \\
\text { Number }\end{array}$ & $\begin{array}{c}\text { Modes } \\
\text { Avallable }\end{array}$ & $\begin{array}{c}\text { Modes } \\
\text { Required }\end{array}$ & $\begin{array}{l}\text { Supporting } \\
\text { Components } \\
\end{array}$ & $\begin{array}{l}\text { Weighting } \\
\text { Factor }\end{array}$ & $\begin{array}{l}\text { Fallure Per } \\
\text { Million Hours }\end{array}$ & $\begin{array}{c}\text { Fallure } \\
\text { Mode }\end{array}$ \\
\hline 1 & 1 & 1 & Tank T & 1 & .1 & Excessive leakage \\
\hline 10 & $\begin{array}{l}1 \\
2 *\end{array}$ & 1 & $\begin{array}{l}\text { Valve } V-1 \\
\text { Valve } W-1^{*} \\
\text { Valve } V-2 \\
\text { Valve } W-2^{*}\end{array}$ & $\begin{array}{l}1 \\
1\end{array}$ & $\begin{array}{l}5.0 \\
10.0^{*} \\
5.0 \\
10.0^{*}\end{array}$ & $\begin{array}{l}\text { Fail to open } \\
\text { Fail to open }\end{array}$ \\
\hline 20 & $\mathbf{1}$ & 1 & $\begin{array}{l}\text { Transformer } X-1 \\
\text { Transformer } X-2\end{array}$ & $\begin{array}{l}1 \\
1\end{array}$ & $\begin{array}{ll}5 & 0 \\
5 & 0\end{array}$ & $\begin{array}{l}\text { Fail to supply power } \\
\text { Fa1l to supply power }\end{array}$ \\
\hline $\begin{array}{c}25 \\
\text { (Standby) }\end{array}$ & 2 & 1 & $\begin{array}{l}\text { Bus Tre B-T } \\
\text { (Others see Function } 20 \text { ) }\end{array}$ & 2 & 20 & Fall to close \\
\hline $\begin{array}{l}30 \\
\text { (Interval 1) }\end{array}$ & 1 & 1 & $\begin{array}{l}\text { Pump P-1 } \\
\text { Check Valve C-1 } \\
\text { Pump P-2 } \\
\text { Check Valve C-2 }\end{array}$ & $\begin{array}{l}1 \\
1 \\
1 \\
1\end{array}$ & $\begin{array}{r}10.0 \\
.25 \\
10.0 \\
.25\end{array}$ & $\begin{array}{l}\text { Fail to } s \operatorname{tart} \\
\text { Blockage } \\
\text { Fail to } s \operatorname{tart} \\
\text { Blockage }\end{array}$ \\
\hline $\begin{array}{l}35 \\
\text { (Interval 2) }\end{array}$ & 1 & 1 & $\begin{array}{l}\text { Pump } P^{\prime}-1 \\
\text { Check Valve C-1 } \\
\text { Pump } P^{\prime}-2 \\
\text { Check Valve C-2 }\end{array}$ & $\begin{array}{l}1 \\
1 \\
1 \\
1\end{array}$ & $\begin{array}{l}5.0 \\
.25 \\
5.0 \\
.25\end{array}$ & $\begin{array}{l}\text { Fail to continue running } \\
\text { Blockage } \\
\text { Fall to continue running } \\
\text { Blockage }\end{array}$ \\
\hline 50 & 2 & 1 & (Same as Function 35) & & & \\
\hline $\begin{array}{l}55 \\
\text { (Intervals } \\
2 \text { and } 3 \text { ) }\end{array}$ & 1 & 1 & (See Function 35) & & & \\
\hline $\begin{array}{c}60 \\
\text { (Standby) }\end{array}$ & 1 & 1 & Check Valve $C^{\prime}-1$ & 1 & 10 & Leakage \\
\hline $\begin{array}{c}65 \\
\text { (Intervals } \\
2 \text { and 3) }\end{array}$ & 1 & 1 & (See Function 35) & & & \\
\hline $\begin{array}{c}70 \\
\text { (Standby) }\end{array}$ & 1 & 1 & Check Valve $C^{\prime}-2$ & 1 & 1.0 & Leakage \\
\hline 75 & 3 & $\begin{array}{l}1 \\
2^{*}\end{array}$ & $\begin{array}{l}\text { Valve } V-5 \\
\text { Valve } V-6 \\
\text { Valve } V-7\end{array}$ & $\begin{array}{l}1 \\
1 \\
1\end{array}$ & $\begin{array}{ll}10 & 0 \\
10 & 0 \\
10 & 0\end{array}$ & $\begin{array}{l}\text { Fail to open } \\
\text { Fail to open } \\
\text { Fail to open }\end{array}$ \\
\hline $\begin{array}{c}80 \\
\text { (Standby for } \\
\text { Interval 1) }\end{array}$ & 6 & $\begin{array}{l}1 \\
2 *\end{array}$ & $\begin{array}{l}\text { Pump P-1 } \\
\text { Pump P-2 } \\
\text { Pump P-3 } \\
\text { Check Valve C-1 } \\
\text { Check Valve C-2 } \\
\text { Check Valve C-3 } \\
\text { Valve V -3 } \\
\text { Valve V -4 } \\
\text { Valve V -5 } \\
\text { Valve V-6 } \\
\text { Valve V -7 }\end{array}$ & $\begin{array}{l}3 \\
3 \\
3 \\
3 \\
3 \\
3 \\
3 \\
3 \\
1 \\
1 \\
1\end{array}$ & $\begin{array}{l}10.0 \\
10.0 \\
10.0 \\
25 \\
.25 \\
25 \\
100 \\
100 \\
100 \\
10.0 \\
10.0\end{array}$ & $\begin{array}{l}\text { Fall to start } \\
\text { Fail to start } \\
\text { Fall to start } \\
\text { Blockage } \\
\text { Blockage } \\
\text { Blockage } \\
\text { Fall to open } \\
\text { Fail to close } \\
\text { Fail to open } \\
\text { Fail to open } \\
\text { Fall to open }\end{array}$ \\
\hline $\begin{array}{l}\quad 85 \\
\text { (Standby for } \\
\text { Interval } 2 \text { ) }\end{array}$ & 6 & $\begin{array}{l}1 \\
2^{*}\end{array}$ & $\begin{array}{l}\text { Pump } P^{\prime}-1 \\
\text { Pump } P^{\prime}-2 \\
\text { Pump } P^{\prime}-3 \\
\text { Check Valve } C^{\prime}-1 \\
\text { Check Valve } C^{\prime}-2 \\
\text { (Others see Function } 80 \text { ) }\end{array}$ & $\begin{array}{l}3 \\
3 \\
3 \\
3 \\
3\end{array}$ & $\begin{array}{ll}5 & 0 \\
5 & 0 \\
5 & 0 \\
1 & 0 \\
1 . & 0\end{array}$ & $\begin{array}{l}\text { Fail to continue running } \\
\text { Fail to continue running } \\
\text { Fail to continue running } \\
\text { Leakage } \\
\text { Leakage }\end{array}$ \\
\hline $\begin{array}{l}90 \\
\text { (Standby for } \\
\text { Interval 3) }\end{array}$ & 3 & $\begin{array}{l}1 \\
2^{*}\end{array}$ & $\begin{array}{l}\text { Pump } P^{\prime}-3 \\
\text { (Others see Functions } 80 \\
\text { and } 85 \text { ) }\end{array}$ & 3 & 5.0 & Fail to continue running \\
\hline
\end{tabular}


TABLE 2.8

TIME PROFILE

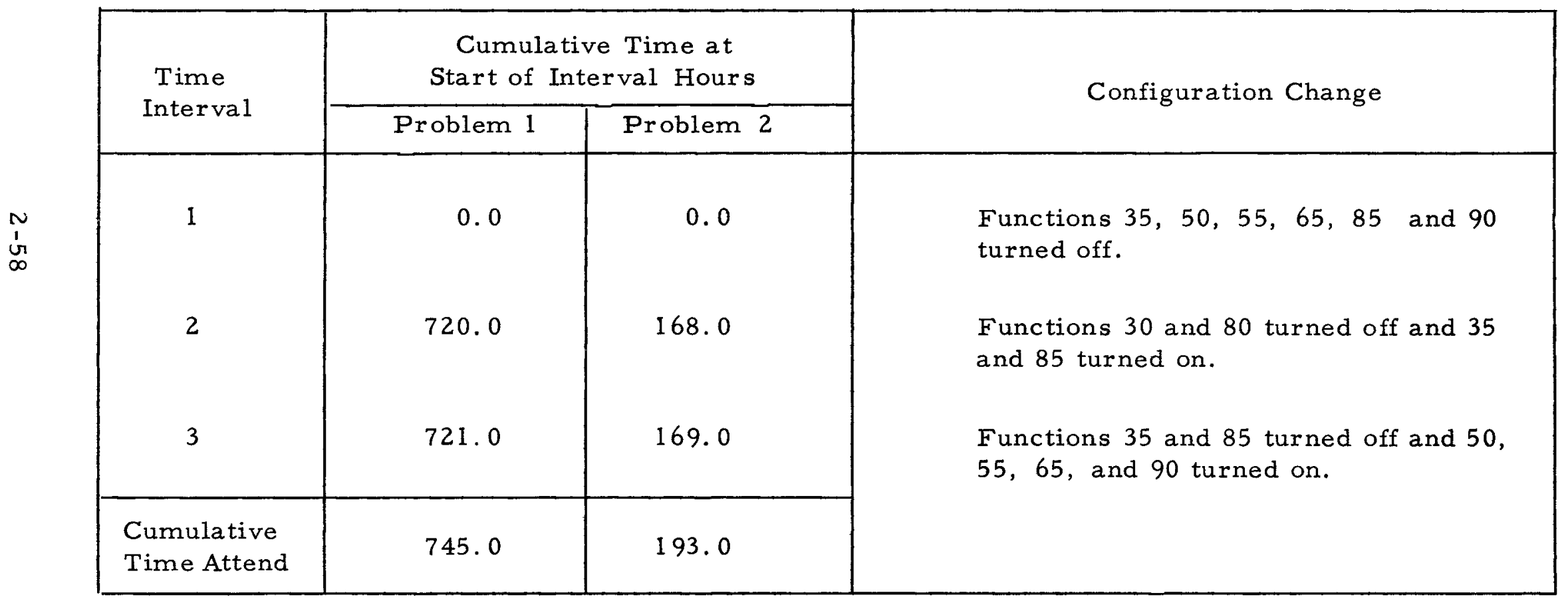


TABLE 2.9

LOGICAL "OR" AND "AND" GATES APPEARING IN DRESDEN -3 EMERGENCY AC POWER SYSTEM FAULT TREE

\begin{tabular}{|c|c|}
\hline $\begin{array}{l}\text { Logical Gate } \\
\text { Identification }\end{array}$ & Conse quence \\
\hline Al & No Power To T-1 \\
\hline $\mathrm{A} 2$ & T-1 Fails Open \\
\hline A3 & Line 12 Dead \\
\hline A4 & Line 11 Dead \\
\hline A5 & No Power To Line 13 \\
\hline A6 & No Power To T-3 \\
\hline A7 & T-3 Fails Open \\
\hline A8 & No Power From $\mathrm{T}-3$ \\
\hline A9 & Line 32 Dead \\
\hline A10 & Line $32 \mathrm{~A}$ Dead \\
\hline All & BAB-32 Source Dead \\
\hline $\mathrm{A} 12$ & No Power To T-2 \\
\hline Al3 & T-2 Fails Open \\
\hline A14 & No Power To Line 21 \\
\hline Al 5 & $\begin{array}{l}\text { No Power To BAB-31 } \\
\text { \& No Power To Line } 21 \mathrm{~A}\end{array}$ \\
\hline A 16 & BAB-31 Source Dead \\
\hline A 17 & No Power To BAB-42 \\
\hline Al 8 & BAB-51 Fails Open \\
\hline A19 & No Power From BAB-5l \\
\hline $\mathrm{A} 20$ & No Power From Diesel Generator \\
\hline A21 & BAB-52 Fails Open \\
\hline $\mathrm{A} 22$ & No Power From BAB-52 \\
\hline A23 & BAB-53 Failed Open \\
\hline $\mathrm{A} 24$ & T-8 Fails Open \\
\hline $\mathrm{A} 25$ & No Power From T-8 \\
\hline A26 & No Power From BAB-53 \\
\hline $\mathrm{A} 27$ & BAB-42 Failed Open \\
\hline A28 & No Power From BAB-42 \\
\hline A29 & BAB-41 Failed Open \\
\hline
\end{tabular}


TABLE 2. 9 (continued)

\begin{tabular}{|c|c|}
\hline $\begin{array}{l}\text { Logical Gate } \\
\text { Identification }\end{array}$ & Consequence \\
\hline A30 & No Power From BAB-41 \\
\hline A3 1 & BAB-61 Fails Open \\
\hline A32 & No Power From BAB-62 \\
\hline A33 & BAB-62 Fails Open \\
\hline A34 & No Power From BAB-62 \\
\hline A35 & No Power To $T-8$ \\
\hline A36 & BAB-63 Fails Open \\
\hline A37 & No Power From BAB-63 \\
\hline A38 & BAB-54 Failed Open \\
\hline A39 & No Power From BAB-54 \\
\hline A40 & No Power To $T-6$ \\
\hline A4l & T-6 Fails Open \\
\hline A42 & No Power From T-6 \\
\hline$A 43$ & No Power To BAB-2A \\
\hline A44 & BAB-2A Fails Open \\
\hline A45 & BUS-2A Dead \\
\hline$A 46$ & BAB-64 Fails Open \\
\hline A47 & No Power From BAB-64 \\
\hline A48 & No Power To $\mathrm{T}-7$ \\
\hline A49 & T-7 Fails Open \\
\hline A 50 & No Power From T-7 \\
\hline A51 & No Power To BAB-2B \\
\hline A 52 & BAB-2B Fails Open \\
\hline A53 & BUS-2B Dead \\
\hline $\mathrm{B} 1$ & No Power To BAB-51 \\
\hline $\mathrm{B} 2$ & BUS-5 Dead \\
\hline B3 & No Power to $\mathrm{BAB}-61$ \\
\hline B4 & BUS 6 Dead \\
\hline B5 & Loss of $4160 \mathrm{~V}$ Power \\
\hline B6 & Loss of $480 \mathrm{~V}$ Power \\
\hline B7 & Loss of Emergency AC Power \\
\hline
\end{tabular}


TABLE 2.10

COMPONENT FAILURE AND REPAIR DATA FOR DRESDEN-3 EMERGENCY AC POWER SYSTEM

\begin{tabular}{|c|c|c|c|}
\hline $\begin{array}{c}\text { Component } \\
\text { I. D. }\end{array}$ & Failure & MTTF (hours) & MTTR (hours) \\
\hline $\mathbf{x l}$ & T-1 $345 \mathrm{KV}$ Bus Connection Fails & $1.0 \times 10^{6}$ & $10 \pm 5$ \\
\hline $\mathrm{x} 2$ & $345 \mathrm{KV}$ Bus Dead & $1.0 \times 10^{7}$ & $15 \stackrel{ \pm}{ \pm} .5$ \\
\hline $\mathbf{x} 3$ & T-l Primary Fails Open & $1.11 \times 10^{5}$ & $48 \pm 12$ \\
\hline $\mathrm{X} 4$ & T-l Secondary Fails Open & $1.11 \times 10^{5}$ & $48 \stackrel{t}{-} 12$ \\
\hline $\mathbf{X} 5$ & Unit 3 Generator Fails & $1.0 \times 10^{4}$ & $334 \pm 50$ \\
\hline $\mathrm{x} 6$ & Line 11 Broken & $1.0 \times 10^{6}$ & $5 \pm 2.5$ \\
\hline $\mathrm{x} 7$ & T-3 Bus Connection Fails & $1.0 \times 10^{6}$ & $10 \pm 5$ \\
\hline $\mathrm{X} 8$ & T-3 Primary Fails Open & $1.11 \times 10^{5}$ & $48 \pm 12$ \\
\hline $\mathrm{x} 9$ & T-3 Secondary Fails Open & $1.11 \times 10^{5}$ & $48 \pm 12$ \\
\hline $\mathrm{X} 10$ & Line 32 Broken & $1.0 \times 10^{6}$ & $5 \pm 2.5$ \\
\hline $\mathrm{X} 11$ & Line 32A Broken & $1.0 \times 10^{6}$ & $5 \pm 2.5$ \\
\hline $\mathrm{X} 12$ & BAB-32 Fails Open & $2.0 \times 10^{5}$ & $8 \stackrel{+}{-} 4$ \\
\hline $\mathrm{X} 13$ & Line 13 Broken & $1 \times 10^{6}$ & $5 \pm 2.5$ \\
\hline $\mathrm{X} 14$ & T-2 Primary Fails Open & $1.11 \times 10^{5}$ & $48 \pm 12$ \\
\hline $\mathbf{X} 15$ & T-2 Secondary Fails Open & $1.11 \times 10^{5}$ & $48 \pm 12$ \\
\hline $\mathrm{X} 16$ & Line 21 Broken & $1 \times 10^{6}$ & $5 \pm 2.5$ \\
\hline $\mathbf{X} 17$ & BAB-31 Fails Open & $2.0 \times 10^{5}$ & $8 \pm 4$ \\
\hline $\mathrm{X} 18$ & Line $21 \mathrm{~A}$ Broken & $1 \times 10^{6}$ & $5 \pm 2.5$ \\
\hline $\mathrm{X} 19$ & BAB-51 Failed Open & $2.0 \times 10^{5}$ & $8 \pm 4$ \\
\hline$\times 20$ & BAB-5l Manual Actuator Fails & $2.0 \times 10^{5}$ & $3 \pm 1.5$ \\
\hline $\mathrm{X} 21$ & BAB-52 Failed Open & $2.0 \times 10^{5}$ & $8 \stackrel{+}{-4}$ \\
\hline $\mathrm{X} 22$ & BAB-52 Auto-Actuator Fails & $1.0 \times 10^{5}$ & $3 \pm 1.5$ \\
\hline $\mathrm{X} 23$ & Diesel Generator Fails & $1.0 \times 10^{5}$ & $200 \pm 50$ \\
\hline $\mathrm{X} 24$ & Line To Diesel Generator Broken & $1.0 \times 10^{6}$ & $5 \pm 2.5$ \\
\hline$\times 25$ & BAB-53 Auto-Actuator Failed & $1.0 \times 10^{5}$ & $3 \pm 1.5$ \\
\hline $\mathrm{X} 26$ & BAB-53 Failed Open & $2.0 \times 10^{5}$ & $8 \pm 4$ \\
\hline $\mathbf{X} 27$ & T-8 Secondary Fails Open & $1.11 \times 10^{5}$ & $48 \pm 12$ \\
\hline $\mathbf{X} 28$ & T-8 Primary Fails Open & $1.11 \times 10^{5}$ & $48 \pm 12$ \\
\hline
\end{tabular}


TABLE 2.10 (continued)

\begin{tabular}{|c|c|c|c|}
\hline $\begin{array}{l}\text { Component } \\
\text { I. D. }\end{array}$ & Failure & MTTF (hours) & MTTR (hours) \\
\hline $\mathrm{x} 29$ & Line From $\mathrm{T}-8$ Broken & $1.0 \times 10^{6}$ & $5 \pm 2.5$ \\
\hline $\mathrm{x} 30$ & BAB-42 Failed Open & $2.0 \times 10^{5}$ & $8 \pm 4$ \\
\hline X31 & BAB-42 Auto-Actuator Fails & $1.0 \times 10^{5}$ & $3 \pm 1.5$ \\
\hline$\times 32$ & BAB-4l Failed Open & $2.0 \times 10^{5}$ & $8 \pm 4$ \\
\hline $\mathrm{X} 33$ & BAB-4l Auto-Actuator Fails & $1.0 \times 10^{5}$ & $3 \pm 1.5$ \\
\hline$\times 34$ & BAB-61 Fails Open & $2.0 \times 10^{5}$ & $8 \pm 4$ \\
\hline $\mathrm{X} 35$ & BAB-61 Manual Actuator Fails & $2.0 \times 10^{5}$ & $3 \pm 1.5$ \\
\hline$\times 36$ & BAB-62 Fails Open & $2.0 \times 10^{6}$ & $8 \pm 4$ \\
\hline$\times 37$ & BAB-62 Auto-Actuator Fails & $1.0 \times 10^{5}$ & $3 \pm 1.5$ \\
\hline $\mathbf{X} 38$ & Underground Feeder To T- 8 Failed & $1.0 \times 10^{6}$ & $70 \pm 35$ \\
\hline$\times 39$ & $34.5 \mathrm{KV}$ Outage & $1.0 \times 10^{5}$ & $10 \pm 5$ \\
\hline $\mathrm{X} 40$ & BAB-63 Fails Open & $2.0 \times 10^{5}$ & $8 \pm 4$ \\
\hline $\mathrm{X} 41$ & BAB-63 Auto-Actuator Fails & $1.0 \times 10^{5}$ & $3 \pm 1.5$ \\
\hline $\mathrm{X} 42$ & BAB-54 Failed Open & $2.0 \times 10^{5}$ & $8 \pm 4$ \\
\hline $\mathrm{X} 43$ & BAB-54 Auto-Actuator Failed & $1.0 \times 10^{5}$ & $3 \pm 1.5$ \\
\hline $\mathrm{X} 44$ & Line To T-6 From BAB-54 Broken & $1.0 \times 10^{6}$ & $5 \pm 2.5$ \\
\hline $\mathrm{X} 45$ & T-6 Primary Fails Open & $1.11 \times 10^{5}$ & $48 \pm 12$ \\
\hline $\mathrm{x} 46$ & T-6 Secondary Fails Open & $1.11 \times 10^{5}$ & $48 \pm 12$ \\
\hline $\mathrm{x} 47$ & Line To T-6 Broken & $1.0 \times 10^{6}$ & $5 \pm 2.5$ \\
\hline $\mathrm{X} 48$ & BAB-2A Fails Open & $2.0 \times 10^{5}$ & $8 \pm 4$ \\
\hline $\mathrm{X} 49$ & BAB-2A Auto-Actuator Fails & $1.0 \times 10^{5}$ & $3 \pm 1.5$ \\
\hline $\mathrm{X} 50$ & BAB-64 Failed Open & $2.0 \times 10^{5}$ & $8 \pm 4$ \\
\hline$\times 51$ & BAB-64 Auto-Actuator Failed & $1.0 \times 10^{5}$ & $3 \pm 1.5$ \\
\hline$\times 52$ & Line From BAB-64 Broken & $1.0 \times 10^{6}$ & $5 \pm 2.5$ \\
\hline$\times 53$ & T-7 Primary Fails Open & $1.11 \times 10^{5}$ & $48 \pm 12$ \\
\hline $\mathrm{X} 54$ & T-7 Secondary Fails Open & $1.11 \times 10^{5}$ & $48 \pm 12$ \\
\hline $\mathrm{X} 55$ & Line To $T-7$ Broken & $1.0 \times 10^{6}$ & $5 \pm 2.5$ \\
\hline $\mathrm{x} 56$ & BAB-2B Fails Open & $2.0 \times 10^{5}$ & $8 \pm 4$ \\
\hline$\times 57$ & BAB-2B Auto-Actuator Fails & $1.0 \times 10^{5}$ & $3 \pm 1.5$ \\
\hline
\end{tabular}




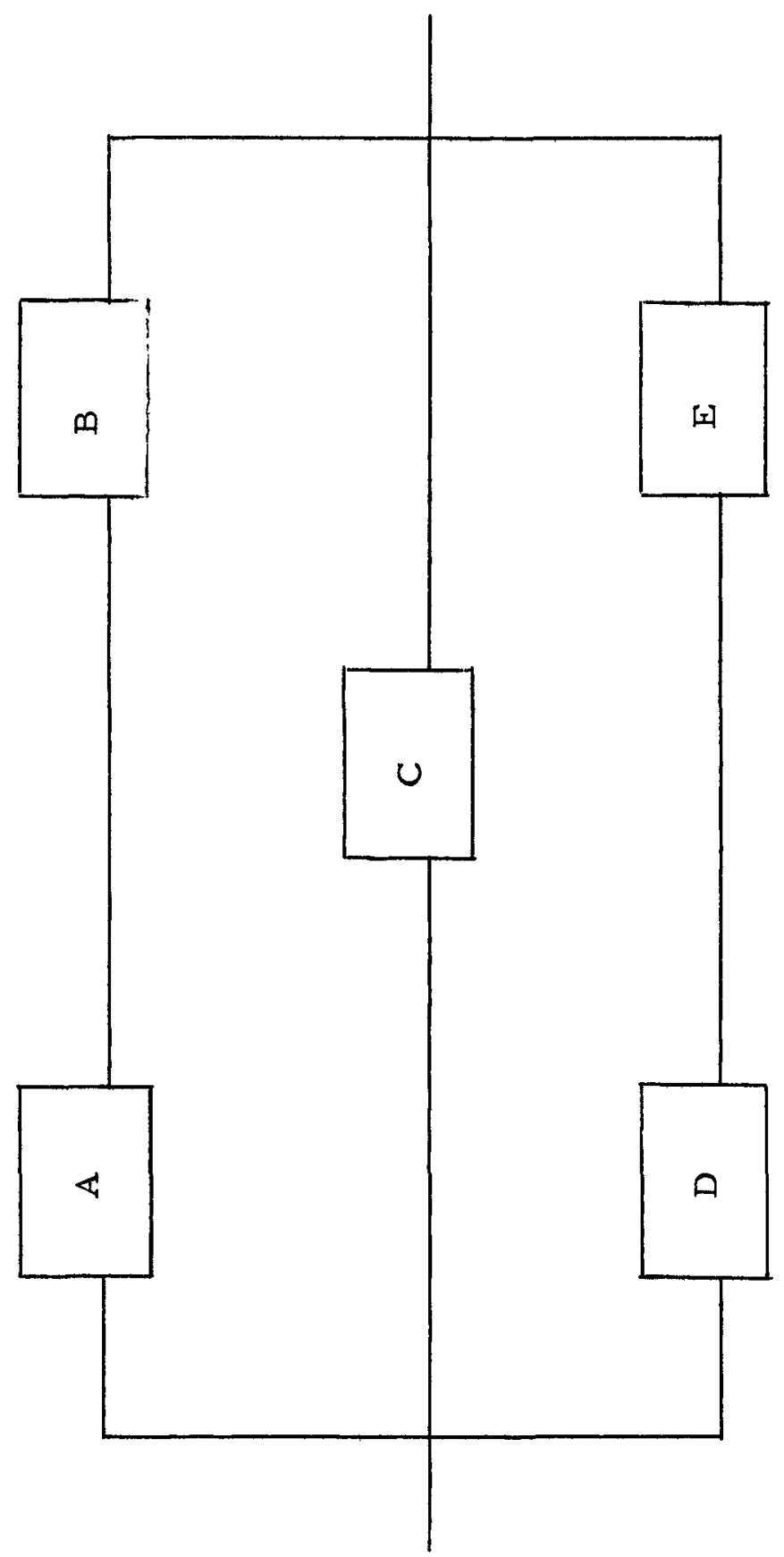

FIGURE 2.1

EXAMPLE SYSTEM

$2-63$ 
Page _ of _

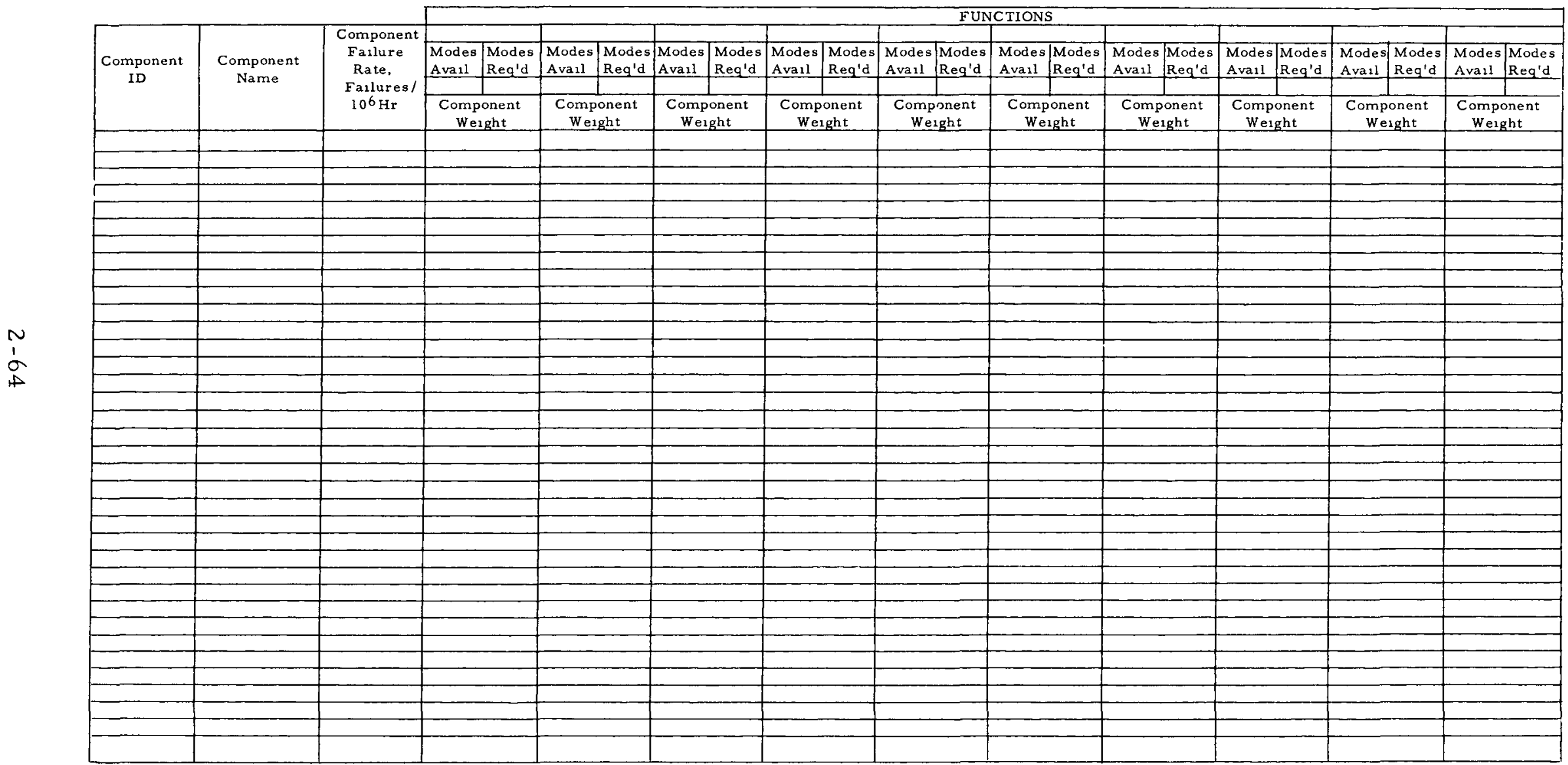

FIGURE 2.2

\section{FUNCTION-COMPONENT SUMMARY SHEET}




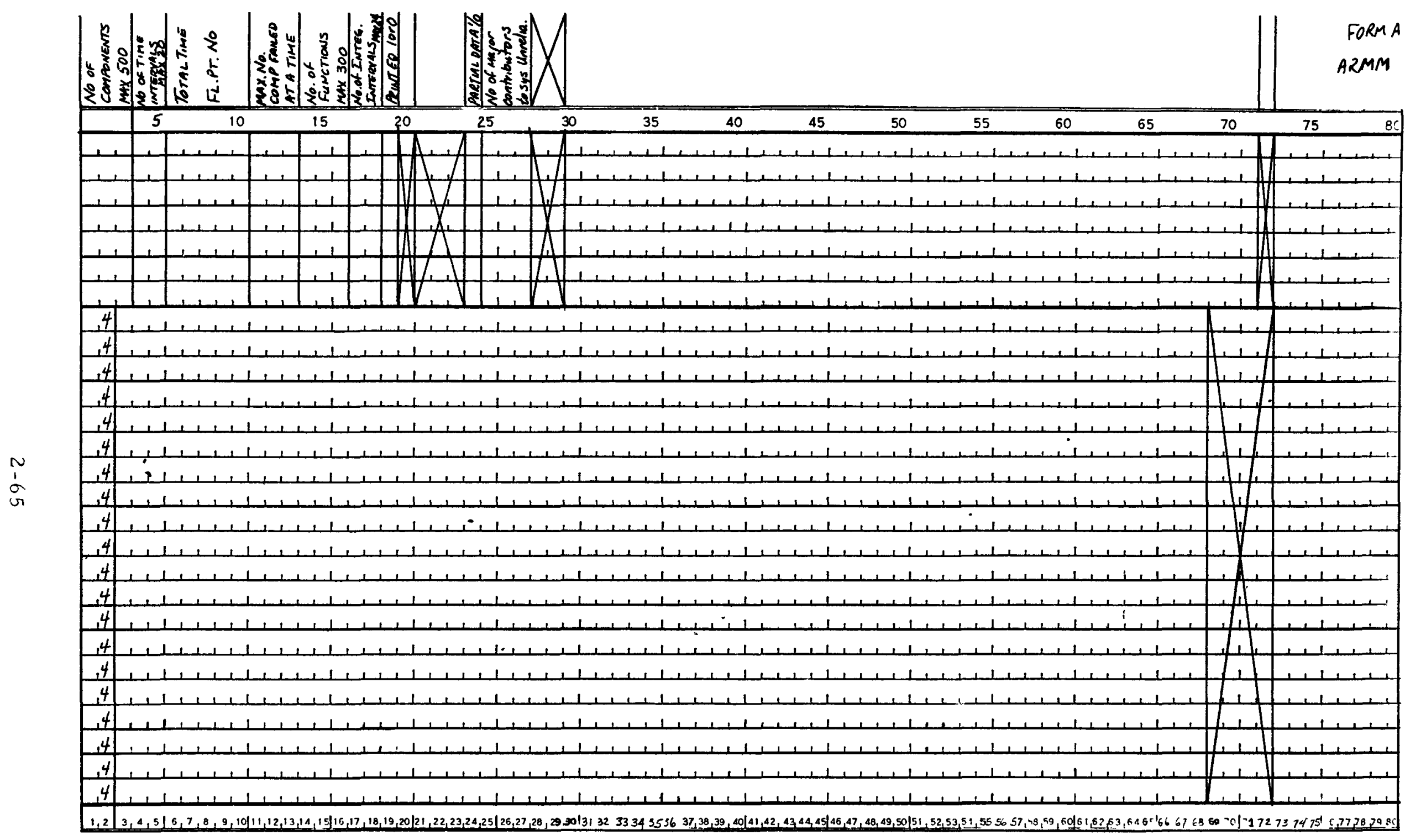

FIGURE 2.3

INPUT FORM A 


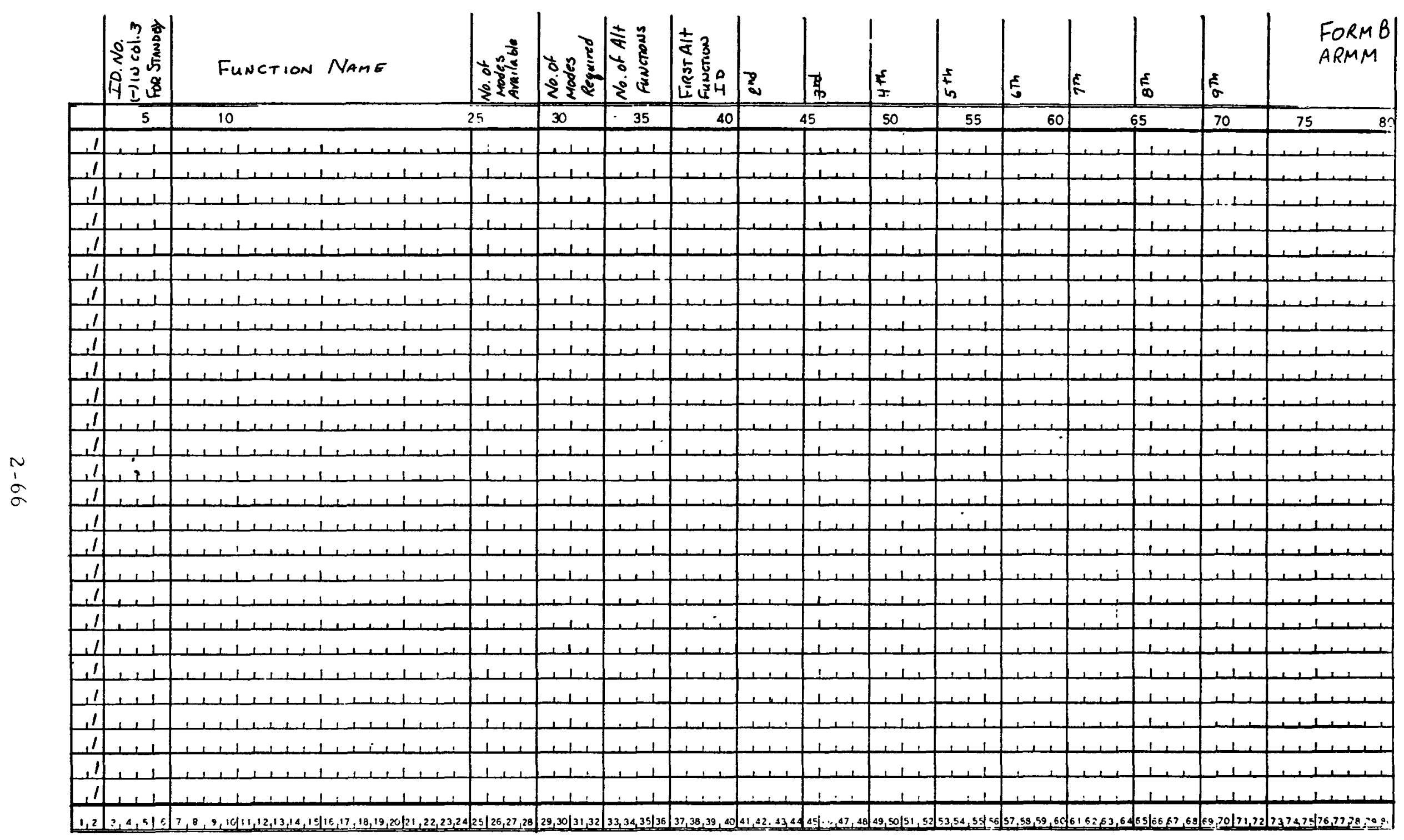

FIGURE 2.4

INPUT EORM B 


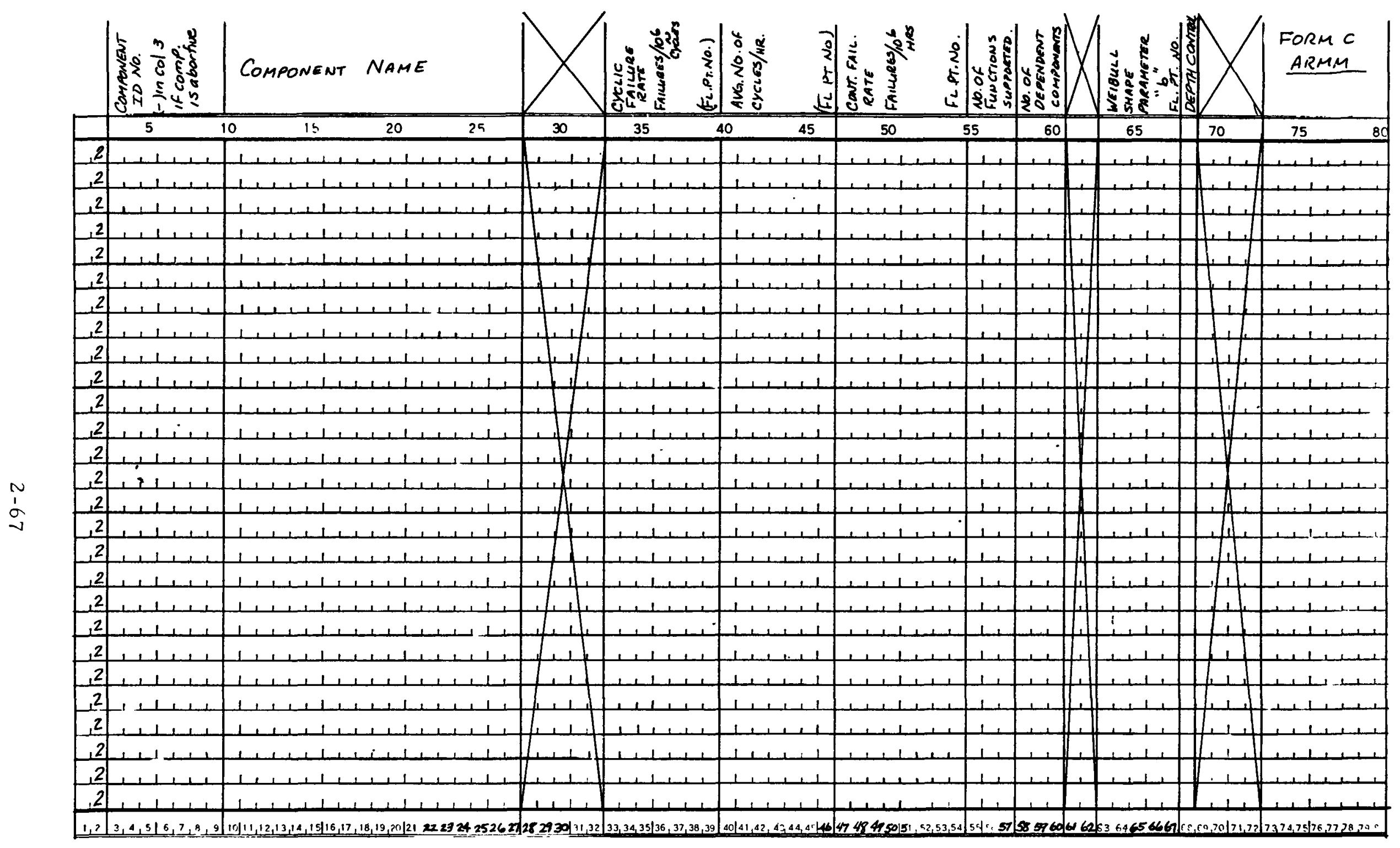

FIGURE 2.5

INPUT FORM C 


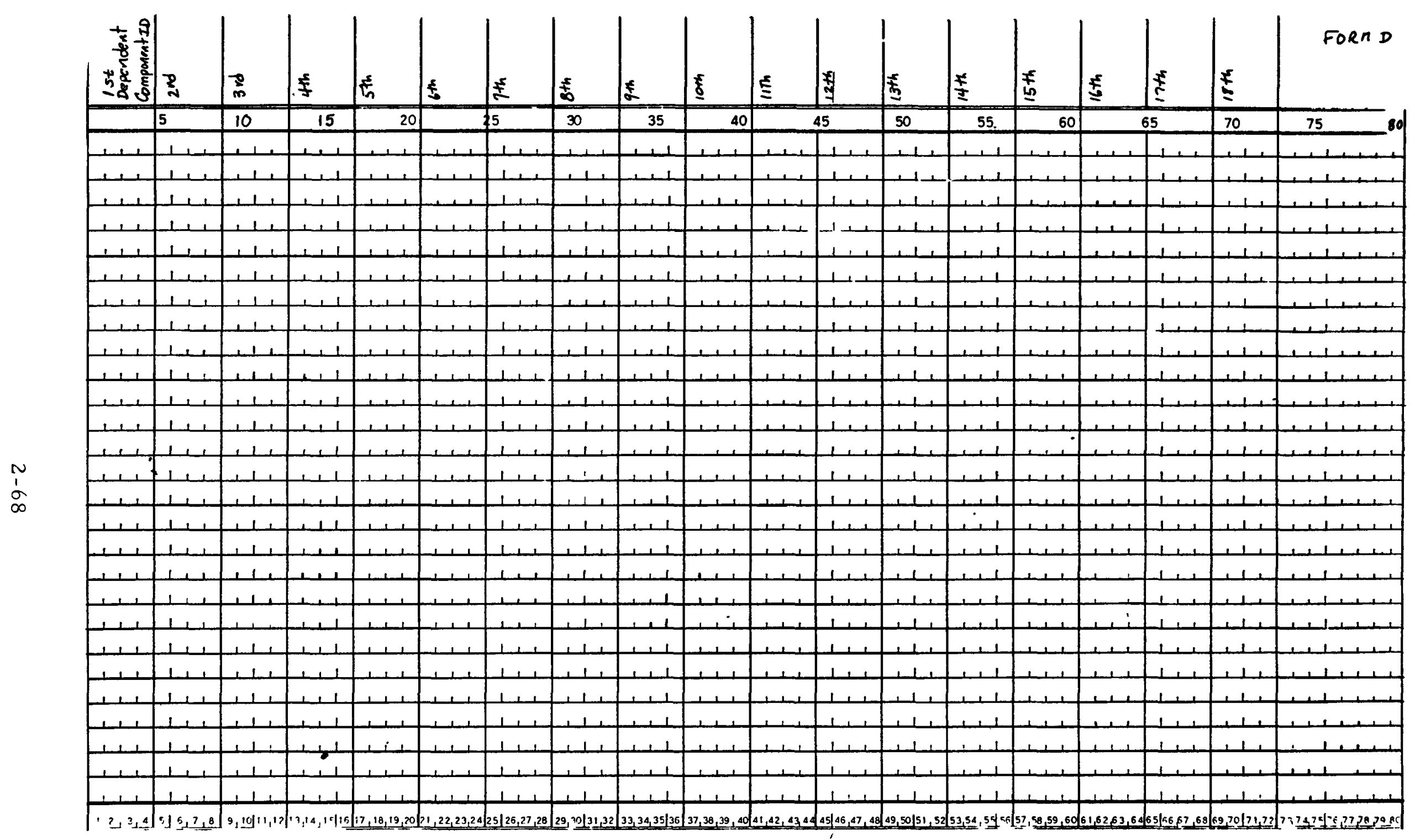

FIGURE 2.6

INPUT FORM D 


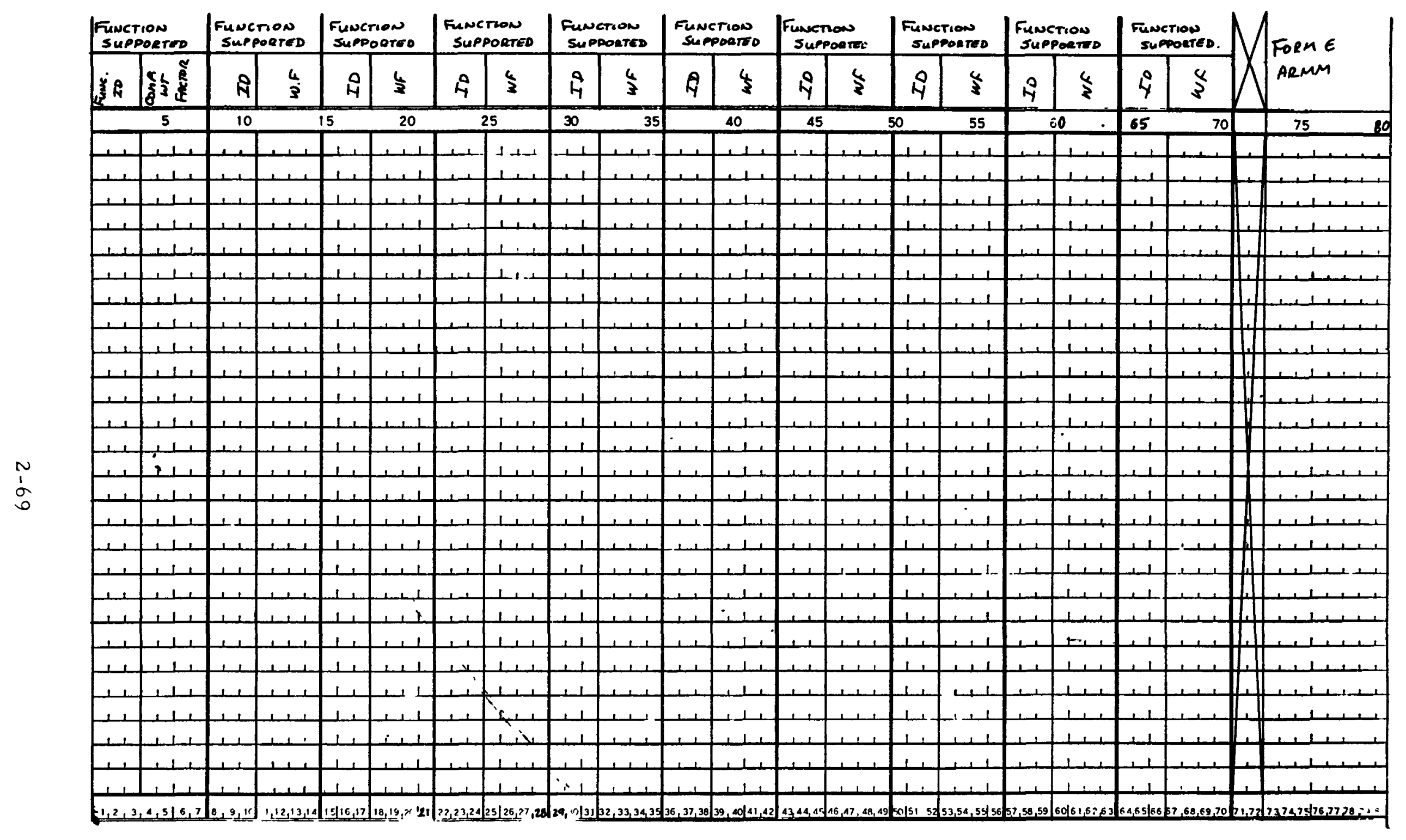

FIGURE 2.7

INPUT FORM E 


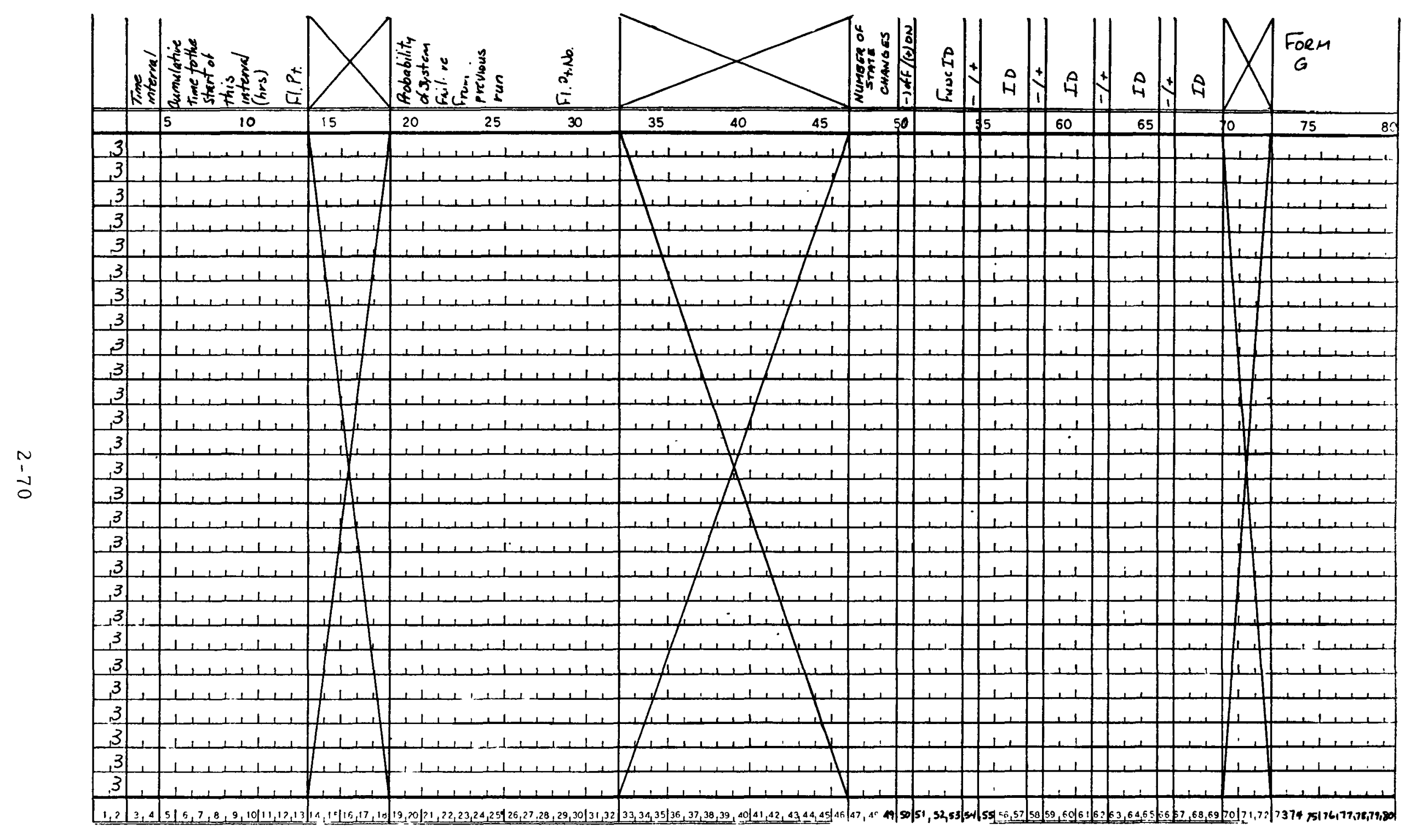

FIGURE 2.8

INPUT FORM G 


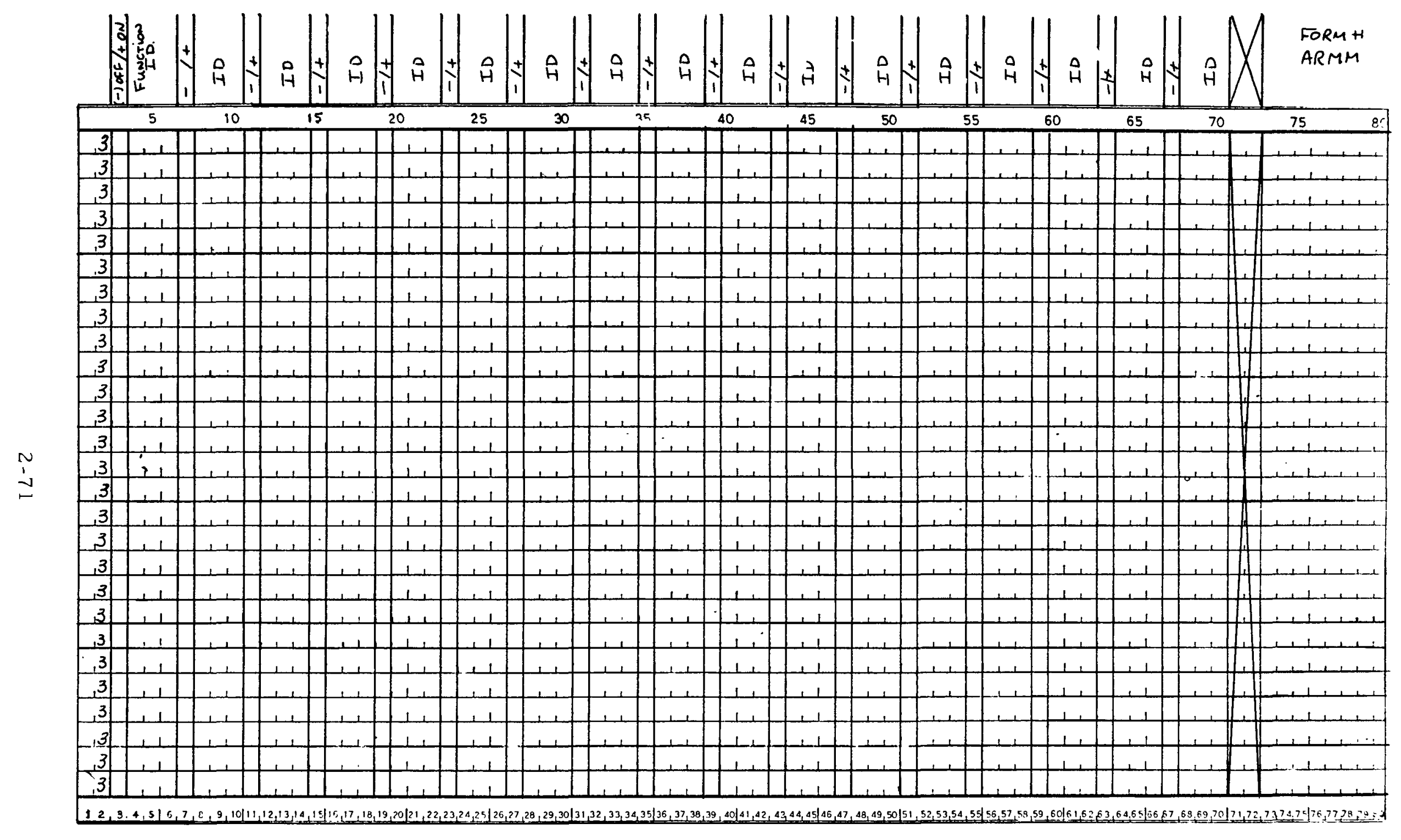

FIGURE 2.9

INPUT FORM H 


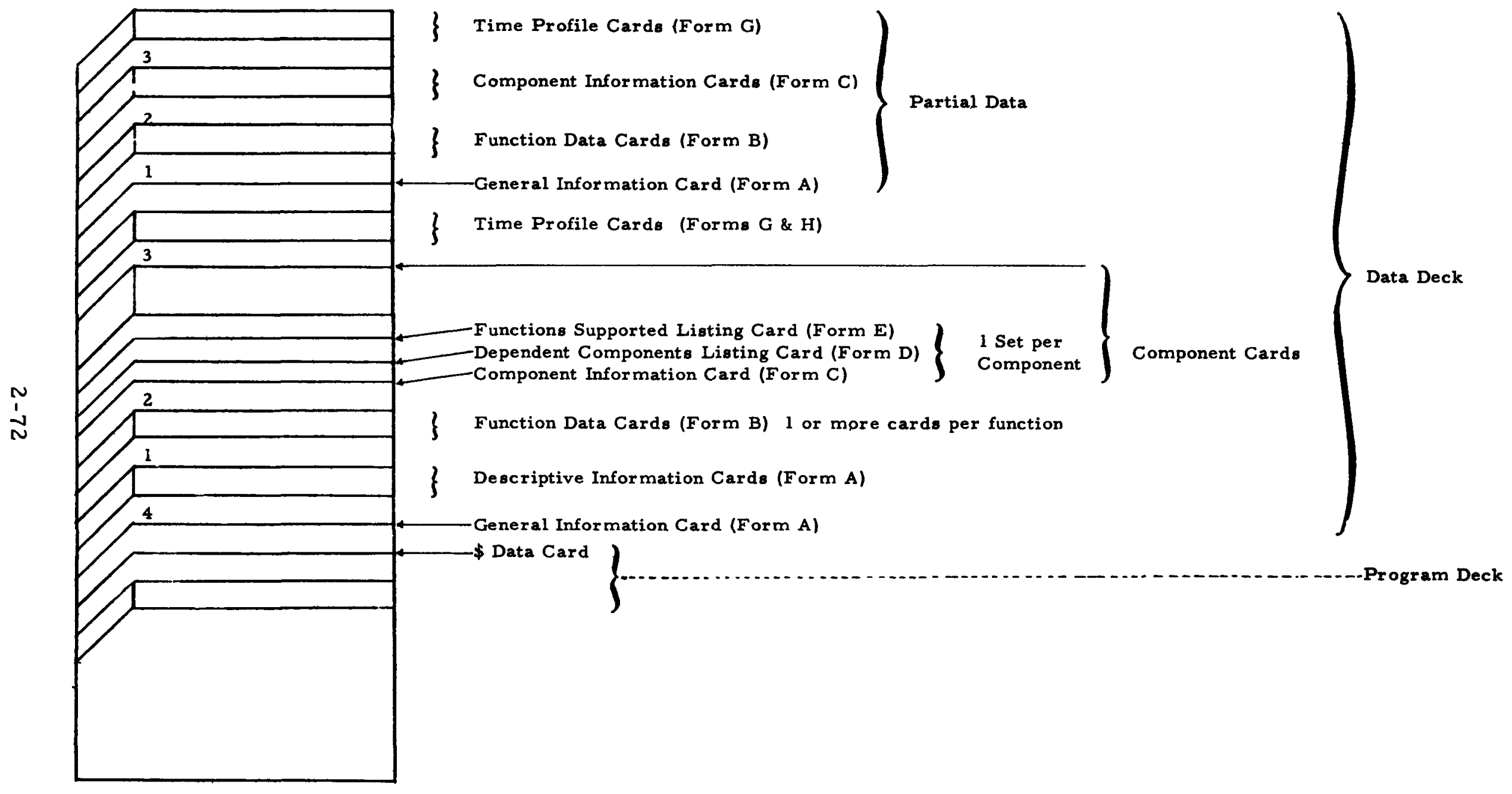

FIGURE 2.10 


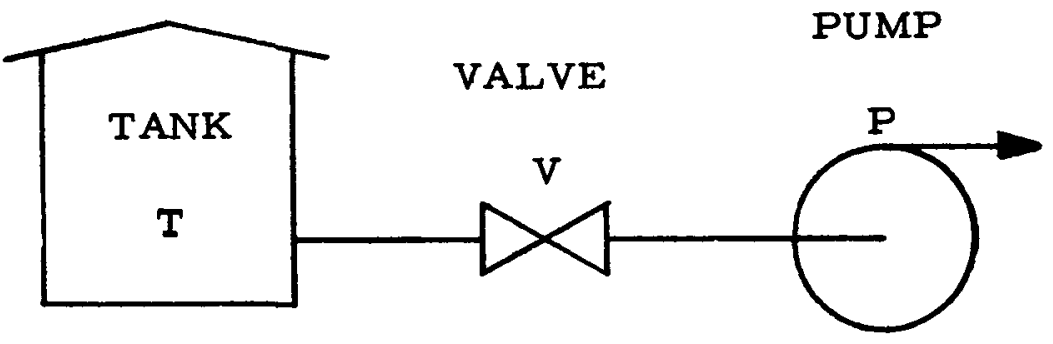

2. $11 \mathrm{~A}$ FLOW DIAGRAM

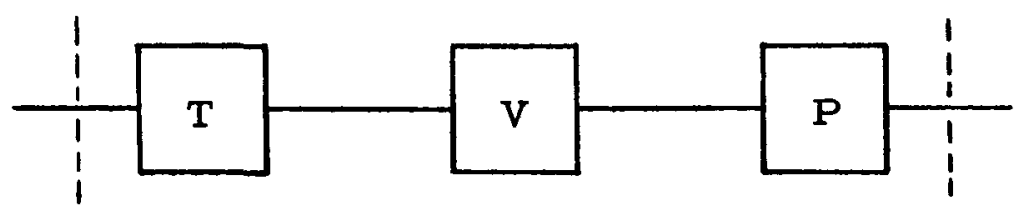

(1) One Function System

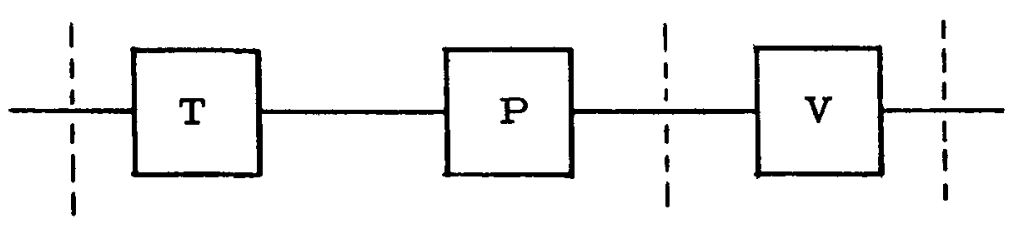

(2) Two Function System

\subsection{B BLOCK DIAGRAMS}

NOTE: --- indicates division of functions.

1,2 , and 3 indicate function number.

FIGURE 2.11

SERIES SYSTEM 
VALVES

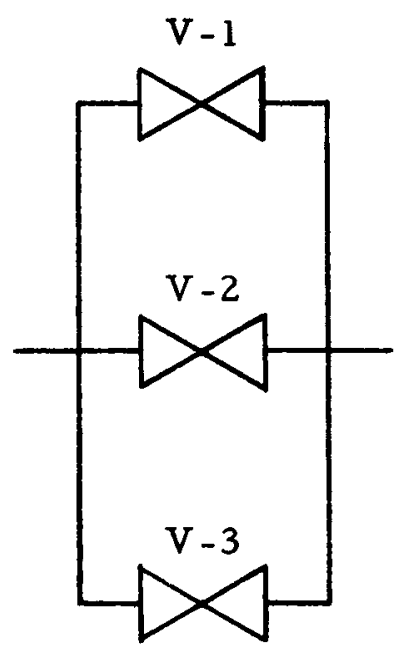

2:12A FLOW DIAGRAM

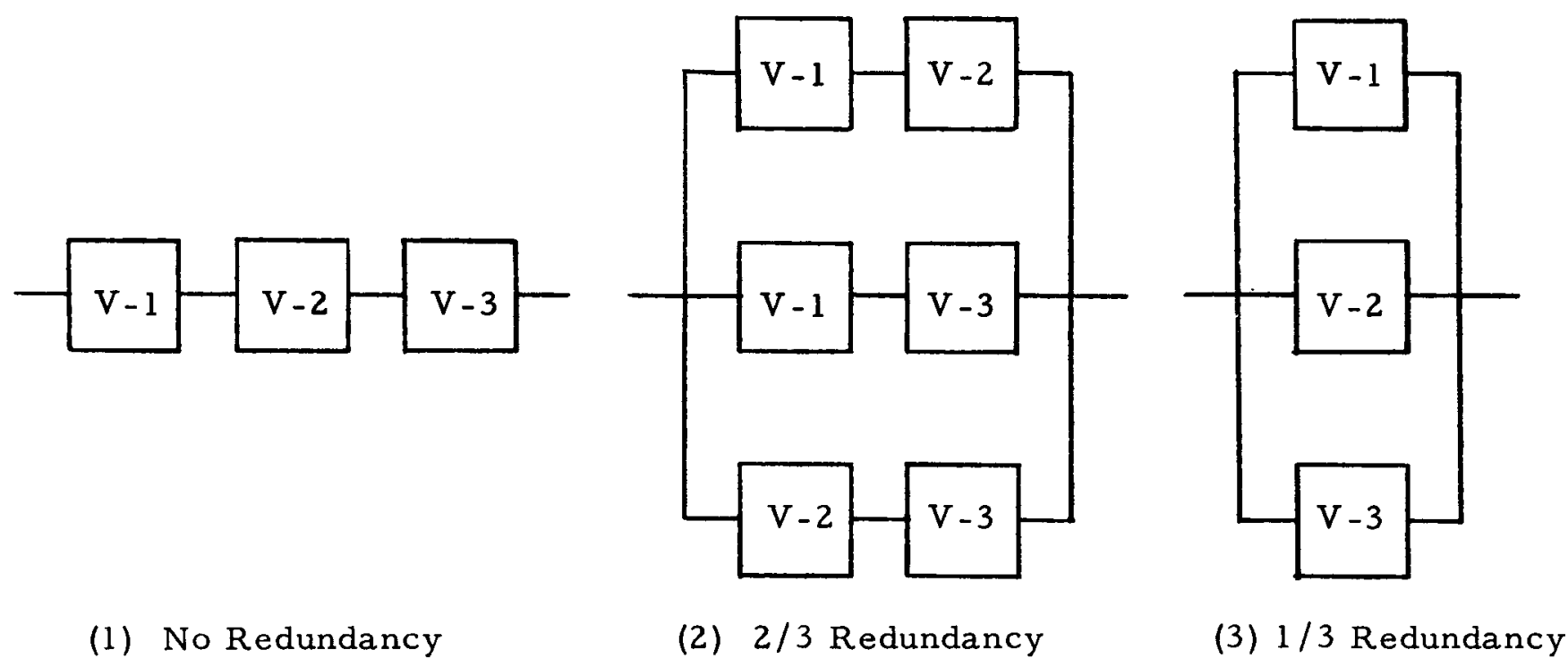

\section{2:12B BLOCK DIAGRAMS}

FIGURE 2.12

PARALLEL SYSTEM 


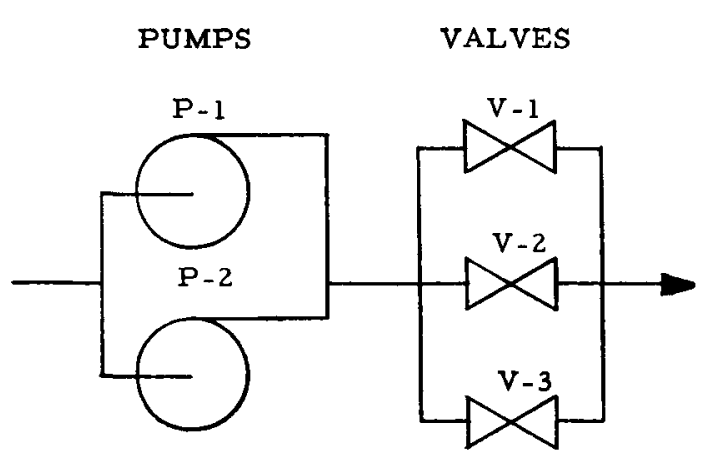

2:13A FLOW DIAGRAM
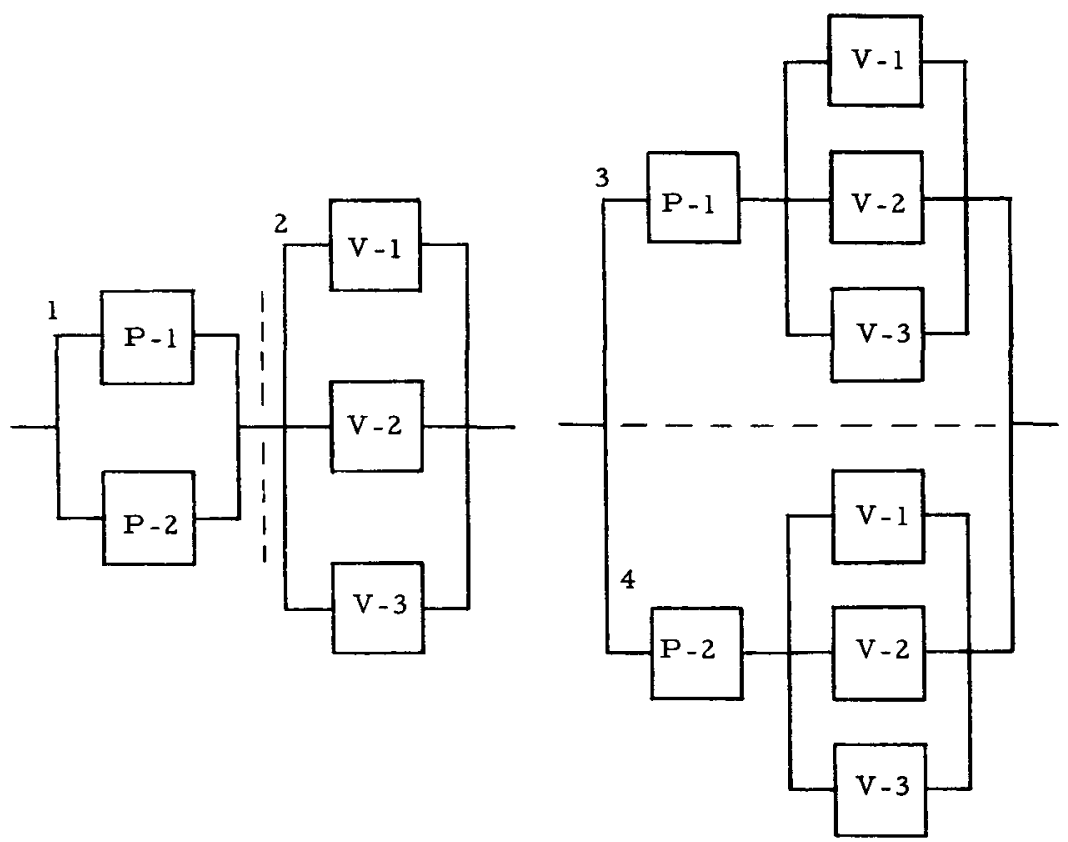

(1) Two Functions in Series
(2) Two Functions in Parallel

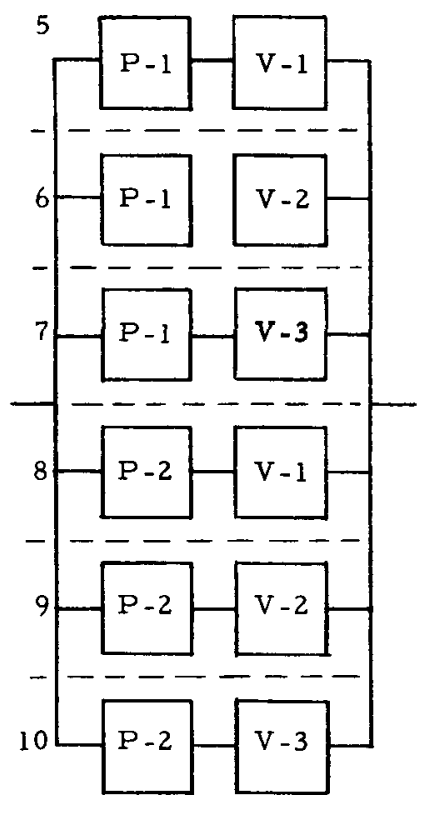

(3) Six Functions in Parallel

2:13B BLOCK DIAGRAMS

FIGURE 2.13

COMPOSITE SYSTEM 


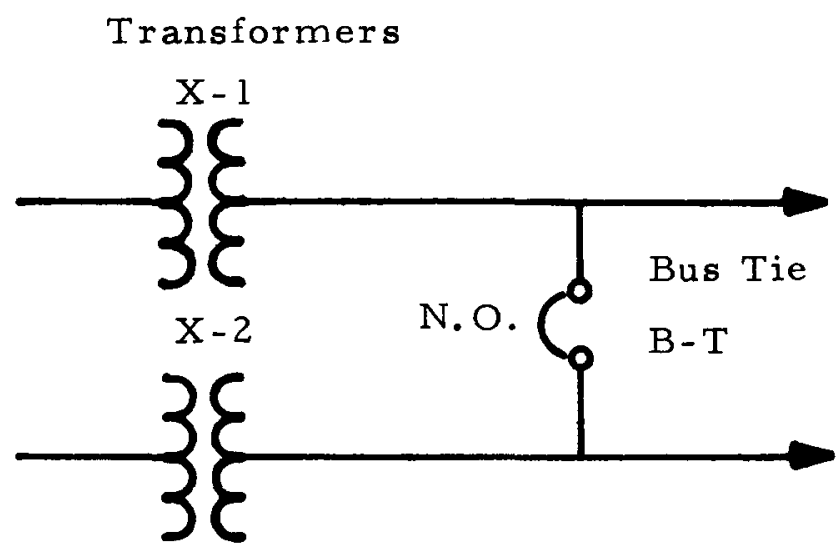

2. 14A LINE DIAGRAM

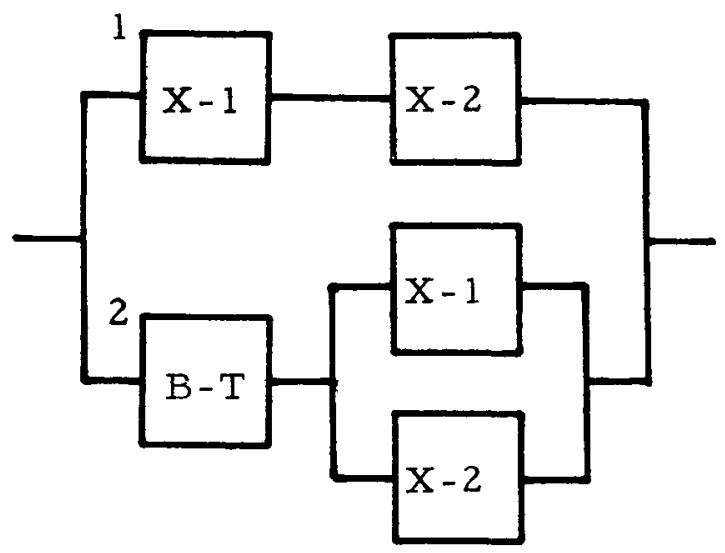

2. 14B BLOCK DIAGRAM

FIGURE 2. 14

ELECTRICAL SYSTEM 


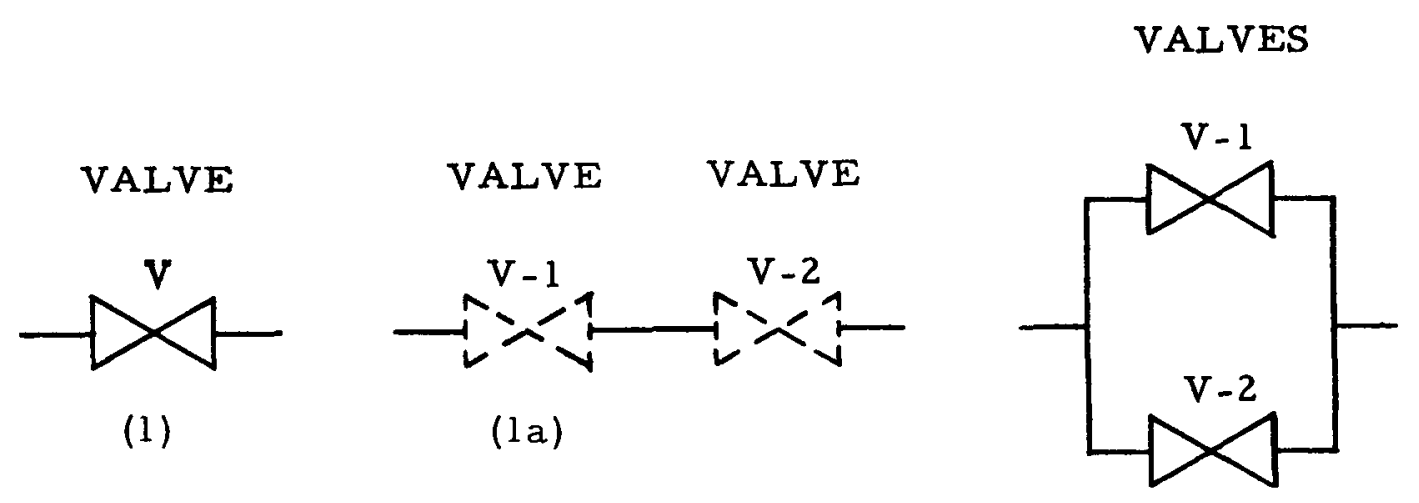

(2)

\section{15A FLOW DIAGRAMS}
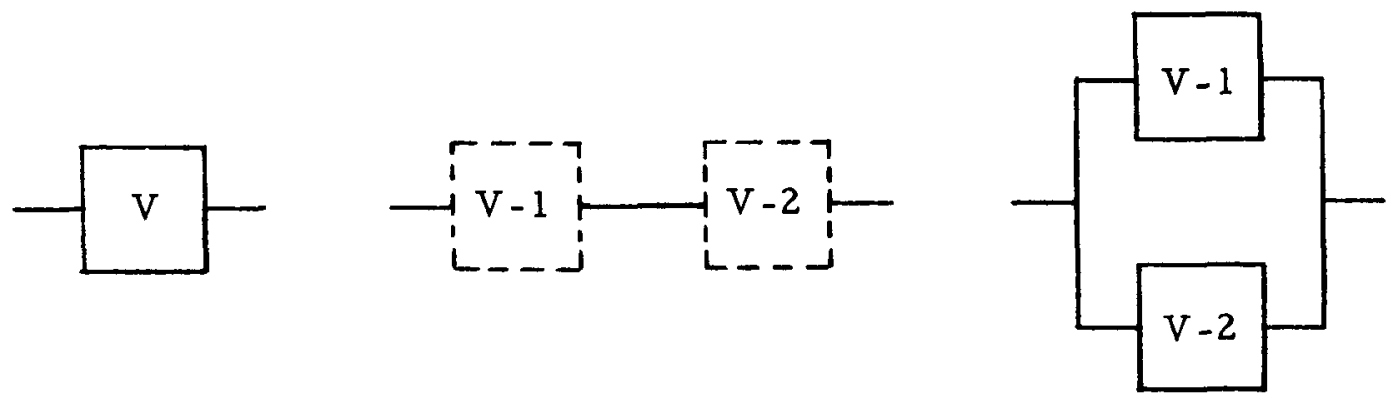

(1) Single Valve
(1a) Two Valves in Series (Fictitious Case)

(2) Two Valves in Parallel

FIGURE 2.15

CHANGE FROM NONREDUNDANT TO REDUNDANT COMPONENTS BY PARTIAL DATA 
PUMPS CHECK VALVES

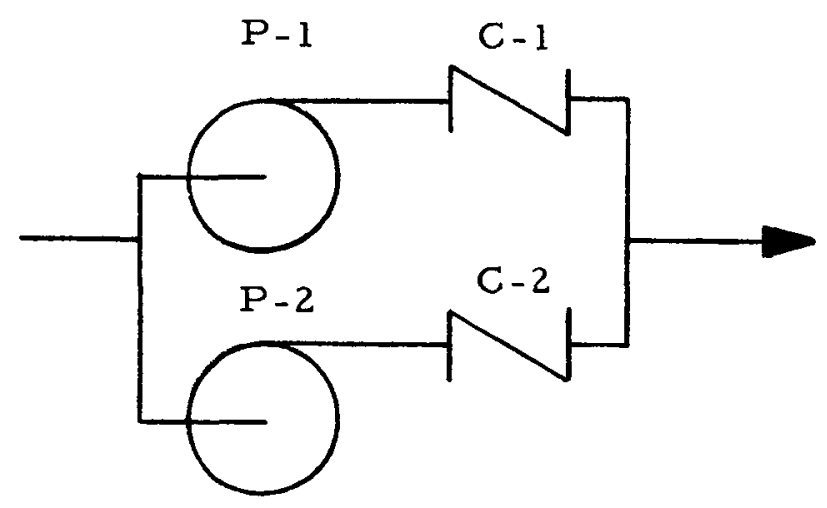

2.16A FLOW DIAGRAM

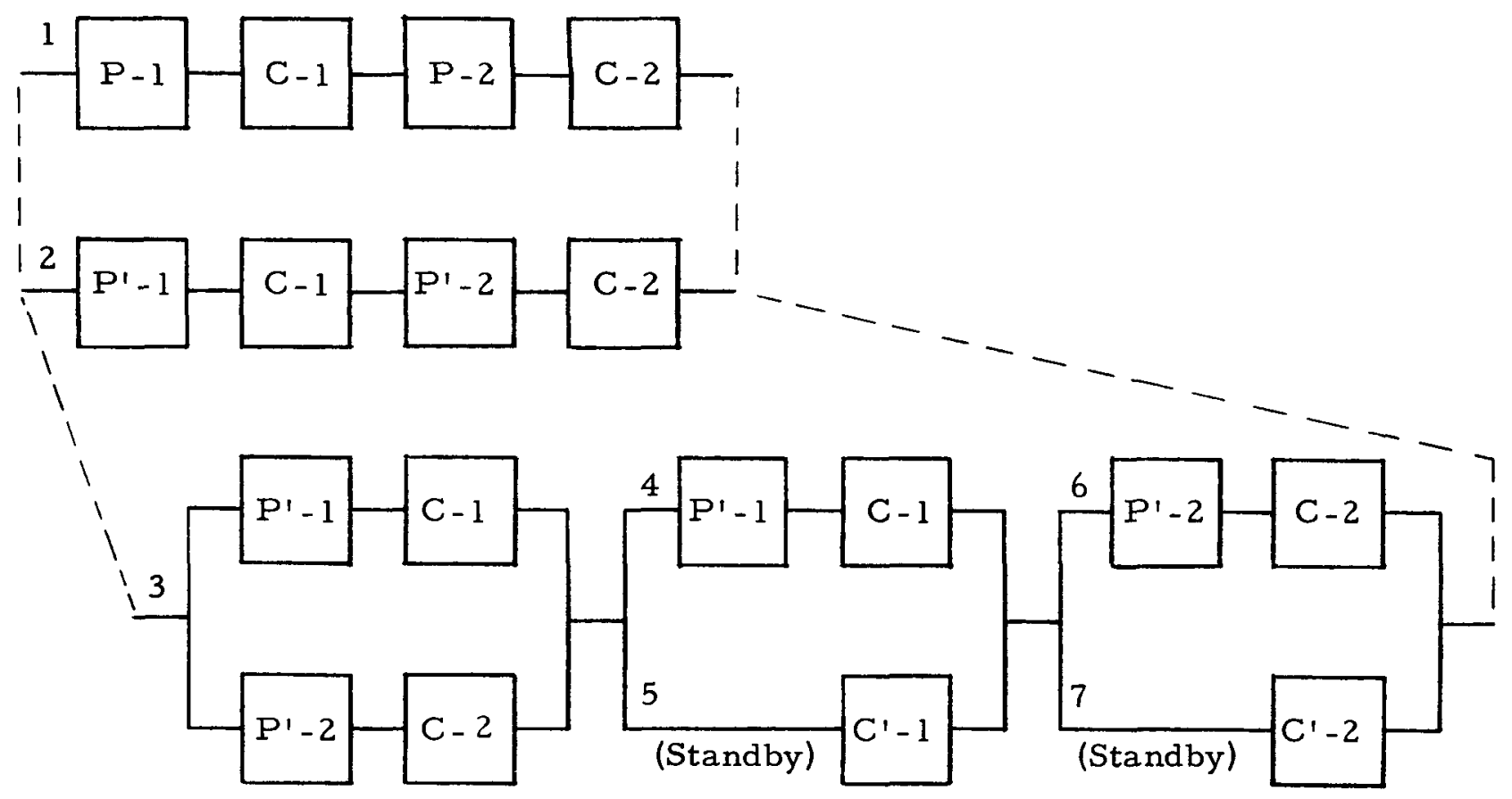

\section{16 B BLOCK DIAGRAM}

NOTE: --- indicates configuration change.with time intervals.

FIGURE 2.16

HY DRAULIC SYSTEM 


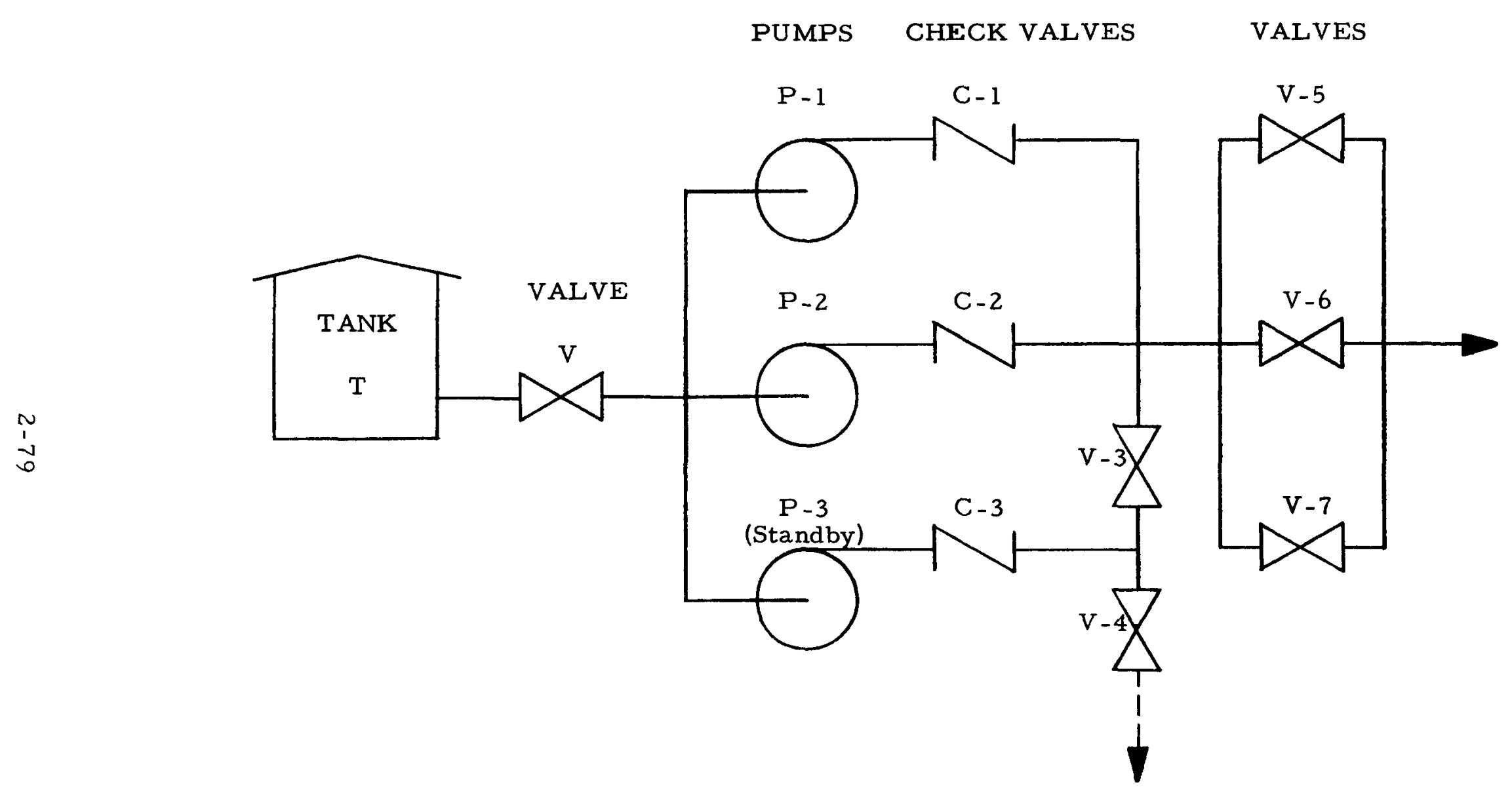

FIGURE 2.17

HY DRAULIC SYSTEM FLOW DIAGRAM 


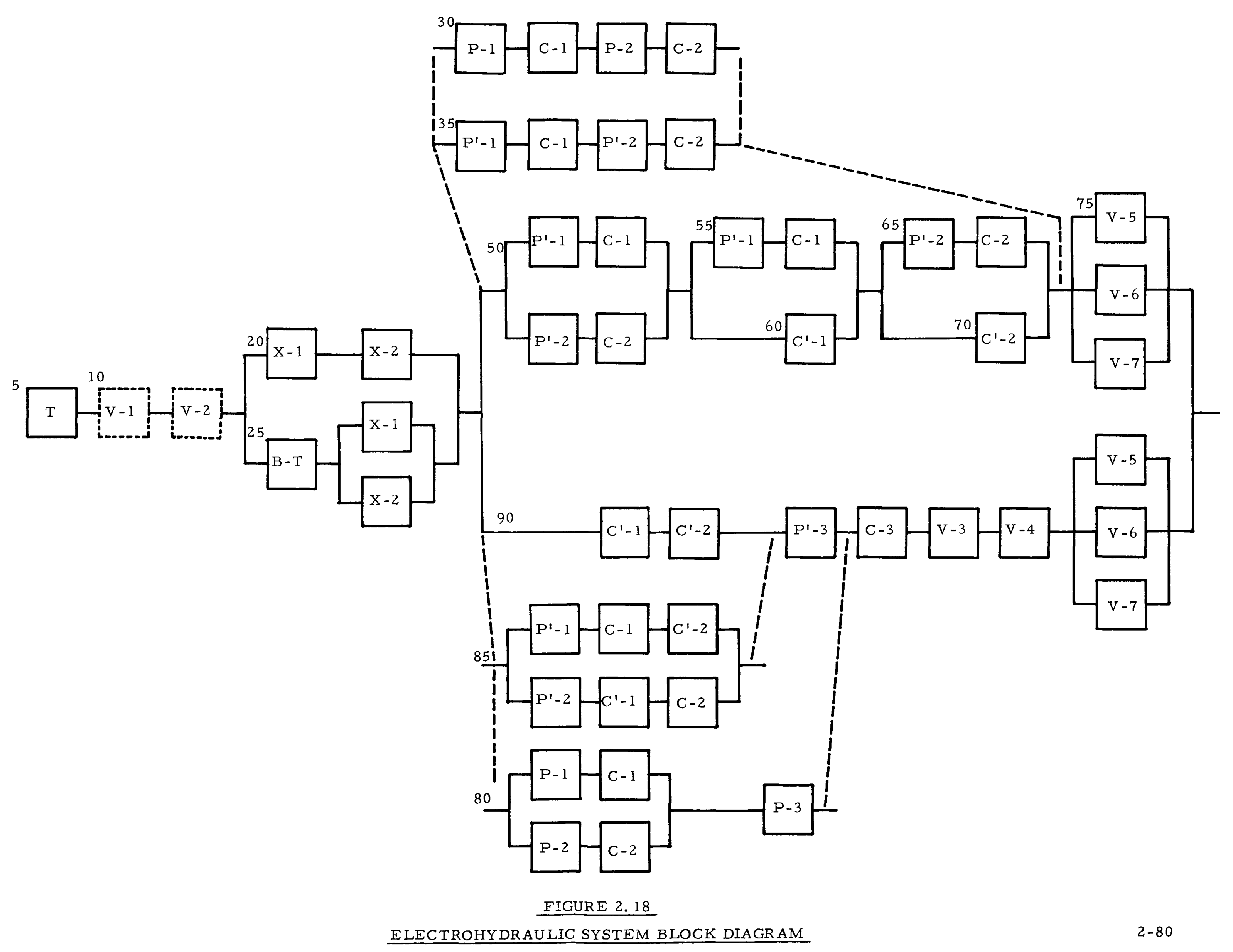




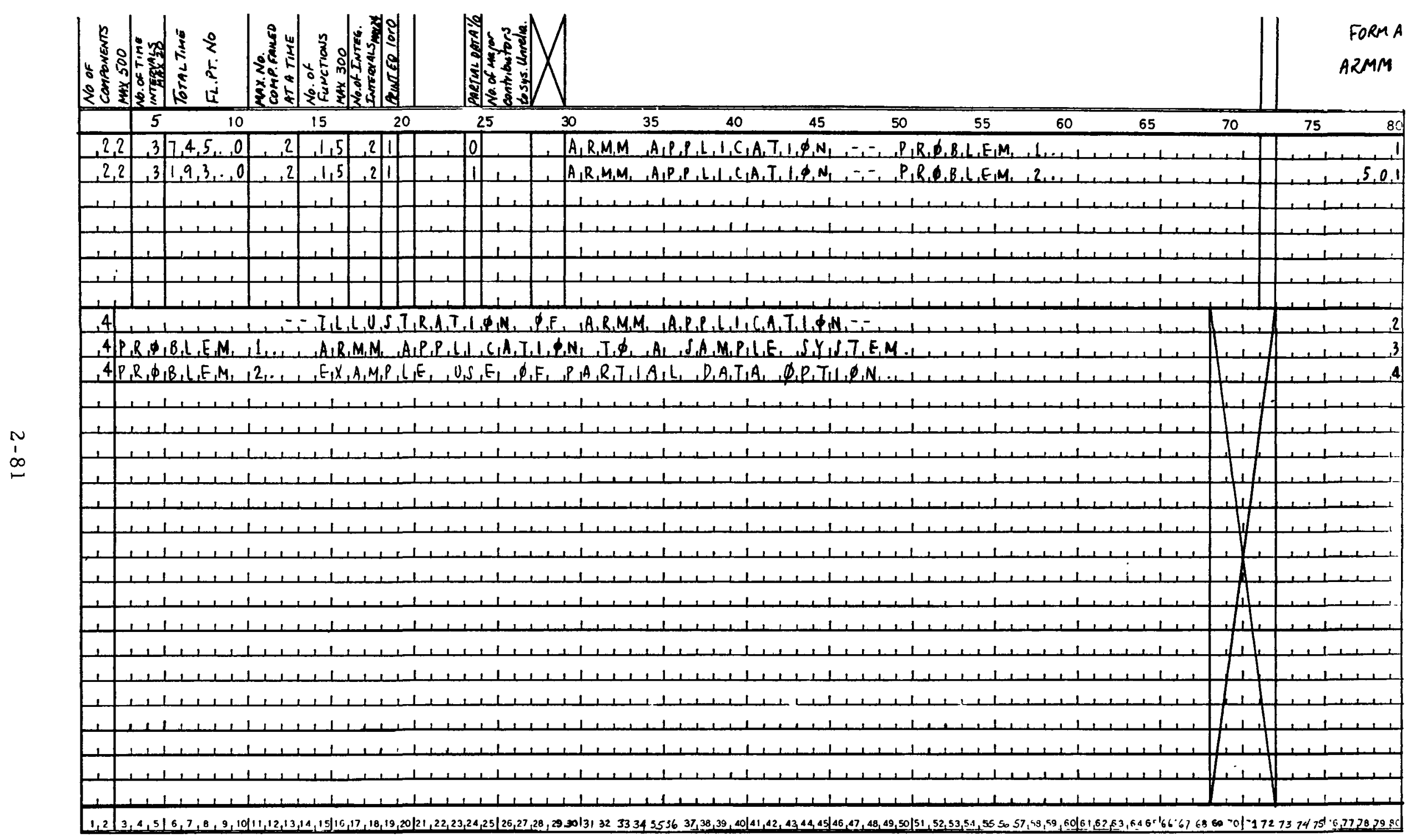

FIGURE 2.19

ARMM INPUT LOADSHEETS 


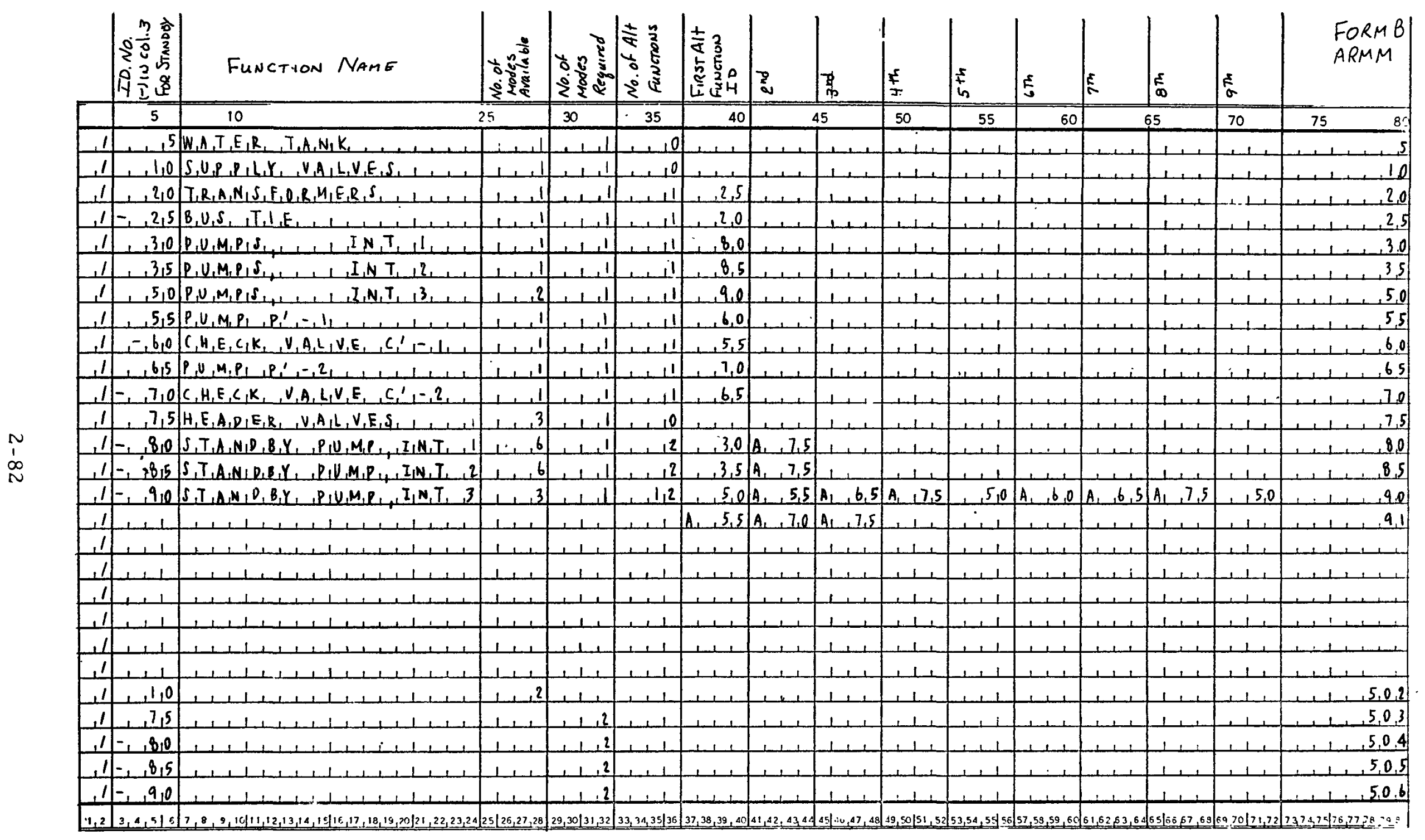

FIGURE 2.19 (continued) 


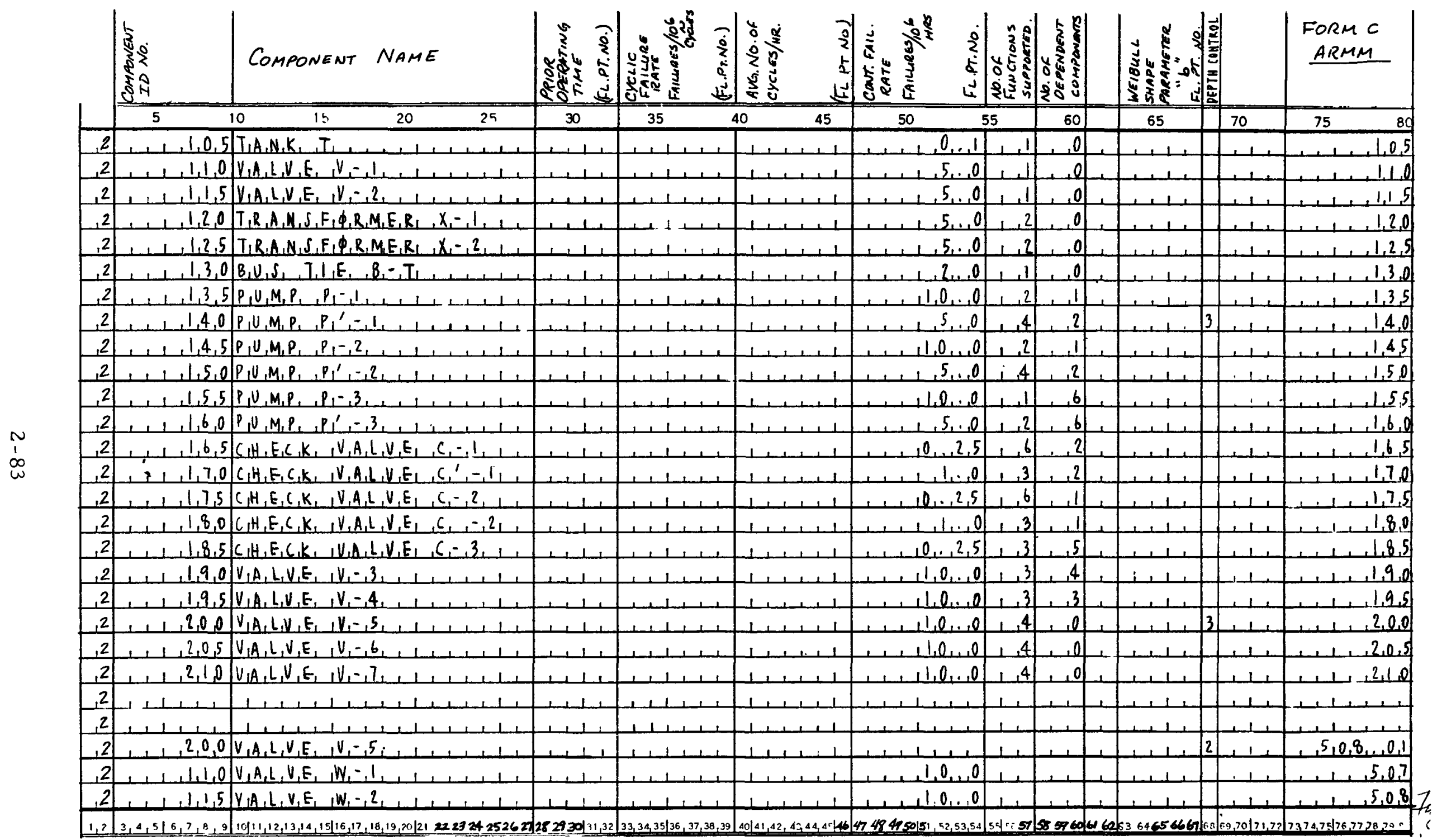

FIGURE 2.19 (continued) 


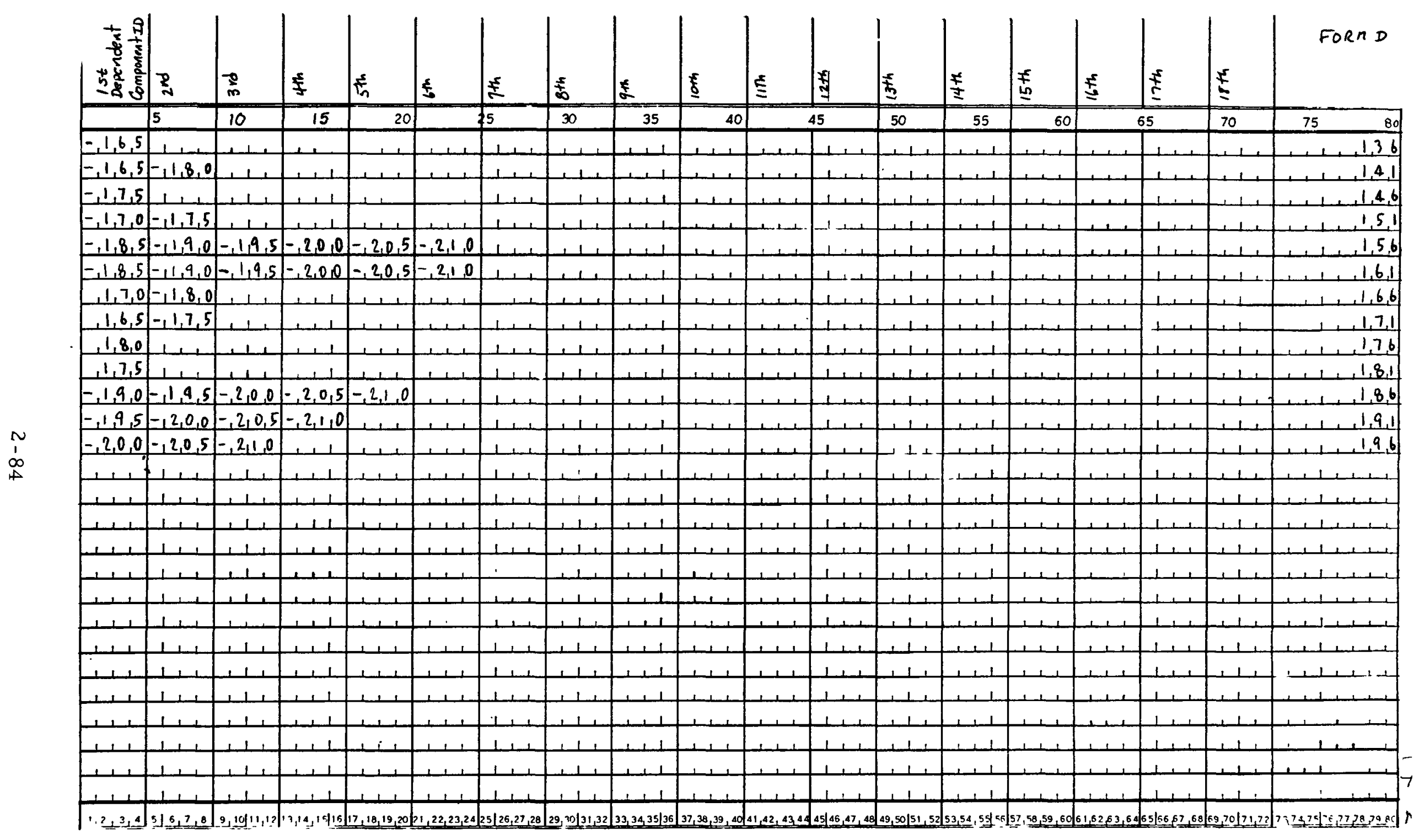

FIGURE 2.19 (continued) 


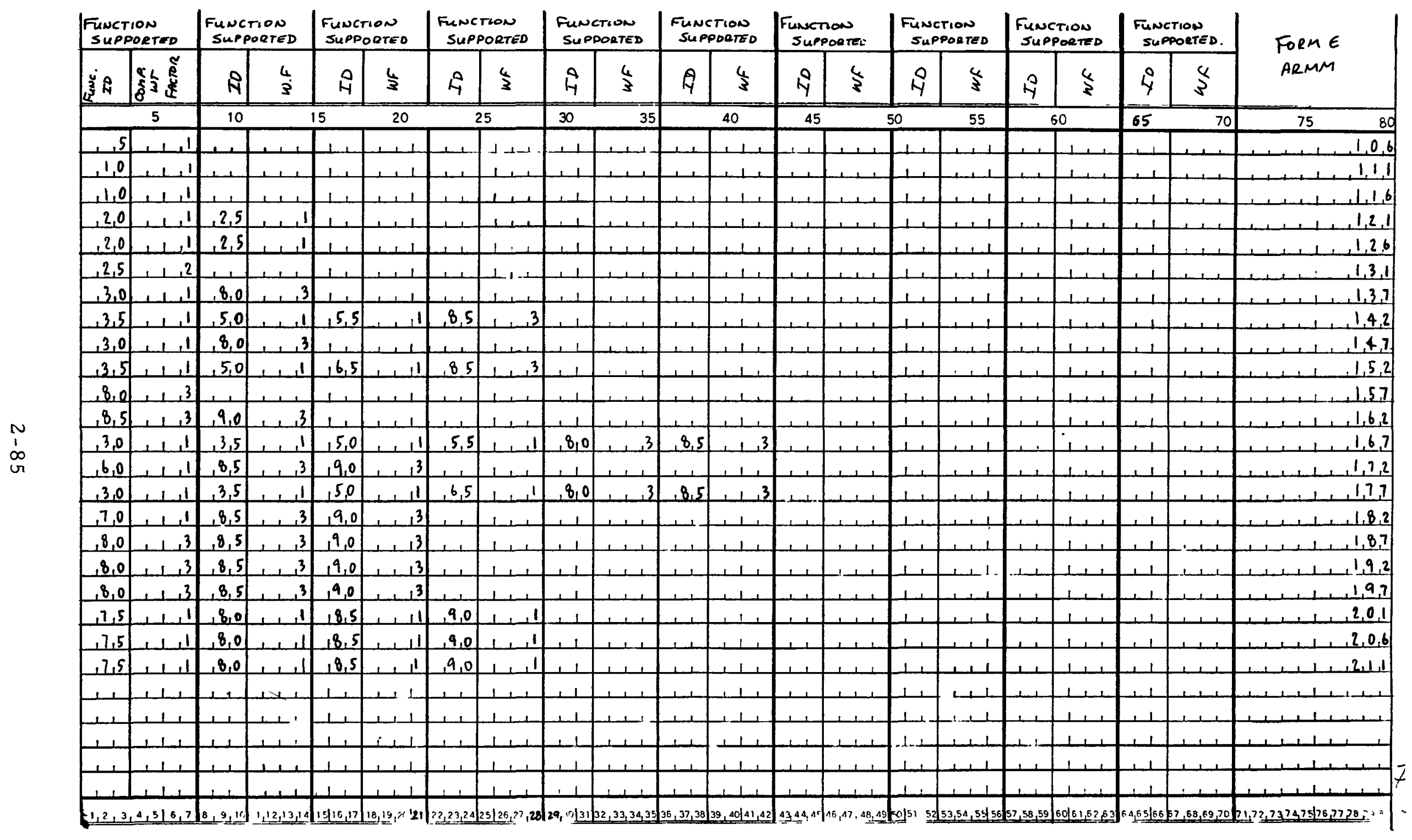

FIGURE 2.19 (continued) 


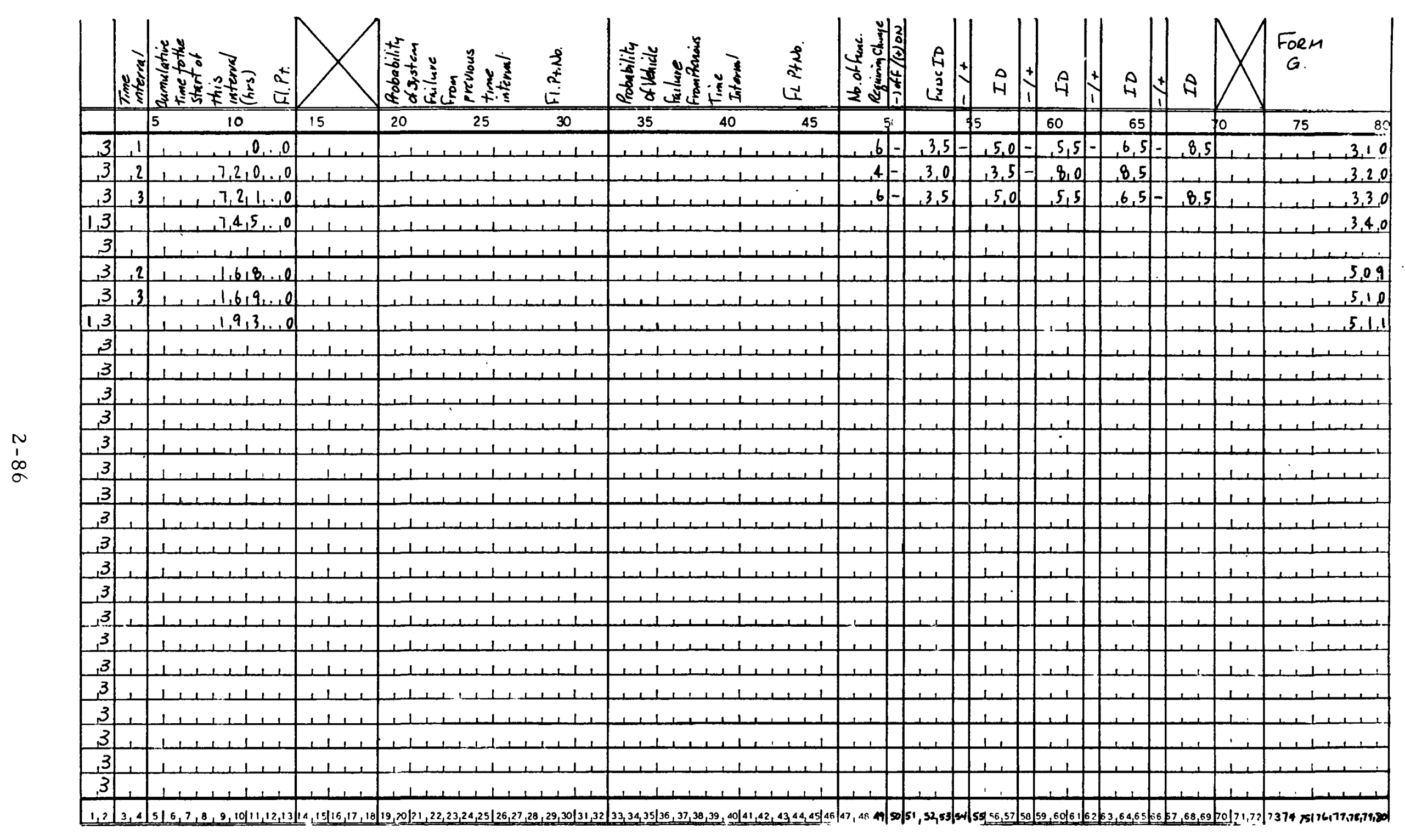

FIGURE 2.19 (continued) 


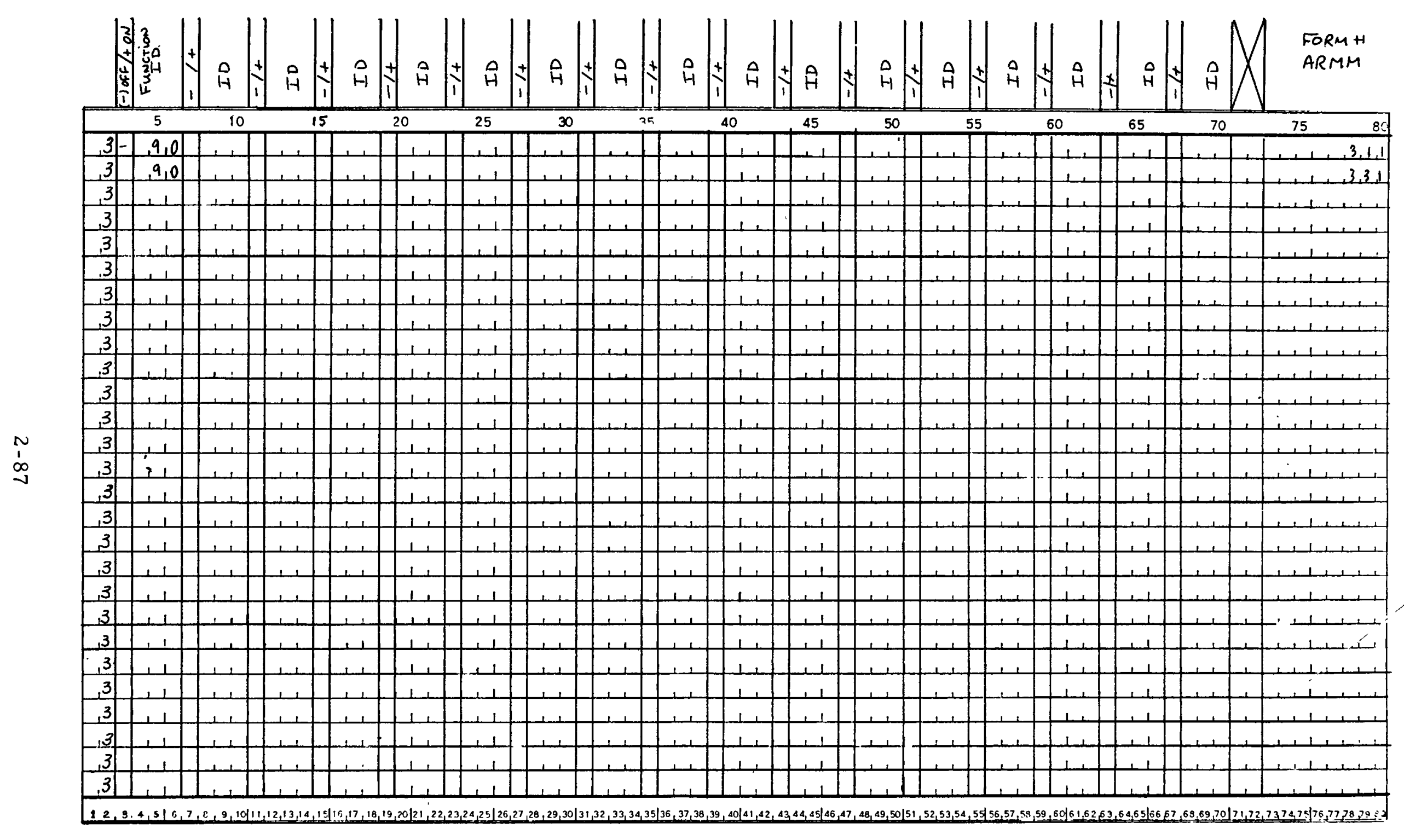

FIGURE 2.19 (continued) 

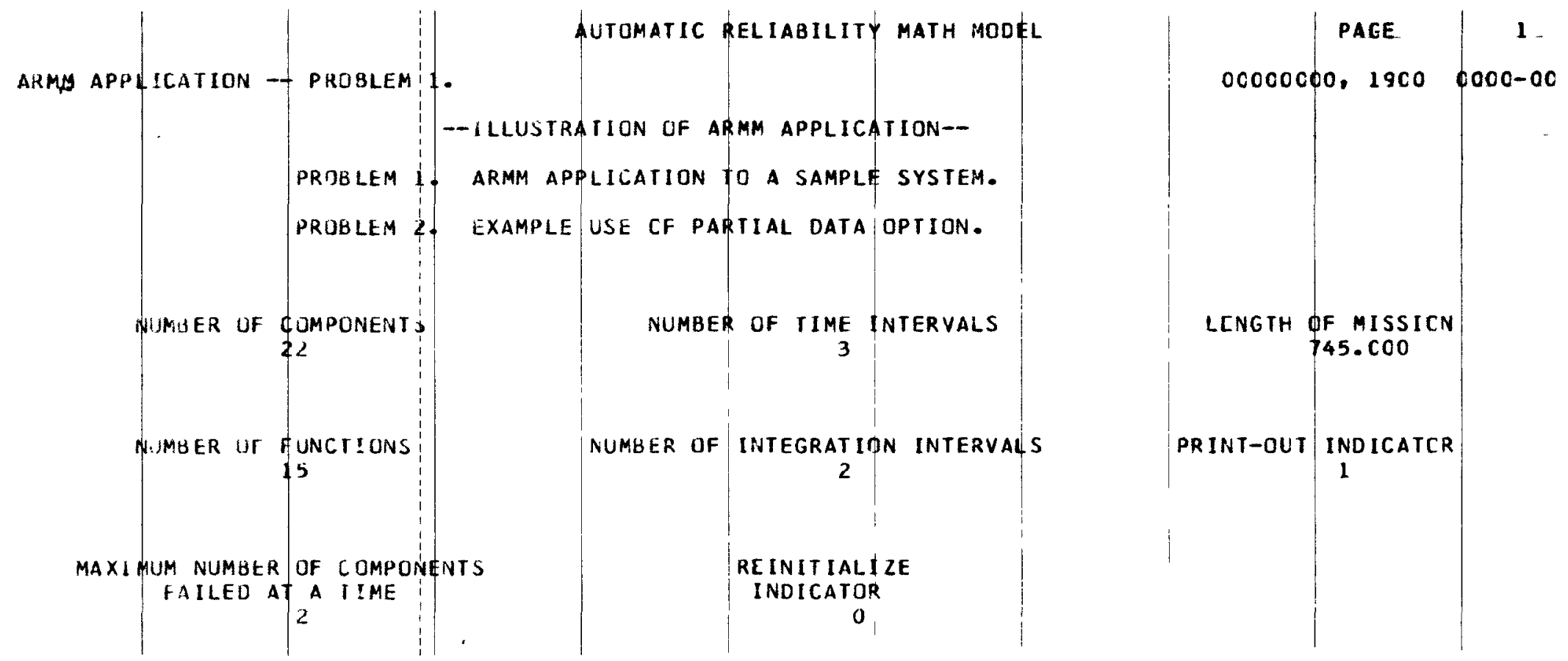

FIGURE 2.20

\section{COMPLETED COMPUTER OUTPUT - PROBLEM 1}



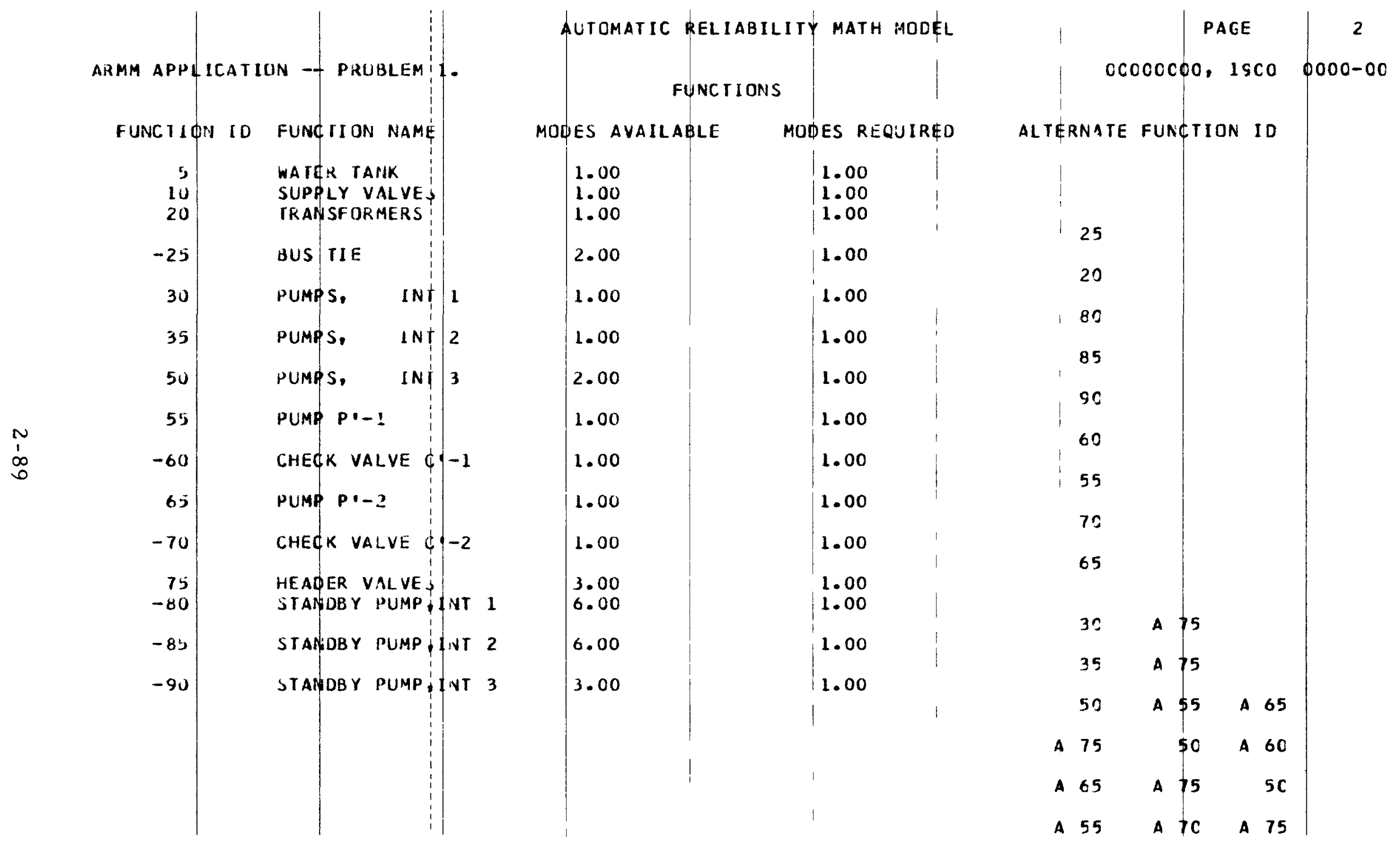


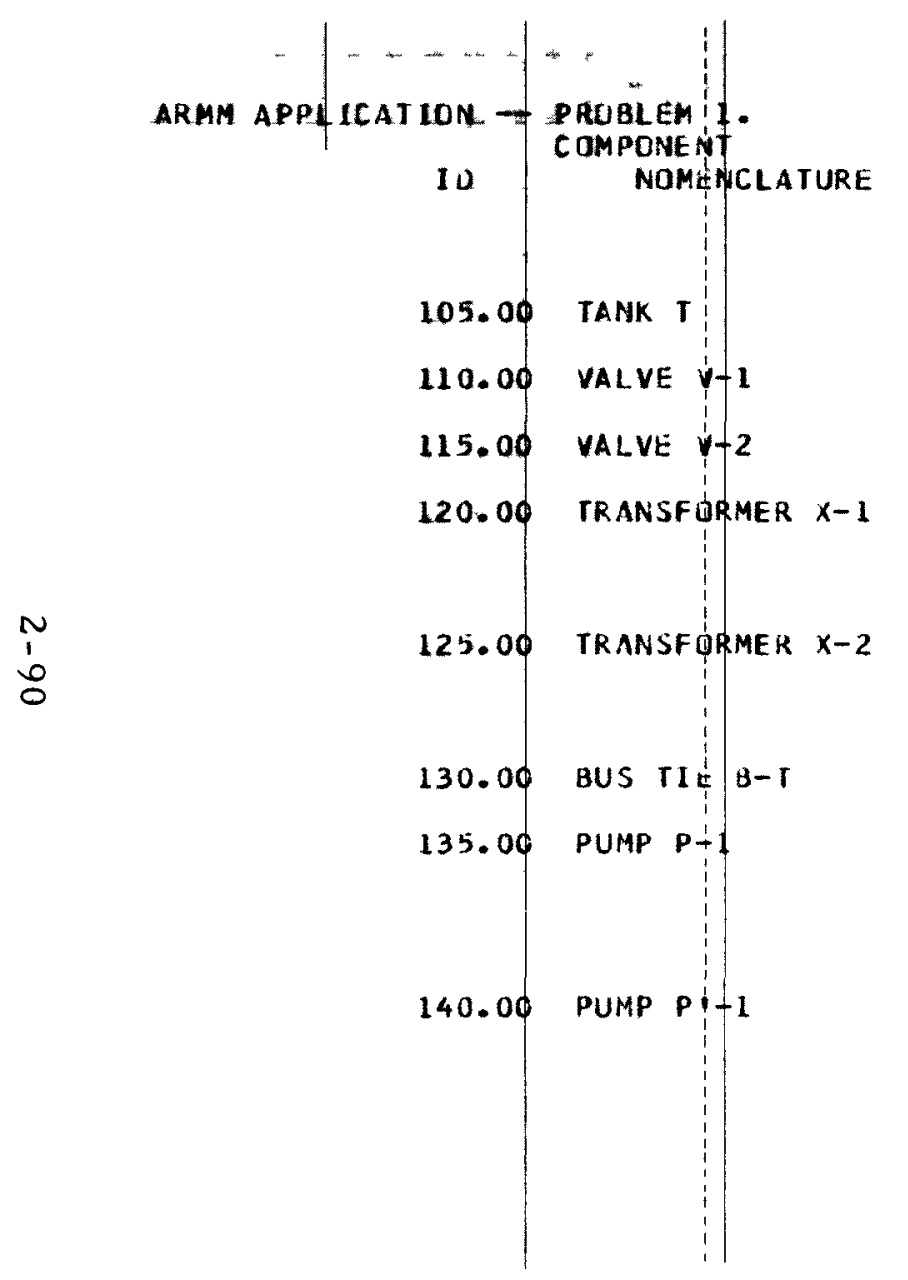

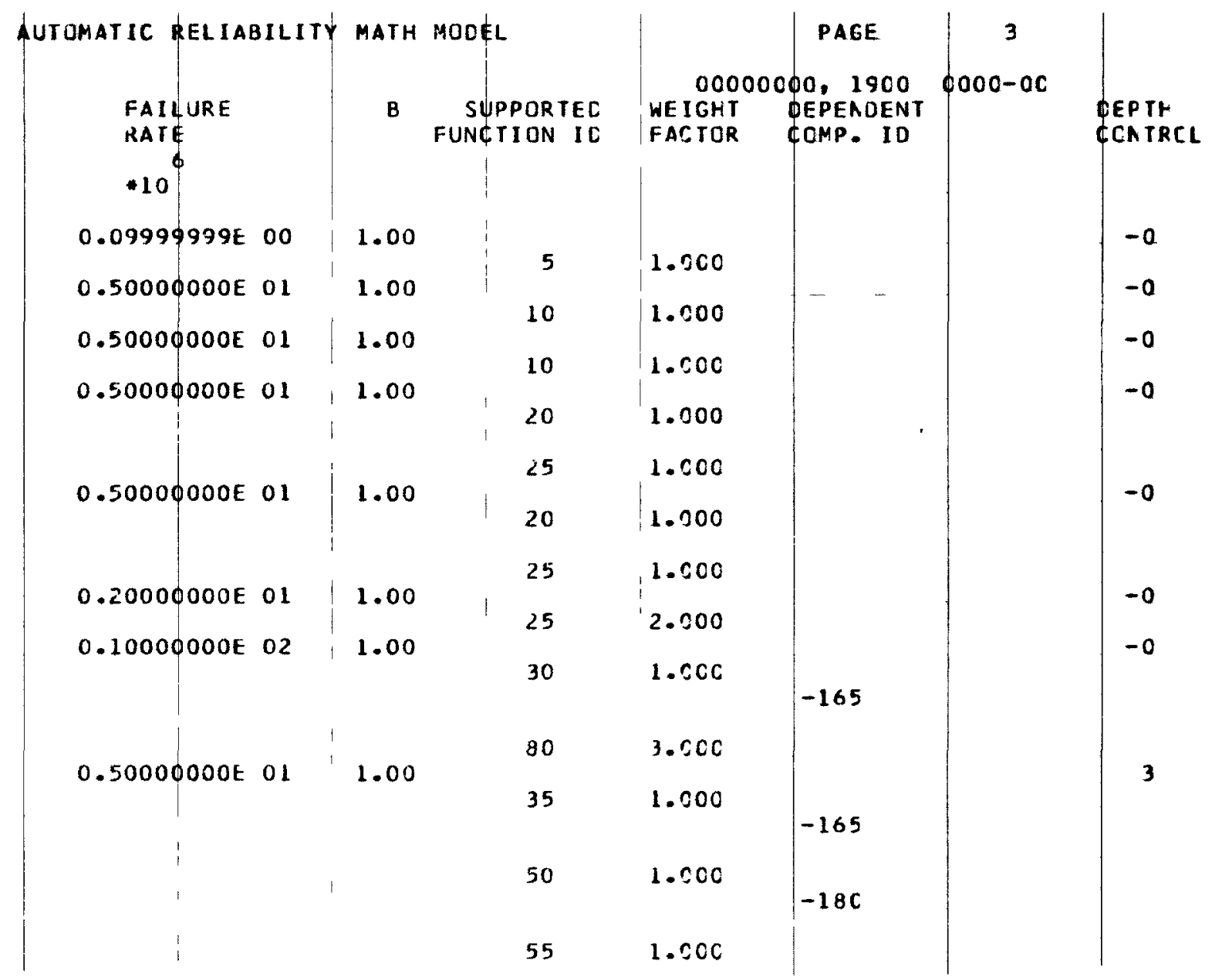

FIGURE 2.20 (continued) 


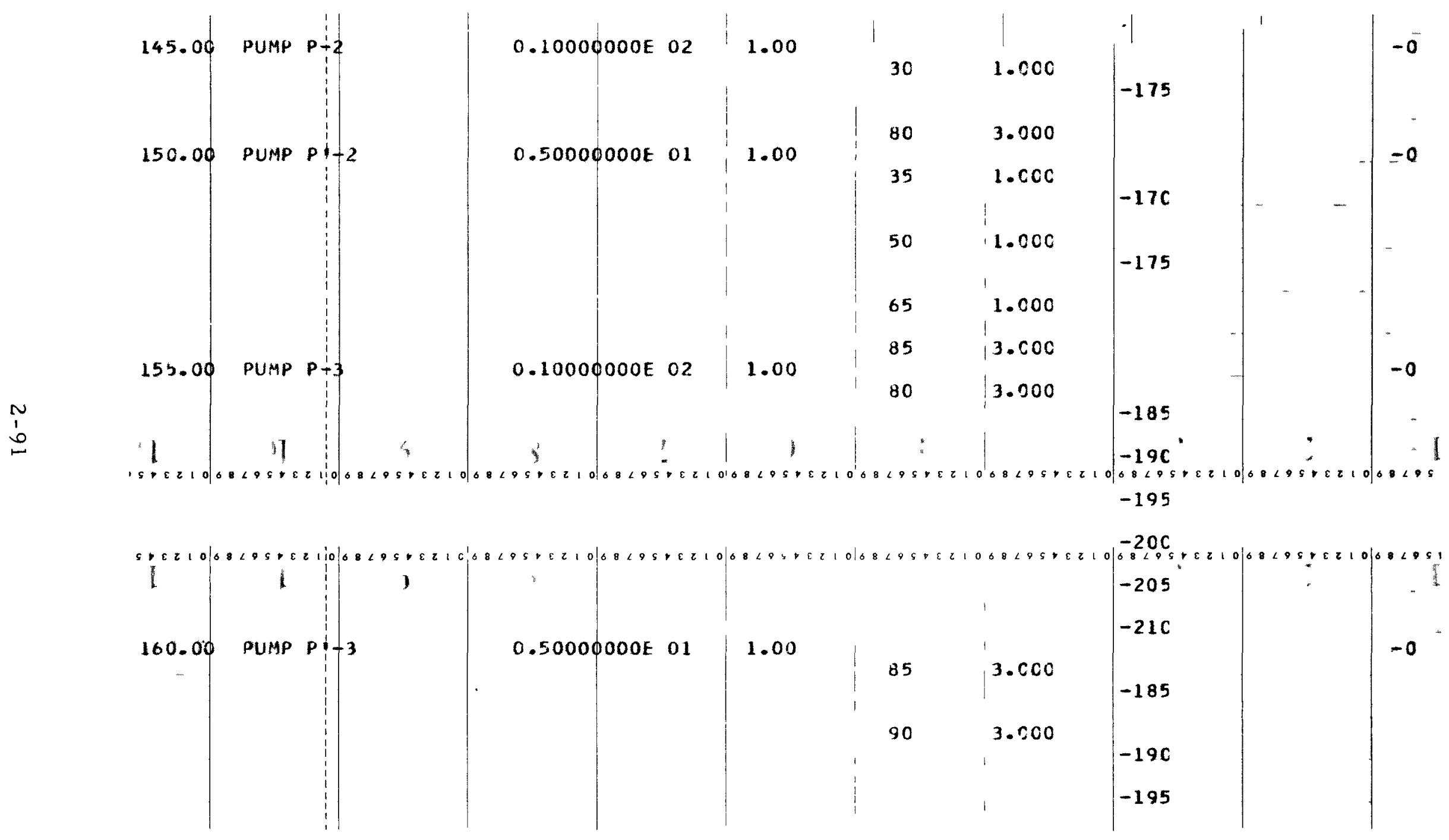

FIGURE 2.20 (continued) 


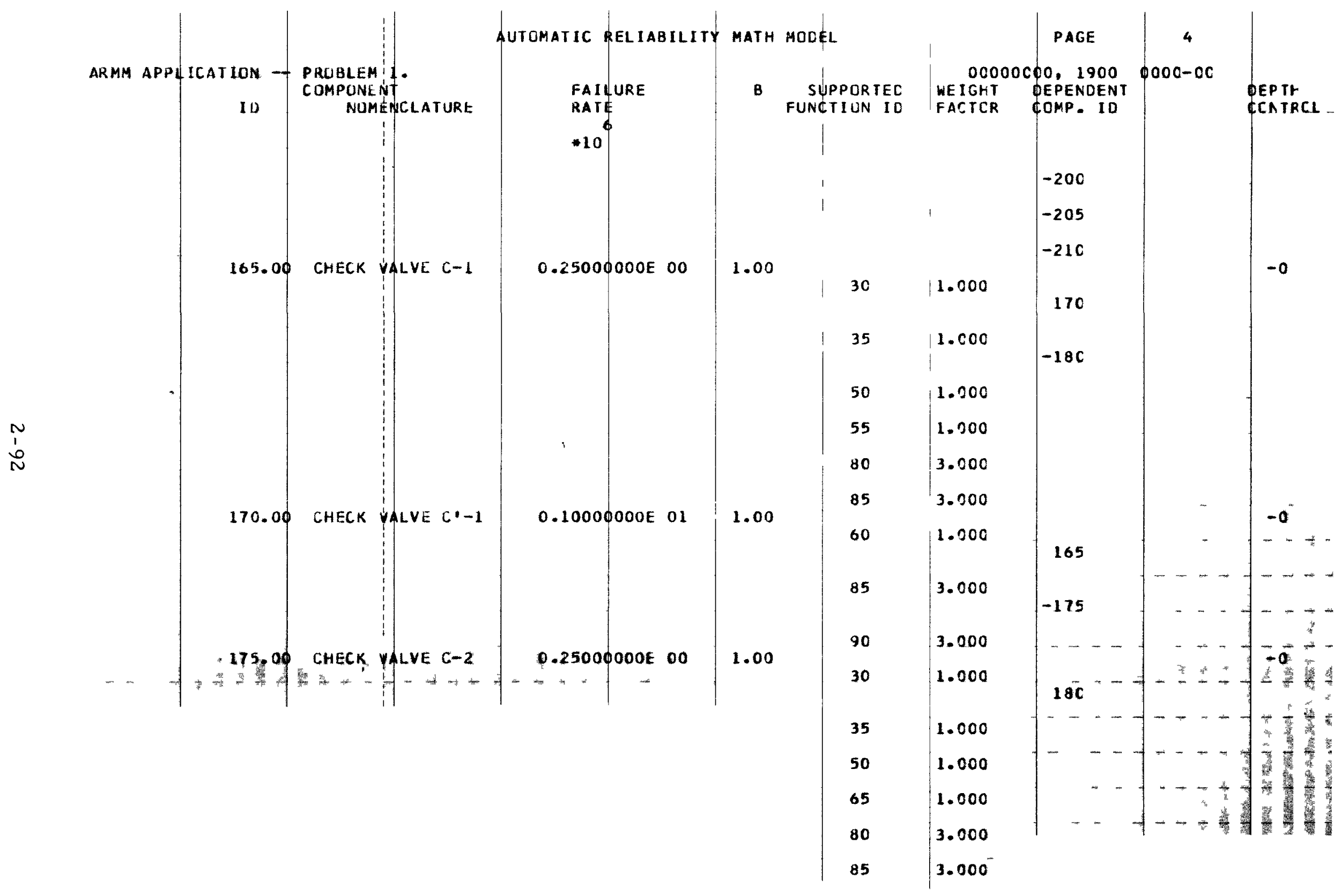




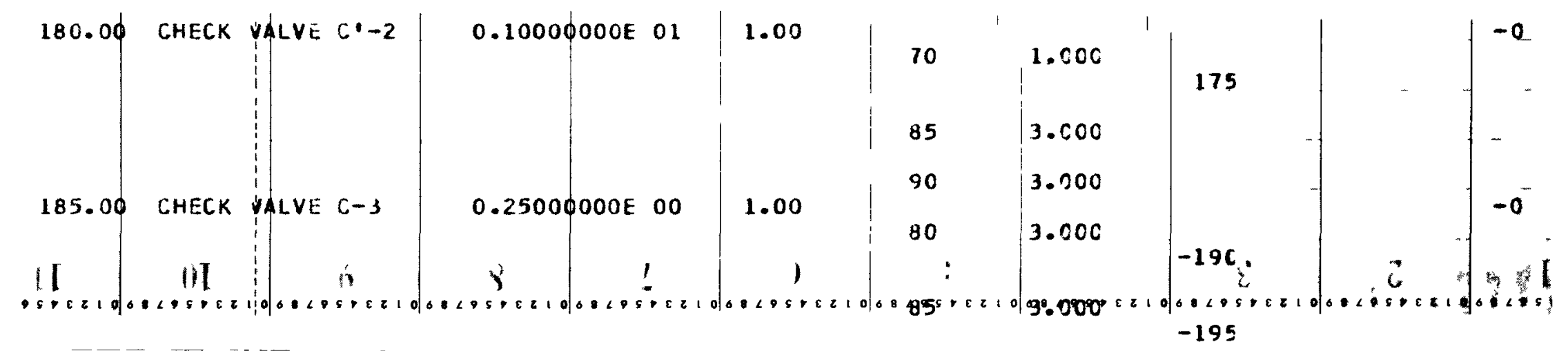

$N$
1
$\omega$

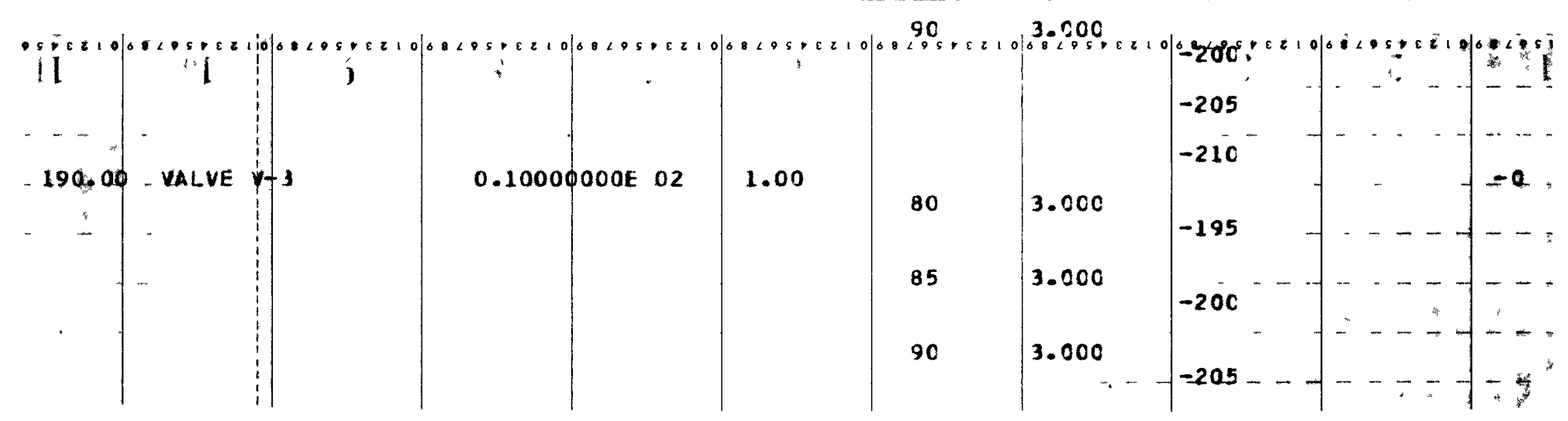

FIGURE 2.20 (continued) 


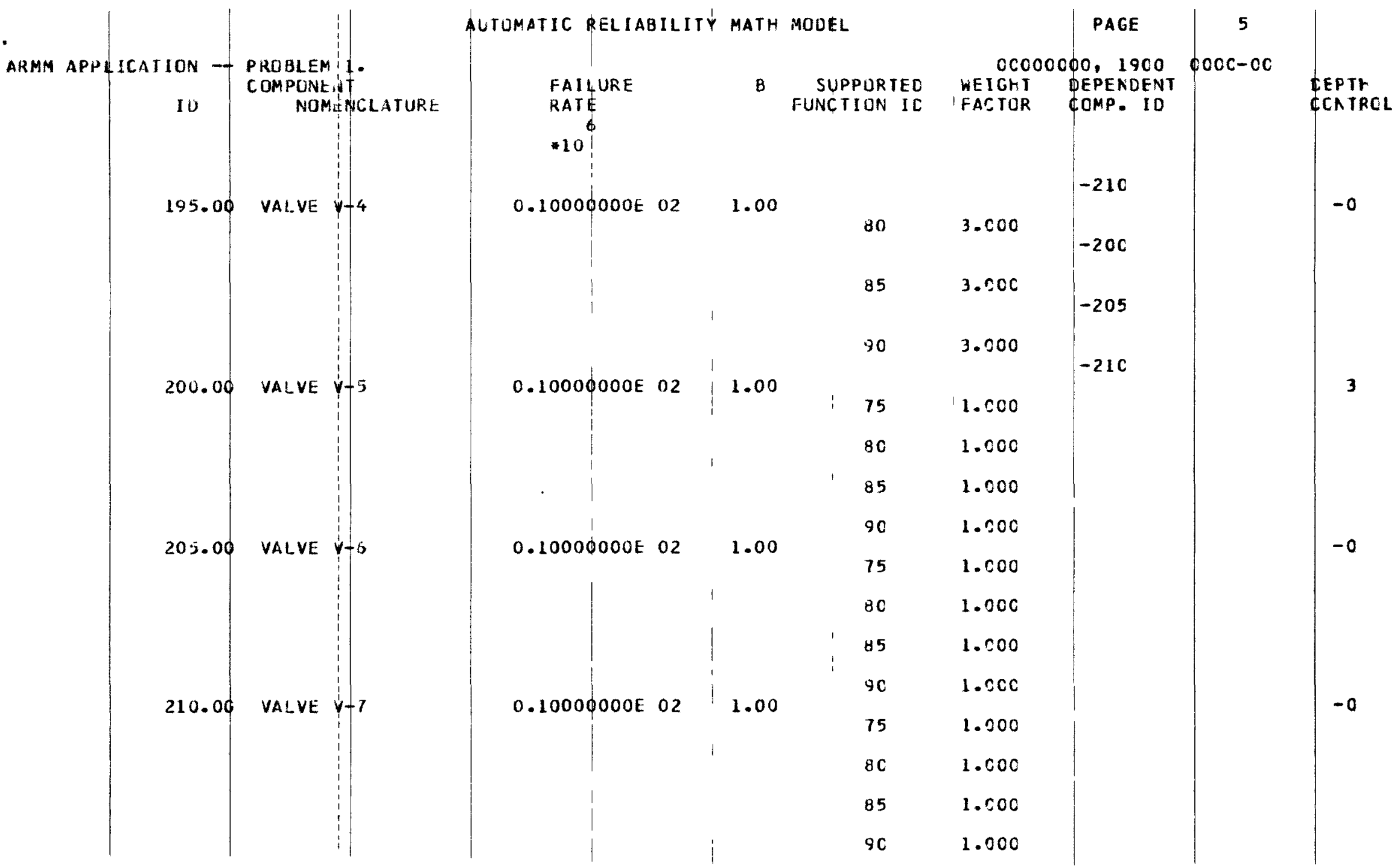

FIGURE 2.20 (continued) 

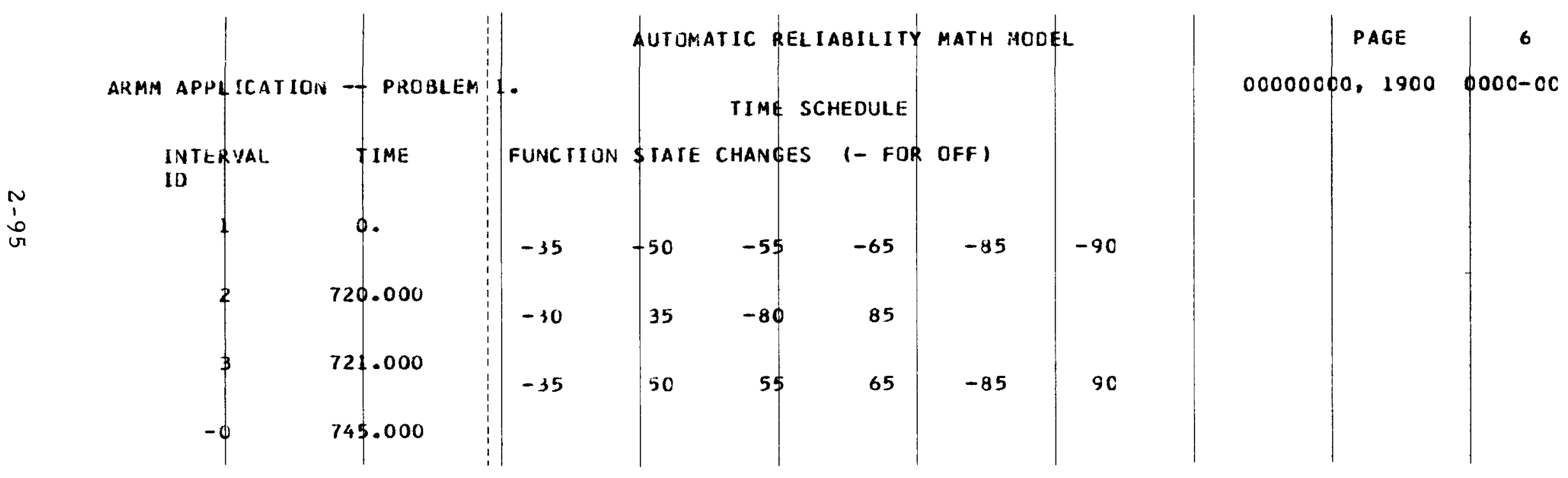

FIGURE 2.20 (continued) 

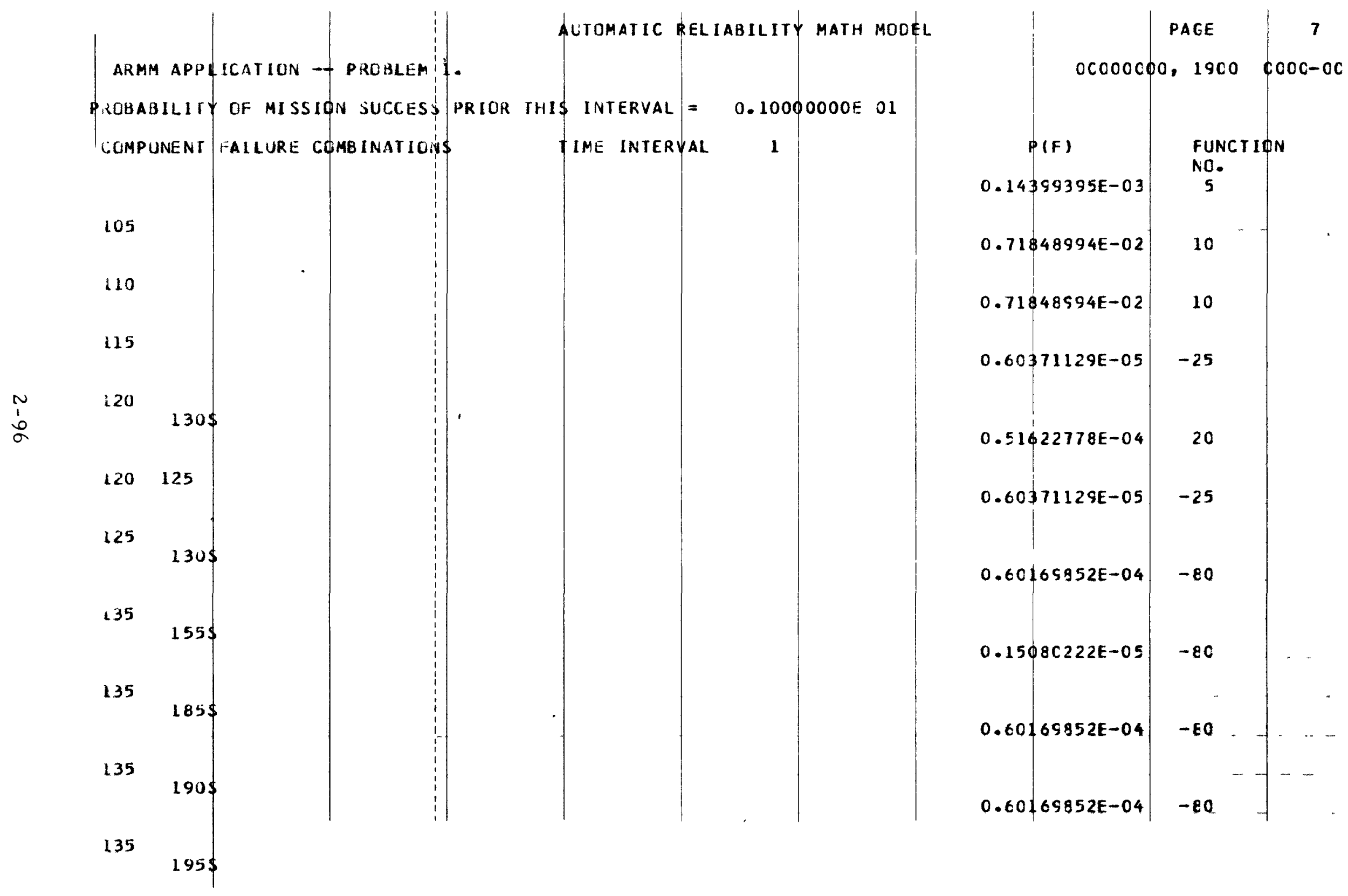

FIGURE 2.20 (continued) 


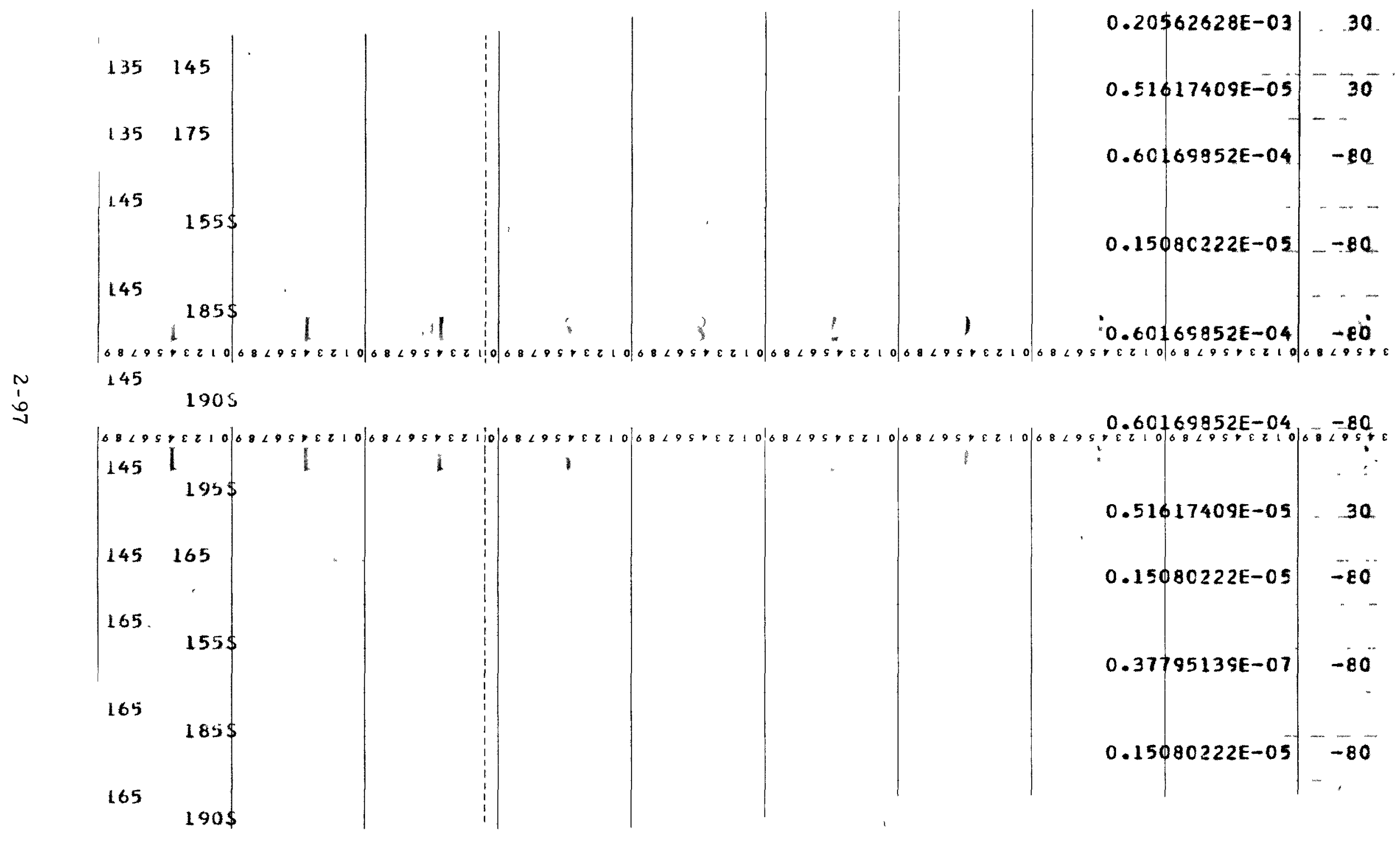

FIGURE 2.20 (continued) 

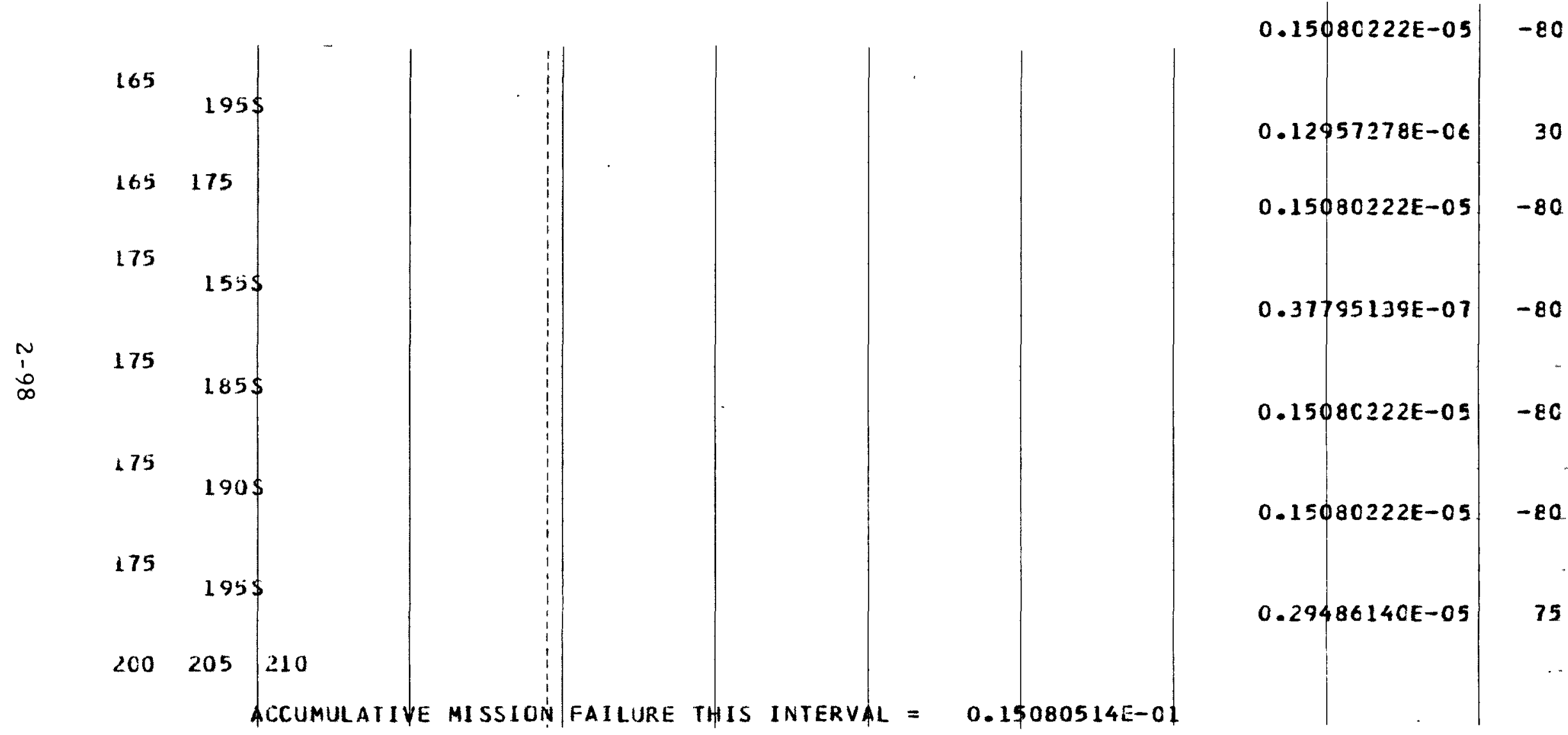

FIGURE 2.20 (continued) 

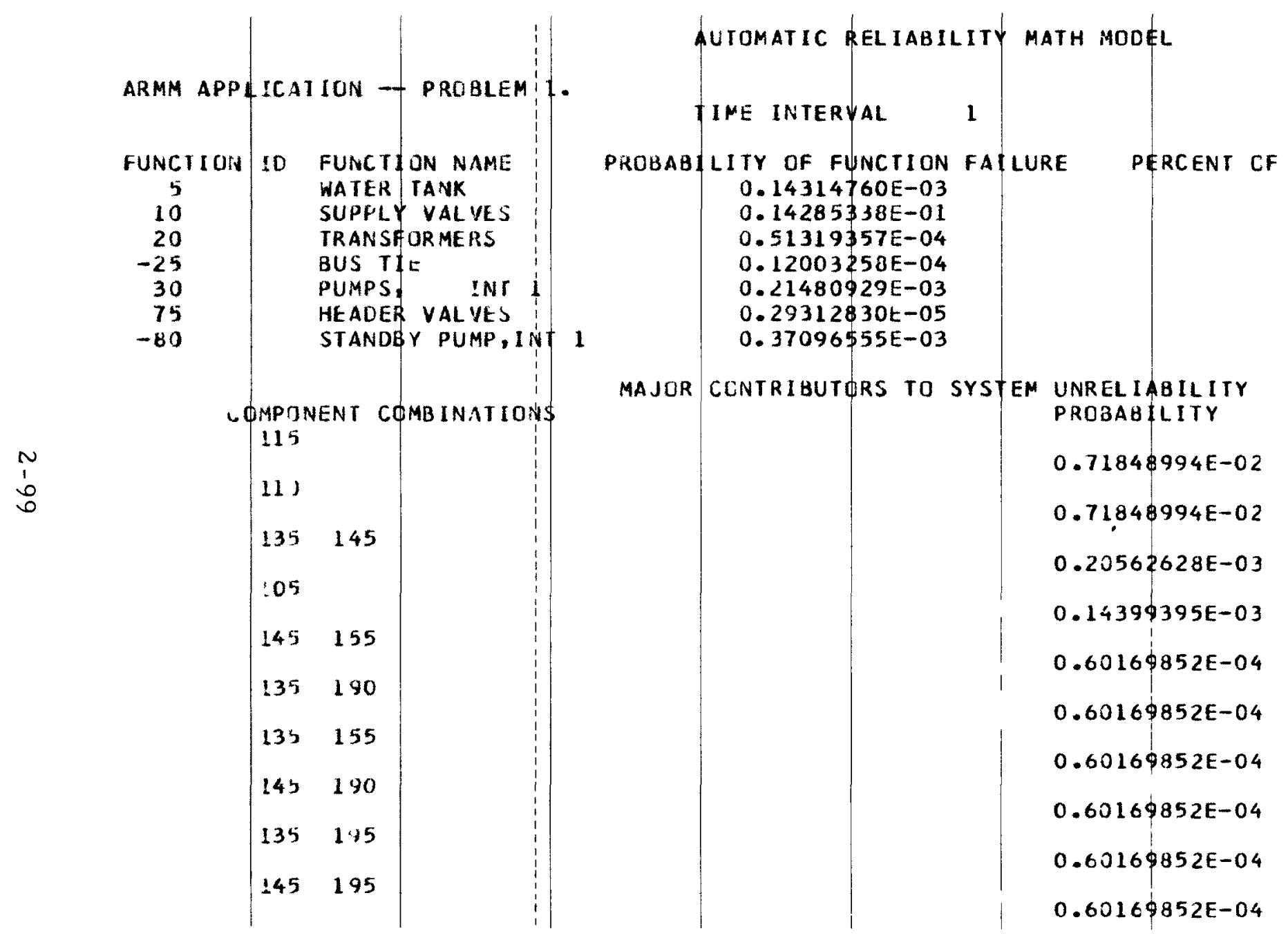

\begin{tabular}{|c|c|c|}
\hline $\mathrm{cccoc}$ & $\begin{array}{c}\text { PAGE } \\
C 0,1900\end{array}$ & $\begin{array}{c}8 \\
0000-00\end{array}$ \\
\hline $\begin{array}{l}\text { YSTEM FAI } \\
2.949 \\
94.727 \\
0.340 \\
0.080 \\
1.424 \\
0.019 \\
2.460\end{array}$ & URE & $\begin{array}{r}\text { RANK } \\
4 \\
1 \\
5 \\
6 \\
3 \\
7 \\
2\end{array}$ \\
\hline PERCE & & \\
\hline 47.36 & & \\
\hline 47.36 & 36 & \\
\hline 1.35 & $\$ 5$ & \\
\hline 0.94 & 92 & \\
\hline 0.39 & 66 & \\
\hline 0.39 & 66 & \\
\hline 0.39 & 66 & \\
\hline 0.39 & & \\
\hline 0.39 & & \\
\hline & & \\
\hline
\end{tabular}

FIGURE 2.20 (continued) 


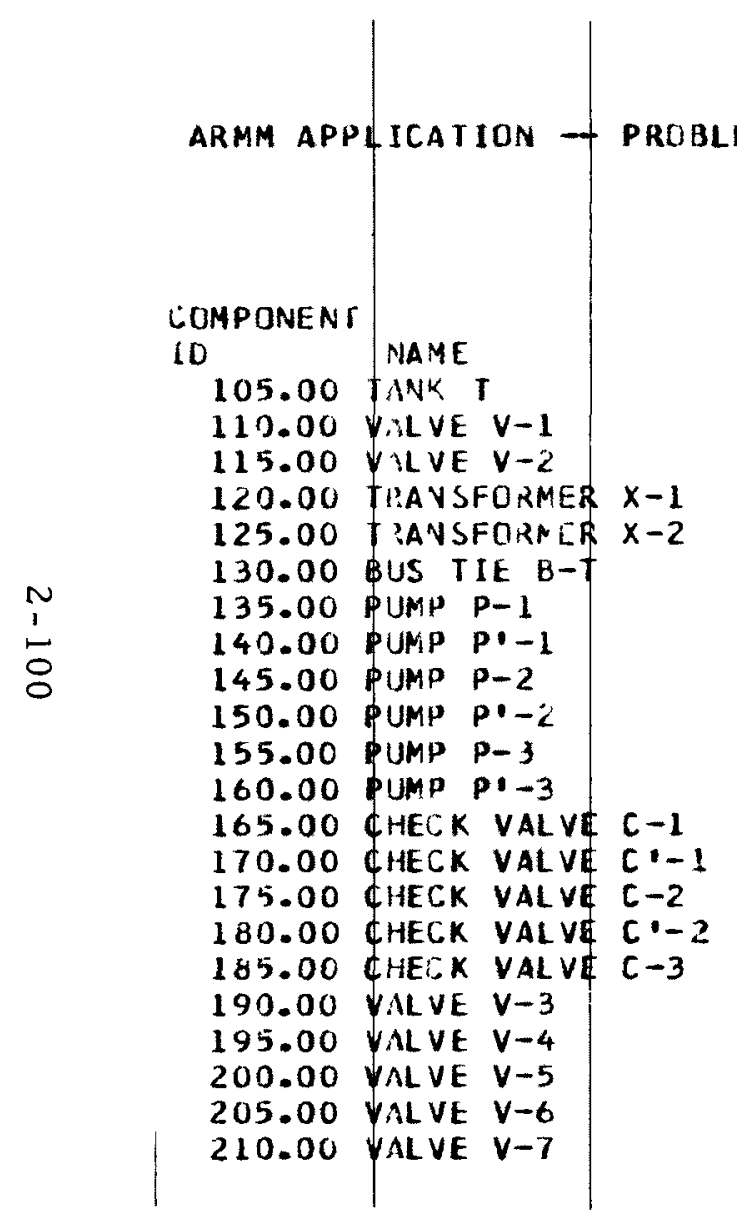

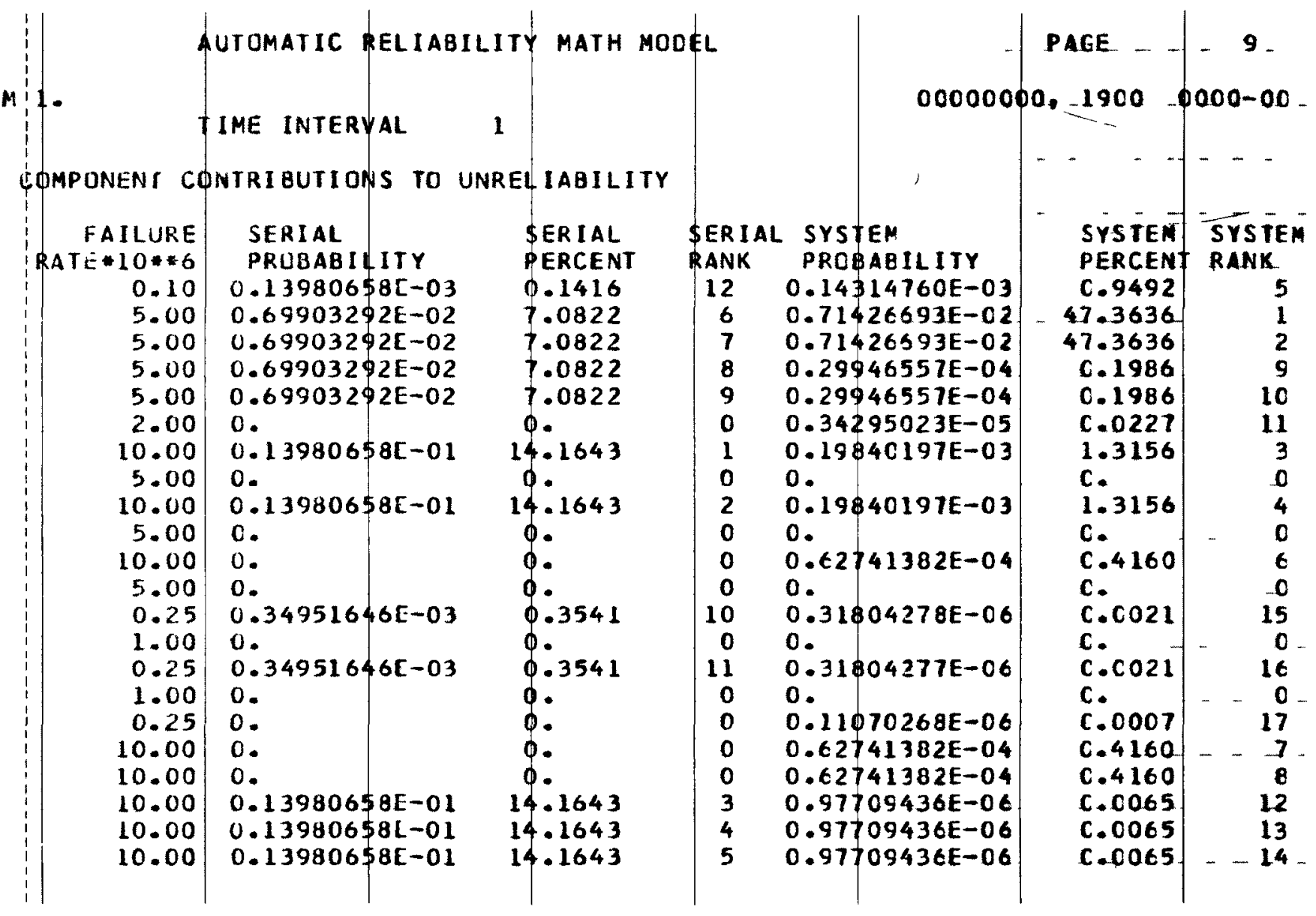




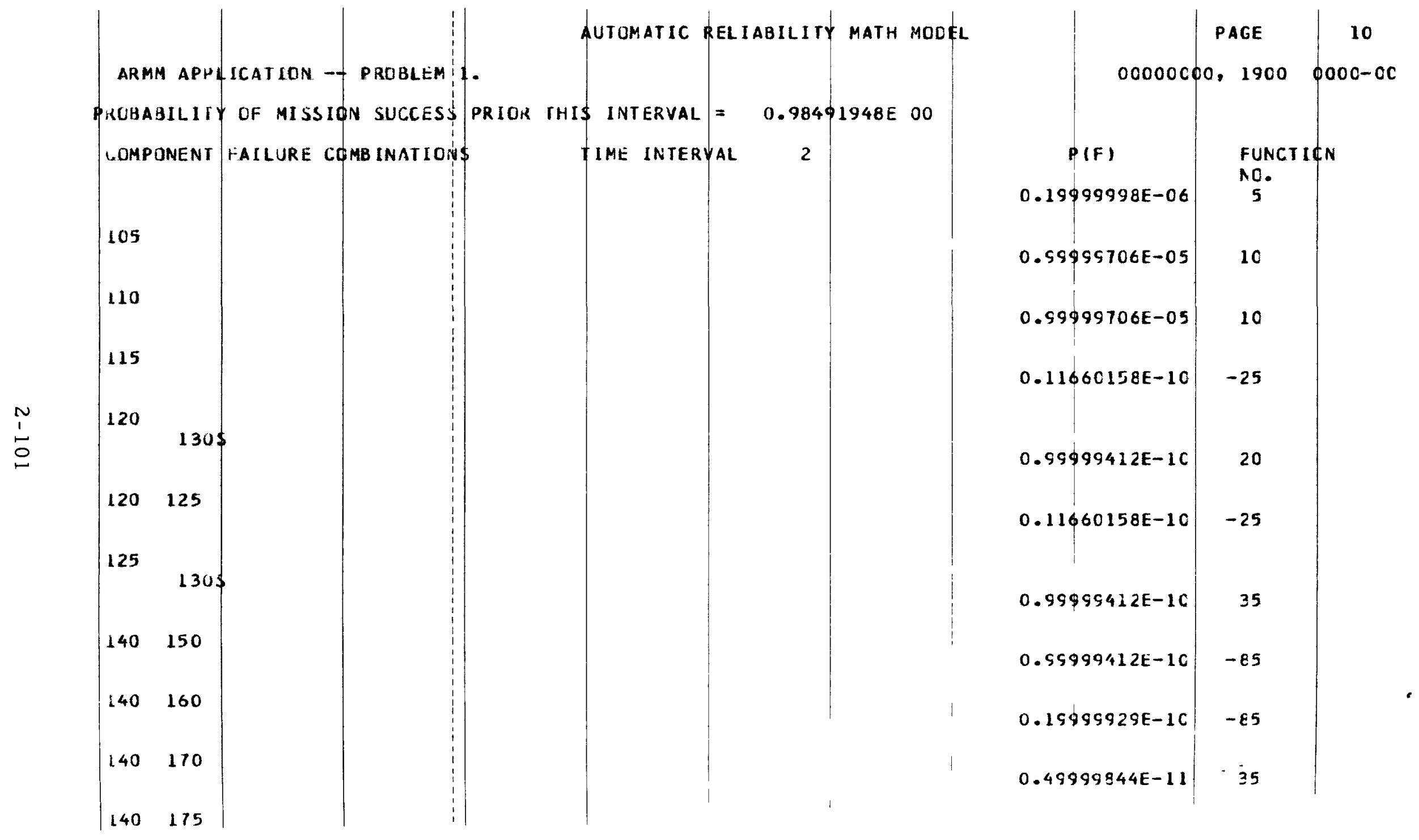

FIGURE 2. 20 (continued) 


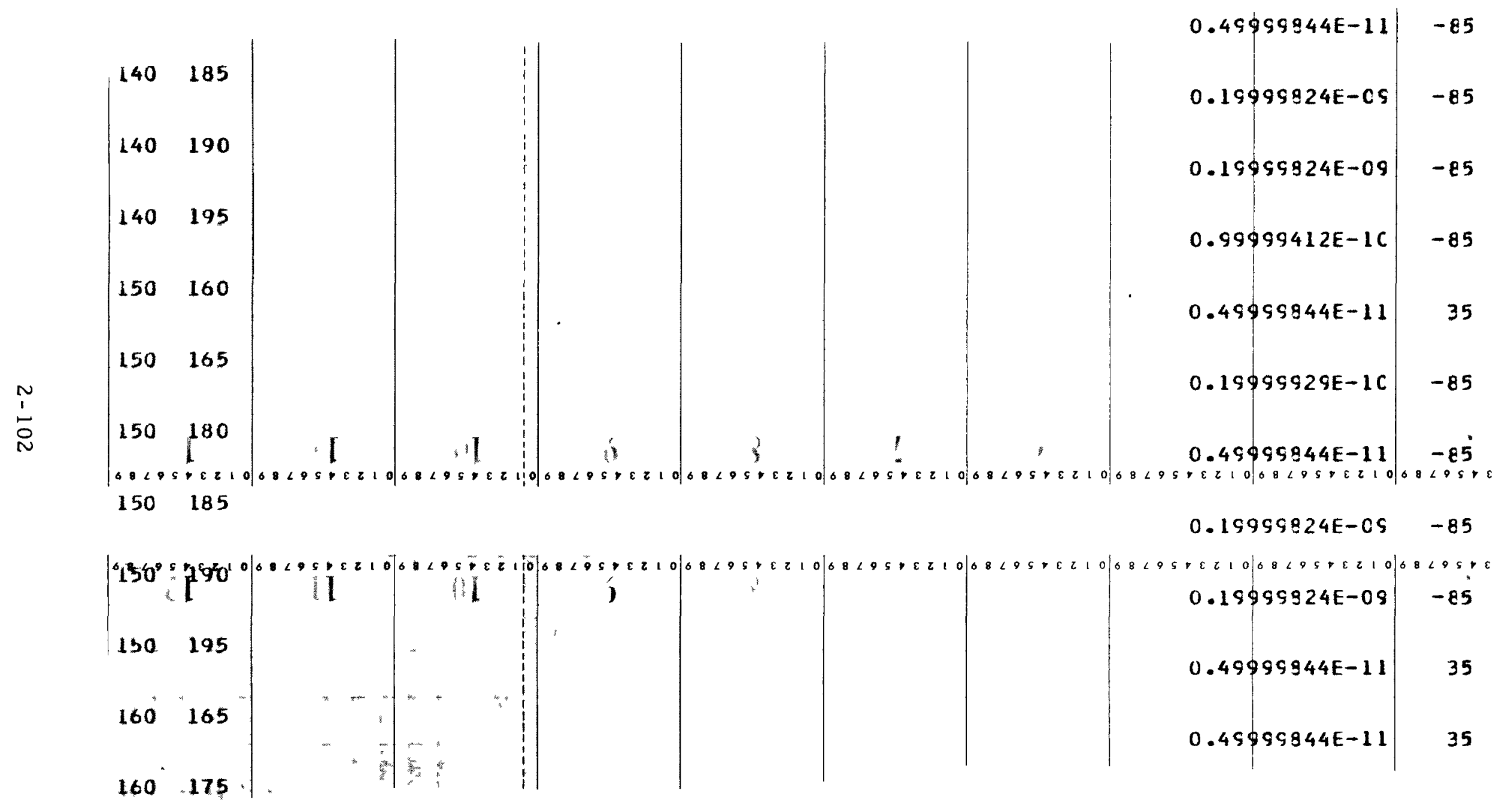

FIGURE 2.20 (continued) 

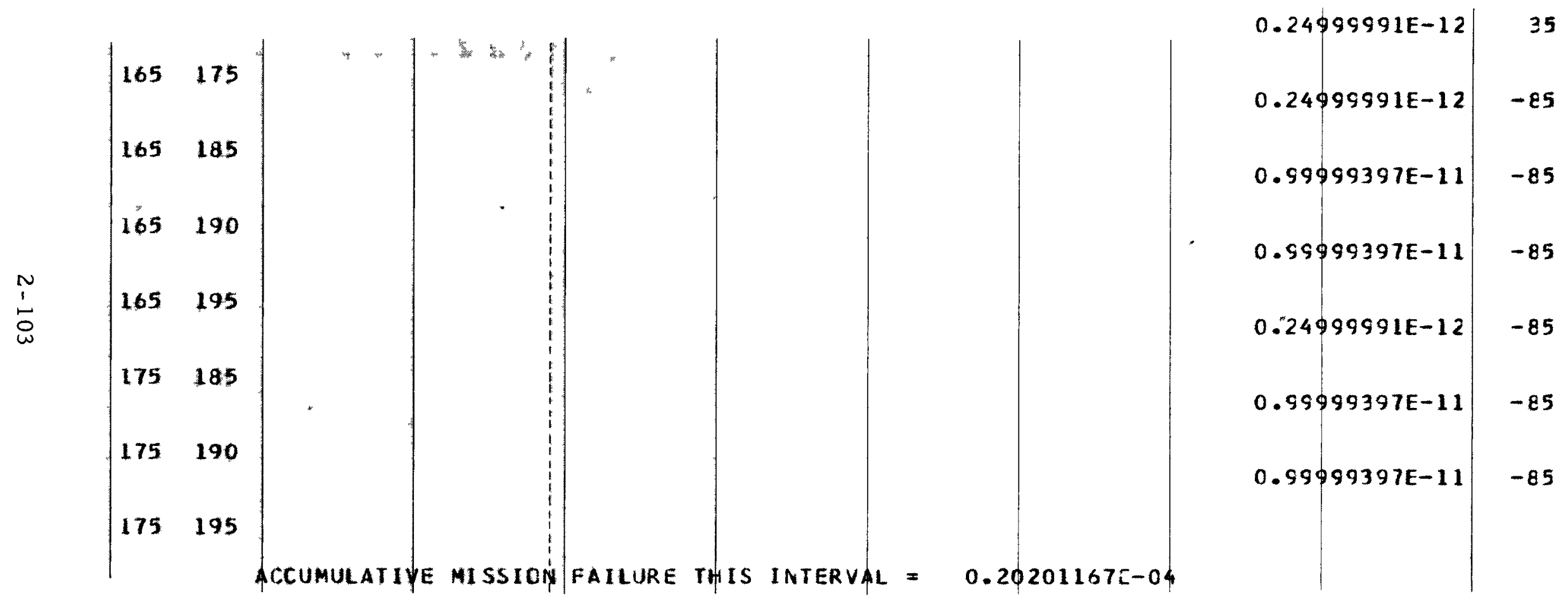

FIGURE 2.20 (continued) 


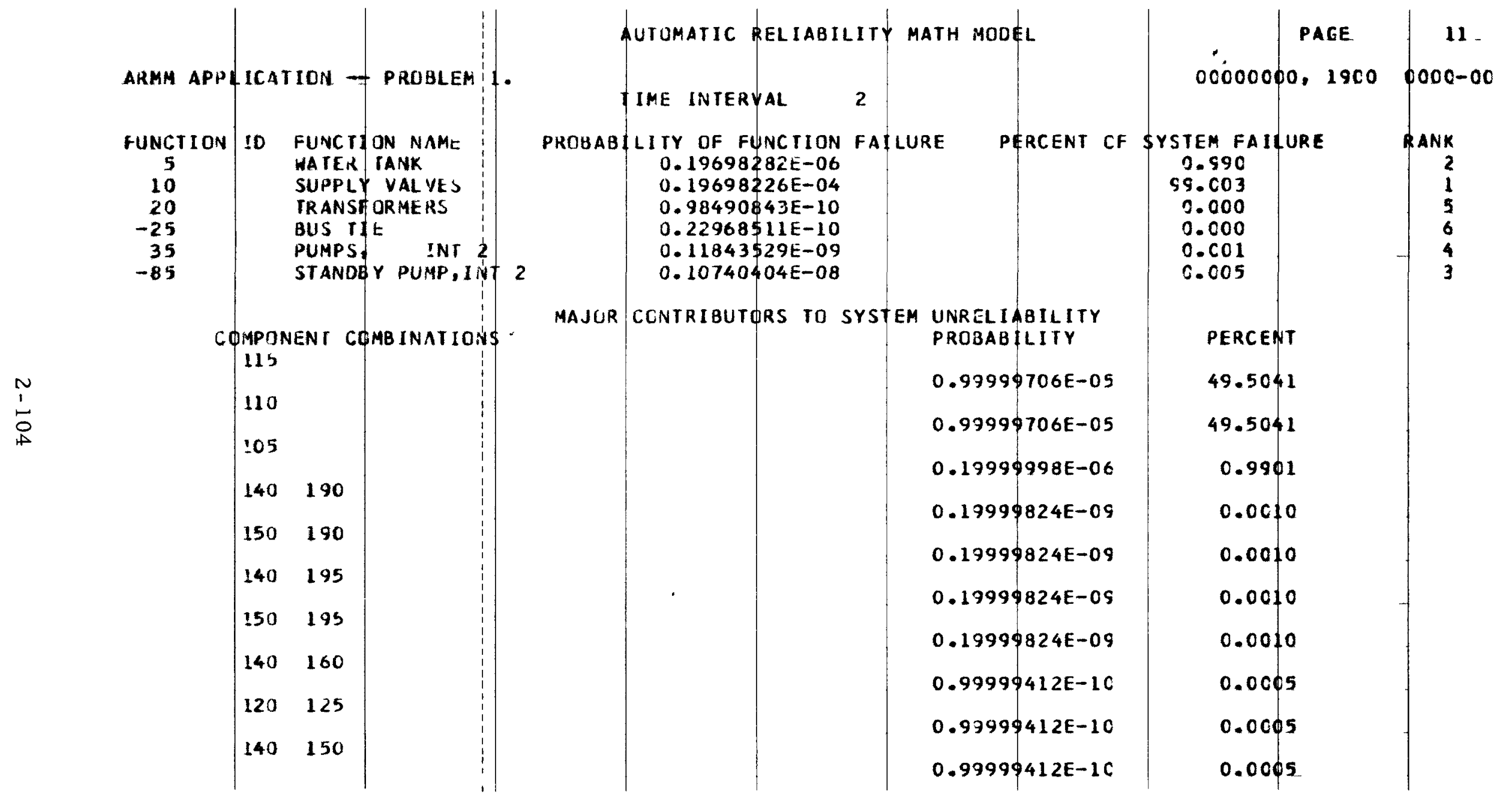

FIGURE 2.20 (continued) 


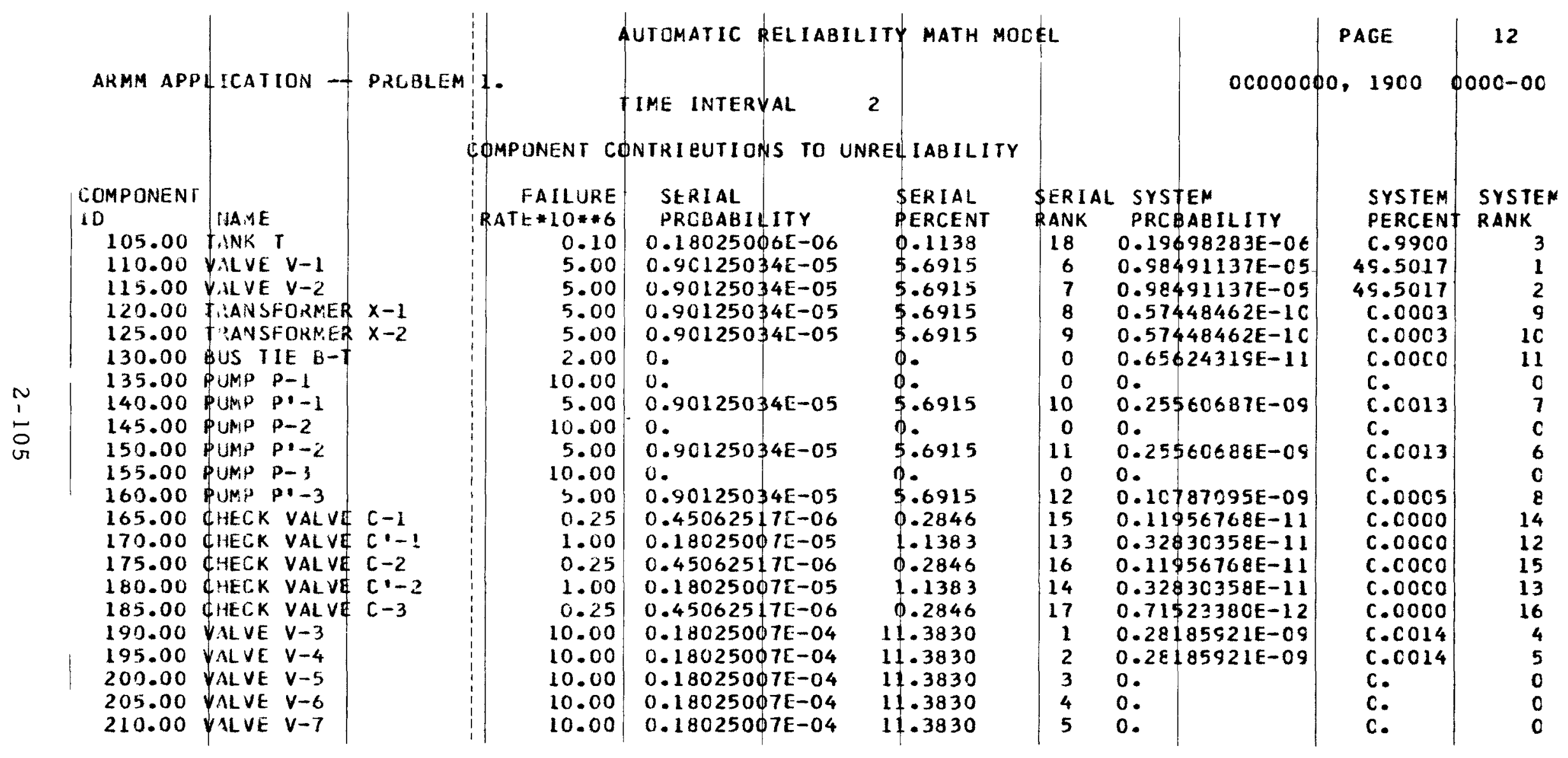

FIGURE 2.20 (continued) 

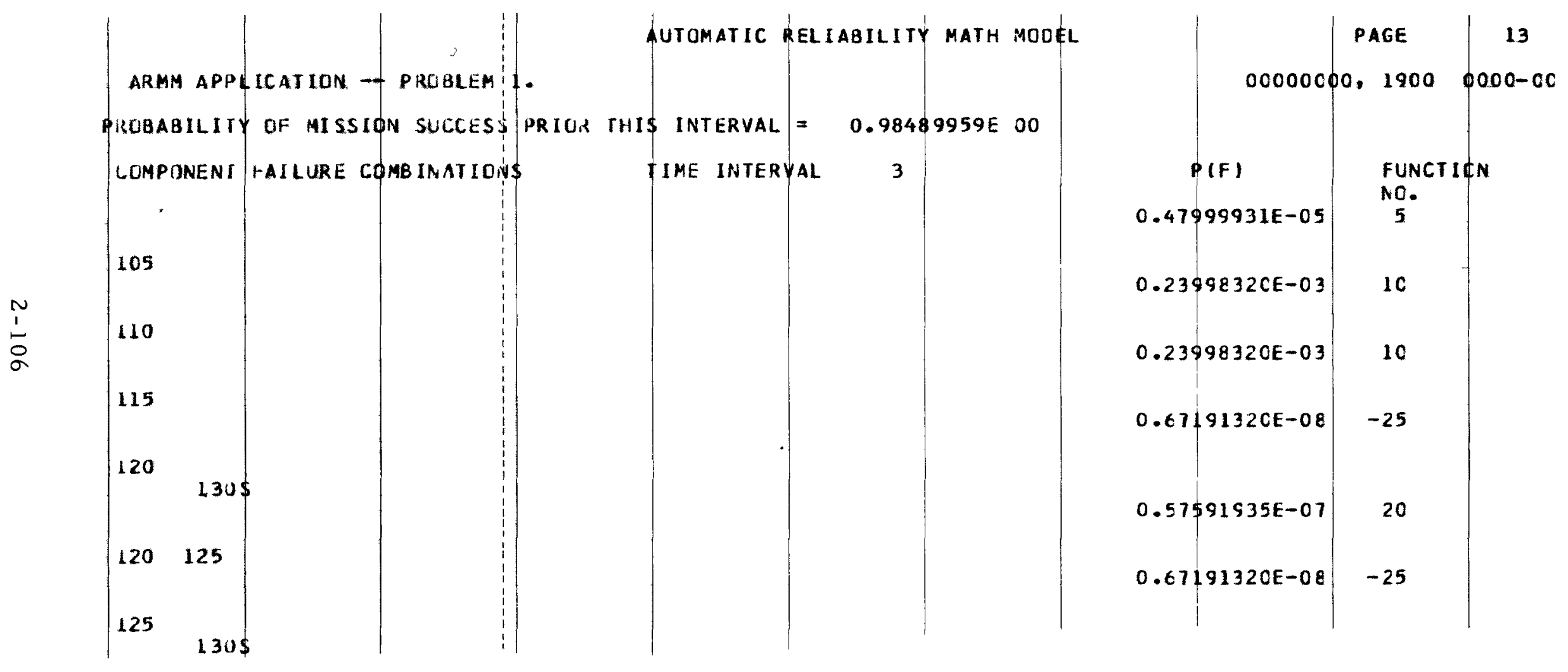

FIGURE 2.20 (continued) 


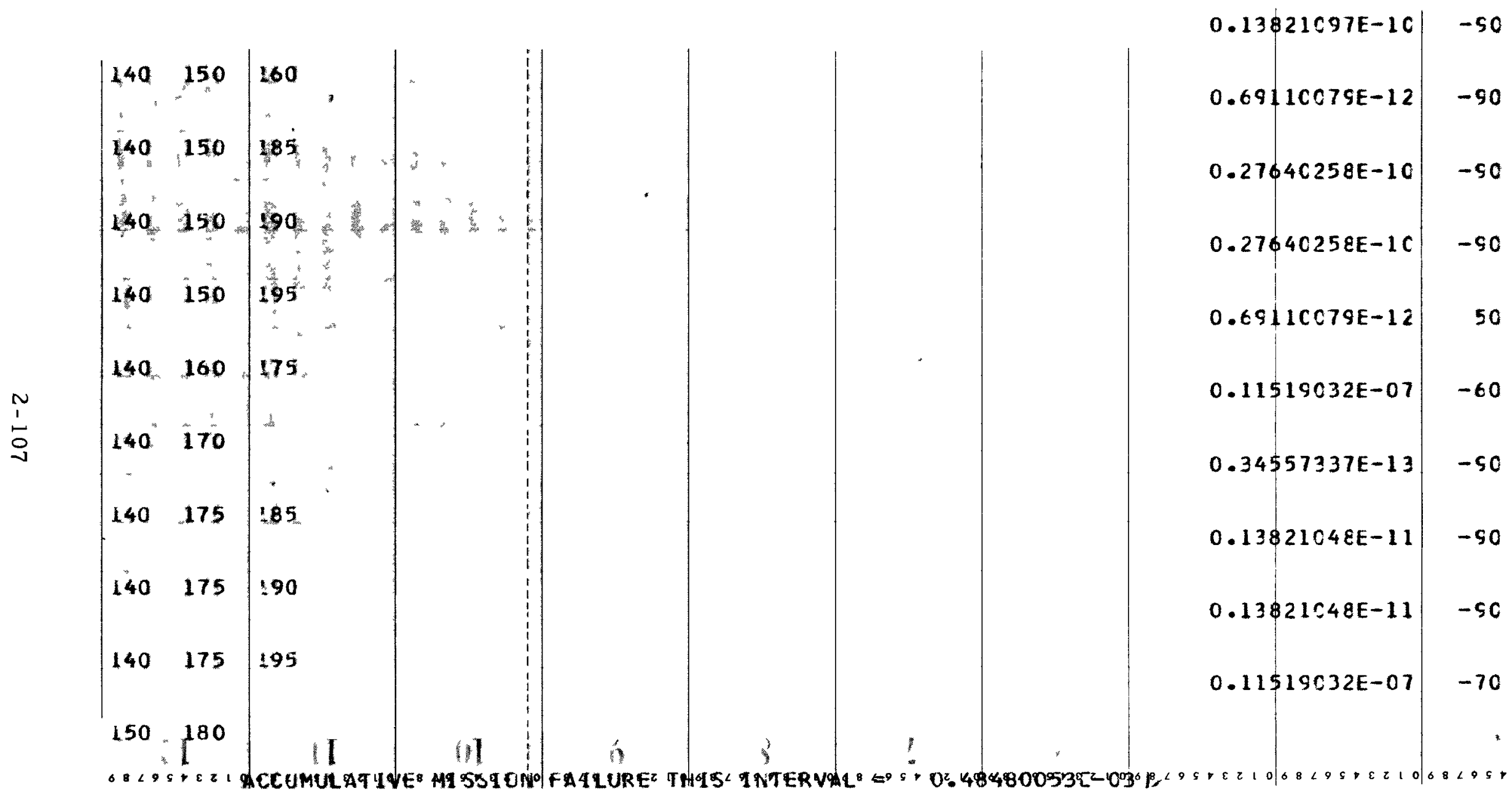

FIGURE 2.20 (continued) 


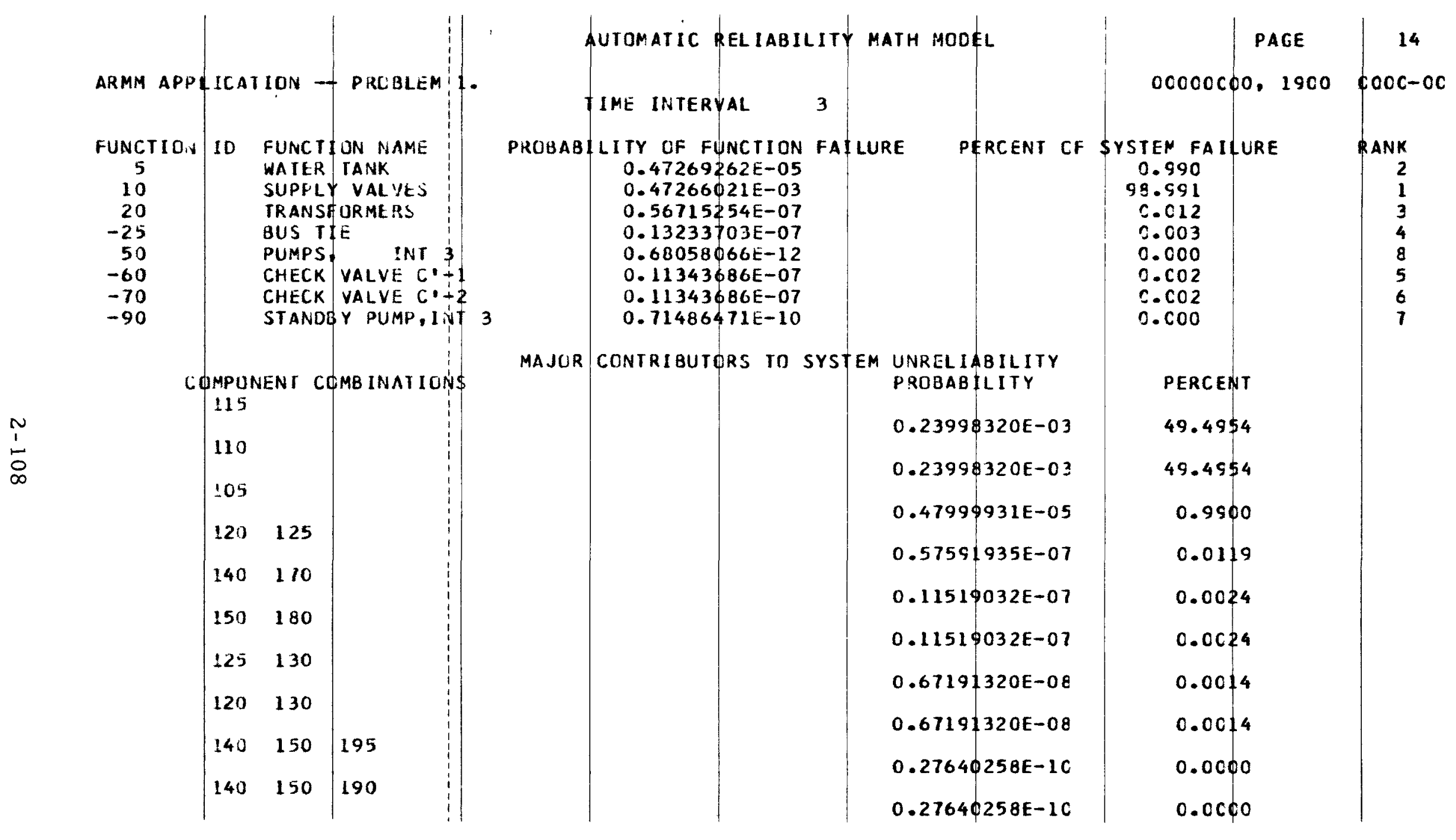

FIGURE 2.20 (continued) 


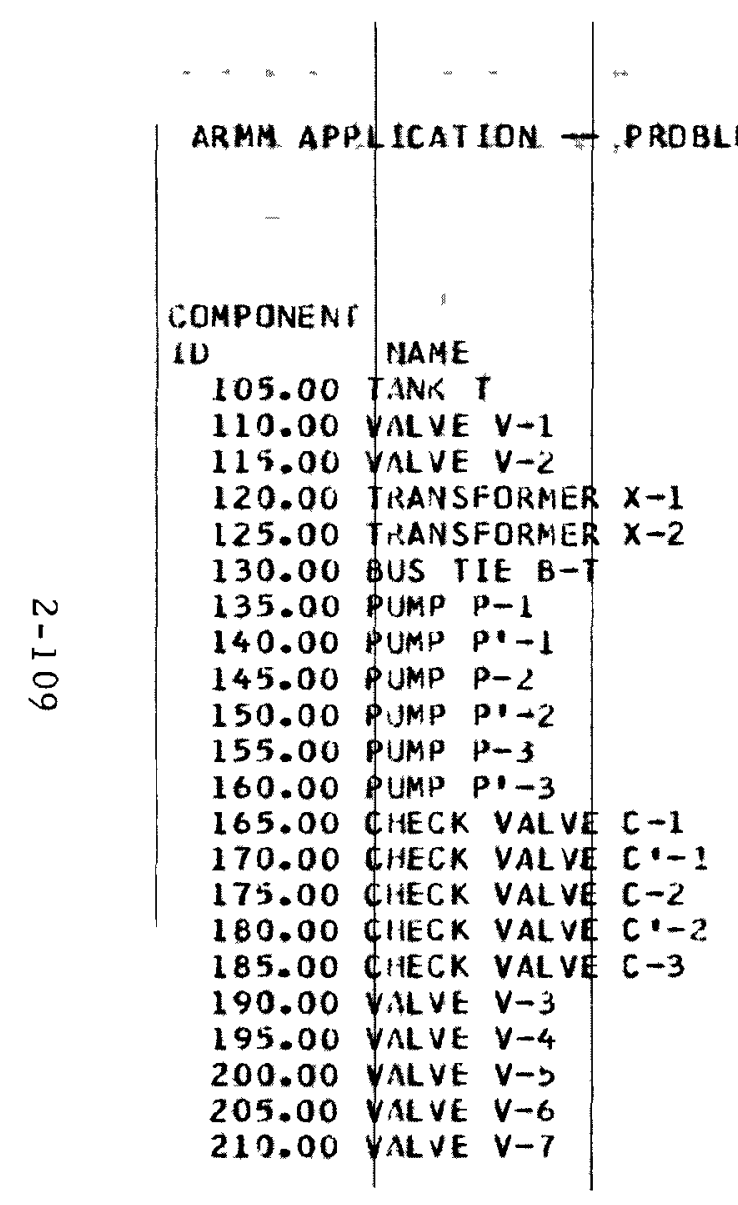

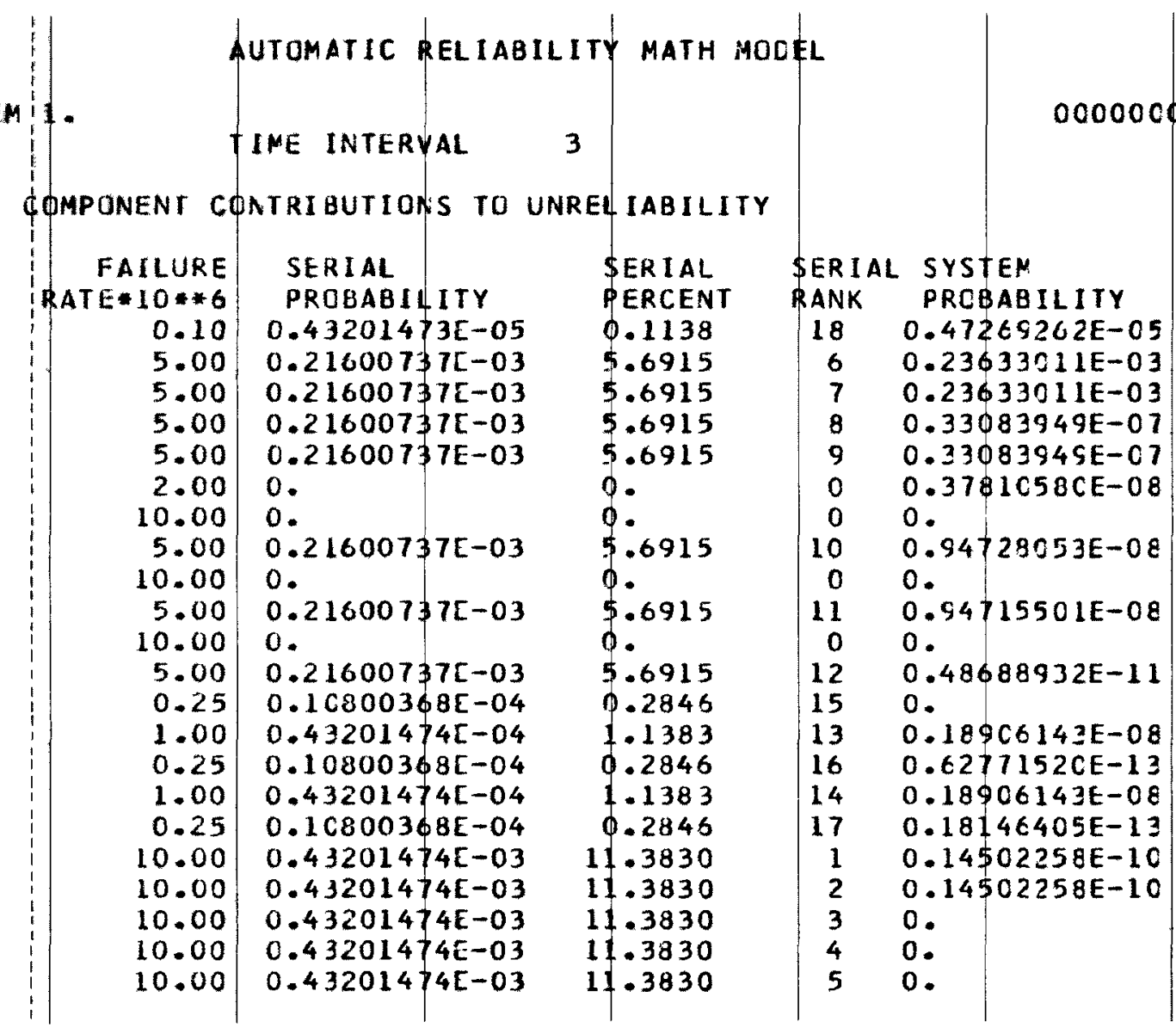

PAGE

15

, $1900 \quad 0000-00$

SYSTEM SYSTEN

PERCENT RANK

C. $99 \mathrm{CO}$

45.4953

49.4953

C. 0069

C. 0069

C. $\operatorname{COC} 8$

C. 0020

0 .

C. 0020

c.

C. $\operatorname{coc} 0$

$c$.

C. $\operatorname{coc} 4$

C. $\mathrm{COCO}$

C. 0004

C. $00 \mathrm{CO}$

C. $\mathrm{COCO}$

C. 0000

c.

c.

c. 


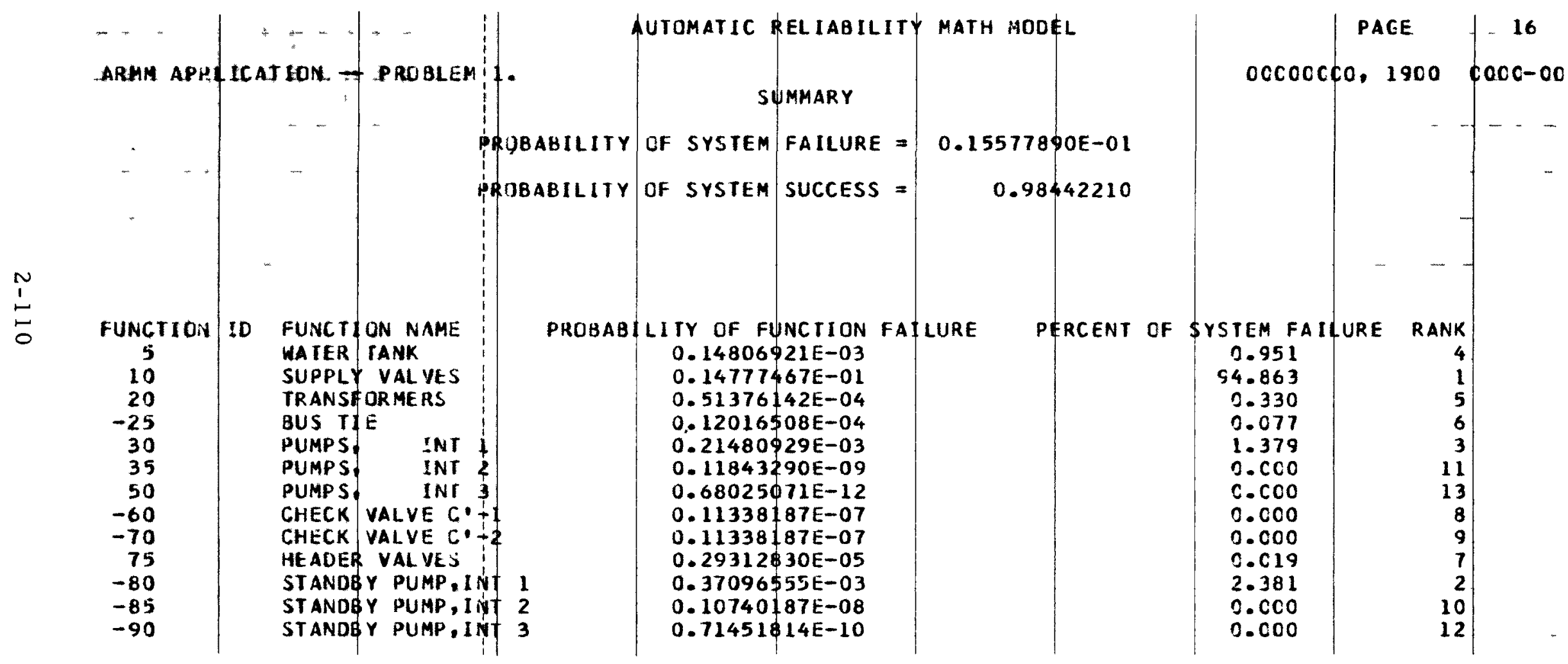

FIGURE 2.20 (continued) 

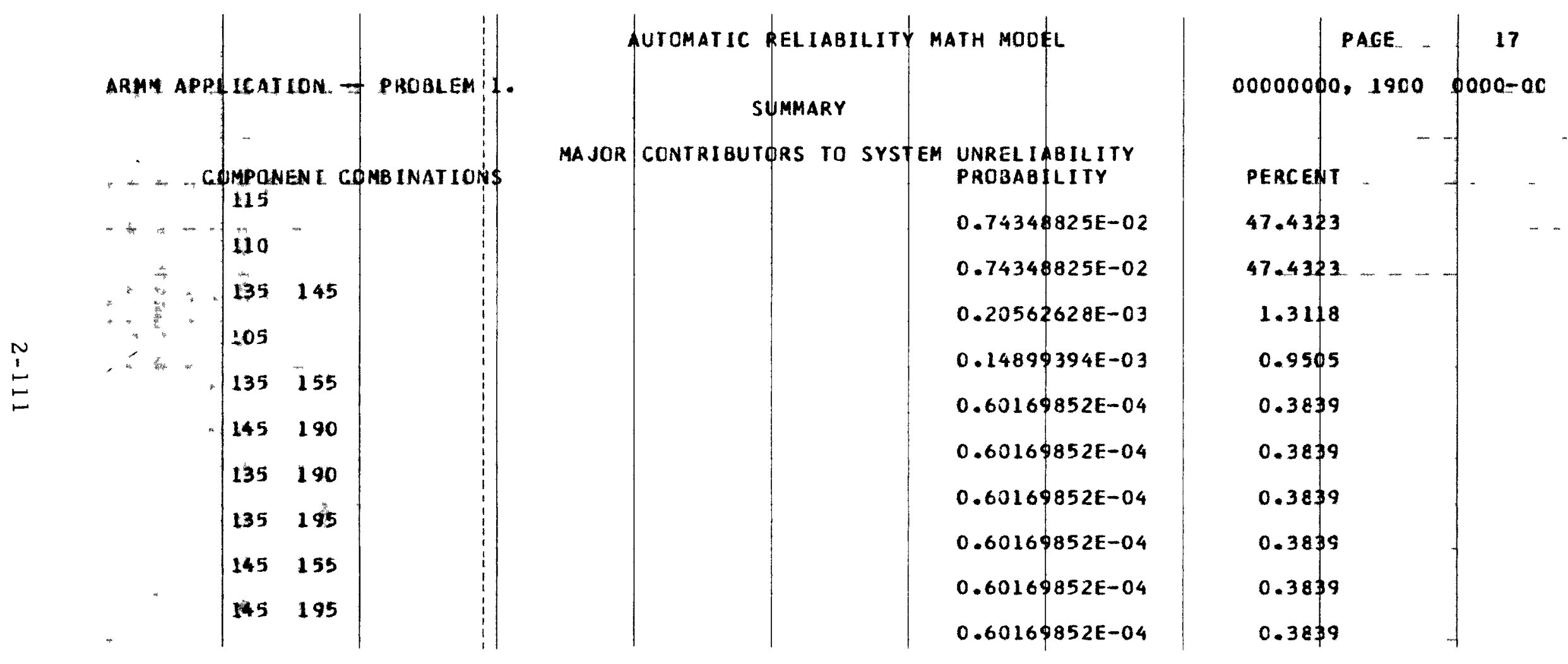

FIGURE 2.20 (continued) 

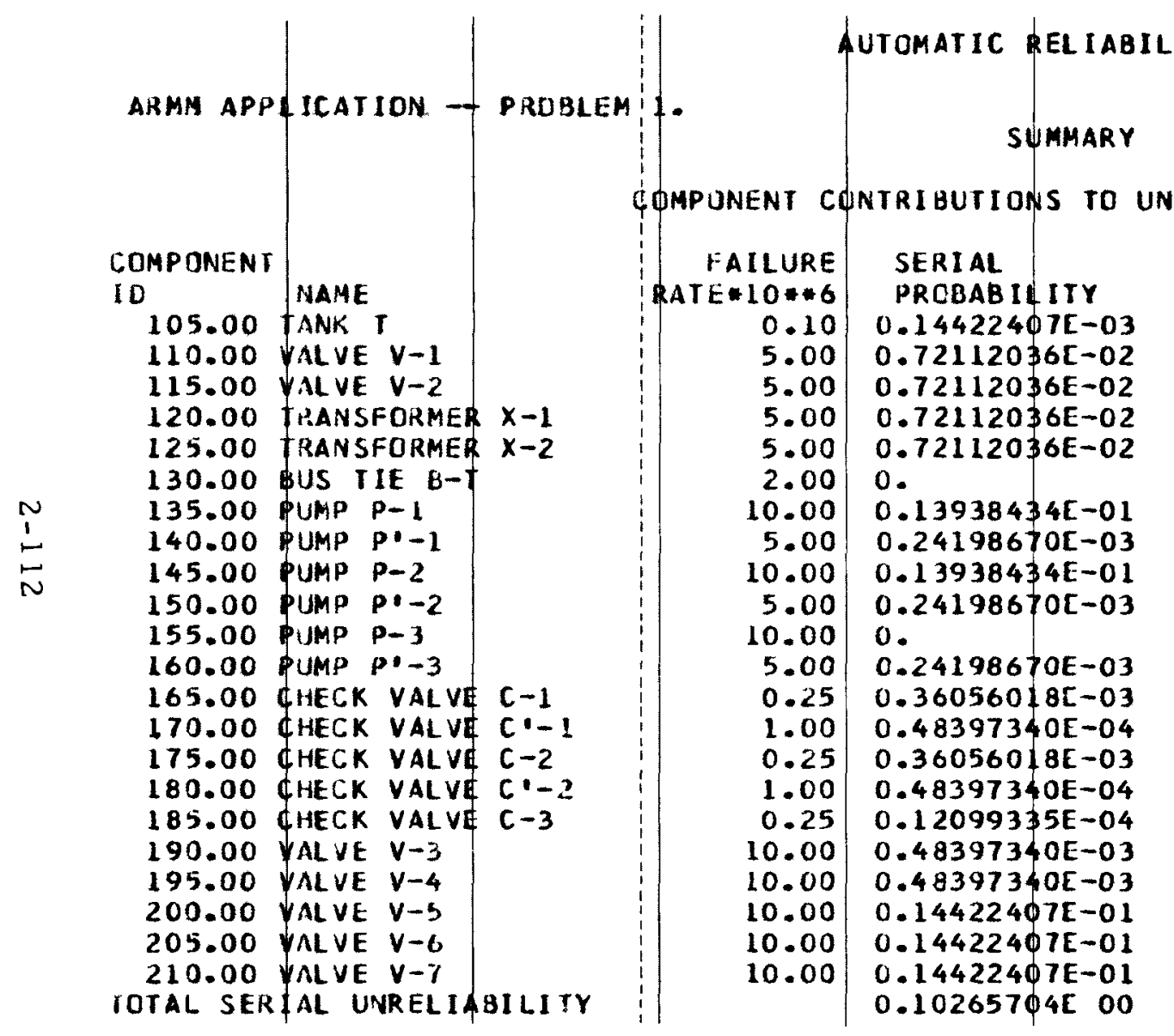

TY MATH MOO
IABILIT
SERIAL
PERCENT
0.1405
7.0246
7.0246
7.0246
7.0246
0.0
13.5777
0.2357
13.5777
0.2357
0.
0.2357
0.3512
0.0471
0.3512
0.0471
0.0118
0.4714
0.4714
14.0491
14.0491
14.0491

SER
SER IAL
RANK
17
6
7
8
9
0
4
14
5
15
0
16
12
18
13
19
20
10
11
1
2
3
3

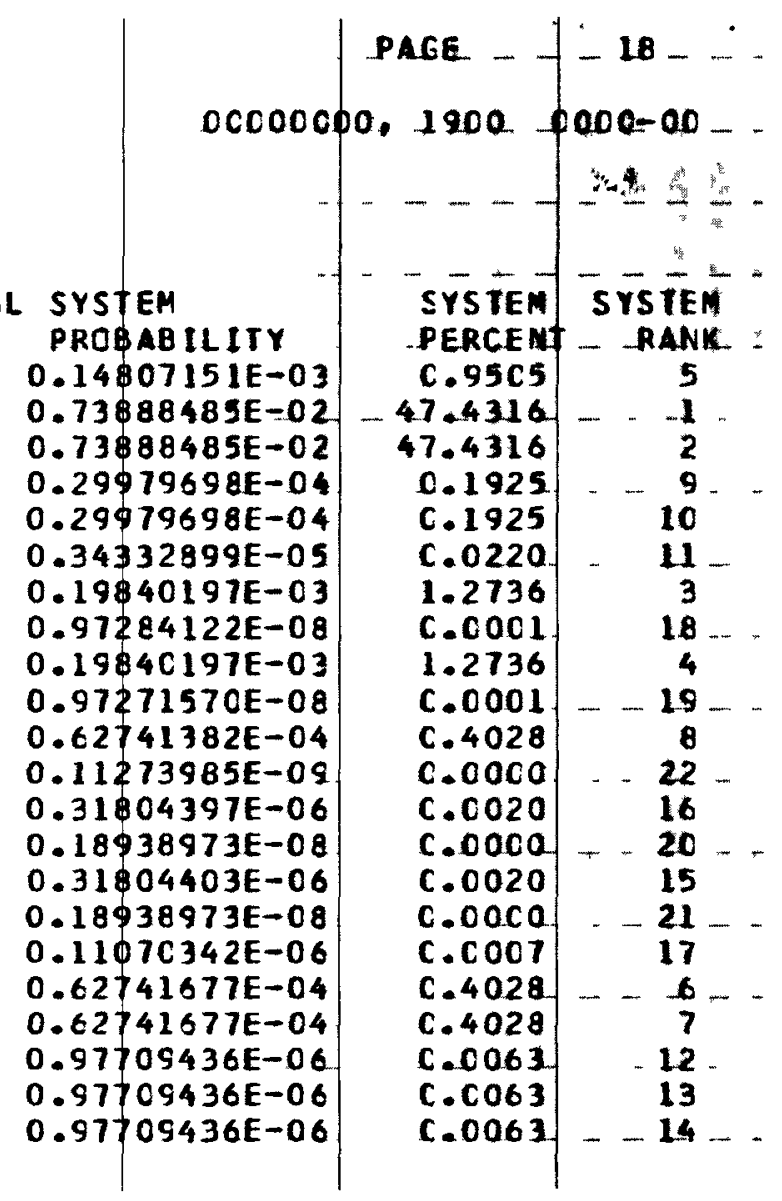

FIGURE 2.20 (continued) 


\section{FIGURE 2.20 (continued)}



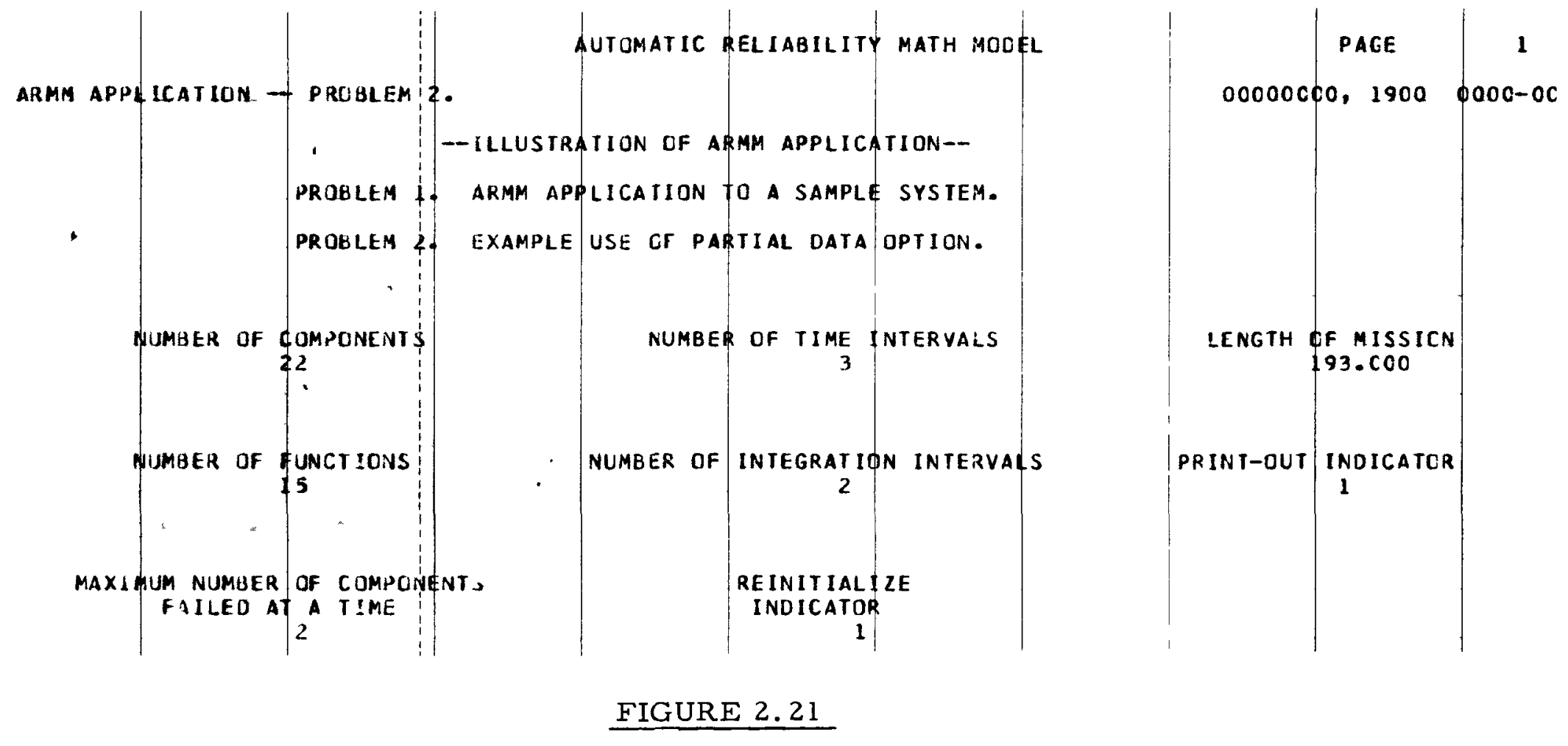

\section{COMPUTER OUTPUT SUMMARY}




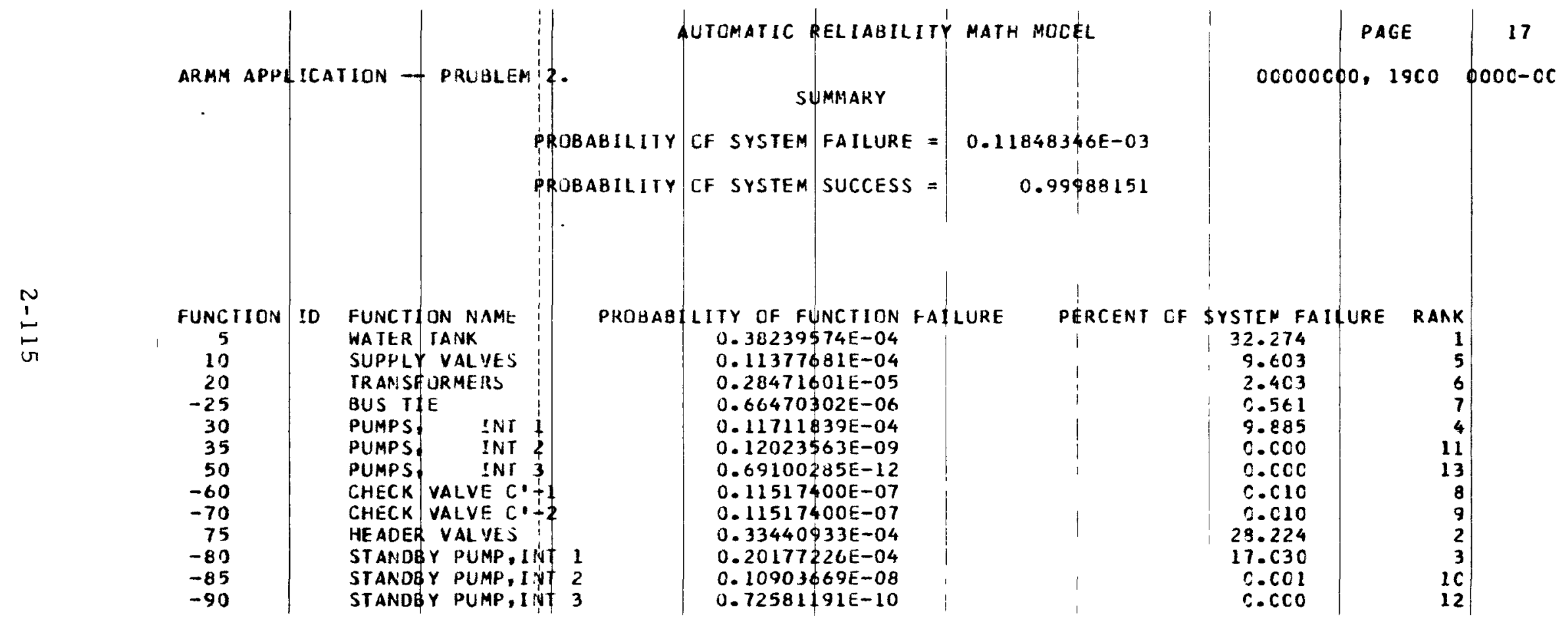

FIGURE 2.21 (continued) 


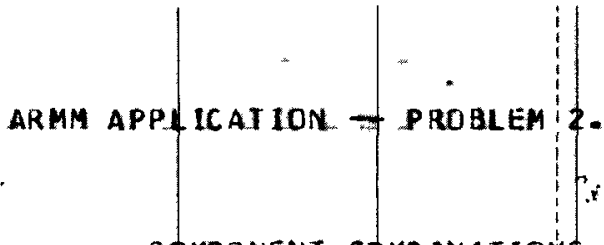

COMPINENT COMBINATION 105

$110 \quad 115$

$200 \quad 210$

200205

135145

205210

135190

$135 \quad 195$

145190

$135 \quad 155$
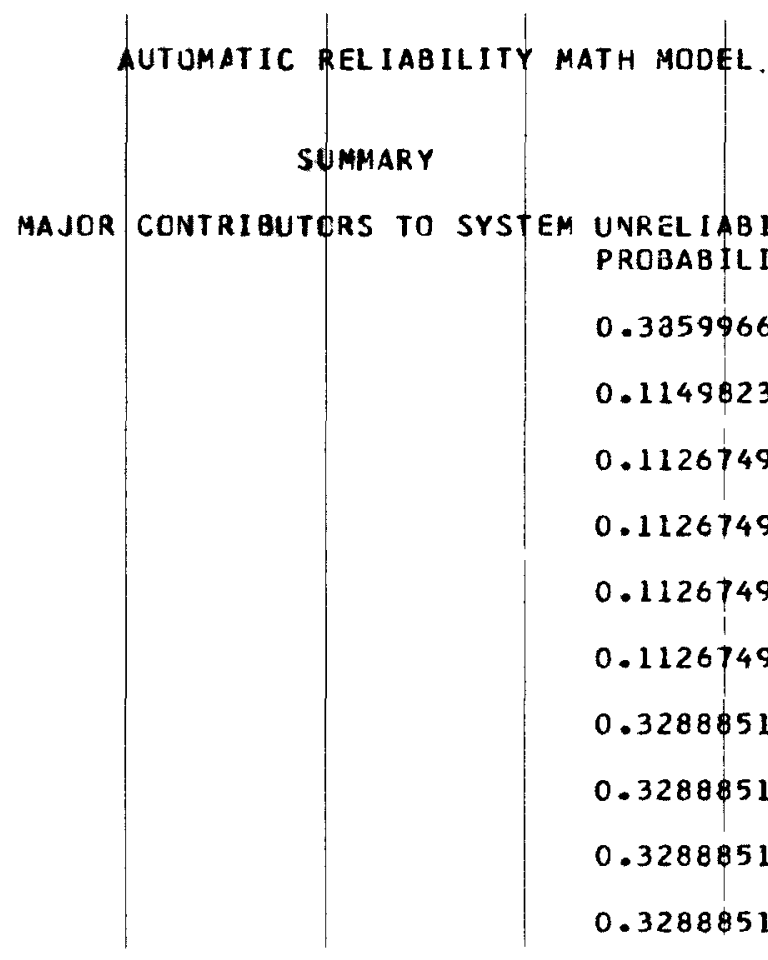

UNREL I AB IL I TY PROBAB IL ITY

$0.3359 \$ 662 E-04$

$0.11498232 E-04$

$0.11267496 E-04$

$0.11267496 E-04$

$0.11267496 E-04$

$0.11267496 \mathrm{E}-04$

$0.3288 \$ 511 E-05$

$0.32888511 E-05$

$0.32888511 E-05$

$0.32888511 E-05$
PAGE

$00000000,1900 \quad$ COCO-CC

PERCENT

32.2450

9.6053

9.4125

9.4125

9.4125

9.4125

2.7474

2.7474

2.7474

2.7474

FIGURE 2.21 (continued) 


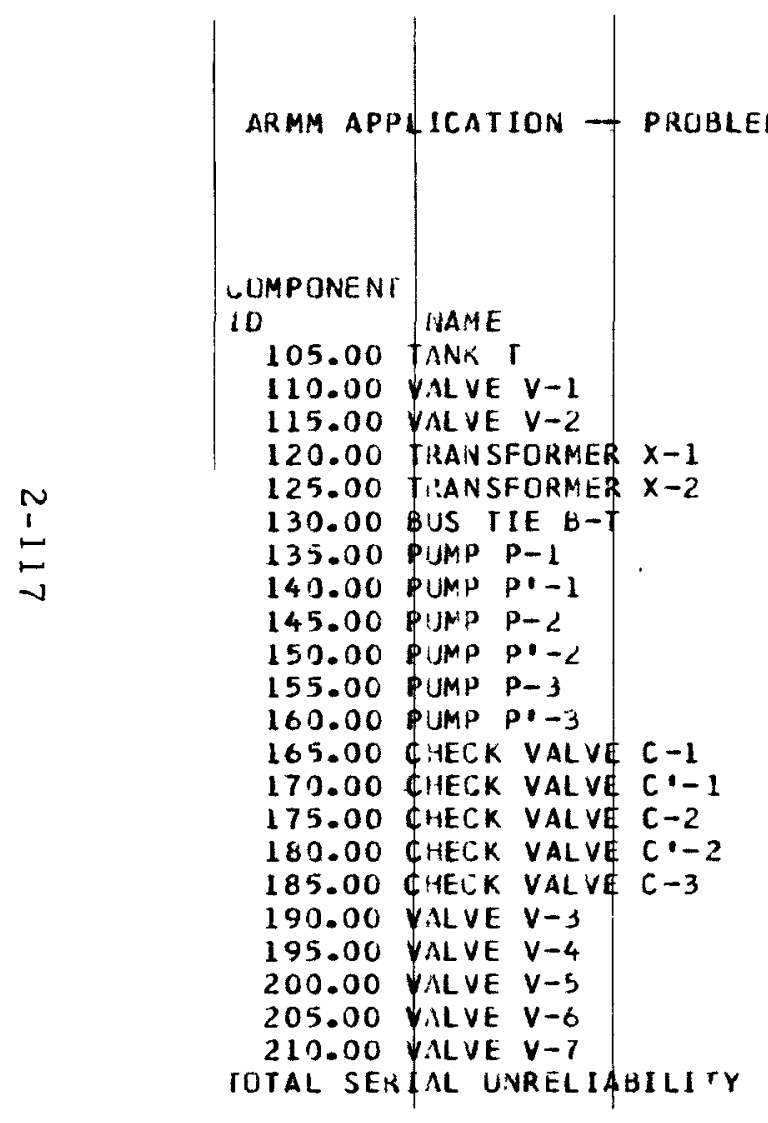

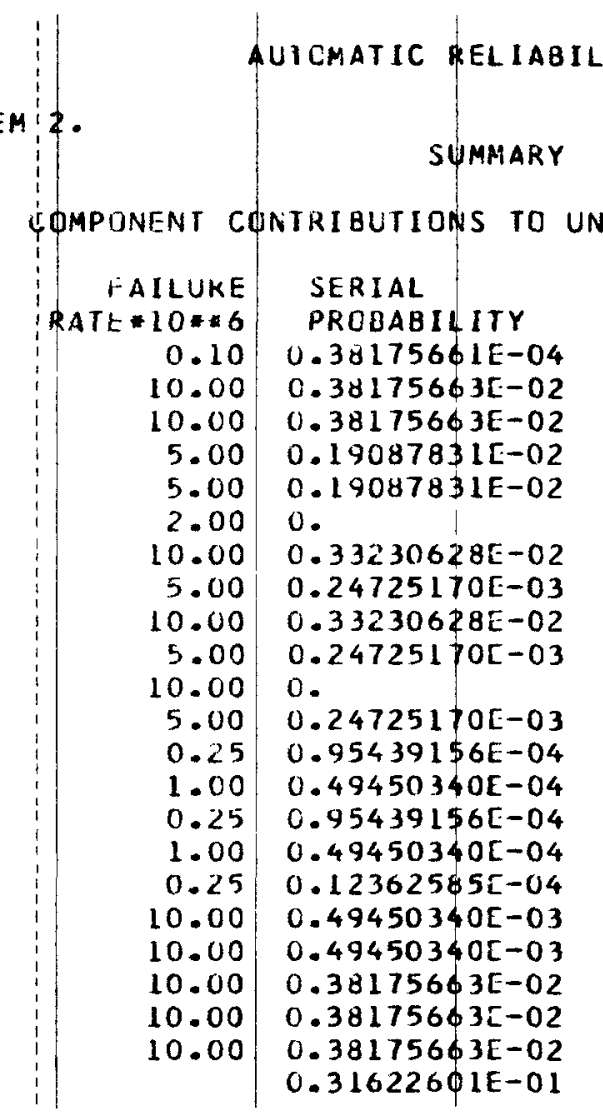

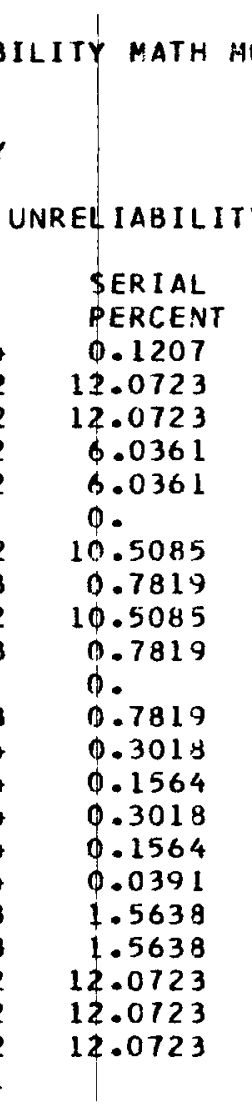

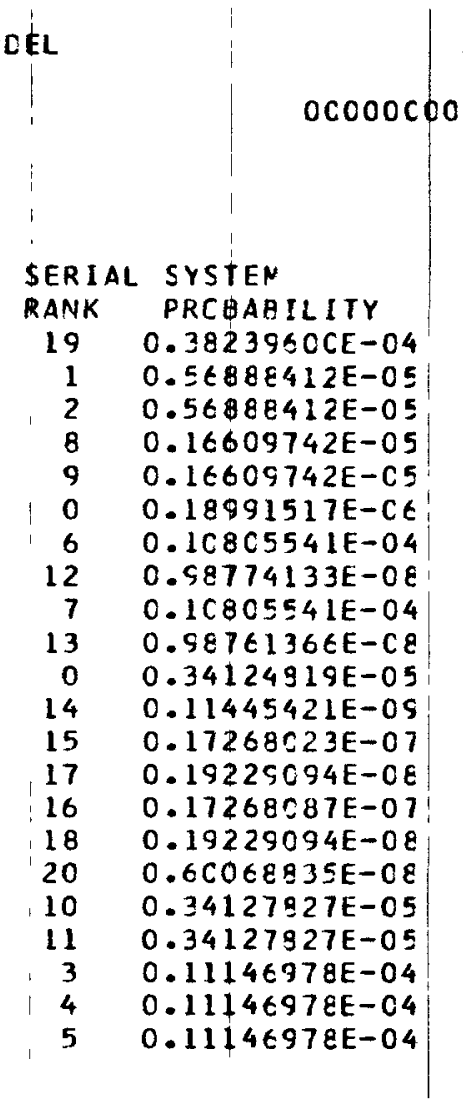

PAGE

19

1900 000C-CC

FIGURE 2.21 (continued)

YSIEM FERCEN 32.2742 4.8014 4.8014

1.4019

1.4019

C. 1603

5.1159

C.0083

9.1199

C. $\cos 3$

$2.88 \mathrm{Cl}$

c. 0001

C.0146

C. $\operatorname{col} 6$

c.0146

c.0016

C.0051

$2.88 \mathrm{C} 4$

2.8804

5.4080

5.4080

5.4080
SYSTEN

RANK

$\frac{1}{7}$

8

12

13

14

5
17

6

18

11

22

16

15

15
21
19

$1 \mathrm{C}$

$1 \mathrm{C}$
2 


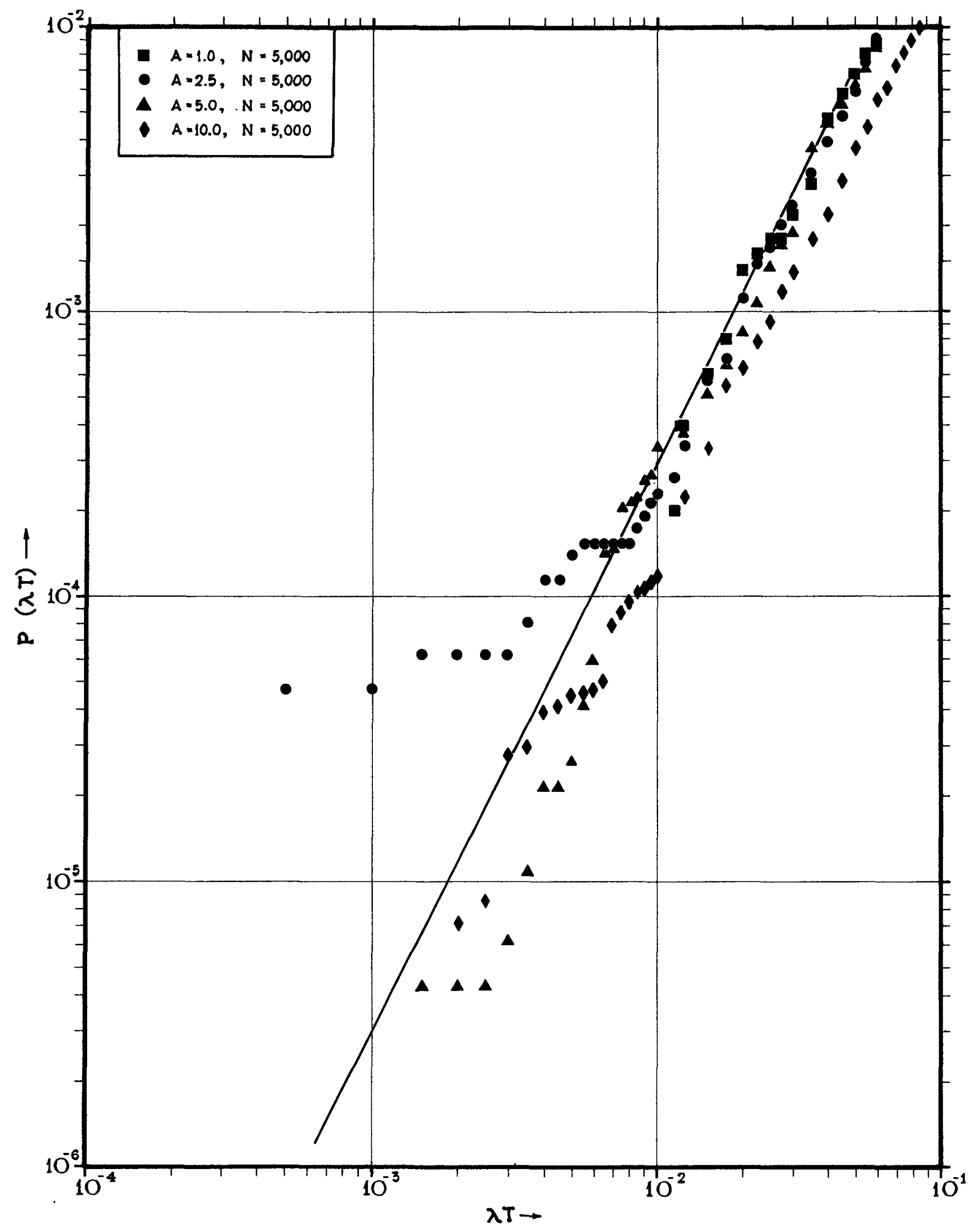

FIGURE 2.22

$P(\lambda T)$ AS A FUNCTION OF $\lambda T$ FOR SEVERAL VALUES OF $A$. THE MODEL IS A TWO-OUT-OF-THREE SYSTEM WITH IDENTICAL COMPONENTS AND NO REPAIR. 


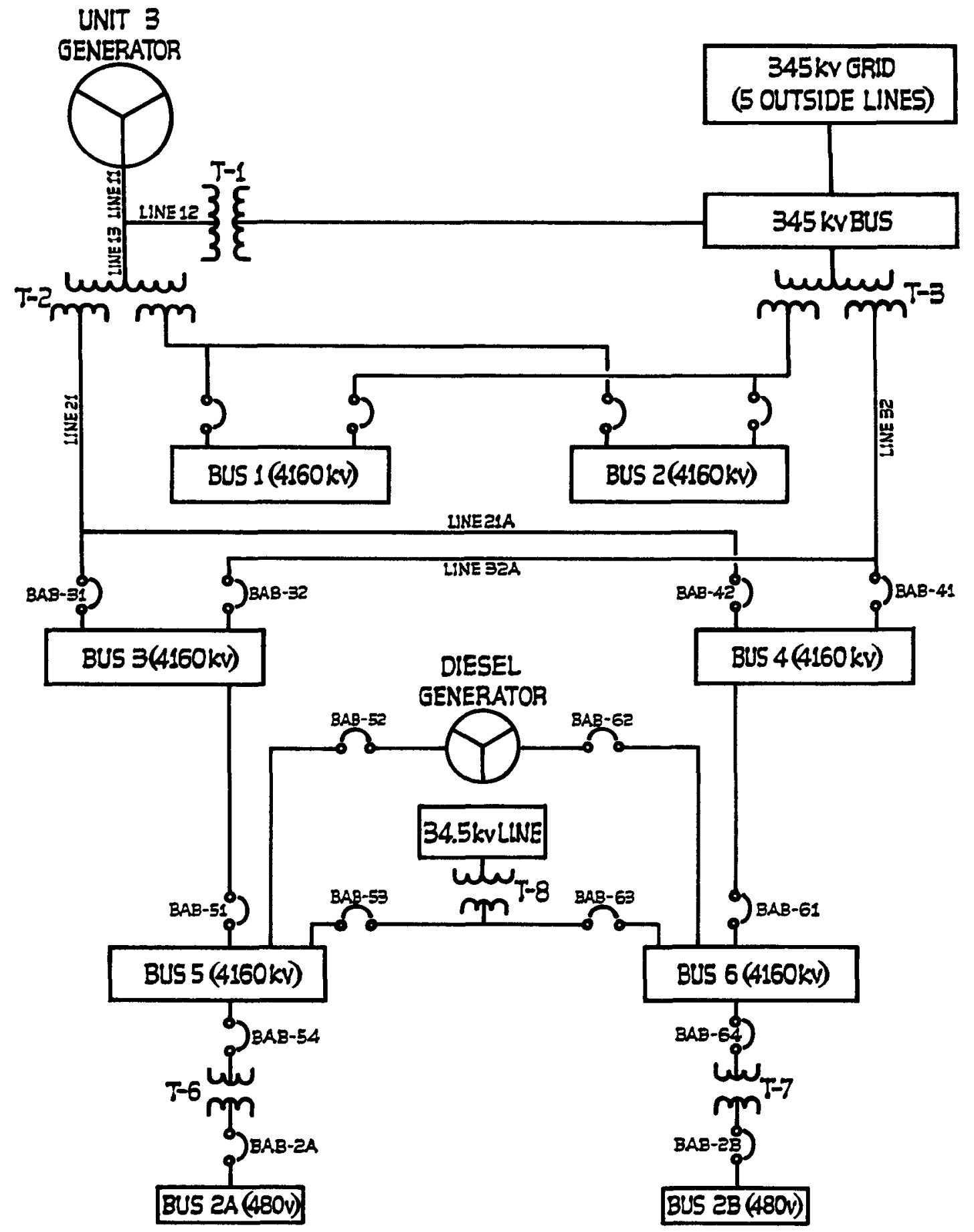

$$
\begin{aligned}
\text { LEGEND } \\
T=\text { TRANSFORMERS } \\
\text { BAB = BUS AIR CIRCUIT BREAKERS }
\end{aligned}
$$

FIGURE 2.23 


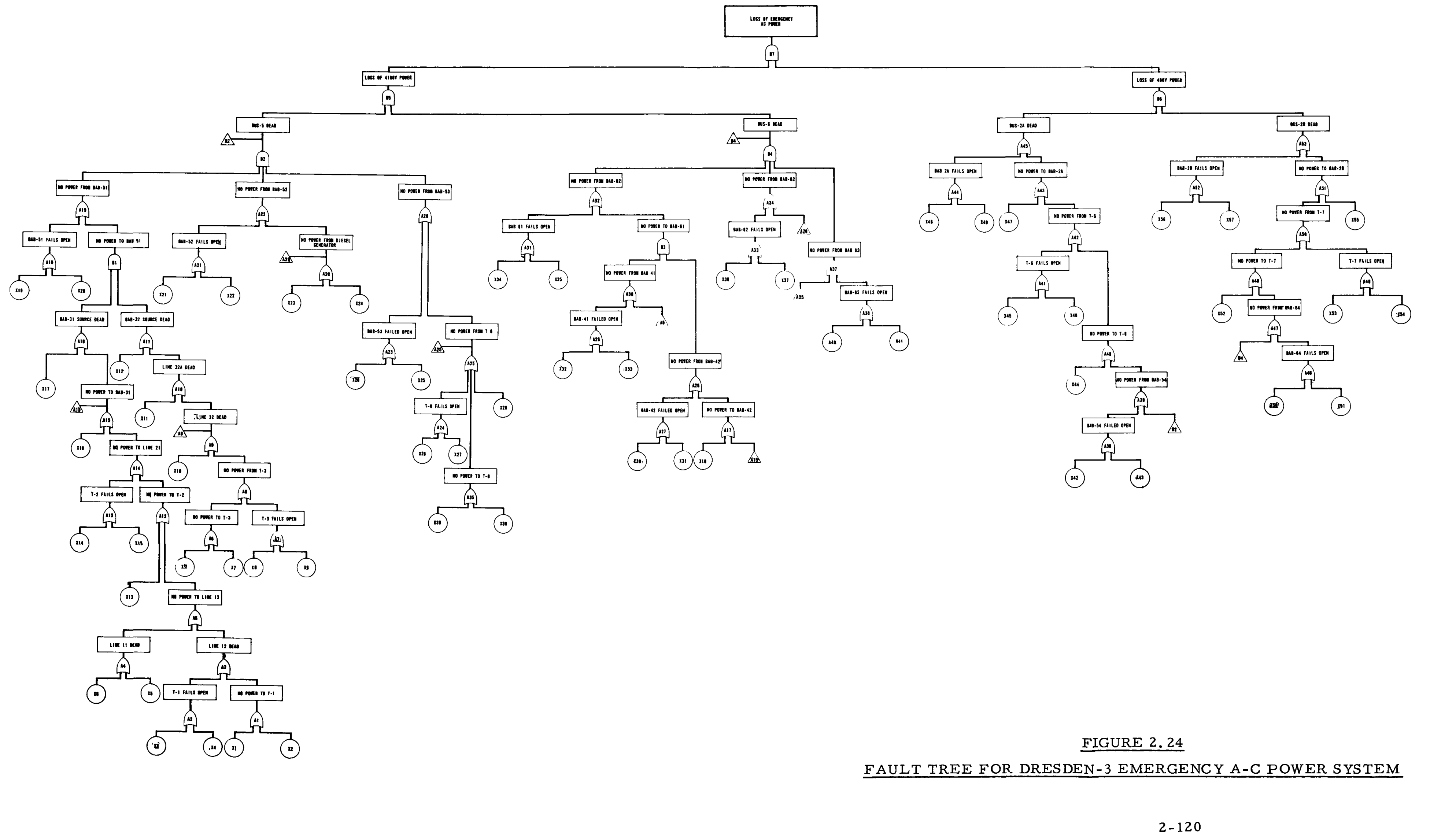




\begin{abstract}
$3000 \quad 57250 \quad 1 \quad 1$ 4.2.0000E 04 1. CONOOE 00 1.00000E OO $1.00000 E 00$

$1.00000 E$ O6 1.00000E $071.11000 E$ O5 1.11000E 05 1.00000E 04 $1.00000 E$ O6 $1.00000 E$ O6 $1.11000 E$ O5 $1.11000 E$ O5 1.00000E $061.00000 E$ O6 2.00000E O5

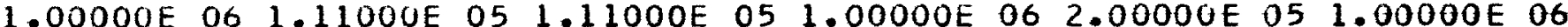
2.00000 E 05 2.00000E $052.00000 E$ O5 1.00000E $051.00000 E$ O5 1.00000E O6 1.00000 E 05 2.00000E 051.11000 E 051.11000 E 051.00000 E 062.00000 E 05 1.00000 E 05 2.00.300E 05 1.00000E 05 2.00000E 05 2.00000E 05 2.00000E 06 1.00000 E 051.00000 E 061.00000 E $05 \quad 2.00000 E-051.00000 E$ O5 $2.00000 E$ OS 1.00000 E 05 1.00000E O6 1.11000 E $051.11000 E$ O5 1.00000E $062.00000 E$ O5 1.00000 O5 2.00000E O5 1.00000E $051.00000 E$ O6 1.11000E 05 1.11000E 05 $1.00000 E$ O6 2.00000E $051.00000 E$ O5

1.00000 E $05 \quad 1.00000$ E 051.00000 E 05 1.00000E $051.00000 E$ OS $1.00000 E 05$

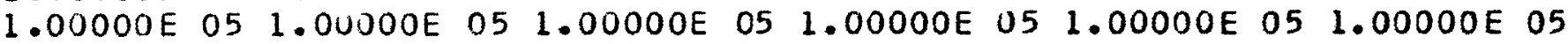

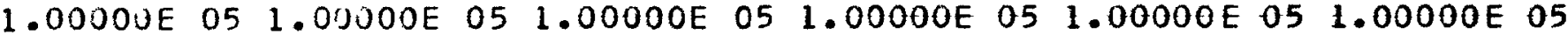

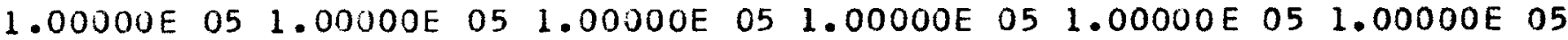

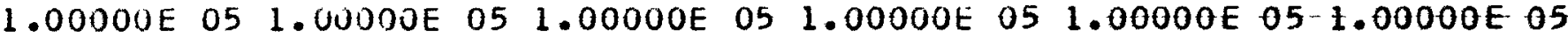

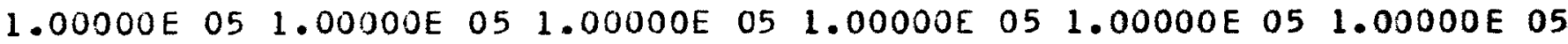
1.00000 E $051.00000 E$ O5 $1.00000 E$ OS 1.00000E 05 1.00000E 05 1.00000E -05

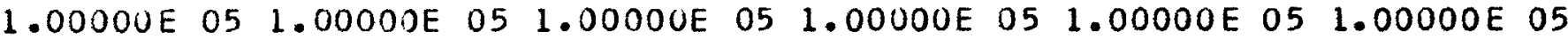
1.00000 E 05 1.00000E 051.00000 E 05 1.00000E 05 1.00000E 05-1.00000E 05 1.00000 E $051.00000 E$ O5 1.00000 E $051.00000 E$ O5 $1.00000 E$ O5 $1.00000 E 05$ 1.00000 E-01 1.0000)E-01 1.00000E-01 1.00000E-01 1.00000E-01-1.00000E-01 $1.00000 E-011.00000 E-011.00000 E-011.00000 E-011.00000 E-011.00000 E-01$ $1.00000 E-011.00000 E-01$ 1.00000E-01 1.00000E-01 1.00000E-01-1.00000E-01 $1.00000 E-01 \quad 1.00000 E-011.00000 E-01 \quad 1.00000 E-011.00000 E-011.00000 E-01$ $1.00000 E-01 \quad 1.00300 E-01 \quad 1.00000 E-01 \quad 1.00000 E-01-1.00000 E-01-1.00000 E-01$ $1.00000 E-01 \quad 1.00000 E-011.00000 E-01 \quad 1.00000 E-011.00000 E-011.00000 E-01$ $1.00000 E-011.00000 E-011.00000 E-01 \quad 1.00000 E-01-1.00000 E-01-1.00000 E-01$ $1.00000 E-011.00000 E-01 \quad 1.00000 E-01 \quad 1.00000 E-01 \quad 1.00000 E-011.00000 E-01$ $1.00000 E-011.00000 E-011.00000 E-01$ 1.00000E-01-1.00000E-01 1.00000E-01 $1.00000 E-01$ 1.00000E-01 1.00000E-01 1.00000E-01 1.00000E-01 1.00000E-01 $1.00000 E 00$
\end{abstract}

0

FIGURE 2. 25

INPUT DATA CARD LISTING FOR SAMPLE CALCULATION (ANALOG) 


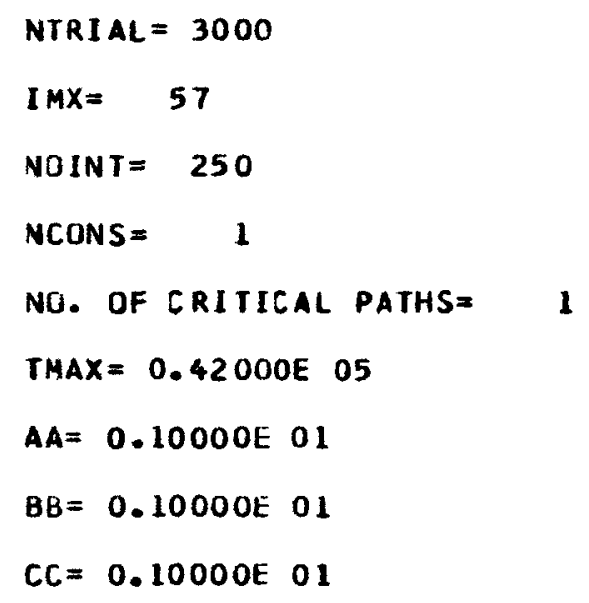

FIGURE 2.26A 


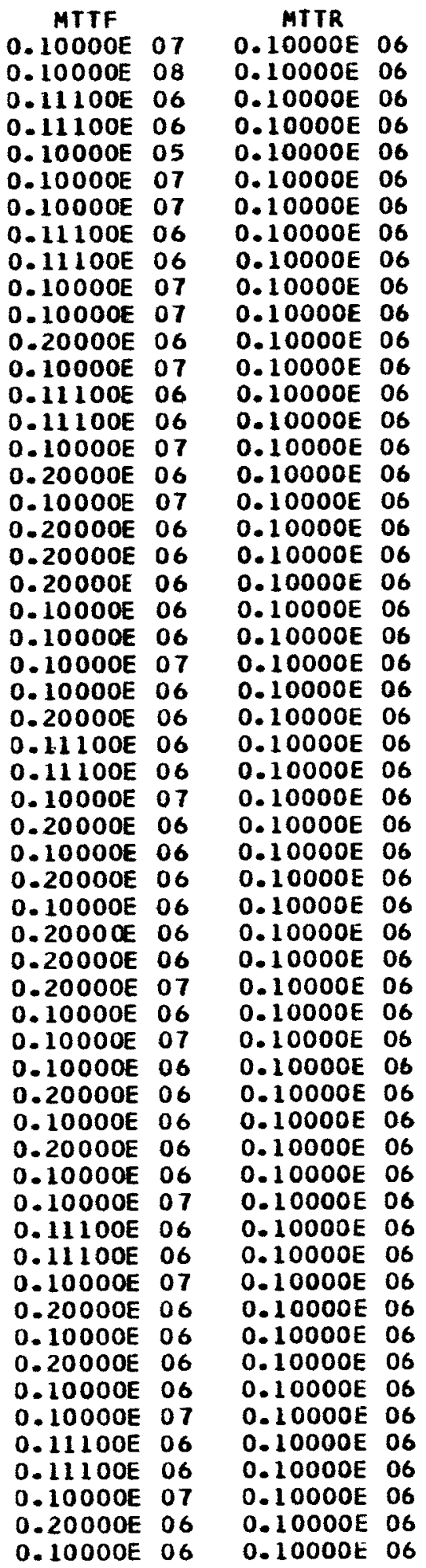

FIGURE 2.26B

SAF TE-I SAMP LE CA LCULATION OUTPUT 


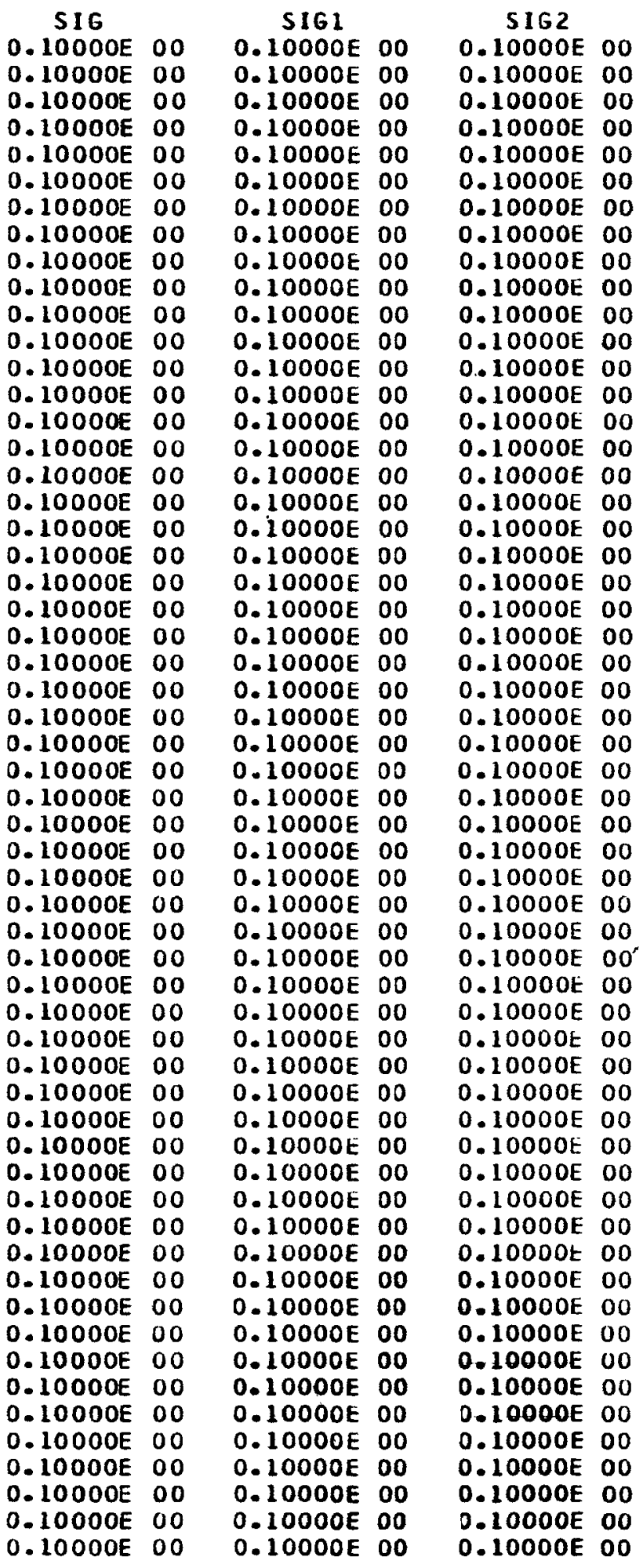

FIGURE 2.26C

SAFTE-I SAMP LE CALCULATION OUTPUT 
PROBABIL ITY OF SYSTEM FAILURE BEFDRE TMAX $=0.25467 E 00$

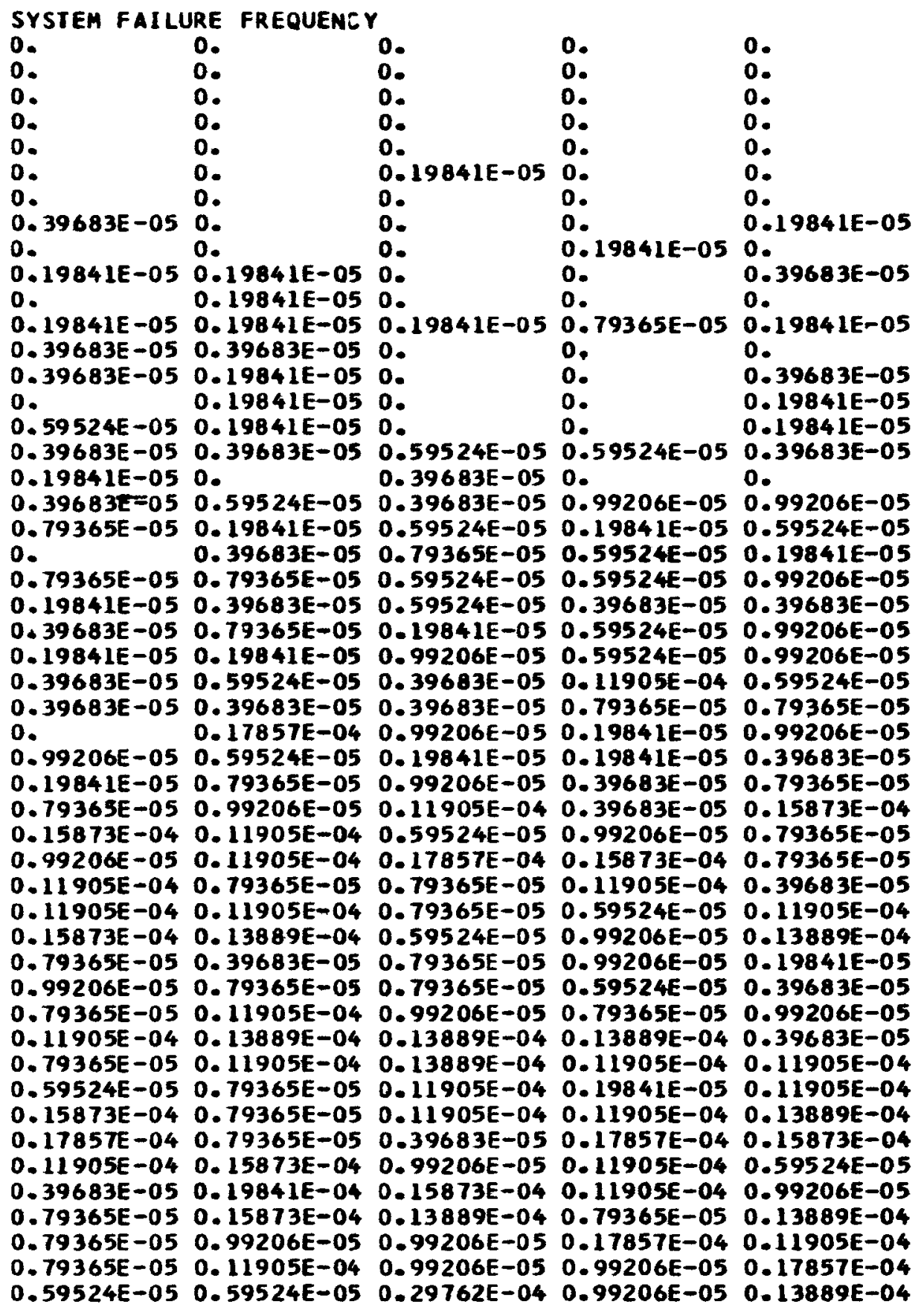

FIGURE 2.26D

SAF TE-I SAMP LE CA LCULA TION OUTPUT 
CUMULATIVE FAILURE DIST.

$\begin{array}{lllll}0 . & 0 . & 0 . & 0 . & 0 . \\ 0 . & 0 . & 0 . & 0 . & 0 . \\ 0 . & 0 . & 0 . & 0 . & 0 . \\ 0 . & 0 . & 0 . & 0 . & 0 . \\ 0 . & 0 . & 0 . & 0 . & 0 . \\ 0.0 & 0.033335-030.33335-030 .\end{array}$

$0.33333 E-03 \quad 0.33333 E-03 \quad 0.33333 E-03$

$0.33333 E-03 \quad 0.33333 E-03 \quad 0.33333 E-03 \quad 0.33333 E-03 \quad 0.33333 E-03$

$0.10000 E-02$ 0. $10000 E-02 \quad 0.10000 E-02 \quad 0.10000 E-02 \quad 0.13333 E-02$

$0.13333 E-02 \quad 0.13333 E-02 \quad 0.13333 E-02 \quad 0.16667 E-02 \quad 0.16667 E-02$

$0.20000 E-02 \quad 0.23333 E-02 \quad 0.23333 E-02 \quad 0.23333 E-02 \quad 0.30000 E-02$

$0.30000 E-02 \quad 0.33333 E-02 \quad 0.33333 E-02 \quad 0.33333 E-02 \quad 0.33333 E-02$

$0.36667 E-02 \quad 0.40000 E-02 \quad 0.43333 E-02 \quad 0.56667 E-02 \quad 0.60000 E-02$

$0.66667 E-02 \quad 0.73333 E-02 \quad 0.73333 E-02 \quad 0.73333 E-02 \quad 0.73333 E-02$

$0.80000 E-02 \quad 0.83333 E-02 \quad 0.83333 E-02 \quad 0.83333 E-02 \quad 0.90000 E-02$

$0.90000 E-02 \quad 0.93333 E-02 \quad 0.93333 E-02 \quad 0.93333 E-02 \quad 0.96667 E-02$

$0.10667 E-010.11000 E-01 \quad 0.11000 E-010.11000 E-010.11333 E-01$

$0.12000 E-0110.12667 E-01 \quad 0.13667 E-01 \quad 0.14667 E-01 \quad 0.15333 E-01$

$0.15667 E-01 \quad 0.15667 E-01 \quad 0.16333 E-010.16333 E-010.16333 E-01$

$0.17000 E-010.18000 E-01 \quad 0.18667 E-01 \quad 0.20333 E-010.22000 E-01$

$0.23333 E-01 \quad 0.23667 E-01 \quad 0.24667 E-01 \quad 0.25000 E-01 \quad 0.26000 E-01$

$0.26000 E-010.26667 E-01 \quad 0.28000 E-01 \quad 0.29000 E-01 \quad 0.29333 E-01$

$0.30667 E-01 \quad 0.32000 E-01 \quad 0.33000 E-01 \quad 0.34000 E-01 \quad 0.35667 E-01$

$0.36000 E-010.36667 E-01 \quad 0.37667 E-01 \quad 0.38333 E-01 \quad 0.39000 E-01$

$0.39667 E-010.41000 E-01 \quad 0.41333 E-01 \quad 0.42333 E-01 \quad 0.44000 E-01$

$0.44333 E-01 \quad 0.44667 E-01 \quad 0.46333 E-01 \quad 0.47333 E-01 \quad 0.49000 E-01$

$0.49667 E-01 \quad 0.50667 E-01 \quad 0.51333 E-01 \quad 0.53333 E-01 \quad 0.54333 E-01$

$0.55000 E-010.55667 E-01 \quad 0.56333 E-01 \quad 0.57667 E-01 \quad 0.59000 E-01$

$0.59000 E-01 \quad 0.62000 E-01 \quad 0.63667 E-01 \quad 0.64000 E-01 \quad 0.65667 E-01$

$0.67333 E-01 \quad 0.68333 E-01 \quad 0.68667 E-01 \quad 0.69000 E-01 \quad 0.69667 E-01$

$0.70000 E-01 \quad 0.71333 E-01 \quad 0.73000 E-010.73667 E-010.75000 E-01$

$0.76333 E-010.78000 E-01 \quad 0.80000 E-01 \quad 0.80667 E-01 \quad 0.83333 E-01$

$0.86000 E-01 \quad 0.88000 E-01 \quad 0.89000 E-01 \quad 0.90667 E-01 \quad 0.92000 E-01$

$0.93667 E-01 \quad 0.95667 E-01 \quad 0.98667 E-01 \quad 0.10133 E \quad 00 \quad 0.10267 E \quad 00$

$0.10467 E$ OO 0.10600 E 00 O.10733E OO $0.10933 E$ OO $0.11000 E$ DO

$0.11200 E$ O $00.11400 E$ OD $0.11533 E$ OO $0.11633 E \quad 00 \quad 0.11833 E \quad 00$

$0.12100 E$ OO $0.12333 E$ OO $0.12433 E$ OO $0.12600 E$ OO $0.12833 E \quad 00$

$0.12967 E \quad 00 \quad 0.13033 E$ DO $0.13167 E$ OO $0.13333 E \quad 00 \quad 0.13367 E$ OO

$0.13533 E$ OO $0.13667 E$ OO 0.13800 E 00 O.1390OE $000.13967 E$ DO

0.141 OOE $00 \quad 0.14300 E$ OO 0.14467 E 00 O.14600E $00 \quad 0.14767 E$ OO

$0.14967 E$ OO $0.15200 E$ OO $0.15433 E \quad 00 \quad 0.15667$ E $00 \quad 0.15733 E \quad 00$

$0.15867 E$ O $0.16067 E$ OO $0.16300 E$ DO $0.16500 E$ OD $0.16700 E$ OO

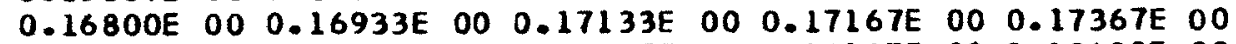

$0.17633 E$ DO $0.17767 E$ OO $0.17967 E$ OO $0.18167 E$ OO $0.18400 E$ DO

$0.18700 E$ OO $0.18833 E$ OO $0.18900 E$ OO $0.19200 E$ OO $0.19467 E$ OO

$0.19667 E$ OO $0.19933 E$ OO $0.20100 E$ OO $0.20300 E$ OO $0.20400 E$ OO

$0.20467 E$ DO $0.20800 E$ OO $0.21067 E$ OO $0.21267 E$ OO $0.21433 E$ OO

$0.21567 E$ O $0.21833 E$ OO $0.22067 E$ OO $0.22200 E$ OO $0.22433 E$ OO

$0.22567 E$ OO $0.22733 E$ OD $0.22900 E$ OO $0.23200 E$ OO $0.23400 E$ DO

$\begin{array}{llllllllllll}0.23533 E & 0 & 0 & 0.23733 E & 00 & 0.23900 E & 0 & 0.24067 E & 0 & 0.24367 E & 00\end{array}$

$0.24467 E$ O $0.24567 E$ O 00 O.25067E $00 \quad 0.25233 E \quad 00 \quad 0.25467 E$ DO

EIGURE 2.26E

SAFTE-I SAMP LE CALCULATION OUTPUT 


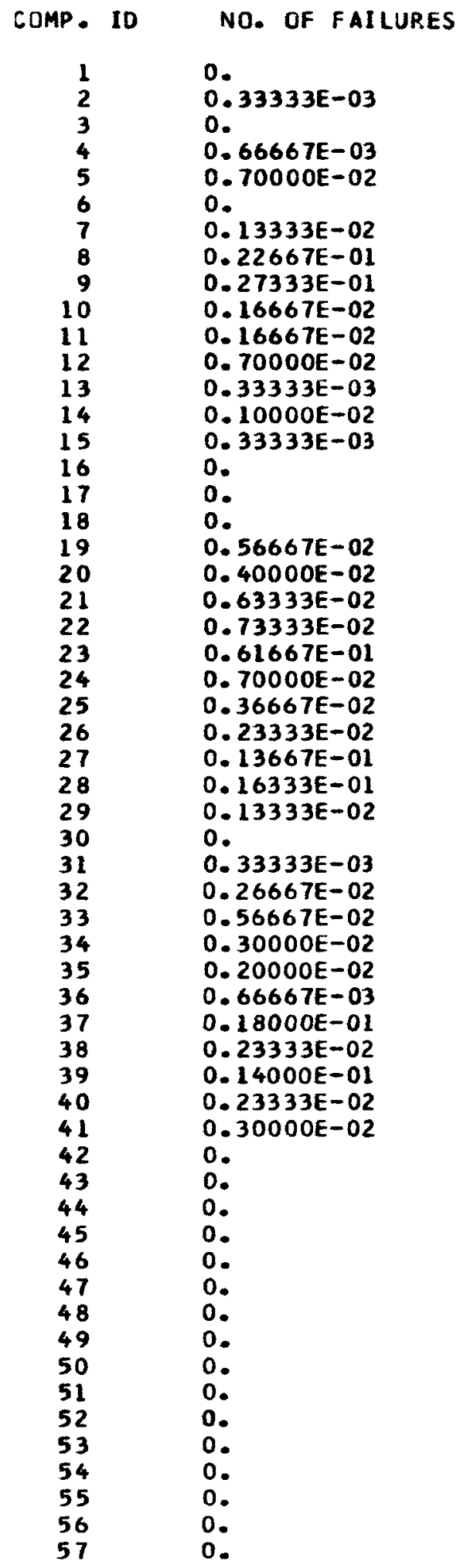

FIGURE $2.26 \mathrm{~F}$

SAF TE-I SAMP LE CALCULA TION OUTPUT 


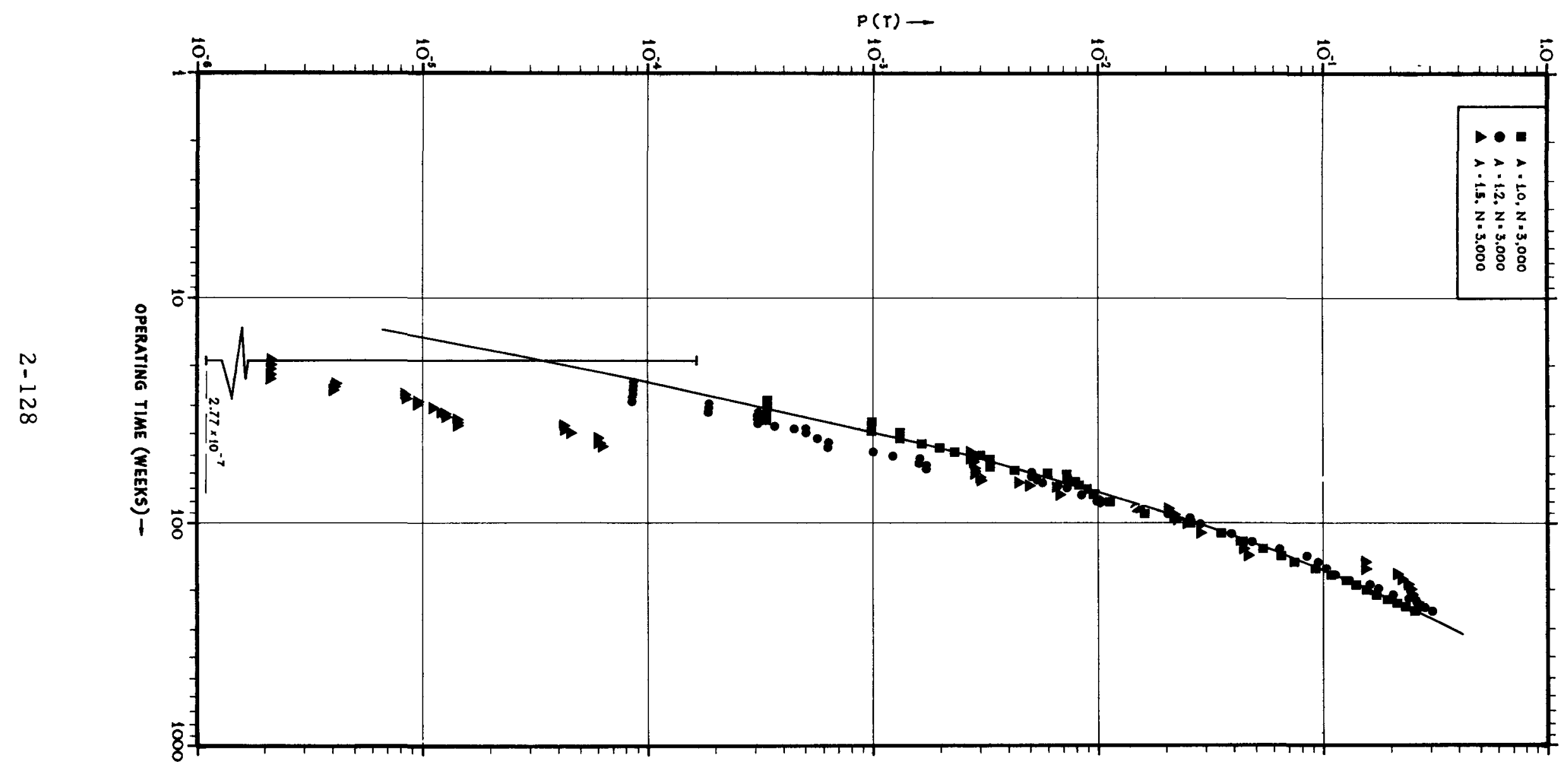

FIGURE 2.27

P(T) AS A FUNCTION OF OPERATING TIME (WEEKS) FOR THE DRESDEN-3 EMERGENCY A-C POWER SYSTEM 
CHAPTER 3

EXAMPLE APPLICATIONS OF RELIABILITY ANALYSIS 


\section{CHAPTER 3}

\section{EXAMPLE APP LICATIONS OF RELIABILITY ANALYSIS}

A system for collection and generation of reliability data has been defined and two methods of performing the quantitative aspects of reliability analysis have been described. This chapter illustrates the steps required in using these tools in reliability analyses for engineered safeguard systems. The intent is to indicate the steps involved in an analysis by example applications to selected PWR and BWR safeguard systems. The examples are not meant to be critical evaluations of the safeguard design. Specific systems for which brief, example reliability applications have been given in following sections are the Dresden-3 primary containment, the Connecticut Yankee safety injection system, the Connecticut Yankee containment cooling system, and the San Onofre safety injection system.

Briefly, the steps involved in a reliability analysis are:

1. Define the system to be analyzed including specific equipment items and their required operating patterns.

2. Define the performance goals for which the probability of success is desired or, conversely, the undesired events for which the probability of occurrence is to be determined.

3. Describe the methods of testing, checking, and maintaining the system, including the time intervals between these activities.

4. Perform an analysis of failure modes and effects for each equipment item in the system identifying the cause of each failure and its importance to system performance goals, and indicating conditions or practices which may eliminate or reduce critical failures.

5. Construct system reliability block diagrams reflecting the knowledge gained in the preceding steps. It is important that this diagram include all critical components. All time intervals of interest should be considered.

6. Obtain failure rate estimates for all components in each failure mode of interest. 
7. Perform numerical reliability evaluations using block diagram logic and appropriate failure rates. These evaluations may include machine or hand calculations or a combination of both.

8. Summarize results of reliability evaluation, identifying the relative importance of various components, the effect of simplifying assumptions and redundancies and the critical phases or intervals in system operation.

9. Identify corrective actions which may be taken to improve system reliability or, conversely, reduce the probability of system failure. These actions may include further investigation of failure rate assumptions, identification of areas for potential use of redundancy or for equipment redesign, modification of operating or maintenance procedures or altered test procedures and frequencies.

The preceding pattern of reliability analysis has been used in each of the following examples.

In following the applications it should be noted that while the goal is a quantitative evaluation, the qualitative information developed in the intermediate steps sheds much light on the potential trouble spots in a system. This is particularly true with respect to performing the failure mode and effect analysis and construction of the appropriate reliability block diagrams. At this stage of the analysis, the potential culprits in a system frequently are obvious to the analyst. Further steps leading to a quantitative result tend to confirm the qualitative evaluation and point out the relative contributions to system unreliability which can be expected from the various components. Thus, numerical reliability estimates provide a means for qualifying the effectiveness of a system and any modifications which may be made in its operation, testing or equipment. In this regard, it should be noted that while corrective actions will be most benefical if applied to prime contributors to unreliability, the lesser contributors must not be overlooked for Murphy's law ${ }^{*}$ tends to prevail in all cases. Consideration of lesser contributors is particularly important if it identifies ways in which these components can be eliminated or the effect of their failures can be made inconsequential to system success.

* If a device can fail, it will fail, (eventually). 


\section{DRESDEN UNIT 3 PRIMAR Y CONTAINMENT}

A preliminary investigation of the reliability of the Dresden-3 containment has been performed. This analysis is based on system descriptions and functional designs indicated in the Unit 3 Plant Design and Analysis Report (PDAR), dated February 1966, and its first three amendments.

\section{SYSTEM DESCRIPTION}

As described in the preceding documents, the Unit 3 containment can be considered as consisting of two major subsystems--a primary containment and a secondary containment. Within each of the se subsystems are assemblies of components designed to perform various functions necessary for the maintenance of overall containment integrity under normal and accident conditions. A schematic crosssection of the overall facility is shown in Figure 3.1 In the Unit 3 PDAR seven containment barriers to fission product release are cited. They are: (1) the high density ceramic $\mathrm{UO}_{2}$ fuel; (2) the high integrity zirconium cladding; (3) the reactor vessel and its connected piping and isolation valves; (4) the drywell suppression chamber primary containment; (5) the reactor building; (6) the reactor building standby gas treatment system utilizing high efficiency and charcoal filters; and (7) elevated discharge of gaseous effluents from a stack. In this discussion, the isolation valves in barrie $r$ 3 and all of barrier 4 are considered the primary containment while barriers 5,6 , and 7 are classed as the secondary containment.

The primary containment is designed to provide a low leakage barrier to the release of radioactivity $(0.5$ percent contained air at 62 psig internal pressure), and the secondary containment is provided for controlled release of any primary containment leakage to a 310-foot stack. The Unit 3 secondary containment also serves as the secondary containment for Dresden Unit 2.

The primary containment houses the reactor vessel, the reactor coolant and recirculating loops, and other service loops connected to the reactor. It consists of a drywell, a pressure suppression chamber which stores a large volume of water, a connecting vent system between the drywell and the water pool, isolation valves, containment cooling systems, and other service equipment. If piping containing reactor coolant or steam fails in the drywell, reactor wate $\mathbf{r}$ and steam would be released into the drywell air space. The resulting increase in drywell pressure would the $n$ force a 
mixture of air, steam, and water through the vent system and into the water stored in the suppression chamber. Appropriate isolation valves are actuated during this period to complete the primary containment. Cooling systems are provided to remove heat from the reactor core, the drywell, and from the water in the suppression chamber; and, thus provide continuous cooling of the primary containment under accident conditions.

A reactor building completely encloses the Unit 2 and Unit 3 primary containments. This structure provides secondary containment when the primary containment is in service, and provides primary containment during periods when the pressure suppression containment system (primary containment) is open. The principal function of the secondary containment is to minimize ground level release of airborne radioactive materials and to provide for controlled, decontaminated, release of the building atmosphere through a stack under accident conditions. This is accomplished by (1) directing leakage from the primary containment to the secondary containment, (2) maintaining the secondary containment at a negative pressure with respect to the outside atmosphere during accident conditions, (3) designing the building for a specified inleakage rate, and (4) discharging the ventilation gases through the 310 -foot stack after they have passed through a series of high efficiency particulate and halogen filters.

Although the Unit 3 containment includes both a primary and a secondary containment, only the primary containment has been considered. The reliability analyses techniques used on the primary containment will apply equally well to the secondary containment.

The most important function of the primary containment and its associated isolation and protective systems is to mitigate rapidly the consequences of postulated accidents involving the reactor primary system. The major assemblies in this containment subsystem were previously listed and are shown functionally in Figure 3.2. Parameters used in developing the primary containment design are listed in Table 3.1 . The design requirements and features of the primary containment components are described briefly in the following sections. More extensive discussion of design considerations is provided in the Unit 3 PDAR, as amended.

\section{Drywell}

As shown in Figure 3.1, the drywell is a steel pressure vessel with a spherical lower portion, 66 feet in diameter and a cylindrical upper portion, 37 feet in diameter. The overall height is approximately 113 feet. 
The drywell is enclosed in the reinforced concrete structure of the reactor building for shielding purposes and to provide additional resistance to deformation and buckling of the drywell over areas where the concrete backs up the steel shell. Shielding at the top of the drywell is provided by a removable, segmented, reinforced concrete plug.

Access to the drywell is provided by the drywell head, one double door airlock, and two equipment hatches. The drywell head and hatch covers are bolted in place and sealed with gaskets. The locking mechanisms on each airlock door are designed so that a tight seal will be maintained when the doors are subjected to either internal or external pressure. The doors are mechanically interlocked so that one door cannot be operated unless the other door is closed and locked. The seals on the doors and the hatches are capable of being tested for leakage.

The drywell will not be entered during power operation, but access is permissible during hot standby operation with the reactor subcritical. The normal operating environment in the drywell is an atmosphere containing less than normal concentrations of oxygen at essentially 0 psig and $135^{\circ} \mathrm{F}$. Temperature control is provided by recirculating the drywell air through cooling units which, in turn, are cooled by a closed loop cooling water system. A functional diagram of the drywell assembly is shown in Figure 3. 3 .

\section{Pressure Suppression Chamber and Vent System}

The functions of the se assemblies are as follows:

1. The vent system will conduct flow of vapors and liquids from the drywell to the suppression chamber, distribute this flow uniformly throughout the pool following a postulated rupture in equipment contained in the drywell, and limit the pressure differentials between the drywell and suppression chamber by use of chamber-to-drywell vacuum breakers.

2. The suppression chamber will receive vapor-liquid flow from the vent system, condense the steam portion of this flow, contain the noncondensable gases and fission products that may be driven into the chamber during the postulated rupture sequence, and provide a source of water for cooling the reactor core and primary containment. 
The vent system consists of eight 8 -foot diameter circular vent pipes connecting the drywell to the suppression chamber. The vent pipes open directly into the drywell and terminate in a 4-foot, 10-inch toroidal vent header in the air-space of the suppression chamber. Jet deflectors are provided in the drywell at the entrance of each vent pipe to prevent possible damage to the vent pipes from jet forces which might accompany a pipe break in the drywell. The pipes are enclosed with sleeves and are provided with expansion joints to accommodate differential motion between the drywell and suppression chamber. Projecting downward from the vent header are 96 downcomer pipes, 24 inches in diameter and terminating 4 feet below the water surface of the suppression chamber pool. Baffles are provided in the suppression chamber to ensure proper interaction of the vent pipe discharge with the suppression pool water.

Six vacuum breakers, which discharge from the suppression pool into the drywell, prevent a backflow of water from the suppression pool into the vent header system. Operation of two of the vacuum breakers is calculated to be adequate for pressure differential control.

The pressure suppression chamber, located below the $d r y w e l l$, is a steel pressure vessel in the shape of a torus with a major diameter of approximately 109 feet and a cross-sectional diameter of 30 feet. It contains approximately 106,000 cubic feet of water and has a net airspace volume above the water pool of approximately 119,500 cubic feet. The suppression chamber is held on supports which transmit vertical and seismic loading to the reinforced concrete foundation slab of the reactor building. Space is provided outside of the chamber for inspection and mainte nance.

Sufficient water is provided in the suppression pool to absorb the initial energy release into the drywell from any postulated pipe failure. The suppression chamber is sized to contain this wate $r$ plus the wate $r$ displaced from the reactor primary system together with the free air initially contained in the drywell.

Access to the pressure suppression chamber for inspection is provided by two 3-foot diameter manhole entrances with double-gasketed, bolted covers. These access ports will normally be bolted closed and will be opened only when the reactor primary coolant temperature is below $212^{\circ} \mathrm{F}$ and the pressure suppression system is not required to be operational. A functional diagram of the suppression chamber and vent system assemblies is shown in Figure 3.4. 


\section{Penetrations}

Openings in the primary containment which permit the entry of pipes, ducts, electrical cable, and the traveling in-core probe (TIP) guide tubes are designed to:

1. Withstand the peak transient pressure which could occur due to the postulated rupture of any reactor primary system pipe inside the drywell.

2. Withstand jet forces equal to that associated with flow from the largest local pipe or connection without failure.

3. Accommodate thermal stresses which may be encountered during all modes of operation without failure.

4. Allow individual leak testing to the extent practical and necessary.

Two general types of pipe penetrations are provided - - those which must accommodate thermal movement, and those which experience relatively little thermal stress. Piping penetrations which provide for movement consist of a penetration sleeve which passes through the concrete and is welded to the primary containment vessel; a process line which passes through the penetration and is connected at one end by a bellows expansion joint to accommodate axial movement; and a guard pipe around the process line to protect the bellows and maintain the penetration seal should the process line fail within the penetration.

The cold piping and ventilation duct penetrations are welded directly to the penetration sleeves. Bellows and guard pipes are not necessary in this design, since the thermal stresses are small and are accounted for in the design of the weld joints.

With the exception of the pipe penetrations which are welded directly to the primary containment shell, it will be possible to leak test individual containment penetrations without pressurizing the entire containment system. Testing may be accomplished by pressurizing the penetration between the double seals utilizing the pressure tap. Leak detection may then be accomplished either by the use of soap suds or by pressure decay techniques. 
Leakage through valves installed in pipelines which open into the containment can be detected by pressurizing between pairs of containment isolation valves. Leakage through valves installed in pipelines that connect to the reactor primary system may be determined when the reactor primary system is pressurized with the containment isolation valves closed.

The electrical penetrations are for electrical power, signal, and instrument leads. Depending on the number of lines for which access is provided two types of electrical penetration cartridges are used. In each case, the penetrating sleeve is welded to the primary containment vessel, and the flanged ends are bolted and sealed with a soft gasket material. A bonding resin is utilized in the seals where the cable emerges from the flange. This arrangement provides a leak-tight configuration which is leak-tested after installation and provides a means for periodic leakage testing.

Isolation valves on the reactor primary system pipe and other ducts or pipes, which penetrate the primary containment, provide a containment barrier in these lines which is considered to be as effective as the primary containment shell.

The criteria on number, type, operation, testing, and location of valves for the various categories of penetrations are as follows:

1. Process pipes which connect to the reactor primary system, and pipes or ducts which penetrate the primary containment and are open to the drywell free air space, shall be provided with at least two isolation valves in series.

Valves in this category shall be designed to close automatically from selected signals, and shall be capable of remote manual actuation from the control room.

2. The valves will be physically separated. On lines connecting to the reactor primary system, one valve shall be located inside the primary containment and the second outside the primary containment as close to the primary containment wall as practical.

3. Lines which penetrate the primary containment and which neither connect to the reactor primary system nor open into the primary containment shall be provided with at least one valve which may be located outside the primary containment. 
Valves in this category shall be capable of manual actuation from the control room.

4. Motive power for the valves on process lines which require two valves shall be physically independent sources to prevent a single accident from inter rupting motive power to both closure devices.

5. Upon loss of motive powe $r$ and when containment closure action of the valve is called for, the valve shall fail closed or shall fail in its existing position.

6. Valve actuation power failure shall be detected and annunciated.

7. The functional performance of valves, sensors, and other automatic devices essential to the isolation of the containment will be periodically tested to demonstrate proper function, correct setpoint of sensors, proper speed of response, and operability of fail-safe features.

The following are exceptions to the above isolation valve criteria:

1. Automatic isolation valves will not be used on the inlet or outlet lines of the core spray, containment spray, and feedwate $r$ supply systems, since operation of the se systems is essential following a loss-of-coolant accident. Check valves located on these lines, inside the drywell, will provide automatic isolation when necessary on inlet lines.

2. One automatic isolation ball valve is provided on each TIP system guide tube outside the primary containment. A second shear isolation valve is provided inside the containment and requires manual actuation.

3. No automatic isolation valves are provided on the control rod drive hydraulic system lines. These lines are isolated by means of the normally closed hydraulic system control valves located in the reactor building and by means of check valves comprising a part of the drive mechanism.

4. Small diameter instrument lines are provided with one manually operated shut-off valve, operable from the reactor building. 
The preceding exceptions are made only in the cases where normal criteria lead to a less desirable situation because of required operation or maintenance of the system in which the valves are located. In these cases, special attention is given to assure that the piping to the isolation valves has an integrity at least equal to the containment.

Table 3.2 is a listing of the principal isolation valves to be used in the piping which penetrates the primary containment. The table indicates the number and service of the valves, location of the valves with respect to the primary containment, the signal which actuates the valves, the motive power which actuates the valve, and the closure time of the valve. Approximate valve locations and arrangements are also shown on Figure 3.5. The re are approximately 550 penetrations in the primary containment.

\section{Containment Spray Cooling}

Two containment spray cooling systems are provided to remove heat from the primary containment system following a blowdown accident. Each system is capable of removing the fission product decay heat at the maximum anticipated rate. This cooling is provided (1) to remove heat from the primary containment and thereby assure that the containment does not become overpressurized and fail; and (2) to reduce rapidly the pressure of the primary containment and hence, the containment leakage rate.

The containment spray cooling system consists of two independent loops each provided with two full-capacity pumps (one a spare), a heat exchanger rejecting heat to the containment cooling service water system, piping and valves, and a containment spray header. One of the loops is shared with Unit 2 as shown in Figure 3.6. The shared loop is on standby and would start automatically if the independent Unit 3 loop failed to start on demand. Both loops can be operated simultaneously. Automatic start is initiated by concurrent trips from drywell high pressure and the low reactor water level.

When operating, this system pump water from the suppression chamber through the heat exchanger and the spray header. The water then flows by gravity back into the suppression chamber. A bypass flow path directly from the heat exchanger discharge line to the suppression chamber air space is provided for test purposes. 


\section{Core Spray Cooling}

R e dundant, full capacity, core spray loops and a core flooding capability are provided to remove decay heat following a blowdown accident. Each of the core spray loops has sufficient capacity to rapidly immerse the fuel assemblies. To continue core cooling following restoration of a water level in the core requires a supply of water from either one or both of the loops, the reactor feedwater system, or the control rod drive feed system.

The core spray system is designed to pump water directly from the suppression chamber into the reactor vessel. Core spray piping from the suppression chamber to the outer isolation valve is fabricated of carbon steel. Safety valves are provided for pressure protection of this piping. Stainless steel piping having a design pressure of 1,250 psig is used from the outer isolation valve to the reactor.

A separate ring header for each of the two loops is located inside the reactor vessel directly above the core and sprays water directly onto the fuel bundles. A strainer is placed ahead of the core spray pump suction to screen out particulates which could interfere with a discharge of water from the nozzles.

Upon receipt of the actuating signal, the pumps in one loop are started automatically and the isolation valve opens. When the reactor pressure drops to approximately $160 \mathrm{psig}$, the check valve opens and water is sprayed onto the top of the fuel bundles.

This system is also designed so that if the pumps in one loop fail to start, the pumps in the other loop are automatically switched into operation. Both the pumps and isolation valves may be actuated from the control room. The electrical loads associated with the se systems are considered in the standby diesel generator size.

Provisions are made for periodically testing the components in the core and containment cooling systems. These tests include:

1. Flow rate measurement in the core spray system. Preoperational testing of the core spray system will demonstrate the core flooding capability of the system. After preoperation testing, no special testing of this feature is planned. 
2. Flow rate measurement in the containment cooling system. In test 1 and 2 each pump of each system can be started individually and water pumped from the suppression chamber through the appropriate supply lines to the outer isolation valve, then returned to the suppression chamber.

3. Exercising and demonstrating operability of all motor operated valves.

4. Blowing air into the containment spray headers and inspecting individual nozzles.

5. Continuous indication in the control room of water level in the suppression chamber.

6. Removal and setpoint testing of safety valves in the low pressure carbon steel lines of the core spray system.

\section{Electrical Power}

For purposes of this example application the Dresden-3 a-c electrical power system is assumed to be that shown in the single line schematic drawing of Figure 3.7. When completed, Dresden-3, as well as Dresden-2, will be tied to the Commonwealth Edison $345 \mathrm{kv}$ network through five $345 \mathrm{kv}$ circuits. At Dresden-3 auxiliary a-c power can be supplied from five separate and independent sources: Units 2 and 3 , the $345 \mathrm{kv}$ network via the $345 \mathrm{kv}$ bus, a standby diesel generator ${ }^{*}$ and a $34.5 \mathrm{kv}$ line. Normal auxiliary power for Dresden-3 is split between the unit auxiliary power transformer, $T-2$, which is connected to the unit generator and the reserve auxiliary transformer, $\mathrm{T}-3$, which is connected to the $345 \mathrm{kv}$ bus. Each auxiliary transformer can carry the full auxiliary load for Dresden-3.

Should the $345 \mathrm{kv}$ bus and the unit generator fail, auxiliary power can be supplied by the standby diesel generator or the $34.5 \mathrm{kv}$ line through auxiliary transformer, T-8. Either of these sources is capable of operating all systems required to shut down and maintain the unit in a safe condition. The general design requirement is to supply duplicate services from different buses. As shown in Figure 3.7, it has been assumed that power for critical loads in the primary containment system is supplied from $4160 \mathrm{v}$ buses 5 and 6 and from $480 \mathrm{v}$ buses subsidiary to buses 5 and 6 . The diesel generator and the $34.5 \mathrm{kv}$ line may be connected to either or both bus 5 and 6 and through them to buses 3 and 4 . Switchgear for the $4160 \mathrm{v}$ buses is metal-clad, indoor type

* There are now three standby diesel generators planned for Dresden Units 2 and 3, one for each unit and one as a standby spare to either unit. This example considers the original condition of only the standby generator available to Unit 3 . 
with circuit breakers operated by a 125-volt d-c, stored/energy mechanism. Transformers and switchgear for the $480 \mathrm{v}$ buses are located in the turbine building.

With loss of normal atxiliary power, the diesel generator starts automatically. As soon as generator voltage is normal, the generator connects to the appropriate low voltage buses to supply essential loads after incoming breakers and nonessential loads are tripped. Other loads may be dispatched by the operator if the system power interruption persists. If the diesel generator is not available, the $34.5 \mathrm{kv}$ supply can be selected as the alternate source. Fuel for the generator is contained in a day tank and a larger tank for prolonged operation.

To protect against environmental conditions which could cause loss of outside power the diesel generator is housed in a concrete block cell in the turbine building and equipment connecting the diesel generator and $34.5 \mathrm{kv}$ line to critical loads is protected by metal enclosure and underground location. The generator is air started and sized to support the loads listed in Table 3.3 Automatically connected loads listed in Table 3.3 will start without operator intervention when normal bus voltage has been restored by the diesel generator. Other loads required for shutdown will be manually connected by the operator in accordance with overload restrictions. To assure operability, this unit will be periodically started and operated under load for sufficient time to assure adequate performance during extended periods of operation.

Direct current loads in the primary containment will be supplied by a 125-volt, station battery, capable of carrying its required connected load for eight hours without recharging. All of the loads connected to the 125 -volt d-c system, except heavy duty loads, also can be supplied by one of two battery chargers. The chargers will be powered from separate a-c buses which are operable from any auxiliary power source.

The battery is located in a ventilated battery room having concrete block walls. The 125-volt d-c system operates ungrounded with a ground detector alarm set to annunciate the first ground. Thus, multiple grounding, as a possible mode of failure, is extremely unlikely. The normal mode of battery failure is a single cell deterioration which is signalled well in advance by the routine of tests which will be performed regularly on the battery. Typical tests and servicings include visual inspection for leaks and corrosion, and checking voltage, and the specific gravity and level of the electrolyte. 


\section{RELIABILITY CONSIDERATIONS}

The reliability of the Unit 3 primary containment must be evaluated with respect to a specific performance requirement. That is, the number of as semblies and components required for successful containment will vary, depending on the type of radioactivity release occurring in this containment subsystem. If the type of release is unspecified, it is probably best to consider the most severe release conditions that can develop in the primary containment. In this example, a major rupture of the reactor coolant system is assumed, i. e., a blow down accident.

Once a release conditions has been identified, the design basis requirements for operation of various parts of the primary containment must be deter mined from the descriptive materials and available accident analyses. In the February 1966 edition of the Unit 3 PDAR, the blowdown accident was analyzed for a number of different containment operability conditions. The results of these analyses are shown as pressure-time curves in Figure 3.8 and as operational assumptions in Table 3.4. As indicated in Table 3.3, the variables in operational assumptions for the analyses were the number of core spray and containment spray systems in operation and the extent of metal-water reaction.

The design basis accident is characterized by the curve given for Case $f$ which assumes operation of one containment spray loop, no core spray, and the maximum metal-water reaction of 27.5 percent. Case $f$ has been used as the basis for the initial reliability analysis for the Unit 3 primary containment. That is, the primary containment reliability has been evaluated on the basis that at least one containment spray functions satisfactorily, and the primary containment achieves and maintains a status which allows only a minimal leakage. This reliability analysis includes all equipment combinations which can be expected to maintain containment pressure below the design pressure. Conversely, it can be used to estimate the probability of a containment pressure greater than design given a blow down accident.

An additional analysis has been made for the condition of at least one containment spray and one core spray operating. This is approximately equivalent to estimating the probability of Case $b$, i. e., one containment spray loop and one core spray loop operable in addition to an adequate isolation condition. To illustrate the value of assuming redundance, a subsidiary analysis has been $r$ un for the core spray alone in which both core spray loops are required to function. In each of the preceding analyses emergency a-c power has been included as an operational requirement by the assumption that either bus 5 or bus 6 and their respective subsidiary $480 \mathrm{v}$ buses, bus $2 \mathrm{~A}$ or bus $2 \mathrm{~B}$, must operate. 


\section{RELIABILITY EVALUATION}

The reliability model has been developed in a number of steps intended to define all systems and components critical to operation of the primary containment and to identify the time dependent status of this equipment. These steps can be summarized as follows:

1. Review design

2. Review accident analysis and characterize accident sequence

3. Perform failure mode and effect analysis

4. Identify critical components

5. Construct reliability block diagram

6. Identify reliability evaluation intervals

7. Identify component requirements for each interval

Step 1 was accomplished by way of the preceding primary containment description. A description of the accident analyses is given in the Unit 3 PDAR. This description is general with respect to characterizing the component operation and sequence of events. It may be briefly summarized as follows:

The reactor is operating at the design thermal output and the outlet leg of a recirculation loop is assumed to be instantly severed in a circumferential break. Flow at critical velocity occurs at the break. Immediately following the break, the large increase in core void fraction due to depressurization sharply decreases reactor power. Scram will be initiated in less than a second by high drywell pressure.

In about four seconds, the subcooled liquid mass below the core will begin flashing vigorously because of depressurization. This will tend to force a steam-water mixture up through the core, as well as backward through the jet pump diffuser pipes.

Calculations indicate that it will take nearly 24 seconds to depressurize the vessel. During a fraction of this time, the bulk of the core will be cooled by a boiling two phase mixture. The recirculation pump in the other leg will continue to inject fluid into the vessel for approximately 4 seconds or at least until the subcooled fluid begins to flash. Low water level in the reactor vessel will initiate actuation of the independent core spray loops about one minute after rupture. 
These loops will begin injecting water into the core when the reactor pressure falls below 150 psig.

The drywell pressure will $\mathrm{r}$ ise to about 39 psig in approximately three seconds. In less than 30 seconds after the break, the pressure in the pressure suppression chamber and drywell will have equalized to about 21 psig, as shown in Figure 3.8. The containment cooling systems are designed so that either of the full capacity independent systems will maintain the pressure of the suppression chamber below the 62 psig design pressure. This analysis assumes that only one of the two independent containment spray systems operates.

For the case in which no core cooling is assumed the core continues to heat up and subsequently undergoes a complete meltdown. The maximum extent of metal-water reaction resulting from the melt down is estimated to be approximately 27.5 percent of all the available metal (channel boxes and cladding) within the core region. It is assumed that there is an unlimited amount of steam available to support the metal-water reaction. The total duration of the reactor core meltdown is approximately one hour. However, the reactor core is effectively melted ( 90 percent) in about half an hour. Table 3.5 indicates the time for various stages of meltdown and metal-water reaction.

On the basis of the preceding accident sequence, the initial reliability model will consider two time intervals. The first interval will evaluate the probability of achieving containment isolation and startup of at least one containment cooling system. The second interval will consider operation of the containment cooling system until primary containment pressure has decayed to essentially atmospheric pressure. The second reliability model is the same as the first with the added requirement that one of the core spray loops also operates. The third, subsidiary model evaluates the probability that both core spray loops will operate.

Since test frequencies have not been defined, it will be assumed for each of the preceding models that the length of the first interval is one month or 720 hours. This is equivalent to assuming that the critical components, which will be subsequently identified, may be assumed to be unmonitored with respect to operability for as long as 720 hours.

The second time interval has been estimated from the pressure-time history for the blowdown accident. Since the time at which the Case f curve in Figure 3.8 decays to 0 psig is not given, the length of the second interval 
has been assumed to be approximately 300 hours. The existing curve indicates a pressure of approximately 17 psig at 278 hours after rupture and; therefore, the 300-hour interval may be somewhat optimistic.

Examples of failure mode and effect analyses on primary containment components are given in Figure 3.9. Based on these analyses and discussions of design, construction, and testing practices contained in the PDAR, it is assumed that the structural integrity of the containment vessels and connecting lines will be high relative to components such as drywell and suppression chamber closure seals, isolation valves in lines open to the containment atmosphere or the primary system and equipment in the containment cooling systems. These latter components will be taken to be the critical components at this time. Reliability block diagrams defining the logical operating requirements for the critical components are given in Figures 3.10 through 3.15.

The isolation valve requirements we re developed on the basis of the guidelines given in Appendix $F$ of this report, the reactor protection system trip functions, and the isolation valve summary in Table 3.2. The reliability evaluation intervals were previously defined. Referring to the reliability block diagrams the functional requirements for the reliability analysis cases in each of these intervals are as follows.

\section{Operable Function Requirements}

$\underline{\text { Interval } 1}$

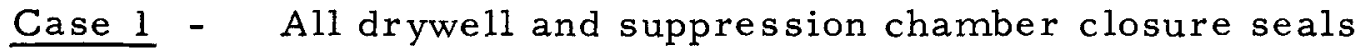
function; all isolation valves actuated by containment isolation protection system trip; operating mode status achieved in one loop of containment cooling system; and emergency a-c power supplied to operable cooling loop.

$\underline{\text { Case } 2}$ - Same as Case 1 with added requirement that operating mode status achieved in one loop of core spray system.

Case 3 - One of two core spray loops achieves operating mode status along with appropriate emergency a-c power train.

Case 4 - Both core spray loops achieve operating mode status and emergency a-c power trains on. 


\section{Interval 2}

Case 1 - Operating mode status continued in one loop of containment cooling system and appropriate emergency a-c powe $r$ train.

Case 2 - Same as Case 1 with added requirement that operating mode status continued in one loop of core spray system.

Case 3 - One of two core spray loops continues operation along with appropriate emergency a-c power train.

Case 4 - Both core spray loops and emergency power trains continue operation.

An important and final step in preparing for the mathematical reliability evaluation is the selection of appropriate failure rates for each component included in the model. In selecting failure rates one must consider the operating environment for each component and the failure mode(s) of importance in each interval. If appropriate failure rates cannot be identified from available reliability data sources such as those listed in Appendix $D$ or from operating experience summaries such as HN-185, then it becomes necessary to assume failure rates and later test the assumption. Limits should be established on assumed failure rate values to evaluate the sensitivity of system reliability to changes in failure rate.

For the present all failure rates have been assumed, as shown in Table 3.6, in order to complete the reliability evaluation illustration. Thus, the reliability analysis presented he re is preliminary with respect to component failure rates. While the failure rates are assumed, they do reflect some consideration of the component status, the appropriate failure mode, and past experience.

Although not a consideration in this example, it should be noted that not all unmonitored components will be tested on the same interval. As a result some failure rate adjustment will be necessary when performing a reliability analysis using a program such as ARMM, which computes success and failure probabilities at the end of fixed intervals for all components. That is, the program computes the reliability of all components under consideration in a time interval on the basis that the component is unmonitored during the time interval with respect to the failure mode of concern. To compensate for the fact that components are tested at different frequencies, the component failure rates should 
be multiplied by a factor equivalent to the ratio of component test interval to reliability evaluation interval.

\section{RESULTS OF ANALYSIS}

Each of the previously listed reliability analysis cases, Cases 1 through 4, has been evaluated by the ARMM computer program. The results of the se evaluations are summarized in Table 3.7. In this summary, Case 1 corresponds to Case $f$ of the Dresden- 3 PDAR. Case 2 is a slightly less restrictive version of Case $b$ in the PDAR; only one of the core spray loops is required to operate. Cases 3 and 4 provide insight into the effect of requiring both core spray loops to operate.

As shown in Table 3.7, the probability of achieving adequate isolation and operation of one of the two containment cooling loops (Case 1) is 0.99981 . If one of the core spray loops is required to operate as well (Case 2), the probability of success is decreased to 0.99970 . The difference between Case 1 and Case 2 represents an increase in the probability of failure of approximately 60 percent.

In both Case 1 and Case 2, the probability failure in the first interval is approximately three orders of magnitude greater than that for the second interval. Thus, if the reliability model and failure rate assumptions are conside red adequate, the most difficult aspect of containment operation in a blowdown accident would be associated with initiating the containment functions. This result derives primarily from the reduction in the number of operating components in the second interval, 162 to 23 in Case 1 and 204 to 31 in Case 2. Requiring a longer period of containment cooling (e.g., 720 instead of 300 hours) in Interval 2 would not significantly effect this estimate. Of course, the se results reflect a preliminary analysis and do not consider the effect of the accident induced environment on component failure rates. Data of this type is currently unavailable.

Cases 3 and 4 provide an indication of the effect of assuming redundancy in the core spray system operation. If such redundancy is not allowed, the $\mathrm{n}$ it is estimated that the reliability of the containment with core spray required would be reduced rather strongly from 0.9997 to approximately 0.9570. Conversely, the unreliability would be excepted to increase by a factor of approximately 200. If Case b were selected as the design basis for the primary containment, the preceding results indicate that it would be desirable to design the core spray system and the containment spray cooling system so that only one loop in each is required for satisfactory operation. 
Further insight on the primary containment reliability can be obtained by considering individual component contributions to system failure. Table 3.8 lists the 20 largest contributors to containment failure in Cases 1 and 2 . Contributions from the remaining components are illustrated by the ARMM output in Figure 3.16. Several important conclusions are implied by this data. First, most of the components listed in Table 3.8 are associated with the supply of emergency a-c power, particularly at the 480 volt level. This may be attributed partly to relative difference between the failure rates for electrical and nonelectrical components. It also results from the serial arrangement of these components in the containment and core spray loops where they provide motive power for proper alignment of these loops. As indicated in Table 3.8, the six largest contributors are the same in both Cases 1 and 2. These components provide 480 volt power to Buses $2 \mathrm{~A}$ and $2 \mathrm{~B}$. In these analyses it has been assumed that these components independently operate the respective buses; components 150,152 , and 154 for Bus 2A, and components 238, 240, and 242 for Bus $2 B$. If the system was arranged so that either set of the preceding components could powe $r$ either bus, the $n$ the system unreliability could be reduced by approximately 67 percent for Case 2 and 78 percent for Case 1. This conclusion as sumes that the equipment used to achieve the redundancy would have an unreliability of the order of $1 \times 10^{-5}$ or less in the se reliability models.

The six major contributors cited in the previous paragraph we re also found to contribute to approximately 62 percent of the system failure probability for Case 4 , both core spray loops in operation. If an actual design review we re being conducted, this result would give additional emphasis to the need for improving the reliability of the 480 volt power supply for checking the analysis for overly conservative estimates of failure rates or for adding redundancy.

Additional information which can be used to scope reliability improvement or monitoring efforts is provided by the ARMM analysis summary give $\mathrm{n}$ in Table 3.9. This table shows that in Case 1 approximately 92 percent of system failure may be attributed to 8 out of 162 components. Similarly, in Case 2 fourteen out of 204 components contribute to approximately 93 percent of system failure. These components are the first 8 and the first 14 components for Cases 1 and 2, respectively, in Table 3.8. If it was desired to concentrate on achieving an order of magnitude reduction in system failure probability, the group contributions to system failure given in Table 3.9 could be used as a guide. For example, an order of magnitude reduction in Case 1 would require concentration of reliability improvement effort largely on 28 of the 162 components. Twenty of these latter components are listed in Table 3.8. 
In conclusion, this example application outlines means whereby potentially troublesome design and equipment can be identified by reliability analysis of an engineered safeguard system. This information can be used in turn to make changes in system design, to set reliability improvement goals for specific equipment, or to suggest altered testing and operational practices; and.thereby minimize the time during which component failures may exist. 


\section{CONNECTICUT YANKEE SAFETY INJECTION SYSTEM}

Reliability analysis of the Connecticut Yankee safety injection system has been performed based on system descriptions and the loss of coolant incident analysis provided in the Facility Description and Safety Analysis (FDSA) for the Haddam Neck Plant, Topical Report No. NYO-3250-5, dated May 1966. This system is one of several systems which might be used to provide emergency core cooling with occurrence of a loss-of-coolant incident.

A loss-of-coolant incident could be caused by rupture of any line or nozzle connected to the reactor coolant system by improper opening of a valve or series of valves, by allowing uncontrolled discharge from the high pressure coolant system, or by material failure in a pipe or component of the reactor coolant system.

For small leaks in the reactor coolant system, the pressurizer level control adjusts the charging rate to maintain a minimum level in the pressurizer. Depletion of coolant in the volume control tank is compensated by automatic makeup control in the chemical and volume control system. Should the automatic makeup malfunction, a low-level signal from the volume control tank-level instrumentation would cause the charge pumps to take suction from the refueling water storage tank instead of the volume control tank.

For larger breaks, in which the discharge rate exceeds the delivery rate of the charge pumps for a prolonged period of time, the level in the pressurizer continues to decrease, eventually exceeding the lowlevel trip point for safety injection. Coincidentally, change in the pressurizer steam volume will cause an accompanying decrease in system pressure. Reactor trip and turbine trip will occur due to low pressurizer pressure. The coincidence of low pressurizer pressure with low pressurizer level, requiring signals from two out of three channels monitoring each parameter, actuates the safety injection system, which automatically delivers borated water to the reactor vessel for cooling the core. With normal incoming electrical power available, the system will prevent melting of the fuel cladding for loss-of-coolant incidents up to and including circumferential failure of the largest reactor coolant line, with only one of the two safety injection pumps, one of two charging pumps, and one of two residual heat removal pumps in operation.

If all outside power sources fail, emergency power will be provided by diesel generators in sufficient numbers to operate at least one residual 
heat removal pump (core deluge) and one of four service water pumps (for recirculation cooling) along with other equipment deemed essential for a loss-of-coolant incident. The system's path of access, mode of cooling water delivery, and design characteristics are such as to assure core cladding immersion in steam and cascading water so that core meltdown and metal-water reactions are highly unlikely.

A summary of safety injection system operation in relation to the type of loss-of-coolant incident is shown in Table 3.10 .

\section{SYSTEM DESCRIPTION}

\section{Equipment}

The primary side of the safety injection system consists of one refueling water storage tank, three pumping trains, and associated valves to properly align the system as shown in Figure 3.17. Pumps are provided with one out of two redundancies in each pumping train, and the isolation valves in all loops entering the reactor vessel are also arranged with various degrees of redundancy. The rated capacities of the safety injection and charge pumps are 1,750 gpm at 1,500 psig and $360 \mathrm{gpm}$ at 2, 300 psig, respectively. These pumps are capable of injecting borated water directly into the reactor vessel. The residual heat removal pumps have rated capacity of $2,250 \mathrm{gpm}$ at $500 \mathrm{psig.} \mathrm{Thus} \mathrm{the} \mathrm{deluge} \mathrm{cooling} \mathrm{of} \mathrm{the}$ reactor core through the vessel head can be provided only when the pressure in the reactor vessel is below the discharge pressure of the residual heat removal pumps.

Piping and motor-operated valves are provided to supply water from the refueling water storage tank to each pump, and from the containment sump to the residual heat removal pumps. Check valves are provided throughout the system to prevent backflow which might adversely affect system operation. All stop valves provided for removal of pumps and other equipment for maintenance purposes are locked-open.

Heat removal from the recirculating water returned from the containment sump is provided by a pair of residual heat exchangers. On the secondary side, the heat exchangers are connected to a service water system through motor-operated valves as shown in Figure 3.18. The system uses four service water pumps, each capable of delivering $6,000 \mathrm{gpm}$, to circulate river water to plant cooling loads. 
Power for the safety injection system is normally supplied by two transmission lines one connecting with the Connecticut Light and Power Company system at the Montville generating station and Haddam substation (line 12500) and the other with the Hartford Electric Light Company system at the Middletown generating station (line 772). These lines along with the $115 \mathrm{kv} / 4,160 \mathrm{v}$ transformers and $4,160 \mathrm{v}$ buses they serve are shown schematically in Figure 3.19. One safety injection pump, one charge pump, and four $4,160 / 480 \mathrm{v}$ station service transformers are served by each of the two $4,160 \mathrm{v}$ buses. Loss of normal supply to either bus section will automatically transfer the dead bus load to the other bus by closing bus tie breaker $2 \mathrm{~T} 3$.

The four station service transformers in turn supply power to four $480 \mathrm{v}$ buses as shown in Figure 3.20. One service water pump is served by each of the four $480 \mathrm{v}$ buses. One residual heat removal pump is served by each of two $480 \mathrm{v}$ buses as are the two sub-buses (No. 5-5 and 5-6) which serve all motor-operated valves and semi-vital buses for controls and instrumentation. Tie breakers between $480 \mathrm{v}$ bus sections permit manual transfer of supplies to adjacent bus sections. They are automatically closed to establish a single bus during an emergency with a total loss of normal $480 \mathrm{v}$ a-c power.

On-site, emergency power is provided by three emergency generators which start automatically if all normal $480 \mathrm{v}$ a-c power is lost. Each generator has a rated capacity of $400 \mathrm{kw}$. These synchronous generators are driven by diesel engines which are equipped with $d-c$ starting motors. Each generator is connected to a section of the $480 \mathrm{v}$ bus by an electrically operated circuit breaker.

\section{Operation}

Components which have been considered initially in evaluating system operation from a reliability viewpoint, are shown in Figure 3.21.

Operation of the safety injection system is initiated by an actuation signal generated as a result of two out of three low pressurizer water level signals coincident with two out of three low pressurizer pressure signals. This actuation is fully automatic and consists of:

1. Opening the refueling water storage tank supply valve.

2. Opening the safety injection isolation valves. 
3. Opening the residual heat exchanger bypass value and throttling value, and the core deluge isolation valves.

4. Opening the control valve and the isolation valves in the charge lines to the reactor coolant loops.

5. Opening the valve in the charge pump suction from the refueling wate $r$ storage tank, and closing the valve in the normal suction line from the volume control tank.

6. Starting the safety injection pumps, charge pumps, and residual heat removal pumps.

The system may also be actuated manually from the main control room. To prevent automatic operation while the reactor is cold and depressurized, the actuation signal is blocked manually when reactor coolant system pressure is below $1,700 \mathrm{psig}$. The signal is unblocked automatically when reactor coolant system pressure rises above 1,700 psig.

Within 10 seconds after the initiation signal is generated, the two safety injection pumps can deliver borated refueling water at full rated flow to a header supplying four independent injection lines, one to the cold leg of each reactor coolant loop. Suction of the two centrifugal charge pumps is automatically transferred from the volume control tank to the refueling water storage tank, and borated refueling water is delivered through the charge lines to the cold leg of loop 2 and the hot leg of loop 4 .

Continued operation of the safety injection will depend upon the leak size and hence the reactor coolant pressure. For small breaks, the system may maintain the reactor coolant pressure at a level sufficiently high to preclude core deluge flow. In this case, the core deluge pumps will be secured and the residual heat removal loop will be realigned to provide high pressure recirculation via the safety injection pumps and charge pumps.

For larger breaks the re may be no reliable indication that the core has been covered with water. In this situation the operator will terminate safety injection and begin low pressure recirculation via the core deluge piping from 150, 000 gallons of borated water have been pumped from the refueling water storage tank. This quantity insures that the core has been covered for any rupture up to and including a double-ended break of a reactor coolant line. 
High pressure recirculation via the safety injection pumps and charge pumps provides a means of using the spilled reactor coolant to keep the reactor core covered with wate $r$ after small leaks or ruptures occur. The borated water draining from a system rupture is collected in a sump located at the lowest point of the reactor containment lower operating level. After several minutes of safety injection operation, sufficient borated wate $r$ will have collected in the sump to permit initiation of recirculation operation. Recirculation flow and cooling is accomplished by the residual heat removal pumps and heat exchangers. Remote operated valves provide the means for (1) transferring the residual heat removal pumps suction from the refueling water storage tank to the reactor containment sump, (2) directing the resulting flow through the heat exchangers, (3) providing service water cooling to the heat exchanger, and (4) transferring discharge from the core deluge heade $r$ to the combined safety injection and charging pump suction header. The cooled borated wate $r$ is then returned to the reactor coolant system via the safety injection pumps and charge pumps.

If a large rupture occurs, low pressure recirculation to the reactor vessel via the core deluge lines is established remotely in the same manner as the high pressure recirculation except that the residual heat pump discharge flow is directed to the core deluge line rathe $r$ than to the suction of the safety injection pumps and charging pumps.

\section{$\underline{\text { Testing }}$}

The-safety injection system may be tested at any time the reactor coolant system is pressurized above the shutoff head of the safety injection pumps (1,400 psig). Water in the safety injection lines is circulated from the refueling water storage tank to the injection system lines during pump testing by using the recirculation lines from each safety injection branch line. Recirculation of water in the safety injection lines insures the proper boron concentration in the lines.

Motor operated isolation valves are tested by manual operation of each valve from the main control room. Remote position indicators in the main control room permit a check of valve operation.

Periodic testing of the core deluge including operation of the residual heat removal pump with emergency powe $r$ is required, and is a part of the periodic test of the safety injection system. 


\section{RELIABILITY CONSIDERATIONS}

The analysis of the loss-of-coolant incident is presented in the FDSA and summarized in Table 3.11 for break sizes corresponding to single ended rupture of $1-1 / 2,3,4,6$, and 10 -inch lines, a 2 square foot rupture, and the hypothetical doubled ended rupture of a reactor coolant loop. In addition, a rupture of a charge line at the connection to the reactor coolant system and a similar rupture of a safety injection line are analyzed. The charge line rupture, in effect, allows all charge flow to spill on the containment floor. The safety injection line break impairs the delivery curve of the injection system. For this special break, delivery to the reactor through the intact lines does not begin until reactor pressure has reduced to a value equal to the pressure drop in the broken line.

In all cases the rupture is assumed to be near a safety injection connection and a charge line connection to the reactor coolant system. Conservatively, $1 / 3$ of the safety injection flow and $1 / 2$ of the charge flow is assumed to be released through the rupture. Delivery curves are adjusted to take this into account. Calculations were made of pressure and volume histories for the various break sizes, and cladding hot spot temperature transients for those cases in which significant core uncovering occurs. The clad hot spot temperature is less than the $2,550^{\circ} \mathrm{F}$. melting temperature in all cases. A typical pressure and volume curve is shown in Figure 3.22 for a 3 -inch charge line break. One of the two safety injection pumps, one of two deluge pumps, and one of two centrifugal charging pumps are assumed to be operating.

Failure Mode and Effect Analysis

Based on the assumptions and the system description given in the FDSA, a failure mode and effect analysis was made as shown in Figure 3.23 on components considered essential to the system reliability.

The refueling water storage tank is equipped with an externally-mounted thermosiphon reboiler-type heat exchangers as shown in Figure 3.17 designed to maintain a refueling water temperature of $55^{\circ} \mathrm{F}$. with an outdoor temperature of $-15^{\circ} \mathrm{F}$. The heating steam is provided by the building service heating system through the auxiliary steam system. Since adequate time can be expected to exist to correct a tank heating failure before the freezing of 250,000 gallons of borated water takes place, the failure rate assigned to the tank was selected with this assumption in mind. 
As for the outside power sources, the possibility of failure of any particular combination of interconnected transmission systems is very remote. For this reason a representative value for their failure rate was chosen.

Pumps have different failure modes, fail to start and fail to continue running, and as such different failure rates were chosen accordingly. As for valves, assigned failure rates are those associated with the failure to open or failure to close modes of operation. Other types of valve failure modes, such as failure to stay open or failure to stay closed, are considered to occur at a rate at least one order of magnitude lower than the first mode. Typical failure rates of all components are listed in Table 3.12.

$\underline{\text { Reliability Model }}$

The reliability model for this analysis has been developed, as shown in Figures 3.24 and 3.25 to include all components critical to operation of the safety injection system under all types of loss-of-coolant incident.

Since motor-operated valves, instrumentation and controls for the safety injection system as well as for other vital systems require Buses No. 5 and 6 to be energized at all times, the reliability block diagram was considerably simplified by isolating components which are fed through these buses from the power network reliability model. The only exceptions to this approach were two service water pumps, since these were in redundancy with two other pumps connected to Buses No. 4 and 7.

\section{RELIABILITY EVALUATION}

Based on the failure mode and effect analysis and the reliability model, loadsheets for an ARMM calculation were prepared as shown in Figure 3.26. As far as the system reliability is concerned, a case which requires longer time to initiate low pressure recirculation will yield slightly lower reliability values. Thus, the case of a charge line rupture shown in Figure 3.22 is demonstrated in the calculation. All other cases will yield a better reliability even though the severity of an incident may be more or less.

Time intervals chosen were based on information in the FDSA and Figure 3.22, and the functions are turned on and off accordingly. The intervals were:

Interval 1 - Since test frequencies have not been defined in the FDSA, it will be assumed that the length of the first interval is one month or 720 hours. This is equivalent to as suming that all components are unmonitored and that if a component fails it will remain in a failed state until detected during a safety injection test. 
Interval 2 - Safety injection is initiated, and after several minutes (assumed 0.1 hour) of operation sufficient borated water will have collected in the sump to permit initiation of high pressure recirculation.

Interval 3 - High pressure recirculation is initiated and approximately 1.400 seconds ( $\sim 0.4$ hours) after initiating safety injection, the reactor coolant pressure will have dropped sufficiently to permit low pressure recirculation through the reactor vessel head.

Interval 4 - Low pressure recirculation is initiated and continues for 24 hours.

As shown in the block diagram in Figure 3.24, there are a number of nonredundant valves, any one of which would contribute significantly to system unreliability if their failure rates were not substantially smaller than other components which are redundantly arranged. In order to improve system reliability, one-out-of-two redundancies were added to these nonredundant components in a second case by using the ARMM program capability for handling a limited number of partial data* changes without changing the basic input. To make this partial data change possible in a single run, additional load sheets were prepared as shown in Figure 3.27, to accomodate those nonredundant components listed in the load sheets shown in Figure 3.26.

In addition, a third case was investigated whereby Interval 1 was shortened to one week (168 hours) simulating a shorter testing interval or shorter interval in which failures could exist. The partial data change is noted in the second half of data entries in Forms $A$ and $G$ of Figure 3.27.

\section{RESULTS OF ANALYSIS}

Table 3.13 presents results of the analys is for three cases and four different time intervals. The overall system unreliability of $0.388 \times 10^{-1}$ (Case 1) for the system described in the FDSA was reduced by 97.9 percent to $0.831 \times 10^{-3}$ (Case 2) for the improved version with all critical in-series valves placed in one-out-of-two redundancies, and by 76.6 percent to $0.908 \times 10^{-2}$ (Case 3) for the system with shorter testing interval. Considering the different time intervals, the analysis shows that the chance of failure at the end of the starting interval is roughly three orders

*See section on ARMM input data Field 10A, Partial Data. 
of magnitude greater than the change of failure of the subsequent safety injection period of 24 hours. Thus, if the system initiates safety injection, the chance of subsequent failure to continue injection is remote.

A summary of component contributions to system unreliability is shown in Figure 3.28 for Cases 1, 2, and 3. Components found least reliable and contributing up to 99 percent of overall system failure in Case 1 are listed in Table 3.14. In Figure 3.29, nonredundant valves were represented by two fictitious valves with only one-half of the true failure rate. Since each of these two valves belonged to a single function, the probability of function failure as shown in Figure 3.29 corresponds to that of a real valve. When these in-series valves were placed in-parallel with one-out-of-two redundancies as in Case 2, their contributions to system failure dropped to as little as $1 / 3$ to $1 / 10$ of those in Case 1. In Case 3 their relative contributions to overall system failure have not changed substantially since the only change was in the initial time interval. 


\section{CONNECTICUT YANKEE CONTAINMENT COOLING}

The Connecticut Yankee plant contains two engineered safeguard systems designed to provide a sufficiently large heat sink to prevent the containment atmosphere pressure buildup resulting from the maximum credible accident from exceeding design limits. These safeguard systems are the air recirculation system and the containment spray system. The following discussion presents a preliminary reliability analysis for these systems.

The analysis is carried to the limit; that is, a reliability model is constructed for the containment cooling system. This model is then used to arrive at a quantitative statement for the probability that, in the event of the maximum credible accident (circumferential failure of the 27.5 inch primary coolant line), the containment design pressure will not be exceeded.

\section{SYSTEM DESCRIPTION}

$\underline{\text { Air Recirculation System }}$

The air recirculation system consists of four (4) 65, $000 \mathrm{cfm}$ fan-cooling coil units and a system of distribution duct work (including inlet and outlet dampers), instrumentation, and controls. A typical unit is shown in Figure 3.30. The fans are direct driven, centrifugal type, and the cooling coils are transverse flow, finned coil banks. The fans take suction from the containment atmosphere and discharge into the system duct work. The duct work distributes the cooled air to the individual reactor coolant loop areas, the refueling cavity area,and the containment dome area. All four fans are normally in operation when the reactor coolant system is above $200^{\circ} \mathrm{F}$. and $300 \mathrm{psig}$.

Two sets of inlet dampers are provided on each air recirculation unit. One set is located on the face of the filters while the other set controls bypass flow directly to the inlet of the cooling coils as shown in Figure 3. 30 .

During normal operation, the dampers on the face of the filters (incident dampers) are closed, while those leading directly from the containment to the cooling coils are open. A high pressure signal from the containment reverses the damper positions, sending $50,000 \mathrm{cfm}$ through the filter bank. In the normal operating position, each set of dampers is 
held against a spring by an energized electrical clutch. Loss of power de-energizes the clutch and the spring forces the dampers into the incident position, i. e., the incident dampers open and the normal dampers close.

The reliability block diagram constructed for a single fan unit is shown in Figure 3. $31 \mathrm{~A}$. Here it was assumed that in the event of failure of the incident flow path, the plant operator has the option of reversing the damper positions, thereby achieving full cooling effectiveness via the normal flow path. Hence, the normal flow path is shown as redundant to the incident flow path in Figure 3.31A. A failure mode and effect analysis for a fan unit is shown in Figure 3. 34A.

\section{Containment Spray System}

The containment spray system utilizes the residual heat pumps, the residual heat exchangers, and a spray header just inside the containment liner to distribute water throughout the containment. Spray water may be drawn either from the refueling cavity water storage tank or from the containment sump.

Containment spray is not automatically initiated in the event of a loss-ofcoolant incident. The residual heat removal pumps would normally deliver water to the reactor vessel in such an incident. To initiate spray, the operator must close a valve in the line leading from the residual heat removal pumps to the reactor vessel and open a valve in the line leading to the spray headers. The path taken by spray water when the residual heat removal system is in this configuration is shown in Figure 3. 32. The reliability block diagram constructed for the spray system is shown in Figure $3.31 \mathrm{~B}$ and the failure mode and effect analysis in Figure 3.34B.

\section{Electrical Power and Service Water Supply Systems}

Electrical power for the fan motors, service water and residual heat removal pumps. and motor operated valves is normally available through the station service supply system. In the event of loss of outside power, three (3) diesel generators are available in the plant to supply power.

Cooling water for the air recirculation system and residual heat exchangers is provided by the plant service water system. The capacity of any one of the four (4) service water pumps is assumed to be sufficient for this purpose. Figure 3.31C shows the reliability block diagram constructed for these systems. 


\section{RELIABILITY CONSIDERATIONS}

Air Recirculation System

Figure 3.33 (extracted from NYO-3250-5) shows the calculated pressure transient in the containment following circumferential failure of a 27.5inch reactor coolant line. It appears that the heat sinks provided by the safety injection and core deluge systems are of small efficacy and that the major burden in controlling the peak pressure is placed on the air recirculation system. Further, as far as the peak pressure is concerned, three (3) fans in operation are seen to be as effective as all four (4) fans in operation. From the point of view of a reliability analysis, it would be desirable to see similar curves for 1 and 2 fans in operation. Lacking this information, a major (conservative) assumption regarding the effectiveness of the air recirculation system was made in the reliability analysis. It was assumed that operation of at least three fan units is required to maintain the pressure transient within design pressure.

Containment Spray System

The containment spray system is installed as a backup for the air recirculation system for depressurization of the containment following a loss-of-coolant accident.

The spray system is not considered in the calculation of the containment pressure transient. To quote from the Plant Design and Analysis Report (NYO-3250-5), "No quantitative credit is taken for the spray system in the analysis of the hypothetical accident because the experimental work done to date is not considered extensive enough to assess accurately the effect of the spray under the conditions which would exist in the containment after such an accident." Nevertheless, in this study it was assumed that the spray system is an effective standby system for the air recirculation system and would be started in the event of failure of more than one fan unit.

\section{RELIABILITY EVALUATION}

\section{Overall Reliability Block Diagram}

Based on the above discussion of system effectiveness, the reliability block diagram for the complete containment cooling system takes the form shown in Figure 3.31D. 
Reliability Calculation

Two separate reliability calculations were made using the ARMM program, one for the combined power supply and service water supply systems and one for the combined air recirculation and containment spray systems. The components were "aged" for a period of 1,020 hours. This assumes that there is a component testing interval of 1 month (720 hours) and that coolant system operation is required for a period of 300 hours following an incident.

Failure rates assumed for the components of Figures $3.31 \mathrm{~A}, \mathrm{~B}$, and C are shown in Table 3.15.

The criteria used for grouping of components into functions as shown in Figures $3.31 \mathrm{~A}, \mathrm{~B}$, and $\mathrm{C}$ was such as to lead to the minimum number of functions necessary for system description in the ARMM input. All components to the right of a function number ( $F$.__ ) belong to that function. For example, in Figure $3.31 \mathrm{~B}$ components $5 \overline{6,57}, 58,59,60$, and 61 belong to function F. -51 , (the minus indicates that the function is listed in standby capacity).

RESULTS OF ANALYSIS

Power Supply

As seen on Figure $3.31 \mathrm{C}$, there are seven paths, not all independent, by which 480-volt power may be supplied. Failure of components 60,90 (common to five of the paths) and 130 lead to system failure. All other component failure combinations resulting in system failure are of higher order; ${ }^{*}$ i.e., they require four or more component failures. Since the probability of failure of a typical single component is of the order of $10^{-2}$, it may be anticipated that the lowest order failure combination contributes most of the total system failure probability. This is clearly evident in the results obtained in the present case, as shown in Table 3. 16.

\section{$\underline{\text { Service Water Supply System }}$}

The reliability, or conversely the unreliability, of the service water supply system has been evaluated on the basis that the critical components are the four service water pumps. Other components in the system were assumed

* The order of a component failure combination has been defined as the number of components failed in order to achieve system failure; e. g., a third order combination is a unique combination of three failed components which will cause system failure. 
to contribute negligibly to the system unreliability. System failure was assumed to require failure of all four pumps (i. e., one service water pump was assumed to supply adequate cooling capacity to the tube sides of the air recirculation unit cooling coils and the residual heat exchangers). The probability of all four service water pumps failing is calculated to be $0.21 \times 10^{-6}$.

\section{Air Recirculation and Containment Spray Systems}

This system incorporates a high degree of redundancy. The three out of four fan units operating condition on the air recirculation system with the containment spray system in standby (See Figure 3.31D) implies that a minimum of 3 components must fail to result in system failure.

The failure probability of the combined system was calculated in two ways. First, a complete ARMM run was made with the containment spray system described in standby capacity to three out of four air recirculation units. As anticipated, the run time was high due to the large number of component failure combinations that contribute to system failure (in this system, 270 third order and 3,222 fourth order combinations).

The results of the ARMM run are shown in Table 3.17A. The seventeen components listed are those involved in third order failure combinations. They are seen to contribute over ninety percent of the total system failure probability. The remaining forty-four components comprising the system are involved only in fourth or higher order failure combinations.

Because of some uncertainties in the present ARMM treatment of standby functions, an approximate hand calculation of the system failure probability, as described below, was made. The simplifying assumptions made are seen to eliminate almost all anaiytical difficulties, allowing the calculation to be readily performed by hand. Without these assumptions, the calculation would have become prohibitive.

The probability of single component failure, $q$, is

$$
\begin{aligned}
& q=1-e^{-\lambda t}, \\
& q \cong \lambda t, \quad \lambda t<<1
\end{aligned}
$$


where

$$
\begin{aligned}
& \lambda=\text { component failure rate, and } \\
& t=\text { operating or aging time. }
\end{aligned}
$$

In this particular problem $t=1,020 \mathrm{hr}$ and $\lambda \max =10 \times 10^{-6} \mathrm{hr}^{-1}$, hence, $(\lambda t)_{\max }=1.02 \times 10^{-2}$, implying $q=\lambda t$ within 1 percent and the error is conservative. Further, for $\mathrm{n}$ components in series, each with failure probabilities $<10^{-2}$, the failure probability $Q$ for the series combination is

$$
Q=\sum_{i=1}^{n} q(i)
$$

to within 1 percent (again, the error is conservative).

Using these approximations and the reliability block diagram shown in Figure $3.31 \mathrm{D}$, the failure probability for a single air recirculation unit operating for time $T$ (in millions of hours) is

$$
\begin{aligned}
Q_{\text {unit }} & =\left(\lambda_{16}+\lambda_{17}+\lambda_{18}\right) \mathrm{T}+\left(\lambda_{14}+\lambda_{15}\right)\left(\lambda_{10}+\lambda_{11}+\lambda_{12}+\lambda_{13}\right) \mathrm{T}^{2} \\
& =1.53 \times 10^{-2}
\end{aligned}
$$

using $T=1,020$ hours and $\lambda$ values from Table 3.15 .

Failure of the air recirculation system requires failure of two or more fan units. Hence, the failure probability of the air recirculation system $Q_{a r s}$, is given by

$$
\begin{aligned}
Q_{\text {ars }} & =\sum_{i=2}^{4} \frac{4 !}{i !(4-i) !}\left(Q_{\text {unit }}\right)^{i}\left(1-Q_{\text {unit }}\right)^{4=i} \\
& \cong 6 Q_{\text {unit }}^{2} \quad \text { within } 1 \text { percent. }
\end{aligned}
$$

Using the value computed above,

$$
\mathrm{Q}_{\mathrm{ars}} \cong 14 \times 10^{-4}
$$


or conversely, the air recirculation system reliability is

$$
\mathrm{R}_{\mathrm{ars}} \cong 0.9986
$$

The failure probability of the containment spray system, to the same level of approximation as above, is

$$
\begin{aligned}
& Q_{\text {cs }}=T \sum_{i=70}^{74} \lambda_{i}+T^{2}\left(\sum_{i=54}^{55} \lambda_{i} \sum_{i=50}^{53} \lambda_{i}+\sum_{i=56}^{58} \lambda_{i} \sum_{i=59}^{61} \lambda_{i}+\sum_{i=62}^{\underline{6}=} \lambda_{i} \sum_{i=66}^{69} \lambda_{i}\right) \\
& =3.8 \times 10^{-2} \text {, }
\end{aligned}
$$

with $T=1,020$ hours and $\lambda$ values from Table 3.15 .

Conversely, the containment spray system reliability is

$$
R_{\text {css }} \stackrel{\sim}{=} 0.962
$$

The containment cooling system failure probability, $Q$, then becomes

$$
Q \cong Q_{\text {ars }} Q_{\text {css }} \cong 5.3 \times 10^{-5}
$$

Conversely, the estimated system reliability is

$$
R=0.999947
$$

This assumes (conservatively) that the spray system is alternate to the air recirculation system. If the spray system is treated in standby capacity the estimate of $Q$ will be lowe $r$, since components in standby are not aging until the standby function is required to operate.

The general equation for the system failure probability with the spray system in standby to the air recirculation system is

$$
Q(\operatorname{standby})=\int_{0}^{T} \dot{Q} \text { ars } i_{0}^{T-t^{\prime}} \dot{Q}_{c s s} d t^{\prime} d t .\left(\dot{Q}=\frac{d Q}{d t}\right)
$$

At the level of approximation being considered in this case, $Q_{\text {ars }} \propto t^{2}$ and $Q_{c s s} \propto t, i . e .$, the $t^{2}$ terms in the expression for $Q_{\text {unit }}$ and $Q_{c s s}$ are ner irible. Hence, with the containment spray system in standby 


$$
\begin{aligned}
Q(\text { standby }) & =\frac{1}{3} Q_{\text {ars }} Q_{\text {css }} \\
& =1.8 \times 10^{-5}
\end{aligned}
$$

or the estimated system reliability, with the containment spray in standby is

$$
R(\text { standby) }=0.999982 \text {. }
$$

As previously indicated, there are 270 third order and 3, 222 fourth order failure combinations resulting in system failure. Table $3.17 \mathrm{~B}$ shows the estimated percentage contribution of these combinations to the probability of system failure. This estimate was arrived a* as follows:

Within the framework of the above approximations, we may write

$$
\begin{aligned}
& Q_{\text {unit }}=a_{3 u} T+a_{4 u} T^{2} \\
& Q_{\text {css }}=a_{3 c} T+a_{4 c} T^{2}
\end{aligned}
$$

where the a's are coefficients relating to the component $\lambda$ values.

Then,

$$
\begin{aligned}
& Q(\text { standby }) \propto\left(Q_{\text {unit }}\right)^{2}\left(Q_{\text {css }}\right) \\
& Q(\text { standby })=a_{3} T^{3}+a_{4} T^{4}+a_{5} T^{5}+a_{6} T^{6} .
\end{aligned}
$$

The $\mathrm{T}^{3}$ term gives the contribution from third order failures. The $\mathrm{T}^{4}$ term gives the fourth order contribution and so on.

Comparison of Tables 3.17 A and 3.17B shows good agreement between the ARMM and hand calculated allocation of system failure probability to third and higher order failure combinations. Both methods attribute approximately 90 percent of the system failure probability to third order failure combinations. However, the system failure probability calculated by ARMM is a factor of two higher than the hand calculated value. This disagreement can be expected when considering the way ARMM presently handles standby functions. 


\section{Conclusions}

The results displayed in Tables $3.16,3.17 \mathrm{~A}$, and $3.17 \mathrm{~B}$ indicate the following conclusions. In this complex system containing redundant paths and components with failure probabilities $\approx 10^{-2}$, the system unreliability may be attributed almost entirely to those components involved in the lowest order failure combinations. It may be anticipated that components involved in a higher order failure combination will contribute significantly to the system unreliability only if that combination contains a component whose failure rate is at least an order of magnitude greater than that of the components in the lower order combination. This was not the case in the present study.

In this model of the Connecticut Yankee containment cooling system, the greatest contributors to the calculated system unreliability turn out to be the fans and fan motors in the air recirculation units, (Components 17 and 18 in Figure $3.31 \mathrm{~A}$ ) and the valves channeling coolant to the spray header rings in the containment spray system (Components 71 and 72 in Figure 3.3lB). Hence, the system reliability estimate will be most sensitive to the particular assumptions made regarding these components. Modification of these assumptions will strongly affect the computed system reliability.

For example, it was assumed in the analysis that the fans and fan motors were being aged over the whole testing interval (720 hours), as well as during the period of incident operation ( 300 hours). It could be argued that since these components are in continuous operation during the testing interval, their failure would be observed and repair would be effected. Using this reasoning, these components might be considered to be aging only for a period corresponding to the required repair time (say, I day) plus incident operating time. The total aging time for these components would then

be a factor of three $\left(\frac{300+24}{300+720}\right)$ less than previously assumed and the estimate of system reliability would be increased by a factor of approximately 10 .

Regardless of the assumptions used, the primary contributors to system failure are expected to be the fans, fan motors, and spray system valves. If increased reliability is desired in the containment cooling system, it appears that this goal can be most effectively gained through

1. Modifying these components to significantly reduce their failure rates, if further investigation of failure rate assumptions reveal no significant error, and

2. Consideration of additional redundancy in spray loop valves, if this redundancy will not significantly degrade the reliability of emergency core cooling functions. 
The San Onofre safety injection system is designed to limit core damage due to overheating following a loss-of-coolant accident in the reactor coolant system and to insert negative reactivity, in the form of borated water, during rapid cooldown of the reactor following a turbine-cycle-side steam line rupture. Design criteria used to assure accomplishment of these functions are:

1. The system shall prevent release of fission products from the fuel rods following a rupture of the reactor coolant system for any size break, up to complete severance of the largest line connecting to the reactor coolant loops, i.e., the 10 -inch nominal size pressurizer surge line.

2. The system shall limit fission product release for hypothetical break sizes (which are not considered credible), up to the complete severance of a reactor coolant pipe, assuming discharge of coolant from both ends of the pipe.

3. The system shall provide a means of cooling the core for extended periods, following a loss-of-coolant accident.

4. The system shall provide a means of inserting negative reactivity following a loss-of-coolant or a turbine-cycle-side steam line break.

5. The system design shall assure that no inadvertent system operation can be postulated which would constitute a hazard, such as would result from unlimited addition of unborated water wo the reactor coolant system.

6. The system shall operate satisfactorily with second-order mechanical equipment failures, i.e., with the loss-of-coolant accident as a first order condition; the failure of any component to respond actively in the prescribed manner can be tolerated without loss of ability to provide the necessary protection. Numerous third order or multiple failures can also be tolerated, but the design is not intended to cover all such cases.

7. Power shall be continuously available to safety injection system equipment. 
8. The design of the equipment in the safety injection system and supporting systems shall be in accordance with the seismic ground motion criteria established for the plant.

\section{SYSTEM DESCRIPTION}

The safety injection system is made up of two essentially separate systems; one for the initial injection of borated water to the reactor coolant system, and the other for subsequent cooling and recirculation of spilled water back to the reactor coolant system for long-term removal of the core residual heat. These two systems are shown in Figure 3. 35.

\section{Injection}

The injection system consists of two separate and independent pumping trains for delivery of water, borated at refueling concentration, into the reactor coolant system. This philosophy of equipment separation has been extended so that equipment in each injection train is supplied from independent 4,160-volt and 480 -volt buses.

Each injection train uses one of the two feedwater pumps which deliver feedwater to the steam generators during normal operation. These pumps are Byron-Jackson, Type DVMX, two-stage pumps with horizontally split case and double volute. Pump design features are listed in Table 3.18. The pumps are designed to operate at any point over their delivery curve from a minimum flow near shutoff to flows in excess of the nominal $10,500 \mathrm{gpm}$ injection requirement. The pump design was selected for its low net positive suction head requirement and its ability to continue delivery at high flows with some cavitation.

For safety injection service, the feedwater pumps are each supplied with borated refueling water by ore of the two safety injection pumps. The safety injection pumps are single-stage, high-capacity, low head units. As shown in Figure 3.35, each pump is designed to supply $10,500 \mathrm{gpm}$ of borated water from the refueling water storage tank to one feedwater pump at a pressure sufficient to prevent feedwater pump cavitation. Each pumping train obtains its borated water from the 240, 000 -gailon refueling water storage tank (approximately 11 times the reactor vessel volume) and discharges into the injection header, from which three separate injection lines run into the containment sphere and discharge into each of the three reactor coolant loops. The injection lines between the feedwater pump discharge isolation valves and the reactor coolant system isolation valves are always kept filled with borated water. Similarly, the safety injection line to the suction of these pumps is also kept filled with borated water. 
Although the accident analysis has shown additional injection flow to be unnecessary, both centrifugal charging pumps in the chemical and volume control system are placed in service to augment injection system flow. The charging pumps, which are in service (one operating and the other standby) during normal operation of the plant, inject into the reactor coolant system upon initiation of safety injection. As shown in Table 3.18, rated flow for each of the two centrifugal charging pumps is $213 \mathrm{gpm}$. The combined output of both pumps fulfills the requirement for purification flow plus the seal water flow required when all reactor coolant pumps are operating on breakdown bushings and both charging pump bypass lines are open. The discharge pressure of each pump is indicated in the control room and a pressure switch on the pump discharge header automatically starts the standby pump if the header pressure falls below 2, 200 psig.

The charging flow is arranged so that it can inject into each of the reactor coolant loops, thereby providing three additional flow paths. A charging pump alone will prevent the release of core fission products for break sizes up to approximately 6 inches and acts as a complete backup for the safety injection system in this range.

Additional injection flow can be provided by the refueling water pumps. These pumps are arranged so that they can inject into the reactor coolant system through the recirculation lines. For this service the pumps have a capacity of $500 \mathrm{gpm}$. Their primary function, however, is for containment spray. The injection function of these pumps is provided primarily as a backup for recirculation operation after the initial injection phase. Either recirculation pump is capable of delivery of water at a rate in excess of that needed for decay heat removal. Design parameters for these pumps are listed in Table 3. 18.

Recirculation

The design basis for recirculation is to provide sufficient water to the reactor core for long-term, post-accident cooling, when operation of the injection subsystem is discontinued. The equipment provided will accomplish this basic purpose with any one component failing to respond as required.

The recirculation system consists of recirculation pumps which take spilled water from the containment sphere sump and pump it through the recirculation heat exchanger to the charging pumps. The charging pumps then return the water to the reactor coolant system via a line from each of the reactor coolant pump seal-water injection lines. The recirculation pumps also supply water for long-term operation of the containment sphere spray system. 
The recirculation pumps are canned-motor, submersible units, designed to pump spilled water from the containment sphere sump. Each pump has a flow capacity approximately double that needed to compensate for boil-off of water covering the core due to residual heat generation. The pump design also permits periodic dry-test starts. Design parameters for these pumps are summarized in Table 3.18. The recirculation heat exchanger is a shell and tube unit. It removes decay heat from recirculated water by heat exchange with component cooling water circulating on the shell side. Operating conditions for this heat exchanger vary according to the severity of the loss-of-coolant accident and the equipment available for operation following the accident. The design is conservatively based on the set of parameters shown in Tabie 3.19.

\section{Electrical Power}

Electric power for safety injection system operation is supplied by the plant auxiliary electric power system. As previously indicated, power for each injection pumping train is supplied from independent 4, 160-volt and 480-volt buses connected to the station switchyard. If an accident requiring safety injection system operation occurs, power will continue to be supplied to all electrically powered safety injection equipment (pumps, valves, and instrumentation) from the same sources supplying the buses prior to the emergency. No bus transfers are required. For this example application, the plant has been assumed to be provided with two independent sources of power for the auxiliary electrical system. * These originate from:

1. The main turbine-generator unit at the San Onofre Nuclear Generating Station and the Southern California Edison Company's 220-kv system.

2. The San Diego Gas \& Electric Company's 138-kv system.

In the event of a loss-of-coolantincident the reactor will scram, the turbine will trip, and the main breakers tying to the 220-kv switchyard will open. However, the $138-\mathrm{kv}$ system will continue to supply power to the buses connected to safety injection equipment. As an emergency safety injection operating procedure, the operator will restore the $220-\mathrm{kv}$ system as an independent backup source of power into the plant

*The existing facility also is provided with emergency diesel generating capability; however, the power system defined in the Final Engineering Report and Hazard Analysis has been used here. 
following reactor coolant pump coastdown operation. This source is not required for safety injection power supply, but is made available as standby. Both the $138-\mathrm{kv}$ and $220-\mathrm{kv}$ systems are adequate to supply the electrical power requirements for safety injection. Safety injection system power requirements are summarized in Table 3.20. Figure 3.36 is a single-line diagram showing the electrical power supply to the pumps in the safety injection and recirculation systems. As shown, Bus 3 serves as an alternate power source to either of the normally used $480 \mathrm{v}$ buses (Buses 1 and 2).

A loss-of-coolant accident, coincident with the occurrence of major equipment electrical faults, is not considered credible. The electrical system is arranged, however, so that a large degree of protection if provided should a major electrical fault occur at some time following a safety injection incident.

\section{Injection System Operation}

Operation of the injection system is initiated automatically upon coincidence of low pressurizer pressure and low pressurizer water level, when the plant is at power or in the hot shutdown condition. The safety injection signal will initiate a reactor scram if the reactor has not already scrammed from an independent low pressure signal.

To assure reliability of the injection signal and freedom from spurious safety injection signals, the actuation circuit uses three independent pressure channels and three independent pressurizer water level channels. A signal initiating injection system operation is generated when two out of three low water level and two out of three low pressure signals occur. This arrangement allows testing of the actuation circuitry during operation.

When the plant is at normal power operation, the feedwater pumps deliver feedwater to the steam generators. The safety injection signal starts the two safety injection pumps and the standby charging pump, and stops ail four condensate pumps and the two heater drain pumps. Motor-operated valves also are actuated to align the pumping flow path for safety injection service and to isolate the feedwater condensate system. All valves act immediately, with the exception of the valves at the point of feedwater pump discharge into the injection header (valves $851 \mathrm{~A}$ and $\mathrm{B}$ ). These valves are interlocked to remain closed until the condensate isolation valves at feedwater pump suction (valves $854 \mathrm{~A}$ and $\mathrm{B}$ ) have completely closed. This ar rangement assures that borated water, from the safety injection pumps, will replace the significant portion of unborated water between the feedwater pump isolation valves before a flow path to the reactor coolant loops is opened. Should valve 854 fail to close, the interlock will prevent delivery of unborated water from the affected train into the injection header. 
Is olation valves, which must function in order to align the injection system for operation, are gate valves with Limitorque operators. Each valve is designed to operate under the pressure differentials expected during safety injection and has a motor operator design compatible with operating time requirements and, where applicable, with post-accident ambient conditions within the containment sphere. Reliability of valve operation is assured by a program of testing both in the manufacturer's shop and periodically throughout the plant lifetime.

With the pumps started and the injection flow path aligned, the injection system delivers refueling water at a $r$ ate dependent upon break size and reactor coolant system pressure. The feedwater pumps can begin delivery at pressures as high as 1,157 psig. A system delivery of approximately $7,000 \mathrm{gpm}$ is required to meet performance criteria in the case of the hypothetical severance of a reactor coolant pipe. The large feedwater pump capacity assures this delivery even if the hypothetical break occurs at an injection line connection and one injection train fails to function.

When the containment sphere sump level indicator shows an adequate water level for initiation of recirculation, and when the water level in the refueling water storage tank indicates that an adequate amount of refueling water has been delivered into the reactor coolant system, injection flow will be terminated by the operator and the recirculation subsystem placed in operation. For small breaks, the restoration of water level in the pressurizer provides additional indication that the reactor coolant system has been refilled.

The operator terminates injection system operation by blocking the automatic actuation signal, switching the four safety injection relays to the off position, and then individually turning off the feedwater pumps and the safety injection pumps in sequence. With occurrence of a low level in the refueling water storage tank an alarm will sound in the control room before the injection system empties the refueling water storage tank.

\section{Recirculation System Operation}

This phase of operation is initiated by the operator after the core has been covered with borated water by the injection system and a sufficient quantity of water has accumulated in the containment sphere sump. 
The recirculation subsystem is placed in operation by starting the recirculation pumps and operating appropriate valves to open the recirculation flow paths. The valve isolating the refueling water storage tank is closed. Containment spray also can be initiated using the refueling water pump.

The operator has ample time to initiate recirculation. The refueling water storage tank has a minimum of ten minutes supply of borated water, even in the case of the hypothetical break, when both feedwater pumps deliver at their maximum capability. An additional time of approximately 10 minutes is available for initiating recirculation before the water in the reactor vessel would be evaporated below a level sufficient to assure core cooling. For the credibif maller piping ruptures, injection system operation will continue for longer periods before recirculation system operation is required.

\section{RELIABILITY EVALUATION}

Based on the preceding system descriptions and operational information, a preliminary reliability analysis has been performed for the San Onofre safety injection system. The basis which has been chosen for this analysis is the probability of successful operation in conjunction with a postulated break in a 10-inch line in the reactor coolant system. The analysis is made over three time intervals. The first interval is one month or 720 hours; the second interval is 10 minutes; and the third interval is 24 hours. In the first interval, the system is in standby. The feedwater pumps are operating to supply water to the steam generators, and one of the charging pumps is operating as part of the chemical and volume control system. The other charging pump is standby to the first. The break in a 10-inch line occurs at the end of the first interval. During the second interval, the safety injection system pumps the borated water from the refueling water tank to the reactor coolant loops. This requires the startup of the safety injection pumps and the alignment of the flow path by means of operation of the valves. Also, the refueling water pumps and the second charging pump are turned on, although for this size break only the safety injection pumping trains have the capacity to adequately cover the reactor core. However, the two charging pump trains and the two refueling pump trains can contribute to system unreliability during this interval, since at least one of them is required in the third interval. If all four of these latter pumps should fail in the second interval, they would not be available for the third interval. During the third interval, the borated water which has spilled from the break and collected in the containment sump is recirculated into the reactor for the removal of fission product decay heat. For this purpose one of the two recirculation pumps, which were not on during the first two intervals, must operate and, as stated before, one of the charging pump trains or refueling pump trains must work. 
During the second and third intervals, the flow through the system is changed by means of actuation of valves and the startup of pumps. To properly consider the contribution of the se components to the unreliability during this phase of their operation, they have been assumed to perform this operation at the end of the preceding interval. The pumps have been assigned two failure rates, one for their startup and one for their continuing operation. To do this using the ARMM program, the pumps are described as two components, each with one of the failure rates. The component with the startup failure rate is on during the preceding interval and off the following intervals during which the component with the operating failure rate is on. In effect, the failure rate for the startup component reflects the effect of aging on the likelihood of a pump accomplishing a successful startup. Valves must open or close to align the system properly. They have been assumed to perform this operation at the end of the interval prior to the interval during which this proper alignment is required. For example, the safety injection loop valves must be open diring the second interval, so they are assumed to open at the end of the first interval. Once a valve has performed its operation successfully, it is assumed to be perfectly reliable. For example, a valve which is required to open will not fail closed once it has opened. Its only failure is to fail to open in the first place. For this reason, a valve is "on" only during the interval at the end of which it is required to function. Therefore, in this example, the valves on the safety injection loop do not contribute to system unreliability in any but the first time interval. As with the startup of a pump, the opening and closing of a valve is a cyclic operation. It is evaluated by ARMM by using a continuous failure rate which approximates the probability of a single cycle failure at the end of the interval of interest.

The length of the first and third intervals were chosen somewhat arbitrarily. The first interval of one-month duration was assumed to be the interval between tests for all components of the safety injection system. This would not be the procedure followed in the actual plant operation. The feedwater pumps, for example, are operafing continuousiy, so, in a sense, they are continuously monitored and tested. The only time they contribute to the unreliability of the system in the test interval is within a length of time prior to a loss-of-coolant accident equal to the length of time it takes to repair or replace a failed pump, assuming plant operation would continue with one feedwater pump out of service. To reflect this condition in a reliability analysis with ARMM, the failure rates for these pumps would have to be multiplied by the ratio of the repair time to the interval length. The failure rates of other components which have more frequent tests than represented by the interval length would need to be multiplied by the ratio of their test interval to the program interval. This modification was not done in this calculation. The ten minute duration of the second interval was based on the minimum supply of borated water in the refueling water tank assuming the two safety injection pumping trains operate at maximum capability. The length of 24 hours for the third interval was, as stated above, arbitrary. It could easily have taken any other value. 
Using the preceding considerations, a failure mode and effect analysis was performed on the safety injection system components to identify the critical nature of component failures. This analysis is illustrated for major system components in Figure 3.37. Such an analysis identifies the cause and effect of component failures, the conditions under which the failures become significant, and the actions which can be taken to eliminate or mitigate such failures. The analysis also was used to assure that subsequent steps in the reliability analysis identify the important failure modes in the equipment being considered.

Based on the preceding work a reliability block diagram was prepared for the safety injection system as shown in Figure 3.38. The diagram was used, in turn, to guide preparation of a reliability model input for analys is by the ARMM program. As shown in Figure 3. 38, the number above each block is the component ID used in the ARMM analysis. Several pumps have two ID numbers. These represent the pump during its startup and then during the continuous operation which follows.

In the reliability block diagram in Figure 3.38 each electrical bus is placed in line with the pump or pumps it powers, with one exception. In that case, the 480 volt buses (numbers 1 and 2) appear by themselves and as alternates (in redundancy) to each other in the block diagram immediately following the safety injection loop valves. This allows the reliability analysis to consider the possibility of failure of both buses in this configuration during the first interval. During the second and third intervals, these two buses have this configuration combined with the two recirculation pumps. During the first interval, these two buses are also combined with the refueling water pumps. However, in this combination they have as additional alternates the two charging pump trains.

For this analysis the $220 \mathrm{kv}$ system was assumed to be connected to the 4,160 volt buses, $1 \mathrm{~A}$ and $1 \mathrm{~B}$, and from these buses to the two breakers, $11 \mathrm{C} 01$ and $12 \mathrm{C01}$, to the 4,160 volt buses, $1 \mathrm{C}$ and $2 \mathrm{C}$. As previously indicated in the system description, the $220 \mathrm{kv}$ system is not reconnected until after the reactor coolant pump coastdown.

The intervals during which each component contributes to system unreliability is given in Table 3.21 .

The failure rates used for the components of the safety injection system are given in Table 3.22, As shown in Table 3.22, the tank, sump, and headers were given very low failure rates since they were not expected to fail at rates comparable to those assigned to other active components. If the failure rates for these static components were increased significantly, their serial location in the system reliability model would make them major contributors to system unreliability. 


\section{RESULTS OF ANALYSIS}

The components which make the largest contribution to system unreliability are listed in Table 3.23 for each time interval and for the total time. Included in the list is the probability that each component might cause system failure and the percentage contribution that a component might be expected to make to system unreliability for the interval or for the entire time.

A comparison of the data for Interval 1 and for the entire time shows that Interval $l$ is the period of greatest unreliability. There are several reasons for this. First, the length of the first interval is much greater than the two following intervals. This illustrates the potential value of increasing the frequency of tests. Another reason is the number of components which were considered during the first interval compared with the number in the other two intervals. As shown in Table 3.21, 70 components were considered in the first interval, 42 in the second, and 31 in the third.

The primary contributors to system unreliability in the first interval are the startup components of the two safety injection pumps, the two feedwater pumps, the auxiliary transformer $C$, and the main transformer. Together they contribute 97.4 percent of total unreliability during that interval.

During the second interval the three major contributors are series components, i.e., components not supported by a redundancy. In the third interval, the major contributor, the recirculation heat exchanger, is also a series component.

This example analysis has shown how the components which can be expected to be major contributors to the unreliability of a given system can be identified. For the San Onofre safety injection system, these components are listed in the summary of Table 3.23. Within the accuracy of the reliability model and failure rate assumptions, these results point out the components which either (1) need better failure rate data, (2) need to be redesigned or replaced, (3) need additional redundancy, or (4) need more frequent testing.

Because the major contributors to the unreliability of the safety injection system are part of redundant units, Number (3) above would not appear to be the solution to better reliability. Because of the long duration of the first interval, Number (4), more frequent testing appears to be the best solution (assuming the failure rate data is reasonably accurate). 


\section{TABLE 3.1}

PRINCIPAL DESIGN PARAMETERS

FOR DRESDEN-3 PRIMARY CONTAINMENT

Pressure Suppression Chamber Internal Design Pressure

62 psig

-1 psig

Drywell Internal Design Pressure

62 psig

-2 psig

Initial Suppression Chamber Pressure Rise

21 psi max.

Initial Suppression Chamber Temperature Rise $50^{\circ} \mathrm{F}$.

Downcomer Vent Pressure Loss Factor

6.2

Break Area/Vent Pipe Area

.019

Submergence of Vent Pipe Below Pressure Suppression Pool Surface

4 feet

Drywell Free Volume

$\sim 158,000 \mathrm{ft}^{3}$

Pressure Suppression Pool Free Volume

$\sim 119,500 \mathrm{ft}^{5}$

Pressure Suppression Pool Water Volume

$\sim 106,000 \mathrm{ft}^{3}$ 
TABLE 3.2

\section{PRINCIPAL PENETRATIONS OF PRIMARY CONTAINMENT AND ASSOCIATED ISOLATION VALVES}

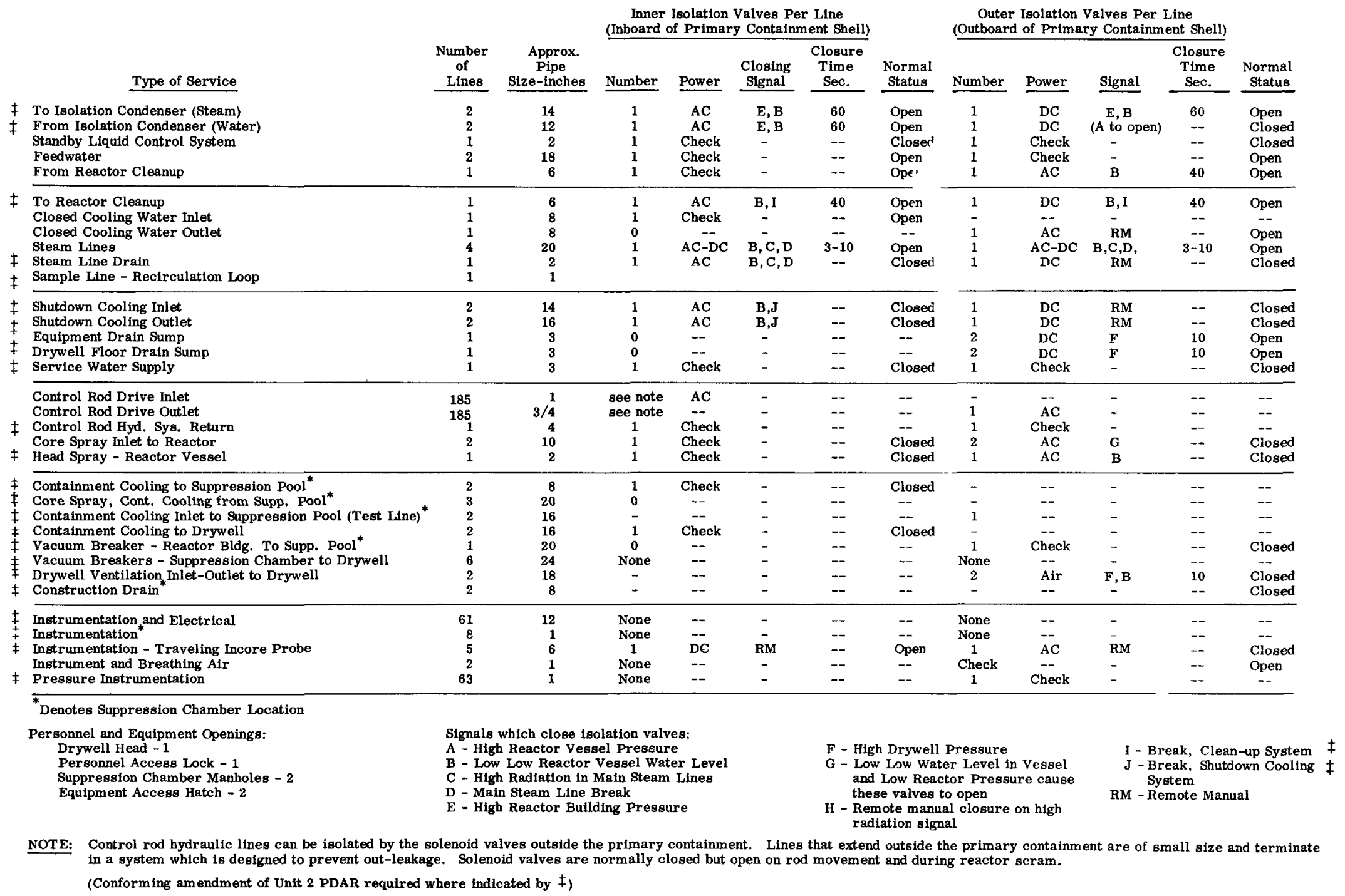

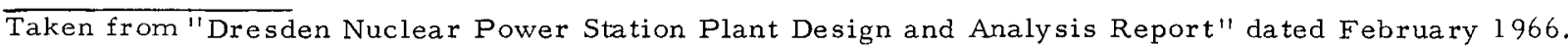


TABLE 3.3

DRESDEN-3 STANDBY DIESEL GENERA TOR

LOADING REQUIREMENTS

\begin{tabular}{|c|c|}
\hline LOAD & $\begin{array}{l}\text { ES TIMA TED POWER } \\
\text { REQUIRED (hp) }\end{array}$ \\
\hline \multicolumn{2}{|l|}{ Automatically Connected Shutdown Loads } \\
\hline One Core Spray Pump & 700 \\
\hline Two Containment Cooling Pumps & 700 \\
\hline Two Containment Cooling Service Water Pumps & 880 \\
\hline Standby Gas Treatment Equipment & 150 \\
\hline All Power Operated Valves Not on D-C & 80 \\
\hline $\begin{array}{l}\text { Emergency A-C Lighting } \\
\text { (time delay transfer from battery) }\end{array}$ & 150 \\
\hline Instrumentation and Control Motor-Generator & 25 \\
\hline Total & $2,685 \mathrm{hp}$ \\
\hline \multicolumn{2}{|l|}{ Manually Connected Shutdown Loads } \\
\hline Standby Liquid Control Pump & 100 \\
\hline Drywell Cooling Blowers & 80 \\
\hline Reactor Building Cooling Water System & 400 \\
\hline $\begin{array}{l}\text { Service Water System (that portion } \\
\text { connected to the reactor building cooling water) }\end{array}$ & 900 \\
\hline Emergency A-C Lighting & 150 \\
\hline Instrument Air Compressor & 150 \\
\hline Fuel Pool Cooling System & 100 \\
\hline Battery Charger & 40 \\
\hline Condensate Transfer Pump & 50 \\
\hline $\begin{array}{l}\text { Control Valves as Required by Above Systems } \\
\text { but not included as battery load }\end{array}$ & 15 \\
\hline Instrumentation and Control Motor-Generator & 25 \\
\hline Total & $2,010 \mathrm{hp}$ \\
\hline
\end{tabular}


TABLE 3.4

\section{BLOWDOWN ACCIDENT SUMMARY}

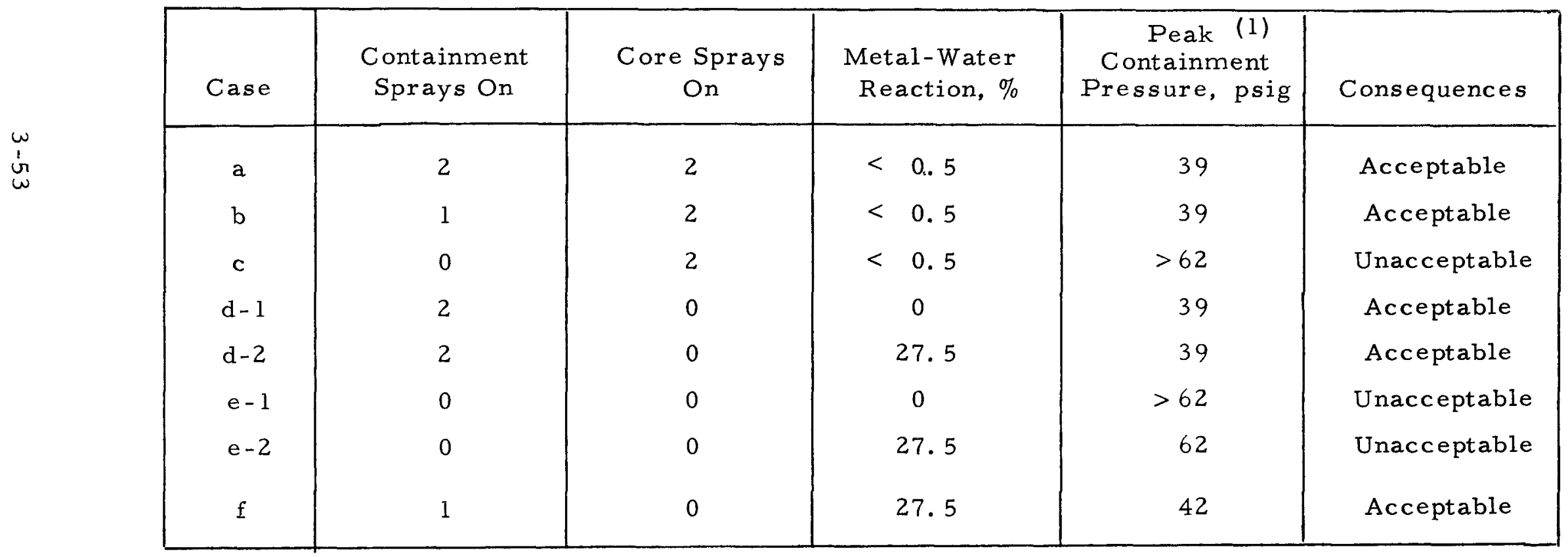

(1) Containment designed for overpressure of 62 psig. 
TABLE 3.5

TIME SEQUENCE FOR BLOWDOWN ACCIDENT

\begin{tabular}{|l|c|}
\hline \multicolumn{1}{|c|}{ Event } & $\begin{array}{c}\text { Time After Main } \\
\text { Recirculation Line Break }\end{array}$ \\
\hline $\begin{array}{l}\text { First Peak in Drywell Pressure } \\
\text { (39 psig) } \\
\text { Perforation of lst Rod }\end{array}$ & 3 sec. \\
$\begin{array}{l}\text { End of Blowdown } \\
\text { Start of Containment Spray System }\end{array}$ \\
$\begin{array}{l}\text { Equalization of Drywell and Suppression } \\
\text { Chamber Pressure (21 psig) }\end{array}$ \\
$\begin{array}{l}\text { Completion of Containment Isolation } \\
\text { (longest valve closure time from end of } \\
\text { blowdown 60 sec.) }\end{array}$ \\
$\begin{array}{l}\text { Start of Metal-Water Reaction } \\
\begin{array}{l}\text { Second Peak in Drywell Pressure (42 psig) } \\
\text { Perforation of All Fuel Rods }\end{array}\end{array}$ \\
$\begin{array}{l}\text { End of Metal-Water Reactor ( 25 percent } \\
\text { reaction) }\end{array}$
\end{tabular}


TABLE 3.6

FAILURE RATE ASSUMPTIONS

\begin{tabular}{|c|c|c|}
\hline Component & Failure Mode & Failure $/ 10^{6} \mathrm{Hr}$. \\
\hline Hatch Closure Seals & Leak Excessively & .01 \\
\hline Personnel Lock Doors & Open & .1 \\
\hline Personnel Lock Door Seals & Leak Excessively & .1 \\
\hline Personnel Lock Interlock & No Door Lock & .01 \\
\hline $\begin{array}{l}\text { Air Operated Valves } \\
\text { (Normally Closed) }\end{array}$ & $\begin{array}{l}\text { Fail Open or } \\
\text { Leaks }\end{array}$ & $\begin{array}{c}.05 \text { (Designed to } \\
\text { fail closed) }\end{array}$ \\
\hline $\begin{array}{l}\text { Air Operated Valves } \\
\text { (Normally Open) }\end{array}$ & $\begin{array}{l}\text { Fail Open or } \\
\text { Leaks }\end{array}$ & $\begin{array}{r}\text { (Designed to } \\
\text { fail closed) }\end{array}$ \\
\hline $\begin{array}{l}\text { Motor Operated Valves } \\
\text { (Normally Closed) }\end{array}$ & $\begin{array}{l}\text { Fail Open or } \\
\text { Leaks }\end{array}$ & $\begin{array}{c}.01 \text { (Designed to } \\
\text { fail as-is) }\end{array}$ \\
\hline $\begin{array}{l}\text { Motor Operated Valves } \\
\text { (Normally Open) }\end{array}$ & Fail Closed & \\
\hline & $\begin{array}{l}\text { Fail Open or } \\
\text { Leaks }\end{array}$ & $\begin{array}{c}.5 \text { (Designed to } \\
\text { fail as-is) }\end{array}$ \\
\hline $\begin{array}{l}\text { Check Valves (Normally } \\
\text { Closed) }\end{array}$ & $\begin{array}{l}\text { Fail to Seat or } \\
\text { Leaks }\end{array}$ & $\begin{array}{l}\text {. } 1 \text { Designed to } \\
\text { close with outflow }\end{array}$ \\
\hline & $\begin{array}{l}\text { Fail Open or } \\
\text { Leaks }\end{array}$ & $\begin{array}{l}\text { 1.0 (Designed to } \\
\text { fail as-is) }\end{array}$ \\
\hline Suction Strainers & Fail Open or Clogged & .01 \\
\hline Pumps (Normally Off) & Fail to Start & 10.0 \\
\hline Heat Exchanger & $\begin{array}{l}\text { Fail to Continue } \\
\text { Leak Excessively or } \\
\text { Badly Fouled }\end{array}$ & 5.0 \\
\hline $\begin{array}{l}\text { Spray Headers } \\
\text { Block Valves (Normally }\end{array}$ & Plugged & .1 \\
\hline Closed) & Fail Open & 1.0 \\
\hline Automatic Trip Devices & Fail to Trip & 1.0 \\
\hline Manual Trip Devices & Fail to Trip & 0.1 \\
\hline
\end{tabular}


TABLE 3.7

SUMMARY OF RESULTS FOR

DRESDEN - 3 PRIMARY CONT AINMENT

RELIABILITY EVALUATION

\begin{tabular}{|c|c|c|c|c|}
\hline Case Variables & Case 1 & Case 2 & Case 3 & Case 4 \\
\hline OPERATIONAL CONSIDERATIONS & & & & \\
\hline $\begin{array}{l}\text { Containment Isolation Achieved } \\
\text { Containment Cooling System On } \\
\text { Core Spray System On } \\
\text { Emergency AC Power On }\end{array}$ & $\begin{array}{l}\text { Yes } \\
1 \text { of } 2 \text { Loops } \\
\text { No } \\
\text { Yes }\end{array}$ & $\begin{array}{l}\text { Yes } \\
1 \text { of } 2 \text { Loops } \\
1 \text { of } 2 \text { Loops } \\
\text { Yes }\end{array}$ & $\begin{array}{l}\text { No } \\
\text { No } \\
1 \text { of } 2 \text { Loops } \\
\text { Yes }\end{array}$ & $\begin{array}{l}\text { No } \\
\text { No } \\
2 \text { of } 2 \text { Loops } \\
\text { Yes }\end{array}$ \\
\hline PROBABILITY OF SYSTEM SUCCESS & & & & \\
\hline Interval 1 & 0.99981 & 0.99970 & 0.99977 & 0.95746 \\
\hline Interval 2 & 0.99999 & 0.99999 & 0.99999 & 0.99998 \\
\hline Overall & 0.99981 & 0.99970 & 0.99977 & 0.95744 \\
\hline PROBABILITY OF SYSTEM FAILURE & & & & \\
\hline Interval 1 & $0.18599 \times 10^{-3}$ & $0.29780 \times 10^{-3}$ & $0.22831 \times 10^{-3}$ & $0.42544 \times 10^{-1}$ \\
\hline Interval 2 & $0.32450 \times 10^{-7}$ & $0.33073 \times 10^{-7}$ & $0.32549 \times 10^{-7}$ & $0.17925 \times 10^{-4}$ \\
\hline Overall & $0.18602 \times 10^{-3}$ & $0.29783 \times 10^{-3}$ & $0.22835 \times 10^{-3}$ & $0.42561 \times 10^{-}$ \\
\hline NO. OF COMPONENTS CONSIDERED & 162 & 204 & 66 & 66 \\
\hline
\end{tabular}




\section{TABLE 3.8}

MAJOR CONTRIBUTORS TO PRIMARY CONTAINMENT FAILURE

\begin{tabular}{|c|c|c|c|c|c|c|}
\hline & \multicolumn{3}{|c|}{ CASE 1} & \multicolumn{3}{|c|}{ CASE 2} \\
\hline $\begin{array}{c}\text { System } \\
\text { Rank }\end{array}$ & $\begin{array}{l}\text { ARMM } \\
\text { I. D. } \\
\text { No. }\end{array}$ & Component & $\begin{array}{l}\text { Percent } \\
\text { System } \\
\text { Failure }\end{array}$ & $\begin{array}{l}\text { ARMM } \\
\text { I. D. } \\
\text { No. }\end{array}$ & Component & $\begin{array}{l}\text { Percent } \\
\text { System } \\
\text { Failure }\end{array}$ \\
\hline 1 & 152 & Transformer 6 & 25.8092 & 152 & Transformer 6 & 20.6034 \\
\hline 2 & 240 & Transformer 7 & 16.0593 & 240 & Transformer 7 & 15.1461 \\
\hline 3 & 150 & Bus 6 - T6 Breaker & 11.3768 & 150 & Bus 5 - T6 Breaker & 9.2601 \\
\hline 4 & 154 & T6 - Bus 2A Breaker & 11.3768 & 154 & T6 - Bus 2A Breaker & 9.2601 \\
\hline 5 & 238 & Bus 6 - T7 Breaker & 6.9255 & 238 & Bus 6 - T7 Breaker & 6.7644 \\
\hline 6 & 242 & T7 - Bus 2B Breaker & 6.9255 & 242 & T7 - Bus 2B Breaker & 6.7644 \\
\hline 7 & 216 & MOV-31 Breaker & 6.9255 & 328 & MOV-20 Breaker & 5.9851 \\
\hline 8 & 220 & MOV-32 Breaker & 6.9255 & 294 & MOV-10 Breaker & 5.9093 \\
\hline 9 & 140 & Service Water Valve Breaker & 0.8867 & 216 & MOV-31 Breaker & 4.0008 \\
\hline 10 & 144 & Spray Test Valve Breaker & 0.8867 & 220 & MOV-32 Breaker & 4.0008 \\
\hline 11 & 224 & MOV -22 Breaker & 0.5067 & 326 & Relief Valve & 1.4630 \\
\hline 12 & 228 & STV-21 Breaker & 0.5067 & 336 & Manual Valve Switch & 1.4630 \\
\hline 13 & 232 & SWV-2 Breaker & 0.5067 & 292 & Core Spray Relief Valve & 1.4119 \\
\hline 14 & 76 & MOV -104 & 0.3782 & 302 & Manual Switch & 1.4119 \\
\hline 15 & 170 & Containment Cooling Pump Motor & 0.3344 & 140 & Service Water Valve Breaker & 0.5122 \\
\hline 16 & 172 & Containment Cooling Pump & 0.3344 & 144 & Spray Test Valve Breaker & 0.5122 \\
\hline 17 & 182 & Service Water Pump Motor & 0.3344 & 332 & MOV-21 Breaker & 0.4596 \\
\hline 18 & 184 & Service Water Pump & 0.3344 & 298 & MOV-11 Breaker & 0.4512 \\
\hline 19 & 164 & Containment Cooling Pump Motor & 0.3344 & 224 & MOV-22 Breaker & 0.2927 \\
\hline 20 & 166 & Containment Cooling Pump & 0.3344 & 228 & STV-21 Breaker & 0.2927 \\
\hline & & Total & 98.0022 & & Total & 95.9839 \\
\hline
\end{tabular}


TABLE 3.9

COMPONENT GROUPING BY PERCENTAGE CONTRIBUTION TO SYSTEM FAILURE

\begin{tabular}{|c|c|c|c|c|}
\hline $\begin{array}{c}\text { System Failure } \\
\text { Percentage } \\
\text { Group } \\
\end{array}$ & $\begin{array}{l}\text { Number Of } \\
\text { Components } \\
\text { In Group }\end{array}$ & $\begin{array}{l}\text { Percent Of } \\
\text { All Components } \\
\text { In Group }\end{array}$ & $\begin{array}{l}\text { Probability Of } \\
\text { System Failure } \\
\text { For Group } \\
\end{array}$ & $\begin{array}{c}\text { Percentage Contribution } \\
\text { ToSystem Failure } \\
\text { By Group }\end{array}$ \\
\hline $\begin{array}{l}\text { Case } 1 \\
20-30 \\
10-20 \\
1-10 \\
0.1-1 \\
0.01-0.1 \\
0.001-0.01 \\
<0.001 \\
\text { Case } 2 \\
20-30 \\
10-20 \\
1-10 \\
0.1-1 \\
0.01-0.1 \\
0.001-0.01 \\
<0.001\end{array}$ & $\begin{array}{r}1 \\
3 \\
4 \\
20 \\
15 \\
13 \\
106 \\
\\
\\
\\
1 \\
1 \\
12 \\
23 \\
23 \\
10 \\
134\end{array}$ & $\begin{array}{r}0.6 \\
1.9 \\
2.5 \\
12.3 \\
9.3 \\
8.0 \\
65.4 \\
\\
\\
0.05 \\
0.05 \\
5.9 \\
11.3 \\
11.3 \\
4.9 \\
65.6\end{array}$ & 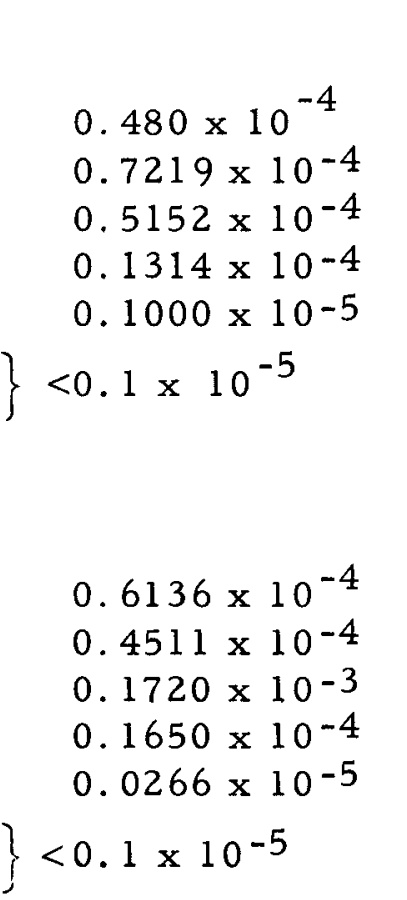 & $\begin{array}{r}25.81 \\
38.81 \\
-27.70 \\
7.68 \\
7.68 \\
7.68 \\
\\
\\
20.60 \\
15.15 \\
57.72 \\
6.53 \\
6.53 \\
6.53\end{array}$ \\
\hline
\end{tabular}


TABLE 3.10

LOSS OF COOLANT INCIDENT AND SAFETY INJECTION SYSTEM SUMMARY

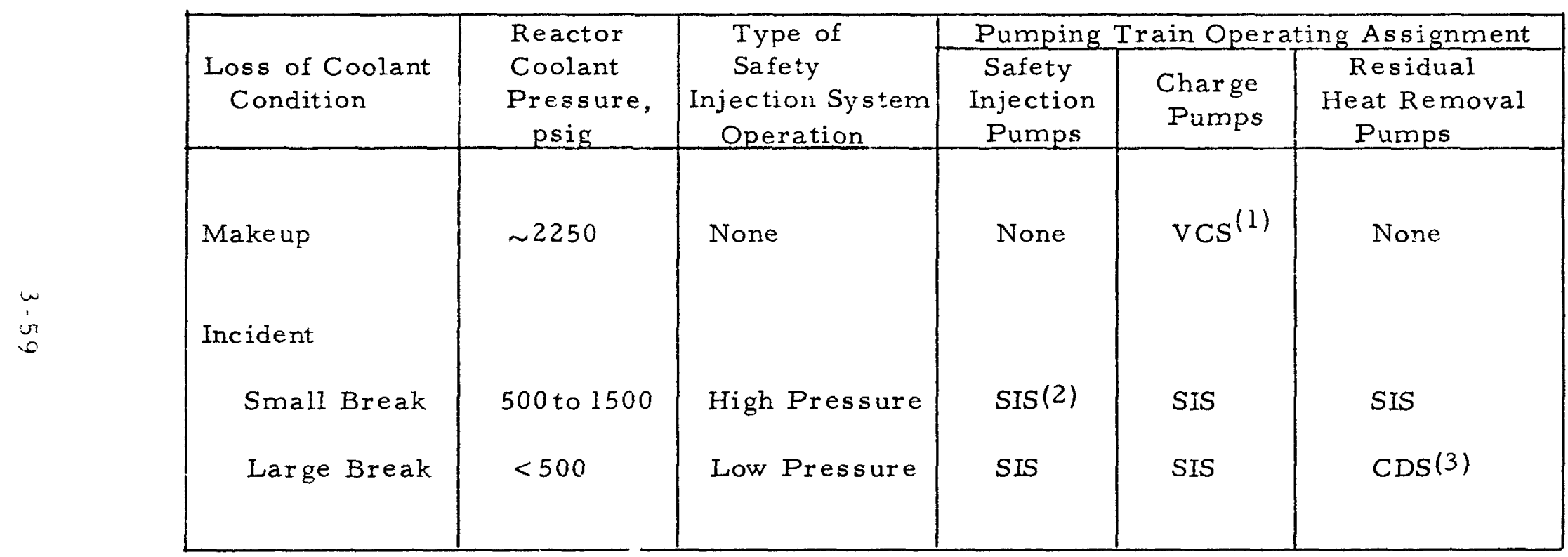

(1) VCS = Volume Control System

(2) SIS = Safety Injection System

(3) $\mathrm{CDS}=$ Core Deluge Subsystem 
TABLE 3.11

RUPTURED PIPE SIZES AND SAFETY INJECTION SYSTEM OPERATION

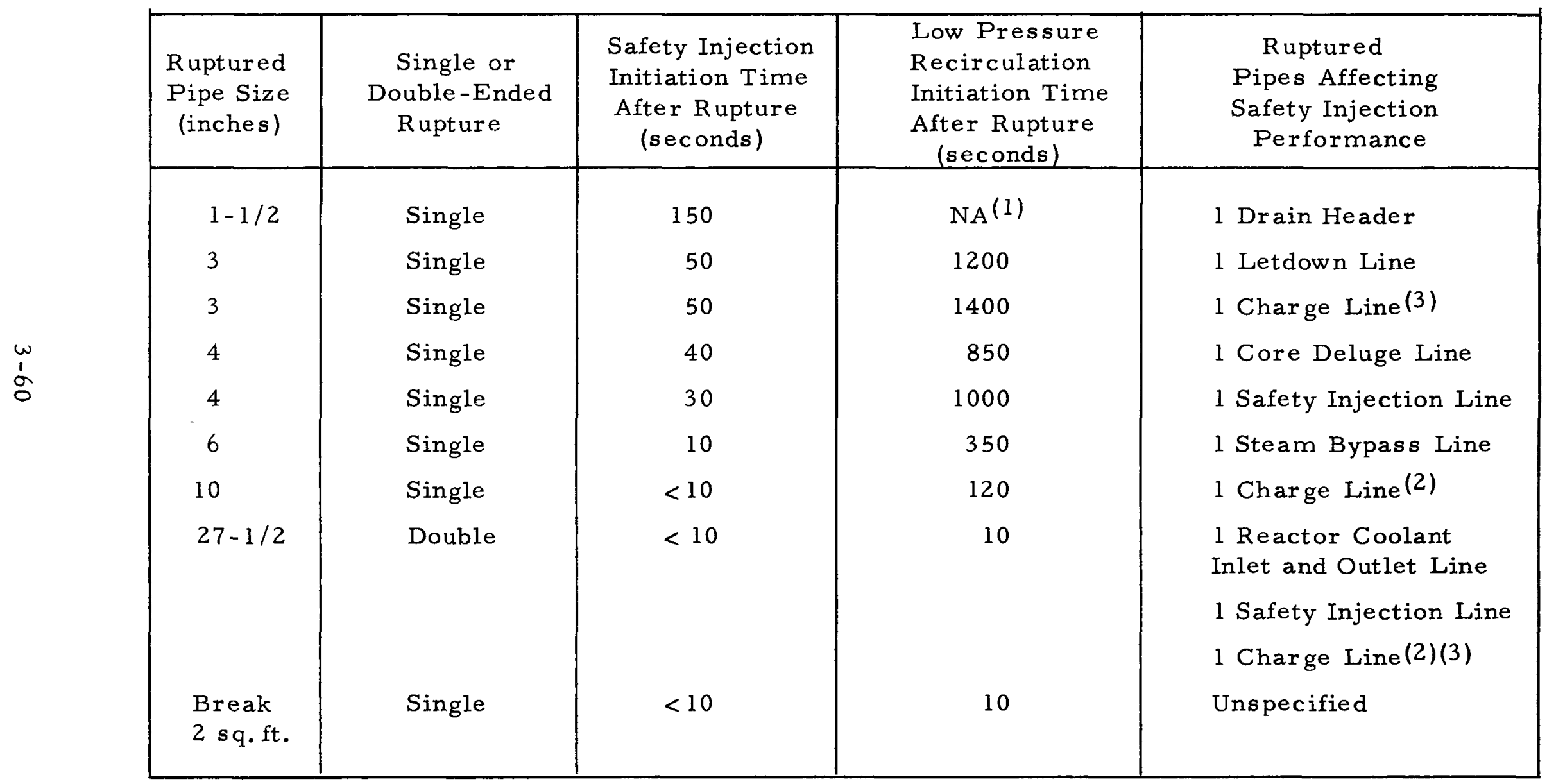
(1) Not Available
(2) Coolant Loop 2
(3) Coolant Loop 4 
TABLE 3.12

FAILURE RATE SUMMARY

\begin{tabular}{|c|c|c|}
\hline Component & Failure Mode & $\begin{array}{l}\text { Failure per } \\
\text { Million Hours }\end{array}$ \\
\hline Storage Tank & Rupture or no flow & 0.1 \\
\hline Pump & $\begin{array}{l}\text { Fail to start } \\
\text { Fail to continue running }\end{array}$ & $\begin{array}{r}10.0 \\
5.0\end{array}$ \\
\hline $\begin{array}{l}\text { Motor Operated } \\
\text { Valve }\end{array}$ & Fail to open or close & $\begin{array}{c}10.0 \\
\text { (designed to failas -is) }\end{array}$ \\
\hline Solenoid Valve & Fail to open & $\begin{array}{c}5.0 \\
\text { (designed to fail open) }\end{array}$ \\
\hline Check Valve & Fail to seat & $\begin{array}{c}1.0 \\
\text { (designed to close } \\
\text { with backflow) }\end{array}$ \\
\hline Heat Exchanger & Rupture & $\begin{array}{l}0.3 \\
\text { (designed to ASME } \\
\text { code) }\end{array}$ \\
\hline $\begin{array}{l}\text { Outside Power } \\
\text { Source }\end{array}$ & Fail to supply power & 0.1 \\
\hline Transformer & Fail to supply power & 5.0 \\
\hline Bus Tie & Fail to close & 2.0 \\
\hline Automatic Switch & Fail to trip & 5.0 \\
\hline Manual Switch & Fail to trip & 5.0 \\
\hline Pressure Switch & Fail to trip & 15.0 \\
\hline Level Switch & Fail to trip & 15.0 \\
\hline
\end{tabular}


TABLE 3.13

SUMMARY OF SYSTEM UNRELIABILITY

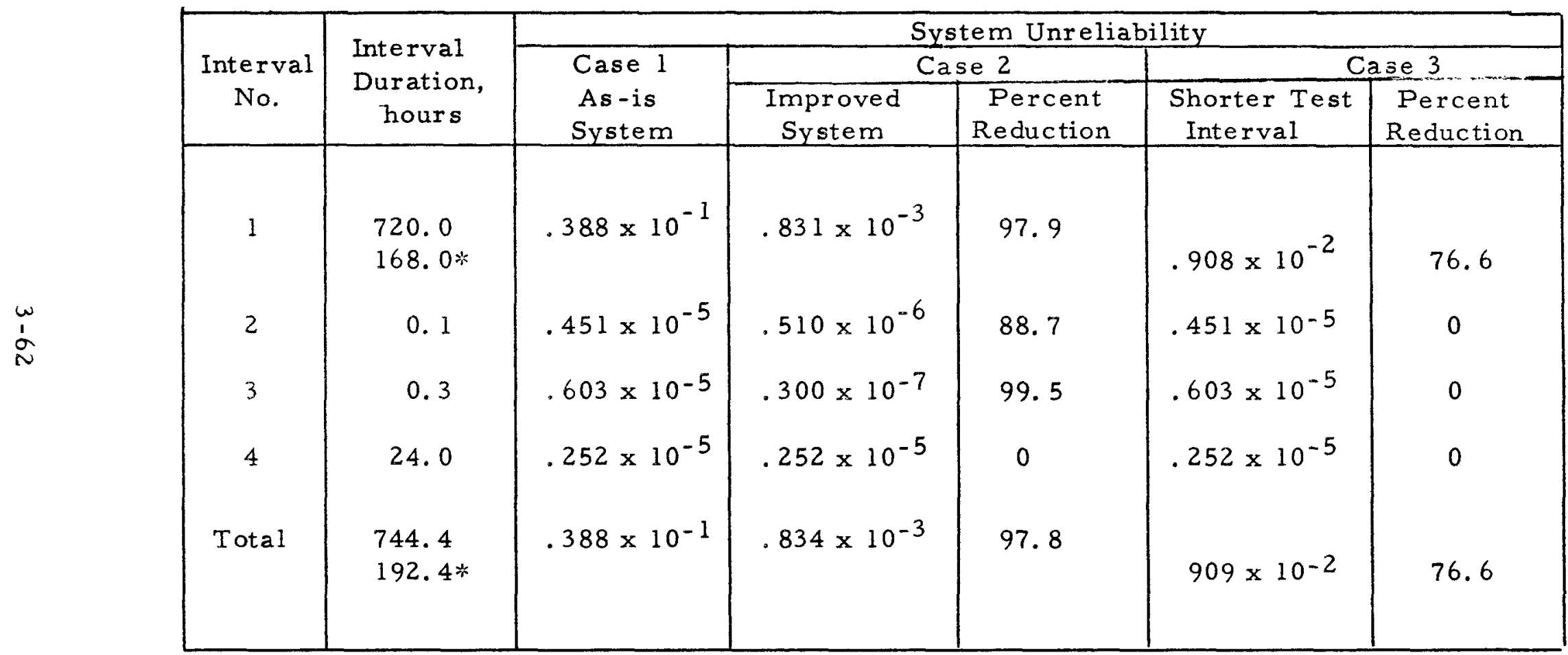

*Applies to Case 3 only. 
TABLE 3.14

MAJOR CONTRIBUTORS TO SYSTEM UNRELIABILITY

\begin{tabular}{|l|c|c|c|}
\hline \multirow{2}{*}{ Component } & \multicolumn{3}{|c|}{ Percent System Failure } \\
\cline { 2 - 4 } Refueling Water Supply Valve & Case 1 & Case 2 & Case 3 \\
Safety Injection Recirculation Valve & 18.2 & $5.6(1)$ & 18.4 \\
Containment Sump Valve & 18.2 & $5.6(1)$ & 18.4 \\
Residual Heat Throttle Valve & 18.2 & $5.6(1)$ & 18.4 \\
Residual Heat Pump Suction Valve & 9.1 & $5.6(1)$ & 18.4 \\
Residual Heat Bypass Valve & 9.1 & $1.4(1)$ & 9.2 \\
Charge Pump Suction Valve & 2.7 & $1.4(1)$ & 9.2 \\
Volume Control Valve & 2.7 & $.35(1)$ & 2.7 \\
Charge Line Control Valve & 1.8 & $.35(1)$ & 2.7 \\
Safety Injection Valves(2) & .78 & 33.4 & 1.8 \\
Refueling Water Storage Tank & .19 & 8.1 & .19 \\
\hline
\end{tabular}

(1) 2 valves with 1 out of 2 redundancy

(2) 4 valves with 3 out of 1 redundancy 
TABLE 3.15

CONNECTICUT YANKEE

CONTAINMENT COOLING SYSTEM

COMPONENT FAILURE RATES

\begin{tabular}{|c|c|c|}
\hline $\begin{array}{l}\text { Figure } \\
\text { Number }\end{array}$ & $\begin{array}{c}\text { ARMM } \\
\text { Component I. D. }\end{array}$ & $\begin{array}{l}\text { Component Failure Rate, } \\
\text { Failures per } 106 \text { hour }\end{array}$ \\
\hline $3.31 \mathrm{~A}$ & $10,11,12,13$ & 1.0 \\
\hline $3.31 \mathrm{~A}$ & 14 & 2.0 \\
\hline $3.31 \mathrm{~A}$ & 15,18 & 10.0 \\
\hline $3.31 \mathrm{~A}$ & 16 & 0.1 \\
\hline $3.31 \mathrm{~A}$ & 17 & 5.0 \\
\hline $3.31 \mathrm{~B}$ & 50 & 0.0001 \\
\hline $3.31 \mathrm{~B}$ & $51,52,54,71,72$ & 10.0 \\
\hline $3.31 \mathrm{~B}$ & $\begin{array}{l}53,55,64,68,70 \\
73,74\end{array}$ & 5.0 \\
\hline $3.31 \mathrm{~B}$ & $\begin{array}{ll}56, & 58,59,61,62, \\
65, & 66,69\end{array}$ & 1.0 \\
\hline $3.31 \mathrm{~B}$ & 57,60 & 30.0 \\
\hline $3.31 \mathrm{~B}$ & 63,67 & 0.1 \\
\hline $3.31 \mathrm{C}$ & 10,30 & 0.1 \\
\hline $3.31 \mathrm{C}$ & $\begin{array}{l}20,40,60,70 \\
100,110\end{array}$ & 5.0 \\
\hline $3.31 \mathrm{C}$ & $50,80,90,120$ & 2.0 \\
\hline $3.31 \mathrm{C}$ & $130,140,150$ & 90.0 \\
\hline $3.31 \mathrm{C}$ & $160,170,180,190$ & 30.0 \\
\hline
\end{tabular}


TABLE 3.16

COMPONENT FAILURE COMBINATIONS LEADING TO POWER SYSTEM FAILURE*

\begin{tabular}{|c|c|}
\hline $\begin{array}{c}\text { Component Combinations } \\
\text { (Component I. D. Numbers Refer } \\
\text { to Figure 3.31 C) }\end{array}$ & $\begin{array}{c}\text { Percentage Contribution to Power System } \\
\text { Failure Probability }\end{array}$ \\
\hline & \\
$60+90+130$ (Third Order) & 99.7 \\
$20+40+90+130$ (Worst Fourth Order) & 0.15 \\
All Others & 0.15 \\
\hline
\end{tabular}

*Power System Failure Probability $=0.27 \times 10^{-9}$. 
TABLE $3.17 \mathrm{~A}$

COMPONENT CONTRIBUTIONS TO SYSTEM UNRELIABILITY

System: 3-Out-of-4 Air Recirculation Units Plus

Containment Spray System in Standby

(ARMM Calculation)

\begin{tabular}{|c|c|c|}
\hline $\begin{array}{l}\text { Figure } \\
\text { Number }\end{array}$ & $\begin{array}{l}\text { Component } \\
\text { I. D. No. }\end{array}$ & $\begin{array}{c}\text { Contribution to System Failure } \\
\text { Probability (Percent) }\end{array}$ \\
\hline $3.31 \mathrm{~A}$ & $\begin{array}{l}16 \text { (4 identical } \\
\text { components) }\end{array}$ & .008 \\
\hline $3.31 \mathrm{~A}$ & $\begin{array}{l}\text { (4 identical } \\
\text { components) }\end{array}$ & 13.4 \\
\hline $3.31 \mathrm{~A}$ & $\begin{array}{ll}18 & \text { (4 identical } \\
\text { components) }\end{array}$ & 53.3 \\
\hline $3.31 \mathrm{~B}$ & 70 & 2.5 \\
\hline $3.31 \mathrm{~B}$ & 71 & 8.0 \\
\hline $3.31 \mathrm{~B}$ & 72 & 8.0 \\
\hline $3.31 \mathrm{~B}$ & 73 & 2.5 \\
\hline $3.31 \mathrm{~B}$ & 74 & 2.5 \\
\hline \multicolumn{2}{|c|}{ Total } & 90.5 \\
\hline
\end{tabular}

*Probability of system failure $=4.11 \times 10^{-5}$.

System reliability $=0.9999589$. 
TABLE $3.17 \mathrm{~B}$

COMPONENT COMBINATIONS CONTRIBUTING

TO SYSTEM FAILURE

(HAND ESTIMATE)

\begin{tabular}{|c|c|}
\hline $\begin{array}{c}\text { Number of Components } \\
\text { Failed }\end{array}$ & $\begin{array}{c}\text { Contribution to Sys tem } \\
\text { Failure Probability (Percent) }\end{array}$ \\
\hline 3 & 90.5 \\
4 & 9.5 \\
5 or greater & Negligible \\
\hline
\end{tabular}

*Probability of system failure $=1.8 \times 10^{-5}$.

System reliability $=0.999982$. 
TABLE 3.18

COMPONENTS - MECHANICAL FEATURES

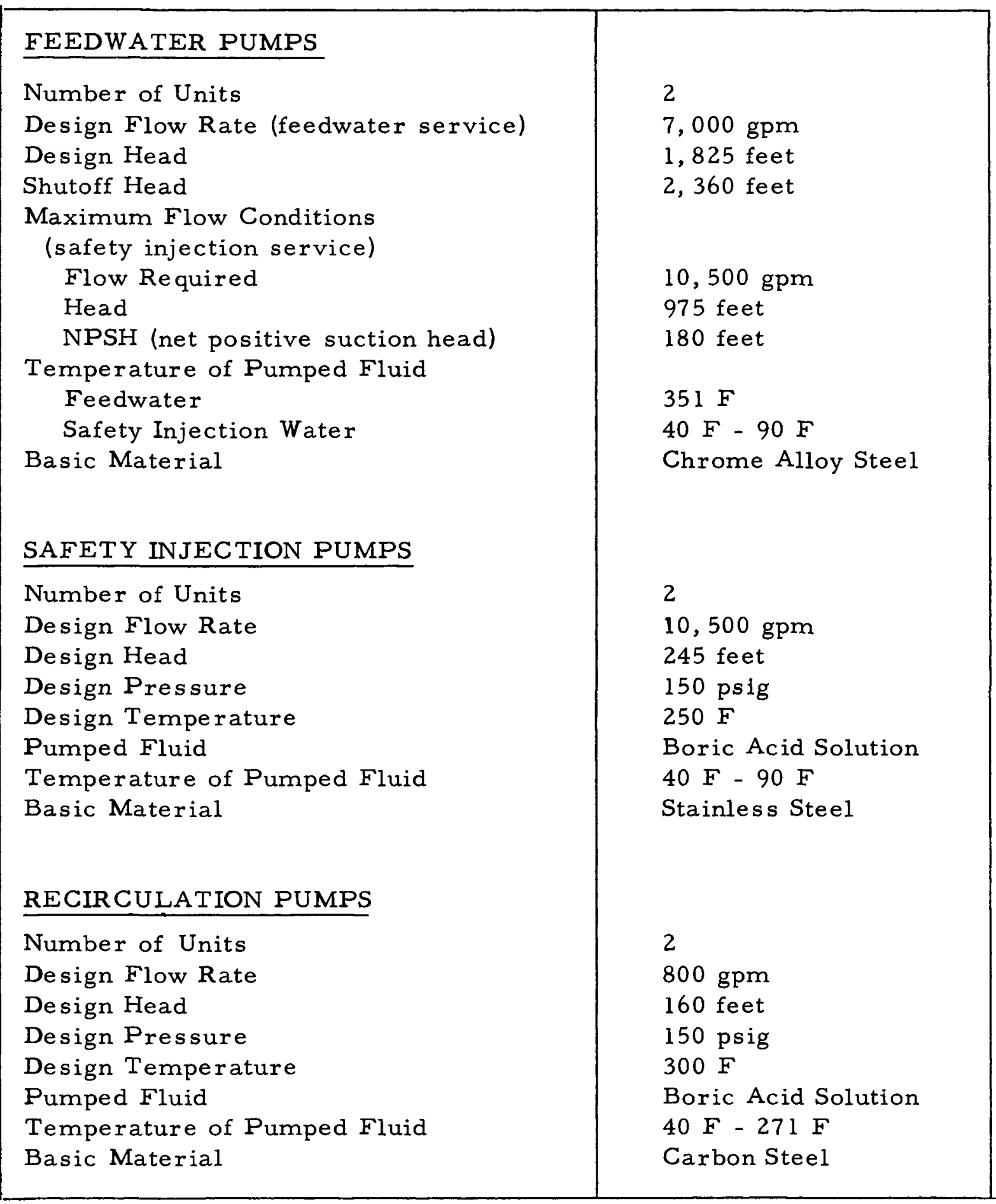


TABLE 3.18 (continued)

\begin{tabular}{|l|l|}
\hline CHARGING PUMPS & \\
Number of Units & 2 \\
Design Flow Rate & $213 \mathrm{gpm}$ \\
Design Head & $5,325 \mathrm{feet}$ \\
Design Pressure & $2,735 \mathrm{psig}$ \\
Design Temperature & $250 \mathrm{~F}$ \\
Pumped Fluid & Boric Acid Solution \\
Temperature of Pumped Fluid & \\
(normal plant operation) & $130 \mathrm{~F}$ \\
Basic Material & Stainless Steel \\
\hline
\end{tabular}

Note: The centrifugal charging pumps are part of the chemical and volume control system. The following parameters are based upon requirements of that system. 
TABLE 3.19

RECIRCULATION HEAT EXCHANGER

\begin{tabular}{|l|l|c|}
\hline \multirow{2}{*}{ Fluid } & Shell Side & Tube Side \\
\cline { 2 - 3 } & $\begin{array}{c}\text { Component } \\
\text { Cooling } \\
\text { Water }\end{array}$ & $\begin{array}{c}17 \%-2.2 \% \text { by } \\
\text { Weight Boric Acid } \\
\text { Solution }\end{array}$ \\
\hline Flow Rate & $1,000 \mathrm{gpm}$ & $1,600 \mathrm{gpm}$ \\
Temperature In & $110 \mathrm{~F}$ & $271 \mathrm{~F}$ \\
Temperature Out & $158 \mathrm{~F}$ & $241 \mathrm{~F}$ \\
Operating Pressure & $50 \mathrm{psig}$ & $100 \mathrm{psig}$ \\
Pressure Loss, & $15 \mathrm{psi}$ & $10 \mathrm{psi}$ \\
Maximum & $150 \mathrm{psig}$ & $150 \mathrm{psig}$ \\
Design Pressure & $200 \mathrm{~F}$ & $300 \mathrm{~F}$ \\
Design Temperature & Approximately & $23.8 \times 10^{6} \mathrm{Btu} / \mathrm{hour}$ \\
Heat Exchanged & \multicolumn{2}{|c|}{} \\
\hline
\end{tabular}


TABLE 3.20

\section{COMPONENTS - ELECTRICAL PARAMETERS}

\begin{tabular}{|l|c|c|}
\hline \multicolumn{1}{|c|}{ Pump Motor } & Horsepower & Voltage \\
\hline Feedwater Pump & 3500 & 4160 \\
Safety Injection Pump & 700 & 4160 \\
Recirculation Pump & 60 & 480 \\
Charging Pump & 600 & 4160 \\
Refueling Water Pump & 150 & 480 \\
\hline
\end{tabular}


TABLE 3.21

INTERVAL COMPONENT CONTRIBUTES TO SYSTEM UNRELIABILITY

\begin{tabular}{|c|c|c|c|c|}
\hline \multirow{2}{*}{$\begin{array}{c}\text { ARMM } \\
\text { ID } \\
\end{array}$} & \multirow{2}{*}{ COMPONENT } & \multicolumn{3}{|c|}{ INTER VAL } \\
\hline & & 1 & 2 & 3 \\
\hline 10 & Refueling Water Tank & $\mathrm{X}$ & $\mathrm{X}$ & \\
\hline 20 & Safety Injection Header & $\mathrm{X}$ & $\mathrm{X}$ & \\
\hline 30 & MOV $850 \mathrm{~A}$ & $\mathrm{X}$ & & \\
\hline 40 & MOV $850 \mathrm{~B}$ & $\mathrm{X}$ & & \\
\hline 50 & MOV $850 \mathrm{C}$ & $\mathrm{X}$ & & \\
\hline 60 & RMS for MOV 883 & & $\mathrm{X}$ & \\
\hline 70 & MOV 883 & & $\mathrm{X}$ & \\
\hline 80 & Recirculation Header & $\mathrm{X}$ & $\mathrm{X}$ & $\mathrm{X}$ \\
\hline 90 & Containment Sump & $\mathrm{X}$ & $\mathrm{X}$ & $\mathrm{X}$ \\
\hline 100 & Recirculation Heat Exchanger & & & $\mathrm{X}$ \\
\hline 110 & FCV $1115 \mathrm{D}$ & $\mathrm{X}$ & & \\
\hline 120 & MOV 356 & $\mathrm{X}$ & & \\
\hline 130 & FCV $1115 \mathrm{E}$ & $\mathrm{X}$ & & \\
\hline 140 & MOV 357 & $\mathrm{X}$ & & \\
\hline 150 & FCV $1115 \mathrm{~F}$ & $\mathrm{X}$ & & \\
\hline 160 & MOV 358 & $\mathrm{x}$ & & \\
\hline 250 & Main Transformer & $\mathrm{X}$ & $\mathrm{X}$ & $\mathrm{X}$ \\
\hline 260 & Auxiliary Transformer C & $\mathrm{X}$ & $\mathrm{X}$ & $\mathrm{X}$ \\
\hline 270 & Circuit Breaker $12 \mathrm{CO} 2$ & $\mathrm{X}$ & $\mathrm{X}$ & $\mathrm{X}$ \\
\hline 280 & Circuit Breaker $11 \mathrm{CO} 2$ & $\mathrm{X}$ & $\mathrm{X}$ & $\mathrm{X}$ \\
\hline 290 & Tie Breaker $11 \mathrm{C} 11$ & $\mathrm{x}$ & $\mathrm{X}$ & $\mathrm{X}$ \\
\hline 300 & Tie Breaker $12 \mathrm{C} 11$ & $\mathrm{X}$ & $\mathrm{X}$ & $\mathrm{X}$ \\
\hline 310 & Auxiliary Transformer B & $\mathrm{x}$ & $\mathrm{X}$ & $\mathrm{X}$ \\
\hline 320 & Auxiliary Transformer A & $\mathrm{x}$ & $\mathrm{X}$ & $\mathrm{X}$ \\
\hline 330 & $\mathrm{CB} 11 \mathrm{~B} 04$ & $\mathrm{X}$ & $\mathrm{X}$ & $\mathrm{X}$ \\
\hline 340 & TB $12 \mathrm{C} 01$ & $\mathrm{X}$ & $\mathbf{x}$ & $\mathrm{X}$ \\
\hline 350 & $\mathrm{CB} 11 \mathrm{A04}$ & $\mathrm{x}$ & $\mathrm{X}$ & $\mathrm{X}$ \\
\hline 360 & $\mathrm{~TB} 11 \mathrm{CO} 1$ & $\mathrm{X}$ & $\mathrm{X}$ & $\mathrm{X}$ \\
\hline 370 & Safety Injection Pump G50A (Start) & $\mathrm{X}$ & & \\
\hline 380 & Safety Injection Pump G50A (Operate) & & $\mathrm{X}$ & \\
\hline 390 & MOV 853 A & $\mathrm{X}$ & & \\
\hline
\end{tabular}


TABLE 3.21 (continued)

INTER VAL COMPONENT CONTRIBUTES TO SYSTEM UNRELIABILITY

\begin{tabular}{|c|c|c|c|c|}
\hline \multirow{2}{*}{$\begin{array}{c}\text { ARMM } \\
\text { ID }\end{array}$} & \multirow{2}{*}{ COMPONENT } & \multicolumn{3}{|c|}{ INTERVAL } \\
\hline & & 1 & 2 & 3 \\
\hline 400 & Feedwater Pump G-3A & $\mathrm{X}$ & $\mathrm{X}$ & \\
\hline 410 & MOV 854 A & $\mathrm{X}$ & & \\
\hline 420 & MOV $852 \mathrm{~A}$ & $\mathrm{X}$ & & \\
\hline 430 & MOV $851 \mathrm{~A}$ & $\mathrm{X}$ & & \\
\hline 440 & Interlock $A$ & $\mathrm{x}$ & & \\
\hline 450 & CV 36 & $\mathrm{X}$ & & \\
\hline 460 & $\mathrm{CV} 875 \mathrm{~A}$ & $\mathrm{X}$ & & \\
\hline 470 & Safety Injection Pump G 50B (Start) & $\mathrm{X}$ & & \\
\hline 480 & Safety Injection Pump G 50B (Operate) & & $\mathrm{X}$ & \\
\hline 490 & MOV 853 B & $\mathrm{X}$ & & \\
\hline 500 & Feed Water Pump G-3B & $\mathrm{X}$ & $\mathrm{X}$ & \\
\hline 510 & MOV 854 B & $\mathrm{X}$ & & \\
\hline 520 & MOV 852 B & $\mathrm{X}$ & & \\
\hline 530 & MOV 851 B & $\mathrm{X}$ & & \\
\hline 540 & Interlock B & $\mathrm{X}$ & & \\
\hline 550 & $\mathrm{CV}-37$ & $\mathrm{X}$ & & \\
\hline 560 & $\mathrm{CV}-875 \mathrm{~B}$ & $\mathrm{X}$ & & \\
\hline 570 & Station Service Transformer \#3 & $\mathrm{X}$ & $\mathrm{X}$ & $\mathrm{X}$ \\
\hline 580 & Station Service Transformer \#2 & $\mathrm{X}$ & $\mathrm{X}$ & $\mathrm{X}$ \\
\hline 590 & Station Service Transformer \# 1 & $\mathrm{X}$ & $\mathrm{X}$ & $\mathrm{X}$ \\
\hline 600 & $\mathrm{CB}-11 \mathrm{C} 10$ & $\mathrm{X}$ & $\mathrm{X}$ & $\mathrm{X}$ \\
\hline 610 & $\mathrm{CB}-12 \mathrm{C} 10$ & $\mathrm{X}$ & $\mathrm{X}$ & $\mathrm{X}$ \\
\hline 620 & CB-1 102 & $\mathrm{X}$ & $\mathrm{x}$ & $\mathrm{x}$ \\
\hline 630 & CB-1 202 & $\mathrm{X}$ & $\mathrm{X}$ & $\mathrm{X}$ \\
\hline 640 & CB-1 303 & $\mathrm{X}$ & $\mathrm{X}$ & $\mathrm{X}$ \\
\hline 650 & TB-1 103 & $\mathrm{X}$ & $\mathrm{X}$ & $\mathrm{X}$ \\
\hline 660 & TB-1203 & $\mathrm{X}$ & $\mathrm{X}$ & $\mathrm{X}$ \\
\hline 670 & Manual Start Recirculation Pumps & & $\mathrm{X}$ & \\
\hline 680 & Recirculation Pump G-45A (Start) & & $\mathrm{X}$ & \\
\hline 690 & Recirculation Pump G-45B (Start) & & $\mathrm{X}$ & \\
\hline
\end{tabular}


TABLE 3.21 (continued)

INTER VAL COMPONENT CONTRIBUTES TO SYSTEM UNRELIABILITY

\begin{tabular}{|c|c|c|c|c|}
\hline \multirow{2}{*}{$\begin{array}{c}\text { ARMM } \\
\text { ID }\end{array}$} & \multirow{2}{*}{ COMPONENT } & \multicolumn{3}{|c|}{ INVER VAL } \\
\hline & & 1 & 2 & 3 \\
\hline 700 & Recirculation Pump G-45A (Operate) & & & $\mathrm{X}$ \\
\hline 710 & Recirculation Pump G-45B (Operate) & & & $\mathrm{X}$ \\
\hline 720 & MOV 866 A & & $\mathrm{X}$ & \\
\hline 730 & MOV 866 B & & $\mathrm{X}$ & \\
\hline 740 & Refueling Water Pump G 27 (Start) & $\mathrm{X}$ & & \\
\hline 750 & Refueling Water Pump G 27 (Operate) & & $\mathrm{X}$ & $\mathrm{X}$ \\
\hline 760 & Refueling Water Pump G 27 S (Start) & $\mathrm{X}$ & & \\
\hline 770 & Refueling Water Pump G 27 S (Operate) & & $\mathrm{X}$ & $\mathrm{X}$ \\
\hline 780 & $\mathrm{CV} 81$ & $\mathrm{X}$ & & \\
\hline 790 & CV 112 & $\mathrm{X}$ & & \\
\hline 800 & MOV 880 & $\mathrm{X}$ & & \\
\hline 810 & MOV $1100 \mathrm{~B}$ & $\mathrm{X}$ & & \\
\hline 820 & MOV $1100 \mathrm{D}$ & $\mathrm{X}$ & & \\
\hline 830 & Charge Pump G 8A (Operate) & $\mathrm{X}$ & $\mathrm{X}$ & $\mathrm{X}$ \\
\hline 840 & Charge Pump G 8B (Start) & $\mathrm{X}$ & & \\
\hline 849 & Charge Pump G 8B (Operate) & & $\mathrm{X}$ & $\mathrm{X}$ \\
\hline 850 & Level Sensor \#1 & $\mathrm{X}$ & & \\
\hline 851 & Level Sensor \#2 & $\mathrm{X}$ & & \\
\hline 852 & Level Sensor \#3 & $\mathrm{X}$ & & \\
\hline 853 & Pressure Sensor \# 1 & $\mathrm{X}$ & & \\
\hline 854 & Pressure Sensor \#2 & $\mathrm{X}$ & & \\
\hline 855 & Pressure Sensor \#3 & $\mathrm{X}$ & & \\
\hline 856 & Automatic Actuate of SIS & $\mathrm{X}$ & & \\
\hline 857 & Manual Actuate of SIS & $\mathrm{X}$ & & \\
\hline & Total Number of Components Considered & 70 & 42 & 31 \\
\hline
\end{tabular}


TABLE 3.22

FAILURE RA TE DATA USED

FOR SAN ONOFRE SAFETY INJECTION SYSTEM

\begin{tabular}{|c|c|}
\hline COMPONENT & $\begin{array}{c}\text { FAILURE RATE } \\
\text { PER MILLION HOURS }\end{array}$ \\
\hline Pumps & \\
\hline Start & 10 \\
\hline Operate & 5 \\
\hline Valves & \\
\hline Open & 0.01 \\
\hline Close & 0.01 \\
\hline Auto Trip Devices & 1.0 \\
\hline Manual Trip Devices & 0.1 \\
\hline Level Sensors & 15 \\
\hline Pressure Sensors & 15 \\
\hline Tank, Sump & 0.0001 \\
\hline Headers & 0.0002 \\
\hline Interlock & 0.01 \\
\hline Transformers & 5 \\
\hline Circuit Breakers & 5 \\
\hline Tie Breakers & 5 \\
\hline Heat Exchanger & 0.1 \\
\hline
\end{tabular}


MAJOR CONTRIBUTORS TO UNRELIABILITY OF SAN ONOFRE SAFETY INJECTION SYSTEM DURING THREE TIME INTER VALS

\section{INTERVAL 1 (720 HOURS)}

Probability of System Success Prior to this Interval: 1.0

Probability of System Failure During this Interval: $1.279 \times 10^{-4}$

\begin{tabular}{|l|c|c|}
\hline \multicolumn{1}{|c|}{ MAJOR CONTRIBUTOR } & SYSTEM PROBABILITY & PERCENT \\
\hline S. I. Pump G50A (Start) & $4.176 \times 10^{-5}$ & 32.0 \\
S. I. Pump G50B (Start) & $4.176 \times 10^{-5}$ & 32.0 \\
Feedwater Pump G-3A & $1.468 \times 10^{-5}$ & 11.5 \\
Feedwater Pump G-3B & $1.468 \times 10^{-5}$ & 11.5 \\
Auxiliary Transformer C & $6.986 \times 10^{-6}$ & 5.5 \\
Main Transformer & $6.239 \times 10^{-6}$ & 4.9 \\
& & \\
\hline
\end{tabular}

\section{INTERVAL 2 (10 MINUTES)}

Probability of System Success Prior to this Interval: 0.99987210 Probability of System Failure During this Interval: $\quad 3.518 \times 10^{-8}$

\begin{tabular}{|l|c|c|}
\hline \multicolumn{1}{|c|}{ MAJOR CONTRIBUTOR } & SYSTEM PROBABILITY & PERCENT \\
\hline RMS for MOV 883 & $1.670 \times 10^{-8}$ & 47.5 \\
Manual Start Recirc. Pumps & $1.670 \times 10^{-8}$ & 47.5 \\
Refuel Tank Valve MOV 883 & $1.670 \times 10^{-9}$ & 4.7 \\
\hline
\end{tabular}


TABLE 3.23 (continued)

MAJOR CONTRIBUTORS TO UNRELIABILITY OF SAN ONOFRE SAFETY INJECTION SYSTEM DURING THREE TIME INTERVALS

\section{INTER VAL 3 (24 HOURS)}

Probability of System Success Prior to this Interval: 0.99987206

Probability of System Failure During this Interval: $2.436 \times 10^{-6}$

\begin{tabular}{|l|c|c|}
\hline \multicolumn{1}{|c|}{ MAJOR CONTRIBUTOR } & SYSTEM PROBABILITY & PERCENT \\
\hline Recirculation Ht. Exch. & $2.400 \times 10^{-6}$ & 98.5 \\
Auxiliary Transformer C & $7.226 \times 10^{-9}$ & 0.3 \\
\hline
\end{tabular}

\section{SUMMARY}

Probability of System Success: 0.99986962

\begin{tabular}{|l|c|c|}
\hline \multicolumn{1}{|c|}{ MAJOR CONTRIBUTOR } & SYSTEM PROBABILITY & PERCENT \\
\hline S. I. Pump G50A (Start) & $4.176 \times 10^{-5}$ & 32.0 \\
S. I. Pump G50B (Start) & $4.176 \times 10^{-5}$ & 32.0 \\
Feedwater Pump G-3A & $1.468 \times 10^{-5}$ & 11.3 \\
Feedwater Pump G-3B & $1.468 \times 10^{-5}$ & 11.3 \\
Auxiliary Transformer C & $6.993 \times 10^{-6}$ & 5.4 \\
Main Transformer & $6.247 \times 10^{-6}$ & 4.8 \\
Recirculation Ht. Exch. & $2.400 \times 10^{-6}$ & 1.8 \\
\hline
\end{tabular}




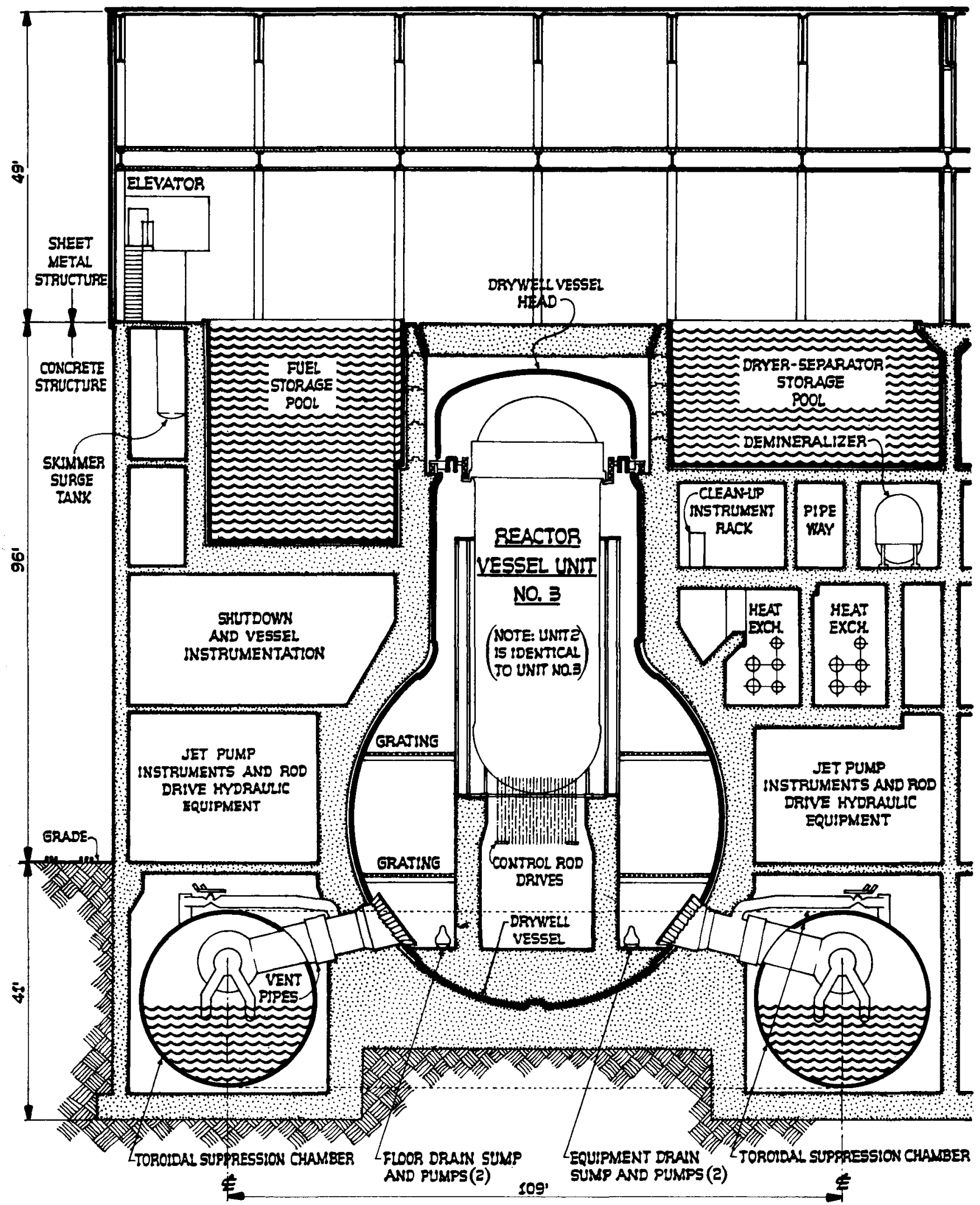

FIGURE 3.1

SCHEMATIC CROSS-SECTION OF DRESDEN - 3 CONTAINMENT 


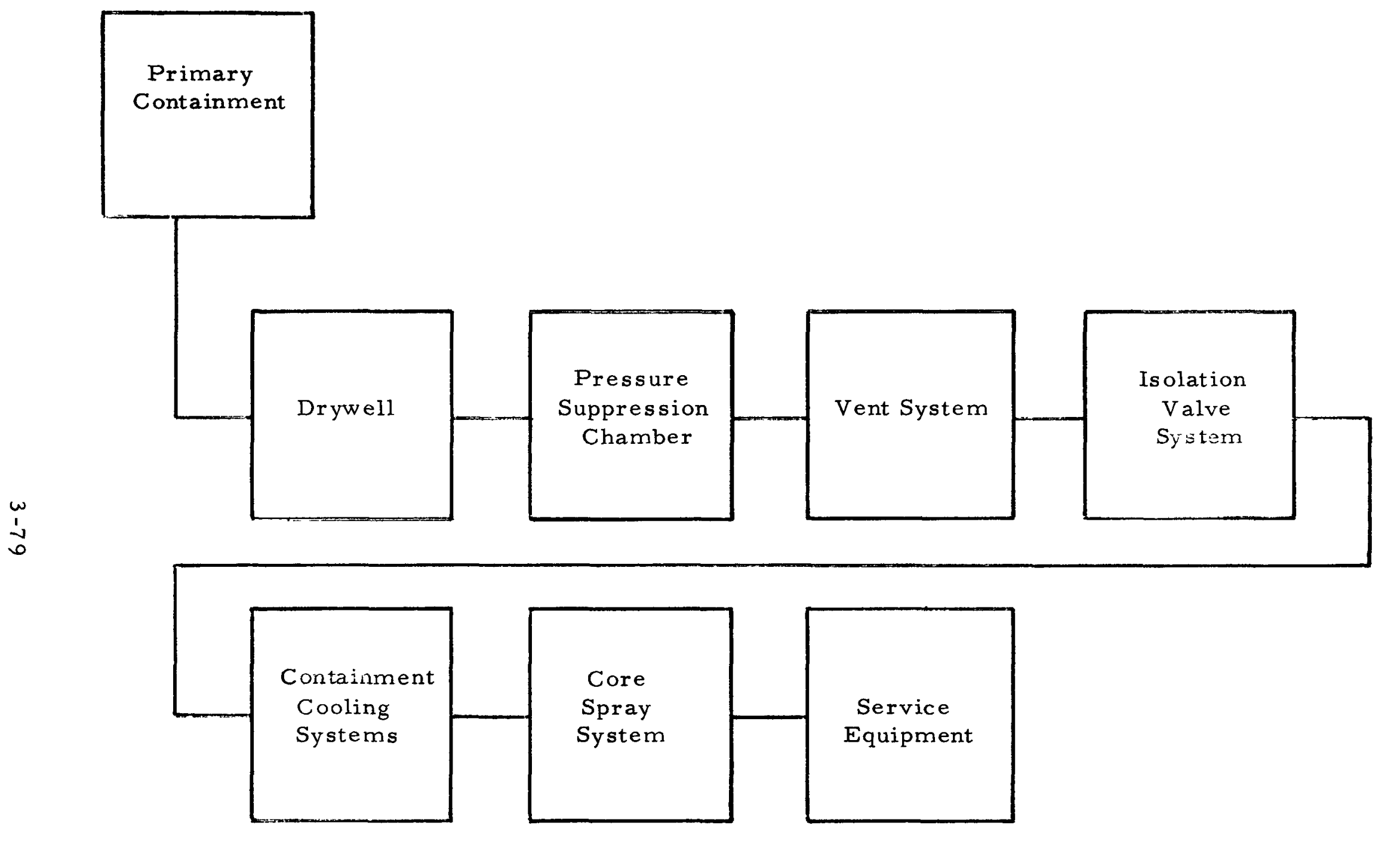

FIGURE 3.2

FUNCTIONAL DIAGRAM - PRIMARY CONTAINMENT 


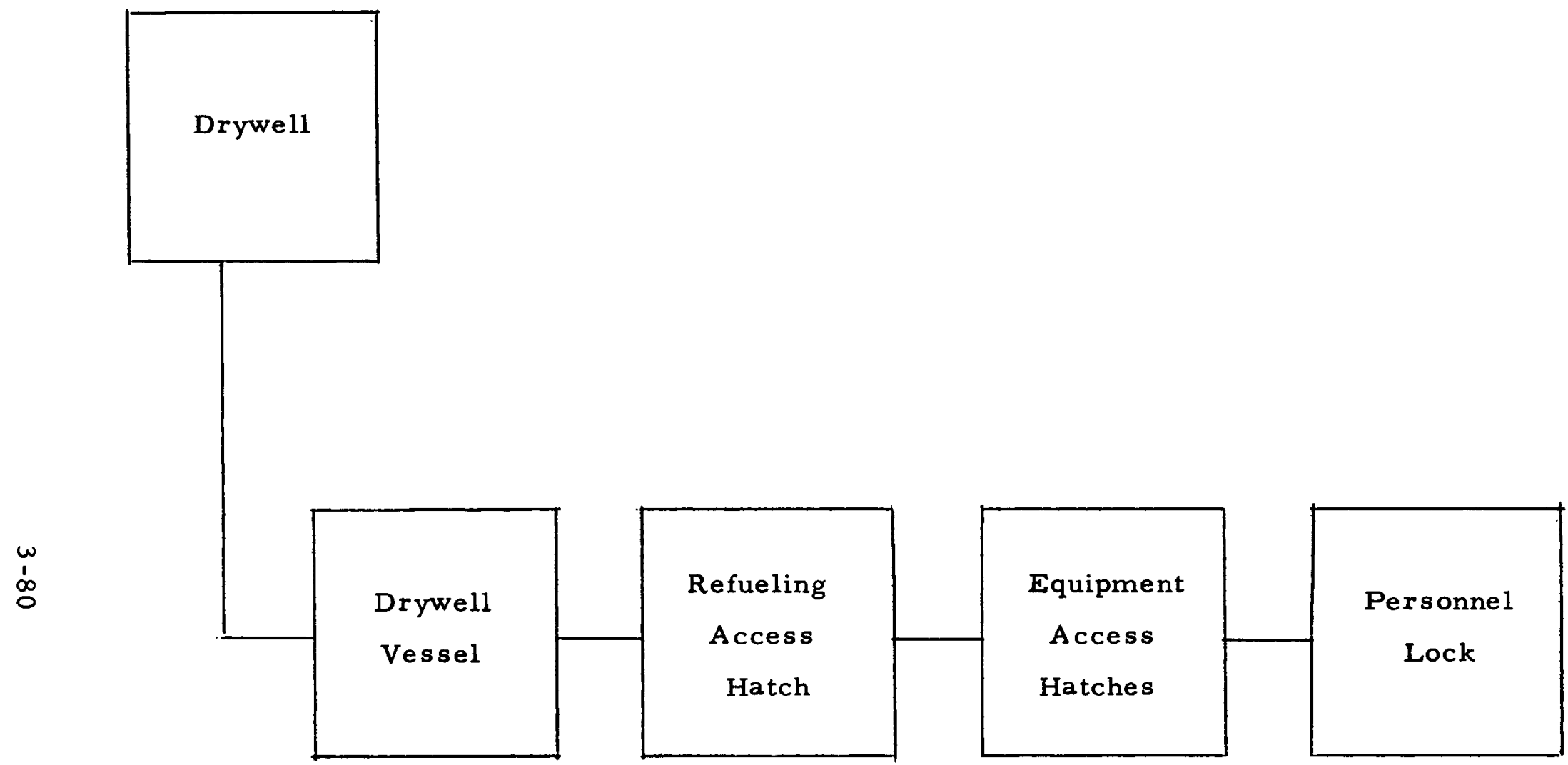

FIGURE 3.3

FUNCTIONA L DIAGRAM - DRYWELL ASSEMBLY 


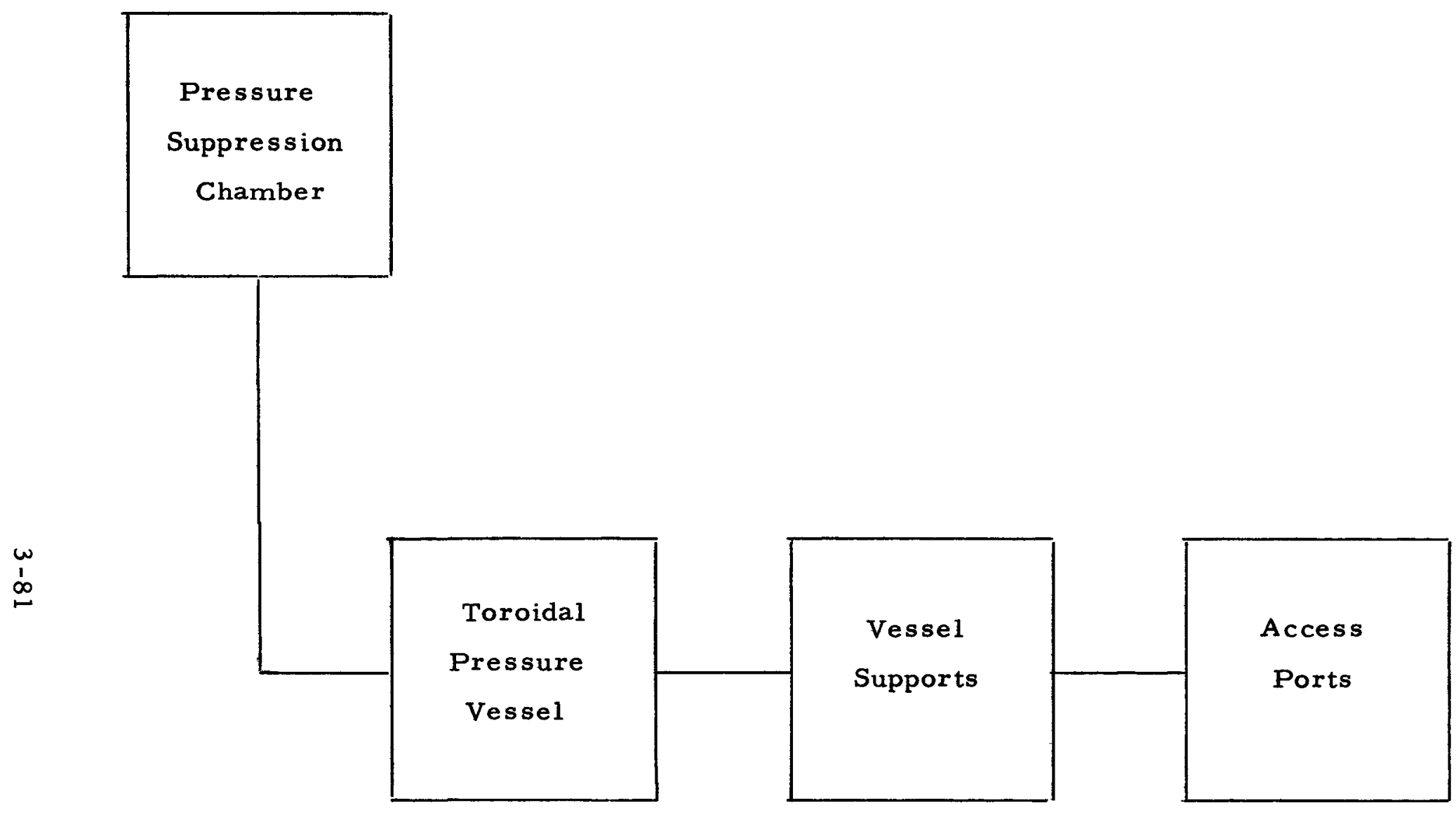

FIGURE 3.4

FUNCTIONAL DIAGRAM - PRESSURE SUPPRESSION CHAMBER 


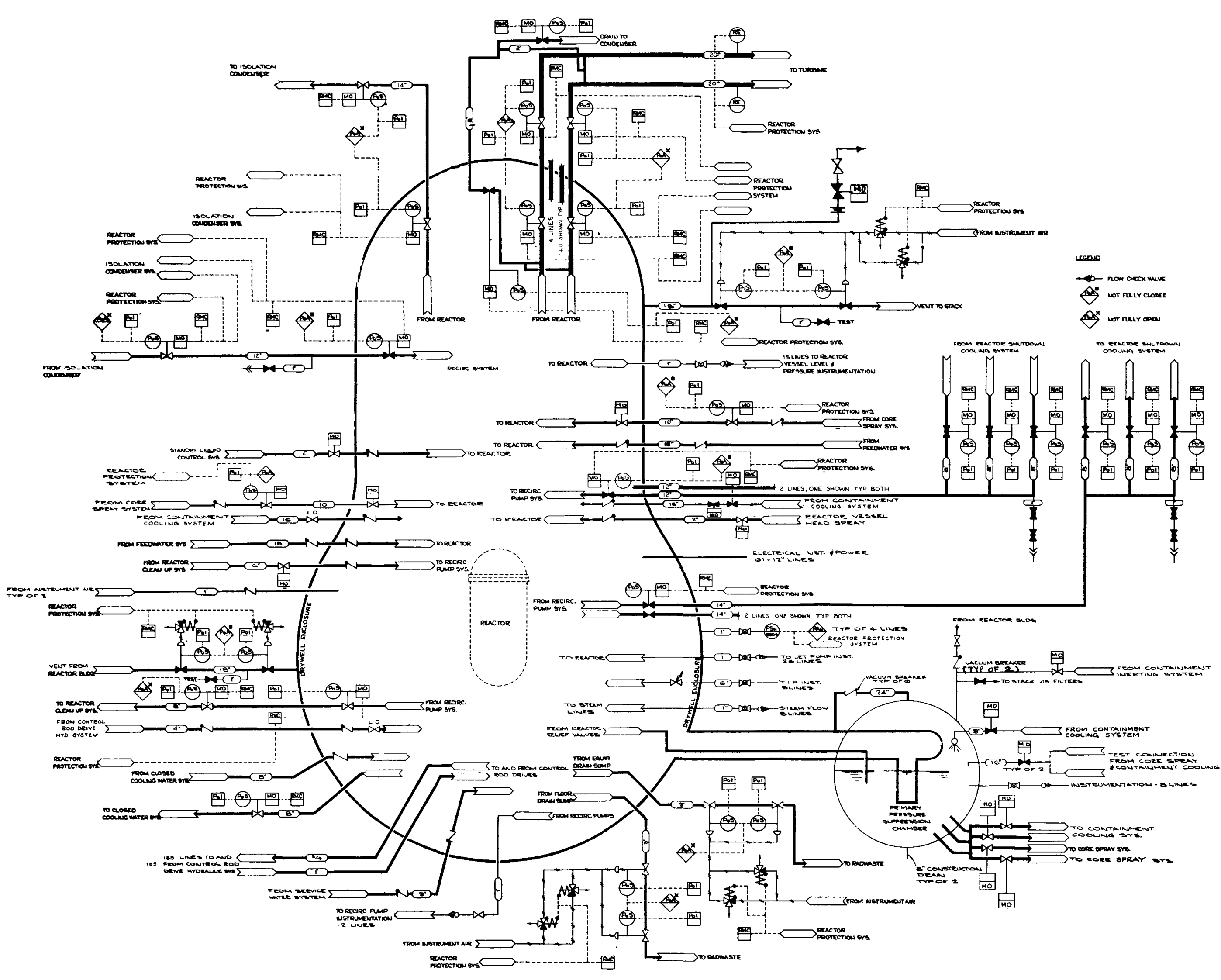

FIGURE 3.5

Taken from "Dresden Nuclear Power Station Unit 3 Plant

DRESDEN-3 PRIMARY

Design and Analysis Report" dated February 1966.

CONT AINMENT PENETRATIONS 


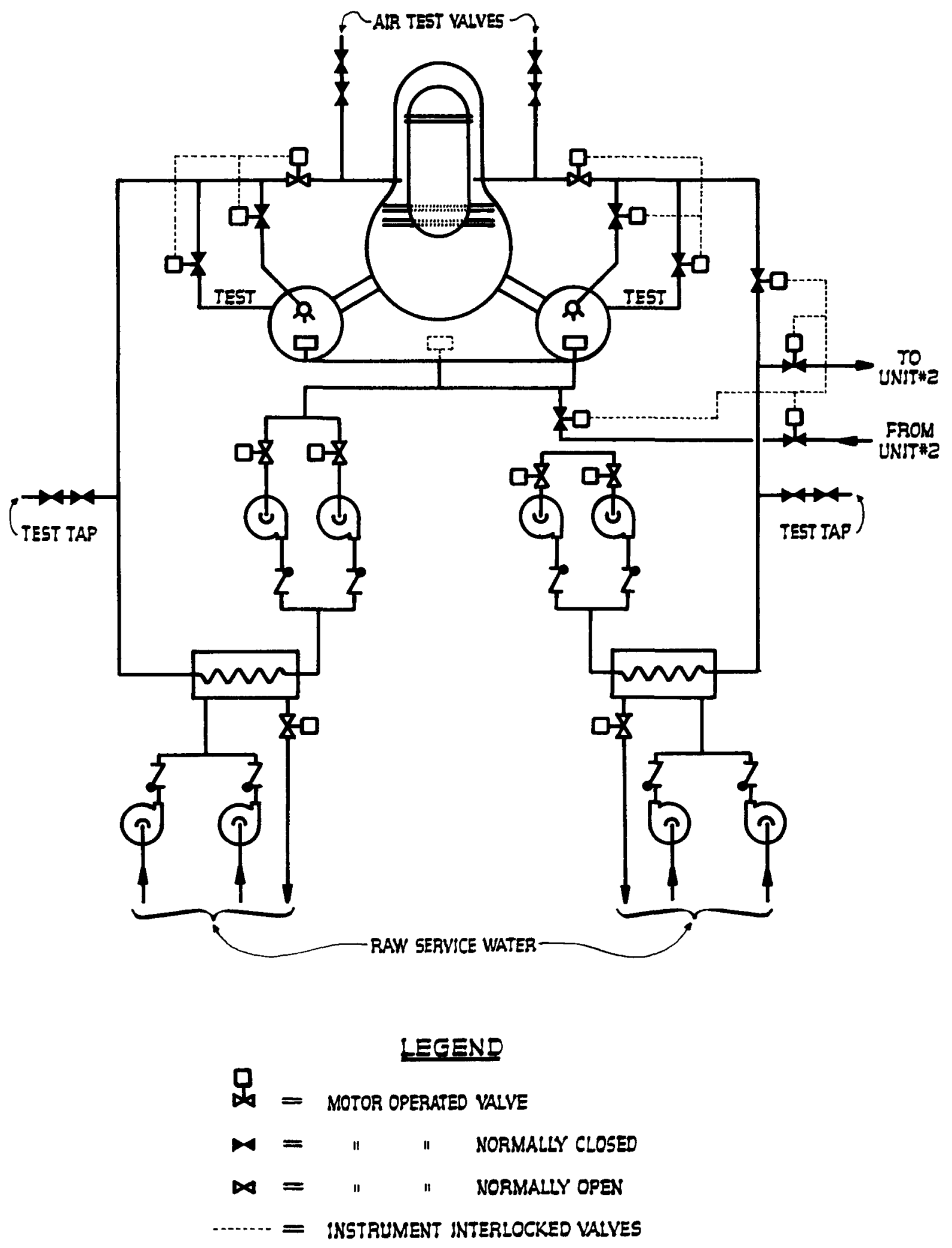

FIGURE 3.6

CONT AINMENT SPRAY COOLING SYSTEM 


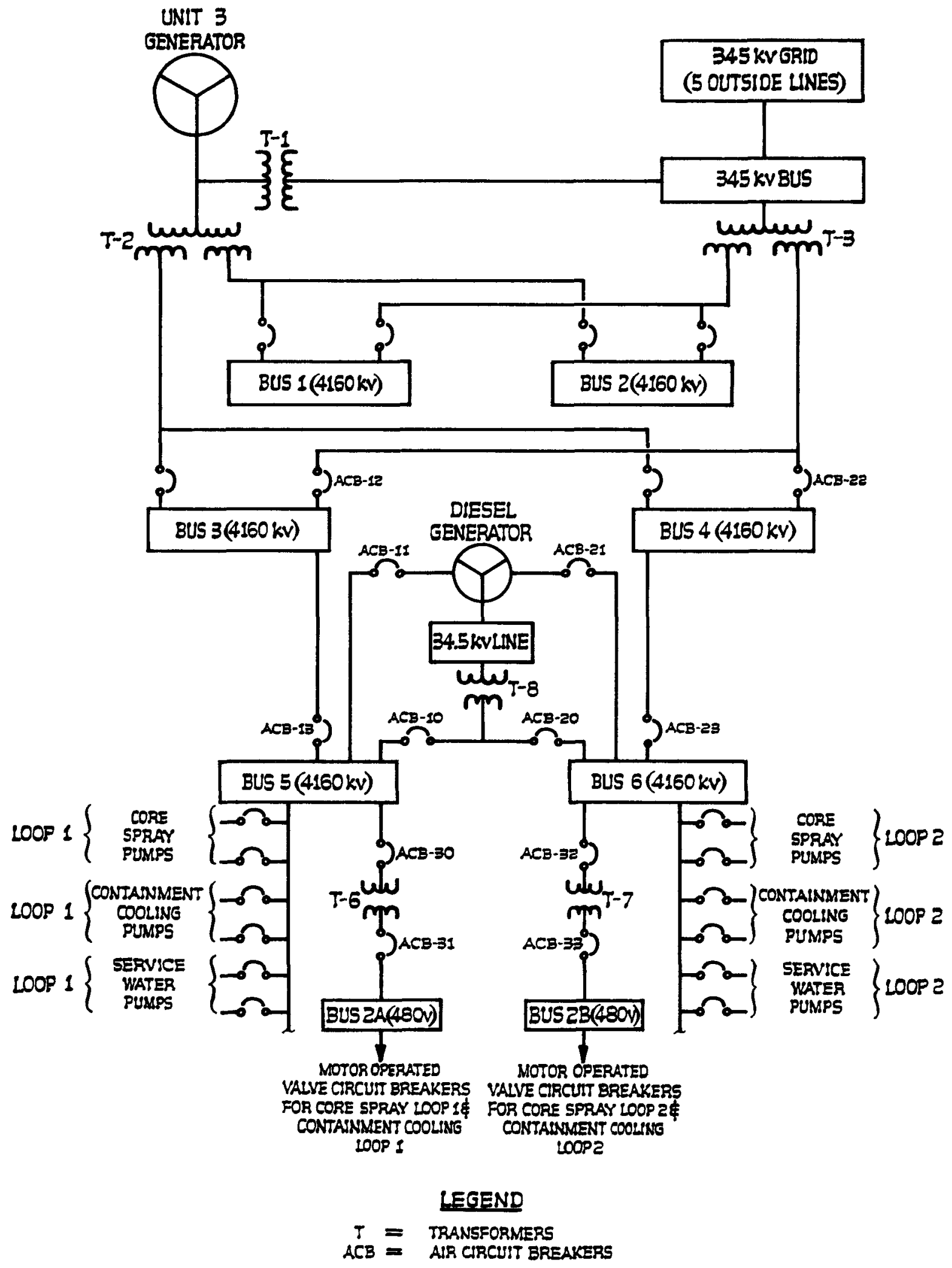

FIGURE 3.7 


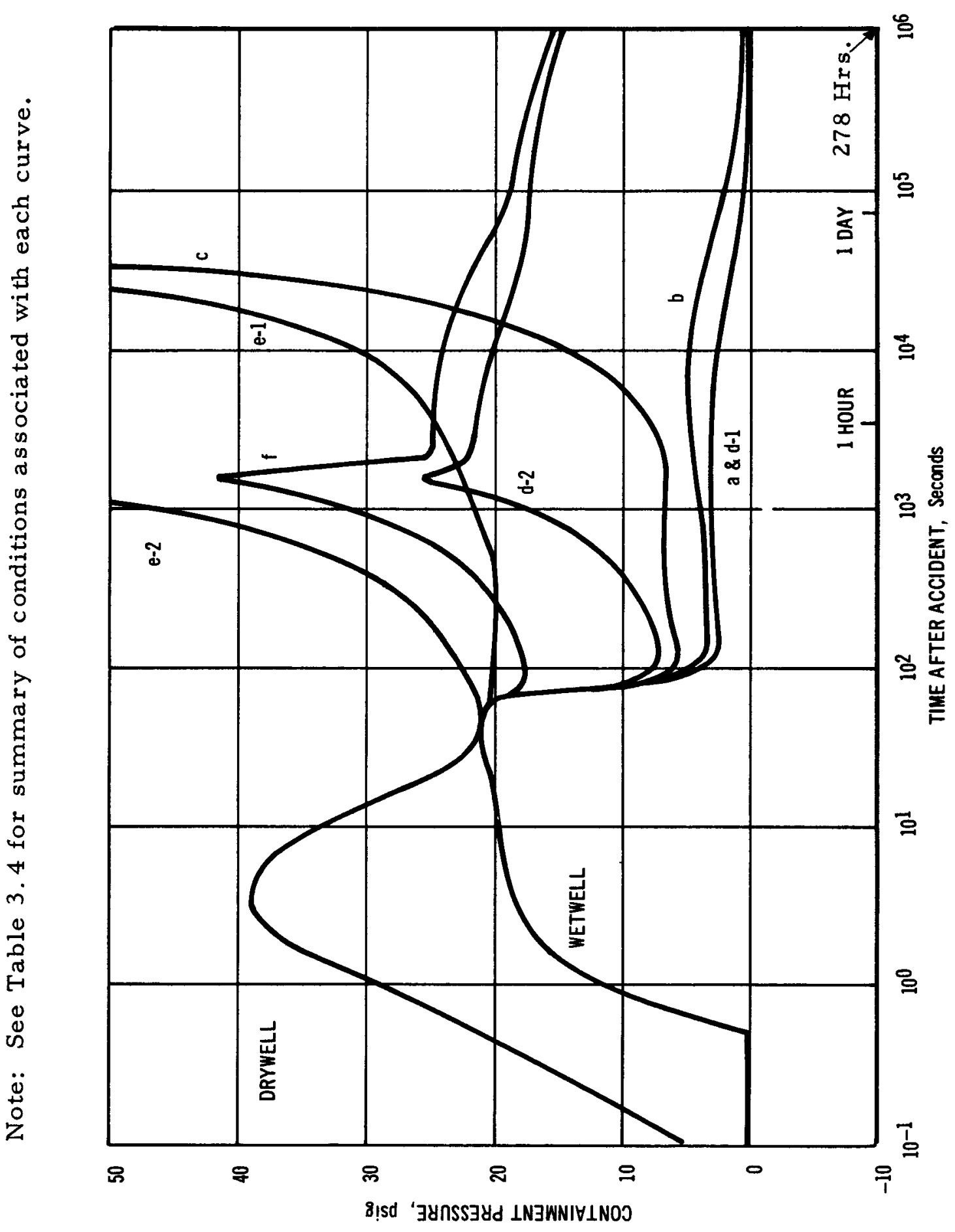

FIGURE 3.8

CONTAINMENT PRESSURE FOR VARIOUS AVAILABLE ENGINEERED SAFEGUARDS 


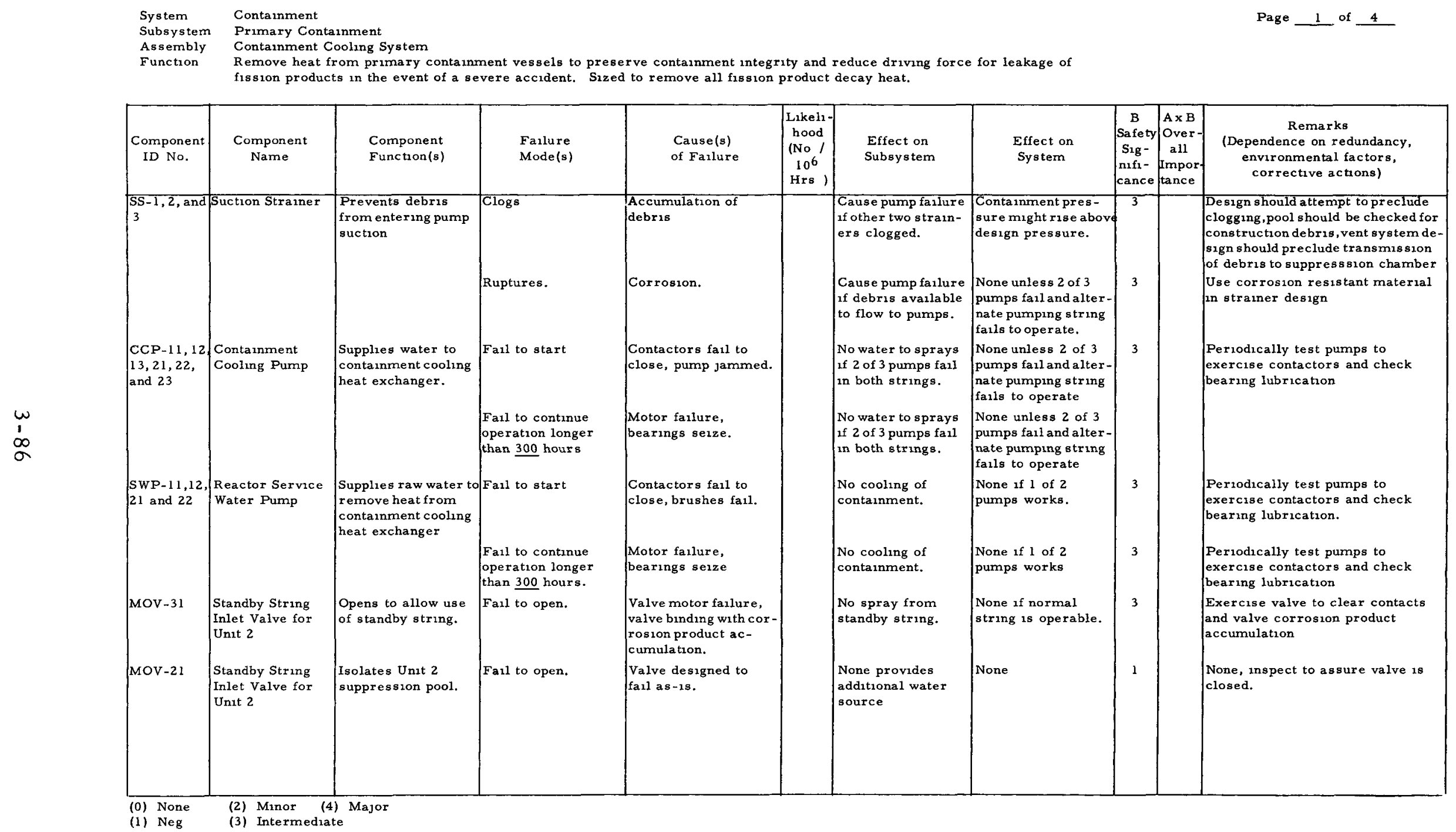

FIGURE 3.9

EXAMPLE OF FAILURE MODE AND EFFECTS ANALYSIS 


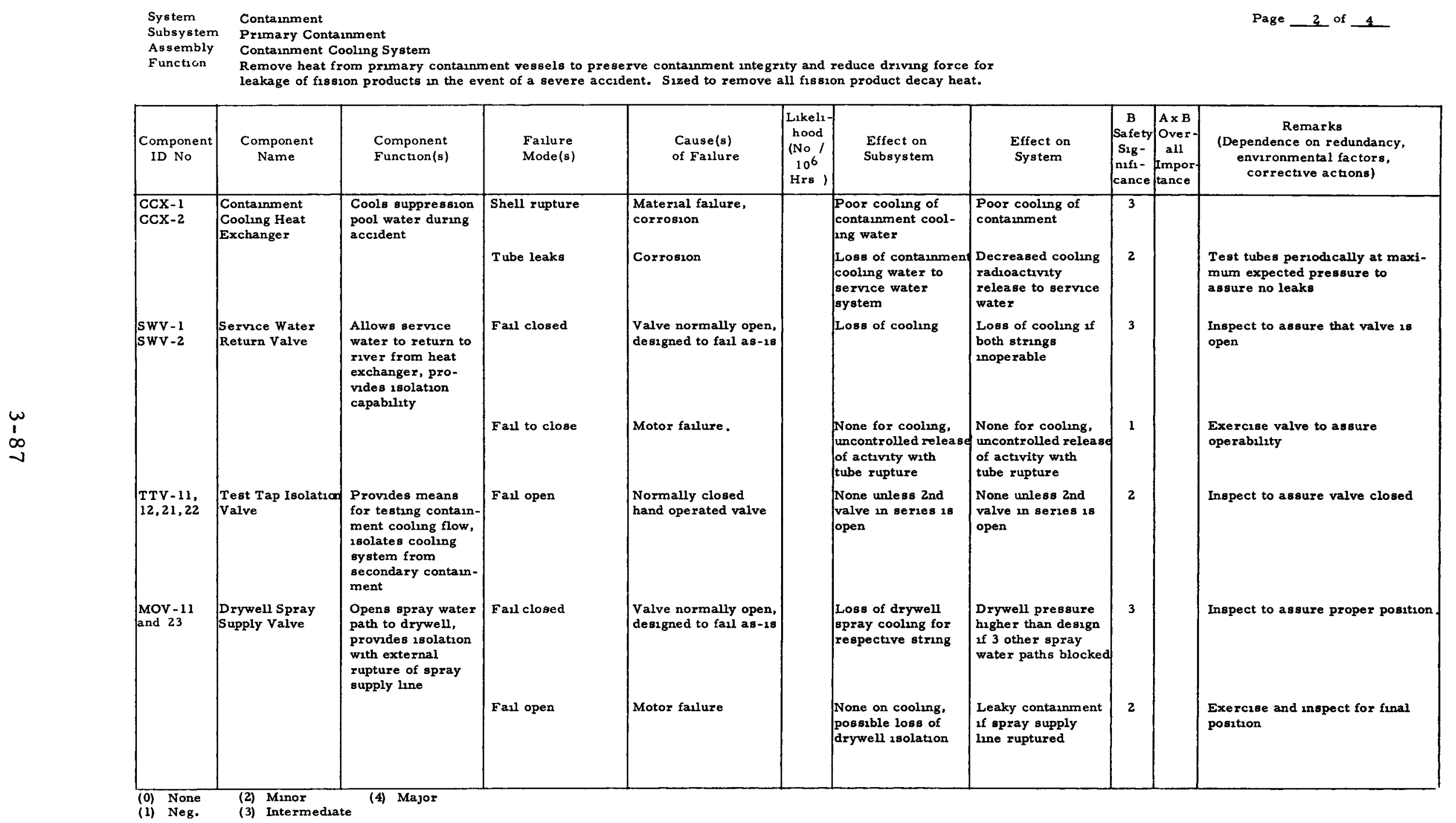

FIGURE 3.9 (continued) 
Contaunment Coolung System

\begin{tabular}{|c|c|c|c|c|c|c|c|c|c|c|}
\hline $\begin{array}{l}\text { Component } \\
\text { ID No }\end{array}$ & $\begin{array}{l}\text { Component } \\
\text { Name }\end{array}$ & $\begin{array}{l}\text { Component } \\
\text { Function (s) }\end{array}$ & $\begin{array}{l}\text { Fallure } \\
\text { Mode(s) }\end{array}$ & $\begin{array}{l}\text { Cause(s) } \\
\text { of Fallure }\end{array}$ & $\begin{array}{c}\text { Likel1 } \\
\text { hood } \\
\text { (No } 1 \\
10^{6} \\
\text { Hrs ) }\end{array}$ & $\begin{array}{l}\text { Effect on } \\
\text { Subsystem }\end{array}$ & $\begin{array}{l}\text { Effect on } \\
\text { System }\end{array}$ & $\mid \begin{array}{c}\text { B } \\
\text { Safety } \\
\text { Sig- } \\
\text { nifi- } \\
\text { cance }\end{array}$ & \begin{tabular}{|c|} 
A $\times B$ \\
Over \\
all \\
Impor \\
tance
\end{tabular} & $\begin{array}{c}\text { Remarks } \\
\text { (Dependence on redundancy } \\
\text { environmental factors, } \\
\text { corrective actions) }\end{array}$ \\
\hline MOV -32 & $\begin{array}{l}\text { Standby Core } \\
\text { Spray Unit } 3 \\
\text { Inlet Valve }\end{array}$ & $\begin{array}{l}\text { Allows standby } \\
\text { spray system } \\
\text { water to go to } \\
\text { Unit } 3\end{array}$ & $\begin{array}{l}\text { Fall to open } \\
\text { Fall to close }\end{array}$ & $\begin{array}{l}\text { Motor fallure } \\
\text { Normally closed, } \\
\text { falls as-18 }\end{array}$ & & $\begin{array}{l}\text { No containment } \\
\text { cooling by standby } \\
\text { system possible } \\
\text { None }\end{array}$ & $\begin{array}{l}\text { Overpressure if } \\
\text { normal spray } \\
\text { system does not } \\
\text { work } \\
\text { None }\end{array}$ & 1 & & $\begin{array}{l}\text { Exercise periodically, provide } \\
\text { manual backup to assure capa- } \\
\text { bulity to open }\end{array}$ \\
\hline \multirow[t]{2}{*}{ MOV-22 } & $\begin{array}{l}\text { Standby Core } \\
\text { Spray Unit } 2 \\
\text { Inlet Valve }\end{array}$ & $\begin{array}{l}\text { Prevents standby } \\
\text { spray system from } \\
\text { going to Unit } 2\end{array}$ & Fall to close & $\begin{array}{l}\text { Normally closed, } \\
\text { falls as-1s }\end{array}$ & & $\begin{array}{l}\text { Insufficient contain- } \\
\text { ment cooling by } \\
\text { standby spray } \\
\text { system }\end{array}$ & $\begin{array}{l}\text { Overpressure if } \\
\text { normal spray sys- } \\
\text { tem does not work }\end{array}$ & 3 & & Inspect to insure closure \\
\hline & & & Fall to open & Motor fallure & & None & None & 0 & & $\begin{array}{l}\text { Should be exercised periodically } \\
\text { to assure that standby system } \\
\text { is also avallable for Unit } 2\end{array}$ \\
\hline \multirow[t]{2}{*}{$\begin{array}{l}\text { MOV-12 } \\
\text { and 24 }\end{array}$} & $\begin{array}{l}\text { Suppression } \\
\text { Chamber Spray } \\
\text { Inlet Valve }\end{array}$ & $\begin{array}{l}\text { Isolates suppressan } \\
\text { chamber, provides } \\
\text { alternate spray } \\
\text { path }\end{array}$ & Fanl to close & $\begin{array}{l}\text { Normally closed, } \\
\text { falls as-18 }\end{array}$ & & $\begin{array}{l}\text { Reduces spray } \\
\text { efficiency in dry } \\
\text { well. }\end{array}$ & None & 1 & & Inspect to assure closure \\
\hline & & & Fail to open & Motor faslure & & $\begin{array}{l}\text { Prevents spray } \\
\text { cooling if drywel } \\
\text { isolated }\end{array}$ & $\begin{array}{l}\text { Containment over- } \\
\text { pressure if other } \\
\text { spray system } \\
\text { failed }\end{array}$ & 2 & & $\begin{array}{l}\text { Exercise periodically, provide } \\
\text { manual backup for valve opera- } \\
\text { tion }\end{array}$ \\
\hline \multirow[t]{2}{*}{$\begin{array}{l}\text { DWS }-1,2 \\
\text { SCS }-1,2\end{array}$} & Spray Header & $\begin{array}{l}\text { Distribute spray } \\
\text { water }\end{array}$ & Plugged & & & $\begin{array}{l}\text { No cooling if other } \\
3 \text { headers plugged }\end{array}$ & Some overpressure & 2 & & \\
\hline & & & Leaks & $\begin{array}{l}\text { Corrosion or poor } \\
\text { assembly }\end{array}$ & & Reduced efficiency & None & 0 & & $\begin{array}{l}\text { Inspect and test with water on } \\
\text { mitial installation }\end{array}$ \\
\hline $\begin{array}{l}\mathrm{TTV}-13, \\
14,23 \text { and } \\
24\end{array}$ & $\begin{array}{l}\text { Spray Header } \\
\text { Test Valve }\end{array}$ & $\begin{array}{l}\text { Permit spray } \\
\text { headex test, pre- } \\
\text { vent spraywater } \\
\text { leakage }\end{array}$ & Fall open & Left open & & Poor spray cooling & Some overpressure & 2 & & $\begin{array}{l}\text { Inspect periodically to assure } \\
\text { valve closed, provide lock on } \\
\text { valve }\end{array}$ \\
\hline & & & Fanl closed & Normally closed & & None & None & 0 & & \\
\hline
\end{tabular}
(0) None
(2) Minor

(4) Major

\section{FIGURE 3.9 (continued)}


Function See Page 1

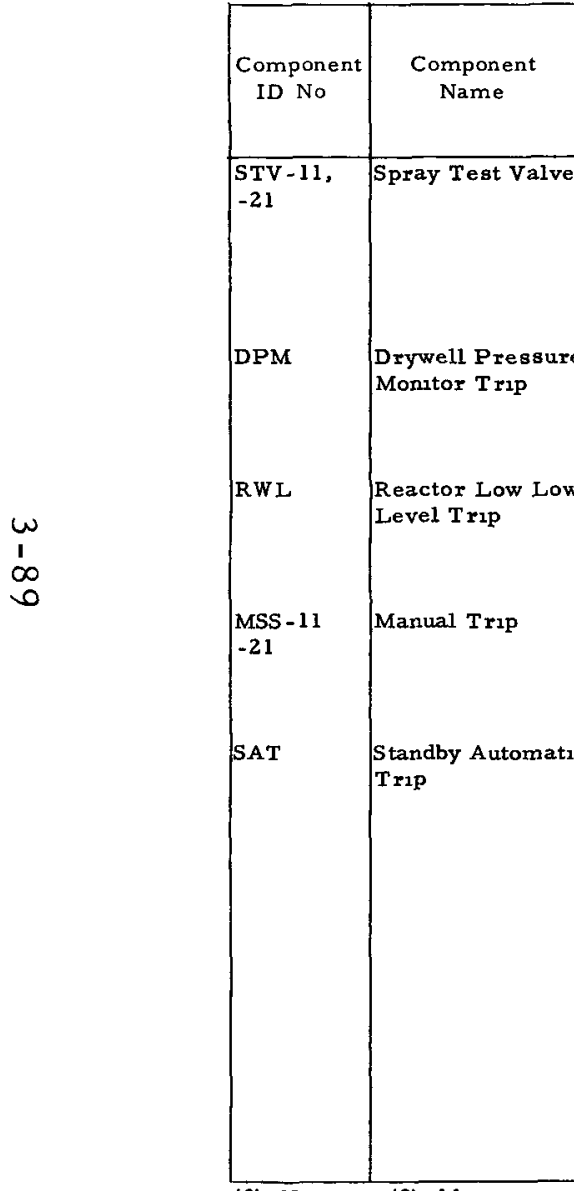

\begin{tabular}{|l|l|}
\hline $\begin{array}{c}\text { Component } \\
\text { Function(s) }\end{array}$ & \multicolumn{1}{|c|}{$\begin{array}{c}\text { Fa1lure } \\
\text { Mode(s) }\end{array}$} \\
\hline $\begin{array}{c}\text { Permit spray flow } \\
\text { test, prevent spray }\end{array}$ & Fail open \\
\hline
\end{tabular}

Cause(s)
of Fallure

\begin{tabular}{|c|c|c|} 
Likel1- & \\
hood & Effect on & Effect on \\
(No $/$ & Subsystem & System \\
$10^{6}$ & Hubstem & \\
Hrs & &
\end{tabular}

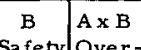

Sig ty Over-

(Dep

\section{Remarks}

Sig all (Dependence on redundancy nif1- Impor environmental factors,

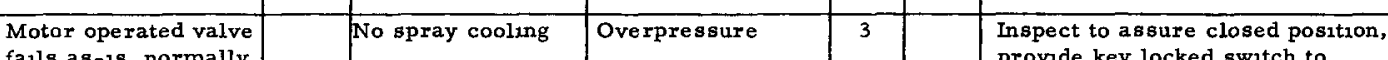

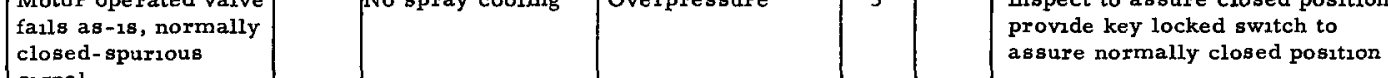

test, prevent spray

signal

Fall closed Motor fallure,

normally closed

Actuates spray $\quad$ Fall to trip

Relay fazlure

None

None

assure normally closed position

Trip spuriously

Relay fallure

Actuas spray

Fall to trip

system

(0) None

4) Major

Test periodically, provide fallure alarms on sensors

May cause water damage and shutdown

Test periodically, provide fallure alarms on sensors

May cause wate $r$ darnage and shutdown

Test perrodically

\begin{tabular}{l|l} 
Actuates spray & Fall to trip
\end{tabular}

System

Trip spuriously

relay fallure

Actuates standby

Fasl to trip

Operator error

No normal spray

cooling

0

spray

Relay faulure

None

o

Overpressure

None

No standby spray

cooling

Ncue if manual

0

Cause water damage and shut-

down

Test with spray system test by

actual shutdown of normal

Trip spuriously

Relay fallure

None

trip works

0

Cause water damage

FIGURE 3.9 (continued) 


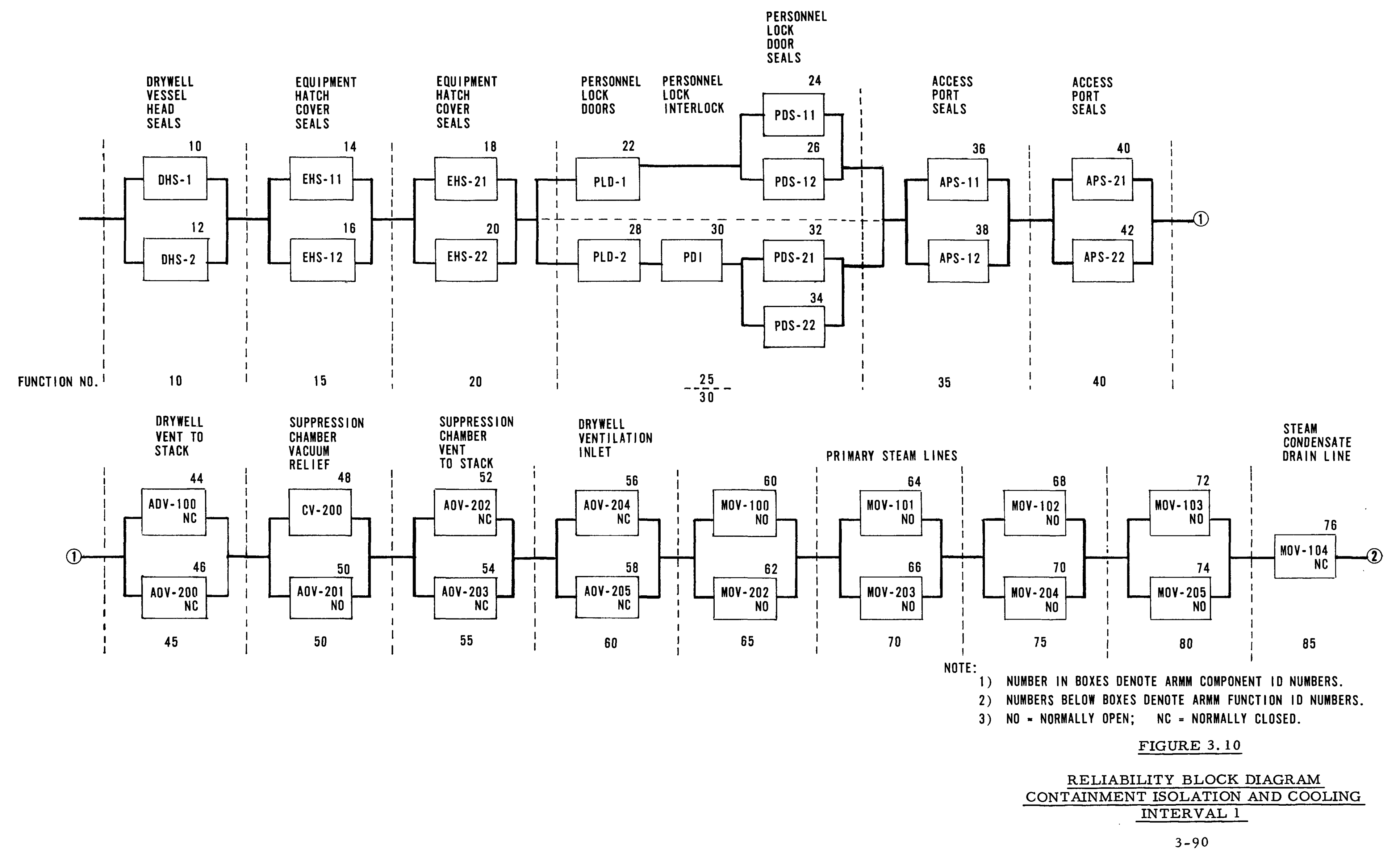




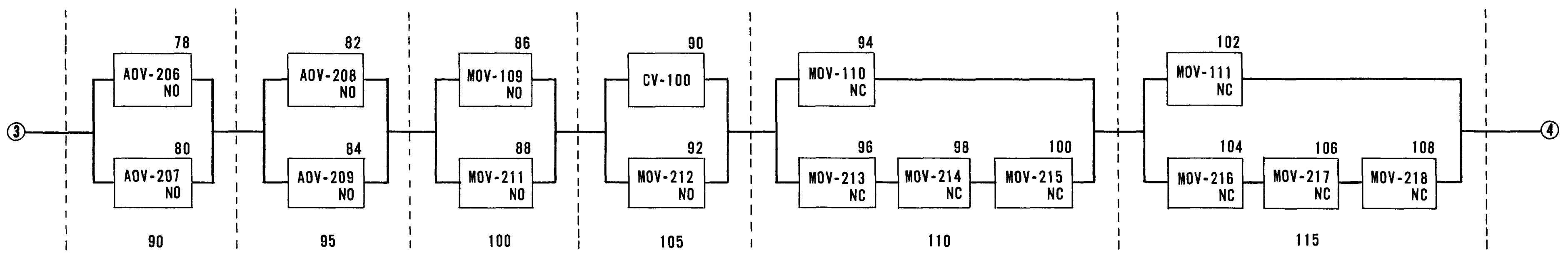

RECIRCULATING PUMP SHUTDOWN COOLING WATER RETURN

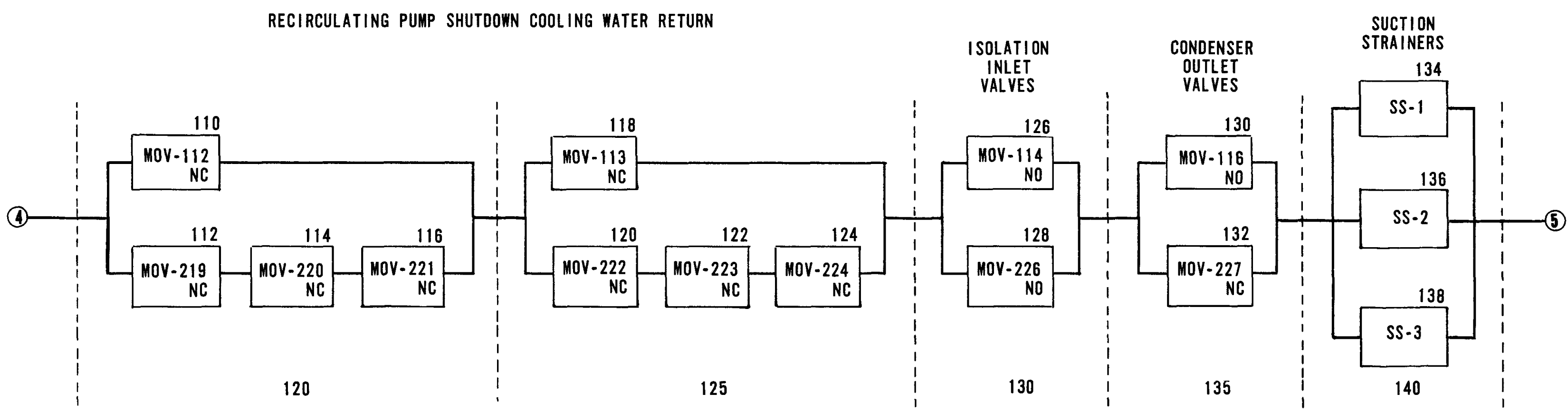


CONTA I NMENT

SPRAY LOOP

ALIGNMENT

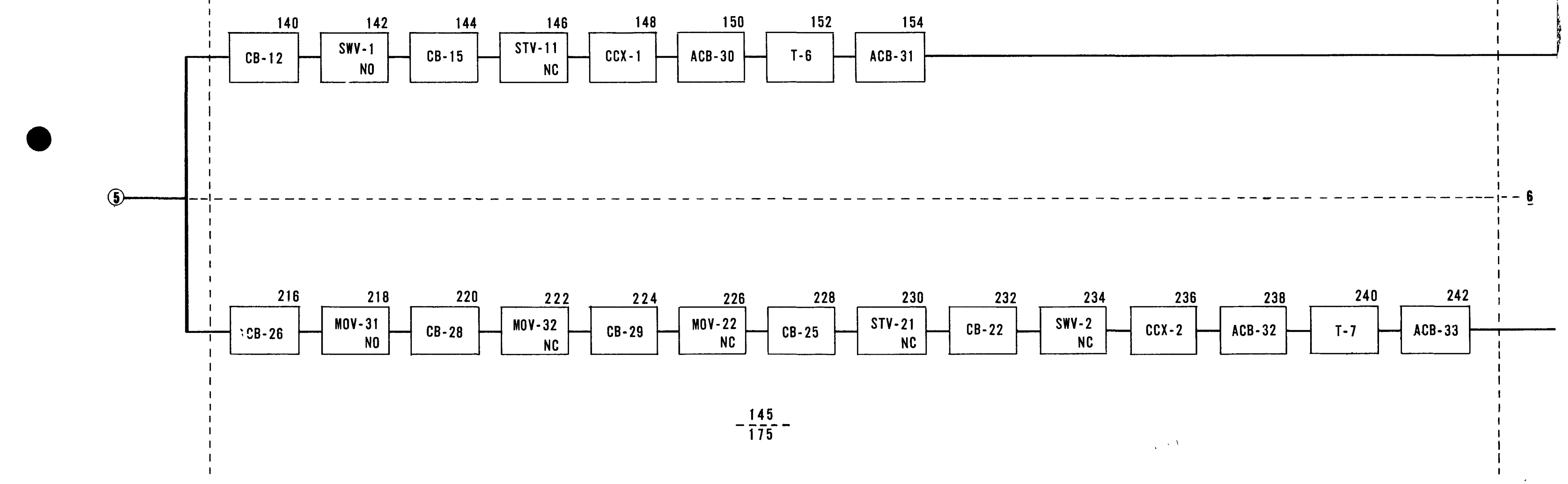

FIGURE 3.10 (continued) 


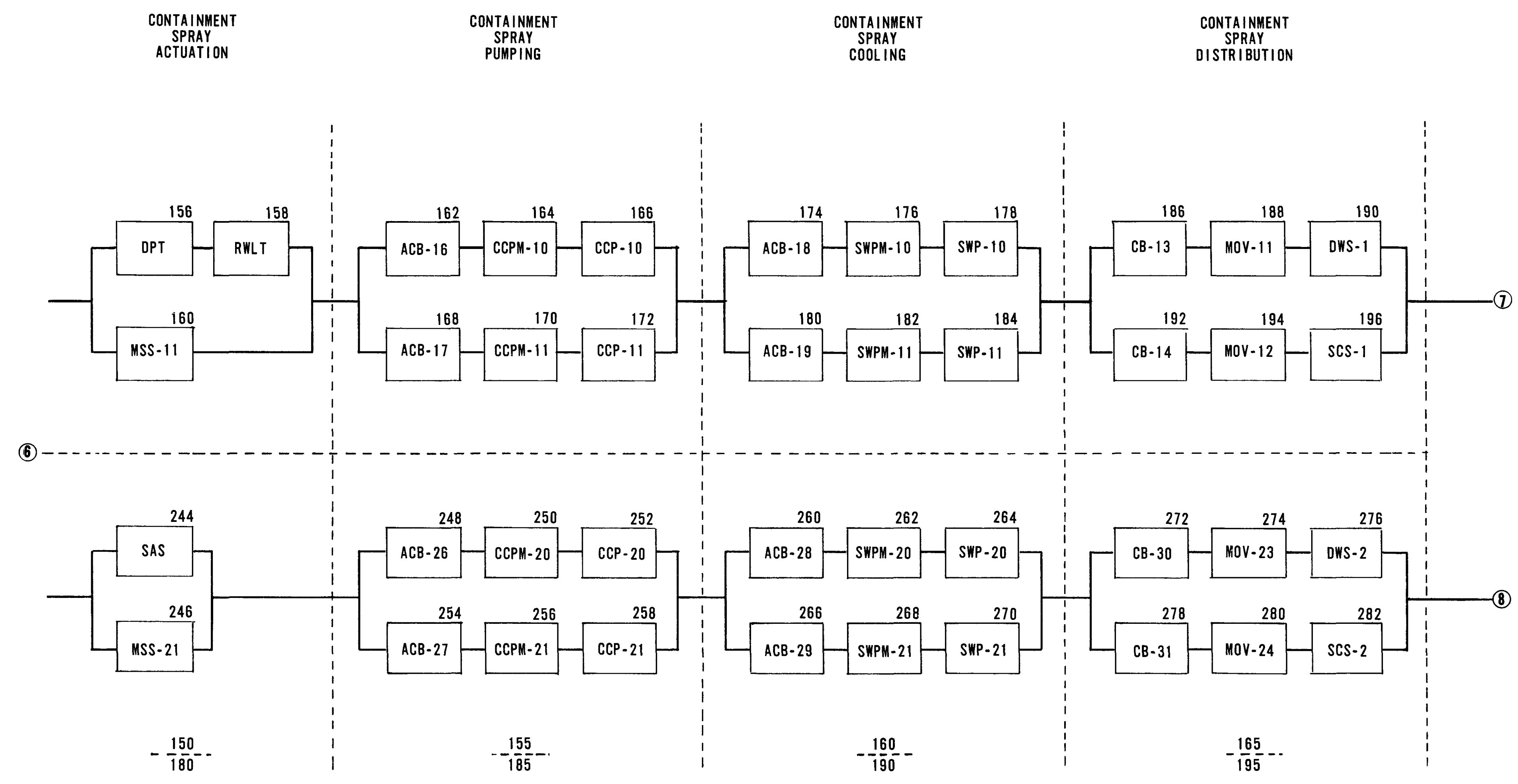

FIGURE 3.10 (continued) 
POWER SUPPLY SOURCES

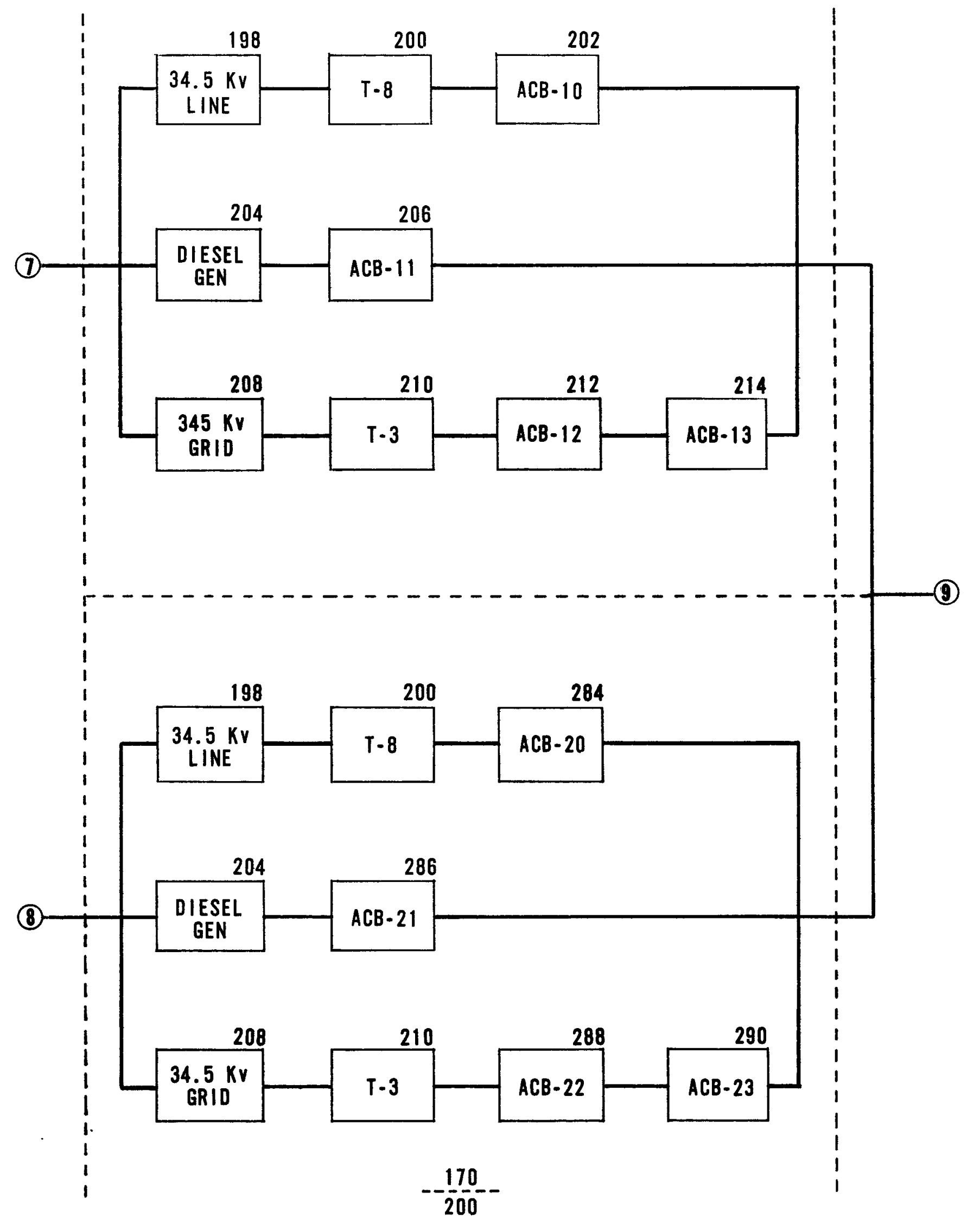

FIGURE 3.10 (continued) 


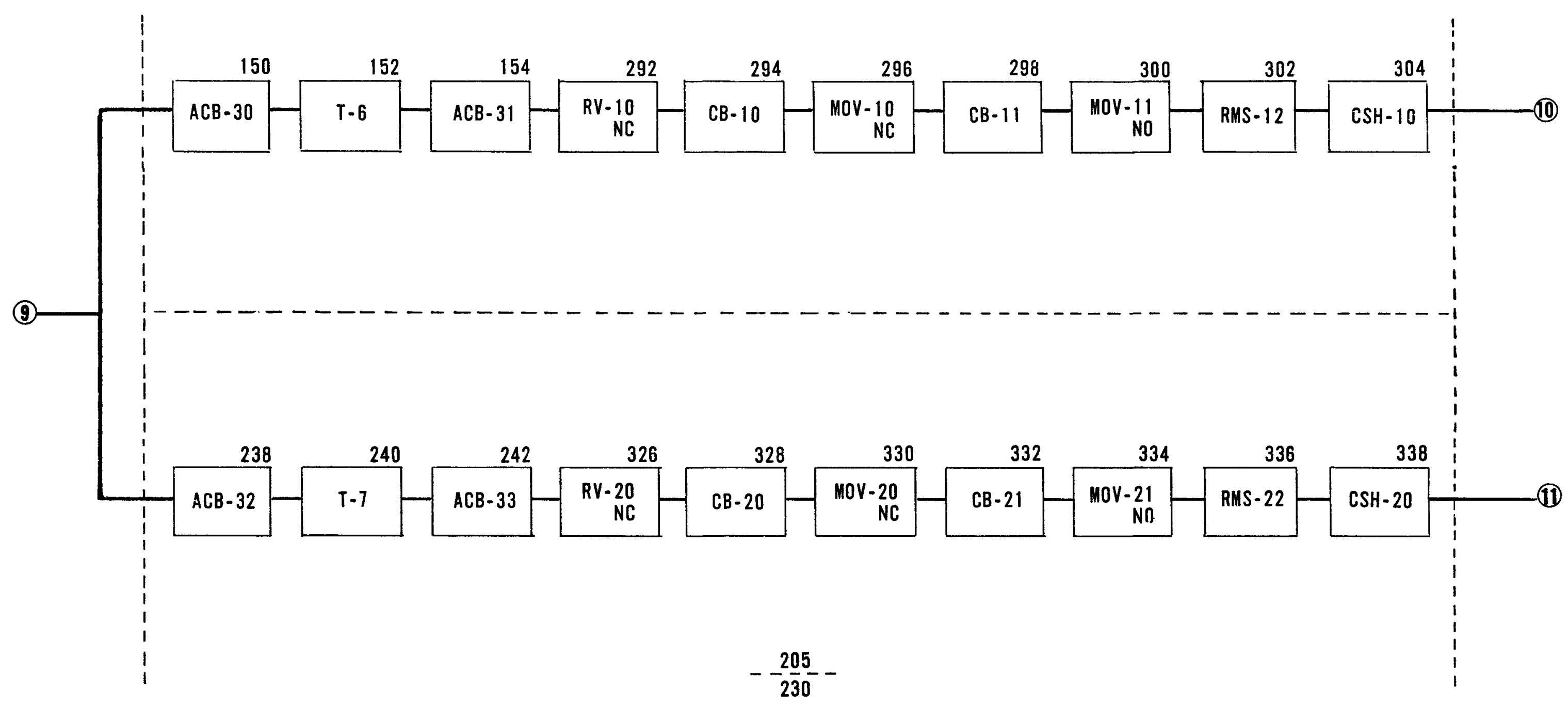

FIGURE 3.11

RELIABILITY BLOCK DIAGRAM 


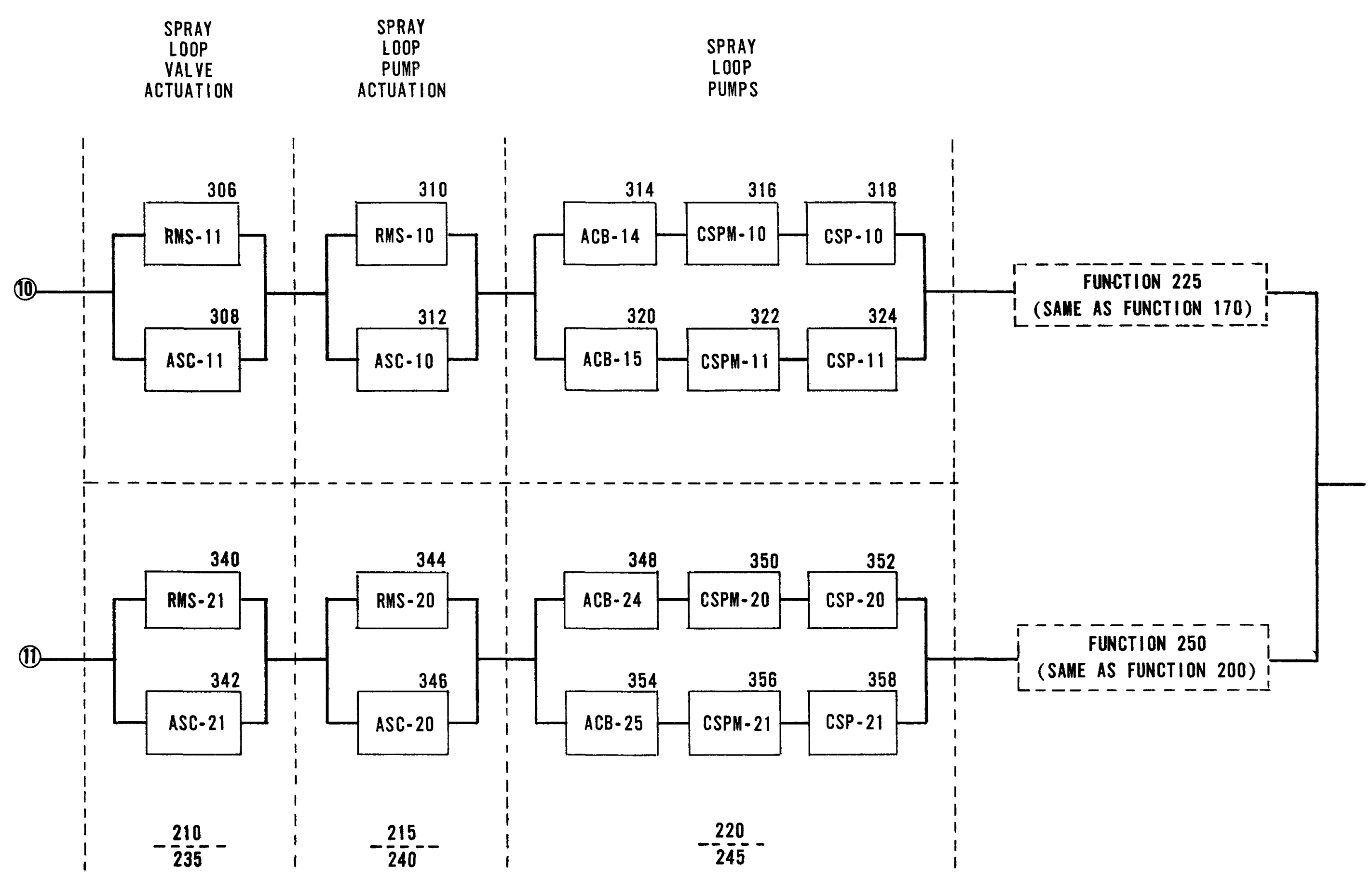

FIGURE 3.11 (continued) 


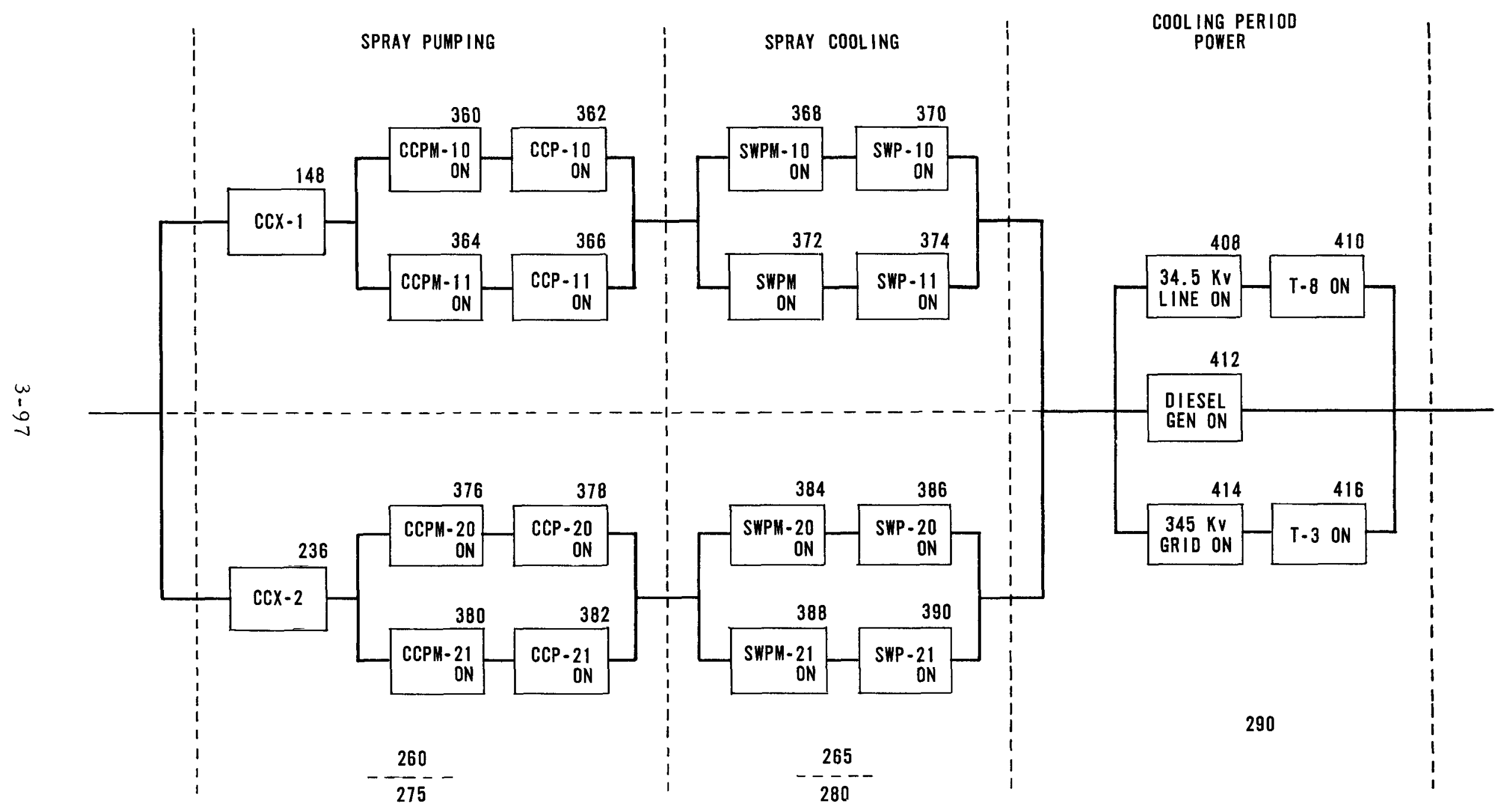

FIGURE 3.12

RELIABILITY BLOCK DIAGRAM

CONTAINMENT COOLING 


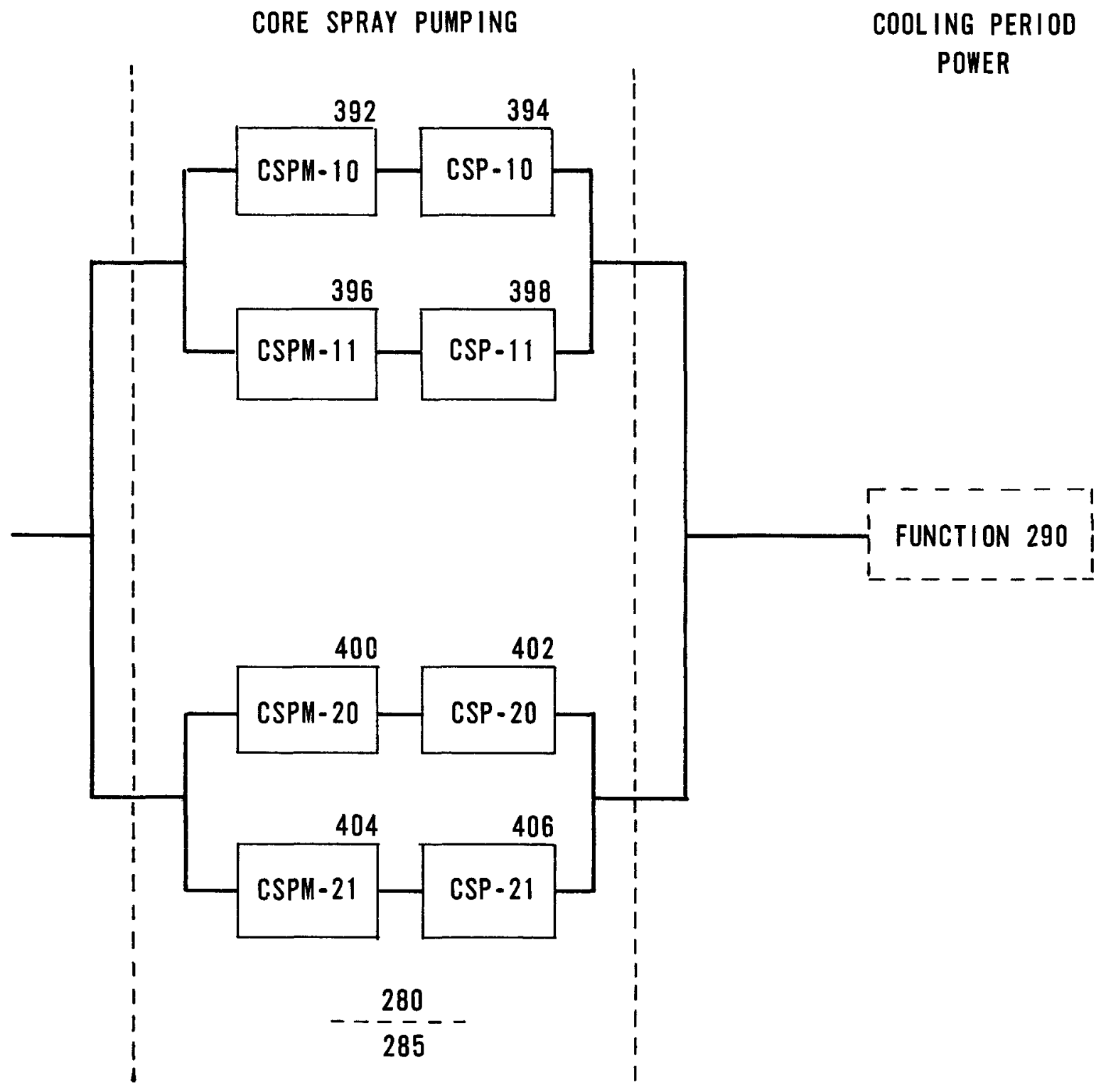

FIGURE 3.13

RELIABILITY BLOCK DIAGRAM CORE SPRAY - INTERVAL 2

(REDUNDANT OPERATION) 


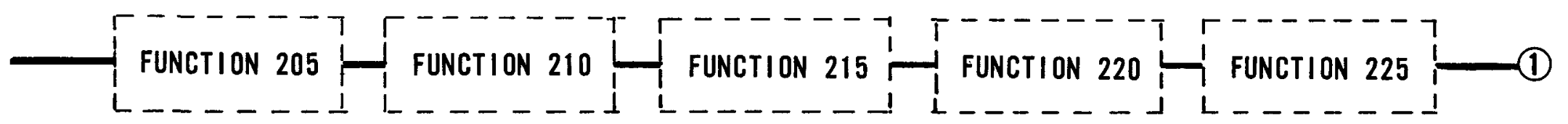

$\omega$
1
0

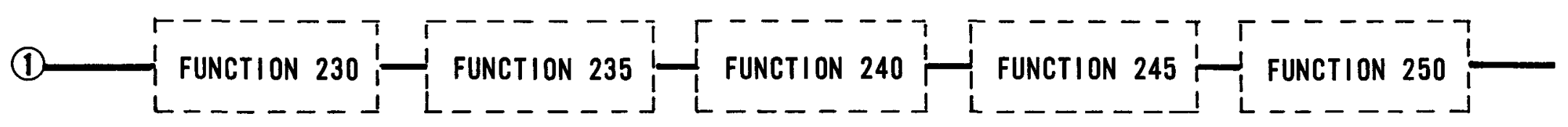

FIGURE 3.14

\section{RELIABILITY BLOCK DIAGRAM \\ CORE SPRAY - INTERVAL 1 \\ (BOTH LOOPS REQUIRED)}




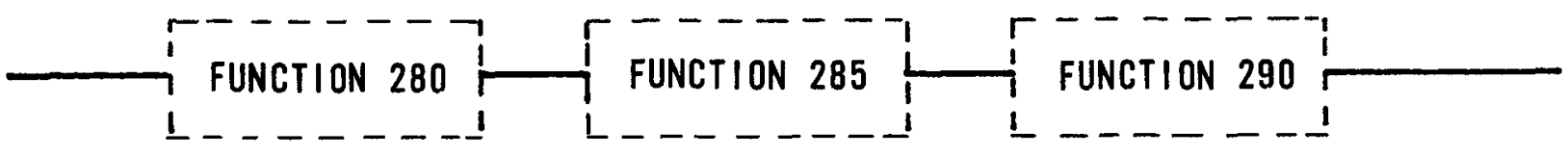

FIGURE 3.15

RELIABILITY BLOCK DIAGRAM CORE SPRAY - INTERVAL 2

(BOTH LOOPS REQUIRED) 
URESUEN 3 PKIMARY CONTAINMENT CASE 2

PRIMARY CDNTAINMENT LASE 2 CUNSIDERS PRIM. CUNT. CASE 1 AND CORE SPRAY CASE 1 TCGETHER

TWO TIME INTEPVALS CCNSIUERED

INTERVAL I ISULATION AND INITIATIUN PHASE AT END OF 30 DAYS

INTERVAL 2 CUNTINUED CUULING FOR $300 \mathrm{HR}$

VALVES, BREAKERS AND SWITCHES NOT CONSIDERED TO FAIL IN INTERVAL 2

IF UPERATED PRLIPERLY IN INTERVAL I

$\stackrel{1}{2}$

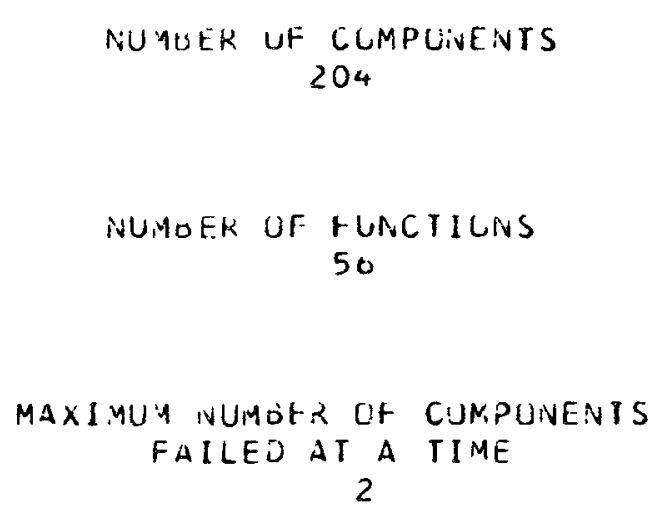

$$
50
$$

PRINT-RUT INUICATOH

FIGURE 3.16 


\author{
PRLIBAEILITY CF SYSTEM FAILUFE $=0.29782789 \mathrm{E}-03$ \\ PRCEAGILITY LF SYSTEY SUCCESS $=0.99970210$
}

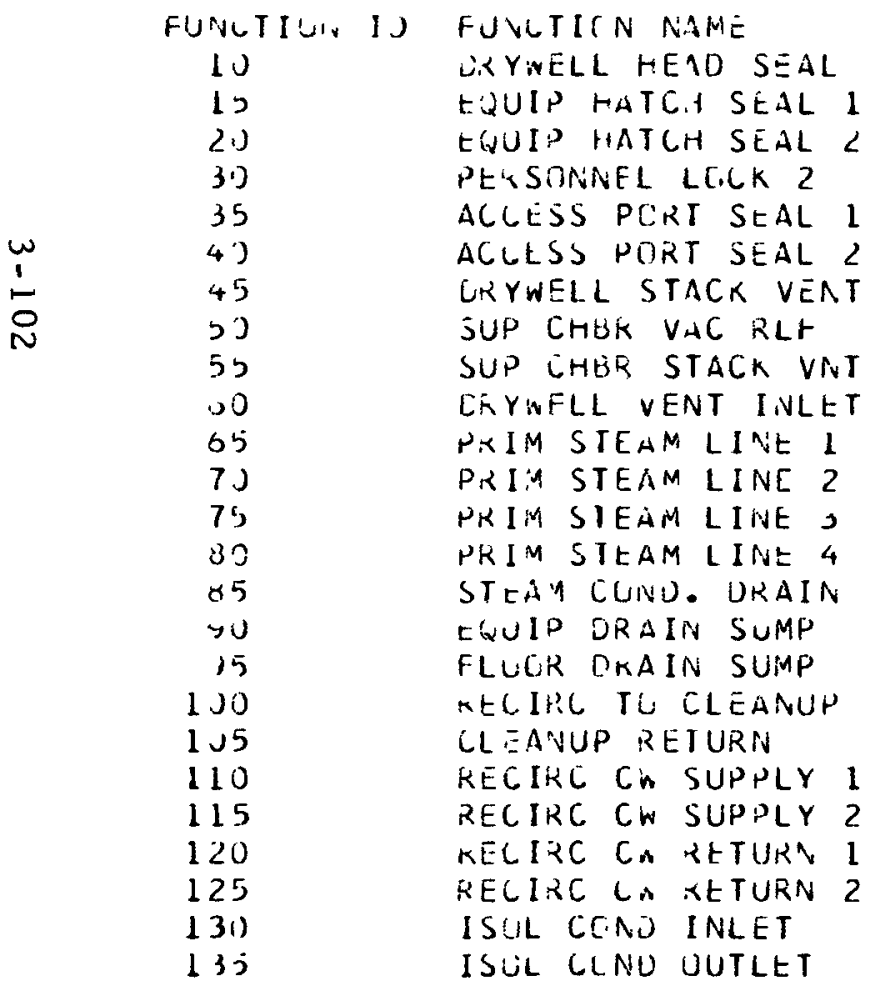

\begin{tabular}{|c|}
\hline 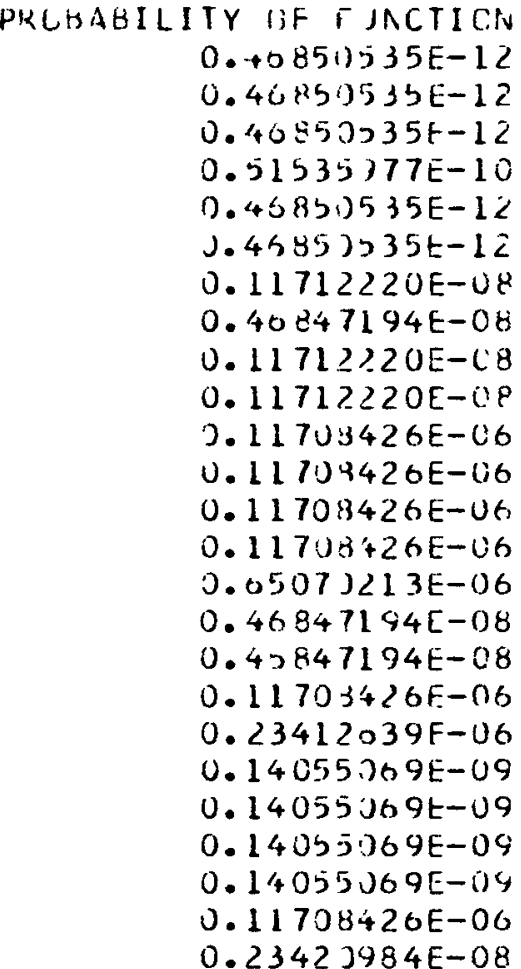 \\
\hline
\end{tabular}

$\begin{array}{cr}\text { PERCENT OF SYSTEM FAILURE } & \text { RANK } \\ 0.000 & 33 \\ 0.000 & 34 \\ 0.000 & 35 \\ 0.000 & 29 \\ 0.000 & 36 \\ 0.000 & 37 \\ 0.000 & 19 \\ 0.002 & 12 \\ 0.000 & 20 \\ 0.000 & 21 \\ 0.034 & 5 \\ 0.039 & 6 \\ 0.039 & 7 \\ 0.039 & 8 \\ 0.218 & 3 \\ 0.002 & 13 \\ 0.002 & 14 \\ 0.039 & 9 \\ 0.079 & 4 \\ 0.000 & 23 \\ 0.000 & 24 \\ 0.000 & 25 \\ 0.000 & 26 \\ 0.039 & 10 \\ 0.001 & 18\end{array}$




\begin{tabular}{cll} 
& FUNCTION ID & FUNCTIUN NAME \\
& 140 & SUCTION STRAINER \\
170 & CONT COOL BUS 5PWR \\
-175 & CUNT COOL VALVES \\
-180 & CONT COUL ACTUATE \\
-185 & CONT COUL PUMP \\
-140 & SERVICE WTR PUMP \\
& -195 & CUNT CGOL SPRAY \\
& -230 & CORE SPRAY VALVES \\
& -235 & VALVE ACTUATE \\
& -240 & PUMP ACTUATE \\
& -245 & CURE SPRAY PUMP \\
$\omega$ & -270 & CONT COOL PUMP ON \\
1 & -275 & SERVICE WTR PUMPON \\
\hline & -285 & CORE SPRAY UN \\
$\omega$ & 290 & COUL PLKIOU PWR
\end{tabular}

LOMPONENT COMBINATIONS

$\begin{array}{ll}152 & 240 \\ 152 & 238 \\ 152 & 216 \\ 150 & 240 \\ 154 & 240 \\ 152 & 328 \\ 152 & 242 \\ 152 & 220 \\ 294 & 240 \\ 150 & 220\end{array}$

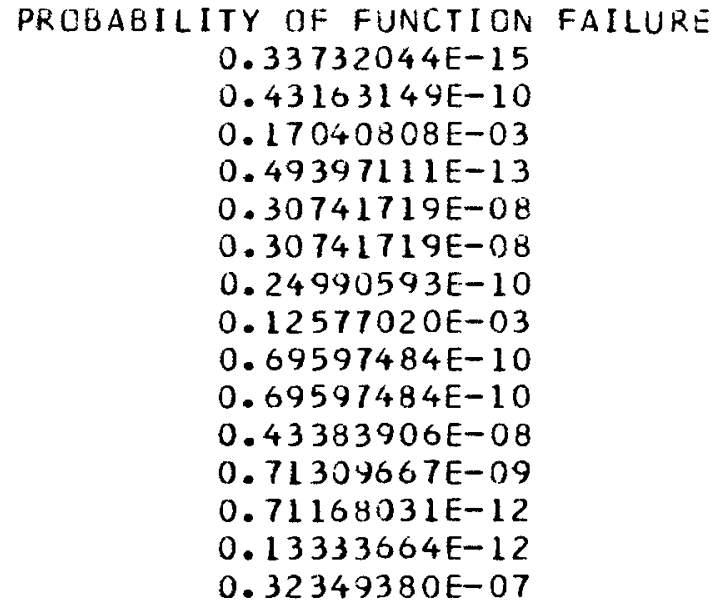

$\begin{array}{cr}\text { SYSTEM FAILURE } & \text { RANK } \\ 0.000 & 40 \\ 0.000 & 30 \\ 57.217 & 1 \\ 0.000 & 39 \\ 0.001 & 16 \\ 0.001 & 17 \\ 0.000 & 31 \\ 42.229 & 2 \\ 0.000 & 27 \\ 0.000 & 28 \\ 0.001 & 15 \\ 0.000 & 22 \\ 0.000 & 32 \\ 0.000 & 38 \\ 0.011 & 11\end{array}$

MAJCR CONTRIBUTORS TO SYSTEM UNRELIABILITY

$$
\begin{aligned}
& \text { PROBABILITY } \\
& 0.20904720 E-04 \\
& 0.11624881 E-04 \\
& 0.11624981 E-04 \\
& 0.11624981 E-04 \\
& 0.11624881 E-04 \\
& 0.11624881 E-04 \\
& 0.11624881 E-04 \\
& 0.11624881 E-04 \\
& 0.11624881 E-04 \\
& 0.64644687 E-05
\end{aligned}
$$

PERCENT

6.3435

3.5276

3. 5276

3.5276

3.5276

3.5276

3.5276

3.5276

3. 5276

1.9616

FIGURE 3.16 (continued) 
CGMPONENT CONTRIBUTIONS TO UNREL IABILITY

\begin{tabular}{|c|c|c|}
\hline $\begin{array}{l}\text { CUIA PUNENT } \\
\text { ID }\end{array}$ & IVAYE & \\
\hline$(0.1 \mathrm{~J}$ & LRY YWELL & SEAL I \\
\hline 12.00 & ORYNELL HEAL & SEAL 2 \\
\hline 14.00 & EQЛIP HAICH & SEAL 11 \\
\hline 10.00 & HATCH & StAL 12 \\
\hline 13.01$)$ & HATCH & SEAL 21 \\
\hline 20.00 & EQUIP HATCH & StAL 22 \\
\hline 36.00 & ACitSS PORT & $S E A L 11$ \\
\hline 38.00 & ACEESS PLRT & SF AL 21 \\
\hline 40.00 & ACCESS PORT & SEAL 21 \\
\hline 42.00 & ACLESS PORT & SEAL 22 \\
\hline $4+.00$ & $A O V-100$ & \\
\hline 40.00 & $A C I V-200$ & \\
\hline 48.00 & $C V-200$ & \\
\hline 50.00 & $A C: V-201$ & \\
\hline $52 \ldots 0$ & $A() V-202$ & \\
\hline 54.00 & $A U V-203$ & \\
\hline 56.00 & $A 0 v-204$ & \\
\hline 53.50 & $A U V-2 O B$ & \\
\hline 60.00 & MUV-1UO & \\
\hline $62.0 \mathrm{~J}$ & MOV -202 & \\
\hline 64.00 & NuV-101 & \\
\hline 66.00 & $M U V-203$ & \\
\hline 68.00 & MOVV- 102 & \\
\hline 70.00 & MuV -204 & \\
\hline 72.00 & intiv $v-103$ & \\
\hline 74.00 & $M G V-2 C S$ & \\
\hline 76.00 & MI) V -104 & \\
\hline 70.00 & $A \cup V-206$ & \\
\hline 80.00 & $A O V-207$ & \\
\hline
\end{tabular}

$\begin{array}{cc}\text { FAILURE } & \text { SERIAL } \\ \text { RATE IO\$0 } & \text { PYJBA3ILITY } \\ 0.00 & 0.65394789 E-06 \\ 0.00 & 0.65394789 E-06 \\ 0.00 & 0.65394739 E-06 \\ 0.00 & 0.05394789 E-06 \\ 0.00 & 0.65394739 E-06 \\ 0.00 & 0.65394789 E-06 \\ 0.00 & 0.05394739 E-06 \\ 0.00 & 0.65394789 E-06 \\ 0.00 & 0.65394789 E-06 \\ 0.00 & 0.65394739 E-06 \\ 0.05 & 0.32697394 E-04 \\ 0.05 & 0.32697394 E-04 \\ 0.10 & 0.65394788 E-04 \\ 0.10 & 0.65394738 E-04 \\ 0.05 & 0.32697394 E-04 \\ 0.05 & 0.32697394 E-04 \\ 0.05 & 0.32697394 E-04 \\ 0.05 & 0.32697394 E-04 \\ 0.50 & 0.32697394 E-03 \\ 0.50 & 0.32697394 E-03 \\ 0.50 & 0.32697394 E-03 \\ 0.50 & 0.32697394 E-03 \\ 0.50 & 0.32697394 E-03 \\ 0.50 & 0.32697394 E-03 \\ 0.50 & 0.32697394 E-03 \\ 0.50 & 0.32097394 E-03 \\ 0.00 & 0.65394139 E-06 \\ 0.10 & 0.65394788 E-04 \\ 0.10 & 0.65394788 E-04\end{array}$

SERIAL
PERCENT
0.0004
0.0004
0.0004
0.0004
0.0004
0.0014
0.0004
0.0034
0.0004
0.0004
0.0134
0.0134
0.0368
0.0308
0.0194
0.0184
0.0184
0.0184
0.1840
0.1840
0.1840
0.1840
0.1840
0.1840
0.1840
0.1840
0.0004
0.0368
0.0368

SERIAL SYSTEM

RANK PRJBABILITY
122
$0.23425270 \mathrm{E}-12$

$0.23425270 E-12$

$0.23425270 E-12$

$0.23425270 E-12$

$0.23425270 E-12$

$0.23425270 \mathrm{~F}-12$

$0.23425270 E-12$

$0.23425270 E-12$

$0.23425270 E-12$

1). $23425270 \mathrm{E}-12$

$0.53561105 E-09$

$0.53561105 \mathrm{E}-09$

$0.23423600 E-08$

$0.234236 J 0 E-08$

$0.58561105 E-09$

$0.53561105 E-09$

$0.58561105 \mathrm{E}-09$

$0.59561105 E-09$

$0.58542139 E-07$

$0.53542139 E-07$

$0.58542139 E-07$

$0.58542139 \mathrm{E}-07$

$0.53542139 E-07$

$0.58542139 E-07$

$0.58542139 E-07$

$0.58542139 \mathrm{E}-07$

$0.6507022 \mathrm{JE}-06$

$0.2342360 \mathrm{OE}-08$

$0.23423600 E-08$

FIGURE 3.16 (continued) 
COMPINENT CONIRI HUTIONS TU UVRELIAHILITY

COMPUNEVI

\begin{tabular}{|c|c|}
\hline ive & \\
\hline 0 & NAML \\
\hline $8<.00$ & $A L V-20 B$ \\
\hline 84.00 & $A r i v-2 \cup y$ \\
\hline 86.00 & MOV-10t \\
\hline 84.150 & $M \cup v-211$ \\
\hline $90 . J)$. & $(v-1) 0$ \\
\hline 92.00 & $M O V-212$ \\
\hline 94.00 & ate $)-110$ \\
\hline 96.00 & $M 1 \cdot V-213$ \\
\hline 96.10 & $A O B V-214$ \\
\hline 100.00 & $M C \sqrt{ }-21 b$ \\
\hline 102.00 & $M L V-111$ \\
\hline 104.00 & $M U v-210$ \\
\hline 100.00 & ML $V-217$ \\
\hline 108.00 & $M L v-218$ \\
\hline 110.10 & nus 1112 \\
\hline 112.00 & MiUV -219 \\
\hline 114.00 & $M L v-220$ \\
\hline 116.00 & $M L V-22 L$ \\
\hline 114.30 & $M L s-11 s$ \\
\hline $1<0.00$ & $M U v-222$ \\
\hline 122.00 & $M L^{\prime} V-223$ \\
\hline $1<4.0 J$ & $M(1 v-224$ \\
\hline 126.00 & $M(\sqrt{ }-114$ \\
\hline $1<A .30$ & $M[: V-\angle<6$ \\
\hline 130.20 & $w v-116$ \\
\hline 132.00 & $\mathrm{NiUV}-227$ \\
\hline $150.0 \mathrm{v}$ & LRYAELL PRESS TRIP \\
\hline 128.100 & WATER LLVEL TKIP \\
\hline
\end{tabular}

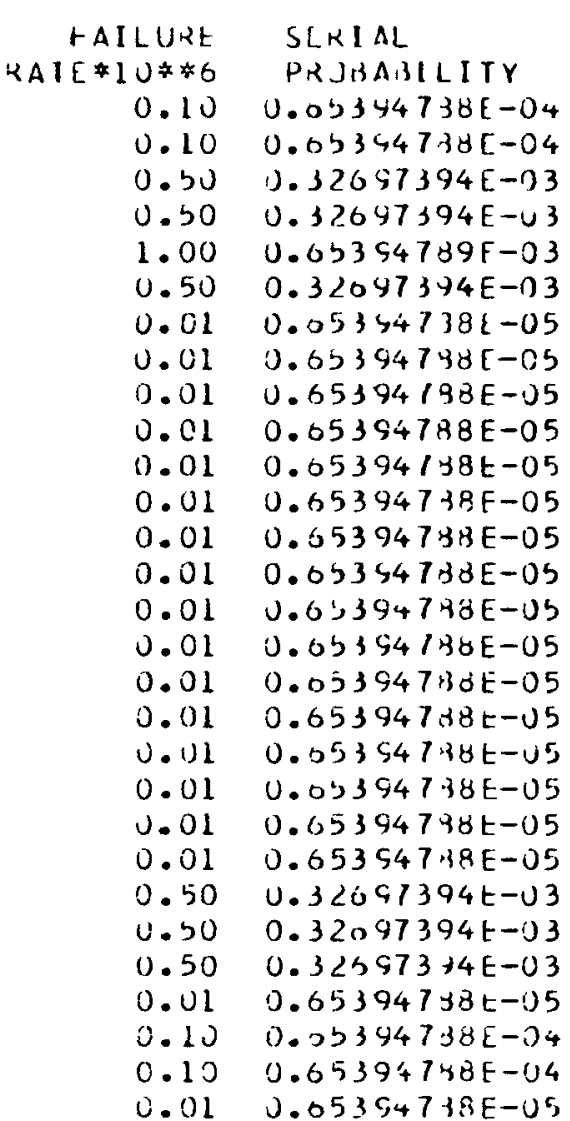

\begin{tabular}{|c|c|c|}
\hline $\begin{array}{l}\text { SER IAL } \\
\text { PERCENT }\end{array}$ & $\begin{array}{l}\text { SEK I IL } \\
\text { RANK. }\end{array}$ & 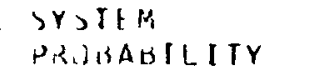 \\
\hline 0.0368 & 80 & $3.234236) \cup(-74$ \\
\hline 0.0308 & B 1 & 1). $2342360135-73$ \\
\hline 0.1340 & 53 & $7.535421335-07$ \\
\hline $0.18+0$ & 64 & $3.35^{3} 4 / 133 t-07$ \\
\hline 0.3590 & 50 & $0.150084775-00$ \\
\hline $0.18 \div 0$ & 65 & $0.710421375-07$ \\
\hline 0.0037 & 91 & $0.70<753 \rightarrow 3 F-12$ \\
\hline 0.10037 & $9 ?$ & $0.23425113 F-10$ \\
\hline 0.0037 & 93 & $0.23425119 t-13$ \\
\hline 0.0037 & 94 & $0.234231(35-1)$ \\
\hline 0.0037 & 95 & $0.7 J<733 b(-1)$ \\
\hline 0.0037 & 96 & $0.23425113(-1)$ \\
\hline 0.0037 & 97 & $0.23425113 E-1)$ \\
\hline 0.0037 & 98 & $0.234251(3 t-1)$ \\
\hline 0.11037 & 34 & $0.13275353 E-13$ \\
\hline 3.0037 & 100 & $0.23425113 F-1 J$ \\
\hline 0.0737 & 101 & $0.23425113 E-1)$ \\
\hline 0.0037 & 102 & 0.7342511 3t -1$)$ \\
\hline 0.0037 & 103 & $0.7,275353 t-10$ \\
\hline 0.0737 & 174 & $0.23425113 t-1)$ \\
\hline 0.0037 & 105 & $0.23425113 E-1)$ \\
\hline 0.0037 & 106 & $0.234251(8 t-1)$ \\
\hline 0.1840 & 66 & 0.5354213 tt-07 \\
\hline 0.1840 & 67 & $0.5 B>4213+E-27$ \\
\hline 0.1840 & 68 & $0.229617 \supset 1 E-0$. \\
\hline 0.3037 & 107 & $0.45923502 E-10$ \\
\hline $0.03,0$ & 32 & $0.16802122 t-12$ \\
\hline 0.0 & 43 & $0.168021224-12$ \\
\hline 0.0037 & 108 & $0.336(: 4243 L-11$ \\
\hline
\end{tabular}

\begin{tabular}{|c|c|}
\hline$S Y \backslash T F M$ & SYSTEM \\
\hline PERCENT & RANK \\
\hline 3.3704 & 78 \\
\hline 0.10$) 8$ & 79 \\
\hline 3.197 & 57 \\
\hline נ.0197 & $5 \hat{E}$ \\
\hline $7.75 \geq 4$ & 45 \\
\hline 0.0202 & 46 \\
\hline 9.9000 & 111 \\
\hline .7 .001 & 128 \\
\hline 0.1010 & $1 / 4$ \\
\hline 0.2730 & 130 \\
\hline 0. & 112 \\
\hline 1. & 131 \\
\hline 1.9700 & 132 \\
\hline 2.10000 & 133 \\
\hline 0.3000 & 113 \\
\hline 7.3000 & 134 \\
\hline 0.3030 & 135 \\
\hline ח & 136 \\
\hline $3.17 x$ & 114 \\
\hline 0.100 & 137 \\
\hline 1). $1+30$ & $13 i$ \\
\hline נ) נ. & $13 \%$ \\
\hline 1. $\| 1147$ & $\because 5$ \\
\hline 3.1197 &, 0 \\
\hline $0 . \cdot 2 \cap 4$ & $m$ \\
\hline 1.11$)$ n & 119 \\
\hline 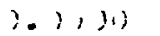 & 150 \\
\hline ). in 1. ! & 131 \\
\hline 1.1114 & 111 \\
\hline
\end{tabular}


COMPONENT CONTRIBUTIONS TO UNRELIABILITY

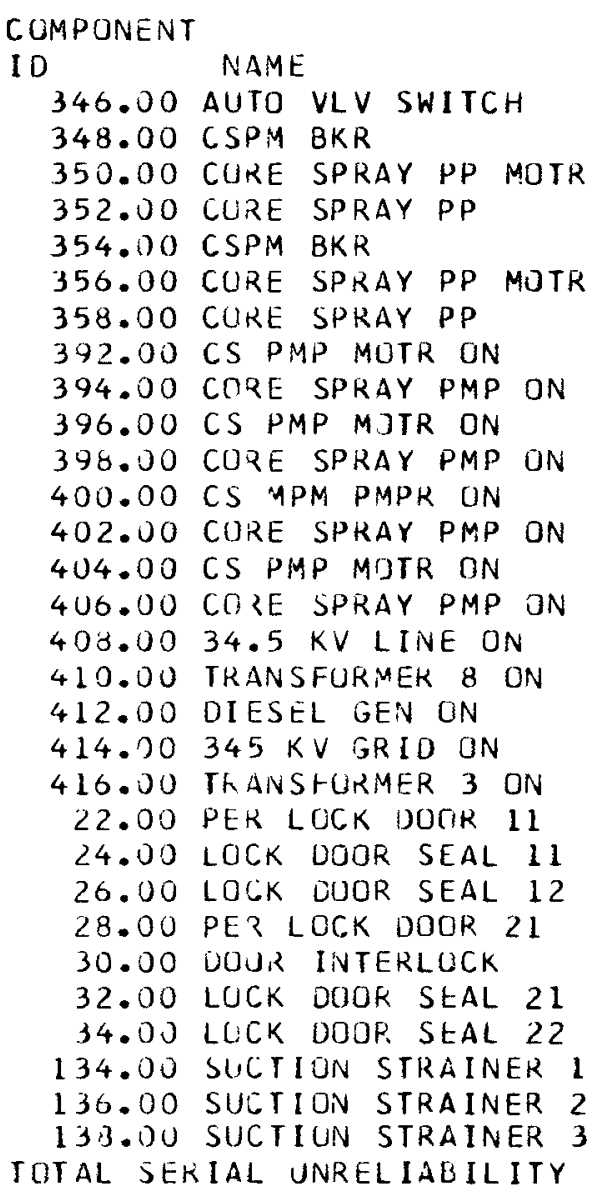

$\begin{array}{lr}\text { SER IAL } & \text { SERI } \\ \text { PERCENT } & \text { RANK } \\ 0 . & 0 \\ 0 . & 0 \\ 0 . & 0 \\ 0 . & 0 \\ 0 . & 0 \\ 0 . & 0 \\ 0 . & 0 \\ 0.7667 & 41 \\ 0.7667 & 42 \\ 0.7667 & 43 \\ 0.7667 & 44 \\ 0 . & 0 \\ 0 . & 0 \\ 0 . & 0 \\ 0.0 & 0 \\ 1.5335 & 30 \\ 0.7667 & 45 \\ 1.3801 & 31 \\ 0.0002 & 136 \\ 1.3801 & 32 \\ 0.0037 & 113 \\ 0.0037 & 114 \\ 0.0037 & 115 \\ 0.0037 & 116 \\ 0.0004 & 135 \\ 0.0037 & 117 \\ 0.0037 & 118 \\ 0.0037 & 119 \\ 0.0037 & 120 \\ 0.0037 & 121 \\ & \end{array}$

SYSTEM

PRIBAABILITY

$0.29086388 E-10$

$0.22953065 E-09$

$0.72095972 E-09$

$0.72095972 \mathrm{E}-09$

$0.22953065 E-09$

$0.72095972 \mathrm{E}-09$

$0.72095972 \mathrm{E}-09$

$0.16667081 E-13$

$0.16667081 E-13$

$0.16667081 E-13$

$0.16667081 E-13$

$0.16667081 \mathrm{E}-13$

$0.16667081 E-13$

$0.16667081 E-13$

$0.16667081 \mathrm{E}-13$

$0.77007144 E-08$

$0.23454976 E-08$

$0.11152539 \mathrm{E}-07$

$0.21195964 E-15$

$0.11150631 \mathrm{E}-07$

$0.27684356 E-10$

$0.12850369 \mathrm{E}-15$

$0.12850369 E-15$

$0.23425230 E-10$

$0.42591422 \mathrm{E}-12$

$0.11244076 \mathrm{E}-15$

$0.11244076 \mathrm{E}-15$

$0.11244015 E-15$

$0.11244015 E-15$

$0.11244015 \mathrm{E}-15$

$\begin{array}{lr}\text { SYSTEM } & \text { SYSTEM } \\ \text { PERCENT } & \text { RANK } \\ 0.0000 & 125 \\ 0.0001 & 102 \\ 0.0002 & 81 \\ 0.0002 & 82 \\ 0.0001 & 103 \\ 0.0002 & 83 \\ 0.0002 & 84 \\ 0.0000 & 172 \\ 0.0000 & 173 \\ 0.0000 & 174 \\ 0.0000 & 175 \\ 0.0000 & 176 \\ 0.0000 & 177 \\ 0.0000 & 178 \\ 0.0000 & 179 \\ 0.0026 & 71 \\ 0.0008 & 73 \\ 0.0037 & 67 \\ 0.0000 & 185 \\ 0.0037 & 68 \\ 0.0000 & 126 \\ 0.0000 & 186 \\ 0.0000 & 187 \\ 0.0000 & 127 \\ 0.0000 & 148 \\ 0.0000 & 188 \\ 0.0000 & 189 \\ 0.0000 & 190 \\ 0.0000 & 191 \\ 0.0000 & 192 \\ & \end{array}$


CCMPONENT CONIRIBUTIONS TU UNRELIABILITY

\begin{tabular}{|c|c|}
\hline$M P I N$ & \\
\hline & NAME \\
\hline 144.00 & VLV $\triangle K R$ \\
\hline 140.03 & SPRAY TEST VLV \\
\hline J. J & COVT CDUL EXCHANGR \\
\hline & BUSD-T6 BKR \\
\hline Jo & TRANSFURMER 6 \\
\hline & $16-3 U S 2 A$ BKR \\
\hline 00 & $M \cup V-31 \quad B K K$ \\
\hline $0 \mathrm{~J}$ & ST JSY LOOP \\
\hline & MLV V-32 BKR \\
\hline & ST DAY LUOP \\
\hline & MOV $-22 \quad B K R$ \\
\hline Ju & STJBY LUCP \\
\hline 00 & STV- $21 \quad 3 K R$ \\
\hline 00 & SPRAY TEST VLV \\
\hline 0 & $S W V-2 \quad B K R$ \\
\hline 00 & SER WTR RTN \\
\hline & CONT COUL EXCHANGA \\
\hline טמ & BUSG-T7 BKR \\
\hline 240 & IRANSFOKMER \\
\hline 24 & T7-BUS2 B BKR \\
\hline 244 & ST JBY AUTO STRT \\
\hline & MAN START Sh \\
\hline 24 & CCPM BKR \\
\hline 25 & CO.VT \\
\hline 252 & COOL \\
\hline & CCPM \\
\hline & CUNT \\
\hline & CUNT CUOL PMP \\
\hline & SER WIR PP MTR \\
\hline
\end{tabular}

$\begin{array}{rl}\text { FAILURE } & \text { SERIAL } \\ \text { RATE*10*0 } & \text { PROSABILITY } \\ 1.00 & 0.65394789 E-03 \\ 0.01 & 0.55394788 E-05 \\ 0.10 & 0.42642616 E-04 \\ 5.00 & 0.32697394 E-02 \\ 9.00 & 0.58855310 E-02 \\ 5.00 & 0.32697394 E-02 \\ 5.00 & 0 . \\ 0.50 & 0 . \\ 5.00 & 0 . \\ 0.50 & 0 . \\ 1.00 & 0 . \\ 0.01 & 0 . \\ 1.00 & 0 . \\ 0.01 & 0 . \\ 1.00 & 0 . \\ 0.01 & 0 . \\ 0.10 & 0 . \\ 5.00 & 0 . \\ 9.00 & 0 . \\ 5.00 & 0 . \\ 0.10 & 0 . \\ 0.10 & 0 . \\ 5.00 & 0 . \\ 10.00 & 0 . \\ 10.00 & 0 . \\ 5.00 & 0 . \\ 10.00 & 0 . \\ 10.00 & 0 . \\ 5.00 & 0 .\end{array}$

SER I AL
PERCENT
0.3680
0.0037
0.0521
1.8402
3.3123
1.8402
0.
0.
0.
0.
0.
0.
0.
0.
0.
0.
0.
0.
0.
0.
0.
0.
0.
0.
0.
0.
0.
0.
0.

SERIAL SYSTE

PERCENT RANK

$0.5122 \quad 16$

$0.0001 \quad 100$

$0.0080 \quad 61$

$7.2601 \quad 3$

$20.6034 \quad 1$

$9.2601 \quad 4$

$\begin{array}{ll}9.2601 & 4 \\ 0.0008 & 9\end{array}$

$0.0855 \quad 37$

$4.0008 \quad 10$

$0.0855 \quad 38$

$0.2927 \quad 19$

$0.0001 \quad 104$

$0.2927 \quad 20$

$0.0001 \quad 105$

$0.2927 \quad 21$

$0.0001 \quad 106$

$\begin{array}{rrr}0.13100754 \mathrm{E}-07 & 0.0044 & 65 \\ 0.20146422 \mathrm{E}-04 & 6.7644 & 5 \\ 0.45109661 \mathrm{~F}-04 & 15.1461 & 2\end{array}$

$\begin{array}{rrr}0.13100754 \mathrm{E}-07 & 0.0044 & 65 \\ 0.20146422 \mathrm{E}-04 & 6.7644 & 5 \\ 0.45109661 \mathrm{E}-04 & 15.1461 & 2\end{array}$

$\begin{array}{lrl}0.45109661 \mathrm{E}-04 & 15.1461 & 2 \\ 0.20146422 \mathrm{E}-04 & 6.7644 & 6\end{array}$

$0.11692726 \mathrm{E}-14 \quad 0.0000 \quad 181$

$0.11692726 \mathrm{E}-14 \quad 0.0000 \quad 182$

$0.15638201 E-09 \quad 0.0001 \quad 107$

$0.4953201 \mathrm{BE}-09 \quad 0.0002 \quad 91$

$0.49532018 \mathrm{E}-09 \quad 0.0002 \quad 92$

$0.15638201 E-09 \quad 0.0001 \quad 108$

$0.49532018 \mathrm{E}-09 \quad 0.0002 \quad 93$

$0.49532018 E-09 \quad 0.0002 \quad 94$

$0.15638201 \mathrm{E}-09 \quad 0.0001 \quad 109$ 
COMPONENT CONTRIBUTIONS TO UNRELIABILITY

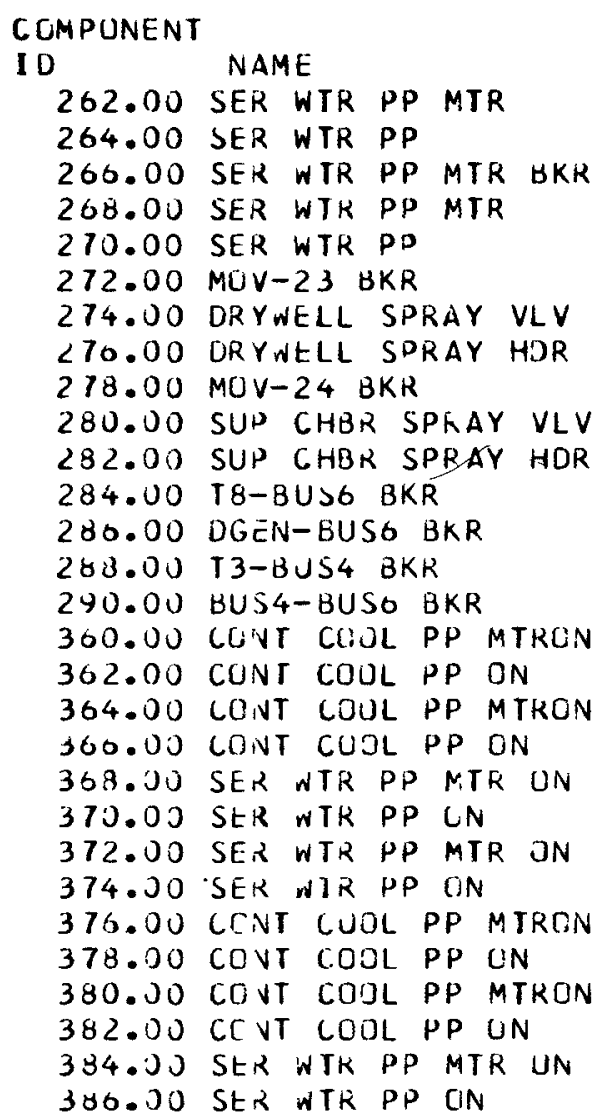

SER IAL
PERCENT
0.
0.
0.
0.
0.
0.
0.
0.
0.
0.
0.
0.
0.
0.
0.
0.7667
0.7667
0.7667
0.7667
0.7667
0.7667
0.7667
0.7667
0.
0.
0.
0.
0.
0.

$\begin{array}{cl}\text { SERIAL } & \text { SYSTEM } \\ \text { RANK } & \text { PROBABILITY } \\ 0 & 0.49532018 E-09 \\ 0 & 0.49532018 E-09 \\ 0 & 0.15638201 E-09 \\ 0 & 0.49532018 E-09 \\ 0 & 0.49532018 E-09 \\ 0 & 0.21288046 E-11 \\ 0 & 0.23508288 E-15 \\ 0 & 0.23586806 E-17 \\ 0 & 0.10725236 E-10 \\ 0 & 0.87469176 E-16 \\ 0 & 0.87733366 E-18 \\ 0 & 0 . \\ 0 & 0 . \\ 0 & 0 . \\ 0 & 0 . \\ 33 & 0.33070159 E-10 \\ 34 & 0.33070159 E-10 \\ 35 & 0.33070159 E-10 \\ 36 & 0.33070159 E-10 \\ 37 & 0.33070159 E-10 \\ 38 & 0.33070159 E-10 \\ 39 & 0.33070159 E-10 \\ 40 & 0.33070159 E-10 \\ 0 & 0.14348443 E-12 \\ 0 & 0.14348443 E-12 \\ 0 & 0.14348443 E-12 \\ 0 & 0.14348443 E-12 \\ 0 & 0.14348443 E-12 \\ 0 & 0.14348443 E-12\end{array}$

$\begin{array}{lr}\text { SYSTEM } & \text { SYSTEM } \\ \text { PERCENT } & \text { RANK } \\ 0.0002 & 95 \\ 0.0002 & 96 \\ 0.0001 & 110 \\ 0.0002 & 97 \\ 0.0002 & 98 \\ 0.0000 & 147 \\ 0.0000 & 184 \\ 0.0000 & 194 \\ 0.0000 & 144 \\ 0.0000 & 193 \\ 0.0000 & 195 \\ 0 . & 0 \\ 0 . & 0 \\ 0.0 & 0 \\ 0.0 & 0 \\ 0.0000 & 116 \\ 0.0000 & 117 \\ 0.0000 & 118 \\ 0.0000 & 119 \\ 0.0000 & 120 \\ 0.0000 & 121 \\ 0.0000 & 122 \\ 0.0000 & 123 \\ 0.0000 & 162 \\ 0.0000 & 163 \\ 0.0000 & 164 \\ 0.0000 & 165 \\ 0.0000 & 166 \\ 0.0000 & 167\end{array}$


SUMMARY

COMPONENT CONTRIBUTIONS TO UNRELIABILITY

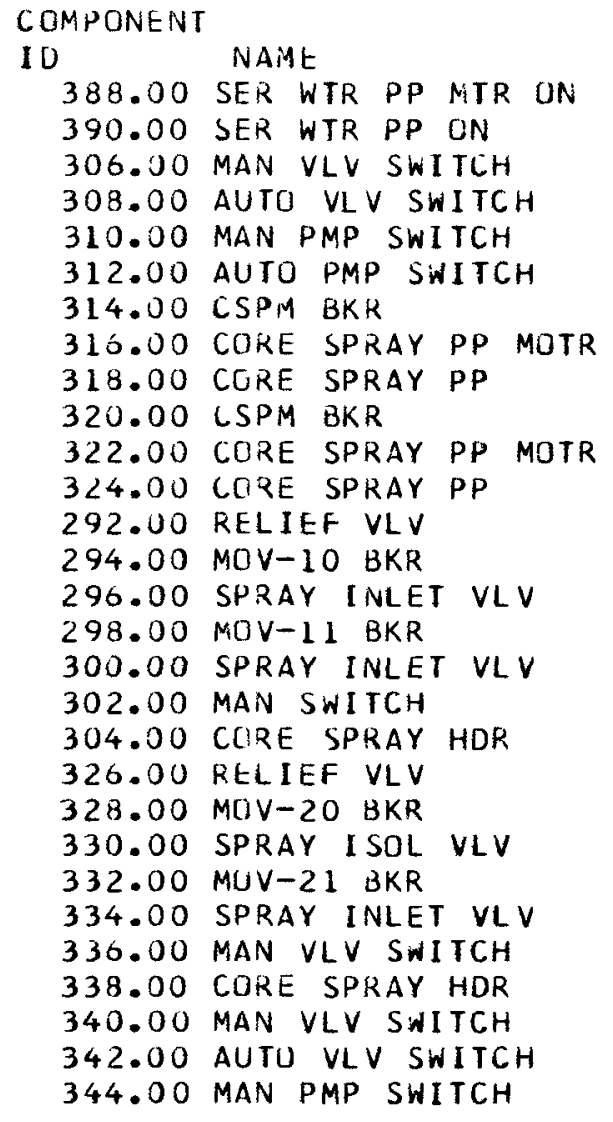

$\begin{array}{rl}\text { FAILURE } & \text { SERI AL } \\ \text { RATE*10*\$6 } & \text { PROBABILI TY } \\ 5.00 & 0 . \\ 5.00 & 0 . \\ 2.00 & 0.13078958 E-02 \\ 5.00 & 0.32697394 \mathrm{E}-02 \\ 2.00 & 0.13078958 \mathrm{E}-02 \\ 5.00 & 0.32697394 \mathrm{E}-02 \\ 5.00 & 0.32697394 \mathrm{E}-02 \\ 10.00 & 0.65394788 \mathrm{E}-02 \\ 10.00 & 0.65394788 \mathrm{E}-02 \\ 5.00 & 0.32697394 \mathrm{E}-02 \\ 10.00 & 0.65394788 \mathrm{E}-02 \\ 10.00 & 0.65394788 \mathrm{E}-02 \\ 2.00 & 0.13078958 \mathrm{E}-02 \\ 5.00 & 0.32697394 \mathrm{E}-02 \\ 0.50 & 0.32697394 \mathrm{E}-03 \\ 1.00 & 0.65394789 \mathrm{E}-03 \\ 0.10 & 0.65394788 \mathrm{E}-04 \\ 2.00 & 0.13078958 \mathrm{E}-02 \\ 0.20 & 0.13078958 \mathrm{E}-03 \\ 2.00 & 0 . \\ 5.00 & 0 . \\ 0.50 & 0 . \\ 1.00 & 0 . \\ 0.01 & 0 . \\ 2.00 & 0 . \\ 0.20 & 0 . \\ 2.00 & 0 . \\ 5.00 & 0 . \\ 2.00 & 0 .\end{array}$

SER I AL
PER CENT
0.
0.
0.7361
1.8402
0.7361
1.8402
1.8402
3.6803
3.6803
1.8402
3.6803
3.6803
0.7361
1.8402
0.1840
0.3680
0.0368
0.7361
0.0736
0.
0.
0.
0.
0.
0.
0.
0.0
0.
0.

\section{SERIAL SYSTEM}

RANK PROBABILITY

$0.14348443 E-12$

$0.14348443 E-12$

$0.83322538 E-08$

$0.20830634 E-07$

$0.83322538 \mathrm{E}-08$

$0.20830634 E-07$

$0.16447568 E-06$

$0.51646828 E-06$

$0.51646828 \mathrm{E}-06$

$0.16440157 \mathrm{E}-06$

$0.51654239 E-06$

$0.51654239 E-06$

$0.42944786 E-05$

$0.17599574 E-04$

$0.39468931 \mathrm{E}-06$

$0.13437097 \mathrm{E}-05$

$0.19508176 \mathrm{E}-07$

$0.42944786 \mathrm{E}-05$

$0.72798377 E-07$

$0.43573654 \mathrm{E}-05$

$0.17825329 E-04$

$0.40486960 E-06$

$0.13687662 E-05$

$0.23039947 E-09$

$0.43573654 E-05$

$0.75731236 E-07$

$0.11634555 \mathrm{E}-10$

$0.29086388 \mathrm{E}-10$

$0.11634555 \mathrm{E}-10$

$\begin{array}{lr}\text { SYSTEM } & \text { SYSTEM } \\ \text { PERCENT } & \text { RANK } \\ 0.0000 & 168 \\ 0.0000 & 169 \\ 0.0028 & 69 \\ 0.0070 & 62 \\ 0.0028 & 70 \\ 0.0070 & 63 \\ 0.0552 & 43 \\ 0.1734 & 33 \\ 0.1734 & 34 \\ 0.0552 & 44 \\ 0.1734 & 31 \\ 0.1734 & 32 \\ 1.4419 & 13 \\ 5.9093 & 8 \\ 0.1325 & 36 \\ 0.4512 & 18 \\ 0.0066 & 64 \\ 1.4419 & 14 \\ 0.0244 & 48 \\ 1.4630 & 11 \\ 5.9851 & 7 \\ 0.1359 & 35 \\ 0.4596 & 17 \\ 0.0001 & 101 \\ 1.4630 & 12 \\ 0.0254 & 47 \\ 0.0000 & 142 \\ 0.0000 & 124 \\ 0.0000 & 143\end{array}$


CUMPCNENT CONTRIBUTIONS TO UNRELIABILITY

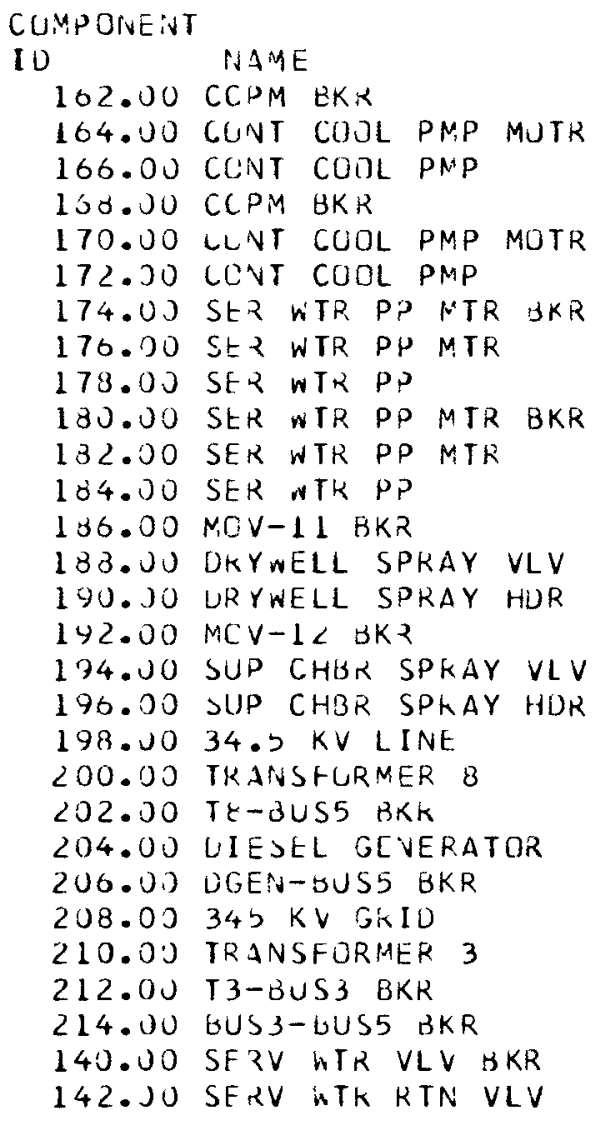

$\begin{array}{rc}\text { FAILURE } & \text { SERIAL } \\ \text { RATE } 10 * \$ 6 & \text { PROBABILITY } \\ 5.00 & 0.32697394 E-02 \\ 10.00 & 0.63354738 E-02 \\ 10.00 & 0.65344738 E-02 \\ 5.00 & 0.32697394 F-02 \\ 10.00 & 0.65354788 E-02 \\ 10.00 & 0.65394788 E-02 \\ 5.00 & 0.32691394 E-02 \\ 10.00 & 0.65394738 E-02 \\ 10.00 & 0.65394788 E-02 \\ 5.00 & 0.32697394 E-02 \\ 10.00 & 0.05394738 E-02 \\ 10.00 & 0.65394738 E-02 \\ 1.00 & 0.55394789 E-03 \\ 0.01 & 0.05394738 E-05 \\ 0.00 & 0.65394789 E-06 \\ 5.00 & 0.32697394 E-02 \\ 0.01 & 0.05394798 E-05 \\ 0.00 & 0.65394789 E-06 \\ 0.40 & 0.26157915 E-03 \\ 0.40 & 0.26157915 E-03 \\ 5.00 & 0.32697394 E-02 \\ 0.40 & 0.26157915 E-03 \\ 3.00 & 0.32697394 E-02 \\ 0.00 & 0.26157915 E-07 \\ 0.40 & 0.26157915 E-03 \\ 5.00 & 0.32697394 E-02 \\ 5.00 & 0.32697394 E-02 \\ 1.00 & 0.05354789 E-01 \\ 0.01 & 0.65394788 E-05\end{array}$

$\begin{array}{lrl}\text { SERIAL } & \text { SERIAL } & \text { SYSTFM } \\ \text { PFRCENT } & \text { RANK } & \text { PROBABILITY } \\ 1.8402 & 14 & 0.18311600 E-06 \\ 3.6303 & 1 & 0.57527821 E-06 \\ 3.6803 & 2 & 0.57527821 E-06 \\ 1.8472 & 15 & 0.18303352 E-00 \\ 3.68 J 3 & 3 & 0.57536070 E-06 \\ 3.6803 & 4 & 0.57536070 E-06 \\ 1.84 J 2 & 16 & 0.18311600 E-06 \\ 3.6803 & 5 & 0.57527821 E-06 \\ 3.68 J 3 & 6 & 0.57527821 E-06 \\ 1.8402 & 17 & 0.18303352 E-06 \\ 3.6803 & 7 & 0.57536070 E-06 \\ 3.6803 & 8 & 0.57536070 E-06 \\ 0.3630 & 51 & 0.25535184 E-08 \\ 0.0037 & 109 & 0.28372733 E-12 \\ 0.0004 & 133 & 0.29412187 E-14 \\ 1.8402 & 18 & 0.12862219 E-07 \\ 0.0037 & 110 & 0.11167798 E-12 \\ 0.0004 & 134 & 0.11208860 E-14 \\ 0.1472 & 70 & 0.71942194 E-11 \\ 0.1472 & 71 & 0.71942194 E-11 \\ 1.8402 & 19 & 0.1 \\ 0.1472 & 72 & 0.14388437 E-10 \\ 1.8402 & 20 & 0.1 \\ 0.0000 & 137 & 0.21501446 E-18 \\ 0.1472 & 13 & 0.14386279 E-10 \\ 1.8402 & 21 & 0 . \\ 1.8402 & 22 & 0 . \\ 0.3630 & 52 & 0.15256264 E-05 \\ 0.0037 & 111 & 0.28867524 E-09\end{array}$

$\begin{array}{lr}\text { SYSTEM } & \text { SYSIEM } \\ \text { PERCENT } & \text { RANK } \\ 0.0615 & 39 \\ 0.1932 & 27 \\ 0.1932 & 28 \\ 0.0615 & 41 \\ 0.1932 & 23 \\ 0.1932 & 24 \\ 0.0615 & 40 \\ 0.1932 & 29 \\ 0.1932 & 30 \\ 0.0615 & 42 \\ 0.1932 & 25 \\ 0.1932 & 26 \\ 0.0009 & 72 \\ 0.0000 & 149 \\ 0.0000 & 180 \\ 0.0043 & 66 \\ 0.0000 & 170 \\ 0.0000 & 183 \\ 0.0000 & 145 \\ 0.0000 & 146 \\ 0.0 & 0 \\ 0.0000 & 140 \\ 0.0 & 0 \\ 0.0090 & 196 \\ 0.0000 & 141 \\ 0 . & 0 \\ 0.0152 & 0 \\ 0.5122 & 15 \\ 0.0001 & 99 \\ & \end{array}$




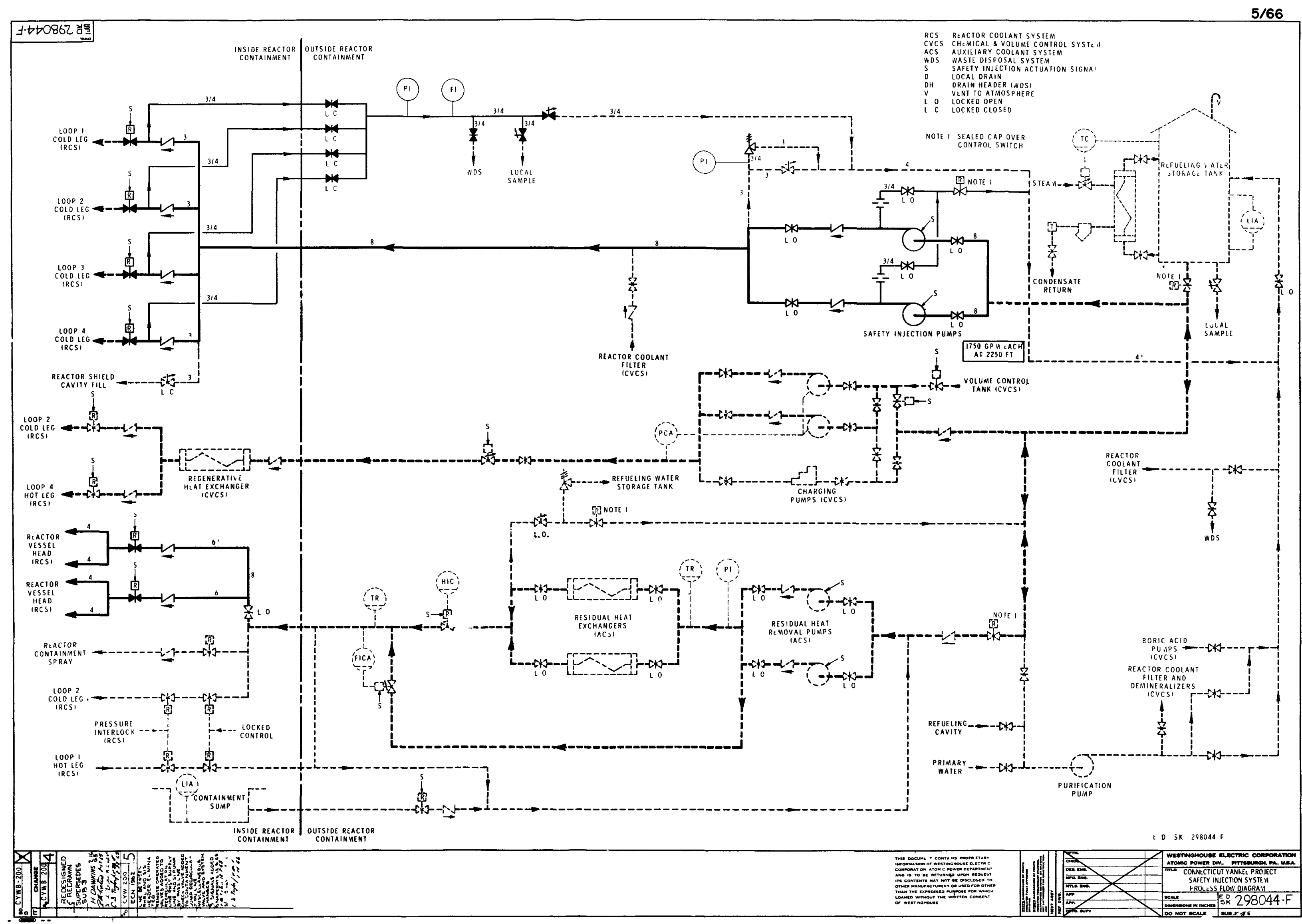

FIGURE 3.17 


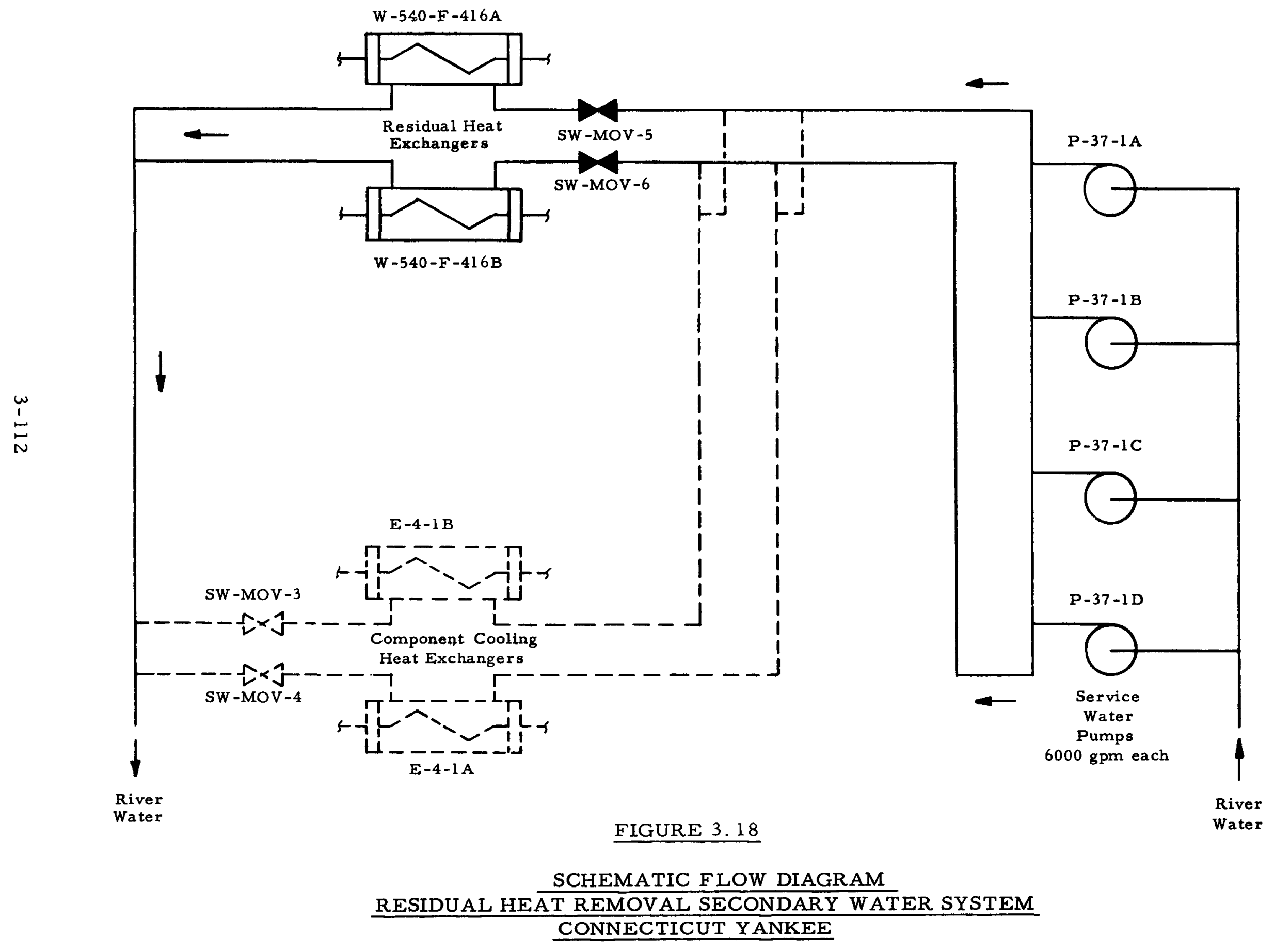


S.l. SAFETY INJECTION

S. S. STATION SERVICE

TRANS TRANSFORMER

S.1. PUMP

$P-15-1 B$

CHARGE PUMP

$P-18-1 B$
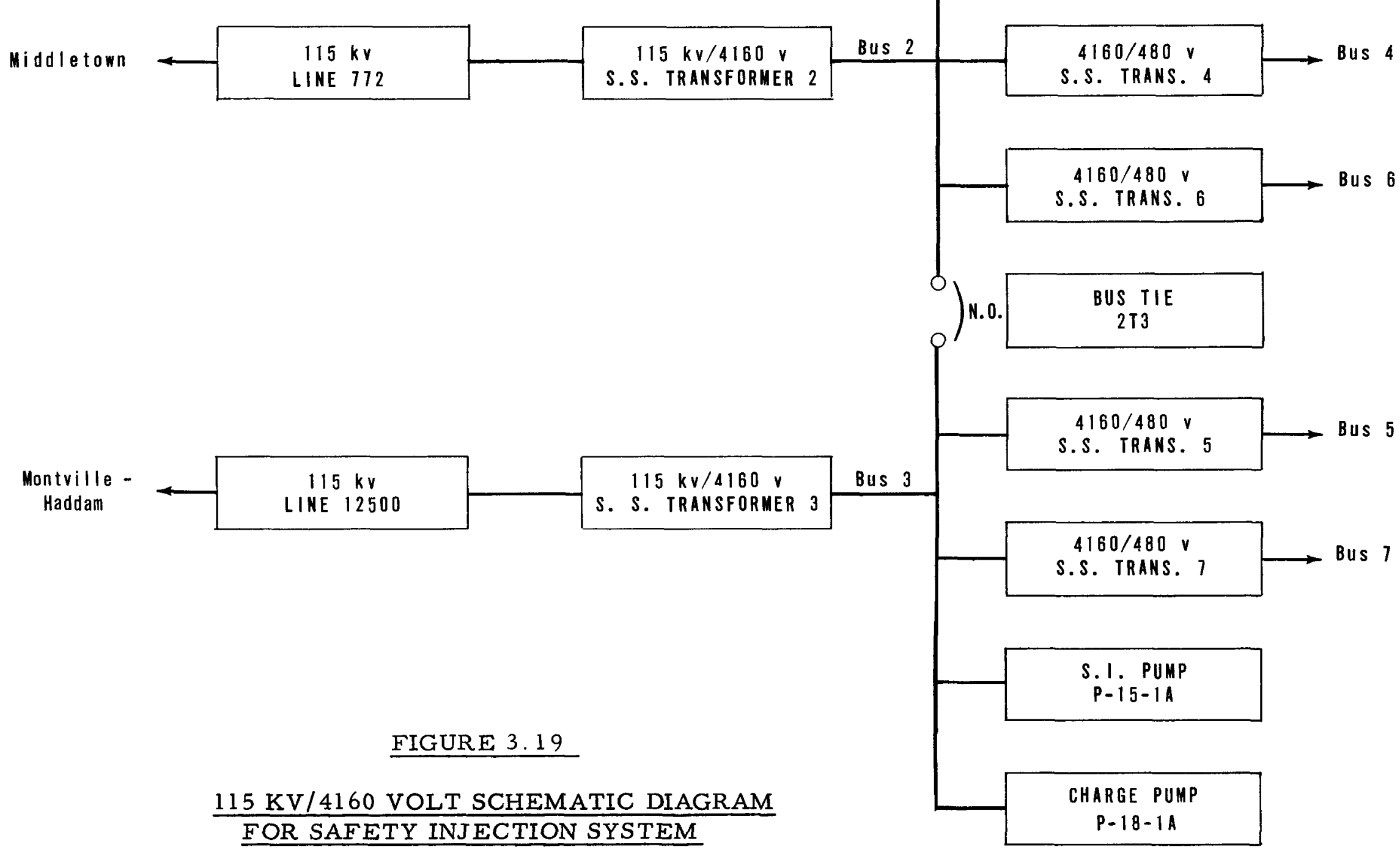


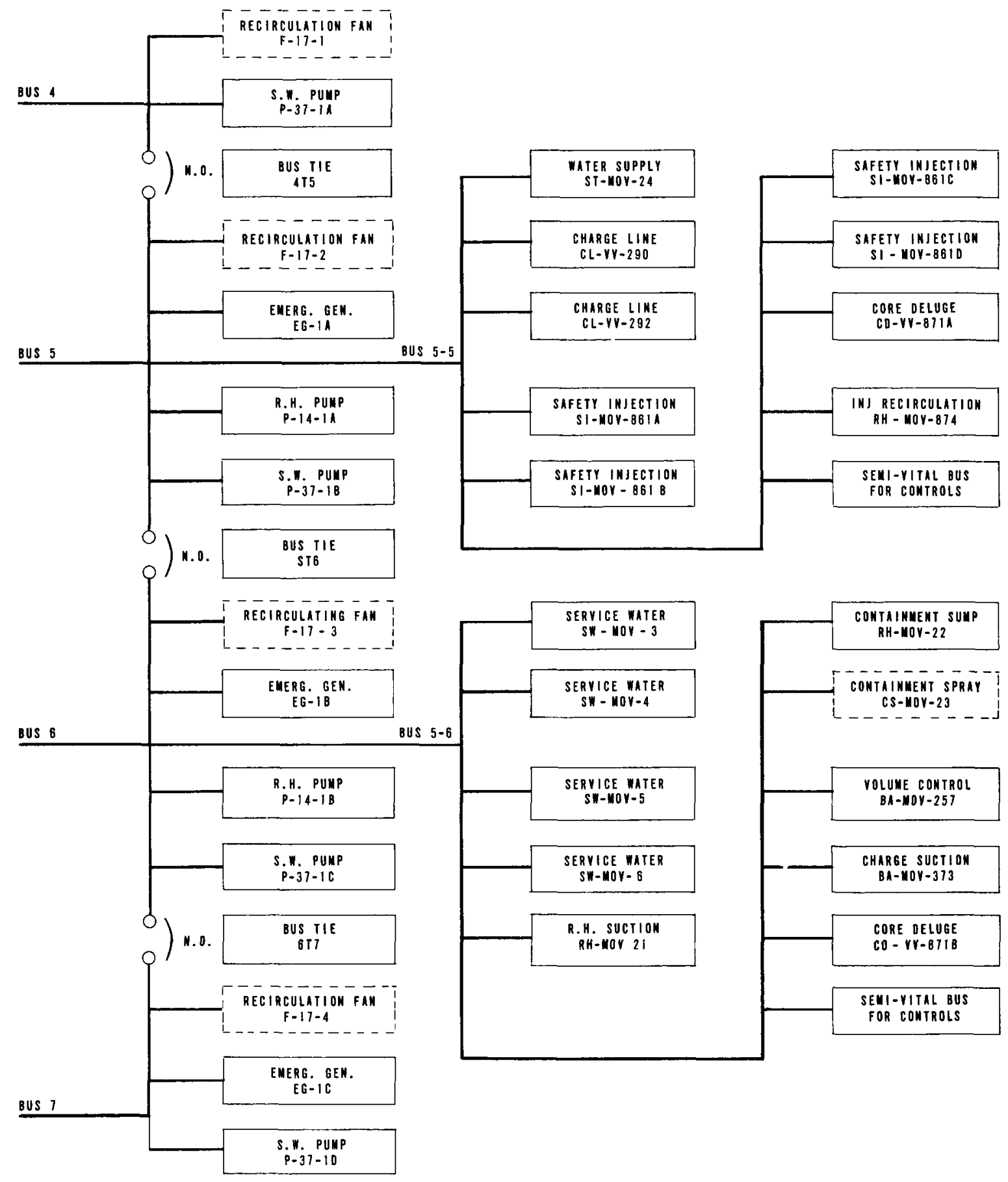

FIGURE 3.20

480 VOLT SCHEMATIC DIAGRAM

FOR SAFETY INJECTION SYSTEM 


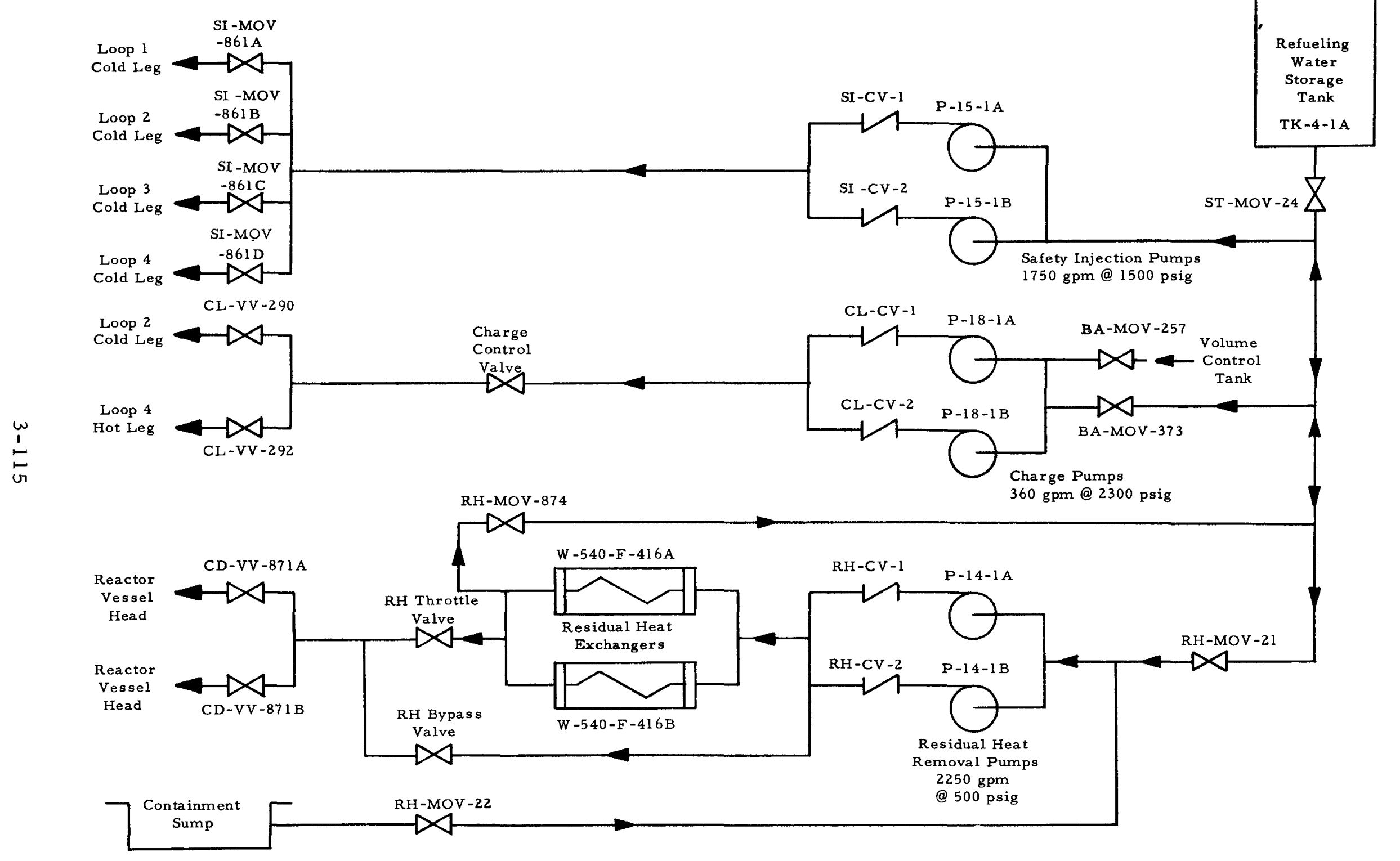

FIGURE 3.21 


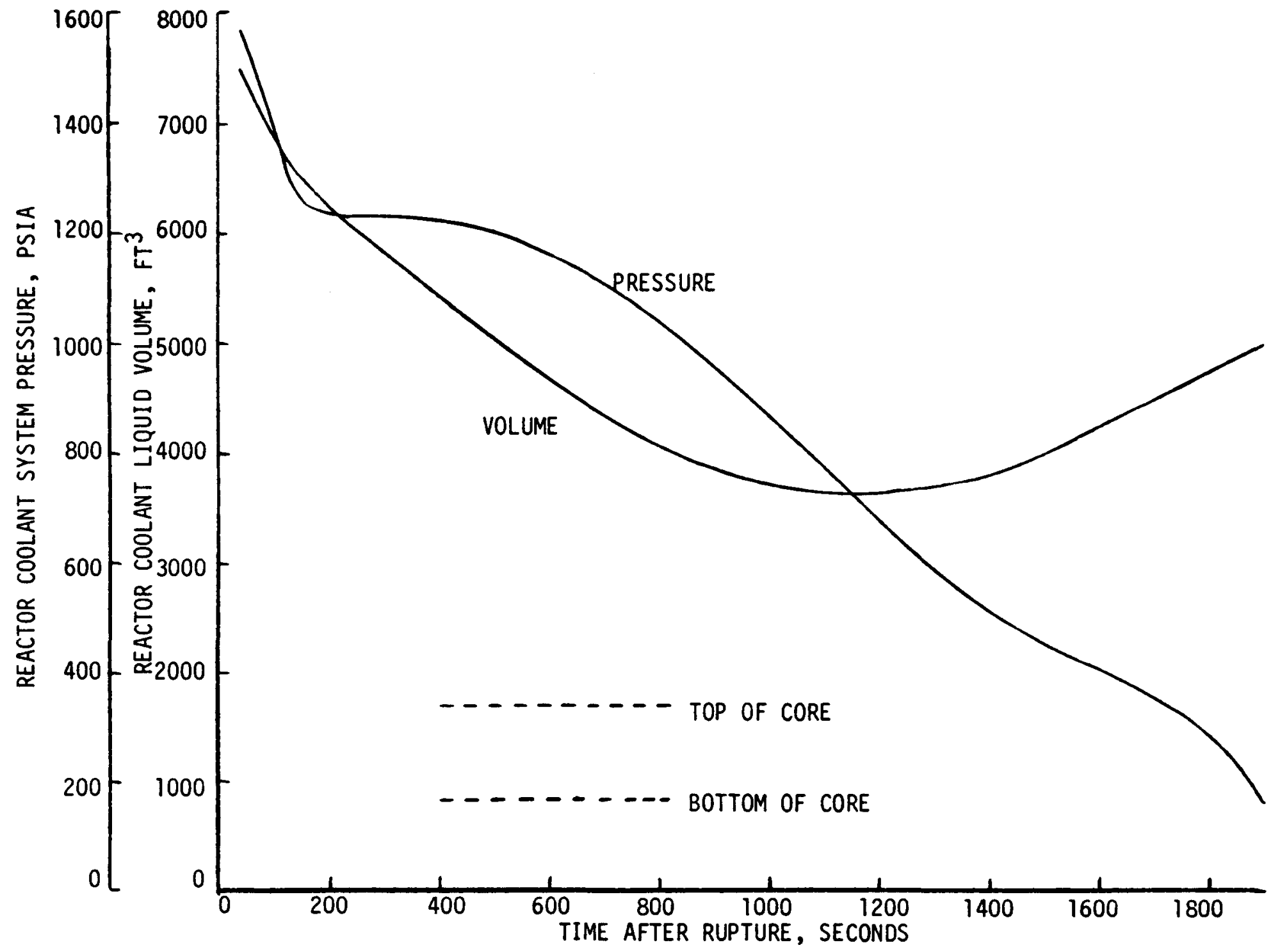

FIGURE 3.22 
Function Deliver borated water to reactor vessel for cooling reactor core following a loss-of-coolant accident

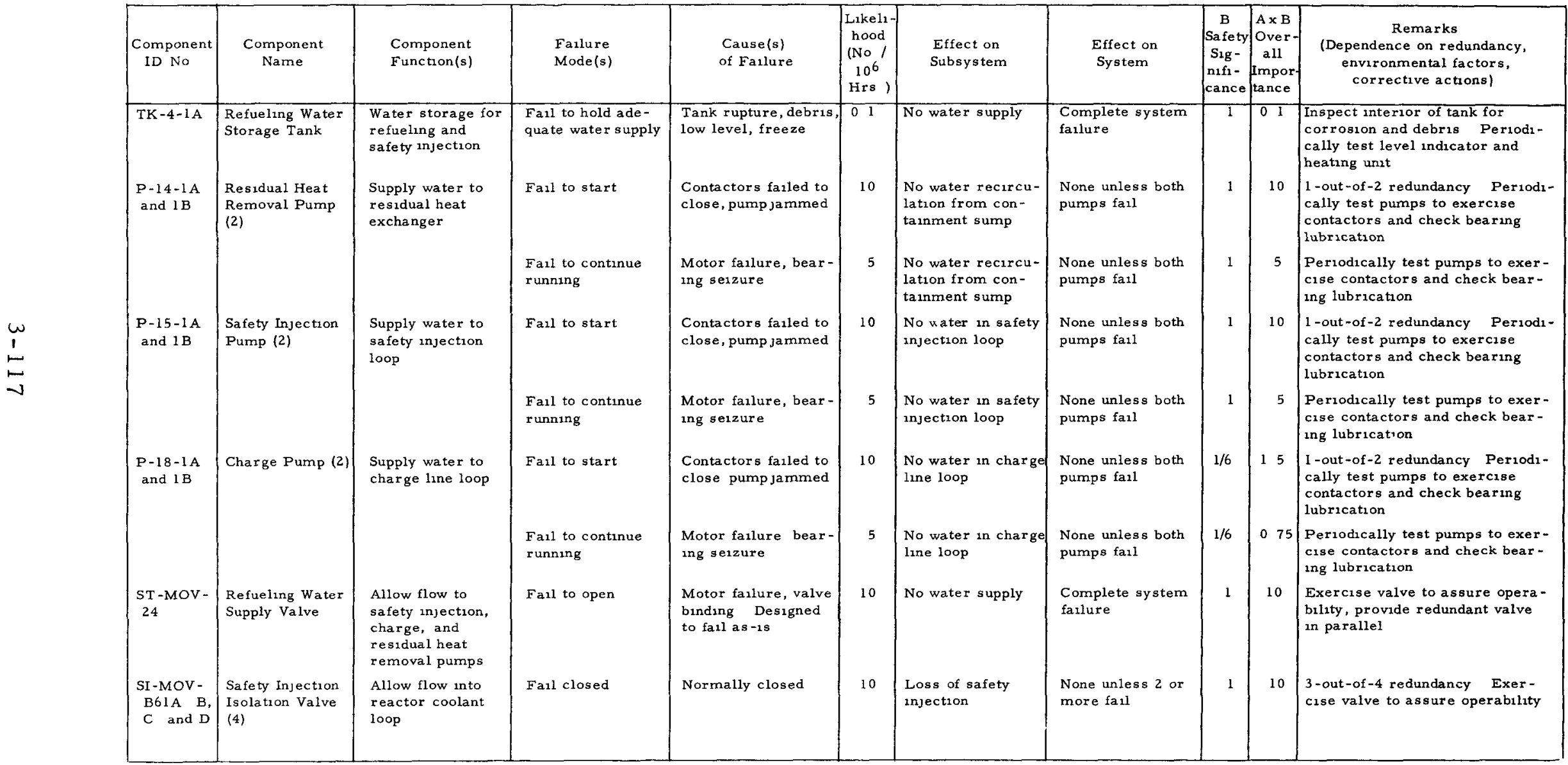




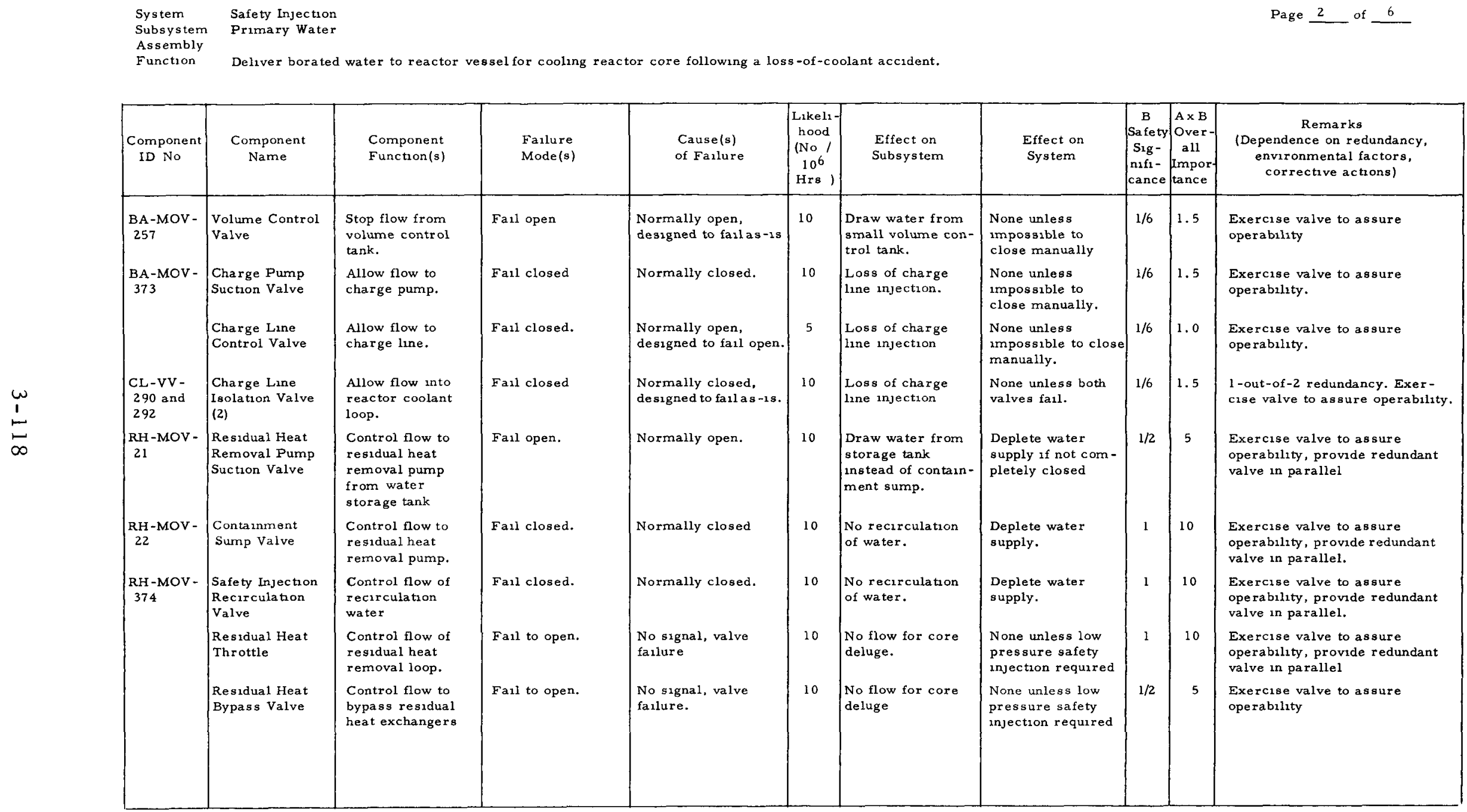

FIGURE 3.23 (continued) 
Deliver borated water to reactor vessel for cooling reactor core following a loss-of-coolant accident

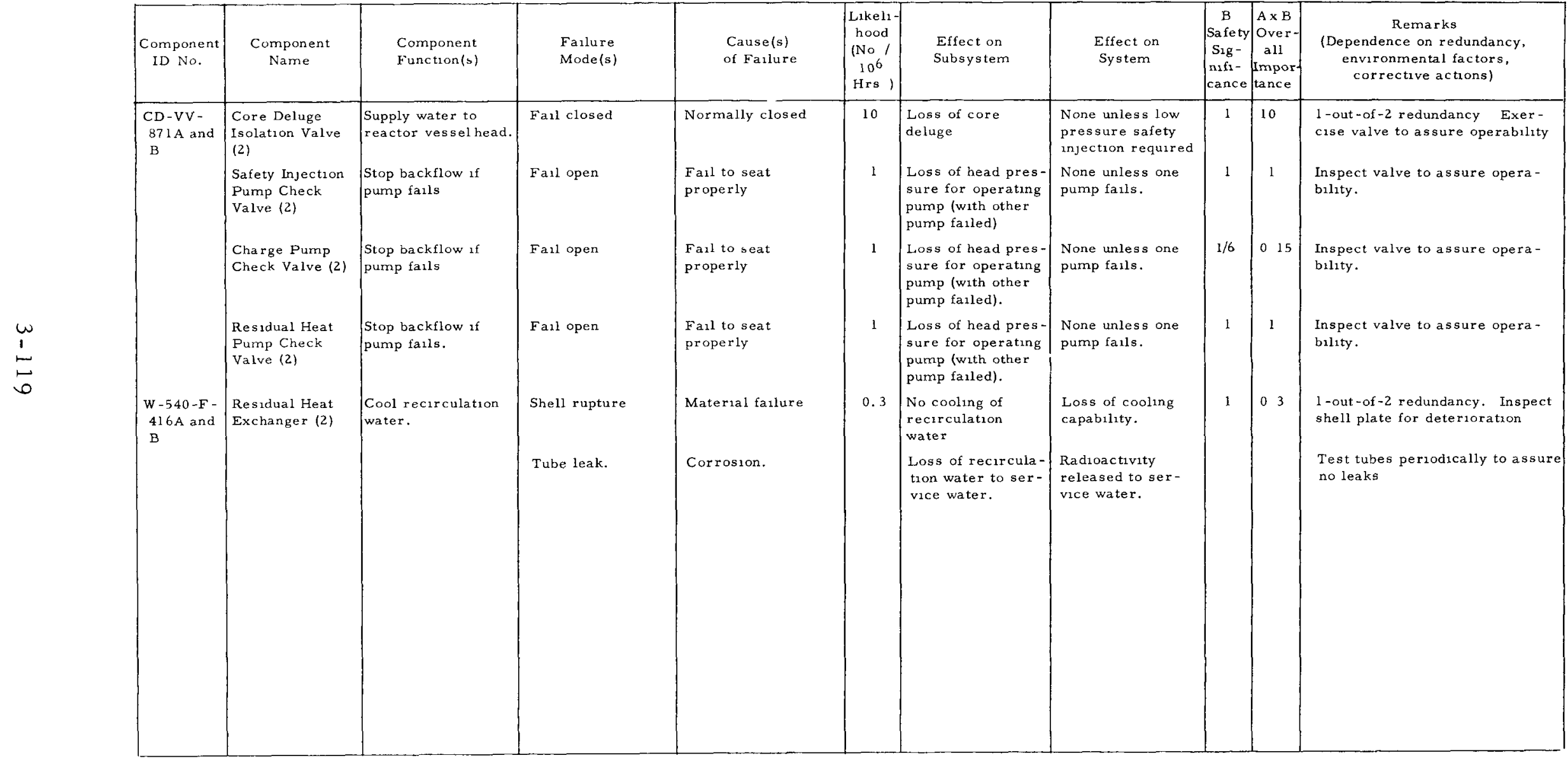


Safety Injection

Page 4 of 6

Deliver service water to residual heat exchanger for cooling (primary) recirculating water

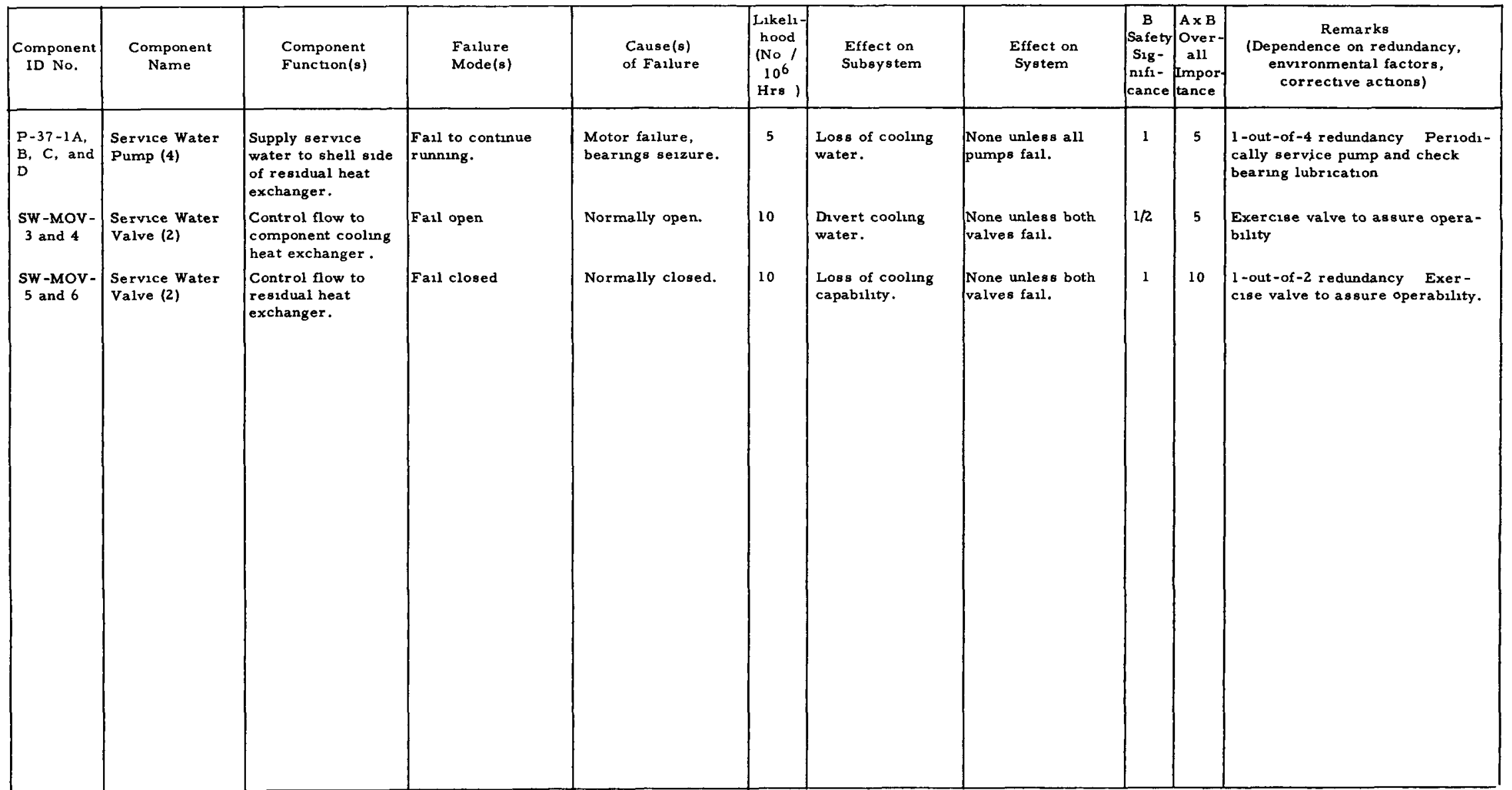

FIGURE 3.23 (continued) 
Provide power and control to mechanical system.

\begin{tabular}{|c|c|c|c|c|c|c|c|c|c|c|}
\hline $\begin{array}{l}\text { Component } \\
\text { ID No. }\end{array}$ & $\begin{array}{l}\text { Component } \\
\text { Name }\end{array}$ & $\begin{array}{l}\text { Component } \\
\text { Function(s) }\end{array}$ & $\begin{array}{l}\text { Faslure } \\
\text { Mode(s) }\end{array}$ & $\begin{array}{l}\text { Cause(s) } \\
\text { of Failure }\end{array}$ & $\begin{array}{c}\text { Likell } \\
\text { hood } \\
\text { (No } / \\
10^{6} \\
\text { Hrs })\end{array}$ & $\begin{array}{l}\text { Effect on } \\
\text { Subsystem }\end{array}$ & $\begin{array}{l}\text { Effect on } \\
\text { System }\end{array}$ & $\left|\begin{array}{c}\text { B } \\
\text { Safety } \\
\text { Sig- } \\
\text { nifi- } \\
\text { cance }\end{array}\right|$ & $\mid \begin{array}{c}A \times B \\
\text { Over } \\
\text { all } \\
\text { Impor } \\
\text { tance }\end{array}$ & $\begin{array}{c}\text { Remarks } \\
\text { (Dependence on redundancy, } \\
\text { environmental factors, } \\
\text { corrective actions) }\end{array}$ \\
\hline $\begin{array}{l}772 \text { and } \\
12500\end{array}$ & Power Line & $\begin{array}{l}\text { Provide outside } \\
\text { power source. }\end{array}$ & $\begin{array}{l}\text { Fall to continue } \\
\text { providing power. }\end{array}$ & $\begin{array}{l}\text { Loss of outside } \\
\text { power source. }\end{array}$ & 0.1 & $\begin{array}{l}\text { Complete loss of } \\
\text { power and control. }\end{array}$ & $\begin{array}{l}\text { None unless both } \\
\text { lines fall. }\end{array}$ & 1 & 0.1 & 1 -out-of -2 redundancy. \\
\hline 2 and 3 & $\begin{array}{l}\text { Station Service } \\
\text { Transformer(2) }\end{array}$ & $115 \mathrm{KV} / 4160$ volt. & $\begin{array}{l}\text { Fail to supply } \\
4160 \text { volt. }\end{array}$ & $\begin{array}{l}\text { Loss of outside } \\
\text { power, transformer } \\
\text { failure. }\end{array}$ & 5 & $\begin{array}{l}\text { Complete loss of } \\
\text { power and control. }\end{array}$ & $\begin{array}{l}\text { None unless both } \\
\text { transformers } \\
\text { fall. }\end{array}$ & 1 & 5 & 1 -out-of-2 redundancy. \\
\hline $\begin{array}{l}4,5,6 \\
\text { and } 7\end{array}$ & $\begin{array}{l}\text { Station Service } \\
\text { Transformer (4) }\end{array}$ & $4160 / 480$ volt. & $\begin{array}{l}\text { Fa1l to supply } \\
480 \text { volt. }\end{array}$ & $\begin{array}{l}\text { Loss of } 4160 \text { volt, } \\
\text { transformer fallure. }\end{array}$ & 5 & $\begin{array}{l}\text { Loss of } 480 \text { volt } \\
\text { power and all con- } \\
\text { trol. }\end{array}$ & $\begin{array}{l}\text { None unless Nos. } 5 \\
\text { and } 6 \text { fail. }\end{array}$ & 1 & 5 & Inspect perrodically. \\
\hline $2 \mathrm{~T} 3$ & Bus Tie & $\begin{array}{l}\text { Bus the between } \\
4160 \text { volt buses. }\end{array}$ & Fall to close. & $\begin{array}{l}\text { Loss of automatic } \\
\text { switching. }\end{array}$ & 2 & $\begin{array}{l}\text { Partial loss of } \\
4160 \text { volt (with } \\
\text { Transformers } 2 \\
\text { or } 3 \text { falled). }\end{array}$ & $\begin{array}{l}\text { None unless } \\
\text { Transformer } 2 \text { or } \\
3 \text { fa1ls. }\end{array}$ & 1 & 2 & $\begin{array}{l}\text { Check automatic switching } \\
\text { periodically. }\end{array}$ \\
\hline $\begin{array}{c}4 \mathrm{~T} 5,5 \mathrm{~T} 6 \\
\text { and } 6 \mathrm{~T} 7\end{array}$ & Bus Tie (3) & $\begin{array}{l}\text { Bus tre between } \\
480 \text { volt buses. }\end{array}$ & Fall to close. & Switchgear Falure. & 2 & $\begin{array}{l}\text { Partial loss of } \\
480 \text { volt (with } \\
\text { Transformers } 4 \\
5,6 \text {, or } 7 \text { falled). }\end{array}$ & $\begin{array}{l}\text { None unless Buses } \\
5 \text { and } 6 \text { are out of } \\
\text { power. }\end{array}$ & 1 & 2 & Check switchgear periodically. \\
\hline \multirow[t]{5}{*}{$\begin{array}{l}\text { EG-1A, B, } \\
\text { and C }\end{array}$} & $\begin{array}{l}\text { Emergency } \\
\text { Generator }(3)\end{array}$ & $\begin{array}{l}\text { Provide power if } \\
\text { outside power fails. }\end{array}$ & Fail to start. & $\begin{array}{l}\text { Diesel engine or } \\
\text { starter motor fa1 - } \\
\text { ure, lack of fuel. }\end{array}$ & & $\begin{array}{l}\text { Loss of } 480 \text { volt } \\
\text { power. }\end{array}$ & $\begin{array}{l}\text { None uniess more } \\
\text { than on generator } \\
\text { falls. }\end{array}$ & 1 & & $\begin{array}{l}\text { Test periodically to assure } \\
\text { operability. }\end{array}$ \\
\hline & & & $\begin{array}{l}\text { Fail to continue } \\
\text { running. }\end{array}$ & $\begin{array}{l}\text { Diesel engine or } \\
\text { generator failure, } \\
\text { lack of fuel. }\end{array}$ & & & & & & \\
\hline & $\begin{array}{l}\text { Automatic } \\
\text { Permissive } \\
\text { Switch }\end{array}$ & $\begin{array}{l}\text { Unblock automatic } \\
\text { operation when } \\
\text { reactor coolant } \\
\text { pressure } 1 \text { s above } \\
1700 \text { psig. }\end{array}$ & Fall open. & Relay failure. & 5 & $\begin{array}{l}\text { No initiation } \\
\text { signal. }\end{array}$ & $\begin{array}{l}\text { No safety injec- } \\
\text { tion. }\end{array}$ & 1 & 5 & $\begin{array}{l}\text { Test periodically, provide } \\
\text { failure indicator. }\end{array}$ \\
\hline & Manual Switch & $\begin{array}{l}\text { Allow manual } \\
\text { actuation. }\end{array}$ & Fa1l to close. & $\begin{array}{l}\text { Contactor fasled to } \\
\text { close. }\end{array}$ & 5 & $\begin{array}{l}\text { No initiation } \\
\text { signal. }\end{array}$ & $\begin{array}{l}\text { None unless auto- } \\
\text { matic operation } \\
\text { fails. }\end{array}$ & 1 & 5 & Test periodically. \\
\hline & & & $\begin{array}{l}\text { Premature opera- } \\
\text { tion. }\end{array}$ & Operator error. & & None. & None. & & & Reactor shutdown. \\
\hline
\end{tabular}




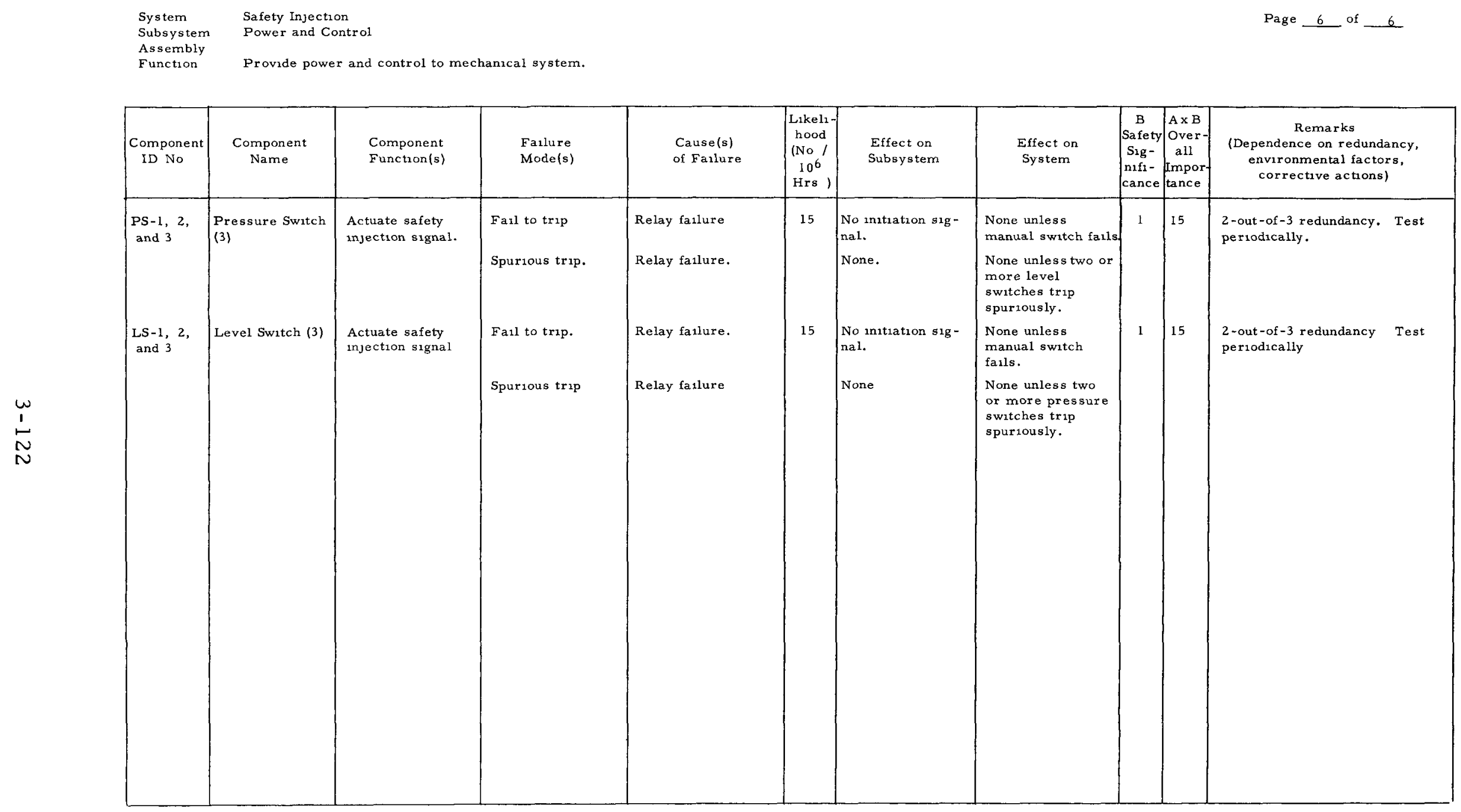

FIGURE 3.23 (continued) 


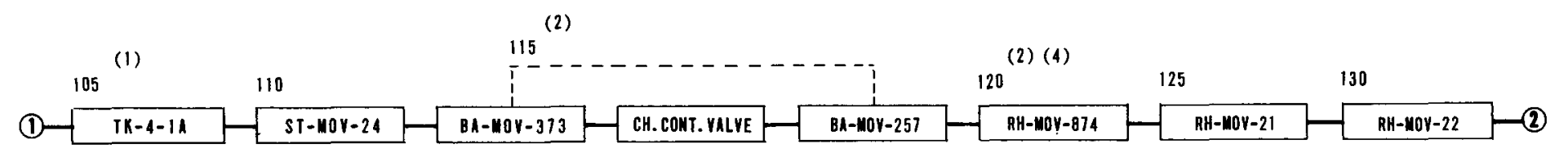

$\stackrel{w}{1}$

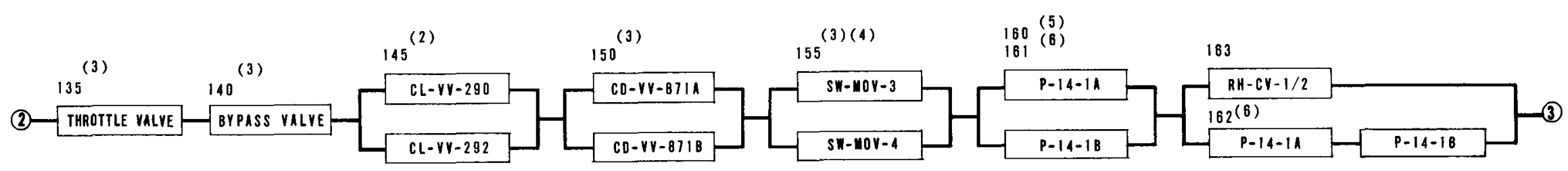
NOTE: (1) TYPICAL FUNCTION 1.D. NO. FOR ARMM: REFER TO FIGURE 11.
(2) FUMCTION NOT REQUIRED For CORE DELUGE ONLY (i.e. WithOUT SAFETY INJECTION)
(3) FUNCTION NOT REQUIRED FOR HIGH PRESSURE SAFETY INJECTION (i.e. WITHOUT CORE DELUGE)
(4) Function NOT REQUIRED FOR LOW PRESSURE SAFETY INJECTION (i.e. WITH CORE DELUGE)
(5) FUNCTION TO START
(6) Function to CONTINUE RUMMING
(7) FUnCTION WITHOUT RECIRCULATION

FIGURE 3.24

\section{RELIABILITY BLOCK DIAGRAM \\ SAFETY INJECTION SYSTEM \\ CONNECTICUT YANKEE}




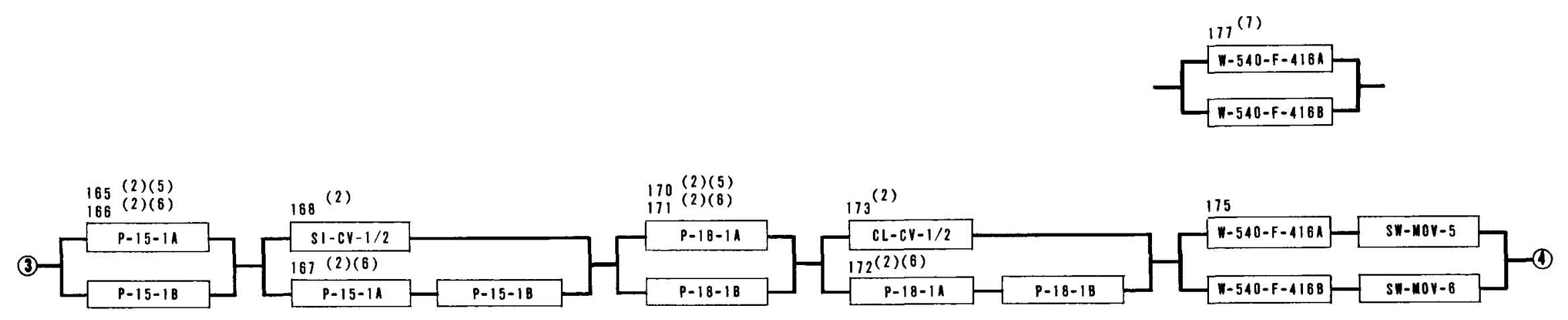

$\omega$
1
$N$
$N$

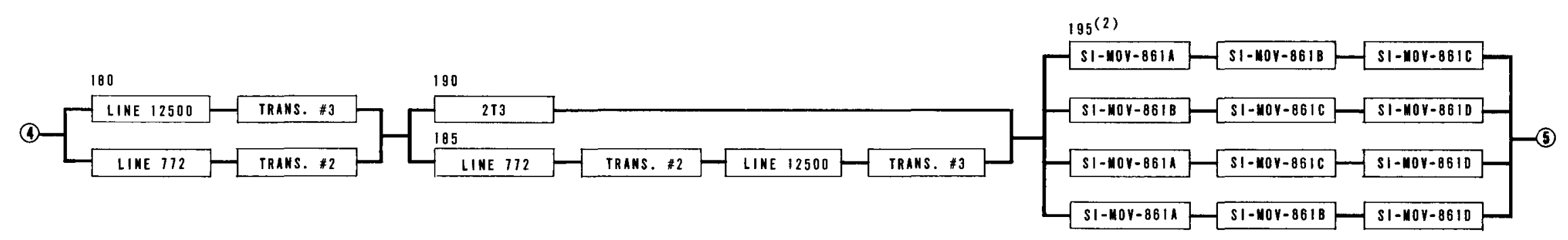

FIGURE 3.24 (continued) 


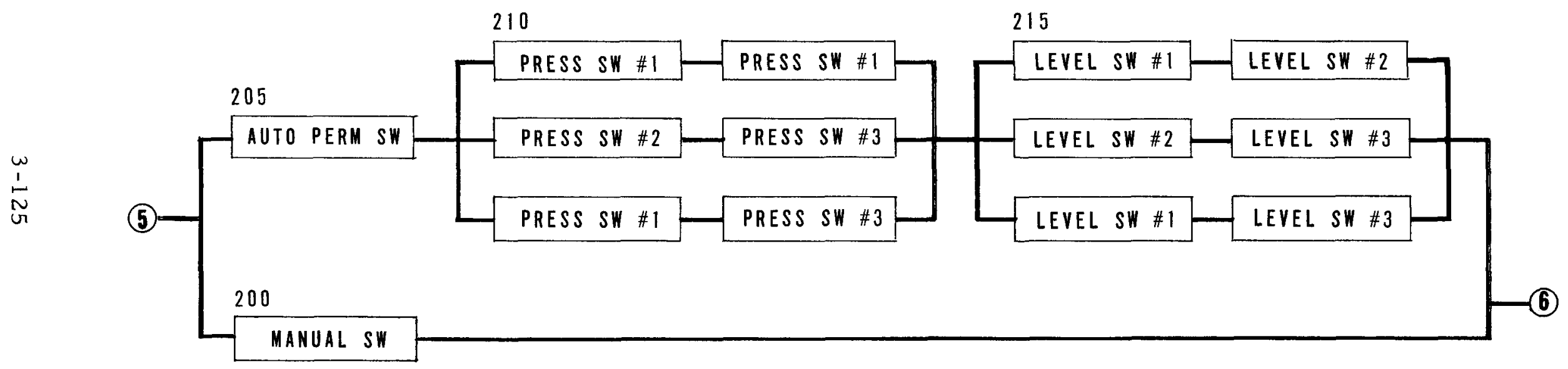

FIGURE 3.24 (continued) 


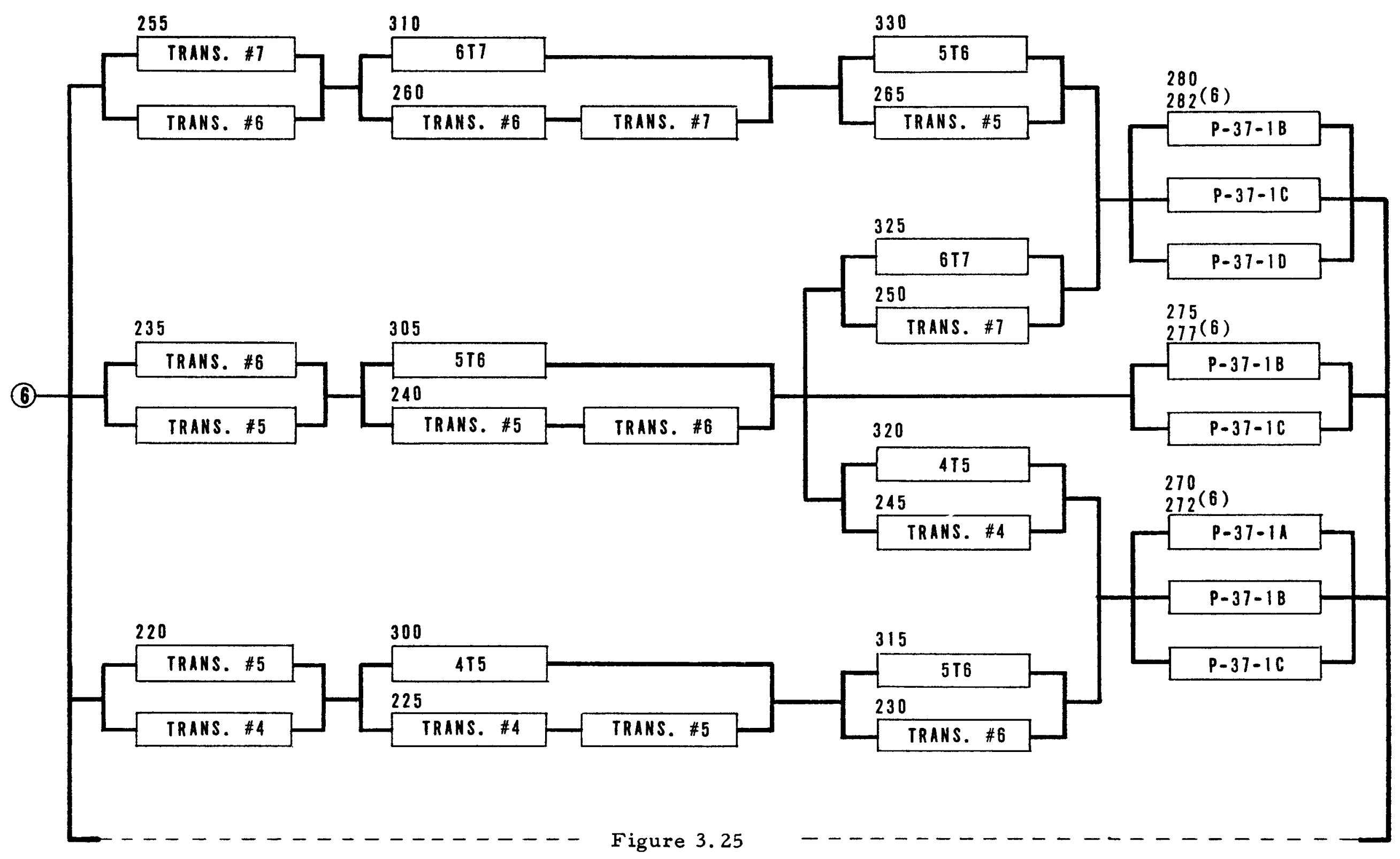




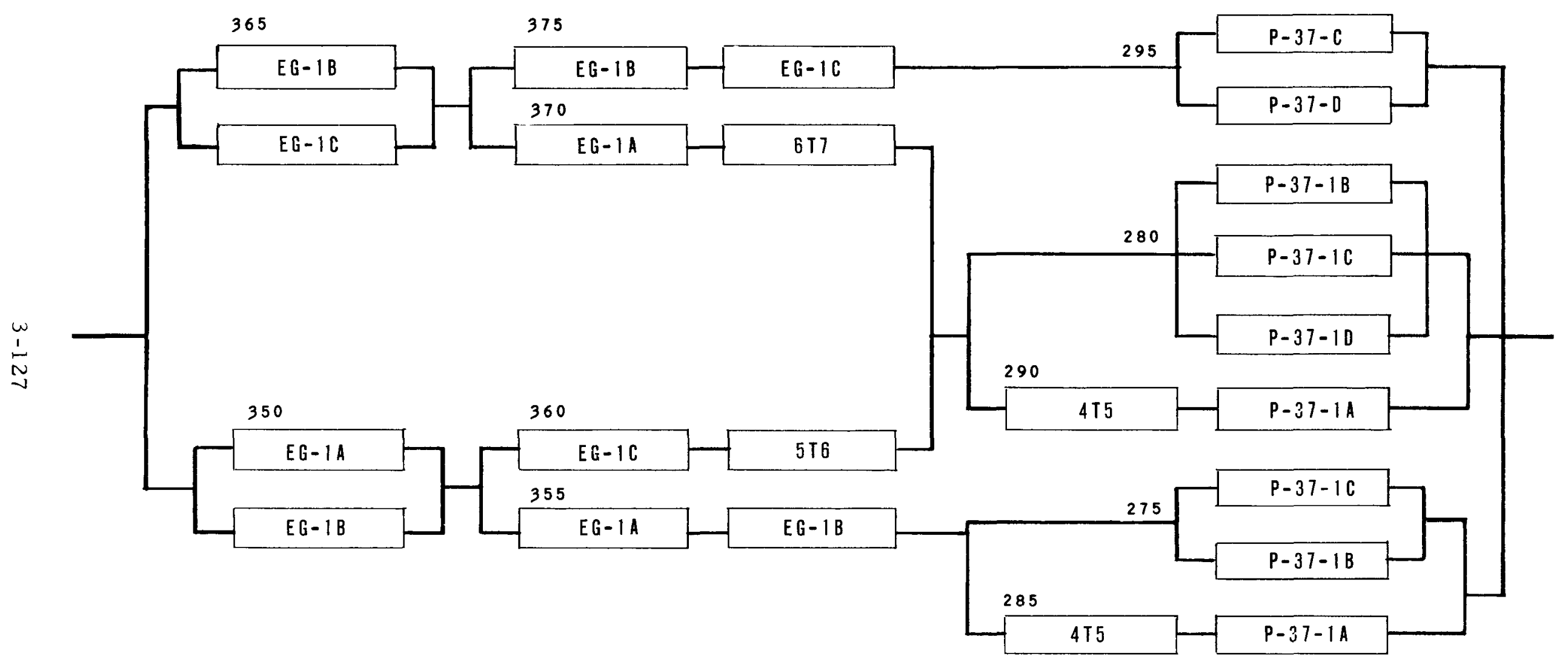

FIGURE 3.25

RELIABILITY BLOCK DIAGRAM EMERGENCY POWER FOR CORE DELUGE SYSTEM 


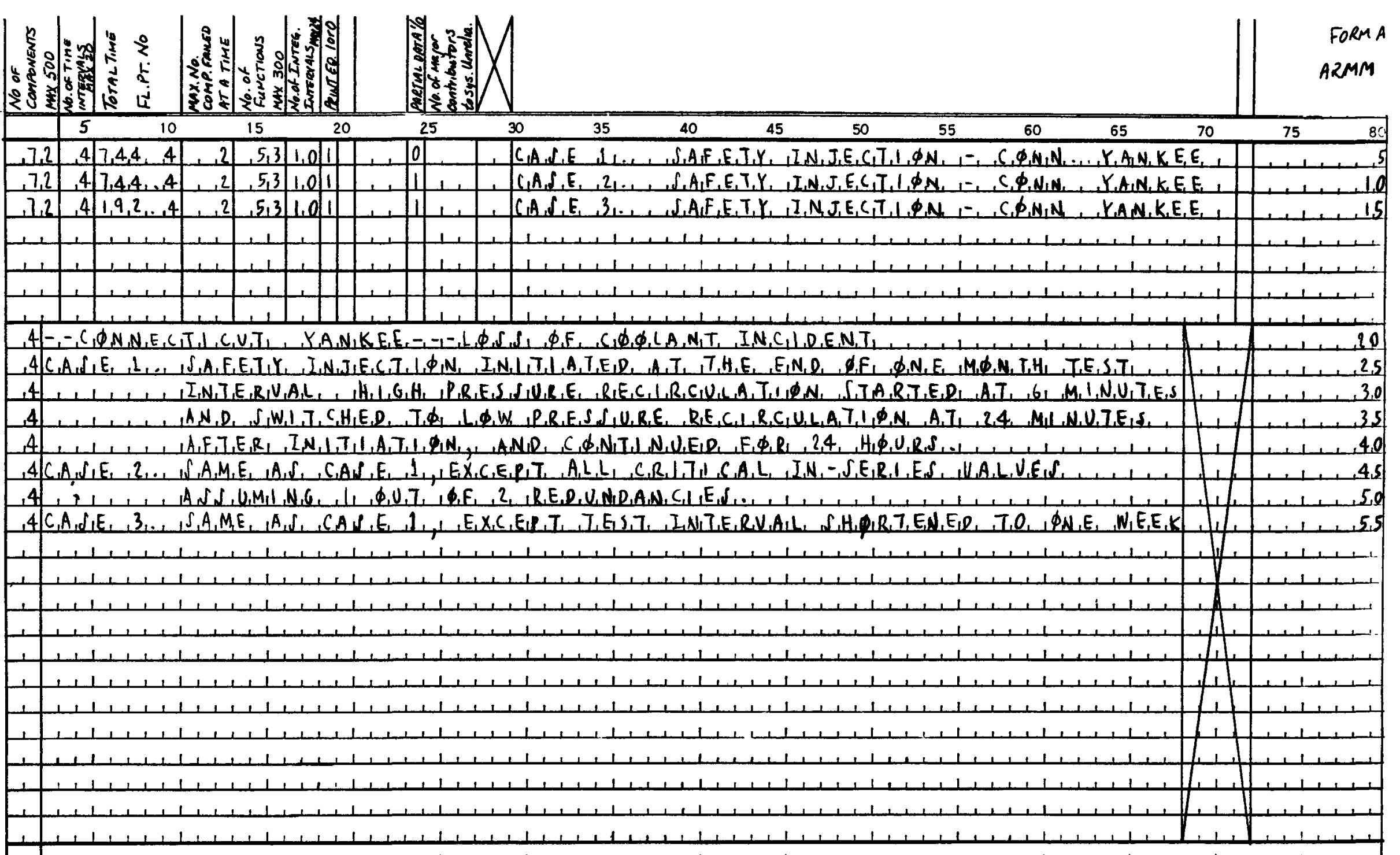

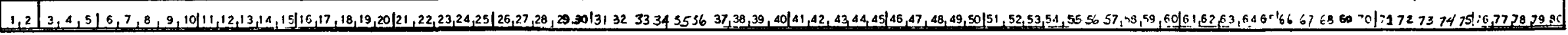

FIGURE 3.26

ARMM INPUT LOADSHEETS - CASE 1 


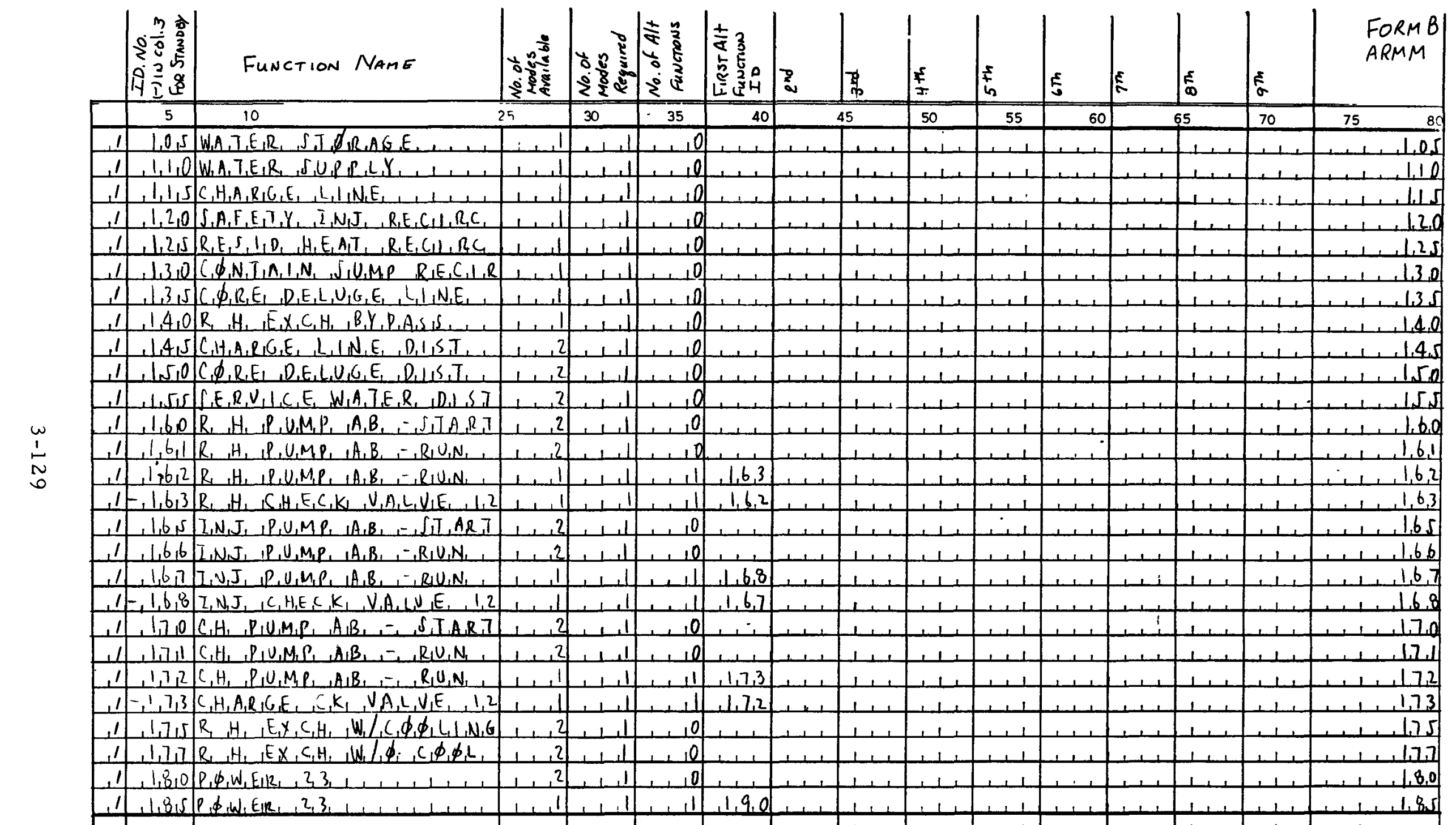

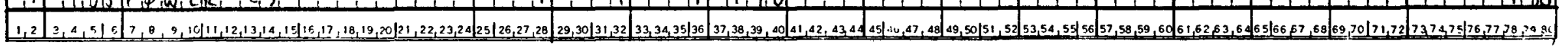

FIGURE 3.26 (continued) 


\begin{tabular}{|c|c|c|c|c|c|c|c|c|c|c|c|c|c|c|c|}
\hline & 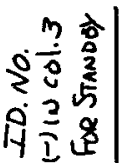 & Function Name & 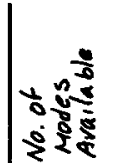 & 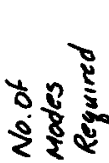 & 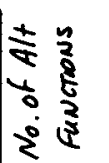 & 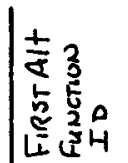 & $\stackrel{2}{a}$ & 尊 & F & $f_{0}$ & $\xi$ & E & $\left.\right|_{\infty} ^{E}$ & |हू & $\begin{array}{l}\text { FoRMB } \\
\text { ARMM }\end{array}$ \\
\hline & $\frac{2}{5}$ & 10 & 25 & 30 & $\frac{35}{35}$ & 40 & & 45 & 50 & 55 & 60 & & 65 & 70 & \\
\hline & -190 & $R, 0, W, E, R, 2, T, 3, \ldots, \ldots$ & & .1 .1 & $\overline{1+11}$ & .1 .8 .5 & & & & +1 & & & & & 190 \\
\hline 1 & $4.9,5$ & SAF,ETYY, IN,J, D, ST, & 1.4 & $.1,3$ & .1 .10 & & & & 1. & 1 & & & 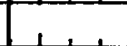 & . & 1.95 \\
\hline 1 & $.2,0,0$ & $M, A, N, V_{1} A_{1} L_{1}, \phi_{1}, P_{1}, R, A_{1} T_{1}, \phi N_{1}$ & 1,1 & .1 .1 & $-2 .+3$ & $.2,0,5$ & A, $2,1,0$ & $A, 2,1,5$ & & . & & & & & $2,1,200$ \\
\hline 1 & $2,0,5$ & $A_{1} \cup, \phi_{1}, \phi, P, \in, R_{1}, A_{2}, \phi_{1}, N_{1}, \ldots$ & 1,1 & .1 .1 & .1 .1 & $., 2,0$ & & & 1 & +1 & & & + & . & 20,5 \\
\hline .1 & $2,2,0$ & $P R_{1} E_{1} S S_{1}, R_{1} E_{1} \in S, E, N, Q_{1} R_{2}$ & 3 & $-1,2$ & 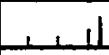 & $, 2,0,0$ & & & 1 & -1 & & & 1. & 1 & $+,+, 2,10$ \\
\hline . & $.2,15$ & $L, E, V, E, L, V, E, N_{1} S, R, R$, & $1,1,3$ & $1+11^{2}$ & 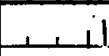 & $2,0,0$ & & & & & & & & & $-1,1,315$ \\
\hline 1 & $2,2,0$ & $p, \phi, W, E, R, 4,5,1, \ldots, 1,1$, & $1+2$ & 1.1 & - & $2,3,5$ & A,2,4,0 & $9,2,4,5$ & $A, 2,0$ & $2,2,3,5$ & $A, 2,4,0$ & $A, 2,75$ & $2,3,5$ & $A, 2 A, 0$ & $=1,2,2,0$ \\
\hline & & & & & -.1. & $A, 2,5,0$ & $A, 2,9,0$ & $2,5,5$ & A, 46,0 & $A, 2,6,5$ & $A, 28,0$ & & & & , $2,2,1$ \\
\hline .1 & $2,2,5$ & $p, \phi, W, E, R_{1}, 4,5, \ldots, \ldots$ & $-1,1$ & ل. لــ. & & $3,0,0$ & & 1, & T., &., 1 & & & 1, & 1 & $, 1,2,2,5$ \\
\hline 1 & 2,30 & $P, \phi, w, E, R, b_{1}, 1, \ldots, 1,1,1$ & & . & 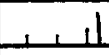 & 3.25 & & & & & & & & 1 & , $1,2,3,0$ \\
\hline & $, 2,3,5$ & $P, \phi, W, E, R, 5,6,1, \ldots, 1, \ldots$ & 1.2 & 1.1 .1 & $\ldots$ & $, 2,20$ & A,22,5 & $A, 2,3,0$ & A, 47,0 & $.25,5$ & $A, 26,0$ & $A, 2,6,5$ & $A, 28,0$ & .1. & $, ., 1,2,35$ \\
\hline .1 & $, 2,4,0$ & $P, \phi, W, E, R_{1}, 5,6,1$, & & & 1,1 & $3,0,5$ & & & $T$ & 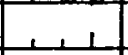 & & & & 1. & $, 1,2,4,0$ \\
\hline .1 & $, 2,4,5$ & $R, \phi, W, G_{1} R, 4, \ldots, \ldots$ & 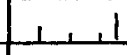 & 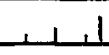 &.,- 1 & $.3,2,0$ & & & . & +1 & & & 1. & .1 & $, 1,2,45$ \\
\hline & $2 \sqrt{10}$ & $P, \phi, w_{1} \in R_{,}, T_{1}$ & & 管 & . & $3,2,5$ & & & & & & & & & $., 1,250$ \\
\hline . & $2,5,5$ & $P, \phi, w, E, R, b, 7, \ldots$, & 1,2 & -1 & 1,5 & $2,2,0$ & $A, 2,2,5$ & $A, 2,3,0$ & A, 2,, 20 & $2,3,5$ & A.240 & A,2,45 & $A, 2,7,0$ & .435 & $, ., 2,255$ \\
\hline & & & & & 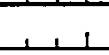 & $A, 2,4,0$ & $A, 2,7,5$ & , 2,3,5 & $n, 2,4,0$ & $A, 25,0$ & $4,2,8,0$ & & & & $1,1,2,56$ \\
\hline 1 & $2,6,0$ & $P, \phi, W, E, R, \quad, \quad, Z$ & لــــ & 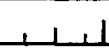 & 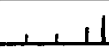 & $3,1.0$ & & 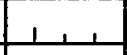 & .1, & . & & 1 & 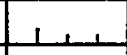 & بـ & $=, 1,2,60$ \\
\hline & $2,6,5$ & $R, \phi, W_{1}, R_{R}, 5,1,1$ & & & & $3,3,0$ & & & & & & & & & $, 1,2,65$ \\
\hline .1 & $, 2,20$ & $S, E, R, V, W, P, U, M, P,, A_{1}, B_{1},{ }_{1}$ & 1,3 & 1 & .1 .8 & $2,3,5$ & $A, 2,4,0$ & $A, 2,5,0$ & $A, 2,8,0$ & 2.55 & $A, 2,6,0$ & A.2, 2,5 & $A, 2,8,0$ & & $=-2,2.0$ \\
\hline & $, 2,2,5$ & 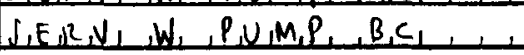 & & & t. & $2,2,0$ & $A, 2,2,5$ & , $, 23,0$ & $A, 2,7,0$ & .235 & $1,2,4,0$ & $A, 4,4,5$ & $A, 2,7,0$ & $.2,3,5$ & $., 1,275$ \\
\hline & & & & & & $A, 2,4,0$ & $A, 2,5,0$ & $A, 2,3,0$ & $, 2,25$ & $A, 2,6,0$ & $A, 2,6,5$ & $A, 2, B, 0$ & & 1. & $,+, 4,2 b$ \\
\hline 1 & $.2,8,0$ & $S_{1} E R, N_{1}, W, P, O M_{1} R_{1}, B_{1}, C_{1} R_{2}$ & & & & $, 3,2,0$ & $A, 2,2,5$ & $4,2,3,0$ & $0,20,0$ & $2,2,3,5$ & $A, 34,0$ & $A, 2,4,5$ & $A, 2,70$ & 1 & $=, 1,28.0$ \\
\hline & $-3,0,0$ & $P, \phi, W E R_{1}, 4, T_{1}, S_{1}, \ldots, 1$, &.,+ 1 & .1 .1 & & $2,2,5$ & $\ldots$ & 1,1, & .1, & -1 & 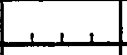 & .... & & 1. & $,-1,3,0,0$ \\
\hline & $-3,0,5$ & $Q, \phi_{1} W_{E, R_{2}}, v_{1} T_{1} \sigma_{1}, \ldots, 1$, & & 1 & & $, 2,4,0$ & & & .1. & 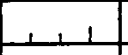 & . ب. & , & 1,1 & . & . \\
\hline 1 & $-3,1,0$ & $P, \phi, W, E, R, b, 7,7, \ldots, 1,1$, & ل. & 1 & 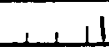 & $2,6,0$ & & & 1. & 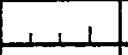 & 1. & וـ & . & 然 & , 3, $3,1,0$ \\
\hline & $-3,1,5$ & $P, \phi, W, E, R, \quad 5,7,6, \ldots, \ldots, 1$, & & 1 & 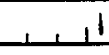 & $3,3,0$ & & 1, & 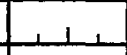 & -1, & & 1,1 & 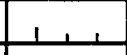 & ـــ. & $=, 3,3,15$ \\
\hline & $-3,2,0$ & $P \phi, W_{1}, R_{1}, 4 I, S_{1}$ & & .1 .1 & & $2,4,5$ & & & 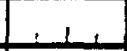 & & & & & & $-1,3,2,0$ \\
\hline
\end{tabular}

FIGURE 3.26 (continued) 


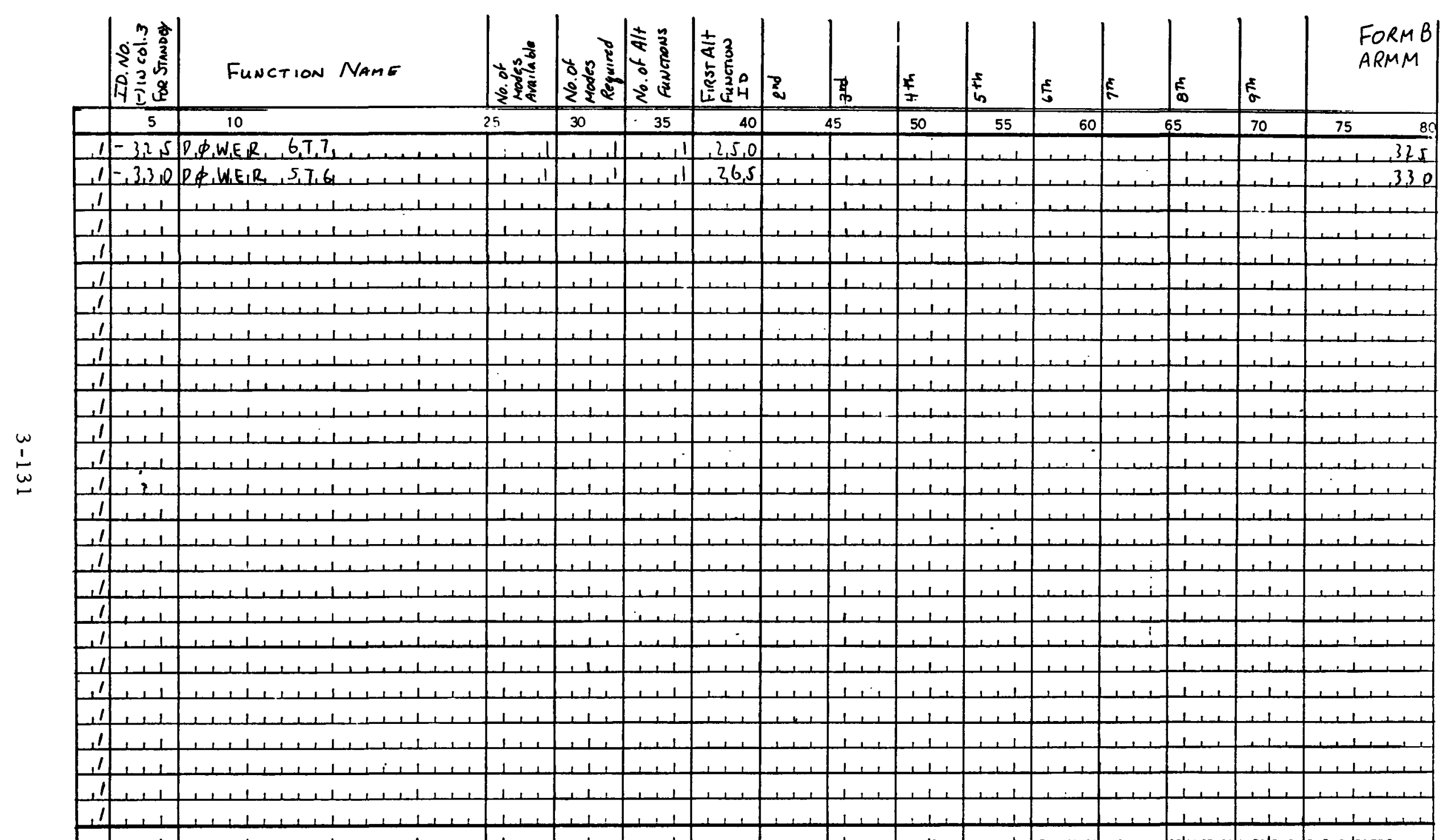

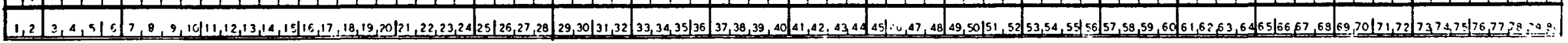

FIGURE 3.26 (continued) 


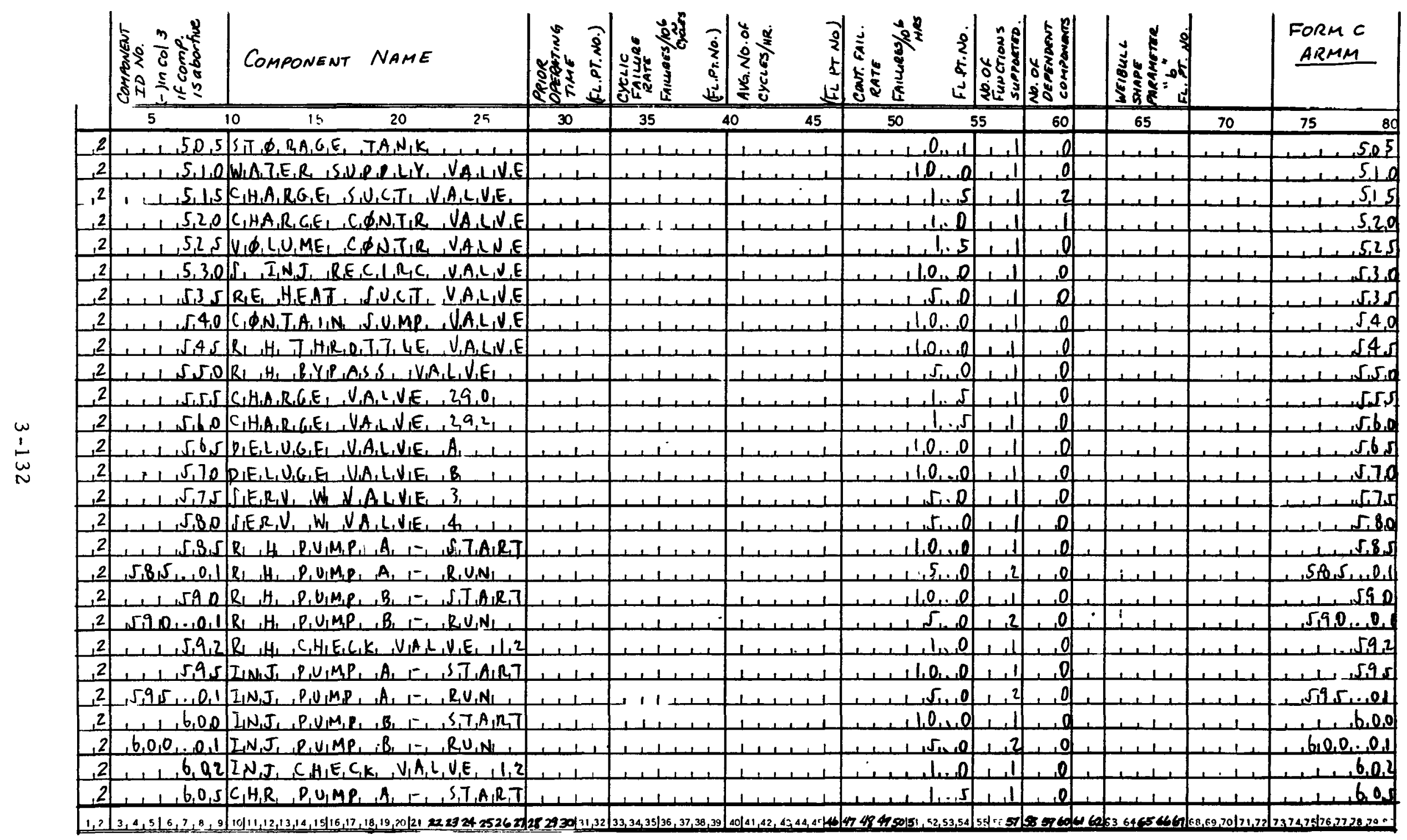

FIGURE 3.26 (continued) 


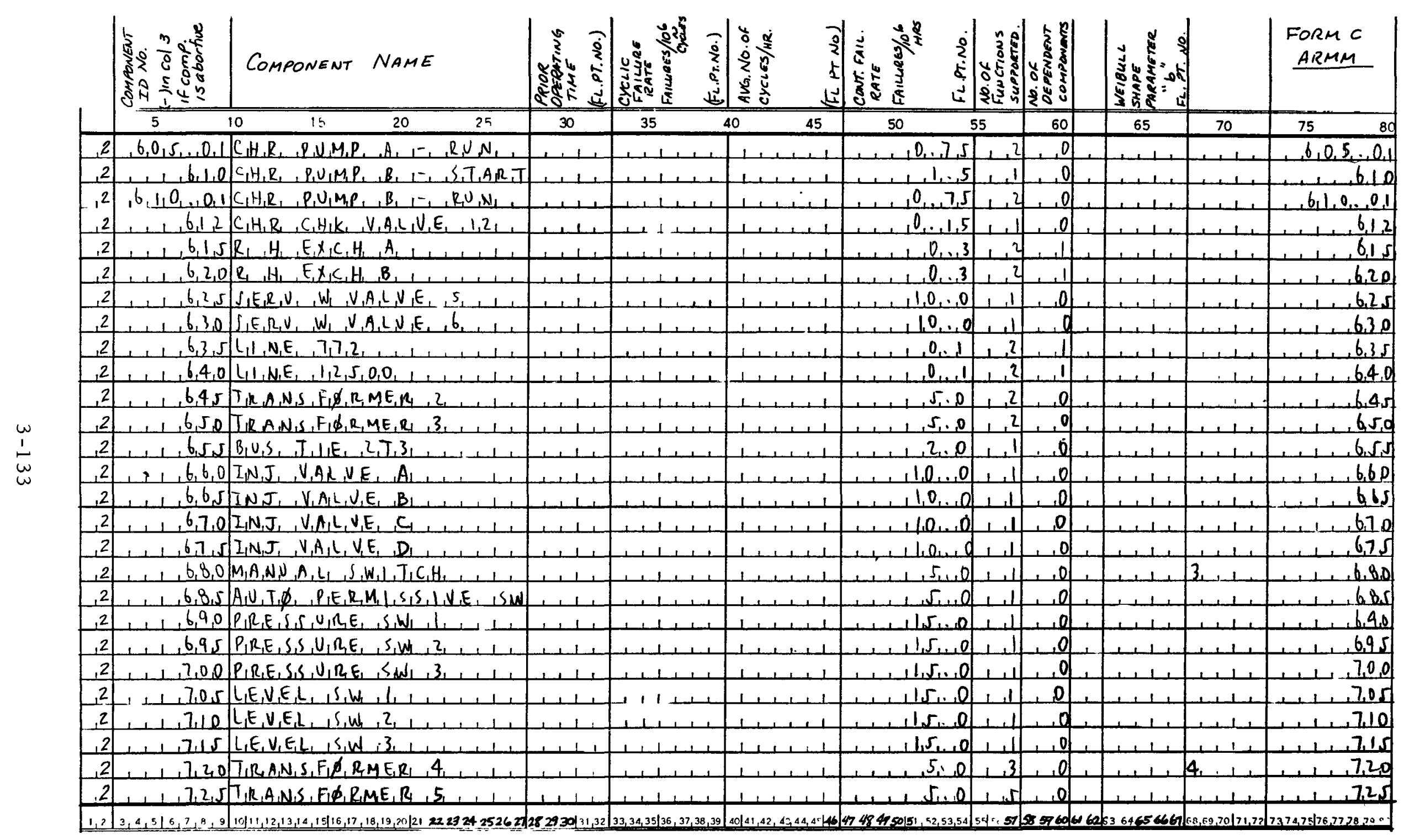

FIGURE 3.26 (continued) 


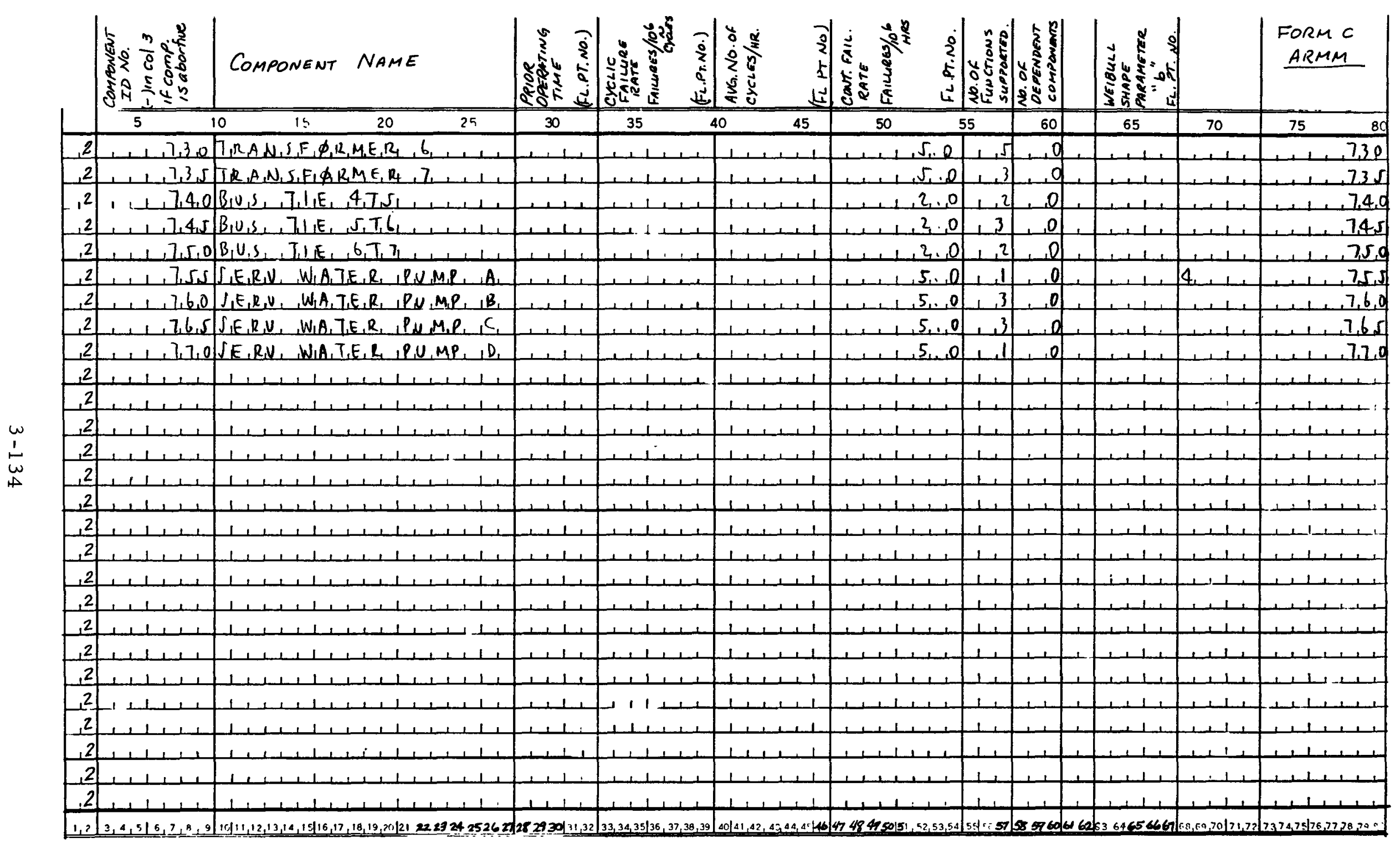

FIGURE 3.26 (continued) 


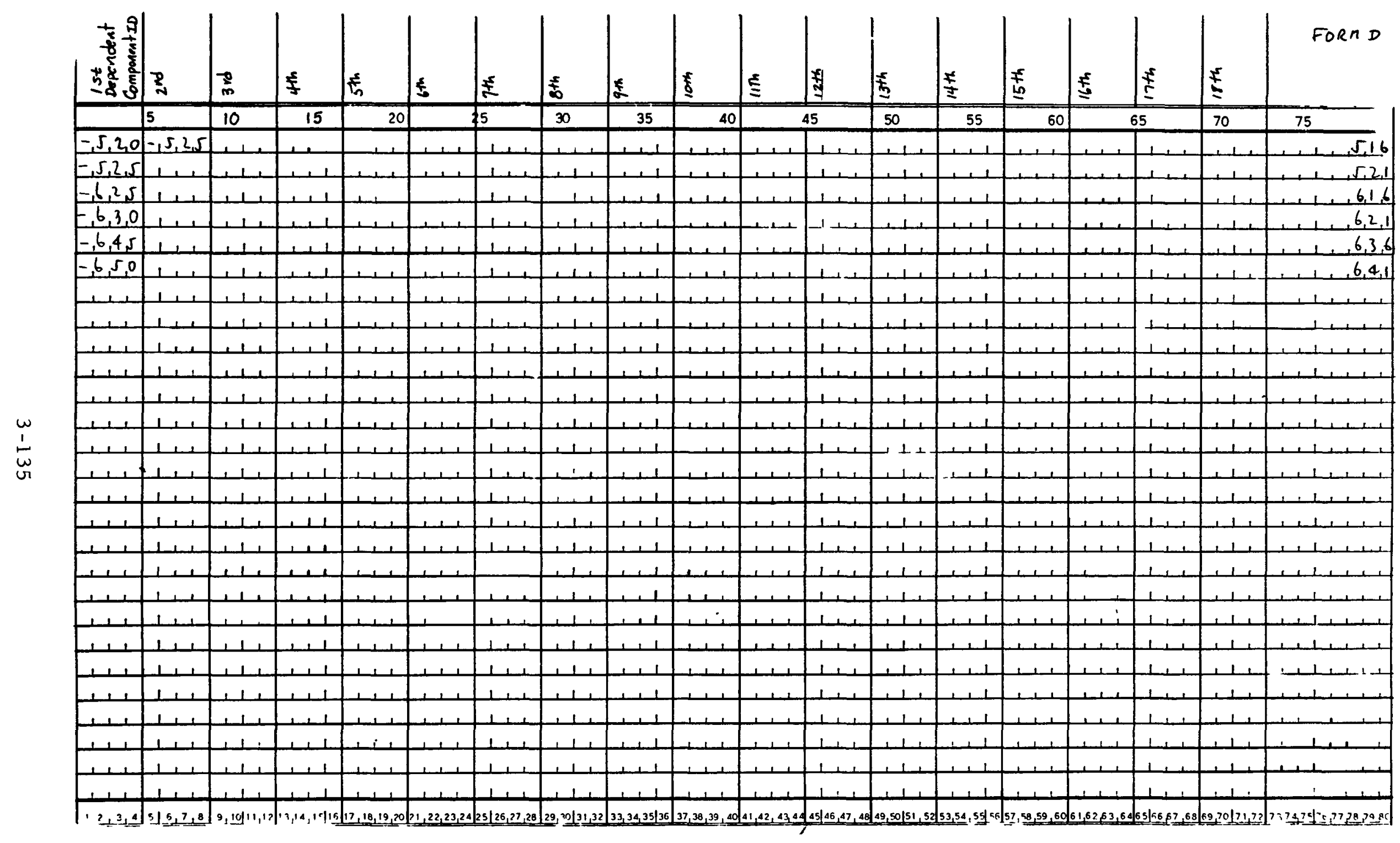

FIGURE 3.26 (continued) 


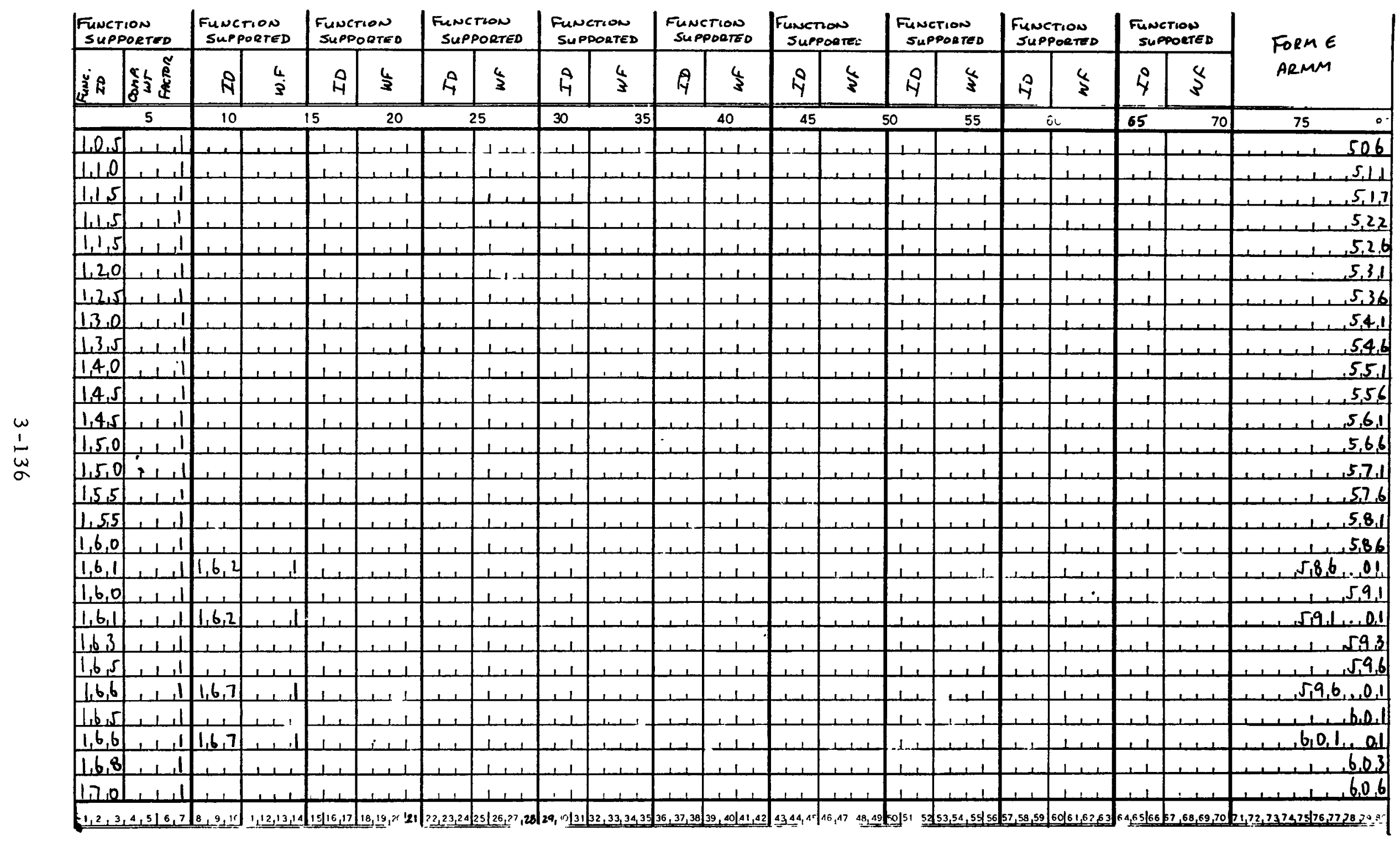

FIGURE 3.26 (continued) 


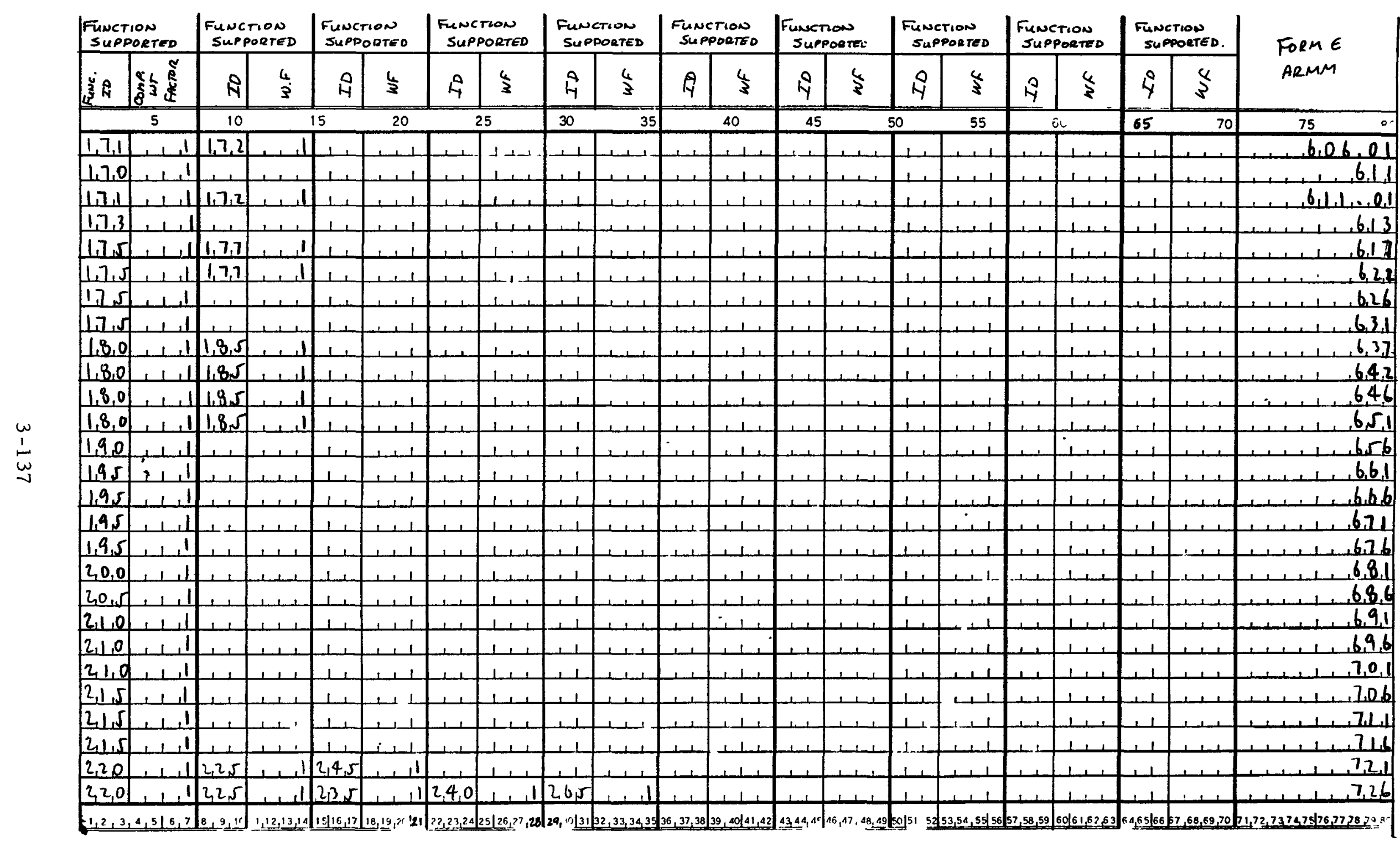

FIGURE 3.26 (continued) 


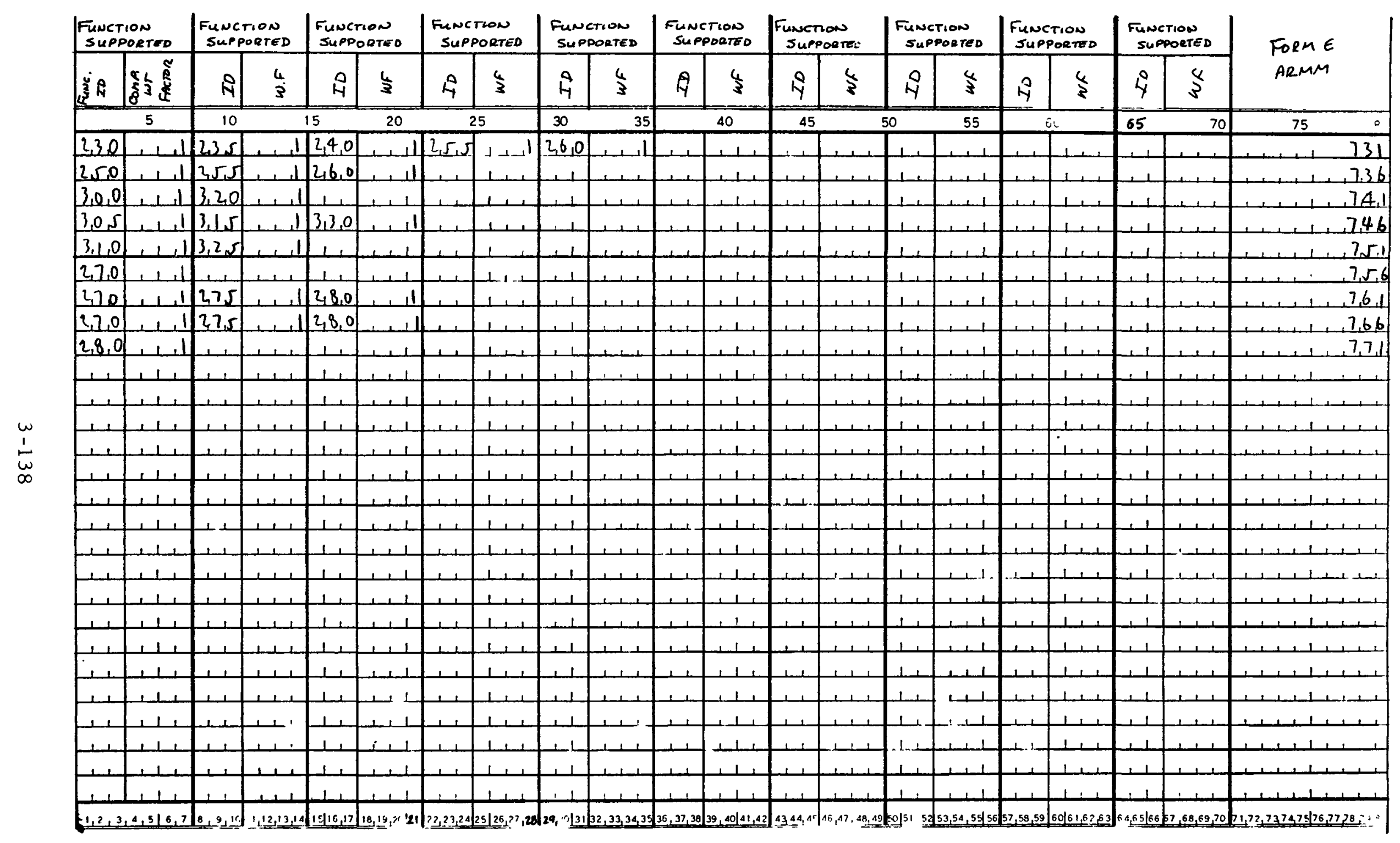

FIGURE 3.26 (continued) 


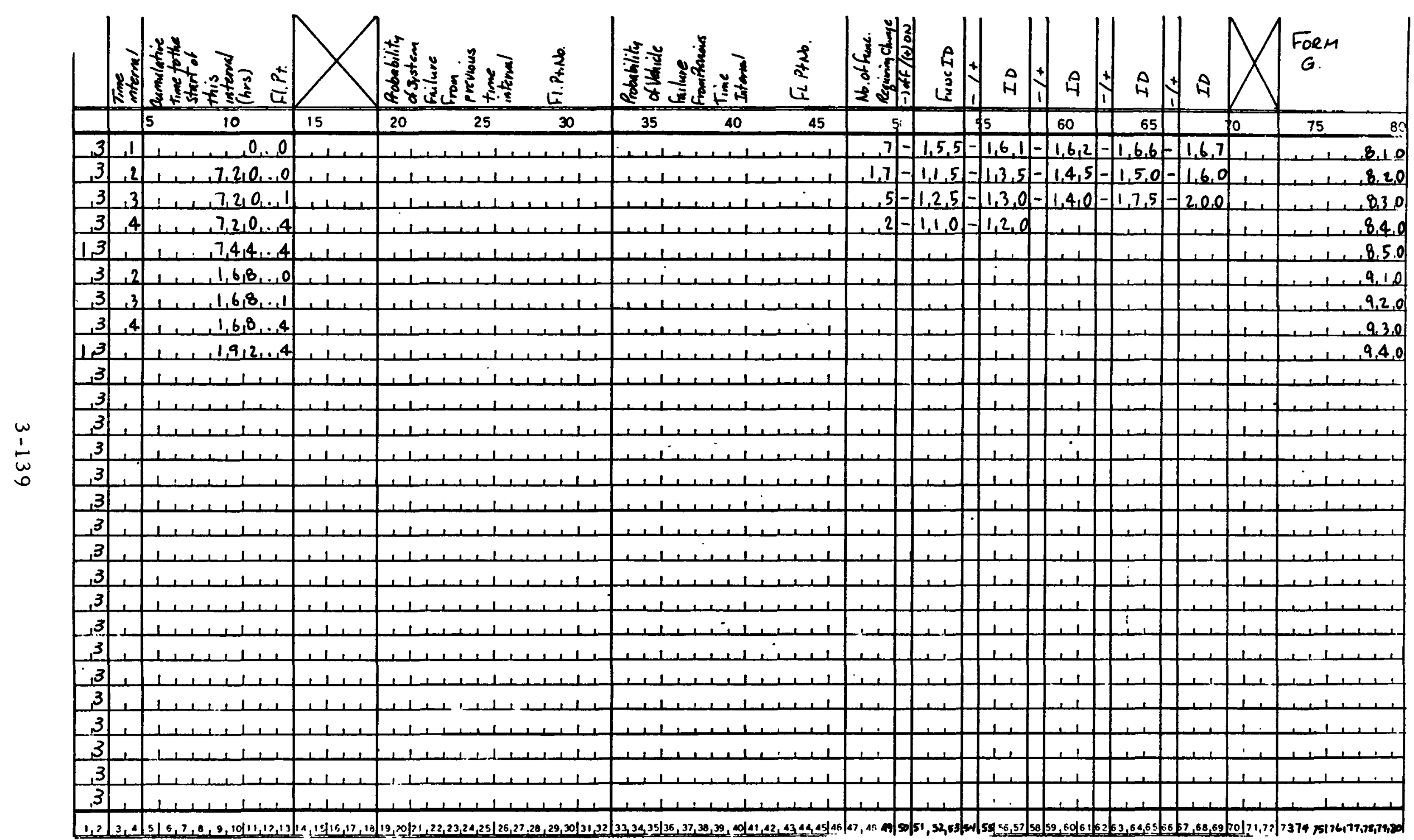

FIGURE 3.26 (continued) 


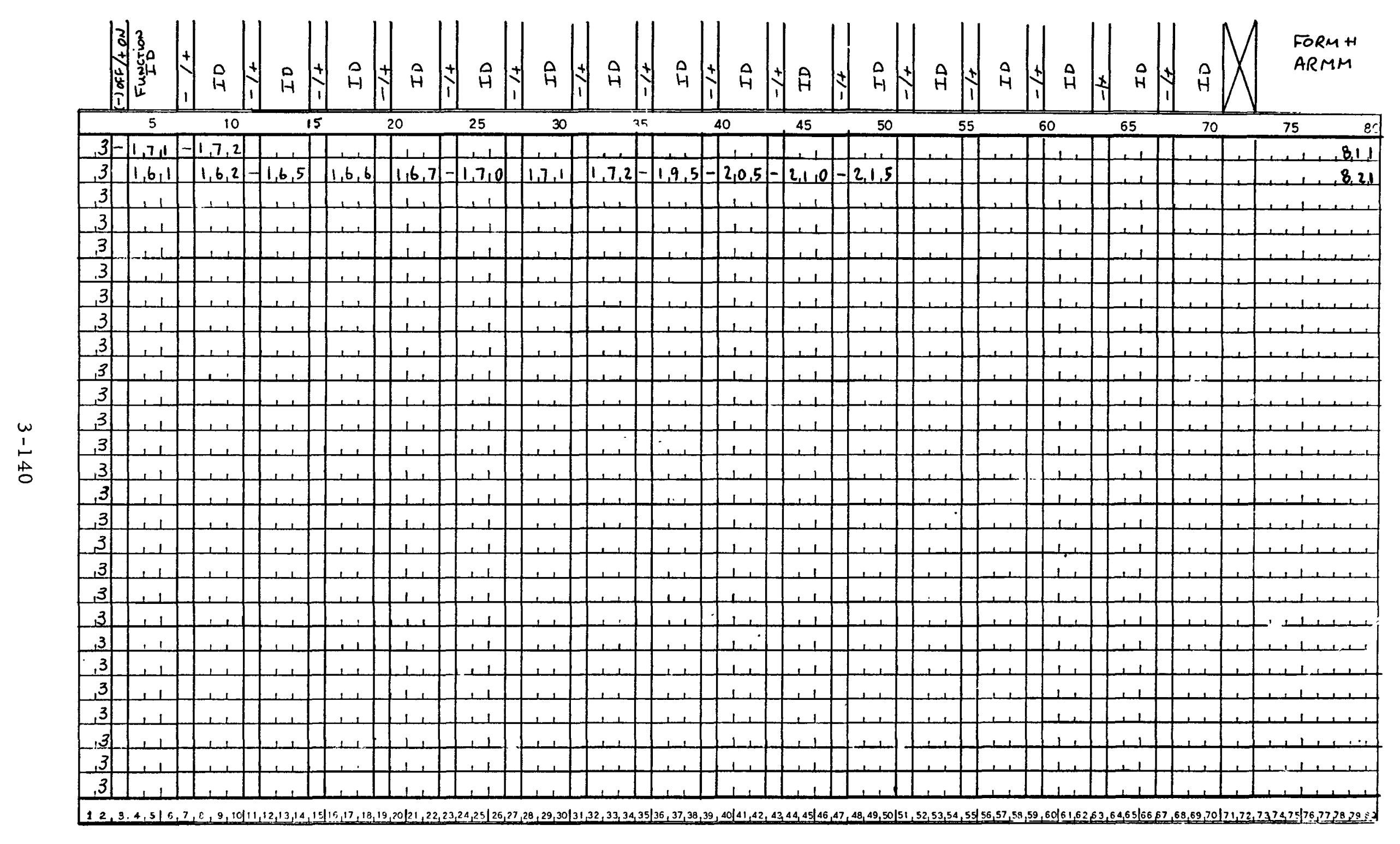

FIGURE 3.26 (continued) 


\begin{tabular}{|c|c|c|c|c|c|c|c|c|c|c|c|}
\hline & 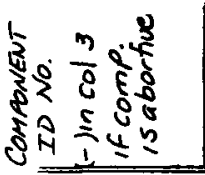 & Component name & 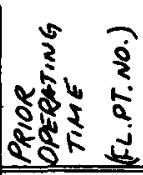 & 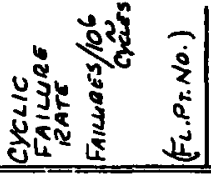 & 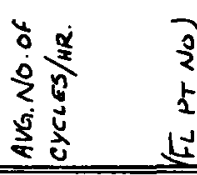 & 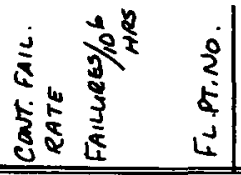 & 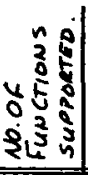 & 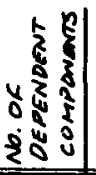 & 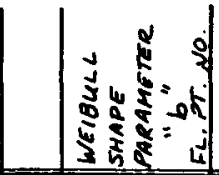 & & $\begin{array}{l}\text { FORM C } \\
\text { ARMM }\end{array}$ \\
\hline & 5 & 20 & 30 & 35 & 45 & 50 & 5 & 60 & 65 & 70 & 75 \\
\hline 2 & $\pm, 5,1,0$ & W,A,Z,E,R, S,U,R,R,L,Y, $V, A, l, V, E$ & 1 & & & $, .5,0$ & & .0 & & & $5,1,0$ \\
\hline 2 & $, 1,5,1,2$ & $W A, T, E, R, \cap S, U, P, P, L Y, V, A, L, V, E$ & & & & $1,0,0$ & & 0 & & & $, 1,5,1,2$ \\
\hline 2 & $1,1,5,1,2$ & 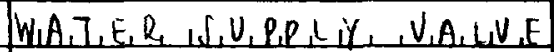 & & & 11 & $1,5,0$ & & 0 & & 1,1 & $, 1,5,1,2$ \\
\hline 2 & $, \quad, 5,3,0$ & $f_{1}, I, N, J, R, E, C_{1}, R_{1} C_{1}, V_{1} A_{1}, \in, E$ & 1 & & & $, 1,5,0$ & & 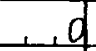 & & & 5,30 \\
\hline .2. & $, 1,5,3,2$ & $S_{1}, I, N, J_{1}, R_{1}, C_{1}, \Omega_{1} C_{-}, V, N, L, V, E$ & & & +1 & 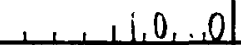 & & .0 & & & 5,32 \\
\hline 2 & $, 1,5,3,2$ & $\delta_{1}, I_{1} N, J, \perp R, E, C, L, R_{1} C, \cup, A, L, V, E$ & 1 & & 1 & $1,5,1,0$ & & .0 & & & $, ., 5,3,2$ \\
\hline 2 & $, 5,3,5$ & $\left.R, E_{1}, H, E, A_{1} T_{1}, S, \cup, C_{1}\right]_{1}, V_{1} A_{1}, V_{1}, E$ & , I & 1 & 1,1 & $,, 1,2,-5$ & & 0 & & 1,1, & $1,1,5,3,5$ \\
\hline 2 & $, 1,5,3,7$ & $R, E, H, E, A, T, S, U, C, T, V, A, L, V, E$ & 1 & .1, & $\perp, 1,1,1$ & $, 1,1,5, .0$ & & . & & 1,1, & $5,5,37$ \\
\hline 2 & $, 1,5,3,7$ & $R, E, H, E, A, I, S, U, C], V, A, L, V, E$ & 1 & $, 1,1$, & $1,1,1,1$ & $, 1,1,2,5$ & & d & 1,1, & +1 & $+, 1,37$ \\
\hline 2 & $1,1,5,4,0$ & $C, \phi, H, T, A_{1}, \omega, \omega, S, \mu_{1}, p, V, P, l, V, E$ & 1 & 1 & 1 & $, 1,5,0$ & & 0 & - L & & 4,540 \\
\hline .2 & $1,1,54,2$ & $C_{1}, N, T, A_{1}, \mathcal{N}, \mathcal{N}, \cup, M_{1} P_{1}, V, M, \in, \cup \in E$ & & & & $1,0,0$ & & 0 & & & $\sqrt{44.2}$ \\
\hline 2 & $, 1, \sqrt{4}, 2$ & $C, \phi, N, T, A, P, N, S, \mathcal{M}, M R,, V, A, L, V, E$ & & & 1 & $1,5,0$ & & .0 & & 1,1 & $., 1, \sqrt{.4,2}$ \\
\hline 2 & $.1,5,4,5$ & $R_{1}, H_{1}, T, H, R, 0,7, T, L, E, V, A, L, V, E$ & & & 1, & $1, \sqrt{1}, 0$ & & 0 & & 1 & $, 1,5,4,5$ \\
\hline 2 & $\therefore, \sqrt{4}, 7$ & $R_{1}, H_{1}, T, H, R_{1}, T, T, L E_{1}, V A L, V, E$ & 1 & 1 & $1,1,1,1$ & $1,0, .0$ & & 0 & & 1,11 & $\therefore, 1,5.4,7$ \\
\hline 2. & $,+, 1,4,1$ & $R_{1}, H_{1}, T, H, R, 0, T, T, L, E, V, A, U, V, E$ & & & $\perp$ & $1,5,0$ & & 0 & & L & $\ldots, 1,4,4,7$ \\
\hline 2 & 1,550 & $R_{1}, H_{1}, B,\left(P, A S S, \cup, A, L, U F_{1}\right.$ & 11 & $1,1,1$ & $1,1,1,1$ & $+, 1,2,5$ & & & & $1+1$ & $-\sqrt{2} \sqrt{10}$ \\
\hline 2 & $1,5,5,2$ & $R_{1}, H, B, Y, P, A, S, \quad V, A, V_{1} E_{1}$ & 1 & 1,1 & $1,1,1$ & $1+1,5$ & & & ith & $1+1$ & $5,5.5 .2$ \\
\hline 2. & $1,5,5,2$ & $R_{1}, H_{1}, B_{1}, P A, S, V, A, L, V, E_{1}$ & +1 & $1,1,1,1$ & $1,1,1$, & $,+, 1,2,5$ & & 0 & $i, 1,1$ & 1,1 & $+1,+\sqrt{1} \sqrt{2}$ \\
\hline 2 & $, 1,5,1,5$ & $C_{1} H, A, R, G, E_{1}, S, U, C, T_{1}, V, A, L, V, E$, & +1 & $1,1,1,1$ & $1,1,1,1$ & $, ., 0,7,5$ & 1,1 & 2 & 1 & 1,1, & $5,5,1,5$ \\
\hline 2 & $, 1,5,1,8$ & $C_{1}, A, A, R, G, E_{\perp}, S, U, C_{,} T_{1}, V, A, L, V_{1} E_{1}$ & 1 & L, L, & $1,1,1$ & $, 1,1,1,5$ & 1,1 & 2 & 1,1 & 1ـ & $-15,1.8,0$ \\
\hline 2 & $, 1,5,1,8$ & $C_{1} H, A, R, G, E_{1}, S, V_{1}, T_{1}, V, A, L, V_{1} E_{1}$ & 11 & $1,1,1$ & Leed & $, \ldots, 10, \ldots, 5$ & & 2 & 1 & , 1, & $, 5,1,8,0$ \\
\hline 2. & $, 1,5,2,0$ & $C H, A, R, G, E, C, \phi, N, T, R, V, A, L, V, E$ & & & & $1,0, \ldots 5$ & & & ten & 1,1 & $.1,5,2,0$ \\
\hline$\perp^{2}$ & $, 5,2,3$ & $K, H, A, R, G, E, C, \phi, N, T, R, V, A, L, V, E$ & & & & $1,1,1,0$ & & & & & $15,2,3,0$ \\
\hline 2) & $, 5,2,3$ & $K_{1} H, A, R, G, E, C, \phi, N, T, R, U, A, L, V, E$ & & $\perp 11$ & & $1,0, \ldots$ & & & & د & $, 5,2,3,-, 0$ \\
\hline 2 & $5,2,5$ & $V, \phi, L, U, M, E, C, \phi_{i}, N_{1}, R_{1}, V, A, L, U, E$ & & & & $, 0,0,7,5$ & 1,1 & , 요 & & & $-, 1,2,5$ \\
\hline 2. & $5,2,8$ & $V, \phi, L, \cup, M, E, C, N, T, R, V, A, L, U, E$ & & & & $1,1,5$ & 1 & & & & $1,5,2,8,0,0$ \\
\hline 2 & $, 5,2,8$ & $V, \phi, L, U, M, E, C, \phi, T, R, V, A, L, U, E$ & & & & $0 ., 7.5$ & 1.1 & 0 & & & $,-5,2,8,0$ \\
\hline & & & & & & & & & & & \\
\hline
\end{tabular}

FIGURE 3.27

ARMM INPUT LOADSHEETS - CASE 2 


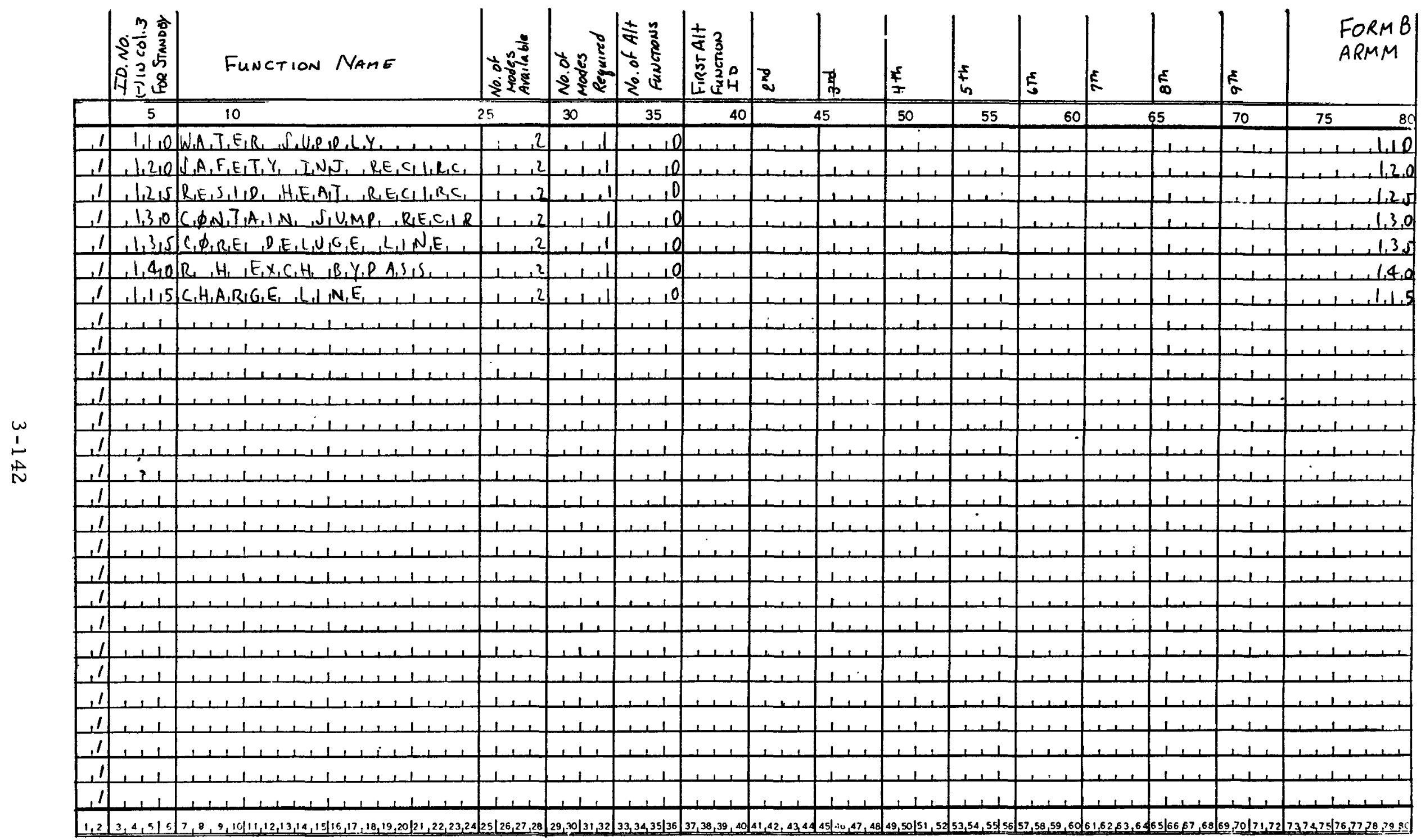

FIGURE 3.27 (continued) 


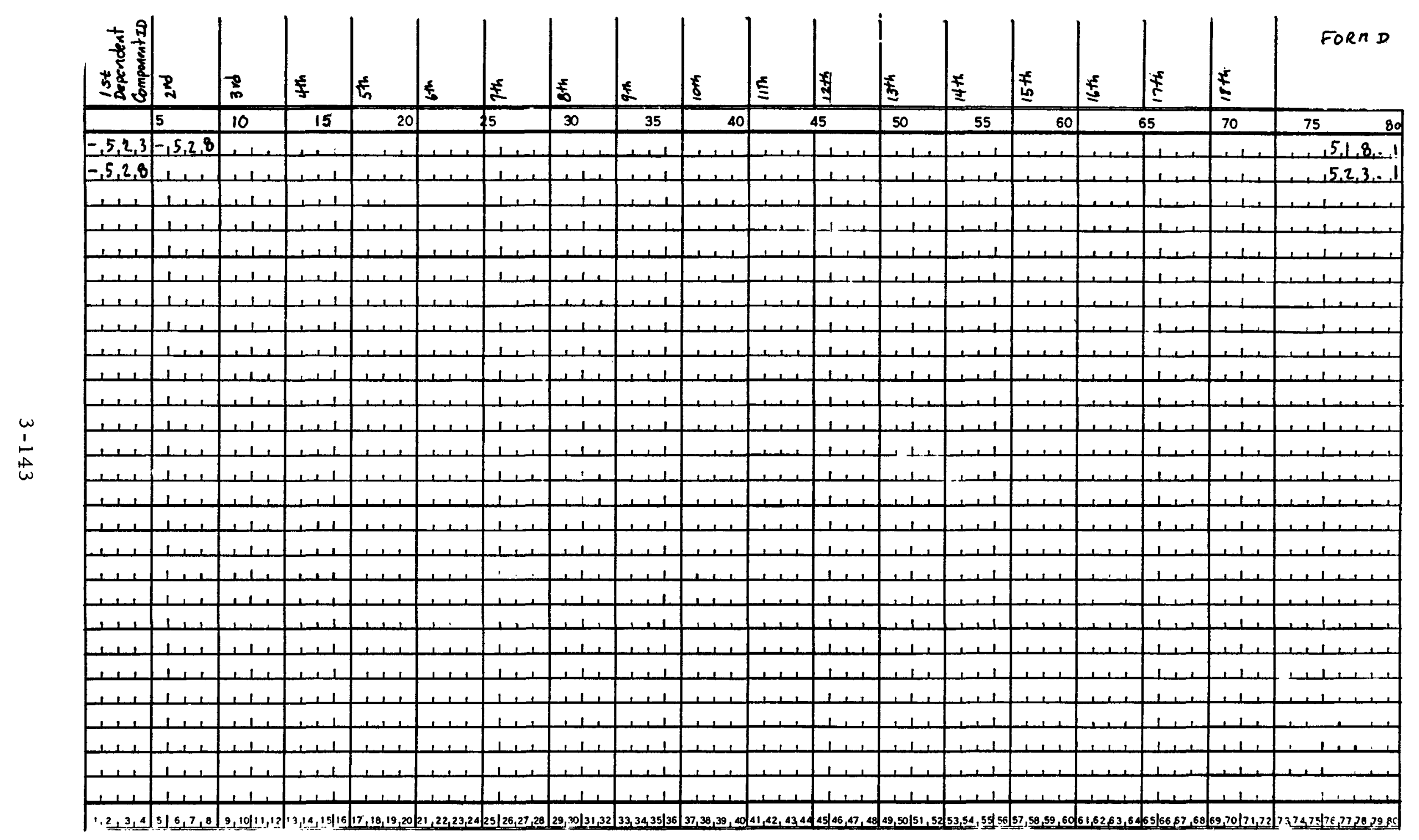

FIGURE 3.27 (continued) 


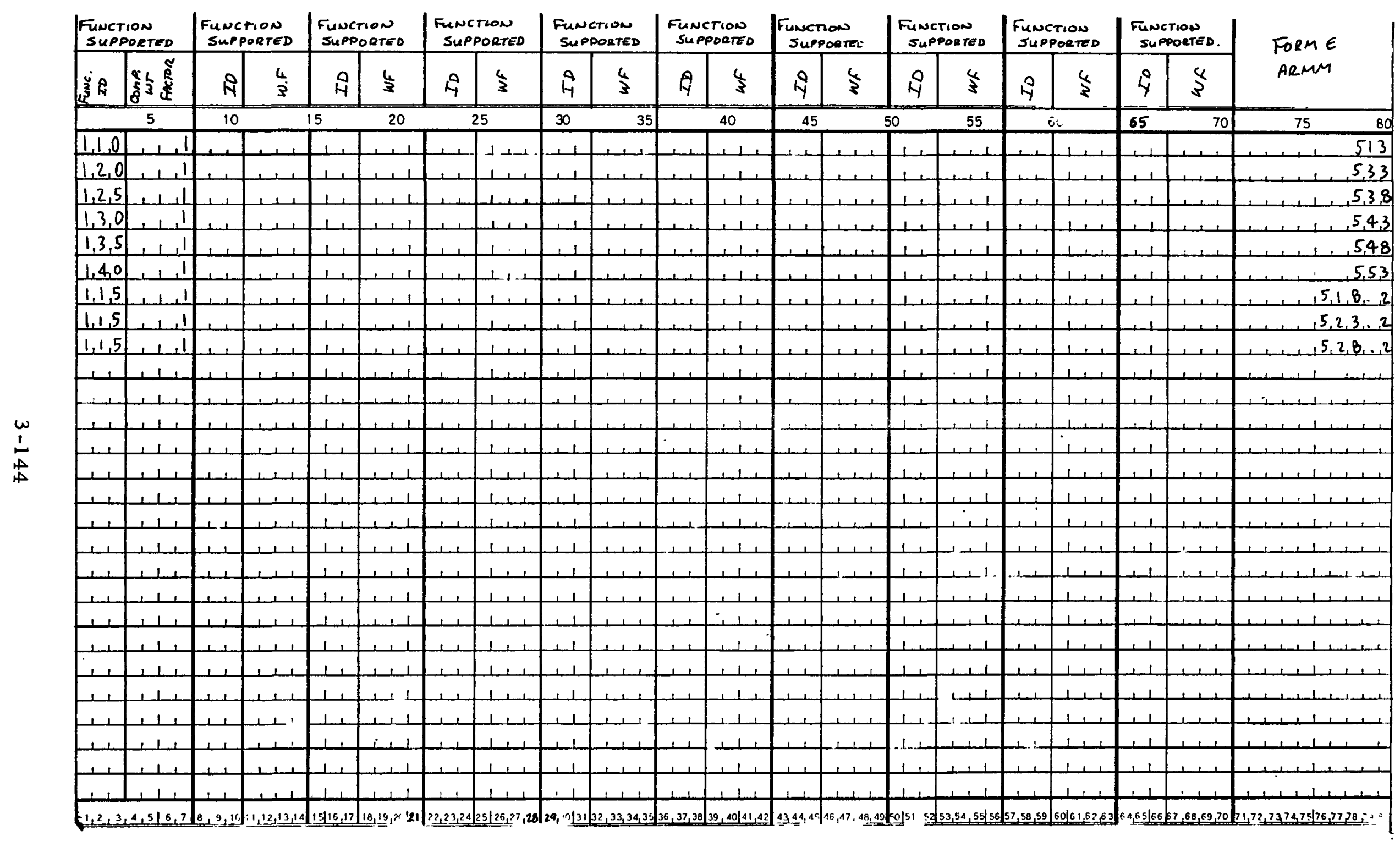

FIGURE 3.27 (continued) 
COMPONENT CONTRIBUTIONS TO UNRELIABILITY

COMPONENT

IO NAME

505.00 STORAGE TANK

510.00 HATER SUPPLY VALVE

512.00 HATER SUPPLY VALVE

515.00 CHARGE SUCT VALVE

518.00 CHARGE SUCT VALVE

520.00 CHARGE CONTR VALVE

523.00 CHARGE CONTR VALVE 525.00 VOLUME CONTR VALVE 528.00 VOLUME CONTR VALVE 530.00 S INJ RECIRC VALVE 532.00 S INJ RECIRC VALVE 535.00 RE HEAT SUCT VALVE 537.00 RE HEAT SUCT VALVE 540.00 CONTAIN SUMP VALVE 542.00 CONTAIN SUMP VALVE 545.00 R H THROTTLE VALVE $547.00 R$ H THROTTLE VALVE $550.00 R$ H BYPASS VALVE 552.00 R H BYPASS VALVE 555.00 CHARGE VALVE 290 560.00 CHARGE VALVE 292 565.00 DELUGE VALVE A 570.00 DELUGE VALVE $B$ 575.00 SERV W VALVE 3 580.00 SERV W VALVE 4 585.00 R H PUMP A - START 585.01 R H PUMP A - RUN 590.00 R H PUMP B - START 590.01 R H PUMP B - RUN

$\begin{array}{rl}\text { FAILURE } & \text { SERIAL } \\ \text { RATE } 10.46 & \text { PROBABILITY } \\ 0.10 & 0.66180061 E-04 \\ 5.00 & 0.32023185 E-02 \\ 5.00 & 0.32023185 E-02 \\ 0.75 & 0.48008106 E-03 \\ 0.75 & 0.48008106 E-03 \\ 0.50 & 0.32005404 E-03 \\ 0.50 & 0.32005404 E-03 \\ 0.75 & 0.48008106 E-03 \\ 0.75 & 0.48008106 E-03 \\ 5.00 & 0.32023185 E-02 \\ 5.00 & 0.32023185 E-02 \\ 2.50 & 0.16004925 E-02 \\ 2.50 & 0.16004925 E-02 \\ 5.00 & 0.32009849 E-02 \\ 5.00 & 0.32009849 E-02 \\ 5.00 & 0.32005404 E-02 \\ 5.00 & 0.32005404 E-02 \\ 2.50 & 0.16004925 E-02 \\ 2.50 & 0.16004925 E-02 \\ 1.50 & 0.96016212 E-03 \\ 1.50 & 0.96016212 E-03 \\ 10.00 & 0.64010809 E-02 \\ 10.00 & 0.640108 .09 E-02 \\ 5.00 & 0 . \\ 5.00 & 0 . \\ 10.00 & 0.64010809 E-02 \\ 5.00 & 0.10846277 E-03 \\ 10.00 & 0.64010809 E-02 \\ 5.00 & 0.10846277 E-03\end{array}$

$\begin{array}{lr}\text { SERIAL } & \text { SER } \\ \text { PERCENT } & \text { RAN } \\ 0.0310 & 59 \\ 1.5007 & 29 \\ 1.5007 & 30 \\ 0.2250 & 47 \\ 0.2250 & 48 \\ 0.1500 & 51 \\ 0.1500 & 52 \\ 0.2250 & 49 \\ 0.2250 & 50 \\ 1.5007 & 31 \\ 1.5007 & 32 \\ 0.7500 & 39 \\ 0.7500 & 40 \\ 1.5000 & 33 \\ 1.5000 & 34 \\ 1.4998 & 36 \\ 1.4998 & 37 \\ 0.7500 & 41 \\ 0.7500 & 42 \\ 0.4499 & 43 \\ 0.4499 & 44 \\ 2.9997 & \\ 2.9997 & 10 \\ 0 . & 0 \\ 0.9 & 0 \\ 2.9997 & 11 \\ 0.0508 & 55 \\ 2.9997 & 12 \\ 0.0508 & 56 \\ \end{array}$

$\begin{array}{lr}\text { SYSTEM } & \text { SYSTEM } \\ \text { PERCENT } & \text { RANK } \\ 0.1883 & 23 \\ 9.0992 & 1 \\ 9.0992 & 2 \\ 1.3662 & 13 \\ 1.3662 & 14 \\ 0.9109 & 17 \\ 0.9109 & 18 \\ 1.3662 & 15 \\ 1.3662 & 16 \\ 9.0992 & 3 \\ 9.0992 & 4 \\ 4.5518 & 9 \\ 4.5518 & 10 \\ 9.0955 & 5 \\ 9.0955 & 6 \\ 9.0942 & 7 \\ 9.0942 & 8 \\ 4.5518 & 11 \\ 4.5518 & 12 \\ 0.0015 & 44 \\ 0.0015 & 45 \\ 0.0651 & 26 \\ 0.0651 & 27 \\ 0 . & 0 \\ 0.0 & 0 \\ 0.0651 & 28 \\ 0.0000 & 58 \\ 0.0651 & 29 \\ 0.0000 & 59\end{array}$


COMPONENT CONTRI QUTIONS TO UNREL IABILITY

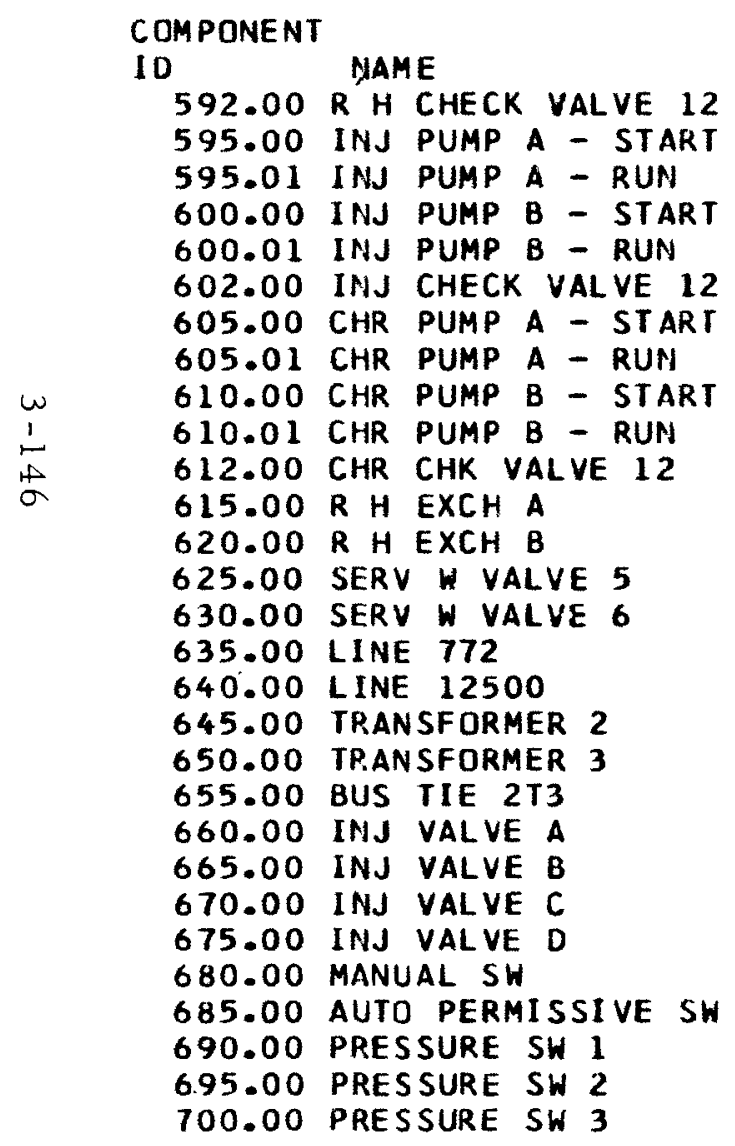

$\begin{array}{rl}\text { FAILURE } & \text { SERIAL } \\ \text { RATE 10.*6 } & \text { PROBABILITY } \\ 1.00 & 0 . \\ 10.00 & 0.64010809 E-02 \\ 5.00 & 0.10846277 E-03 \\ 10.00 & 0.64010809 E-02 \\ 5.00 & 0.10846277 E-03 \\ 1.00 & 0 . \\ 1.50 & 0.96016212 E-03 \\ 0.75 & 0.16269415 E-04 \\ 1.50 & 0.96016212 E-03 \\ 0.75 & 0.16269415 E-04 \\ 0.15 & 0 . \\ 0.30 & 0.19854019 E-03 \\ 0.30 & 0.19854019 E-03 \\ 10.00 & 0.64019698 E-02 \\ 10.00 & 0.64019698 E-02 \\ 0.10 & 0.66180061 E-04 \\ 0.10 & 0.66180061 E-04 \\ 5.00 & 0.33090032 E-02 \\ 5.00 & 0.33090032 E-02 \\ 2.00 & 0 . \\ 10.00 & 0.64010809 E-02 \\ 10.00 & 0.64010809 E-02 \\ 10.00 & 0.64010809 E-02 \\ 10.00 & 0.64010809 E-02 \\ 5.00 & 0.32009849 E-02 \\ 5.00 & 0.32005404 E-02 \\ 15.00 & 0.96016213 E-02 \\ 15.00 & 0.96016213 E-02 \\ 15.00 & 0.96016213 E-02\end{array}$

$\begin{array}{lcl}\text { SERIAL } & \text { SERIAL SYSTEM } \\ \text { PERCENT } & \text { RANK } & \text { PRCBABILITY } \\ 0 . & 0 & 0.46142885 E-09 \\ 2.9997 & 13 & 0.25244723 E-04 \\ 0.0508 & 57 & 0.80746634 E-08 \\ 2.9997 & 14 & 0.25244723 E-04 \\ 0.0508 & 58 & 0.80746634 E-08 \\ 0 . & 0 & 0.46142885 E-09 \\ 0.4499 & 45 & 0.57149072 E-06 \\ 0.0076 & 62 & 0.18169866 E-09 \\ 0.4499 & 46 & 0.57149072 E-06 \\ 0.0076 & 63 & 0.18169866 E-09 \\ 0 . & 0 & 0.10383296 E-10 \\ 0.0930 & 53 & 0.67175526 E-07 \\ 0.0930 & 54 & 0.67175526 E-07 \\ 3.0001 & 7 & 0.26720431 E-04 \\ 3.0001 & 8 & 0.26720431 E-04 \\ 0.0310 & 60 & 0.99503046 E-08 \\ 0.0310 & 61 & 0.99503046 E-08 \\ 1.5507 & 19 & 0.84050844 E-05 \\ 1.5507 & 20 & 0.84050844 E-05 \\ 0 . & 0 & 0.15490359 E-05 \\ 2.9997 & 15 & 0.75734169 E-04 \\ 2.9997 & 16 & 0.75734169 E-04 \\ 2.9997 & 17 & 0.75734169 E-04 \\ 2.9997 & 18 & 0.75734169 E-04 \\ 1.5000 & 35 & 0.71631931 E-05 \\ 1.4998 & 38 & 0.63339216 E-05 \\ 4.4995 & 1 & 0.34865525 E-06 \\ 4.4995 & 2 & 0.34865525 E-06 \\ 4.4995 & 3 & 0.34865525 E-06\end{array}$

SYSTEM SYSTEM PERCENT RANK $0.0000 \quad 62$ $0.0651 \quad 30$ C. $0000 \quad 60$ C.0651 31 C. $0000 \quad 61$ $0.0000 \quad 63$ $0.0015 \quad 46$ C.0000 64 C. $0015 \quad 47$ $0.0000 \quad 65$ $0.0000 \quad 70$ C. $0002 \quad 54$ C. $0002 \quad 55$ $0.0689 \quad 24$ $0.0689 \quad 25$ C.0000 56 C. $0000 \quad 57$ $0.0217 \quad 34$ $0.0217 \quad 35$ C. $0040 \quad 40$ C. $1954 \quad 19$ $0.1954 \quad 20$ $0.1954 \quad 21$ $0.1954 \quad 22$ C.0185 38 $0.0163 \quad 39$ $0.0009 \quad 48$ C.0009 49 C. $0009 \quad 50$ 


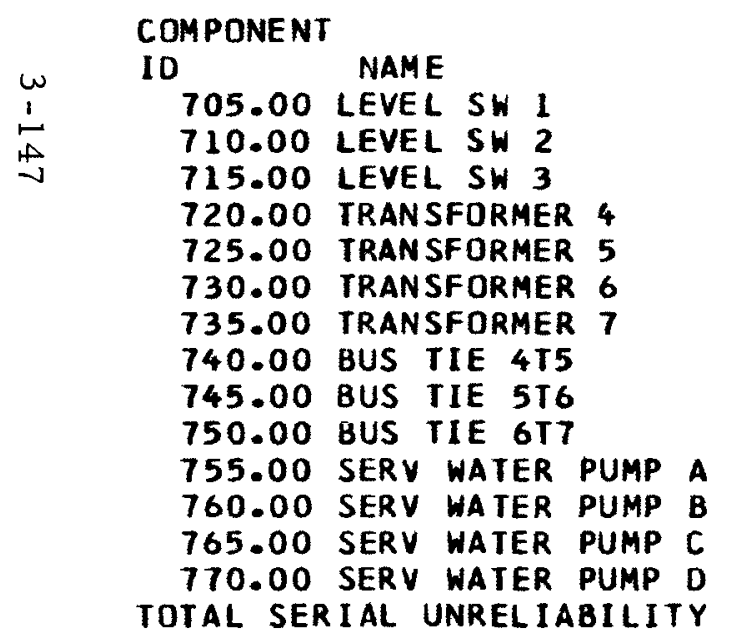

COMPONENT CONTRIBUTIONS TO UNREL IABILITY

\begin{tabular}{|c|c|c|c|c|c|c|}
\hline $\begin{array}{r}\text { FAILURE } \\
\text { RATE } 10 . * 6 \\
15.00 \\
15.00 \\
15.00 \\
5.00 \\
5.00 \\
5.00 \\
5.00 \\
2.00 \\
2.00 \\
2.00 \\
5.00 \\
5.00 \\
5.00 \\
5.00\end{array}$ & $\begin{array}{l}\text { SERIAL } \\
\text { PROBABILITY } \\
0.96016213 E-02 \\
0.96016213 E-02 \\
0.96016213 E-02 \\
0.33090032 E-02 \\
0.33090032 E-02 \\
0.33090032 E-02 \\
0.33090032 E-02 \\
0 . \\
0 . \\
0 . \\
0.33090032 E-02 \\
0.33090032 E-02 \\
0.33090032 E-02 \\
0.33090032 E-02 \\
0.21339339 E-00\end{array}$ & $\begin{array}{l}\text { SERIAL } \\
\text { PERCENT } \\
4.4995 \\
4.4995 \\
4.4995 \\
1.5507 \\
1.5507 \\
1.5507 \\
1.5507 \\
0 . \\
0 . \\
0 . \\
1.5507 \\
1.5507 \\
1.5507 \\
1.5507\end{array}$ & $\begin{array}{l}\text { SER IAL } \\
\text { RANK } \\
4 \\
5 \\
6 \\
21 \\
22 \\
23 \\
24 \\
0 \\
0 \\
0 \\
25 \\
26 \\
27 \\
28\end{array}$ & $\begin{array}{l}\text { SYSTEM } \\
\text { PROBABILITY } \\
0.34865525 E-06 \\
0.34865525 E-06 \\
0.34865525 E-06 \\
0.81559856 E-05 \\
0.16311971 E-04 \\
0.16311971 E-04 \\
0.81559856 E-05 \\
0.14521144 E-05 \\
0.14521144 E-05 \\
0.14521144 E-05 \\
0.4089641 \text { CE- } 10 \\
0.40896410 E-10 \\
0.40896410 E-10 \\
0.40896410 E-10\end{array}$ & $\begin{array}{l}\text { SYSTEM } \\
\text { PERCENT } \\
0.0009 \\
0.0009 \\
0.0009 \\
0.0210 \\
0.0421 \\
0.0421 \\
0.0210 \\
0.0037 \\
0.0037 \\
0.0037 \\
0.0000 \\
0.0000 \\
0.0000 \\
0.0000\end{array}$ & $\begin{array}{c}\text { SYSTEM } \\
\text { RANK } \\
51 \\
52 \\
53 \\
36 \\
32 \\
33 \\
37 \\
41 \\
42 \\
43 \\
66 \\
67 \\
68 \\
69\end{array}$ \\
\hline
\end{tabular}


COMPONENT CONTRIBUTIONS TO UNRELIABILITY

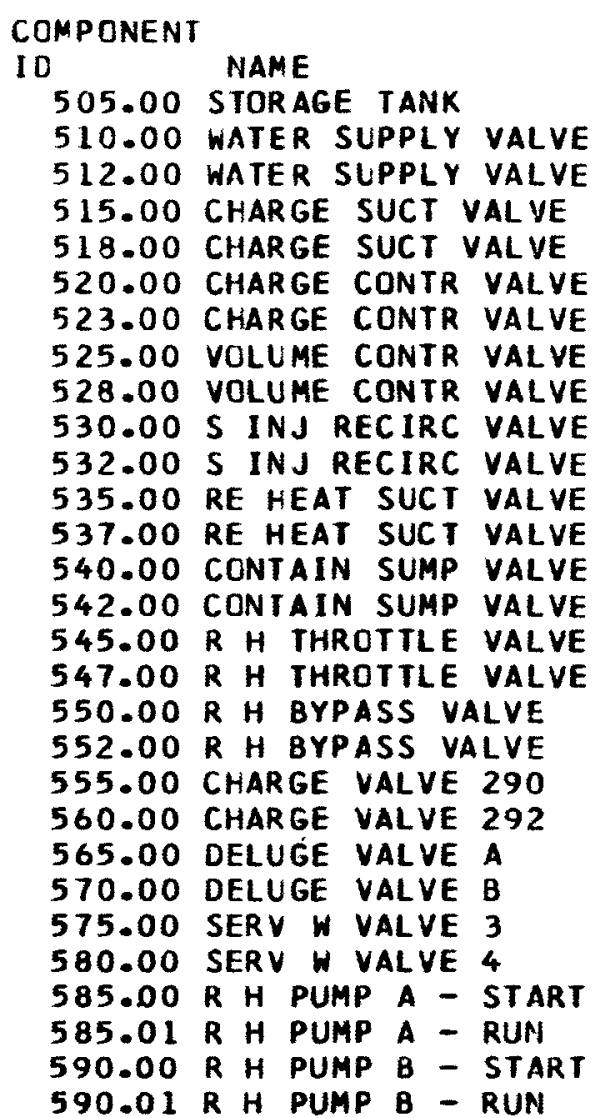

\begin{tabular}{|c|c|c|}
\hline $\begin{array}{l}\text { SERIAL } \\
\text { PERCENT } \\
0.0267 \\
2.5828 \\
2.5828 \\
0.3872 \\
0.3872 \\
0.2581 \\
0.2581 \\
0.3872 \\
0.3872 \\
2.5828 \\
2.5828 \\
1.2909 \\
1.2909 \\
2.5818 \\
2.5818 \\
2.5814 \\
2.5814 \\
1.2909 \\
1.2909 \\
0.3872 \\
0.3872 \\
2.5814 \\
2.5814 \\
0 . \\
0.5 \\
2.5814 \\
0.0437 \\
2.5814 \\
0.0437\end{array}$ & $\begin{array}{l}\text { SER I AL } \\
\text { RANK } \\
59 \\
7 \\
8 \\
43 \\
44 \\
51 \\
52 \\
45 \\
46 \\
9 \\
10 \\
37 \\
38 \\
11 \\
12 \\
15 \\
16 \\
39 \\
40 \\
47 \\
48 \\
17 \\
18 \\
0 \\
0 \\
19 \\
55 \\
20 \\
56\end{array}$ & 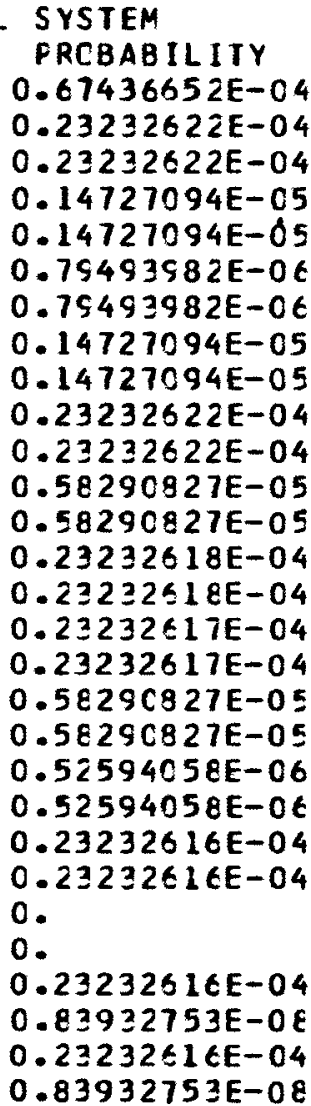 \\
\hline
\end{tabular}

SYSTEM SYSTEN FERCENT RANK

E. 0850

2.7854

2.7854

0.1766

C. 1766

C. 0953

C. 0953

0.1766

C. 1766

2.7854

2.7854

C. 6988

C. 6988

2.7854

$2.78: 4$

2.7854

2.7854

C.6988

C. 6988

0.0631

C. 0631

2.7854

2.7854

0

0 .

2.7854

C. 0010

2.7854

C. 0010 
COMPONENT CONIRIBUTIONS TO UNREL. IABILITY

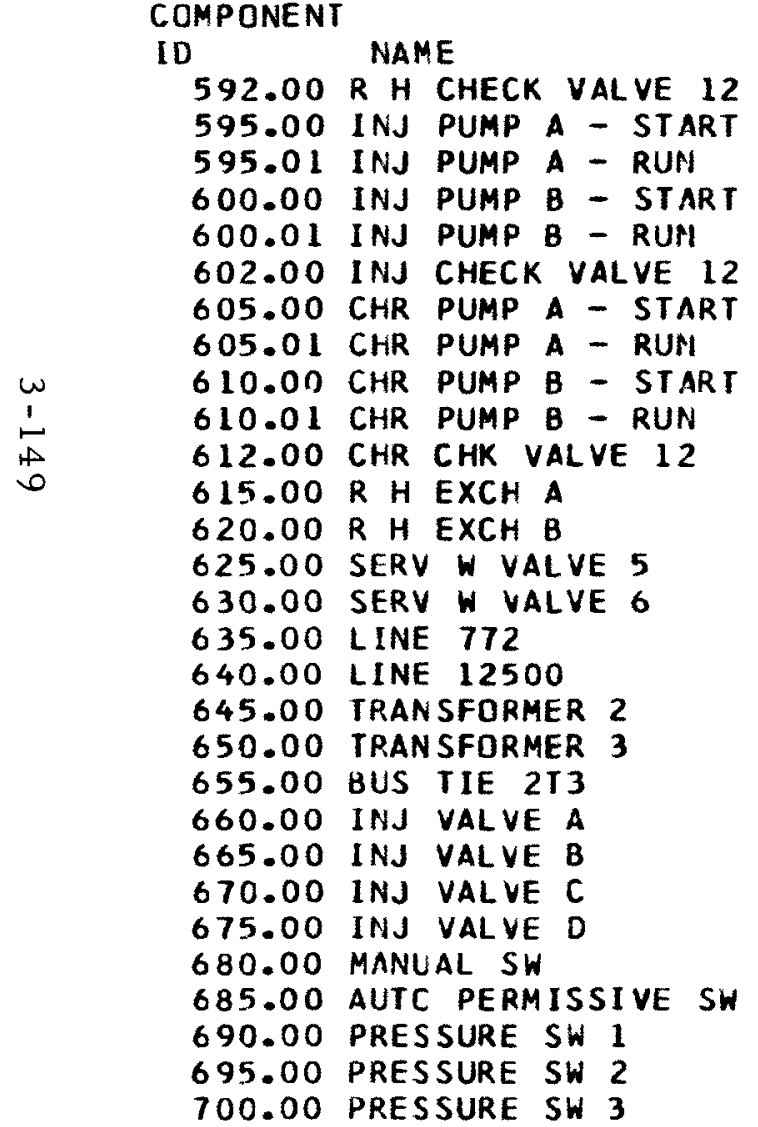

\begin{tabular}{|c|c|c|c|c|}
\hline $\begin{array}{r}\text { FAILURE } \\
\text { RATE*10*6 }\end{array}$ & $\begin{array}{l}\text { SERIAL } \\
\text { PRCBABILITY }\end{array}$ & $\begin{array}{l}\text { SERIAL } \\
\text { PERCENT }\end{array}$ & $\begin{array}{l}\text { SER IA } \\
\text { RANK }\end{array}$ & $\begin{array}{l}\text { SYSTEN } \\
\text { PRCBABILITY }\end{array}$ \\
\hline 1.00 & 0. & 0 & 0 & $0.47963603 \mathrm{E}-09$ \\
\hline 10.00 & $0.62830900[-02$ & 2.5814 & 21 & $0.23232516 \mathrm{E}-04$ \\
\hline 5.00 & $0.10646348 E-03$ & 0.0437 & 57 & $0.83932753 \mathrm{E}-\mathrm{C}$ \\
\hline 10.00 & $0.62830900 E-02$ & 2.5814 & 22 & $0.23232 \leqslant 1 \in \mathrm{E}-\mathrm{C}$ \\
\hline 5.00 & $0.10646348 E-03$ & 0.0437 & 58 & $0.83932753 \mathrm{E}-\mathrm{C}$ \\
\hline 1.00 & 0. & 0 & 0 & $0.47963 \leqslant 03 \mathrm{E}-\mathrm{C}$ \\
\hline 1.50 & $0.94246350[-03$ & 0.3872 & 49 & $0.52594058 \mathrm{E}-$ \\
\hline 0.75 & $0.15969521 E-04$ & 0.0066 & 62 & $0.1888691 \mathrm{iE}-\mathrm{C}$ \\
\hline 1.50 & $0.94246350 E-03$ & 0.3872 & 50 & $0.52594058 E-C$ \\
\hline 0.75 & $0.15969521 E-04$ & 0.0066 & 63 & $0.18886917 \mathrm{E}-\mathrm{C}$ \\
\hline 0.15 & 0 & 0 & 0 & $0.1 C 792996 \mathrm{E}-1$ \\
\hline 0.30 & $0.19488050 E-03$ & 0.0801 & 53 & $0.61824332 \mathrm{E}-\mathrm{C}$ \\
\hline 0.30 & $0.19488050 E-03$ & 0.0801 & 54 & $0.61824332 \mathrm{E}-\mathrm{C}$ \\
\hline 10.00 & $0.62839626 \mathrm{E}-02$ & 2.5818 & 13 & $0.2459 C 704 \mathrm{E}-0$ \\
\hline 10.00 & $0.62839626 E-02$ & 2.5818 & 14 & $0.24590704 E-C$ \\
\hline 0.10 & $0.64960165 E-04$ & 0.0267 & 60 & $0.9158515 E E-C$ \\
\hline 0.10 & $0.64960165 E-04$ & 0.0267 & 61 & $0.9158515 \mathrm{EE}-\mathrm{C}$ \\
\hline 5.00 & $0.32480084 E-02$ & 1.3344 & 27 & $0.77362577 \mathrm{E}-\mathrm{C}$ \\
\hline 5.00 & $0.32480084 E-02$ & 1.3344 & 28 & $0.77362577 \mathrm{E}-\mathrm{C}$ \\
\hline 2.00 & 0 & 0 & 0 & $0.14257726 \mathrm{E}-\mathrm{C}$ \\
\hline 10.00 & $0.62830900[-02$ & 2.5814 & 23 & $0.69697848 \mathrm{E}-\mathrm{C}$ \\
\hline 10.00 & $0.62830900 E-02$ & 2.5814 & 24 & $0.69697848 \mathrm{E}-0$ \\
\hline 10.00 & $0.62830900 \mathrm{E}-02$ & 2.5814 & 25 & $0.69697848 E-0$ \\
\hline 10.00 & $0.62830900 \mathrm{E}-02$ & 2.5814 & 26 & $0.6969784 \varepsilon E-0$ \\
\hline 5.00 & $0.31419813 \mathrm{E}-02$ & 1.2909 & 41 & $0.66495248 \mathrm{E}-\mathrm{C}$ \\
\hline 5.00 & $0.31415450 E-02$ & 1.2907 & 42 & $0.5829 C 823 E-0$ \\
\hline 15.00 & $0.94246349 E-02$ & 3.8721 & 1 & $0.320086600 \mathrm{CE}-\mathrm{C}$ \\
\hline & $0.94246349[-02$ & 3.8721 & 2 & $0.3200 \in E O C E-C$ \\
\hline & $0.94246349 \mathrm{E}-02$ & 3.8721 & 3 & $0.3208660 \mathrm{CE}-\mathrm{C}$ \\
\hline
\end{tabular}

$\begin{array}{lr}\text { SYSTEM } & \text { SYSTEN } \\ \text { FERCENT } & \text { RANK } \\ \text { C.COC1 } & 62 \\ 2.7854 & 2 C \\ C .0010 & 6 C \\ 2.7854 & 21 \\ C .0010 & 61 \\ C .0001 & 63 \\ C .0621 & 46 \\ C .0000 & 64 \\ C .0631 & 47 \\ C . C C C 0 & 65 \\ C . C 000 & 7 C \\ C .0074 & 54 \\ C . C 074 & 55 \\ 2.9482 & 6 \\ 2.9482 & 7 \\ C .0011 & 56 \\ C .0011 & 57 \\ C .9275 & 24 \\ C .9275 & 25 \\ C .1709 & 38 \\ 8.3561 & 1 \\ 8.3561 & 2 \\ 8.3561 & 3 \\ 8.3561 & 4 \\ C .7972 & 28 \\ C .6988 & 33 \\ C .0385 & 4 \varepsilon \\ C .0385 & 49 \\ C .0385 & 50\end{array}$




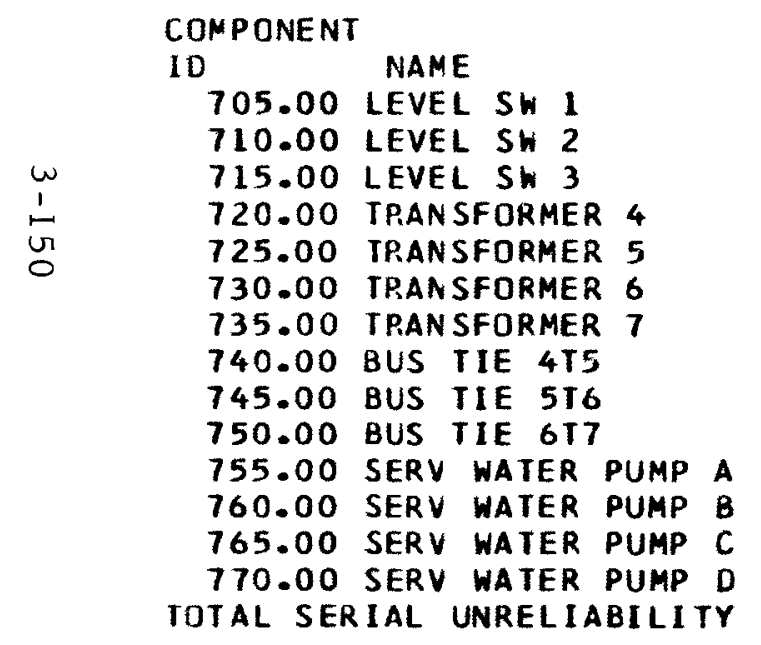

COMPONENT COATRIBUTIONS TO UNRELIABILITY

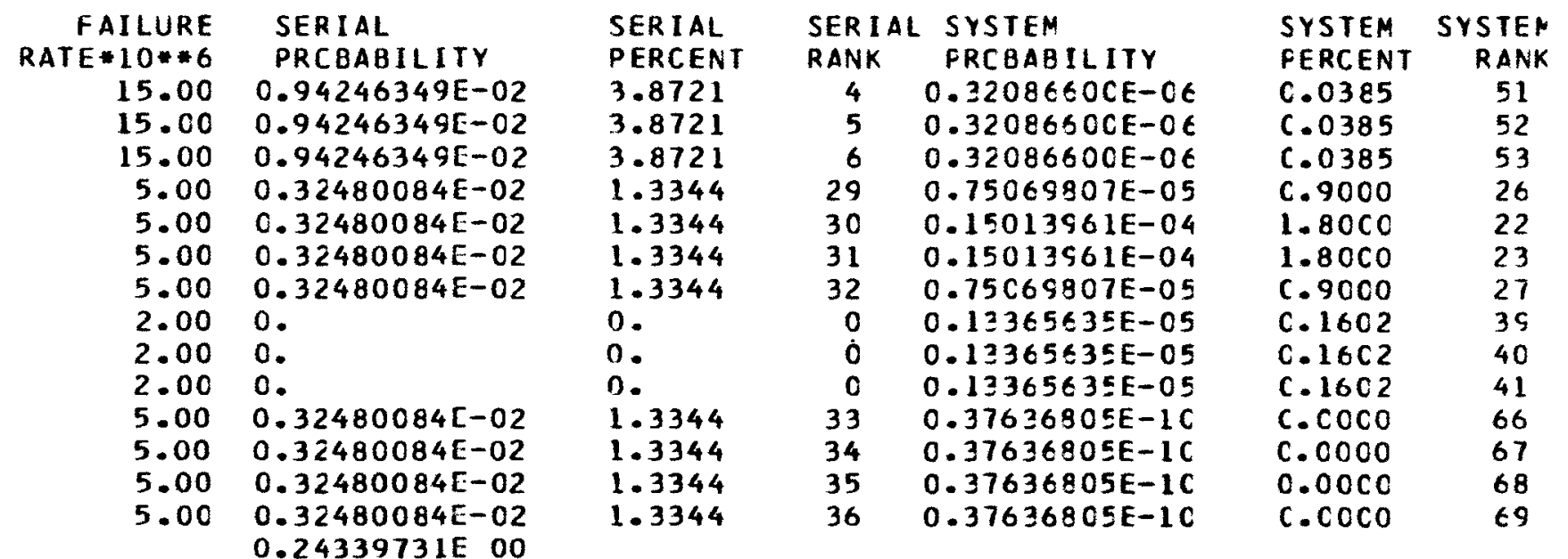


COMPONENT CONTRIBUTIONS TO UNRELIABILITY

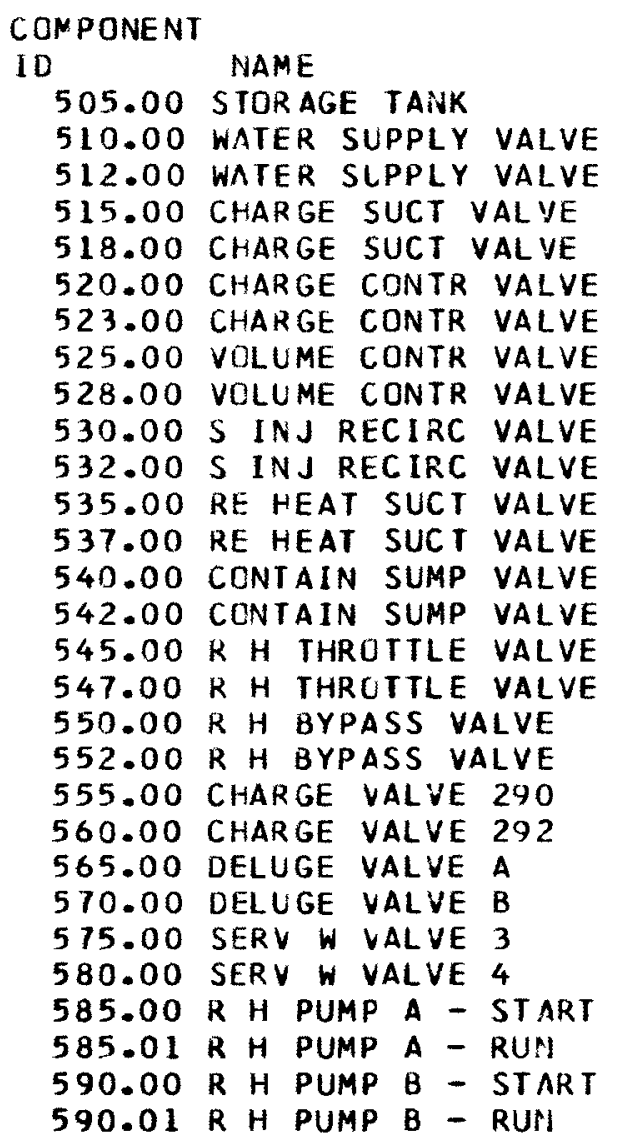

$\begin{array}{rccr}\text { FAILURE } & \text { SERIAL } & \text { SERIAL } & \text { SER } \\ \text { RATE*10.6 } & \text { PRCBABILITY } & \text { PERCENT } & \text { RAN } \\ 0.10 & 0.18698501 E-04 & 0.0335 & 59 \\ 5.00 & 0.81830240 E-03 & 1.4677 & 29 \\ 5.00 & 0.8183024 C E-03 & 1.4677 & 30 \\ 0.75 & 0.12245380 E-03 & 0.2196 & 47 \\ 0.75 & 0.12245380 E-03 & 0.2196 & 48 \\ 0.50 & 0.81635870 E-04 & 0.1464 & 55 \\ 0.50 & 0.81635870 E-04 & 0.1464 & 56 \\ 0.75 & 0.12245380 E-03 & 0.2196 & 49 \\ 0.75 & 0.12245380 E-03 & 0.2196 & 50 \\ 5.00 & 0.81830240 E-03 & 1.4677 & 31 \\ 5.0 C & 0.81830240 E-03 & 1.4677 & 32 \\ 2.50 & 0.4 C 842231 E-03 & 0.7325 & 39 \\ 2.50 & 0.4 C 842231 E-03 & 0.7325 & 40 \\ 5.00 & 0.81684462 E-03 & 1.4650 & 33 \\ 5.0 C & 0.81684462 E-03 & 1.4650 & 34 \\ 5.00 & 0.81635870 E-03 & 1.4642 & 36 \\ 5.00 & 0.81635870 E-03 & 1.4642 & 37 \\ 2.50 & 0.4 C 342231 E-03 & 0.7325 & 41 \\ 2.5 C & 0.4 C 342231 E-03 & 0.7325 & 42 \\ 1.50 & 0.24490761 E-03 & 0.4393 & 43 \\ 1.50 & 0.24490761 E-03 & 0.4393 & 44 \\ 10.00 & 0.16327174 E-02 & 2.9283 & 9 \\ 10.00 & 0.16327174 E-02 & 2.9283 & 10 \\ 5.00 & 0 . & 0.9 & 0 \\ 5.00 & 0.1 & 0.92 & 0 \\ 10.00 & 0.16327174 E-02 & 2.9283 & 11 \\ 5.00 & 0.11856638 E-03 & 0.2127 & 51 \\ 10.00 & 0.16327174 E-02 & 2.9283 & 12 \\ 5.00 & 0.11356638 E-03 & 0.2127 & 52 \\ & & & \end{array}$

R IA
NK
59
30
47
8
55
56
49
0
31
32
39
0
33
34
36
37
4
42
3
4
9
10
0
0
1
11
2
52

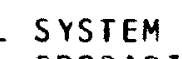

$\begin{array}{lr}\text { SYSTEM } & \text { SYSTEN } \\ \text { FERCENT } & \text { RANK } \\ \text { C.2106 } & 19 \\ 9.2166 & 1 \\ 5.2166 & 2 \\ 1.3797 & 13 \\ 1.3797 & 14 \\ C .9198 & 17 \\ C .9198 & 18 \\ 1.3797 & 15 \\ 1.3797 & 16 \\ 9.2166 & 3 \\ 5.2166 & 4 \\ 4.6011 & 9 \\ 4.6011 & 10 \\ 6.2 C C 2 & 5 \\ 5.20 C 2 & 6 \\ 9.1948 & 7 \\ 9.1948 & 8 \\ 4.6011 & 11 \\ 4.6011 & 12 \\ C .0003 & 44 \\ C .0003 & 45 \\ C .0154 & 26 \\ C .0154 & 27 \\ C . & C \\ 0 . & 0 \\ C .0154 & 28 \\ C . C C C 1 & 48 \\ C .0154 & 25 \\ C .00 C 1 & 49\end{array}$


COMPONENT CONIRIBUTIONS TO UNRELIABILITY

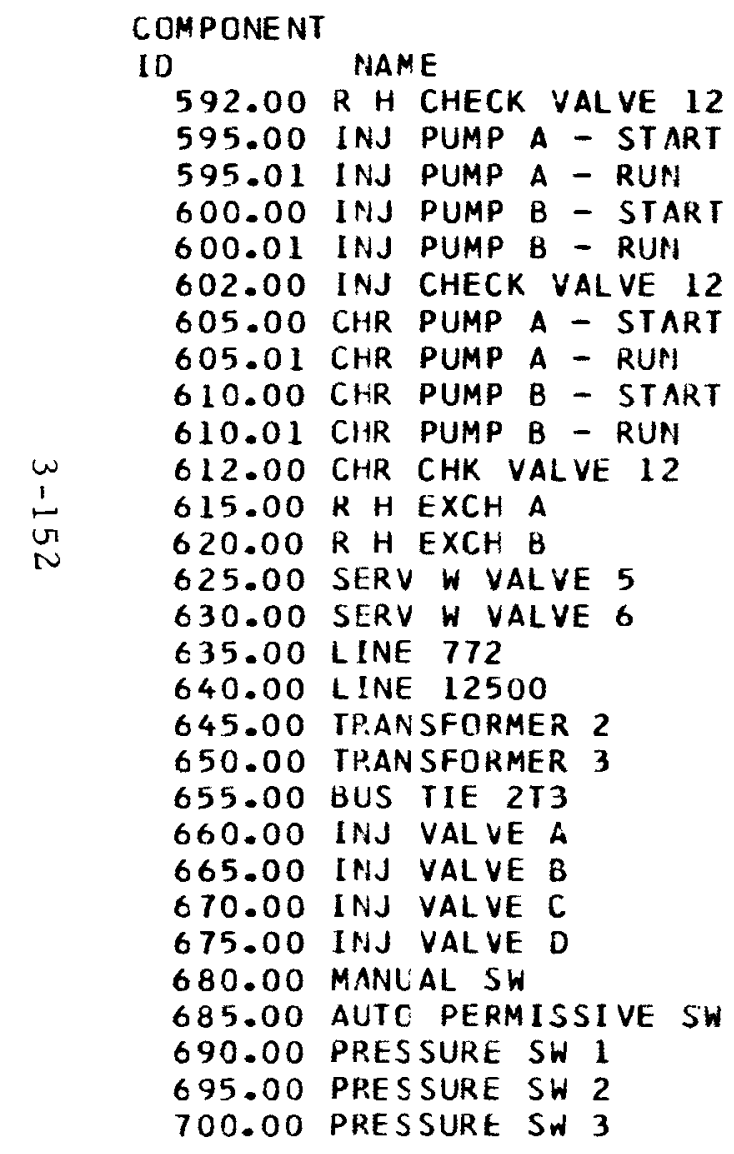

$\begin{array}{rl}\text { FAILURE } & \text { SERIAL } \\ \text { RATE 10.*6 } & \text { PRCBABILITY } \\ 1.00 & 0 . \\ 10.00 & 0.16327174 E-02 \\ 5.00 & 0.11356638 E-03 \\ 10.00 & 0.16327174 E-02 \\ 5.00 & 0.11856638 E-03 \\ 1.00 & 0 . \\ 1.50 & 0.24490761 E-03 \\ 0.75 & 0.17784957 E-04 \\ 1.50 & 0.24490761 E-03 \\ 0.75 & 0.17784957 E-04 \\ 0.15 & 0 . \\ 0.30 & 0.56095504 E-04 \\ 0.30 & 0.56095504 E-04 \\ 10.00 & 0.16336892 E-02 \\ 10.00 & 0.16336892 E-02 \\ 0.10 & 0.18698501 E-04 \\ 0.10 & 0.18698501 E-04 \\ 5.00 & 0.92492507 E-03 \\ 5.00 & 0.93492507 E-03 \\ 2.00 & 0.1 \\ 10.00 & 0.16327174 E-02 \\ 10.00 & 0.16327174 E-02 \\ 10.00 & 0.16327174 E-02 \\ 10.00 & 0.16327174 E-02 \\ 5.00 & 0.81684462 E-03 \\ 5.00 & 0.81635870 E-03 \\ 15.00 & 0.24490761 E-02 \\ 15.00 & 0.24490761 E-02 \\ 15.00 & 0.24490761 E-02\end{array}$

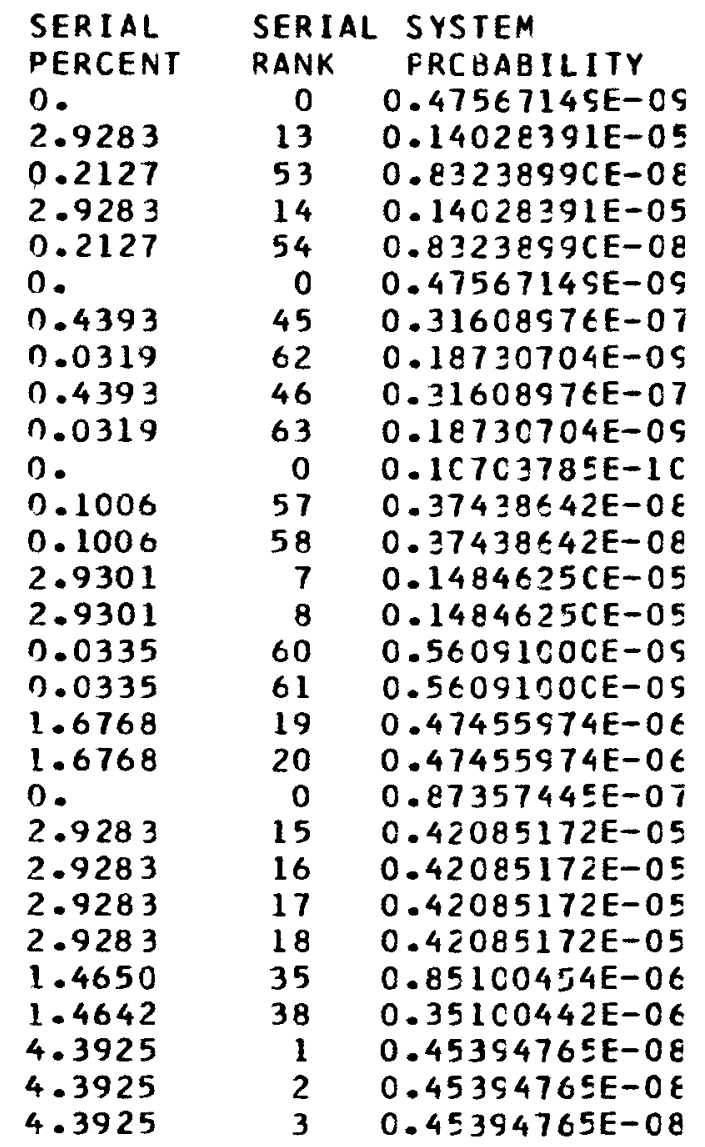

\begin{tabular}{|c|c|}
\hline $\begin{array}{l}\text { SYSTEM } \\
\text { FERCENT } \\
C .00 C O \\
C .0154 \\
C . C O O 1 \\
C .0154 \\
\text { C.0OO1 } \\
C .00 C O \\
C . C 003 \\
C .0000 \\
C .0003 \\
C . C O C O \\
C . C 0 C 0 \\
C . C 000 \\
C .00 C 0 \\
C .0163 \\
C .0163 \\
C .0000 \\
C .0000 \\
C . C 052 \\
C .0052 \\
C . C 010 \\
C .0463 \\
C .0463 \\
C .0463 \\
C .0463 \\
C .0054 \\
C . C 039 \\
C . C 000 \\
C . C 0 C 0 \\
C .00 C 0\end{array}$ & $\begin{array}{c}\text { SYSTEK } \\
\text { RAAK } \\
62 \\
30 \\
50 \\
31 \\
51 \\
63 \\
46 \\
64 \\
47 \\
65 \\
66 \\
58 \\
59 \\
24 \\
25 \\
6 C \\
61 \\
35 \\
36 \\
4 C \\
20 \\
21 \\
22 \\
23 \\
34 \\
39 \\
52 \\
53 \\
54\end{array}$ \\
\hline
\end{tabular}


AUTOMATIC RELIABILITY MATH MOOEL

PAGE

CASE 3. SAFETY INJECTION - CONN. YANKEE

SUMAARY

COMPONENT CONTRI BUTIONS TO UNREL IABILITY

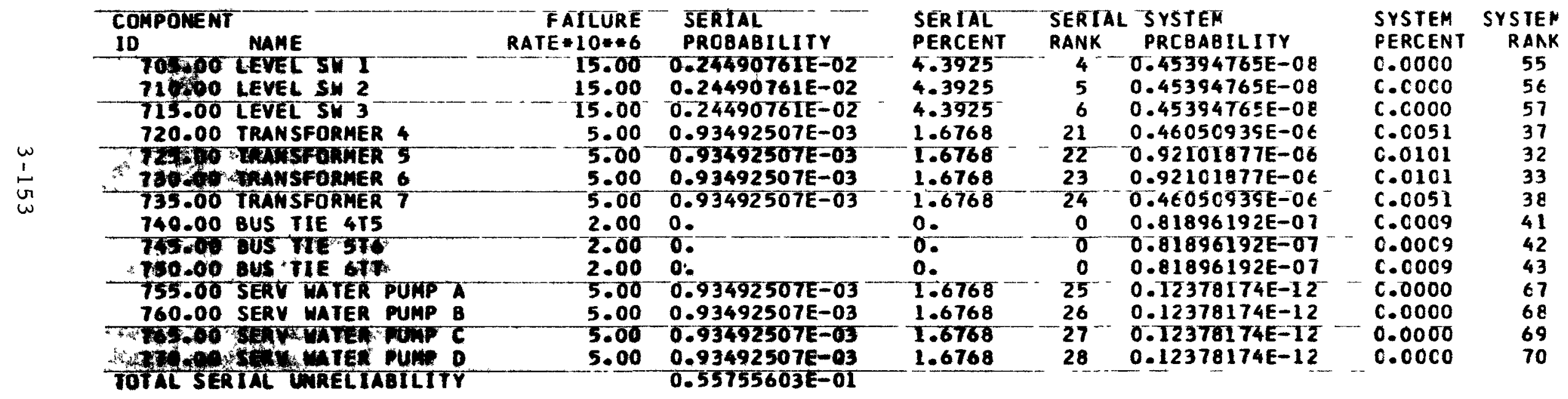


AUTOMATIC RELIABILITY MATH MODEL

CASE 1. SAFETY INJECTION - CONN. YANKEE

SUMMARY

PROBABILITY OF SYSTEM FAILURE $=0.38762840 E-01$

PROBABILITY OF SYSTEN SUCCESS $=0.96123715$
PAGE

36

00000000,1900

$0000-00$

$\begin{array}{cc} & \text { FUNCTION } 10 \\ 105 & \\ 110 \\ 110 \\ 115 \\ 120 \\ 125 \\ 130 \\ \\ w \\ 135 \\ 1 \\ J \\ 140 \\ 145 \\ 150 \\ 160 \\ 161 \\ -163 \\ 165 \\ 166 \\ -168 \\ 170 \\ 171 \\ -173 \\ 175 \\ 177 \\ 180 \\ -190 \\ 195 \\ 200\end{array}$

FUNCTION NAME

HATER STORAGE

WATER SUPPLY

CHARGE LINE

SAFETY INJ RECIRC

RESID HEAT RECIRC

CONTAIN SUMP RECIR

CORE DELUGE LINE

R H EXCH BYPASS

CHARGE LINE DIST

CORE DELUGE DIST

R H PUMP AB - START

$R$ H PUMP AB -RUN

$R$ H CHECK VALVE 12

INJ PUMP AB - START

INJ PUMP AB -RUN

INJ CHECK VALVE 12

CH PUMP AB -START

CH PUMP AB -RUN

CHARGE CK VALVE 12

R H EXCH W/COOLING

R H EXCH W/O COOL

POWER 23

POWER 2 T3

SAFETY INJ DIST

MANUAL OPERATION

PROBABILITY OF FUNCTION FAILURE
$0.72973438 E-04$
$0.70542102 E-02$
$0.28245263 E-02$
$0.70542102 E-02$
$0.35288359 E-02$
$0.70513265 E-02$
$0.70503653 E-02$
$0.35288359 E-02$
$0.11429813 E-05$
$0.50489440 E-04$
$0.50489440 E-04$
$0.13842147 E-07$
$0.27685660 E-08$
$0.50489440 E-04$
$0.13842147 E-07$
$0.27685660 E-08$
$0.11429813 E-05$
$0.31148008 E-09$
$0.62299584 E-10$
$0.53575156 E-04$
$0.49836486 E-10$
$0.13194936 E-04$
$0.51841672 E-05$
$0.30293663 E-03$
$0.48061415 E-06$

PERCENT OF SYSTEM FALLURE RANK 0.188

18.198

7.287

18.198

9.104

18.191

18.188

9.104

0.003

0.130

0.130

0.000

0.000

0.130

0.000

0.000

0.003

0.000

0.000

0.138

0.000

0.034

0.013

0.782

0.001 


\author{
PROBABILITY CF SYSTEM FAILURE $=0.83409745 E-03$ \\ PROBABILITY OF SYSTEM SUCCESS = $0.9991659 \mathrm{C}$
}

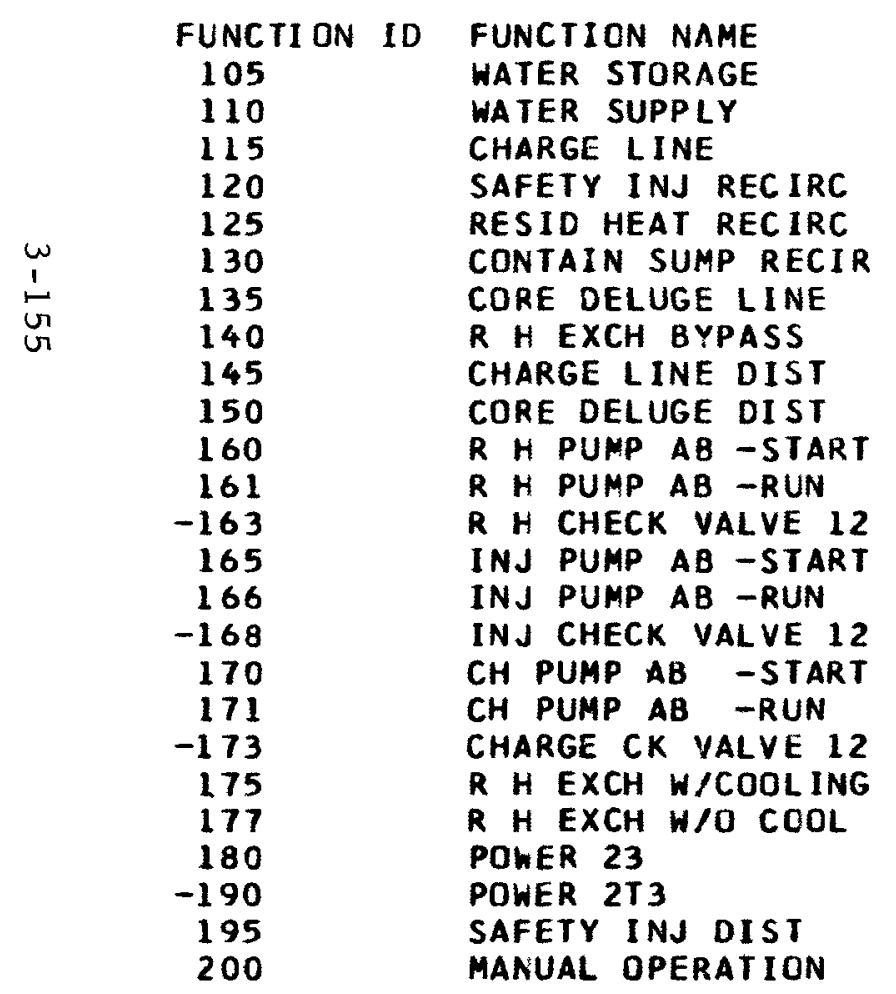

PROBABILITY OF FUNCTION FAILURE
$0.67436637 E-04$
$0.46465238 E-04$
$0.74807162 E-05$
$0.46465238 E-04$
$0.11658164 E-04$
$0.46465230 E-04$
$0.46465229 E-04$
$0.11658164 E-04$
$0.10518810 E-05$
$0.46465226 E-04$
$0.46465226 E-04$
$0.14388334 E-07$
$0.28778088 E-08$
$0.46465226 E-04$
$0.14388334 E-07$
$0.28778088 E-08$
$0.10518810 E-05$
$0.32377052 E-09$
$0.64757814 E-10$
$0.49304999 E-04$
$0.51802944 E-10$
$0.12144962 E-04$
$0.47716412 E-05$
$0.27879136 E-03$
$0.49957641 E-06$

PERCENT CF SYSTEN FAILURE RANK 8. C 85

5.571

C. 897

5. 571

1.398

5. 571

5.571

1.398

0.126

5. 571

5.571

0.002

$0 . \mathrm{COO}$

5.571

C. $\mathrm{CO} 2$

o. $\mathrm{COO}$

0.126

0.000

C. $\mathrm{COO}$

5.911

0.000

1.456

C. 572

33.424

0.060
2

18

16

25

8

9
28

30

$1 \mathrm{C}$

29

21

32
34

34

35

11

19

27 


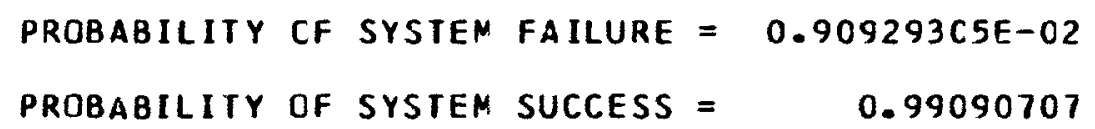

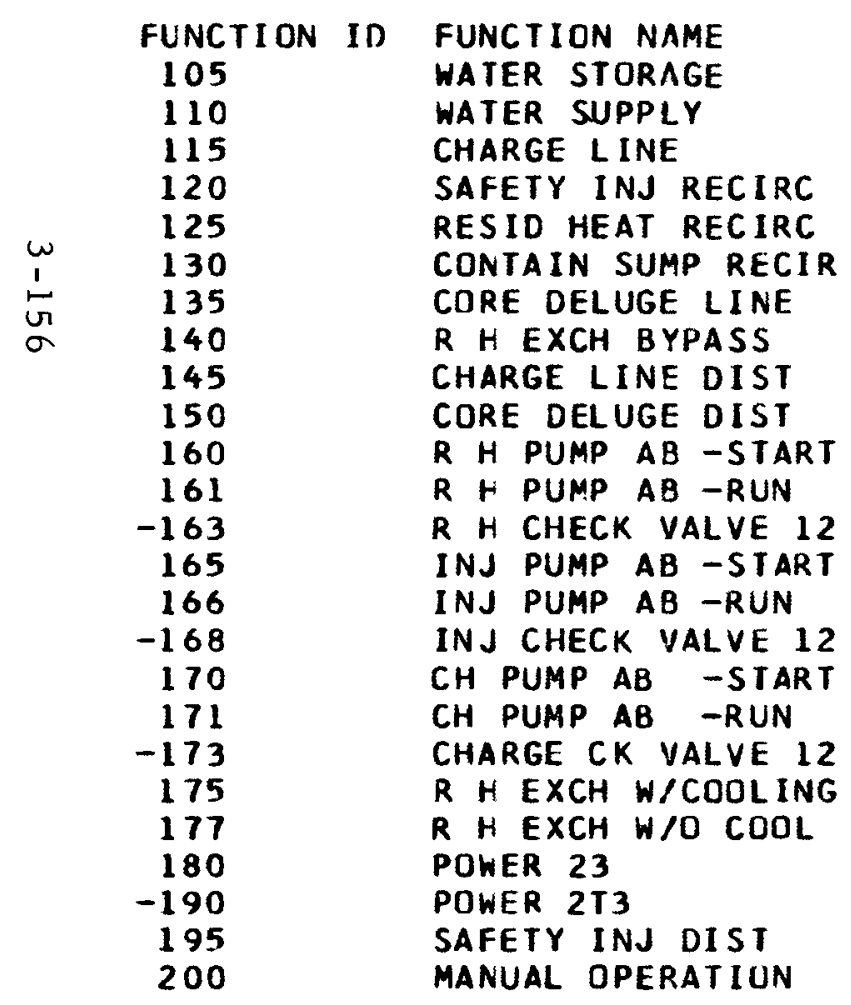

PROBABILITY OF FUNCTION FAILURE
$0.19146139 E-04$
$0.16761153 E-02$
$0.66910295 E-03$
$0.16761153 E-02$
$0.83674685 E-03$
$0.16731426 E-02$
$0.16721516 E-02$
$0.83674685 E-03$
$0.63217946 E-07$
$0.28056779 E-05$
$0.28056779 E-05$
$0.14269404 E-07$
$0.28540217 E-08$
$0.28056779 E-05$
$0.14269404 E-07$
$0.28540217 E-08$
$0.63217946 E-07$
$0.32109434 E-09$
$0.64222547 E-10$
$0.29766860 E-05$
$0.51374757 E-10$
$0.74522764 E-06$
$0.29237095 E-06$
$0.16834067 E-04$
$0.49545842 E-06$

$\begin{array}{cr}\text { PERCENT CF SYSTEN FAILURE } & \text { RAAK } \\ 0.211 & 8 \\ 18.433 & 1 \\ 7.258 & 7 \\ 19.433 & 2 \\ 9.202 & 5 \\ 18.400 & 3 \\ 18.29 C & 4 \\ 5.202 & 6 \\ 0.001 & 24 \\ 0.031 & 11 \\ 0 . C 31 & 12 \\ 0 . C 00 & 28 \\ 0.000 & 30 \\ 0.031 & 13 \\ 0 . C 00 & 29 \\ 0 . C 00 & 31 \\ 0.001 & 25 \\ 0 . C 00 & 32 \\ 0 . C C C & 33 \\ 0.033 & 1 C \\ 0.000 & 34 \\ 0 . C 08 & 14 \\ 0 . C 03 & 20 \\ 0.185 & 9 \\ 0.005 & 19\end{array}$

FIGURE 3.29 (continued) 


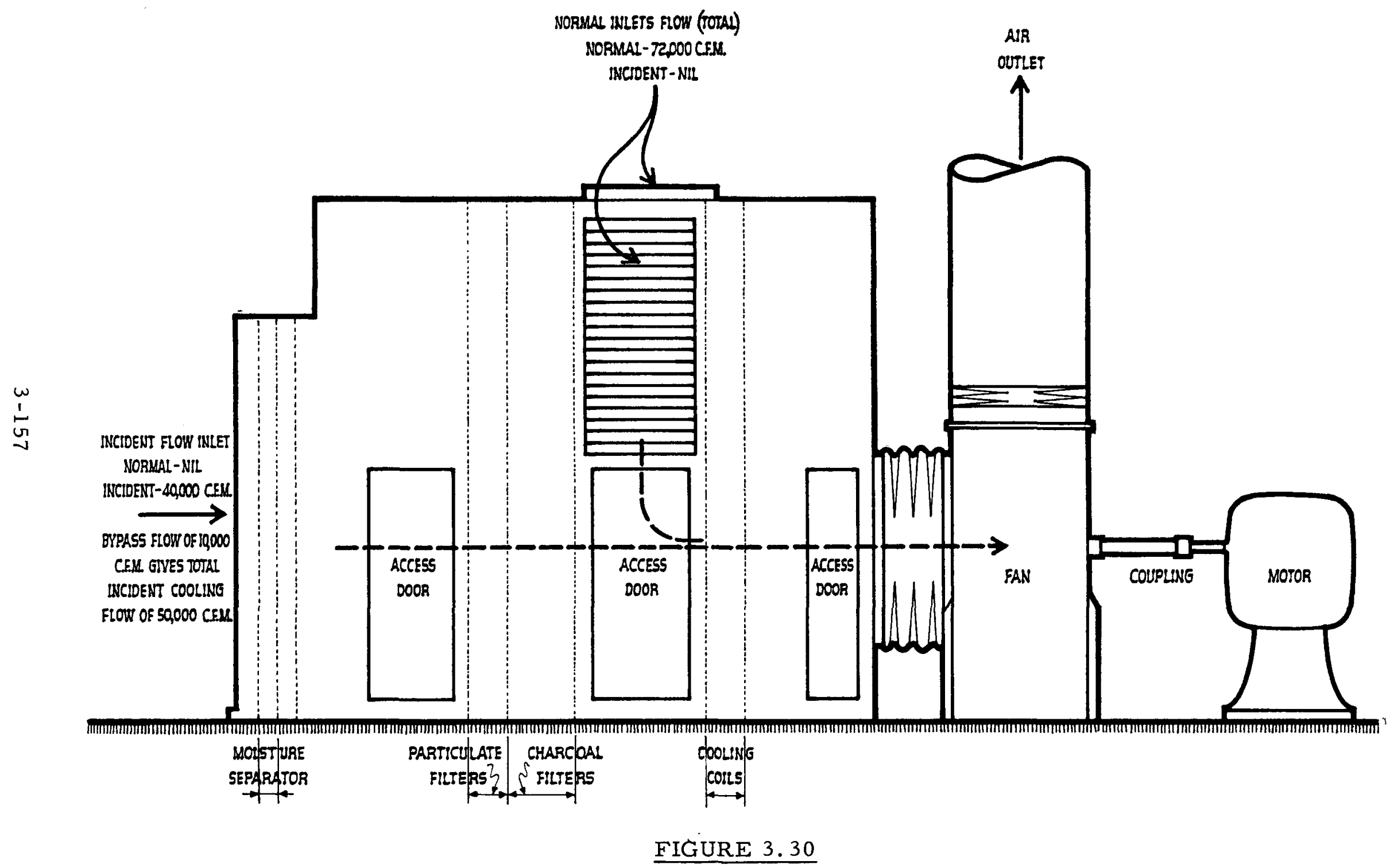

AIR RECIRCULATION FAN - COOLING COIL UNIT CONNECTICUT YANKEE 

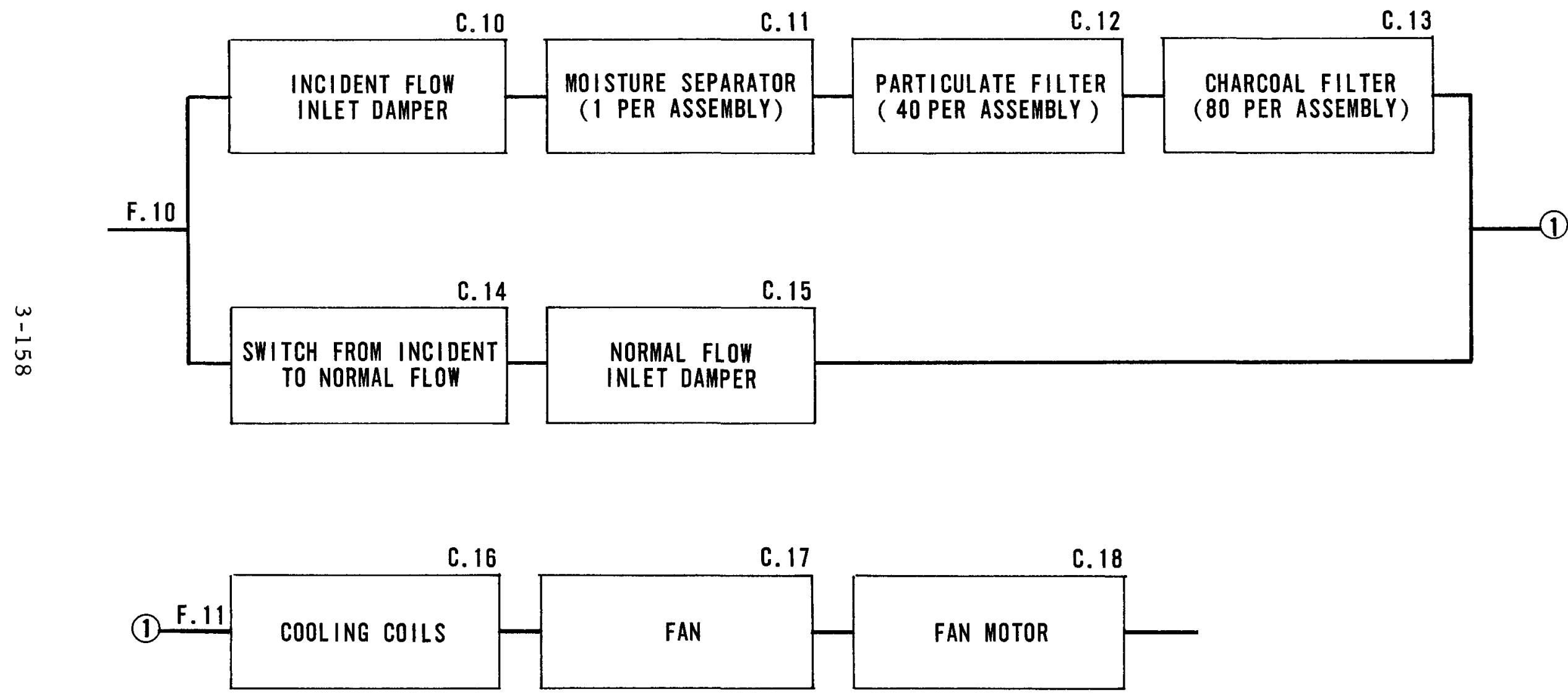

FIGURE $3.31 \mathrm{~A}$

RELIABILITY BLOCK DIAGRAM CONNECTICUT YANKEE - AIR RECIRCULATION UNIT 


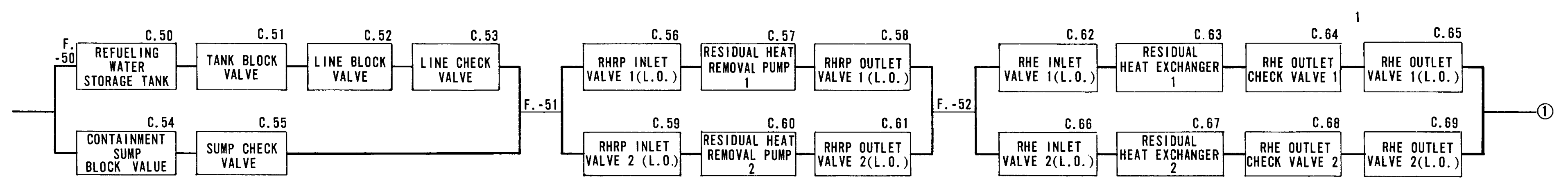

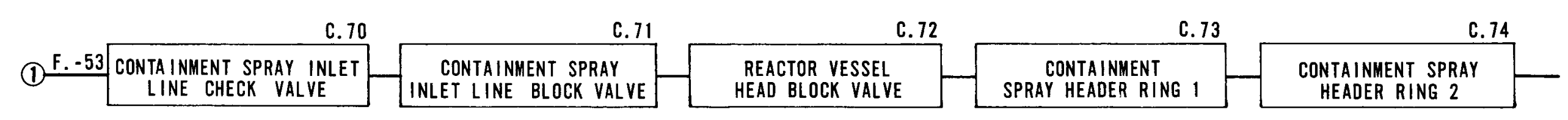




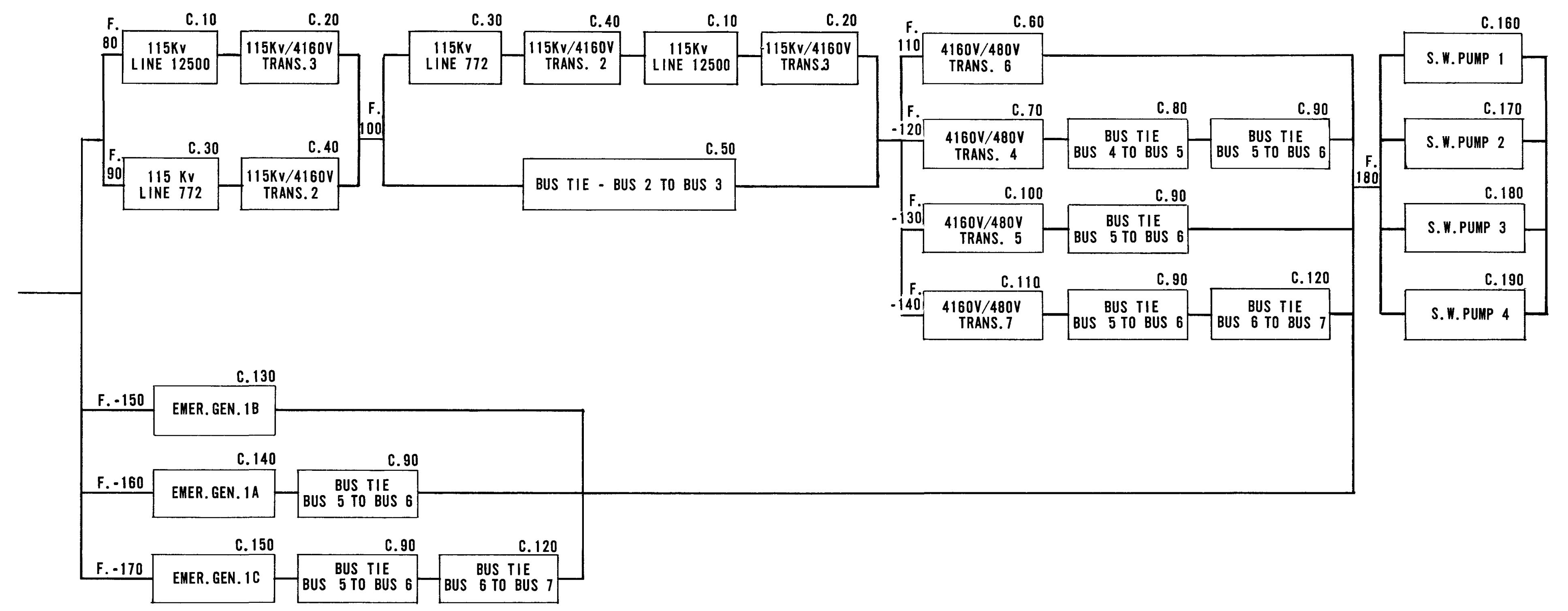

FIGURE $3.31 \mathrm{C}$

RELIABILITY BLOCK DIAGRAM

CONNECTICUT YANKEE

POWER SUPPLY AND SERVICE WATER SYSTEM PUMPS 


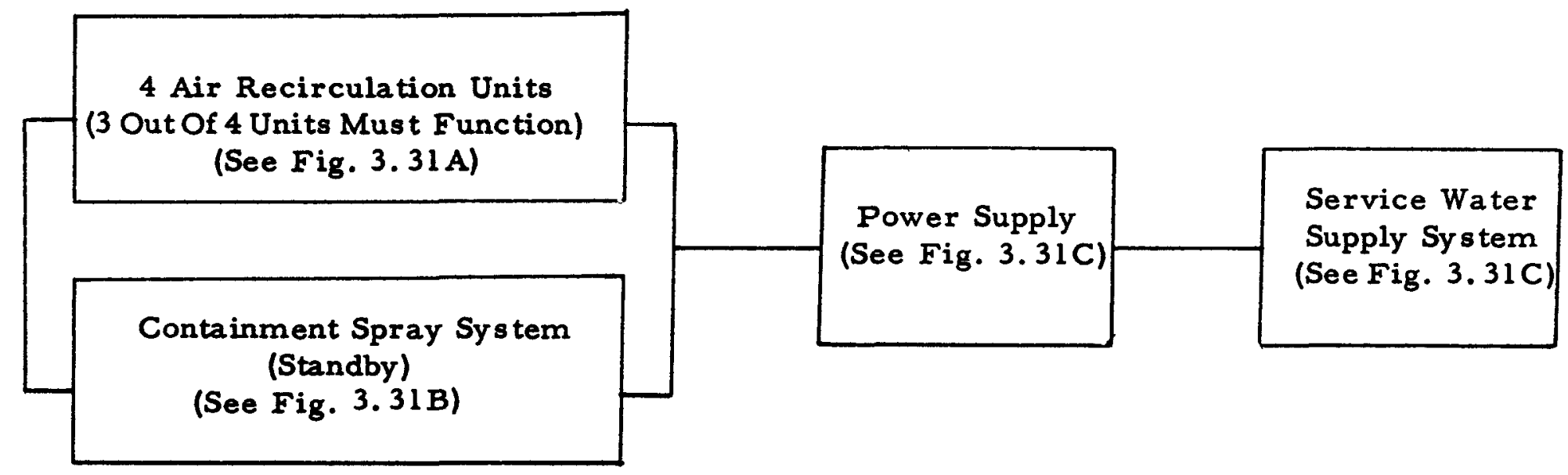

FIGURE $3.31 \mathrm{D}$

CONT AINMENT COOLING SYSTEM RELIABILITY BLOCK DIAGRAM - CONNECTICUTT YANKEE 


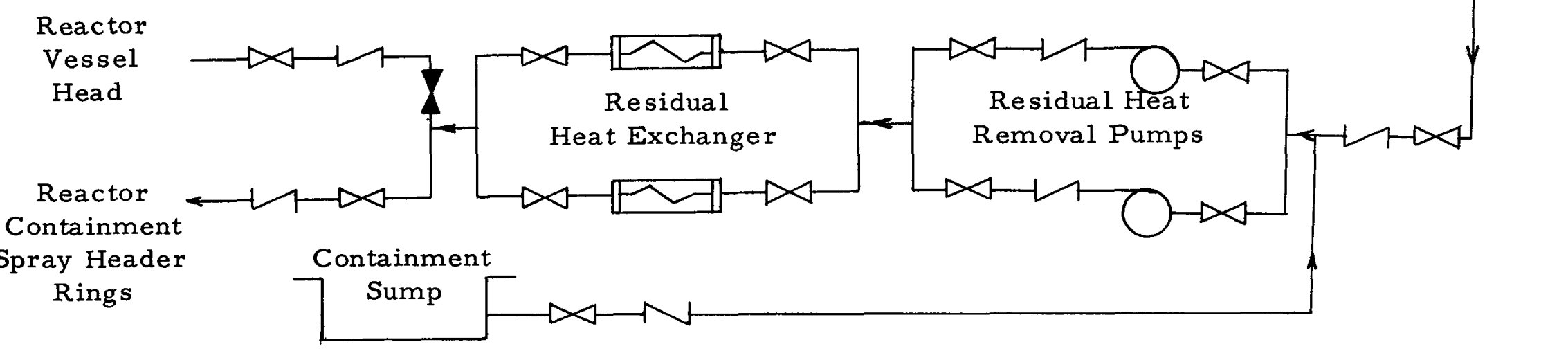




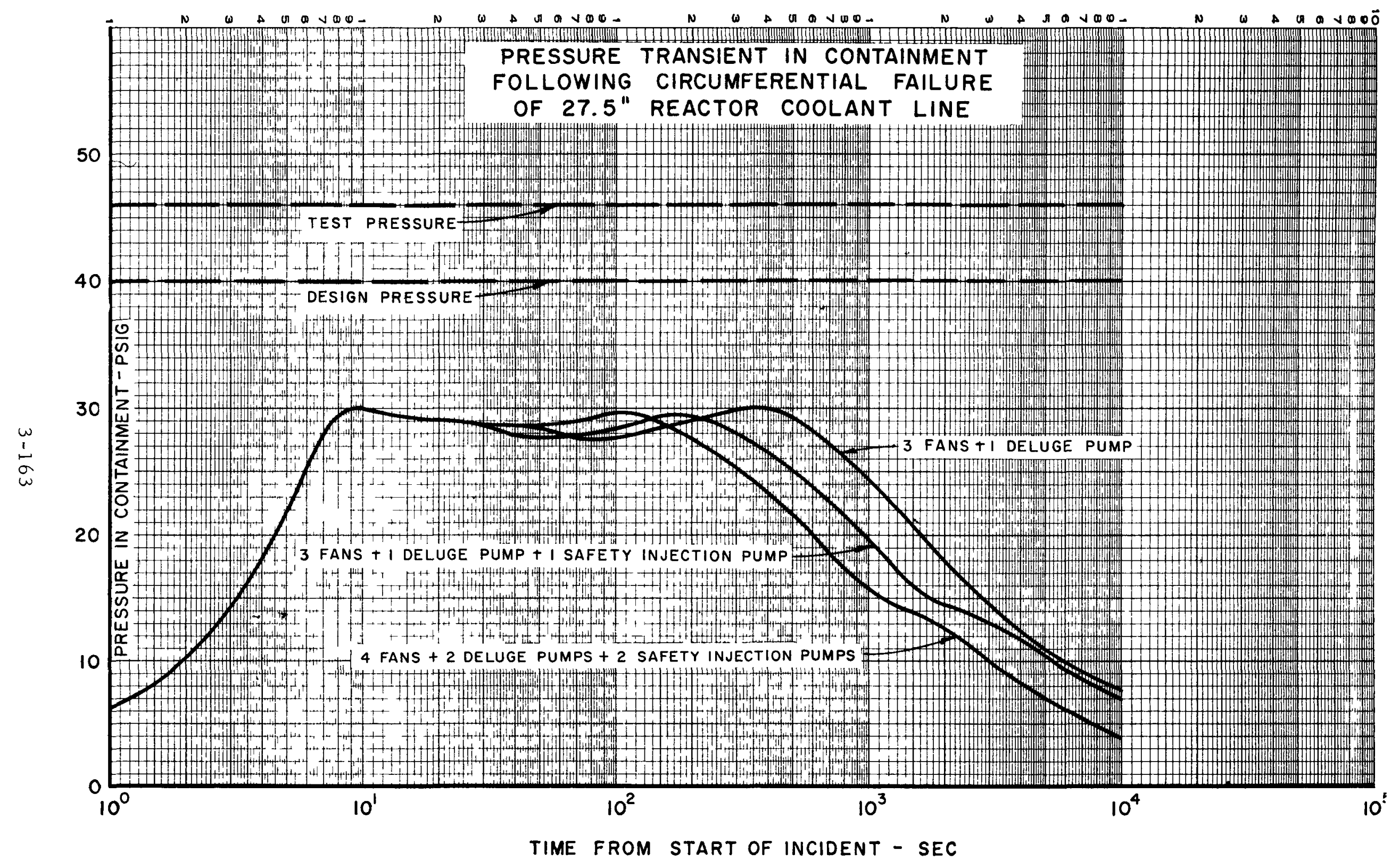

FIGURE 3.35 


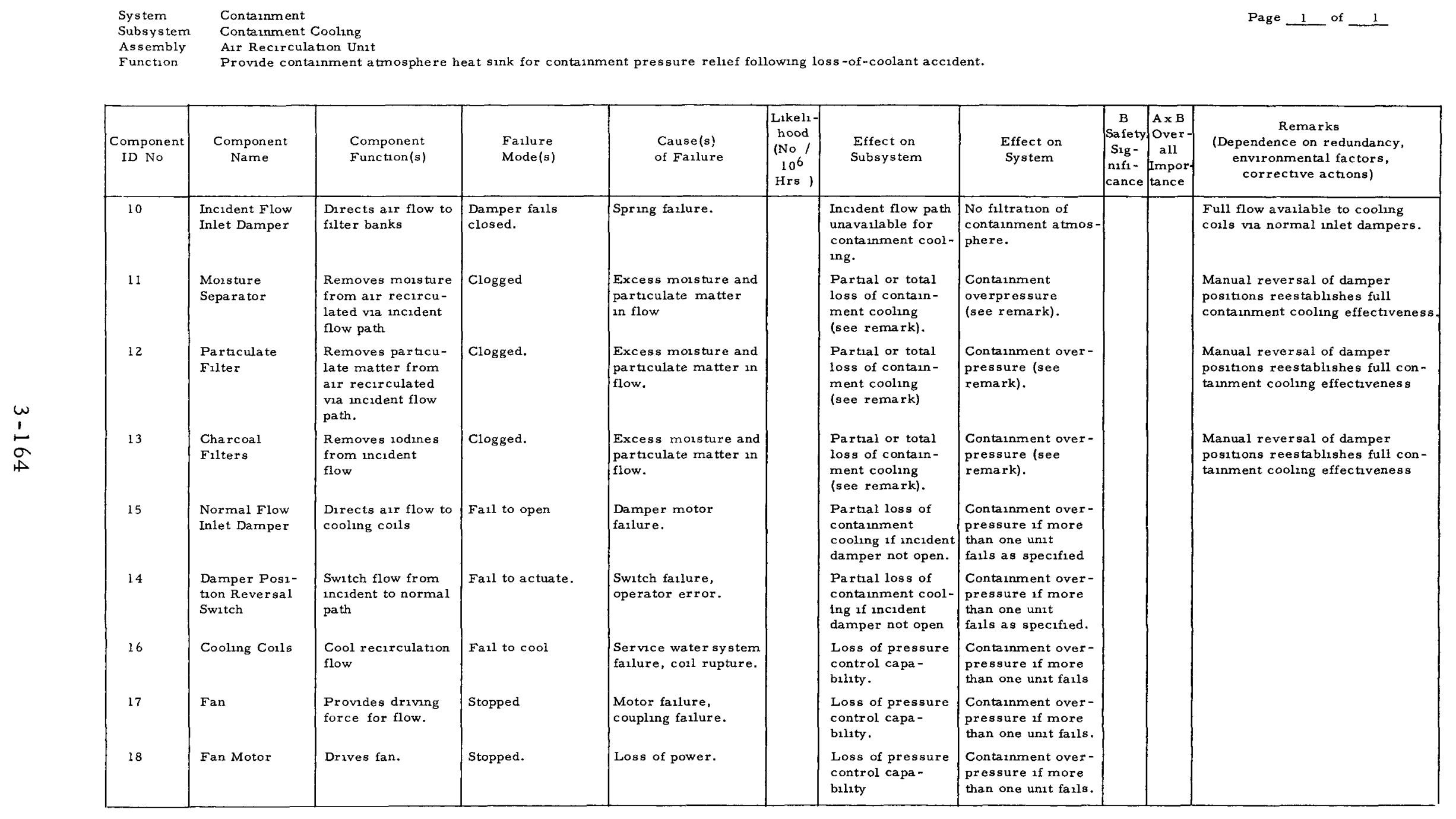

\section{FIGURE $3.34 \mathrm{~A}$}


System Containment

Subsystem Containment Coolin

Page 1 of 2

Containment Spray System

Function Provide containment atmosphere heat sink for containment pressure relief following loss-of-coolant accident.

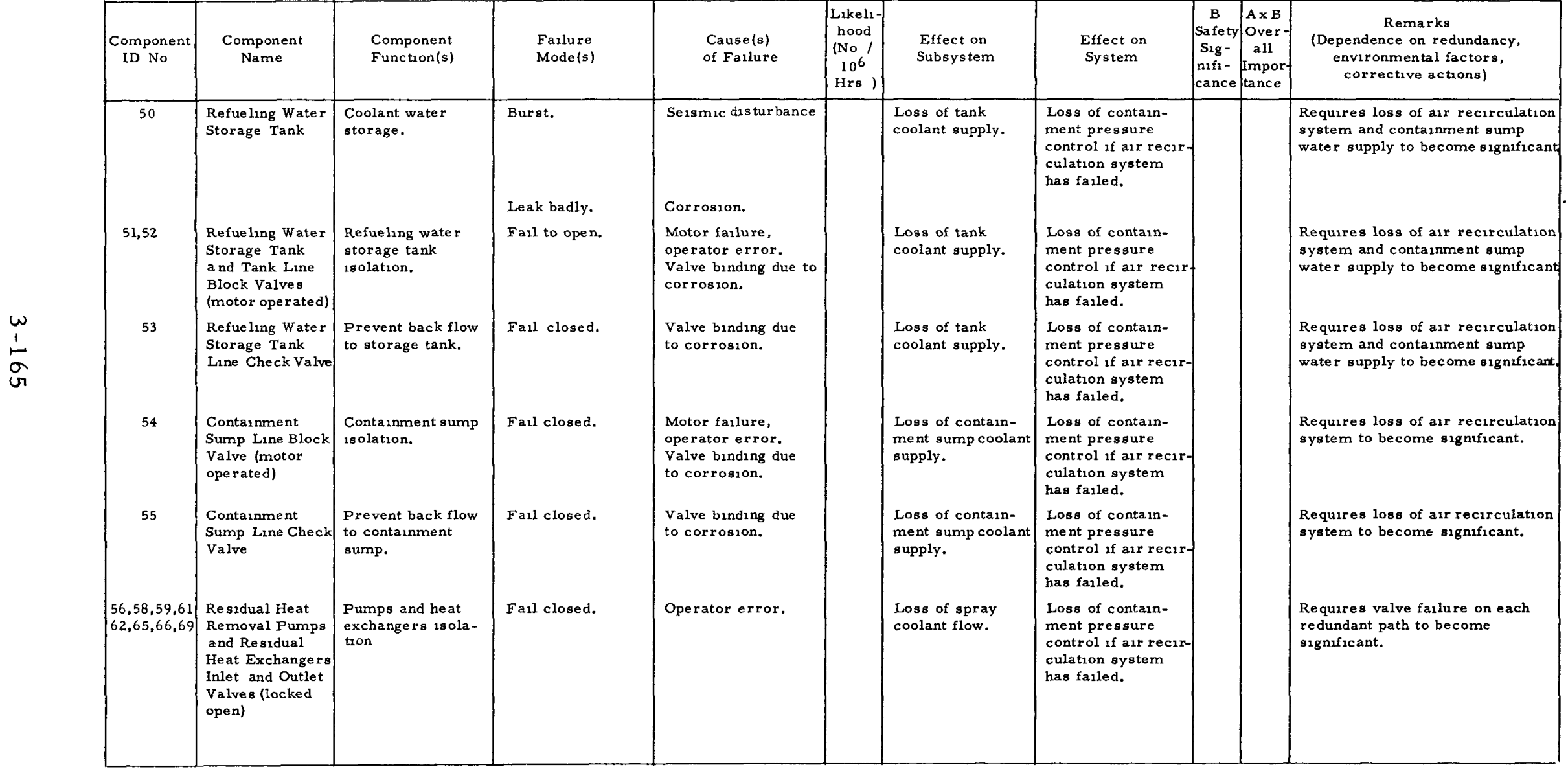

FIGURE 3.34B

FAILURE MODE AND EFFECT ANALYSIS CONNECTICUT YANKEE - AIR RECIRCULATION UNIT 
System

Containment

Page 2 of 2

Assembly Contanment Cooling

Function Provide containment atmosphere heat sink for containment pressure relief following loss-of-coolant accident.

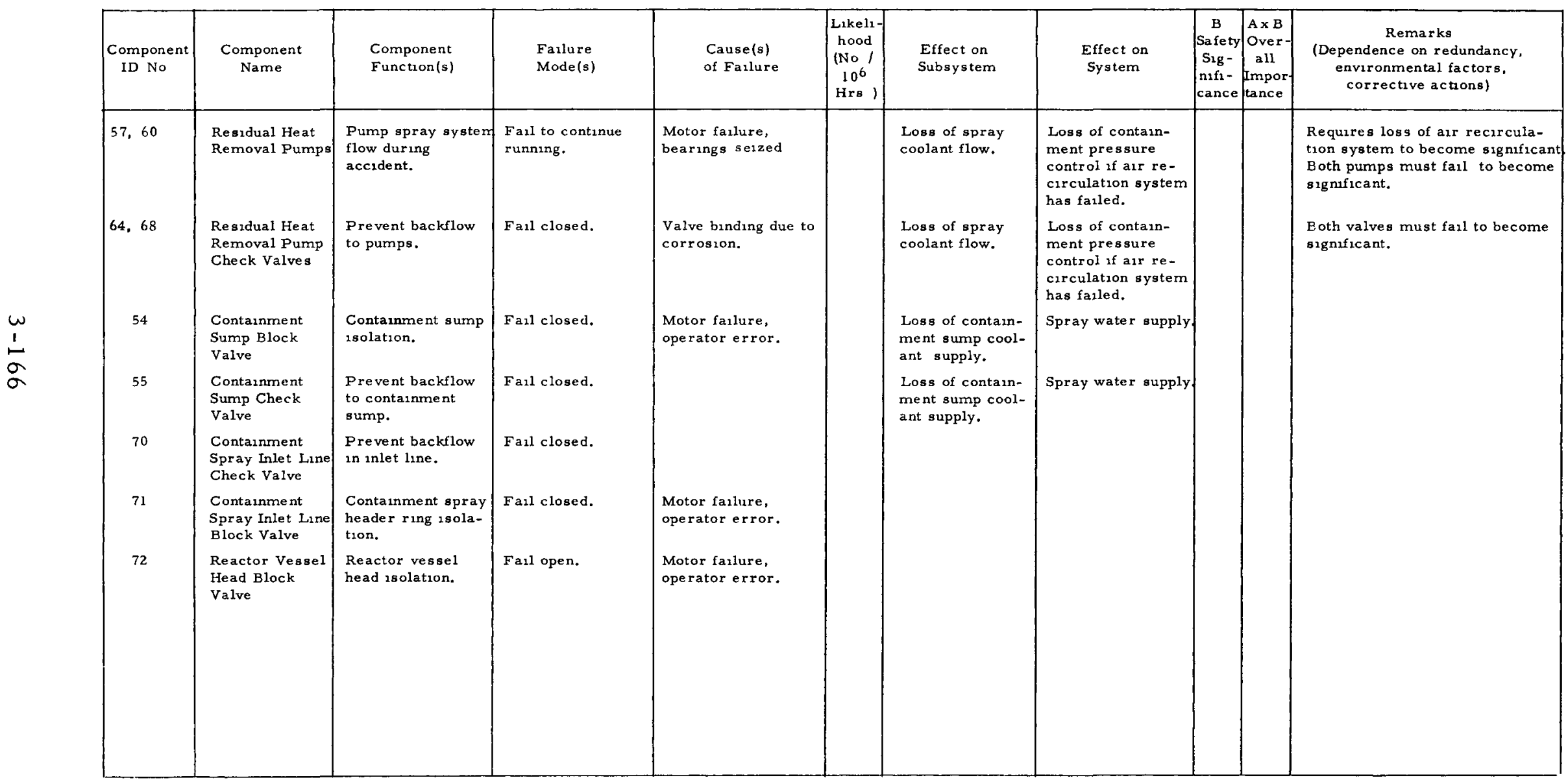

FIGURE 3.34B (continued) 


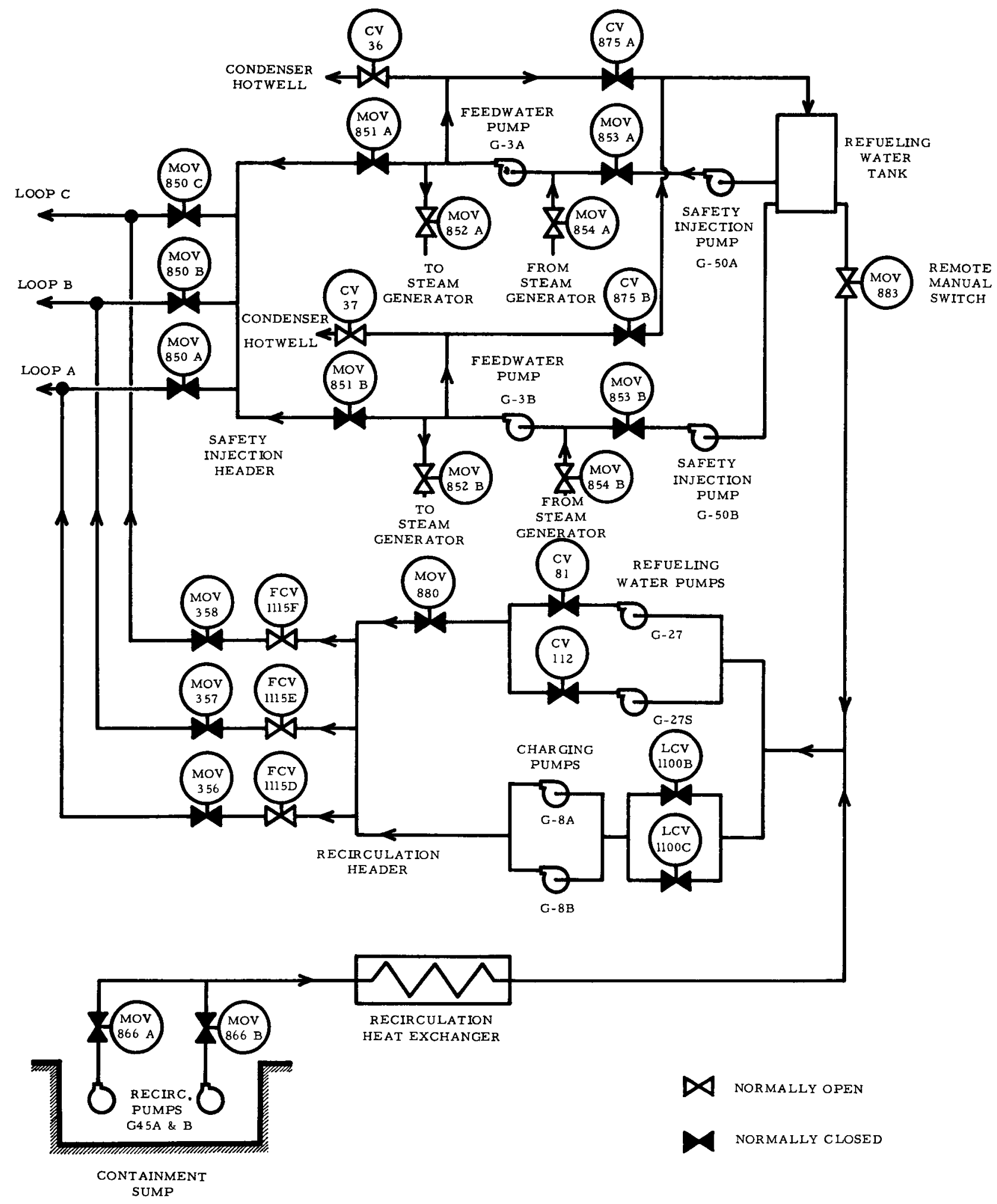

FIGURE 3.35

SCHEMATIC OF SAN ONOFRE SAFETY INJECTION SYSTEM 


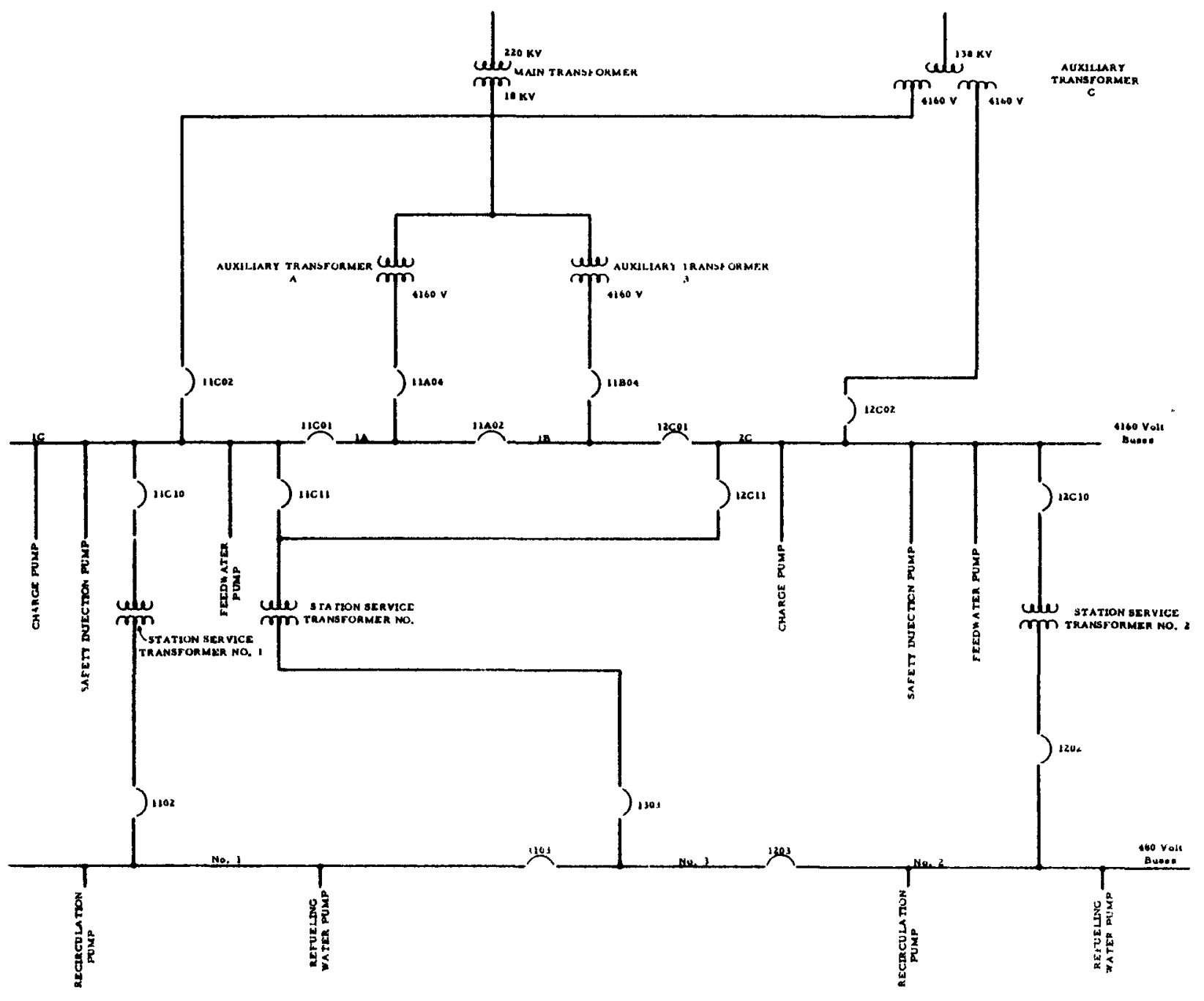

FIGURE 3.36

ELECTRICAL POWER TO SAFETY INJECTION SYSTEM - SAN ONOFRE

$3-168$ 
loss or leakage of reacto colant and to provide secondary means of nuclear shutdown.

\begin{tabular}{|c|c|c|c|c|c|c|c|c|c|c|}
\hline $\begin{array}{c}\text { Component } \\
\text { ID No. }\end{array}$ & $\begin{array}{l}\text { Component } \\
\text { Name }\end{array}$ & $\begin{array}{l}\text { Component } \\
\text { Function(s) }\end{array}$ & $\begin{array}{l}\text { Fallure } \\
\text { Mode(s) }\end{array}$ & $\begin{array}{l}\text { Cause(s) } \\
\text { of Fallure }\end{array}$ & $\begin{array}{c}\text { Likel1- } \\
\text { hood } \\
\text { (Nol } \\
10^{6} \\
\text { Hrs ) }\end{array}$ & $\begin{array}{l}\text { Effect on } \\
\text { Subsystem }\end{array}$ & $\begin{array}{l}\text { Effect on } \\
\text { System }\end{array}$ & $\begin{array}{l}\text { B } \\
\text { Safety } \\
\text { Sig- } \\
\text { nifi- } \\
\text { cance }\end{array}$ & \begin{tabular}{|l|} 
A X B \\
Over \\
all \\
Impor \\
tance
\end{tabular} & $\begin{array}{c}\text { Remarks } \\
\text { (Dependence on redundancy, } \\
\text { environmental factors, } \\
\text { corrective actions) }\end{array}$ \\
\hline \multirow{6}{*}{$\begin{array}{l}\text { MOV-850A } \\
850 \mathrm{~B} \\
850 \mathrm{C}\end{array}$} & $\begin{array}{l}\text { Refueling Water } \\
\text { Tank }\end{array}$ & $\begin{array}{l}\text { Contains } 240,000 \\
\text { gallons borated } \\
\text { water. }\end{array}$ & Large leak & $\begin{array}{l}\text { Disaster causes tank } \\
\text { to crack. }\end{array}$ & & $\begin{array}{l}\text { No water for safety } \\
\text { injection. }\end{array}$ & $\begin{array}{l}\text { No emergency core } \\
\text { cooling a varlable. }\end{array}$ & 4 & & \multirow{8}{*}{ Exercise periodically. } \\
\hline & $\begin{array}{l}\text { Safety Injection } \\
\text { Header }\end{array}$ & $\begin{array}{l}\text { Combine flow from } \\
\text { safety injection } \\
\text { trains and distri- } \\
\text { bute to three } \\
\text { coolant loops. }\end{array}$ & Rupture. & Corrosion, accident. & & No safety injection & $\begin{array}{l}\text { No emergency core } \\
\text { cooling avallable }\end{array}$ & 4 & & \\
\hline & $\begin{array}{l}\text { Safety Injection } \\
\text { Loop Vaives }\end{array}$ & $\begin{array}{l}\text { Direct safety injec } \\
\text { tion to three } \\
\text { coolant loops. }\end{array}$ & Fail to open. & $\begin{array}{l}\text { Stuck from corrosion, } \\
\text { fatled motor. }\end{array}$ & & $\begin{array}{l}\text { Fallure if two } \\
\text { valves fall. }\end{array}$ & $\begin{array}{l}\text { Possible core } \\
\text { melting with loss } \\
\text { of coolant. }\end{array}$ & 3 & & \\
\hline & $\begin{array}{l}\text { Main Trans - } \\
\text { former }\end{array}$ & $220 \mathrm{Kv} / 18 \mathrm{Kv}$ & Cease to operate. & $\begin{array}{l}\text { Fire in transformer, } \\
\text { open windings. }\end{array}$ & & $\begin{array}{l}\text { No direct power to } \\
4160 \text { volt Buses } \\
1 \mathrm{~A} \text { and } 1 \mathrm{~B} \text {. }\end{array}$ & $\begin{array}{l}\text { No effect a s long } \\
\text { as there 1s power } \\
\text { to } 4160 \text { volt Buses } \\
\text { 1C and.2e }\end{array}$ & 2 & & \\
\hline & $\begin{array}{l}\text { Auxiliary Trans - } \\
\text { formers A and B }\end{array}$ & $\begin{array}{l}18 \mathrm{Kv} / 4160 \text { volt } \\
\text { Buses IA and IB }\end{array}$ & Cease to operate. & $\begin{array}{l}\text { Fire in transformer, } \\
\text { open windings. }\end{array}$ & & $\begin{array}{l}\text { No direct power to } \\
4160 \text { volt Buses } \\
1 \mathrm{~A} \text { and } 1 \mathrm{~B}\end{array}$ & $\begin{array}{l}\text { No effect as long } \\
\text { as there } 1 \mathrm{~s} \text { power } \\
\text { to } 4160 \text { volt Buses } \\
\text { IC and 2C }\end{array}$ & 2 & & \\
\hline & $\begin{array}{l}\text { Auxiliary Trans - } \\
\text { former C }\end{array}$ & $\begin{array}{l}138 \mathrm{Kv} / 4160 \text { volt } \\
\text { Buses } 1 \mathrm{C} \text { and } 2 \mathrm{C} \text {. }\end{array}$ & Cease to operate. & $\begin{array}{l}\text { Fire in transformer, } \\
\text { open wandings. }\end{array}$ & & $\begin{array}{l}\text { No direct powe } \\
4160 \text { volt Buses } \\
1 \mathrm{C} \text { and } 2 \mathrm{C}\end{array}$ & $\begin{array}{l}\text { No effect if there } \\
18 \text { power to Buses } \\
\text { IC and } 2 C \text { through } \\
\text { the main trans- } \\
\text { former and Buses } \\
\text { IA and/or } 1 \mathrm{~B} \text {. }\end{array}$ & 3 & & \\
\hline $\begin{array}{l}\mathrm{CB}-11 \mathrm{~A} 04, \\
11 \mathrm{~B} 04 \\
11 \mathrm{C} 02 \\
12 \mathrm{CO}\end{array}$ & Circult Breakers & $\begin{array}{l}\text { Connect power to } \\
4160 \text { volt Buses } \\
\text { IA, IB, IC, and } \\
\text { 2C. }\end{array}$ & $\begin{array}{l}\text { Fanl to close and } \\
\text { stay closed. }\end{array}$ & $\begin{array}{l}\text { Broken parts, } \\
\text { overcur rent. }\end{array}$ & & $\begin{array}{l}\text { No direct power to } \\
\text { corresponding } \\
4160 \text { volt bus. }\end{array}$ & $\begin{array}{l}\text { If thes work, there } \\
\text { are alternate } \\
\text { sources of power } \\
\text { to each bus. }\end{array}$ & 2 & & \\
\hline \begin{tabular}{|}
$\mathrm{TB}-11 \mathrm{C} 11$ \\
$12 \mathrm{C} 11$ \\
$11 \mathrm{C} 01$ \\
$12 \mathrm{C} 01$
\end{tabular} & Tie Breakers & $\begin{array}{l}\text { Tie } 4160 \text { volt buses } \\
\text { together. }\end{array}$ & Faxl to close. & $\begin{array}{l}\text { Broken parts, } \\
\text { overcurrent }\end{array}$ & & $\begin{array}{l}\text { No the between } \\
\text { buses if backup } \\
\text { needed. }\end{array}$ & $\begin{array}{l}\text { Reduced redun- } \\
\text { dancy in power. }\end{array}$ & 2 & & \\
\hline
\end{tabular}




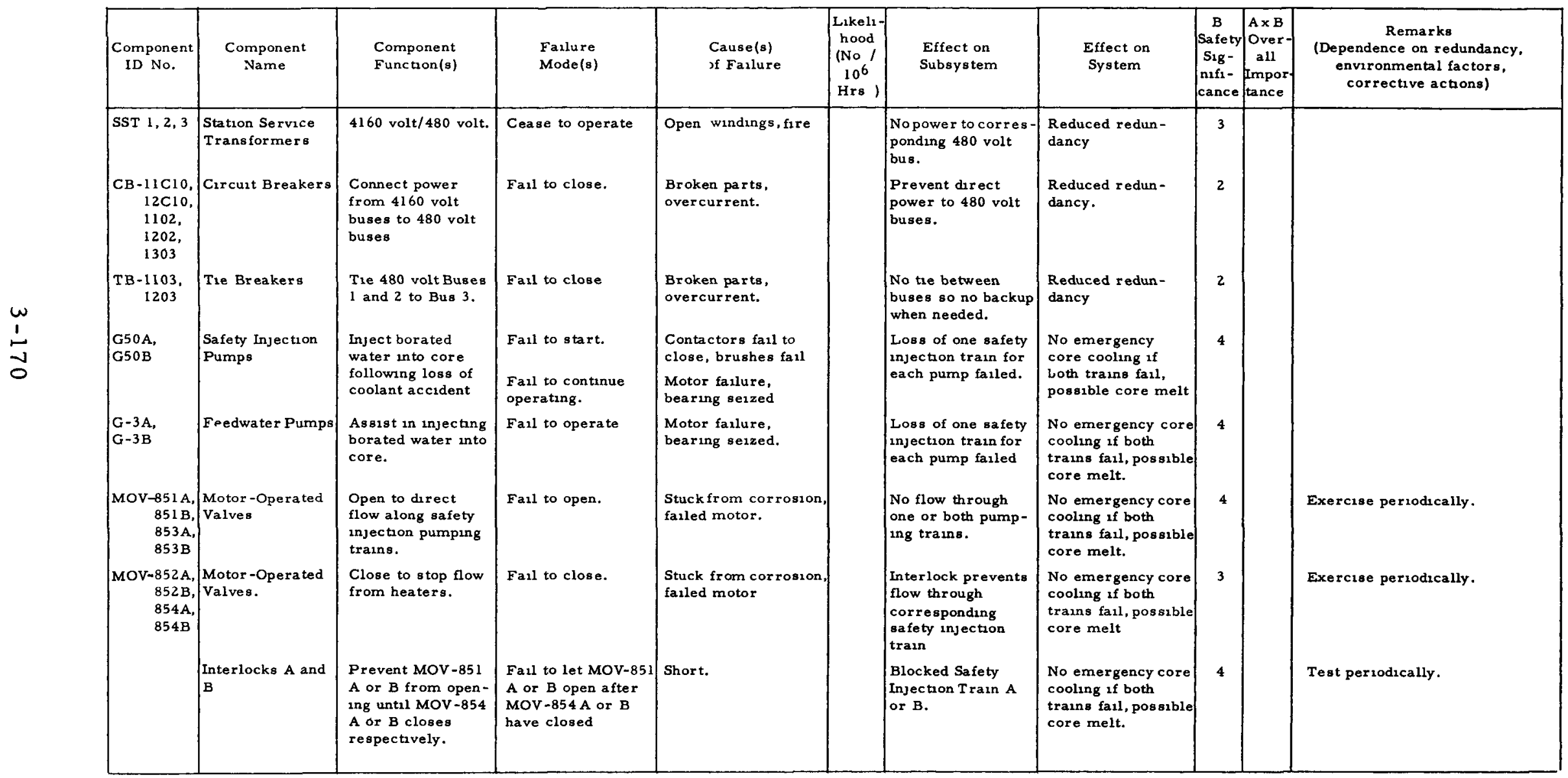

\section{FIGURE 3.37 (continued)}


System Reactor Cooling

Subsystem Safety Injection

Assembly Injection and Recirculation Equipmen

Function Toprovide emergency core coolung with loss or leakage of reactor coolant and to provide secondary means of nuclear shutdown.

\begin{tabular}{|c|c|c|c|c|c|c|c|c|c|c|}
\hline $\begin{array}{l}\text { Component } \\
\text { ID No. }\end{array}$ & $\begin{array}{l}\text { Component } \\
\text { Name }\end{array}$ & $\begin{array}{l}\text { Component } \\
\text { Function(s) }\end{array}$ & $\begin{array}{l}\text { Fallure } \\
\text { Mode(s) }\end{array}$ & $\begin{array}{l}\text { Cause(s) } \\
\text { of Fallure }\end{array}$ & $\begin{array}{c}\text { Likel1 } \\
\text { hood } \\
\text { (Nol } \\
10^{6} \\
\text { Hrs })\end{array}$ & $\begin{array}{l}\text { Effect on } \\
\text { Subsystem }\end{array}$ & $\begin{array}{l}\text { Effect on } \\
\text { System }\end{array}$ & $\begin{array}{c}\text { B } \\
\text { Safety } \\
\text { Sig- } \\
\text { nufi- } \\
\text { cance }\end{array}$ & $\begin{array}{l}\text { A } \times B \\
\text { Over } \\
\text { all } \\
\text { Impor } \\
\text { tance }\end{array}$ & $\begin{array}{c}\text { Remarks } \\
\text { (Dependence on redundancy, } \\
\text { environmental factors, } \\
\text { corrective actions) }\end{array}$ \\
\hline \multirow[t]{2}{*}{$\begin{array}{r}\mathrm{CV}-36 \\
37 \\
875 \mathrm{~A} \\
875 \mathrm{~B}\end{array}$} & Control Valves & $\begin{array}{l}\text { To cut off flow } \\
\text { from feedwater } \\
\text { pumps to condenser } \\
\text { hotwell and recricu- } \\
\text { lation back to refuel- } \\
\text { ing water tank }\end{array}$ & Fail to close. & Stuck from corrosion. & & $\begin{array}{l}\text { Drain part of water } \\
\text { from flow to core. }\end{array}$ & $\begin{array}{l}\text { None unless acci- } \\
\text { dent requires full } \\
\text { capacity of safety } \\
\text { injection system. }\end{array}$ & 1 & & Exercise periodically. \\
\hline & $\begin{array}{l}\text { Recirculation } \\
\text { Header }\end{array}$ & $\begin{array}{l}\text { Combine flow from } \\
\text { charging pumps and } \\
\text { refuel pumps and } \\
\text { distribute to three } \\
\text { coolant loops. }\end{array}$ & Rupture & Corrosion, accident. & & No recirculation & $\begin{array}{l}\text { No decay heat } \\
\text { removal }\end{array}$ & 4 & & Test by flow. \\
\hline \multirow{3}{*}{$\mid \begin{array}{r}\text { FCV-1115D, } \\
1115 E \\
1115 F \\
\text { MOV-356, } \\
357 \\
358\end{array}$} & $\begin{array}{l}\text { Flow Control } \\
\text { Motor-Operated } \\
\text { Valves }\end{array}$ & $\begin{array}{l}\text { Dhrect recircula - } \\
\text { tron to three } \\
\text { coolant loops. }\end{array}$ & Fall to open. & Stuck from corrosion. & & $\begin{array}{l}\text { Block recirculation } \\
\text { to one or more } \\
\text { loops. }\end{array}$ & $\begin{array}{l}\text { If flow to two loops } \\
\text { 2s blocked, no } \\
\text { effective decay } \\
\text { heat removal. }\end{array}$ & 3 & & Exercise periodically \\
\hline & $\begin{array}{l}\text { Containment } \\
\text { Sump }\end{array}$ & $\begin{array}{l}\text { Collect spilled } \\
\text { water for recircu - } \\
\text { lation. }\end{array}$ & $\begin{array}{l}\text { Fanl to hold spilled } \\
\text { water. }\end{array}$ & Na tural disaster. & & $\begin{array}{l}\text { No water for } \\
\text { recirculation. }\end{array}$ & $\begin{array}{l}\text { No decay heat } \\
\text { removal }\end{array}$ & 4 & & \\
\hline & $\begin{array}{l}\text { Recirculation } \\
\text { Heat Exchanger }\end{array}$ & $\begin{array}{l}\text { Cool recirculation } \\
\text { water from sump. }\end{array}$ & Rupture and leak. & Corrosion, accident. & & $\begin{array}{l}\text { Inadequate cooling } \\
\text { of water }\end{array}$ & $\begin{array}{l}\text { Inadequa te decay } \\
\text { heat removal. }\end{array}$ & 3 & & Check periodically for leaks. \\
\hline \multirow[t]{2}{*}{ MOV -883 } & $\begin{array}{l}\text { Motor-Operated } \\
\text { Valve }\end{array}$ & $\begin{array}{l}\text { To atop flow from } \\
\text { refueling water tank } \\
\text { to refuel and charge } \\
\text { pumps and prevent } \\
\text { flow of recircula- } \\
\text { tron water back } \\
\text { into tank }\end{array}$ & Fail to close & $\begin{array}{l}\text { Motor fallure, stuck } \\
\text { from corrosion. }\end{array}$ & & $\begin{array}{l}\text { Uncertain, assume } \\
\text { loss of recir cula- } \\
\text { tion. }\end{array}$ & $\begin{array}{l}\text { Assume system } \\
\text { fanlure }\end{array}$ & 2 & & $\begin{array}{l}\text { Install check valve (which is } \\
\text { actually the case) though not } \\
\text { considered in this analysis. }\end{array}$ \\
\hline & $\begin{array}{l}\text { Remote Manual } \\
\text { Switch }\end{array}$ & Actuate MOV -883 & Fall to connect. & $\begin{array}{l}\text { Poor contacts, } \\
\text { broken line. }\end{array}$ & & $\begin{array}{l}\text { Uncertain, assume } \\
\text { loss of recircula- } \\
\text { tion. }\end{array}$ & $\begin{array}{l}\text { Assume system } \\
\text { fallure. }\end{array}$ & 2 & & Test periodically. \\
\hline
\end{tabular}

\section{FIGURE 3.37 (continued)}


Assembly In

Function To provide emergency core cooling with loss or leakage of reactor coolant and to provide secondary means of nuclear shutdown.

\begin{tabular}{|c|c|c|c|c|c|c|c|c|c|c|}
\hline $\begin{array}{l}\text { Component } \\
\text { ID No. }\end{array}$ & $\begin{array}{l}\text { Component } \\
\text { Name }\end{array}$ & $\begin{array}{l}\text { Component } \\
\text { Function(s) }\end{array}$ & $\begin{array}{l}\text { Fallure } \\
\text { Mode(s) }\end{array}$ & $\begin{array}{l}\text { Cause(s) } \\
\text { of Fallure }\end{array}$ & \begin{tabular}{|c|} 
Likel1- \\
hood \\
(No $/$ \\
$10^{6}$ \\
Hrs $)$
\end{tabular} & $\begin{array}{l}\text { Effect on } \\
\text { Subsystem }\end{array}$ & $\begin{array}{l}\text { Effect on } \\
\text { System }\end{array}$ & $\mid \begin{array}{c}\text { B } \\
\text { Safety } \\
\text { Sig- } \\
\text { nifi- } \\
\text { cance }\end{array}$ & \begin{tabular}{|} 
A $\times$ B \\
Over \\
all \\
Impor \\
tance
\end{tabular} & $\begin{array}{c}\text { Remarks } \\
\text { (Dependence on redundancy. } \\
\text { environmental factors, } \\
\text { corrective actions) }\end{array}$ \\
\hline G-8A & Charging Pumps & $\begin{array}{l}\text { Pump recircula - } \\
\text { tion water to } \\
\text { coolant loops. }\end{array}$ & $\begin{array}{l}\text { Fall to start, fail } \\
\text { to continue } \\
\text { operating }\end{array}$ & $\begin{array}{l}\text { Contactors fall to } \\
\text { close, brushes fail, } \\
\text { motor failure, } \\
\text { bearing selzed. }\end{array}$ & & $\begin{array}{l}\text { Reduced recircula - } \\
\text { tion }\end{array}$ & $\begin{array}{l}\text { If refuel pumps } \\
\text { continue to operate, } \\
\text { no effect }\end{array}$ & 2 & & Monitor frequently. \\
\hline $\begin{array}{l}\text { MOV- } \\
1100 \mathrm{~B} \\
1100 \mathrm{D}\end{array}$ & $\begin{array}{l}\text { Motor-Operated } \\
\text { Valve }\end{array}$ & $\begin{array}{l}\text { Open to allow flow } \\
\text { to charging pumps. }\end{array}$ & Fasl to open. & $\begin{array}{l}\text { Stuck from corrosion, } \\
\text { motor falled. }\end{array}$ & & $\begin{array}{l}\text { Charge pumps out } \\
\text { of operation if both } \\
\text { valves fall. }\end{array}$ & $\begin{array}{l}\text { Reduced decay } \\
\text { heat removal } \\
\text { reliability. }\end{array}$ & 2 & & Exercise periodically \\
\hline $\begin{array}{r}\mathrm{CV}-81 \\
112\end{array}$ & Control Valves & $\begin{array}{l}\text { Refuel water } \\
\text { pumps discharge } \\
\text { valves. }\end{array}$ & Fa1l to open & Stuck fromcorrosion. & & $\begin{array}{l}\text { No flow from } \\
\text { corresponding } \\
\text { refuel water pump. }\end{array}$ & $\begin{array}{l}\text { Reduced decay } \\
\text { heat removal } \\
\text { reliability. }\end{array}$ & 2 & & Exercise periodically \\
\hline G-27, & $\begin{array}{l}\text { Refueling Water } \\
\text { Pumps }\end{array}$ & $\begin{array}{l}\text { Share recircula - } \\
\text { tion duty with } \\
\text { charging pumps. }\end{array}$ & $\begin{array}{l}\text { Fall to start. } \\
\text { Fall to continue } \\
\text { operating. }\end{array}$ & $\begin{array}{l}\text { Contactors fail to } \\
\text { close, brushes fall. } \\
\text { Motor fallure, } \\
\text { bearngs selzed. }\end{array}$ & & $\begin{array}{l}\text { Reduced redun- } \\
\text { dancy in recircula } \\
\text { tion }\end{array}$ & $\begin{array}{l}\text { Reduced decay } \\
\text { heat removal } \\
\text { reliability. }\end{array}$ & 2 & & Test periodically. \\
\hline MOV -880 & $\begin{array}{l}\text { Motor-Operated } \\
\text { Valve }\end{array}$ & $\begin{array}{l}\text { Directs flow from } \\
\text { refueling water } \\
\text { pumps to recircula } \\
\text { tion header. }\end{array}$ & Fazl to open & Same as other valves & & $\begin{array}{l}\text { No flow to recircu- } \\
\text { lation through } \\
\text { refuel pump. }\end{array}$ & $\begin{array}{l}\text { Reduced decay } \\
\text { heat removal } \\
\text { reliability. }\end{array}$ & 3 & & Exercise periodically \\
\hline & Manual Start & $\begin{array}{l}\text { Start recirculation } \\
\text { pumps. }\end{array}$ & Fail to actuate. & $\begin{array}{l}\text { Relay fallure } \\
\text { operator error. }\end{array}$ & & $\begin{array}{l}\text { No pumping from } \\
\text { sump. }\end{array}$ & $\begin{array}{l}\text { No recirculation, } \\
\text { no decay heat } \\
\text { removal. }\end{array}$ & 4 & & Test periodically. \\
\hline $\begin{array}{r}G-45 A \\
45 B\end{array}$ & $\begin{array}{l}\text { Recirculation } \\
\text { Pump }\end{array}$ & $\begin{array}{l}\text { Pump spilled water } \\
\text { from sump through } \\
\text { recirculation } \\
\text { system. }\end{array}$ & $\begin{array}{l}\text { Fall to start. } \\
\text { Fall to operate. }\end{array}$ & $\begin{array}{l}\text { Contactors fail to } \\
\text { close, brushes fall. } \\
\text { Motor fallure, } \\
\text { bearing seized. }\end{array}$ & & No recirculation. & $\begin{array}{l}\text { No decay heat } \\
\text { removal. }\end{array}$ & 4 & & Test periodically. \\
\hline $\begin{array}{r}\text { MOV-866A, } \\
866 \mathrm{~B}\end{array}$ & $\begin{array}{l}\text { Motor-Operated } \\
\text { Valves }\end{array}$ & $\begin{array}{l}\text { Recirculation } \\
\text { pump discharge } \\
\text { valves. }\end{array}$ & Fall to open. & Stuck by corrosion. & & $\begin{array}{l}\text { Block flow from } \\
\text { sump }\end{array}$ & $\begin{array}{l}\text { No recirculation, } \\
\text { no decay heat } \\
\text { removal. }\end{array}$ & 4 & & Exercise periodically. \\
\hline LS $1,2,3$ & Level Sensors & $\begin{array}{l}\text { Sense low pres- } \\
\text { surizer water } \\
\text { level. }\end{array}$ & $\begin{array}{l}\text { Fail to sense } 2 \text { out } \\
\text { of } 3 \text {. }\end{array}$ & Disconnected cable. & & $\begin{array}{l}\text { No actuation of } \\
\text { safety injection } \\
\text { system. }\end{array}$ & $\begin{array}{l}\text { No emergency core } \\
\text { cooling, possible } \\
\text { melt. }\end{array}$ & 4 & & Monitor frequently \\
\hline
\end{tabular}

\section{FIGURE 3.37 (continued)}


Assembly Injection and Recirculation Equipment

To provide emergency core cooling with loss or leakage of reactor

coolant and to provide secondary means of nuclear shutdown

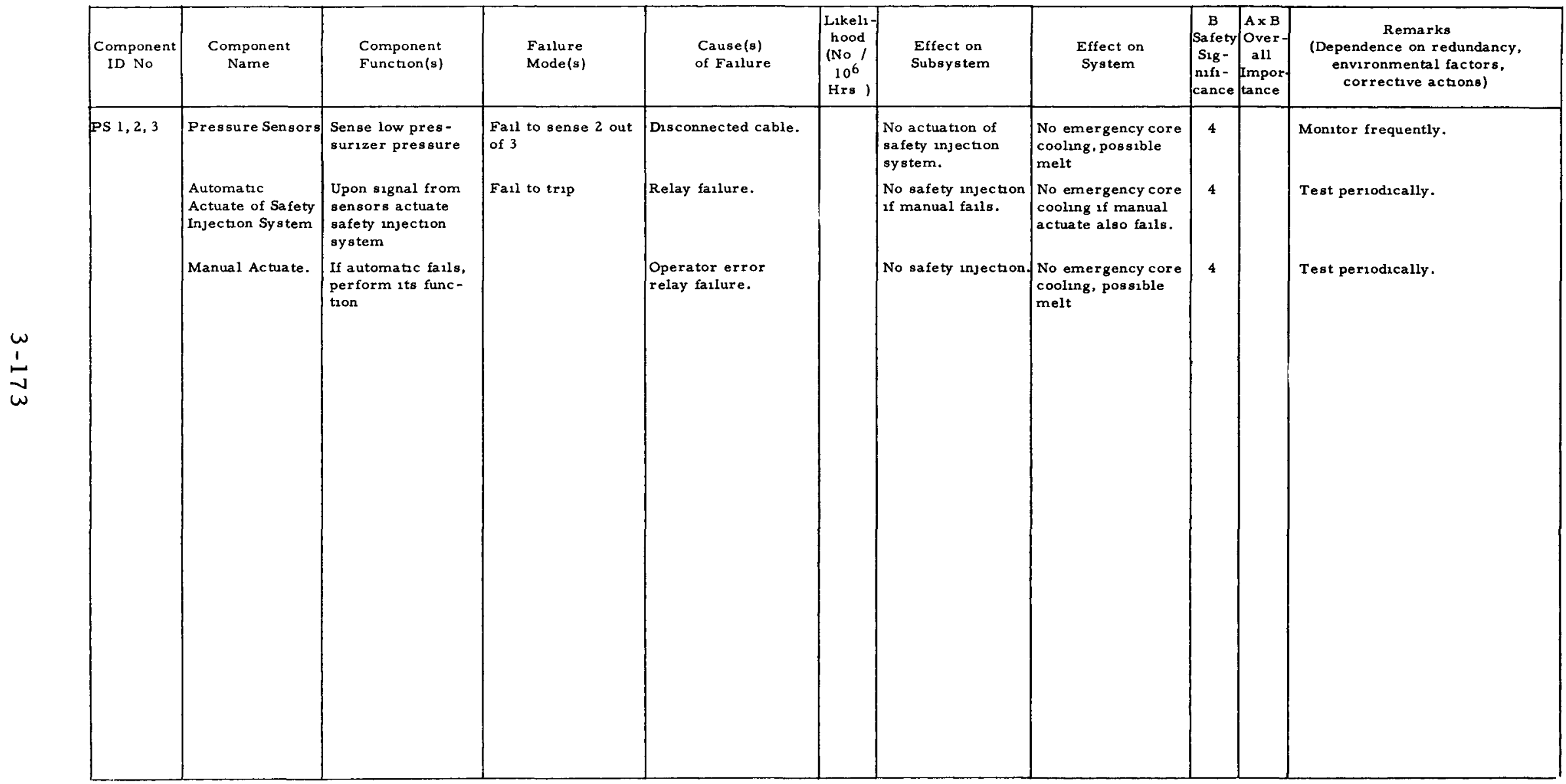

FIGURE 3.37 (continued) 


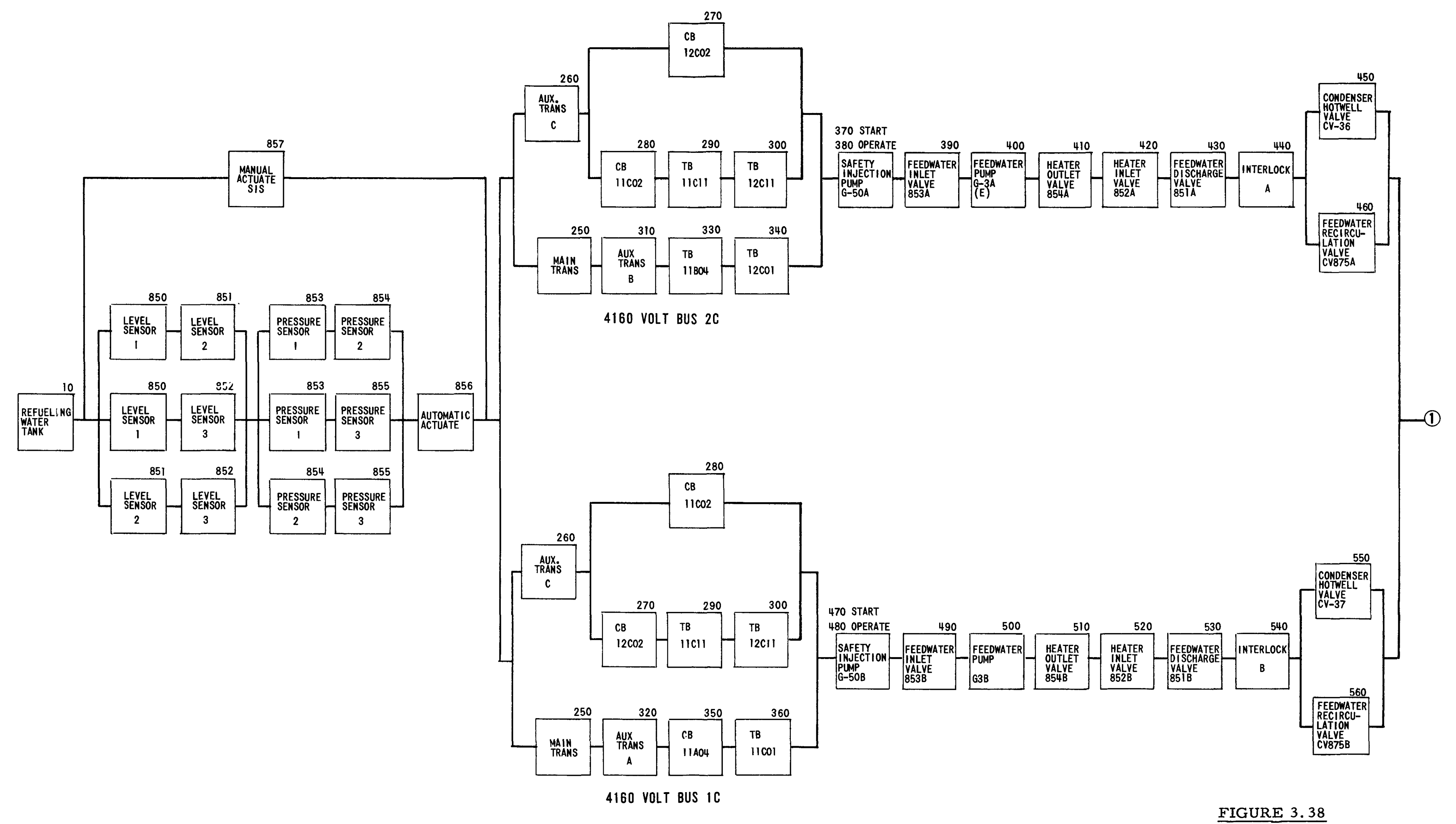

FIGURE 3.38 $\frac{\text { RELIABILITY BLOCK DIAGRAM }}{\text { SAFETY INJECTION SYSTEM }}$ 


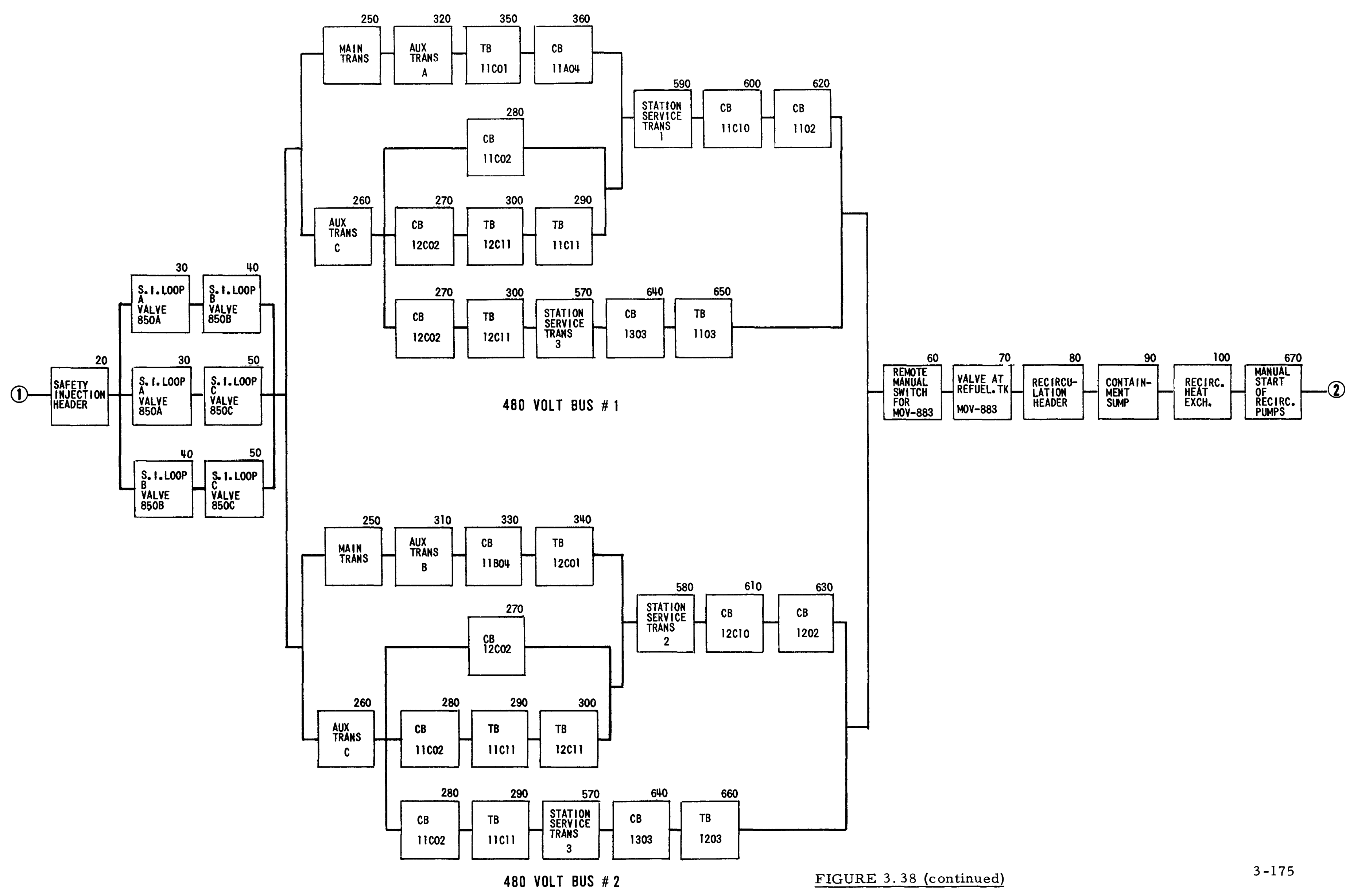




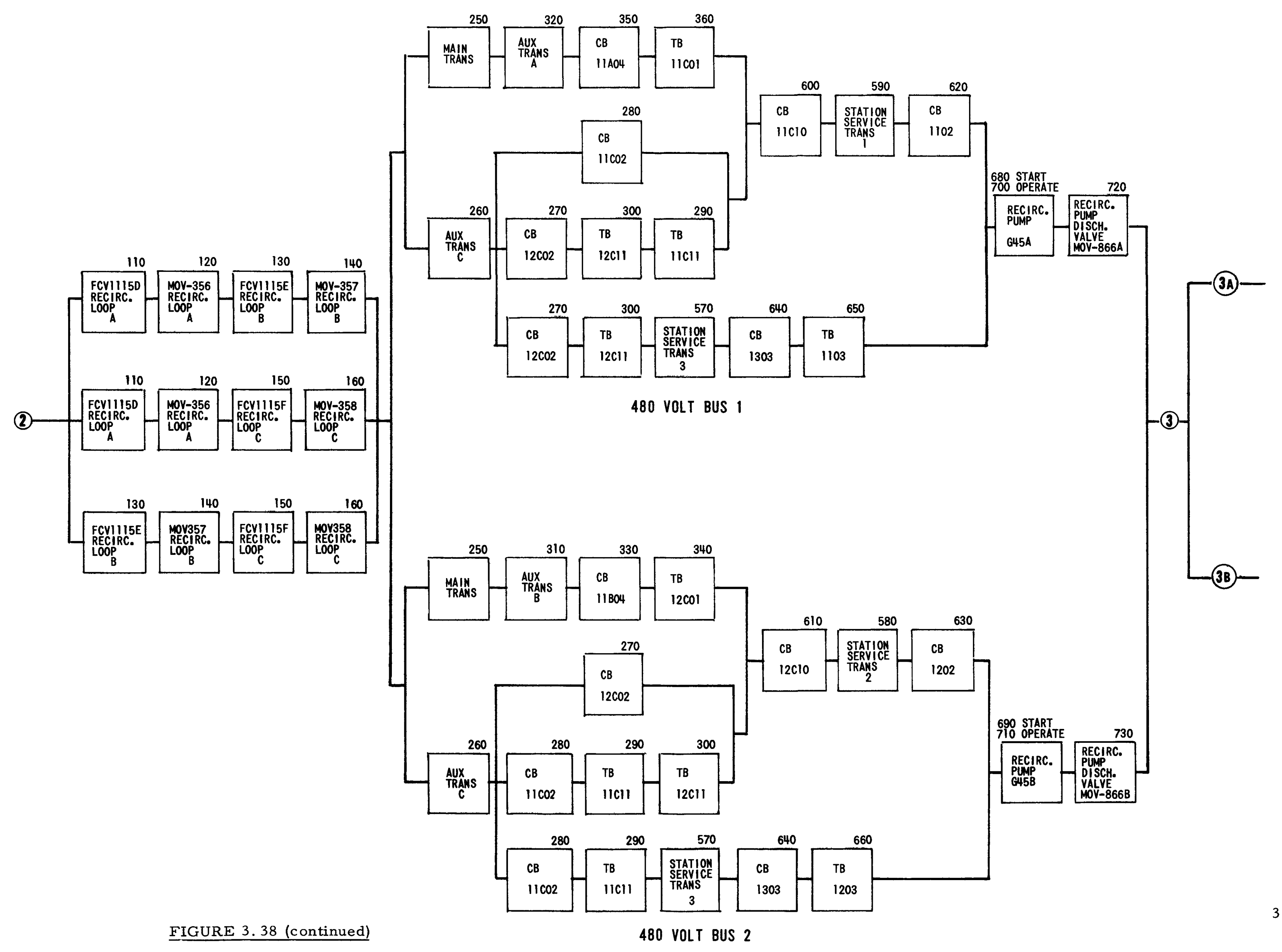




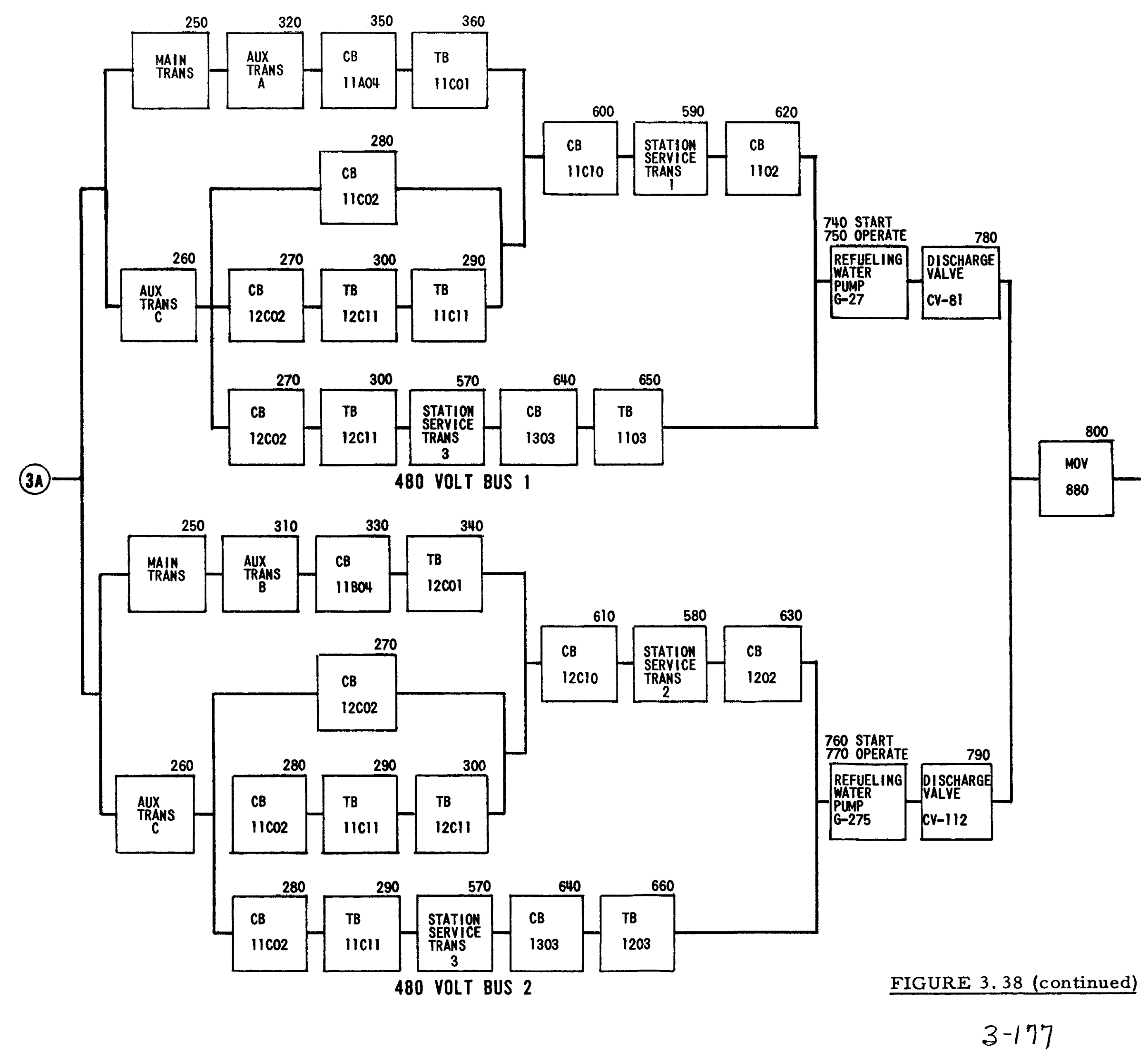




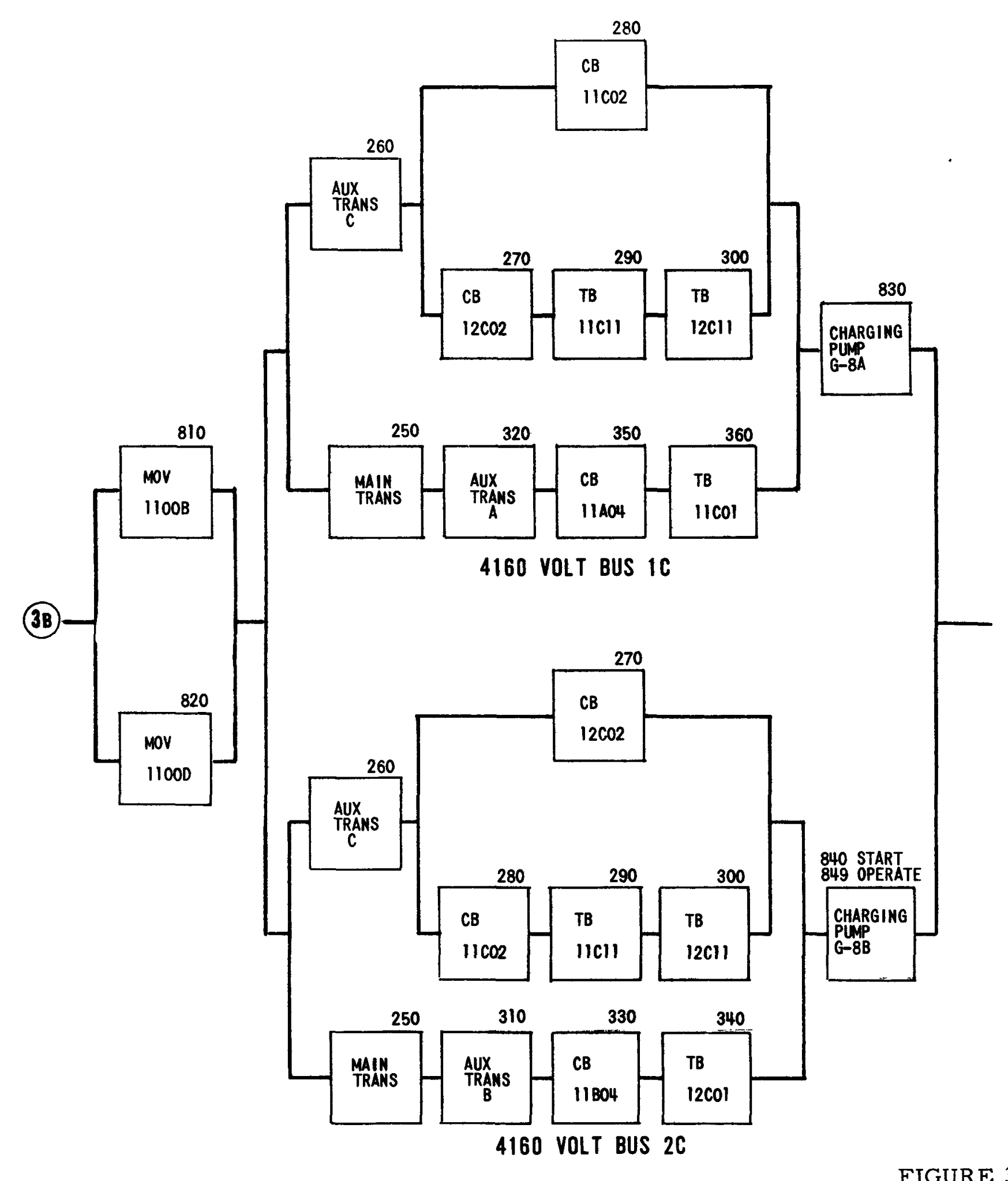

FIGURE 3.38 (continued) 
APPENDIX A

PRESENT DATA COLLECTION PRACTICES IN OPERATING NUCLEAR POWER PLANTS 


\section{APPENDIX A}

\section{PRESENT DATA COLLECTION PRACTICES IN OPERATING NUCLEAR POWER PLANTS}

Data management at five operating nuclear power plants in the United States has been reviewed to determine the applicability of current in-plant forms and procedures to the generation of reliability data. The plants included were:

1. Dresden Nuclear Power Station Unit No. 1.

2. Yankee Atomic Power Station.

3. Indian Point Station.

4. Humboldt Bay Unit No. 3 .

5. Shippingport Atomic Power Station.

As indicated in the following discussion, much of the in-plant information necessary for reliability data is now being collected and recorded. Furthermore, formats closely paralleling the failure event form suggested in this study are now in use. The remaining requirements for adapting present data management practices to reliability data generation are minor modifications and use of one or, at most, two existing forms for the basic reliability data input and specification of procedures which assure consistent, total adherence to completion of these forms.

\section{DRESDEN NUCLEAR POWER STATION}

Operating and Outage Summary Reports

Monthly Operating Reports - Each month data from the preceding logs and reports, as well as various process data sheets, are used to prepare a summary of operations. Preparation of this monthly report includes inputs from the chemistry, thermal, and nuclear engineers on the technical staff as well as maintenance summaries by the mechanical, electrical, and instrument maintenance groups. Emphasis is given to reporting and evaluation of plant and reactor performance during both normal operation and tests and includes a day-by-day listing of significant events. Plant radiation protection, including radwaste inventories, and plant chemistry data are presented in separate sections of the report. 
Outage Reports - These are special reports detailing work performed and results of tests made during extended outages such as refuelings. They are written by the plant engineering staff.

Annual Reports - Subsequent to the first year of operation after rated power tests, the Dresden staff has prepared annual reports for the AEC. These reports, as did the quarterly reports in the first year of operation, describe operating experience pertinent to nuclear safety and changes in facility design, performance characteristics, and operating procedures during the reporting period.

Failure Reports and Maintenance Requests

Maintenance Requests - Dresden uses a system of "maintenance requests" to define work to be done on plant components and to provide administrative control of such work. These maintenance requests reflect work performed on all plant equipment except during outages at which time they are supplanted by outage schedules.

Equipment Maintenance Files - Two separate files exist on equipment maintenance, one on instrument maintenance and the other on mechanical and electrical maintenance. These files represent an organized record of work performed on specific pieces of equipment. They are primarily derived from maintenance request completions.

Maintenance requirements arising during tests and checking operation, or during normal operation, are recorded and transmitted by maintenance work requests. As shown in Figure $A .1$, these requests may be originated by anyone on the station staff. Most frequently they are written by the reactor operators or the shift engineers. Few work requests originate in the maintenance staff. Following the flow shown in Figure A.l, work requests are written in quadruplicate and submitted to the shift engineer who forwards those requests requiring action to the station operating engineers for authorization. The station operating engineers authorize the requests and indicate action to be taken including assignment to electrical, mechanical, or instrument maintenance groups. Since the station maintenance engineer may not be present at Dresden on a continuous basis due to similar commitments at other power stations, the master mechanic directs action on all mechanical and electrical work requests at Dresden. Instrument maintenance is performed by the instrument mechanics under the electrical station operating engineer's direction. Action on a work request may be deferred, when possible, to the next major outage when it is scheduled with other maintenance activities. 
Upon completion of the required maintenance, the four copies of the work requests are signed and dated by the responsible mechanic. Unusual maintenance requirements may be noted on the work request; however, normally only the date of completion is noted. Completed work requests are distributed to the shift engineer, the master mechanic, and the appropriate station operating engineer. The operating engineers and master mechanic maintain files of the completed requests by number and year.

\section{YANKEE ATOMIC POWER STATION}

\section{Operating and Outage Summary Reports}

Operation Report - This report presents a summary of ge neral operations including the status of construction and nonoperating activities such as receipt and shipment of fuel. Only major maintenance activities are reported; and, in general, this information represents some enlargement on similar information in the weekly reports. Summary data includes plant chemistry; turbine plant performance; reactor plant performance; radiation exposure and radioactivity release; and in-plant training activities, primarily for spons or company technical personnel. Other information included in the operation report on an as-available basis are special reports on reactor performance tests and summaries of scheduled and unscheduled plant shutdowns.

Abnormal Occurrence Reports - These reports provide descriptions, analyses, and corrective actions taken for abnormal or unusual events which are of significance in plant operations. Many of the events included have been reported to the $A E C$ under reactor operating license requirements, but this record is not restricted to reportable incidents. Examples of abnormal occurrences are control rod malfunctions, primary coolant leaks or spills, and turbine throttle valve malfunctions.

\section{Failure Reports and Maintenance Requests *}

Maintenance Requests - Equipment deficiencies or malfunctions noted by plant personnel are reported on maintenance requests. These requests are provided with spaces for equipment identification, description of defect, work to be done, materials used, work done, and the names and dates associated with each of the preceding items.

Corrective maintenance is implemented primarily by use of maintenance requests and to a lesser extent by inspection activities associated with 
outage schedules. The flow of maintenance requests is illustrated in Figure A.2, and a blank request form is shown in Figure A. 3. Most maintenance requests are written by the shift supervisors; however, all plant personnel may initiate such requests. The requests are used primarily as a tool to initiate and control maintenance work. Their value as a historical record is limited to information transferred from the requests to equipment history cards. The requests are ultimately returned to the originator who generally disposes of them. The requests also are used by the maintenance supervisors as a guide to maintenance work loads, both past and present.

Equipment History File - Repair and parts replacement for all plant mechanical and electrical equipment, except instrumentation, is recorded in a card file maintained by the administrative assistant. The file is indexed according to the paragraph numbering established in the Final Hazard Summary Report. The maintenance information is obta ined from completed mainte nance requests and, as a result, is dependent on the completeness of information in maintenance requests and the correct handling of the requests. The completeness of information contained on the se cards has improved with time when compared to information in other plant records such as the reactor log and weekly report.

Instrument Maintenance File - Records of parts replacement or repair in components of the instrumentation system are maintained in a card file by the control engineering department. Information included on the cards indentifies the instrument, the date of each maintenance, and the part replaced or repair performed. Information is recorded on the cards by the control engineer or technical assistants. The maintenance file records are augmented by special summaries of significant maintenance activities prepared by the control engineer as time allows.

\section{INDIAN POINT STATION}

\section{Operating and Outage Summary Reports}

Monthly Report - This report contains a summary of plant performance data, major events, and significant health physics data for company management. These reports are prepared by the production staff and approved by the general superintendent. They serve as one input for semiannual reports. 
Semiannual Report - Plant operations (including shutdowns and unusual operating conditions), test results, maintenance and design changes, radiochemistry data, and health physics statistics are summarized each $\mathbf{s i x}$ months. The report is prepared by Consolidated Edis on for the AEC in accordance with requirements in the Provisional Operating License.

Outage Report - Significant testing and maintenance performed during major outages are summarized subsequent to the outage. Important safety information includes repair and replacement data on safety system and safeguard components which may not be recorded elsewhere.

Unit History - A continuous statistical history of operations is maintained in terms of major unit availability, outage causes, and protective system trips. This record is developed from operating logs by the production operating staff.

\section{Failure Reports and Maintenance Requests}

Maintenance Memoranda (MM) - These memos or work requests, a copy of which is shown in Figure A.4, identify equipment found in need of repair by the operating staff. The memos are valuable not only in the identification of equipment malfunctions but also as a record of repairs found necessary.

The use of $M M$ in the initiation and control of routine maintenance may be traced along one of two paths depending upon whether the work requested involved production maintenance personnel or test bureau personnel. In either case, the memoranda are written by the members of the operating staff when deficiencies are noted during inspections, checkoffs, normal operations, or tests. Blank memorandum forms are retained in single books in the central control room and chemical systems control room and are filled out in triplicate (white, yellow, and pink) copies. Although it is possible for other production staff members to write $\mathrm{MM}$, only the general watch foreman or watch foreman may sign and thereby initiate the memo.

For work involving the production mainte nance group, the MM flow is as shown on Figure A.5. With the foreman's signature, the white copy of each memo is forwarded to the reactor engineer or superintendent to receive final approval and priority number. The pink copy is placed in the general watch foreman's "Maintenance Memoranda Pending" file. Where the requested repair is best suited to outage time and may be postponed, the reactor engineer may assign the memo work to the next appropriate outage. With assignment of priority the white copy of the memo is forwarded to the production maintenance group. 
When the maintenance requested on a memo is completed, the general maintenance foreman moves the pink copy of the memo from the "Maintenance Memoranda Pending" file to the "Maintenance Memoranda Completed by Maintenance" file in the general watch foreman's office noting the work done on the memo. These completed MM are reviewed by the general watch foreman and then returned to the production office where they are compared with the third (yellow) copy of each MM which is retained in exhausted MM books from the control rooms. The completed $\mathrm{MM}$ are also used to compile equipment histories.

If an $M M$ item is completed by shift maintenance personnel rather than the production maintenance group, the general watch foreman removes the pink copy from the "Maintenance Memoranda Pending" file, initials the copy, and places it in the "Maintenance Memoranda Completed by the Watch" file. The production maintenance foreman checks this file each morning to eliminate completed work from the collection of MM received. The pink copies in the "Maintenance Memoranda Completed by the Watch" file are also forwarded to the production office for final checkoff and equipment history data. The white copies of these memoranda are retained by the general maintenance foreman.

For work involving test bureau personnel (instrument and control repair), the MM flow is as shown in Figure A. 6. Briefly the flow is the same as for the production mainte nance $\mathrm{MM}$ through the assignment of work priority. Test bureau MM are identified by marking the "Test Bureau" at the top of each applicable form. After assignment of priority the pink and white copies of the $M M$ are sent to the production staff technician who forwards the white copy to the test bureau for action and files the pink copy under "Test Bureau MM" for subsequent follow-up. With completion of the requested work the test bureau notifies operations and the production staff technician who notes the information on the respective $M M$ pink copies and keeps the reactor engineer and superintendent informed of the work status. The white copy of the test bureau MM is retained by the test bureau which maintains records of all work done on controls and instrumentation.

In actual practice, some minor routine maintenance is performed without writing an MM. This work includes adjustment and servicing by operating mechanics in the shift crews and instrument technicians working in the amplifier room. If this minor maintenance is significant to safety or operations, it will be recorded in the general watch foreman's log or the test bureau log maintained in the amplifier room. As a result of these practices, complete equipment histories are available 


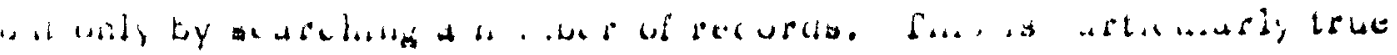

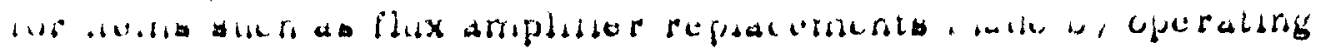

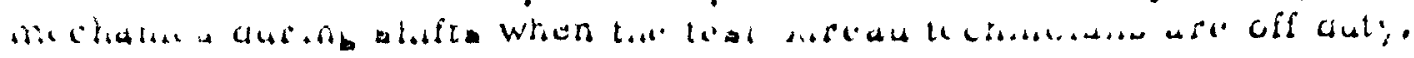

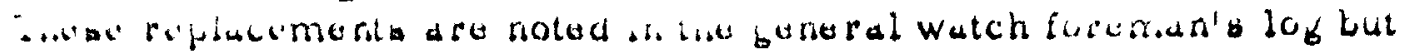

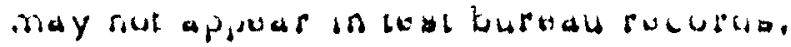

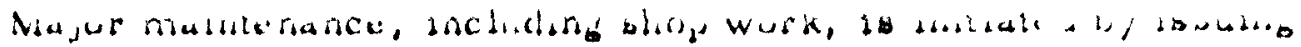

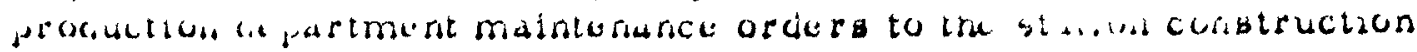

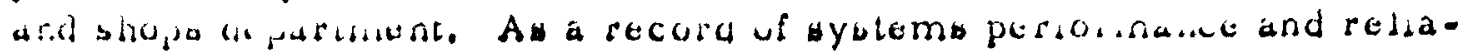
sidiy, ra MM are noore urnportant singo they aro a record of operating

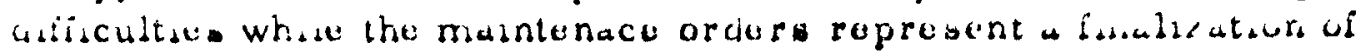
wors requartal ab a realut of operating difflculious or design changes previuubily recorciud in olner blation racorcis.

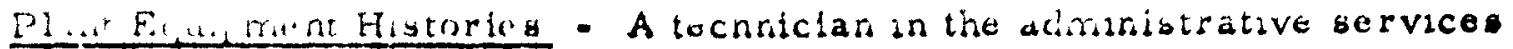

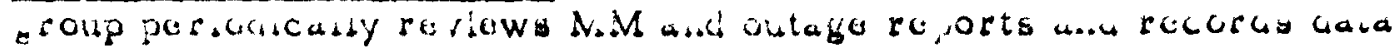
Fom las de docunzents in equipment hasory iates (lococ-jeaf notebooks) to estabiso.. conplete hiotorius on major componersa ta tien process systeris. Indtumertation and control histordub are kept dis the teat unfuaw duge. Thas recording practice is interiued as an asd an schedulinab

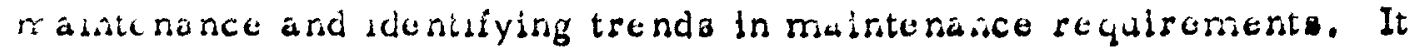
aso serves do a quick means of identifying woak component in various bail olárd byoturis.

\section{A... OH.T IAY UNIT NO, 3}

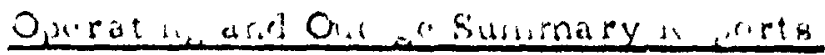

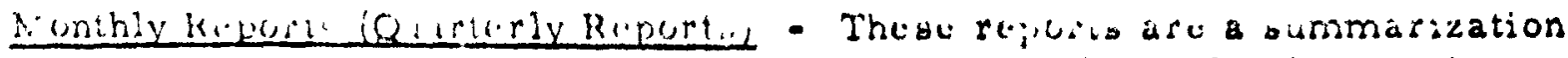
of operations, testing activitios, and sign cant mechanical, electrical, and instrument matritenanco performed auring each bonit. This report is prepared for internal use by PG\&E. Other muceral included in the report as a matater iog which is a day-by-day list of tests, dafety syotem trips, and equipment modificalions and maintenance. Othur information contained in the monthly reports includes individ. : reposts of each scram and outage occurring during the report period, a day-by-day - urrmary of plant performance (Form 51) which is taken from the daily summaries, and summaries of work performed during edch outage rnclicong work chronologies whenever the outage is exunsive. As of

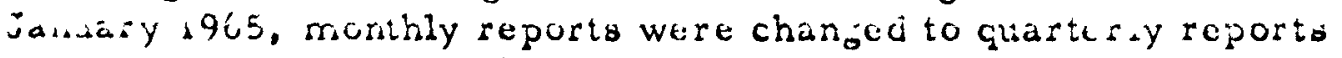
uecause the regularity of operation did not justify reporing on a monthly pirsoc. 
Semiannual Reports to the AEC - The provisional operating license for Unit No. 3 requires a semiannual report of operations. This report is prepared by the on-site staff and the general office and is edited by the general office. It contains general operating data on facility use, electrical output and shutdowns and data on radiation measurements, coolant chemistry, radioactive material releases, discharges and shipments, maintenance and facility changes, tests and test results, and meteorology. This data primarily derives from daily and monthly reports written for internal use.

Annual Operating Report - Operations during each calendar year are summarized in an annual report for internal use by PG\&E. This report is prepared by the on-site staff from monthly or quarterly report data and includes statistical summaries of plant performance in terms of average rates of occurrence or levels. Estimates of fuel usage are also included.

Scram and Outage Reports - PG\&E practices require preparation of individual reports covering the circumstances surrounding each scram and outage. Data included in each scram report are time of scram; type of scram (planned or unplanned, manual or automatic, real or spurious); cause and effect of scram; and corrective action, if any. Data included in outage reports are time of outage, duration of outage, type of outage (scheduled or unscheduled), and reasons for outage.

Failure Reports and Maintenance Requests

Forms 23's (Work Requests) - Maintenance requirements noted by the operating staff during each shift are normally forwarded to the appropriate maintenance group by use of a Form 23 or work request. This form indicates equipment involved; observed malfunctions or operating problems; and, when completed, the work performed by the maintenance staff. Work requests are used to initiate maintenance according to the request flow diagram shown in Figure A. 7. These work requests are primarily a monitoring tool to initiate and assure performance of the desired maintenance including the preparation of outage work schedules. The $y$ are retained as a record until no further surveillance of the pertine nt maintenance is necessary, and then they a re discarded after transferring maintenance data to the appropriate logs or maintenance card files. The completed work requests also are used by the maintenance foremen or instrument engineers to prepare summaries of maintenance work for each monthly report. 
Maintenance Card Files - Each component in the plant has a maintenance card file provided for recording repairs, replacements, and modifications. Routine or scheduled servicings are not indicated on these cards unless a repair or replacement is required during the servicing. The format for the cards varies according to the type of component, e.g., motors, pumps, valves, and electronic instruments; but in all cases a standard entry is provided for the date and work done (see Figures A. 8 and A. 9). The instrumentation cards are kept in a file which visually indicates whether maintenance is being performed by the presence or absence of the card. This file also may be used to schedule routine tests and servicings.

\section{SHIPPINGPORT ATOMIC POWER STATION}

Ope rating and Outage Summary Reports

Summary information on experience of significance to safety may be found in incident reports, monthly (now quarterly) operating reports, quarterly technical progress reports, and summary reports covering approximately the period from startup on one seed to startup on the next seed. The monthly operating reports and summary reports for the operating period of each seed are based on observations and data accumulated in records of daily operation, maintenance, and periodic checks and tests.

Reports of incidents provide complete analyses of unusual occurrences primarily in the reactor plant. These incident reports are summarized in monthly operating reports and summary reports of seed operations. The status of investigations into each incident is monitored by is suance of a quarterly incident status report which indicates incidents that have occurred, planned or accomplished corrective actions, and closes out incidents which were resolved since issuance of the preceding status report.

\section{Failure Reports and Maintenance Requests}

Plant maintenance activities are recorded and regulated by use of work item cards and equipment history cards. The equipment history cards, shown in Figure A. 10a, summarize identification numbers, name plate specifications, and significant repairs or servicings on mechanical and electrical components and all instrumentation. These $5 \times 8 \mathrm{cards}$ also are provided with monthly edge marks to enable use of the cards in a color coded, tickler file for scheduling preventive maintenance. 
Maintenance data entered on the cards is taken from work item cards by a clerk. Only data selected by the foremen or engineers is transferred to the equipment history cards. The equipment history cards we re kept current from their initiation in 1961 until the end of Core 1 operation. Since that time, reduction in the station complement has caused some lag in the transferral of work item data to the equipment history cards.

The work items or work requests are $8-1 / 2 \times 11$ cards filled out by foremen in different plant areas to request and record preventive and corrective maintenance on equipment. Servicings such as lubrication or oil changes are not entered as work items but are initiated from records kept in the control room. Information provided on the work item cards includes identification and location of equipment, nature of work or trouble, time and material requirements, and description of work performed. A blank work item card is shown in Figure A. 10b. The work item cards are reviewed and signed by appropriate supervisory personnel; and, generally, they are retained if they contain significant maintenance information. As previously indicated, this information is transferred to the equipment history cards at the direction of the foremen or engineers. 
ACTION

Maintenance requirement noted and request written

Reviews and authorizes request and forwards request to appropriate Station Operating Engineer

Approve requests, assign priorities, and forward requests to appropriate maintenance group or assign them to outage schedules

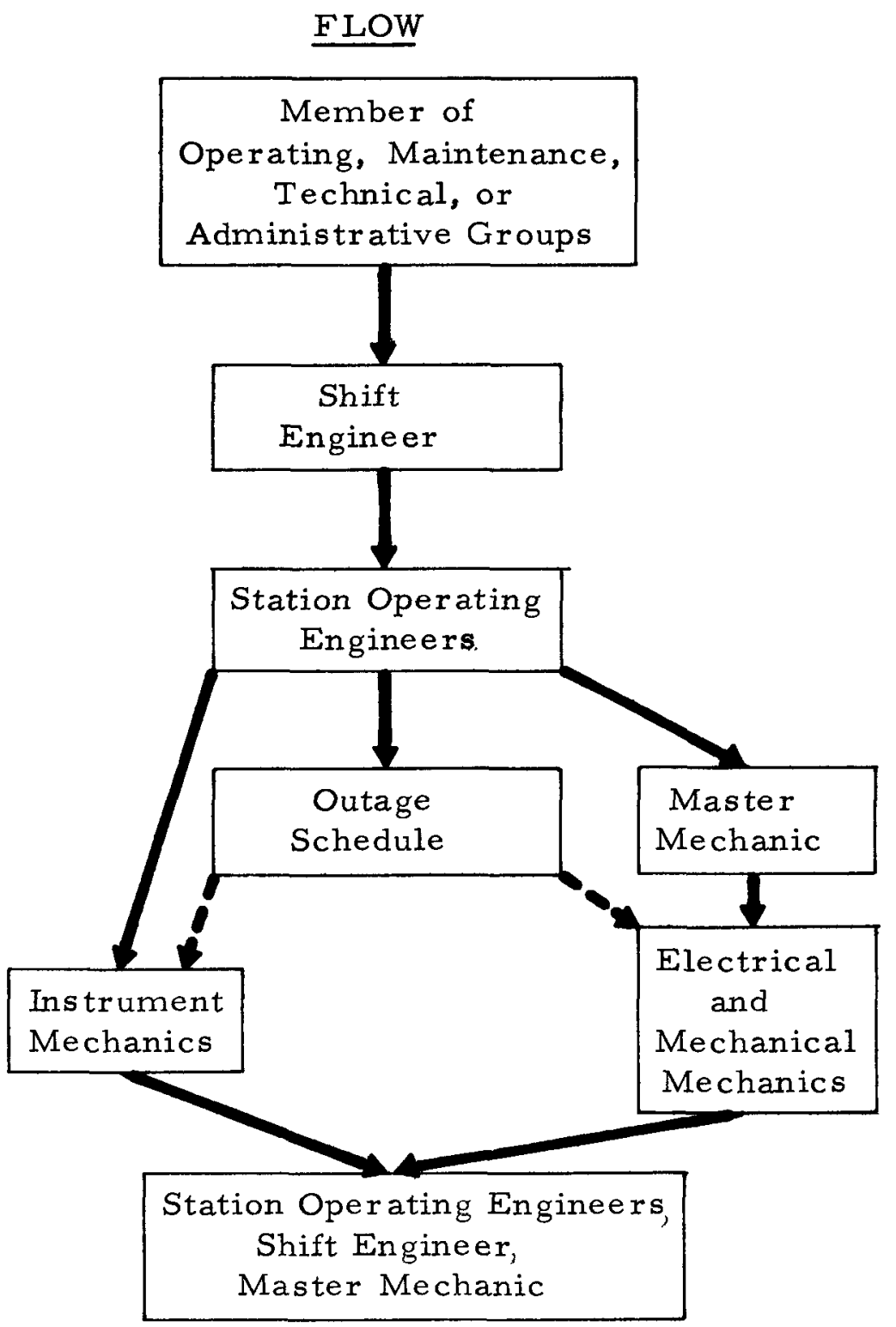

FIGURE A. 1

MAINTENANCE REQUEST FLOW 

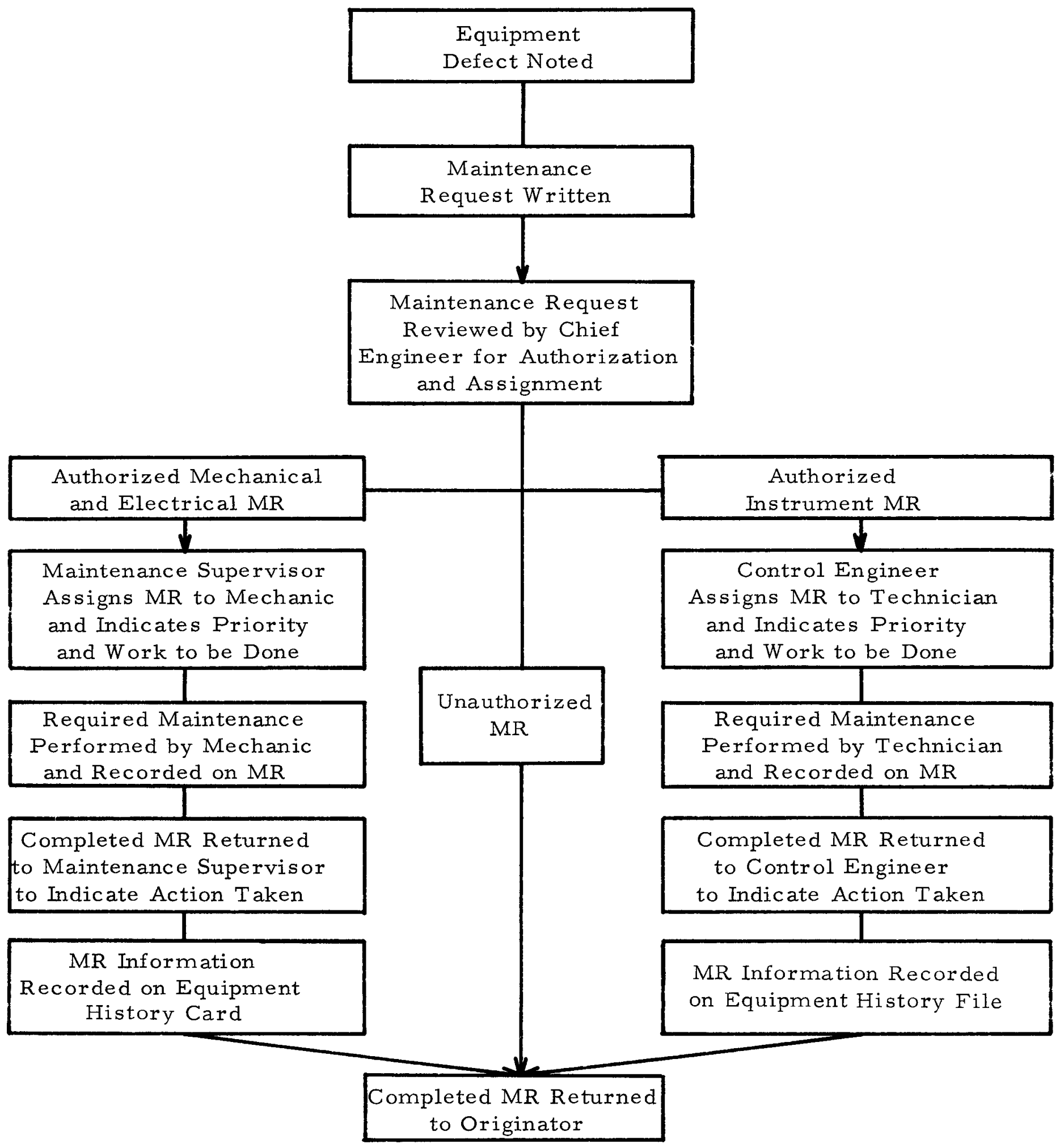

FIGURE A. 2 
YANKEE ATOMIC ELECTRIC COMPANY

MAINTENANCE REQUEST

EQUIPMENT

NATURE OF DEFECT

REPORTED BY DATE

\section{ACTION TAKEN}

INVESTIGATION BY

WORK TO BE DONE

WORK ASSIGNED TO

WORK COMPLETED BY

DATE

DESCRIPTION OF WORK DONE

MATERIAL USED

TO BE ENTERED ON EQUIPMENT CARD YES NO RETURN TO

FIGURE A. 3

MAINTENANCE REQUEST FORM

$$
\text { A }-13
$$




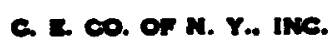

MAINTENANCE MEMORANDUM No 81913

DATE

WATCH

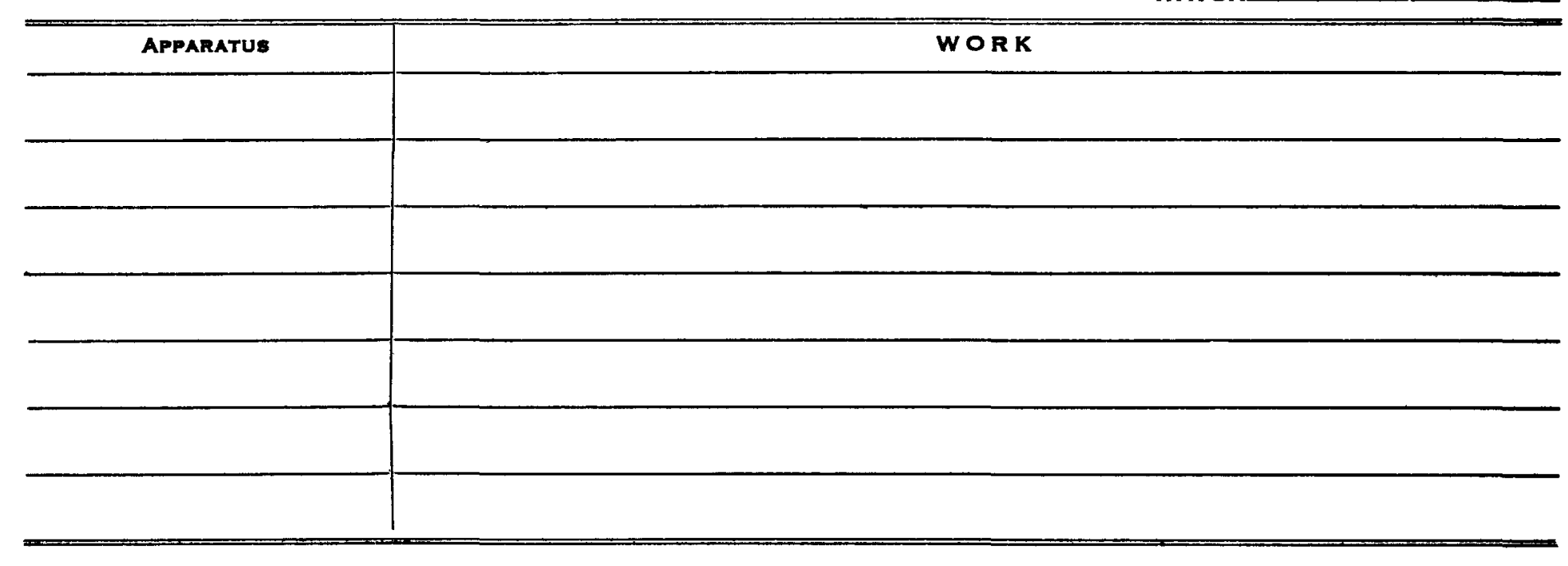

REPORTED EY

WATCH FORIMAM

13-60 (EA.) 7.62 PRODUCTION

APRROVED FOR INTERDEPARTMENTAL WONK REQUET

FIGURE A. 4

MAINTENANCE MEMORANDUM 


\section{ACTION}

Maintenance requirement roted ard memo written

Reviews and initiates memo

Assigns priority to memo

Assigns mechanic to work requested in memo

Performs work and enters description on memo

Notes work on pink copy and advances copy to completed work files; retains white copy in maintenance files

Reviews completed work and forwards memo to Production Office

Transfers work data to equipment histories and files memos

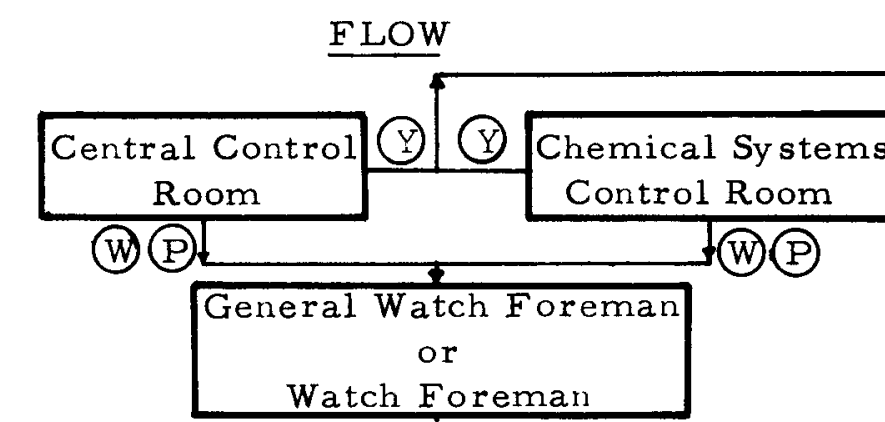

(W)

Reactor Engineer

or

Superintendent

W)

General Maintenance

Foreman:

(W)

Operating Mechanics on Days or Watch

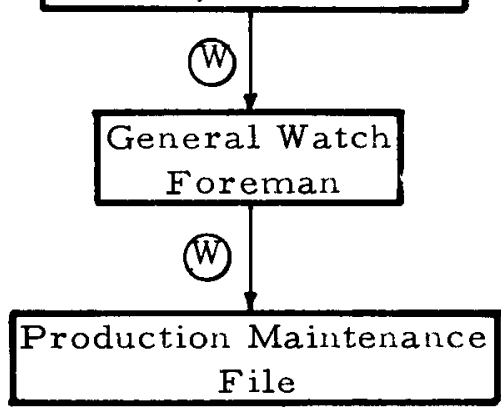

(P)

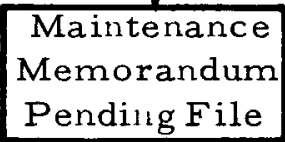

Completed by Maintenance File or Completed by Watch File

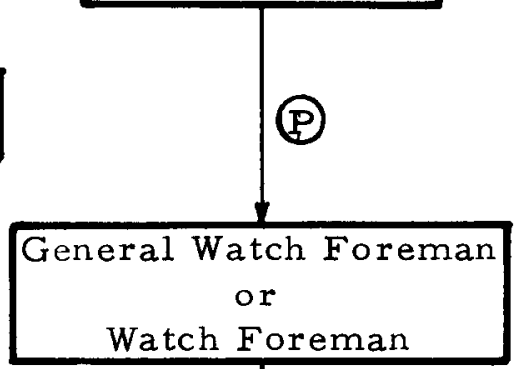

(P) Production Staff Technician

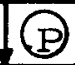

Production Office

File

$\mathrm{Y}-\mathrm{Yellow}$ copy

P - Pink copy

W - Wlite copy

FIGURE A. 5 
Maintenance requirement noted on instruments, "Not Working Properly" tag placed on equipment and memorandum written.

Reviews and initiates memorandum.

Assigns priority to memorandum.

Forwards white copy to Test Bureau and retains pink copy for follow-up.

Performs work, records completed work in log, reports completed work to Production Staff

Technician and Watch and removes "Not Working Properly" tag.

Reports status of Test Bureau work to Reactor Engineer or Superintendent and files completed memos.

Y - Yellow copy

W - White copy

P - Pink copy

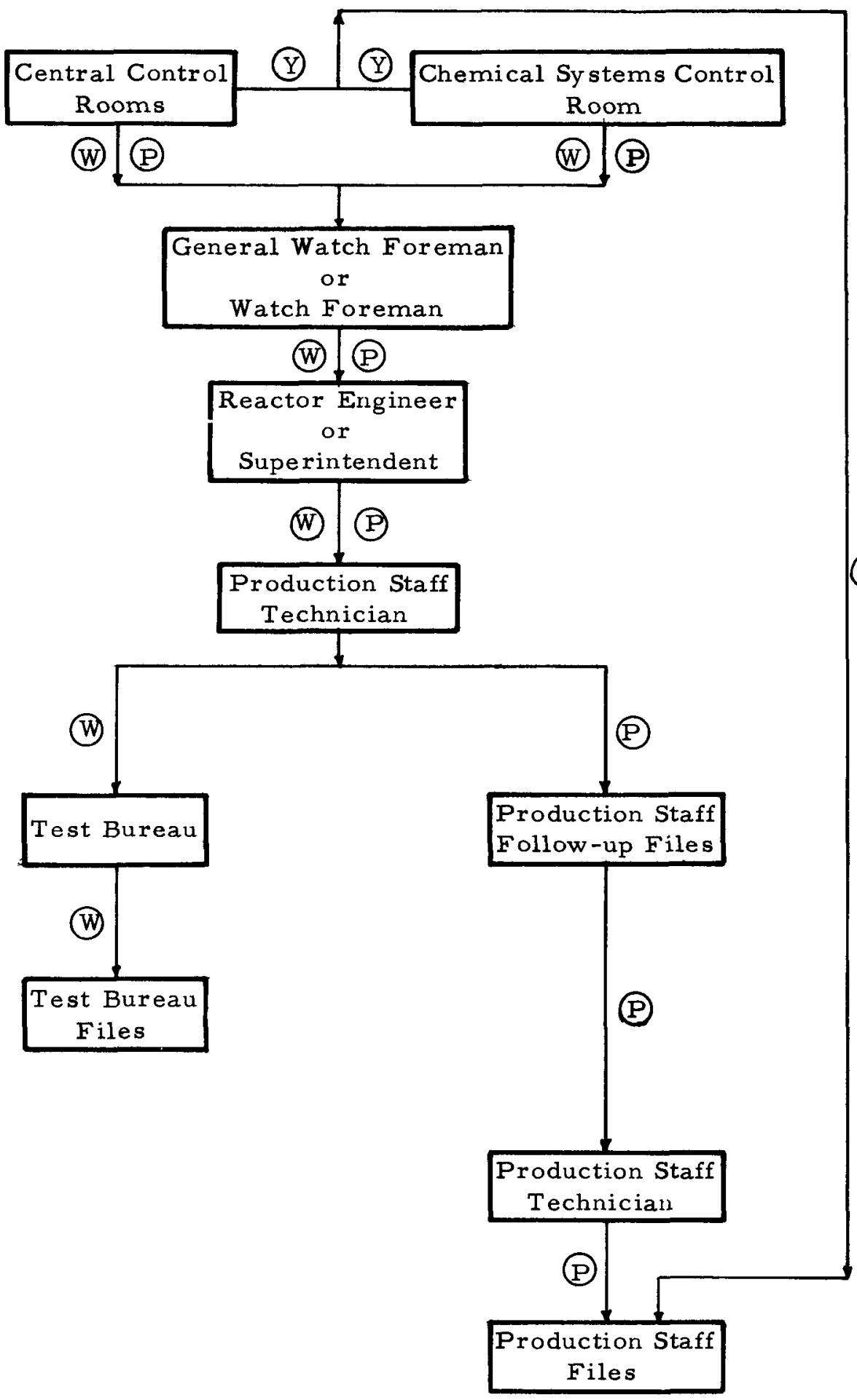

FIGURE A. 6 
Work request made out in triplicate and individual copies forwarded.

Operations retains 1 copy for followup; maintenance group receives second copy for scheduling; Assistant Superintendent or Power Plant Engineer receives third copy, approves request, assigns priority, and provides any additional work directives needed by maintenance groups.

Assigns work to mechanics, electricians, or technicians.

Performs approved work; enters work description in maintenance log or card file and on both copies of work request; forwards both copies of completed requests.

Compares original request to corresponding completed copies; discards original; retains 1 completed copy; forwards other completed copy.

Assistant Superintendent reviews and then discards completed work requests; operations retains completed requests until no further surveillance considered necessary and then discards request.

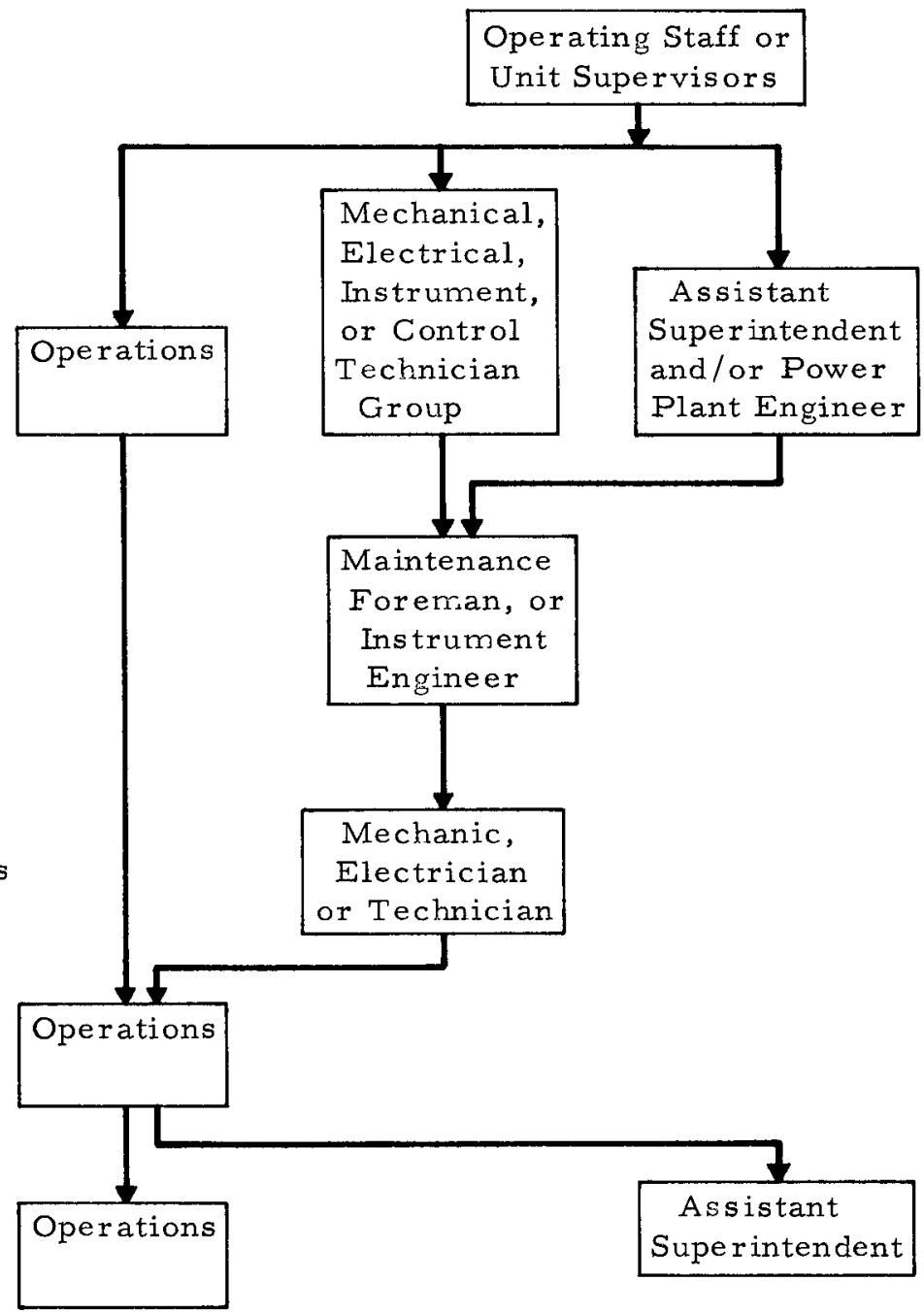

FIGURE A. 7

WORK REQUEST (FORM 23) FLOW 


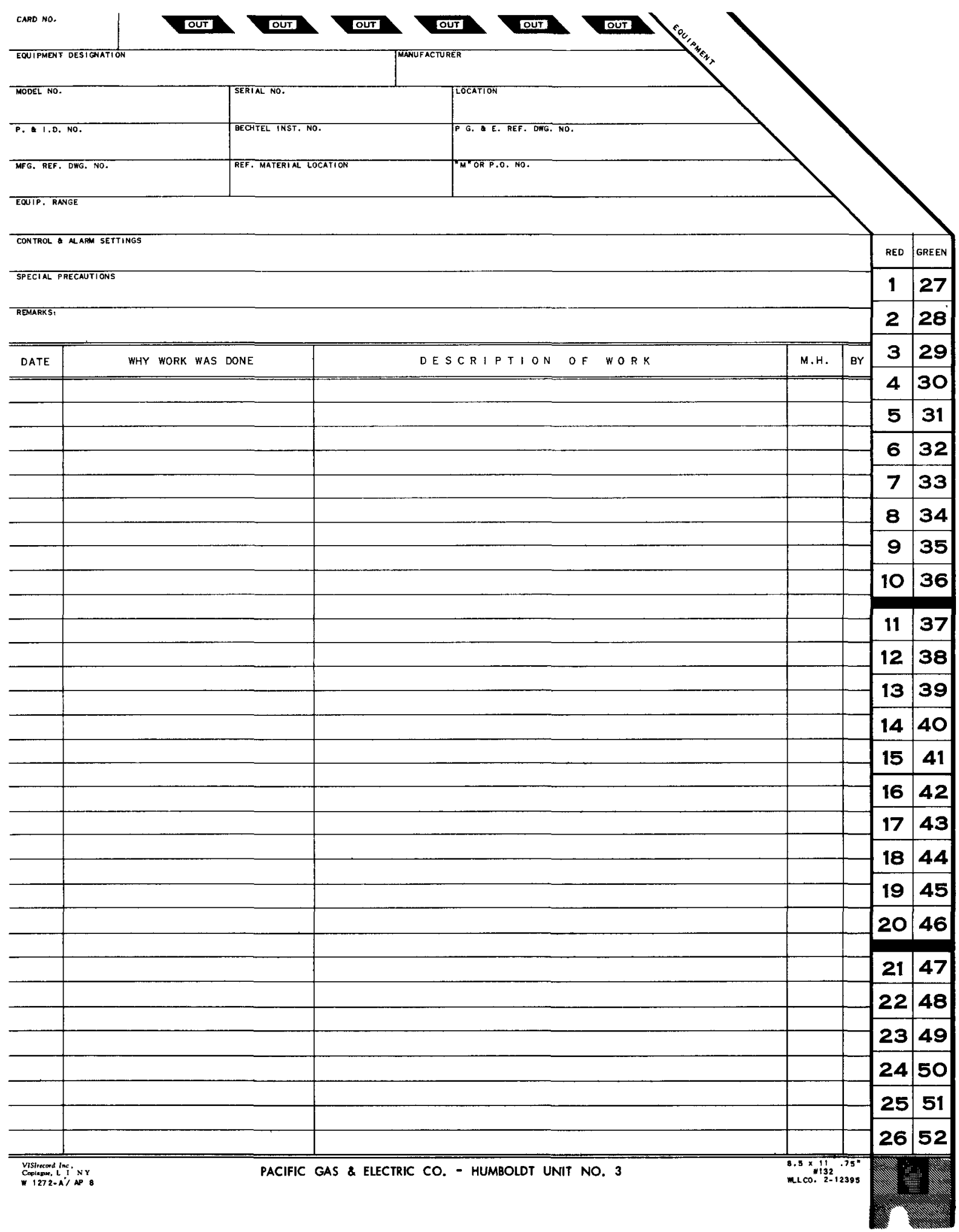

FIGURE A. 8

EQUIPMENT MAINTENANCE RECORD FORM 


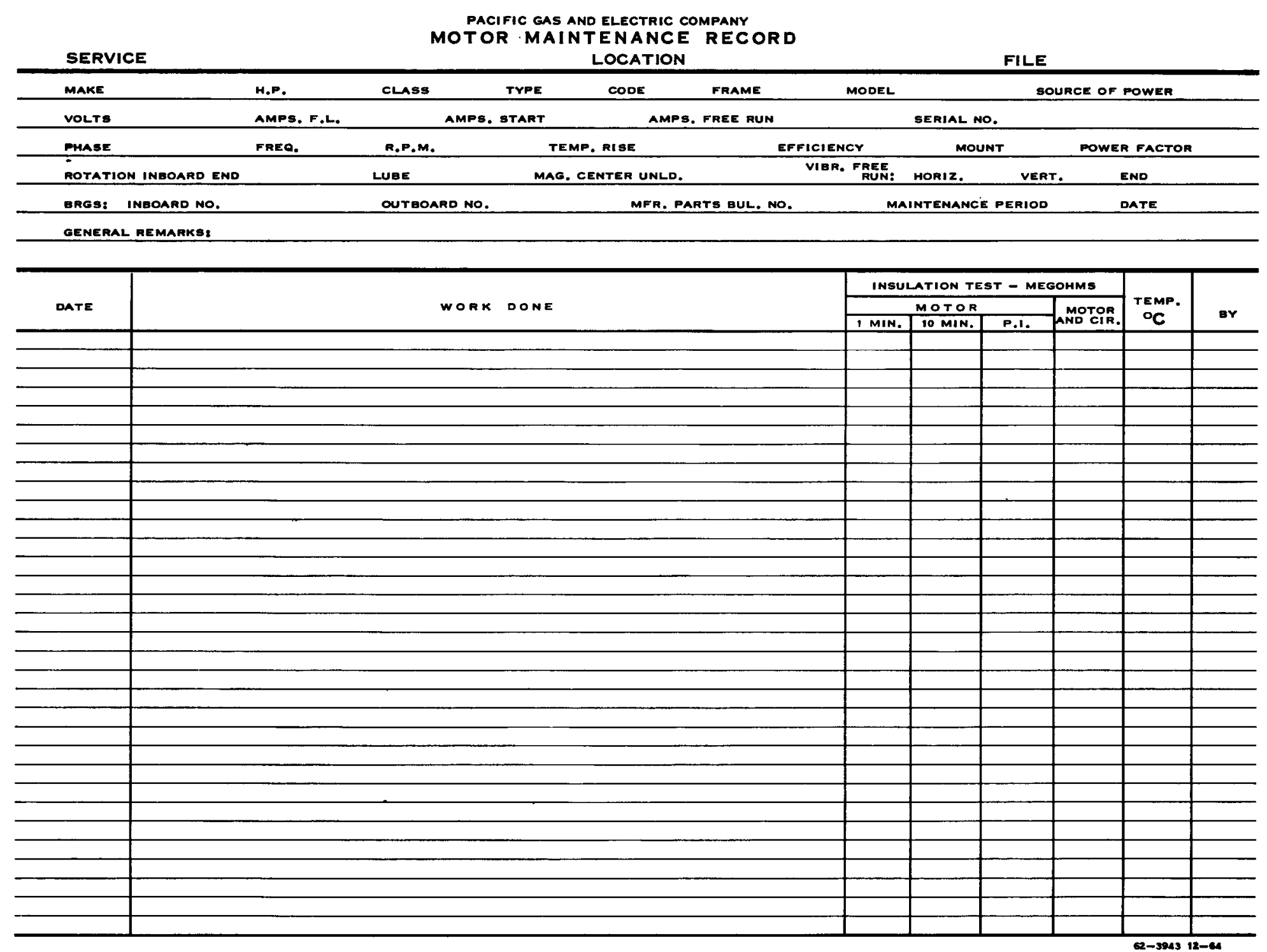

FIGURE A. 9

MOTOR MAINTENANCE RECORD FORM 


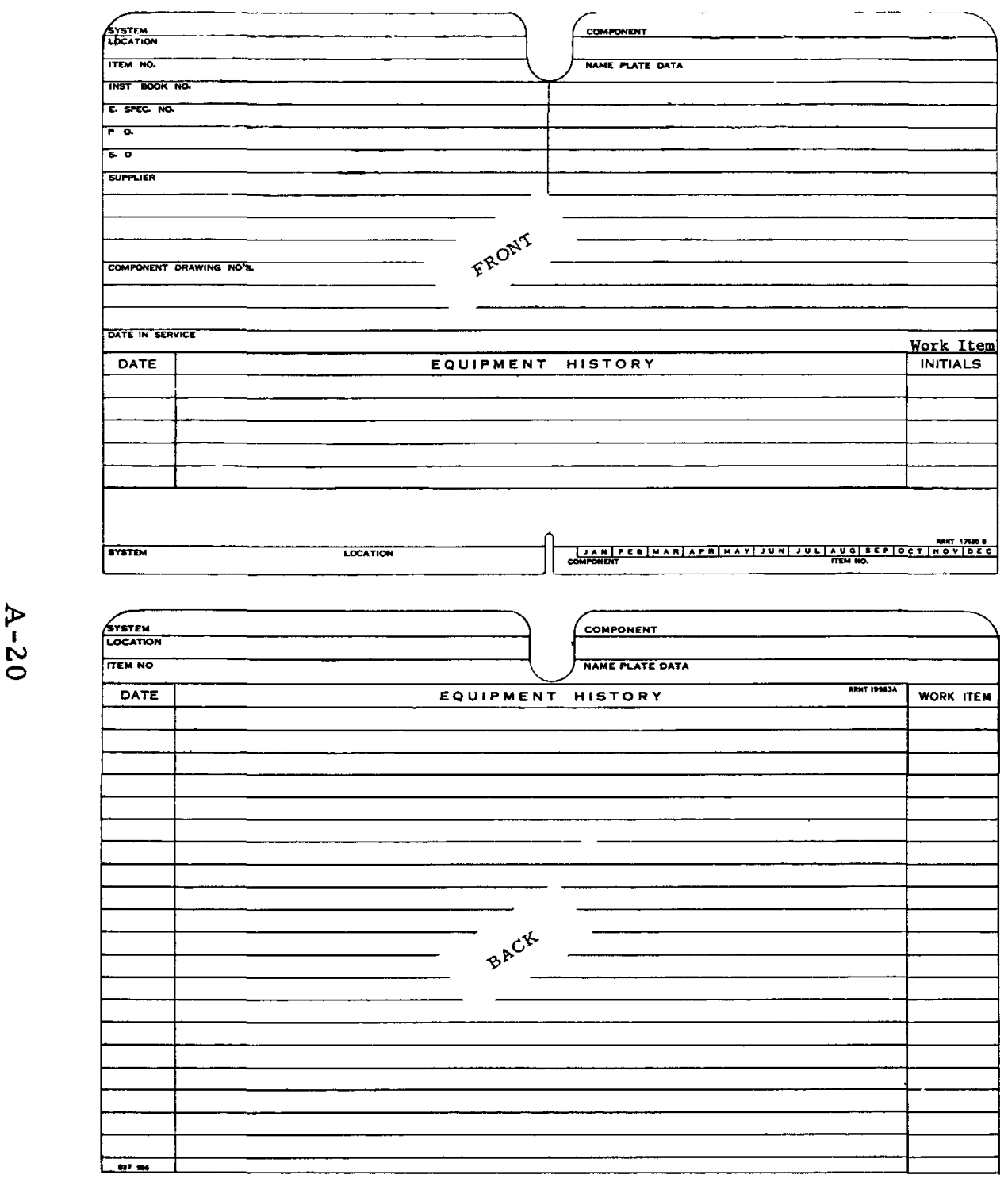

Equipment History Card

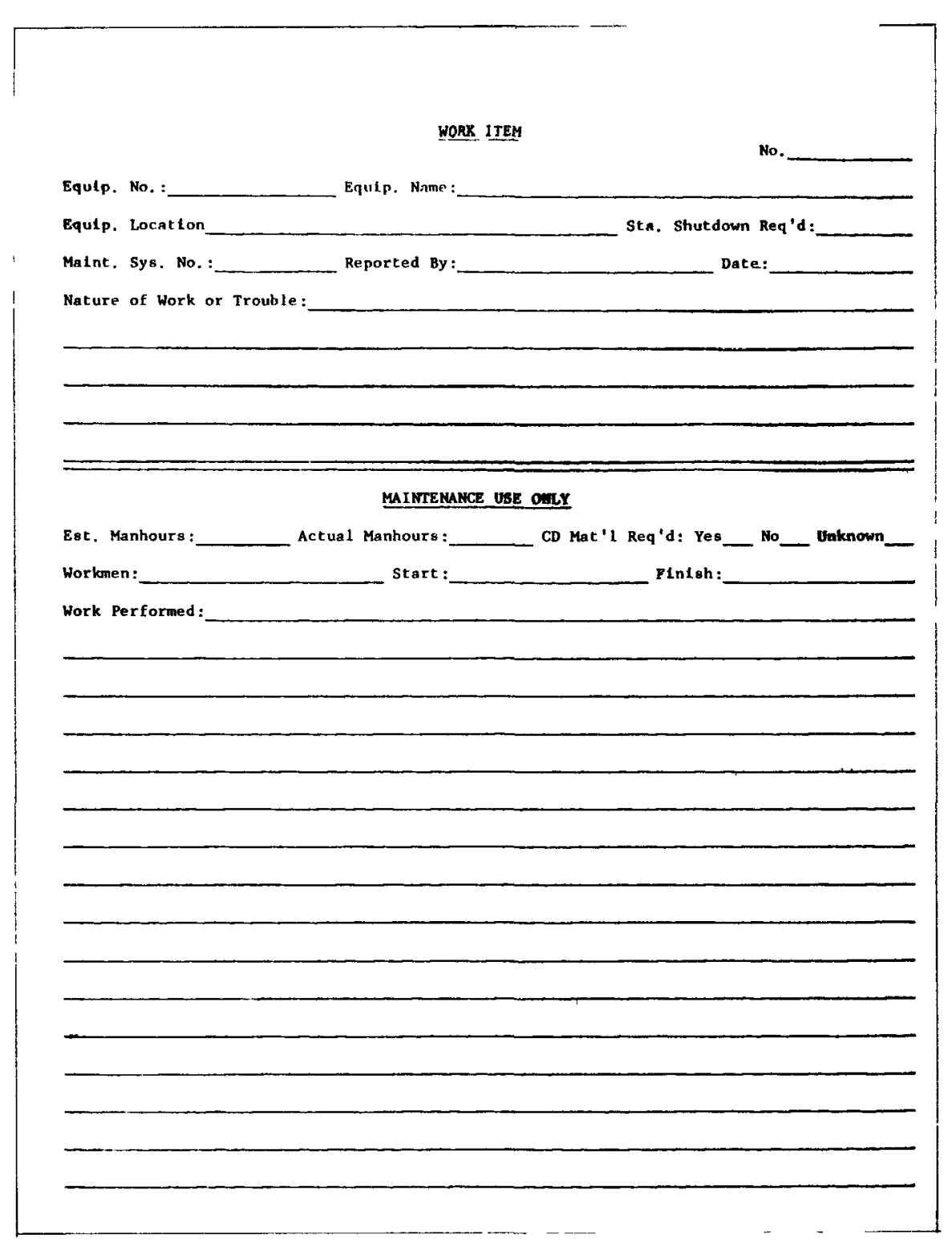

Work Item Card

FIGURE A. 10 
APPENDIX B

DATA MANAGEMENT PRACTICES IN RELATED AREAS 


\section{APPENDIX B}

\section{DATA MANAGEMENT PRACTICES IN RELATED AREAS}

Several reliability data classification and/or collection programs related to the nuclear, utility, aerospace, and merchant marine industries have been reviewed to establish guidelines and formats for the reliability monitoring program data management system. Of these programs, the following have provided the most useful background materials:

1. United Kingdom Atomic Energy Authority (UKAEA) Reactor Fault Reporting System.

2. PM-1 Data Evaluation Program.

3. Maritime Administration (MARAD) Maintenance and Reliability Program.

4. Edison Electric Institute (EEI) Equipment Availability Program.

5. Interservice Data Exchange Program (IDEP), Guided Missile Data Evaluation Program (GMDEP), and Failure Rate Data Program (FARADA).

6. Uniform Subject Index for Nuclear Power Demonstration (NPD) Project.

7. AEC Uniform System of Accounts.

As the name implies, the AEC Uniform System of Accounts was primarily developed for cost accounting and estimating purposes. However, since the breakdown in this system is by functional systems, particularly those related to the reactor plant, it has been reviewed with special interest.

A brief description of each of these programs follows.

\section{UKAEA REAC TOR FAULT REPORTING SYSTEM}

Under the UKAEA Fault Reporting System ${ }^{(1)}$ instituted in 1961, details of reactor faults and incidents which occur on UKAEA reactors are sent to the Safeguards Division of the Authority Health and Safety Branch (AHSB) located at Risley. The details are then coded and stored on punched cards according to the UKAEA data classification system for use in the analysis 
of reactor fault causes and trends. This system has been in operation since September 1964 with some minor modifications of the original class-
ification code.

The UKAEA classification system is based on an 80-column punch card. Column allocation is designated as shown on Table B. 1, and some samples of further breakdowns are shown in Tables B. 2 through B.7. Except for those columns that are self-explanatory, description of column allocations is as follows:

\section{Plant Type}

In the United States plant type generally would be refered to as a system and subsystem level of functional equipment classification. The system and subsystem in which a fault occurred are identified in these columns. The first level breakdown in this classification is:

1. Reactor.

2. Steam Raising Units.

3. Power Plant.

4. Plant Containment.

5. Rigs and Is otope Handling.

6. Electrical Power Supplies.

7. Ancillary Equipment.

Since the classification under "Reactor" encompasses considerably more equipment than what might be normally classified at this level in the United States, this particular level is further amplified as follows:

1. Pressure Vessel.

2. Core.

3. Shield.

4. Reactivity Control Devices.

5. Heat Exchange Circuit.

6. Control Electrical System and Instrumentation Control Circuitry.

As an example, a partial coverage of the control electrical system is shown in Table B. 2 . 


\section{Component Type}

The se columns itemize the components and parts on which the fault occurred. The first-level breakdown, along with a partial coverage of the component type as shown in Table B. 3, illustrates the coverage provided by component type classification.

1. Electrical.

2. Industrial Instrumentation.

3. Mechanical.

4. Chemical Plant.

5. Civil Engineering Work.

6. Component Not Identified.

7. Shielding.

Fault Importance

This portion of the data classification system deals with the safety aspects of each fault. It is basically divided into four broad categories; namely:
A. Lack of safety.
B. Faults or actions which reduce reactor safety.
C. Faults or actions which do not reduce reactor safety.
D. Events which have no safety significance whatsoever.

The definitions and details of the fault importance code are summarized completely in Table B. 4. These definitions were developed during two years of negotiation with reactor operators; and although rather detailed in development, they have been proven workable. The primary reason for the extended negotiations on definition of fault classification is attributed to the fact that power reactor operators and research facility operators tended to have somewhat different attitudes when faced with a given fault. As a result of the classifications shown in Table B.4, most faults will fall in the B and C category class.

In general, assignment of a fault to one of these categories is decided as follows: 
If the reactor protective system functions correctly and the resulting drift or transient is in all respects within the safe operating limit approved for the system, it is a Category C fault. If the reactor protective system functions correctly but the resulting drift or transient exceeds a safe operating limit approved for the system in any respect, it is a Category $B$ fault.

If, in addition to the initiating event, there occurs any malfunction of protective equipment, the fault would be classed as Category $B$ whether any operating limit is exceeded and despite any manual corrective action which has been taken by the operator (unless he takes it so quickly as to prevent a protective circuit action).

Fault Type

Columns 32 to 36 of the classification describe the nature of a fault. Fault types are generally divided into nine basic categories as follows:

1. Electrical.

2. Instrument Effects.

3. Mechanical.

4. Design Fault.

5. Human Error or Maloperation.

6. Undiagnosed Fault.

7. Chemical.

8. Radioactivity.

9. Other Faults.

Subclassifications within each basic fault-type category are illustrated by Table B. 5 .

\section{$\underline{\text { PM-1 DATA EVALUATION PROGRAM }}$}

The U. S. Air Force has established a data collection and classification system for evaluating operating data obtained from land-based military nuclear power plants. The system uses a data management program that catalogs plant performance data in a master file and allows computerized information retrieval and system analysis. (3) Although 
capable of being applied to any military plant, the $\mathrm{PM}=1$ nuclear power plant was chosen as the pilot plant for application of this data management program. The program is capable of producing output reports which assess the reliability, availability, safety, and performance of the plants as follows:

Reliability - Reliability of individual components and plant subsystems.

Availability - Availability of reactor and generating equipment.

Safety - All failures having a potential safety effect.

System Performance - Performance data with respect to steam and electrical output, plant chemistry, radioactive waste production, core lifetime, and economics.

Input to the master file comes from data obtained from the power plant logs. These data are separated by type and grouped into packets of cards, each packet going to form one tape record in the master file. While all data are important in assessing plant performance, only those portions of the program which relate directly to reliability and safety are described here.

The reliability and safety data require preparation of a malfunction packet consisting of three punched cards. The first card is a header card which identifies the type of packet. The second and the third cards, column allocation of which is shown in Tables B. 8 and B. 9, are basically for identifying the part of the plant in which the fault occurred and the type of fault which was caused by the part malfunction.

A code designation is provided for all subsystems and components of consequence in PM-1. Code designations are also provided for all major shutdown causes and all major causes of equipment failure. In addition, all failures are categorized in accordance with their effect on plant safety. Descriptions of column allocation for each data field are shown in Tables B. 8 and B. 9. With the exception of those fields which are self-explanatory, the type of information required is discussed below.

\section{Subsystem and Component Codes}

All plant equipment is classified at two levels; subsystem and component. All subsystems and components are identified by a two or three alphabetic code. For example, the subsystem code for the feedwater 
system is FW, while the component code for an electropneumatic transducer is EPT. Additional examples of subsystem and component codes are given in Table B. 10.

Malfunction, Safety, and Shutdown Codes

As shown in Table B.11, the malfunction and safety codes are developed to designate the effect of faults on reactor operation and safety. The shutdown code relates the cause or reason for a shutdown event.

Failure Code

The failure code used in the program describes component or system faults in terms of four major categories of equipment failure. These categories are mechanical, electrical, instrumentation, and others.

The first three categories are further divided into 20 subcategories, as shown in Table B. 12. In effect the subcategories are an open-ended listing of equipment failure descriptions. More terms can be added as different types of equipment failures are encountered.

A computer program has been developed to compile input data taken from PM-1 records and to generate a number of summary reports. With respect to this study the most important of these reports are the Safety Report, and the Failure Shutdown and Availability Report. Typical printouts of these reports are presented in Figures B. 1 and B. 2 .

MARITIME ADMINIS TRA TION MAINTENANCE AND RE LIABILITY PROGRAM

As a result of increasing costs in the construction and operation of ships subsidized by the United States, the Maritime Administration (MARAD) has developed a systematic approach for evaluating the operating data obtained from these ships. The goal of this program, the MARAD Maintenance and Reliability Program, (4) (5) is to collect data and perform detailed analyses of shipboard maintenance and repair policies, in order to identify areas where economies may be achieved.

In developing the MARAD program it was found that existing classification systems were primarily developed for filing and accounting use and were unsuitable for the objectives of the maintenance and reliability program. Furthermore, there were few sources of information concerned with ships in which the nomenclature agreed 
with the program needs; and nomenclature commonly in use was found to be inconsistent. For example, complete hierarchies of systems withir systems could often be found. It was necessary, therefore, to develop a new classification system.

In the new classification system, a hierarchy of ten levels was established as shown in Table B-13. The first two levels identify the ship and its environment. Levels three through ten provide the functional relationship of the elements of the ship's equipment and structure.

Between the levels the vertical relations are established on the basis of the various degrees of complexity; elements at the same level are expected to have the same degree of complexity.

The basic or fundamental levels in the equipment and structural hierarchy are Level 3-System, Level 6-Components, and Leve1 9-Parts. Intermediate levels are provided primarily for convenience in determining vertical relations between fundamental levels. Every system contains an element at the system level and one or more at the component level. Other elements in the intermediate levels are included to provide a complete classification of normally identifiable equipment. In classifying a system, elements at the component level are listed first; and the $n$ intermediate levels are filled, as necessary. Finally, to maximize consistency, any reference to elements within the hierarchy from system to part includes the name of their level. For example, the steam drum assembly is within the boiler component which is in the steam supply unit of the steam subsystem of the power and propulsion system.

At the system level, Level 3, each system is organized according to the ability of its element to perform, as a group, one of the necessary functions of the ship. A set of 14 general functions have been identified including one for automatic control. At the component level, Level 6, all the elements which are to be considered components under their respective systems are defined. After Level 6 is completed, Levels 5 and then 4 are completed by combining the elements into natural groupings following the appropriate definitions.

Below Level 6, the normal course would be to identify the elements at the parts level, Level 9, and then fill the intermediate Levels, 8 and 7. There are, however, an extremely large number of entries 
at the parts level; and they are subject to variations reflecting manufacturers' alternative designs. Furthermore, this amount of detail was not necessary in the early stages of the MARAD program. Therefore, the components were merely separated into their assemblies at Level 7 , and the classification was not carried beyond that point.

Although this classification process is reasonably straightforward, there are some complicating factors which should be mentioned. First, there are invariably entires which belong at a given level but do not exactly meet the definitions provided for that level. An example at the component level is piping. That heading is used to collect assemblies such as pipes and fittings, valves, traps, strainers, etc., into a group which together perform a specific operation.

However, piping is not a component in the usual sense of the word. The heading "Piping" at the component level, then, is a convenience heading. Other convenience headings include the various accommodation spaces of "associated equipment."

Another complication arises because there are some systems whose functions are not separable into subsystems. That is, all the units in the se systems support the function of the system directly.

Finally, there are a few elements which function within one system but which are structurally or operationally within another system. In classifying these elements, each portion of the element which uniquely supports the function of a system is classified within that system.

\section{Systems Classification Code}

The code which is used with the preceding classification method is in four parts. The first is a "prefix" consisting of the registered hull number designated in the Record of the American Bureau of Shipping (ABS). This prefix allows all data for any one ship at any level to be quickly retrieved. Following the prefix, the coding method for Levels 1 and 2 consists of the MARAD design number and trade route number. For Level 3 and below a letter code is assigned to each system, each subsystem within a system, and each unit within the subsystem. A list of systems and subsystems and their identifying letters appears in Table B. 14. 
Each component is assigned a three-digit component index number which identifies it and also indicates whether the component is unique to a given unit, commonly occurs in several units within a particular system or can occur in any system. Some components, such as pumps, occur in several systems. Since it may be interesting to collect all data on such components, each is given a number from a specific set allotted to that component as shown by the example in Table B. 15.

A second part of this code allows each particular type of component to be identified; for example, as shown in Table B. 16 sectional header, bent tube, and top fired boilers can be distinguished by component type codes $C, D$, and $E$.

The third part of the code is a two-digit assembly index number, which provides information on the relation of assemblies to components, similar to that which the component index provides on the relation of components to unit.

Finally, the fourth part of the code identifies the specific component among the several identical components to which the data applies.

An illustration of the coding using the ship SS President Arthur, whose registered hull number is 264704, MARAD design number C4-S-1, and trade route number 12 , follows. The code number for the steam drum assembly of the bent tube boiler on the starboard side is to be determined. The boilers are classified in the power and propulsion system, P; steam subsystem, B; and steam supply unit, B. The boilers are component 001 in their unit, and the bent tube boilers are designated component type D. The steam drum has 02 assembly designation, and as a rule the starboard boiler is numbered 01 .

The complete code can be written as

\section{$264704 / \mathrm{C} 4 \mathrm{~S} 1 / 12 / \mathrm{PBB} 001 \mathrm{D} 0201$}

\section{Data Collection Program}

The major obstacle in achieving the objectives originally set forth in the Maritime Adminstration program was the lack of adequate data. As a result, a program was established for collecting the required data in the field through direct observation and through cooperative 
efforts of operators. The program specified the data to be reported, its format, and the procedures to be used. Two stepwise approaches to data collection were instituted on a trial basis; (1) initial experimental data collection program by U. S. Merchant Marine Academy cadets during their training year at sea, and (2) subsequent data collection by chief engineers or other operator personnel during the course of their normal duties.

Evaluation of the returns made it apparent that the forms needed to be consolidated and simplified to avoid the confusion. Ambiguous instructions also were identified. Given improved forms, it was concluded that at least half of the cadets and probably more would produce usable reports. Although in some instances the cadets were hampered by officers in their efforts to collect data, this difficulty was traced to inadequate communication with management--a factor which was expected to disappear after the program had become well-established.

Another result of this trial application was the observation that a large number of the cadets were obviously almost wholly dependent on the officers for much of their information and frequently seemed to act mainly as transcribers. Thus, the ship's officers might in many cases have been able to carry out the data task themselves in less time than was required for their "advisory" role. Results of the subsequent experimental data collection program using chief engineers supported such a conjecture. The use of cadets as a continuing source of information can lead to undesirable discontinuities in the data since few ships have cadets present on every voyage. As a result, reliance on random voyage samples makes it impossible to characterize events accurately whose normal cycle approaches or exceeds the normal voyage length--and a significant amount of the information required is of that character. The problem is particularly acute for reliability calculations on equipment having $\mathrm{MTBF}^{\prime} \mathrm{s}$ on the order of a year or more. In this case, an unreported failure during a mission voyage might double the apparent time between failures; and because the total sample size is small for infrequent events, the error introduced by a missing datum is correspondingly larger.

While problems were encountered which led to revision of the data collection forms to the formats shown in Figures B. 3 and B. 4, the results indicated that collection of shipboard mainte nance and reliability data is feasible given proper forms, instructions, indoctrination, follow-through, and encouragement from management. 
Based on initial trials of the data collection program, an integrated data management system has been envisioned, as depicted by the flow chart shown in Figure B. 5 .

EDISON ELEC TRIC INS TITUTE (EEI) EQUIPMENT AVAILABILITY PROGRAM

The Prime Movers Committee of the Edison Electric Institute (EEI) has been collecting outage data based on equipment availability reports submitted by approximately 90 of its member utility companies for a number of years. The program covers more than 500 turbines and boilers for units having a name plate capacity of 50 megawatts or larger and with initial steam conditions of at least $800 \mathrm{psi}$ or $900^{\circ} \mathrm{F}$. Of the total, 91 percent of the units have a single boiler per turbine arrangement. In the near future, the institute plans to report availability and outage data in connection with nuclear units.

Outages are reported by type according to the classification shown in Table B. 17. The cause or reason for each outage is classified as shown in Table B. 18. The general code numbers in Table B: 18 are designated 100 for boilers, 200 for turbines, 300 for generators, 400 for condensers, and 500 for unit outages not caused by any of the previous four categories. Although the list appears to be a component classification, since in many instances the names of equipment are used to describe the code designation, a close $r$ examination reveals an intermixing of component and event data pertaining to a major piece of equipment. A typical summary of forced outages is shown in Figure B.6. Using the data collected in the EEI program analyses of availability reports (6) (7) have been prepared for use by power utility engineers engaged in design, system planning and production management, and by major equipment manufacturers. Basically these reports provide information on equipment availability and repair. As presented, the data in the se reports do not lend themselves to ge nerating component/part failure rate data.

INTERSERVICE DATA EXCHANGE PROGRAM (IDEP), GUIDED MISSILE DATA EVALUATION PROGRAM (GMDEP), AND FAILURE RATE DATA PROGRAM (FARADA)

The Interservice Data Exchange Program (IDEP) was established in 1958 for the free exchange of parts/components data among the Department of Defense ballistic missileactivities. Its principal function is to reproduce and distribute test results submitted by contractors. Each 
participating contractor summarizes the results of his test in a standard report summary sheet which is forwarded, with the complete test results, to the cognizant IDEP office. By multilith process the information on the standard report summary sheet is transferred to a report summary card. In addition, the test data is printed and distributed separately on $16 \mathrm{~mm}$ roll film. A sample of a report summary card is shown in Figure B. 7 .

In 1959 the Navy's Guided Missile Data Evaluation Program (GMDEP), similar in purpose and intent to the IDEP, was initiated at the Fleet Missile Systems Analysis and Evaluation Group (FMSAEG), Corona, California. The significant difference between this program and IDEP is that its prime purpose was to exchange data generated by those Navy contractors engaged in the research, development, and production of guided missiles. As previously indicated, IDEP covered the interchange of ballistic missile and space system information. In July 1964, GMDEP was officially consolidated with IDEP.

Late in 1960 the Naval Ordnance Systems Command requested the Fleet Missile Systems Analysis and Evaluation Group (FMSAEG) at Corona, California, to perform a component part study associated with and as a subtask to the Navy Guided Missile Data Exchange Program (GMDEP). This study was (1) to determine whether failure rate data was available and if it could be obtained on a selected list of electrical, electronic, mechanical, electromechanical, pneumatic, hydraulic, and pyrotechnic parts/components, and (2) to develop a method for the collection, analysis, compilation, presentation, and distribution of failure rate data.

Initially, the possibility of placing failure rate data on the report summary cards being circulated in the existing IDEP and GMDEP programs was explored. However, it was found that test and failure rate data being circulated in those programs lacked sufficient analysis and summarization to facilitate the extraction of failure rates with respect to time and environment. The approach used in GMDEP and IDEP to present results of individual short-term qualification tests performed on parts/components did not lend itself to presenting a rate of failure on those parts/components.

As a result of the study, it was determined that modified or new forms and procedures would be required to obtain this information and to present it uniformly. Plans were then formulated to include as many organizations as were willing to forward a resume of failure rate information from their various in-house programs to a Failure Rate Data (FARADA) 
Information Center at Corona. The Air Force, Naval Ship Systems Command, Army, and NASA also became sponsors of the FARADA program. (8) Flow of FARADA information is shown schematically in Figure B. 8. Qualified data which are submitted to the FARADA Information Ceneter are screened, summarized, compiled, and published in FARADA handbooks. Figures B. 9, B. 10, and B. 11 illustrate completed sample forms of data contribution. Use of the se forms by data contributors, however, is optional since the FARADA Information Cente $r$ will accept generated data from participants in whateve $r$ in-house format available. The FARADA handbooks provide reliability engineers, design engineers, and maintainability engineers with failure rate information in a convenient form.

The FARADA handbooks comprise five volumes encompassing more than 30,000 line entries of tabulated failure rate data; over 2,000 entries of failure mode distribution data; and stress curves on electronic parts/ components. Each volume is updated quarterly, and the presentation of failure data is as follows:

Volume $1 \mathrm{~A} \quad$ Computer printout of electrical and electronic failure rate data. See Figure B. 12.

Volume 1B Computer printout of mechanical, hydraulic, pneumatic, and pyrotechnic failure rate data. See Figure B. 13.

Volume 2 Failure rate data presented as stress curves with environmental and application factors.

Volume 3 Failure rate data background information to support Volumes $1 \mathrm{~A}, 1 \mathrm{~B}, 2$, and 4 .

Volume 4 Failure mode data to implement application of the failure rate data of Volumes $1 \mathrm{~A}, 1 \mathrm{~B}, 2$, and 3 . See Figure B. 14.

Although the IDEP/FARADA programs are not power plant or systems oriented, their generic code system is of value when considering collection of manufactured data.

In the FARADA component code, the first three digits are the major classification corresponding to the part/component designation. These digits are followed by three additional two digit subclass groups which identify service, function, operation, etc. A partial list of major and 
subclassifications is shown in Figures B.15and B.16. For example, from Figure B. 17, the index number for a common globe valve may be 925.60 .75 .84 .

Since several subclasses, such as service, pressure range, power rating, etc., are applicable to a number of parts/components, common subclassifications have also been used according to the breakdown given in Figure B. 17. In some cases, it was necessary to break down the two digit subclasses to the point where each digit gives a subclassification description. For example, in the second subclass for the globe valve mentioned above the first numeral described the pressure range and the second numeral described the function.

UNIF ORM SUBJECT INDEX FOR NUC LEAR POWER DEMONSTRATION (NPD) PROJECT

In 1958, a Uniform Subject Index (USI) was established for the Nuclear Power Demonstration (NPD) project in order to better coordinate files and accounts between different functional groups such as engineering, purchasing, accounting, operating, and maintenance. Prior to establishing this index, a careful review was made of the Hydro-Electric Power Commission (HEPC) breakdowns for hydraulic and thermal projects and the Uniform System of Accounts of the U. S. Federal Power Commission (FPC). Subjects peculiar to nuclear power stations were considered and an index was selected which required minimal changes from project to project.

The resulting USI breakdown is based on a five digit coding system using numbers $r$ the $r$ than letters and is limited to 0 to 9 at each level. For a given system, the numbers 1 through 9 are used to represent the real physical breakdown. Alternative nonphysical breakdowns which an engineer may desire are often provided as a software breakdown in the "0" or general series. This provides further flexibility in extending the index system without affecting the breakdown based on the real physical systems.

Level 1 Breakdown

The Level 1 breakdown fundamentally reflects the major divisions for other projects in the HEPC, and is as follows:

0 - General
1 - Site and Improvement 
2 - Buildings, Structures, and Shielding

3 - Steam Generation (Reactor-boiler and auxiliaries)

4 - Turbine-Generator and Auxiliaries

5 - Electrical

6 - Instrumentation and Control

7 - Common Processes and Services

8 - Plant Construction

9 - Indirect Costs

The scope of each of these major level breakdowns are expected to fit fairly well with any type of nuclear power station. Item 6 is a major exception to the FPC practive on which the USI is based. However, since Item 6 is further subdivided according to the common practice, identification becomes easier.

Shielding which does not form a part of a conventional station has been included as an extension to Item 2. The main generator which is conventionally handled as part of the turbine generator could have been included in either Item 4 or 5 . It has been included under Item 4 . One other exception is the circulating water system. It was considered economically advantage ous to combine water supply requirements, so the circulating water system has been included under water supplies in Item 7.

Level 2 Breakdown

The Level 2 breakdown is set up to conform to a breakdown suitable for a particular type reactor. A breakdown which would be to a large extent the same for different projects employing a heavy water type of nuclear power generating station.

The Level 2 breakdown, as a general rule, corresponds to a group of related systems as shown in the example below:

71 Water Supply Systems

72 Drainage Systems

73 Ventilation Systems 
Level 3 Breakdown

As a general rule, the Level 3 breakdown corresponds to a particular system; for example:

711 Intake Water System

712 Circulating Water System

713 Process Water System

714 Standby Water System

715 Domestic Water System

Level 4 and Level 5 Breakdowns

Further breakdowns into subsystems and types of equipment have been made to suit the needs of Level 3 breakout. As an example, a standard breakdown of fluid systems is shown below:

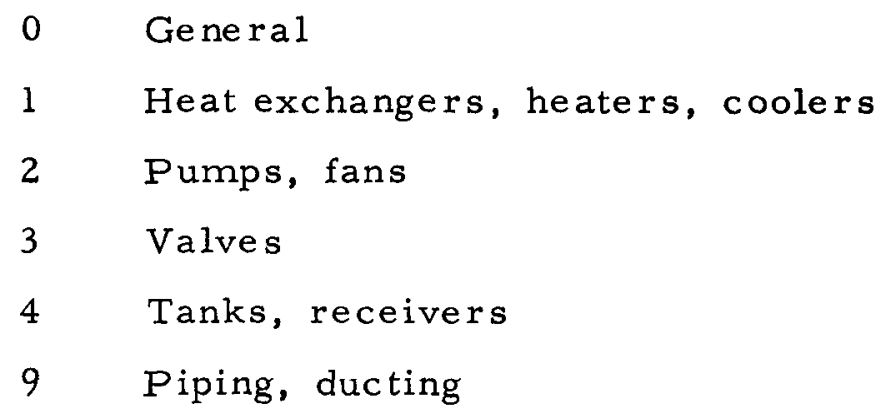

\section{Deficiency Reports}

Based on an operating philosophy of minimizing paper work, deficiency reports have been relied on to collect input data for reliability analysis. Experience has shown that these reports are the best source of data and require a minimum amount of interpretation.

Deficiency reports, a sample form of which is shown in Figure B. 18, are used when deficiencies are noted in equipment, design, procedures or operation to provide:

1. A means of reporting a deficiency to a work unit in order to have it corrected, and

2. A means of recording deficiencies and corrective actions, with minimum details necessary for evaluating system reliability. 
Deficiency reports are handled according to the flow shown in Figure B. 19. The originator completes the upper portion of the form by identifying the system, including its failure mode and shutdown requirements and describing the deficiency itself. The white original and the yellow copy are forwarded to an appropriate work unit which will take corrective action, and the pink copy is retained in the control room for information and follow-up purposes. Upon completion of the work requested, the work unit completes the lower portion of the form and forwards the yellow copy to the originator and his shift supervisor. The white copy is generally retained in the work unit files. If the work has been completed to the satisfaction of the originator and the supervisor, the pink copy is removed from the pending file and destroyed. The yellow copy is placed in the permanent file by the clerical unit for future reference and review including that by the reliability engineer. A space is provided at the bottom of the form for comments by any person during routing.

\section{AEC UNIF ORM S YS TEM OF ACCOUNTS}

In the development of different nuclear power plant designs, many economic data have been submitted to AEC in proposals and studies by industrial organizations; however, direct comparison between different designs were often very difficult since a common set of ground rules for assumptions, site criteria, and format for presenting data were generally lacking. In 1962 the AEC Division of Reactor Development authorized the publication of the first edition of a Guide to Nuclear Power Cost Evaluation $(9)$ to fulfill this need.

In the Guide, a uniform system of accounts is developed for estimating and reporting construction costs for nuclear power and related transmission and general plant facilities. This system was designed by the AEC Division of Finance to provide the means for standard distribution of nuclear power plant costs and to facilitate the comparative economic analyses of various nuclear power plant concepts.

The direct cost accounts have been keyed to the Electric Plant Accounts (10) established by the U. S. Federal Power Commission (FPC) as shown by the example in Table B. 19.

The AEC system of accounts is designed to facilitate cost estimates and production cost analyses for proposed nuclear power plant concepts as well as for completed nuclear power plants. The principal difference between the AEC system of accounts and that of the FPC is that the latter is designed for use in recording costs of completed plants after the 
allocation of indirect construction cost, whereas the AEC system is designed for use during the construction period prior to the allocation of indirect construction costs; and, therefore, provides separate accounts for these costs (account nos. 98 and 99).

A summary of the AEC classification of construction accounts is shown in Table B.20. An example of a detailed listing of the AEC accounts, together with a brief description of the equipment, materials, and services covered by each of the major accounts is shown in Table B. 21 . 


\section{REFERENCES}

1. Parker, E. and K. C. Rushton, "AHSR Reactor Fault Reporting System Reactor Fault Code," AHSB(S)R76, UKAEA, 1964.

2. Ablitt, J. F., "The Contribution of Systematic Incident Evaluation of the Achievement of Reactor Safety," AHSB(S)R89, UKAEA, 1965.

3. "Computer Program to Evaluation of Data from Military Nuclear Power Plants, "AFWL TR-65-14, Westinghouse Electric Corporation, Atomic Power Division, July 1965.

4. Hanifan, D. T., R. A. Westland, L. B. Sklar, and D. A. Atkins, "Maritime Administration Maintenance and Reliability Program," Vols. I-IV, MA-3402, Dunlap and Associates, Inc., 1965.

5. Atkins, D. A. and A. W. Harbaugh, "Merchant Ship Maintenance and Reliability, "Society of Naval Architects and Marine Engineers, Southern California Section, February 11, 1965.

6. "Analytical Report of Equipment Availability for the Seven-Year Period 1955-61." EEI No. 63-42, Edison Electric Institute, May 1963.

7. "Report on Equipment Availability for the Five-Year Period 1960-64," EEI No. 65-35, Edison Electric Institute, May 1965.

8. "Army, Navy, Air Force, and NASA Failure Rate Data (FARADA) Program," Revision 1, May 1966, U. S. Naval Fleet Systems Analysis and Evaluation Group, Corona, California.

9. "Guide to Nuclear Power Cost Evaluation," Kaiser Engineers, TID-7025, March 15, 1962.

10. "Uniform System of Accounts Prescribed for Public Utilities and Licenses of the Federal Power Commission, "January 1, 1961. 
TABLE B. 1

UKAEA FAULT CLASSIFICATION SYSTEM

\begin{tabular}{|c|c|}
\hline Data & Column Allocation \\
\hline Fault Number & $1-5$ \\
\hline \multicolumn{2}{|l|}{ Date } \\
\hline Day & $6-7$ \\
\hline Month & $8-9$ \\
\hline Year & $10-11$ \\
\hline Reactor Identification & $12-13$ \\
\hline Plant Type & $14-21$ \\
\hline Component Type & $22-27$ \\
\hline Fault Importance & $28-31$ \\
\hline Fault Type & $32-36$ \\
\hline Effect & $37-38$ \\
\hline Site Reference Number & $41-45$ \\
\hline Site Run Number & $46-50$ \\
\hline \multicolumn{2}{|l|}{ Time Since Start of Run } \\
\hline $\begin{array}{l}\text { Hours } \\
\text { Fraction of Hours }\end{array}$ & $51-53$ \\
\hline \multicolumn{2}{|l|}{ Time Reactor Out of Operation } \\
\hline $\begin{array}{l}\text { Hours } \\
\text { Fraction of Hours }\end{array}$ & $55-57$ \\
\hline Reactor Power at Occurrence of Fault & $59-61$ \\
\hline Fraction & 62 \\
\hline Unit of Power (MW, $\mathrm{KW}$, or $\mathrm{W})$ & 63 \\
\hline Whether Poisoned Out & 64 \\
\hline Method of Shutdown & $65-66$ \\
\hline Mark Number of Faulty Component & $67-68$ \\
\hline Unused Columns & $39-40,69-74$ \\
\hline
\end{tabular}


TABLE B. 2

PARTIAL LIST OF PLANT TYPES

Control Electrical System and Instrumentation

Control Circuitry

Reactor

Safety Circuits

Start Guard Sequence

Alarms

Emergency Trips Shutdown Button

Reactor Trips

Power Setback

Interlocks

Warnings

Lamp Indication

Position Indication (Safety Rods)

Position Indication (Control Rods and Arms)

Operating Controls

Bypass Circuits

Control Arm Misalignment Circuit

Ancillary Equipment

Door System Indicator

Extract Filter and Seal System

Make-up Plant

M. O. W. or Dewhirst Gear

Nucleonic Channels

Reactor Control

Period Channels

Trip Unit

Period Meter

Reactivity Trip Circuit

Flux Channels

Linear Power

Low Level

High Leve1

Change-over Mechanism

Trip Unit

Log Power

Low Level

High Level

Trip Unit

Integrated Flux

Neutron Source

Position Indicator

\begin{tabular}{|c|c|c|c|c|c|c|}
\hline 1 & 6 & & & & & \\
\hline $1^{\circ}$ & 6 & 1 & & & & \\
\hline 1 & 6 & 1 & 1 & & & \\
\hline 1 & 6 & 1 & 1 & 1 & & \\
\hline 1 & 6 & 1 & 1 & 1 & 1 & \\
\hline 1 & 6 & 1 & 1 & 1 & 2 & \\
\hline 1 & 6 & 1 & 1 & 1 & 3 & \\
\hline 1 & 6 & 1 & 1 & 1 & 3 & 1 \\
\hline 1 & 6 & 1 & 1 & 1 & 4 & \\
\hline 1 & 6 & 1 & 1 & 1 & 5 & \\
\hline 1 & 6 & 1 & 1 & 2 & & \\
\hline 1 & 6 & 1 & 1 & 3 & & \\
\hline 1 & 6 & 1 & 1 & 4 & & \\
\hline 1 & 6 & 1 & 1 & 5 & & \\
\hline 1 & 6 & 1 & 1 & 6 & & \\
\hline 1 & 6 & 1 & 1 & 7 & & \\
\hline 1 & 6 & 1 & 1 & 8 & & \\
\hline 1 & 6 & 1 & 1 & 9 & & \\
\hline 1 & 6 & 1 & 2 & & & \\
\hline 1 & 6 & 1 & 2 & 1 & & \\
\hline 1 & 6 & 1 & 2 & 2 & & \\
\hline 1 & 6 & 1 & 2 & 3 & & \\
\hline 1 & 6 & 1 & 2 & 4 & & \\
\hline 1 & 6 & 2 & & & & \\
\hline 1 & 6 & 2 & 1 & & & \\
\hline 1 & 6 & 2 & 1 & 1 & & \\
\hline 1 & 6 & 2 & 1 & 1 & 1 & \\
\hline 1 & 6 & 2 & 1 & 1 & 2 & \\
\hline 1 & 6 & 2 & 1 & 1 & 3 & \\
\hline 1 & 6 & 2 & 1 & 1 & 4 & \\
\hline 1 & 6 & 2 & 1 & 1 & 5 & \\
\hline 1 & 6 & 2 & 1 & 1 & 6 & \\
\hline 1 & 6 & 2 & 1 & 2 & & \\
\hline 1 & 6 & 2 & 1 & 2 & 1 & \\
\hline 1 & 6 & 2 & 1 & 2 & 1 & 1 \\
\hline 1 & 6 & 2 & 1 & 2 & 1 & 2 \\
\hline 1 & 6 & 2 & 1 & 2 & 1 & 3 \\
\hline 1 & 6 & 2 & 1 & 2 & 1 & 4 \\
\hline 1 & 6 & 2 & 1 & 2 & 2 & \\
\hline 1 & 6 & 2 & 1 & 2 & 3 & \\
\hline I & 6 & 2 & 1 & 2 & 3 & 1 \\
\hline 1 & 6 & 2 & 1 & 2 & 3 & 2 \\
\hline 1 & 6 & 2 & 1 & 2 & 3 & 3 \\
\hline 1 & 6 & 2 & 1 & 2 & 4 & \\
\hline 1 & 6 & 2 & $1^{*}$ & 3 & & \\
\hline 1 & 6 & 2 & 1 & 3 & 1 & \\
\hline 1 & 6 & 2 & 1 & 4 & & \\
\hline 1 & 6 & 2 & 1 & 5 & & \\
\hline
\end{tabular}

*Code numbers left adjusted beginning with Column 14. 
TABLE B. 3

PARTIAL LIST OF COMPONENT TYPES*

\begin{tabular}{|c|c|c|c|c|c|}
\hline Not Applicable & 0 & & & & \\
\hline Electrical & 1 & & & & \\
\hline Control Equipment & 1 & 1 & & & \\
\hline Switches & 1 & 1 & 1 & & \\
\hline Limit & 1 & 1 & 1 & 1 & \\
\hline Micro & 1 & 1 & 1 & 2 & \\
\hline Anti-Vibration & 1 & 1 & 1 & 3 & \\
\hline Reset Position & 1 & 1 & 1 & 4 & \\
\hline Range Selector & 1 & 1 & 1 & 5 & \\
\hline Relays & 1 & 1 & 2 & & \\
\hline Contacts & 1 & 1 & 2 & 1 & \\
\hline Winding & 1 & 1 & 2 & 2 & \\
\hline Sealed & 1 & 1 & 2 & 3 & \\
\hline Moving Coil & 1 & 1 & 2 & 4 & \\
\hline Meters & 1 & 1 & 3 & & \\
\hline Frequency Meter & 1 & 1 & 3 & 1 & \\
\hline Indicating Lamps & 1 & 1 & 4 & & \\
\hline Ordasign & 1 & 1 & 4 & 1 & \\
\hline Alarms & 1 & 1 & 4 & 2 & \\
\hline Overrun Lights & 1 & 1 & 4 & 3 & \\
\hline Voltage Regulators & 1 & 1 & 5 & & \\
\hline Variacs & 1 & 1 & 5 & 1 & \\
\hline Synch ro Equipment & 1 & 1 & 6 & & \\
\hline Magslips & 1 & 1 & 7 & & \\
\hline Transmitters & 1 & 1 & 7 & 1 & \\
\hline Receivers & 1 & 1 & 7 & 2 & \\
\hline Drives & 1 & 1 & 7 & 3 & \\
\hline General Equipment & 1 & 2 & & & \\
\hline Distribution Board Links & 1 & 2 & 1 & & \\
\hline Starters & 1 & 2 & 2 & & \\
\hline Motor Starters & 1 & 2 & 2 & 1 & \\
\hline Interlocks & 1 & 2 & 2 & 1 & 1 \\
\hline U/V Solenoid & 1 & 2 & 2 & 1 & 2 \\
\hline Auxiliary Contacts & 1 & 2 & 2 & 1 & 3 \\
\hline Overload Protection & 1 & 2 & 2 & 1 & 4 \\
\hline Crane Controllers & 1 & 2 & 2 & 2 & \\
\hline Drum Segments & 1 & 2 & 2 & 2 & 1 \\
\hline Commutators & 1 & 2 & 3 & & \\
\hline Components & 1 & 2 & 4 & & \\
\hline Transformers & 1 & 2 & 4 & 1 & \\
\hline Rectifiers & 1 & 2 & 4 & 2 & \\
\hline Suppressors & 1 & 2 & 4 & 3 & \\
\hline Transducers & 1 & 2 & 4 & 4 & \\
\hline Pressure & 1 & 2 & 4 & 4 & 1 \\
\hline Circuit Protection Systems & 1 & 2 & 5 & & \\
\hline Fuses & 1 & 2 & 5 & 1 & \\
\hline Heaters & 1 & 2 & 6 & & \\
\hline Switches & 1 & 2 & 7 & & \\
\hline
\end{tabular}

*Code numbers left adjusted beginning with Column 22 . 
A. Faults or actions which place the reactor in a dangerous state.

110

$1 \quad 1 \quad 1$

A. 1. I Faults or actions resulting in significant hazard or damage to plant and injury to personnel.

$1 \quad 1 \quad 1 \quad 1$

112

to plant or personnel by circumstances outside those envisaged in the equipment design.

113

to plant or personnel by the ultimate available protection.

B. Fiaults or actions which reduce reactor safety.

B. 1 Failure to operate of any trip equipment which reduces reactor safety.

120

121

B. 1.1 Failure to operate of any trip equipment which reduces reactor safety and results in personnel hazard.

$\begin{array}{llll}1 & 2 & 1 & 1\end{array}$

122 equipment (other than trip equipment) which reduces reactor safety.

B. 2.1 Faults or actions affecting the correct functioning of important plant equipment (other than trip equipment) which reduces reactor safety and results in personnel hazard.

B. 3 Incorrect adjustment of plant trip levels which reduces reactor safety.

$\begin{array}{llll}1 & 2 & 2 & 1\end{array}$

123

B. 3. 1 Incorrect adjustment of plant trip levels with the result that the reactor actually trips at a level above the correct value, reducing reactor safety and resulting in personnel hazard.

$\begin{array}{llll}1 & 2 & 3 & 1\end{array}$

$\begin{array}{lll}1 & 2 & 4\end{array}$

have occurred so frequently as to raise doubts on the safety of the system. whether mechanical or human.

125

$1 \quad 2 \quad 5 \quad 1$

20

21

22

$\begin{array}{lll}2 & 2 & 1\end{array}$

$\begin{array}{lll}2 & 2 & 2\end{array}$

23

$\begin{array}{lll}2 & 3 & 1\end{array}$

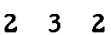

24

25

26

27

28

29

C. 9 Unexpected activity levels.

3

D. Events which have no safety significance whatsoever.

* Code numbers left adjusted beginning with Column 28. 
TABLE B. 5

PARTIAL LIST OF FAULT TYPES*

\begin{tabular}{|c|c|c|c|c|}
\hline Electrical & 1 & & & \\
\hline Mains Supply & 1 & 1 & & \\
\hline Failure & 1 & 1 & 1 & \\
\hline Local to Component & 1 & 1 & 1 & 1 \\
\hline Surge Effects & 1 & 1 & 2 & \\
\hline Voltage Variations & 1 & 1 & 2 & 1 \\
\hline Synch ronization Faults & 1 & 1 & 3 & \\
\hline Standby Supply & 1 & 2 & & \\
\hline Failure & 1 & 2 & 1 & \\
\hline Surge Effects & 1 & 2 & 2 & \\
\hline Synch ronization Faults & 1 & 2 & 3 & \\
\hline Experimental Facilities and Rigs Supply & 1 & 3 & & \\
\hline Failure & 1 & 3 & 1 & \\
\hline Surge Effects & 1 & 3 & 2 & \\
\hline Fault on Component (or Plant) & 1 & 4 & & \\
\hline Failure & 1 & 4 & 1 & \\
\hline Defective & 1 & 4 & 2 & \\
\hline Overheating & 1 & 4 & 2 & 1 \\
\hline A rcing & 1 & 4 & 2 & 2 \\
\hline Short Circuit & 1 & 4 & 2 & 3 \\
\hline Open Circuit & 1 & 4 & 2 & 4 \\
\hline Faulty Operation & 1 & 4 & 2 & 5 \\
\hline No Operation & 1 & 4 & 2 & 6 \\
\hline Reduced Performance & 1 & 4 & 2 & 7 \\
\hline Insulation Breakdown & 1 & 4 & 2 & 8 \\
\hline Adjustment & 1 & 4 & 2 & 9 \\
\hline Incorrect Item & 1 & 4 & 3 & \\
\hline Blown Fuse & 1 & 4 & 4 & \\
\hline Damaged & 1 & 4 & 5 & \\
\hline Timing and Sequence Faults & 1 & 4 & 6 & \\
\hline Earth Faults & 1 & 4 & 7 & \\
\hline Wiring & 1 & 5 & & \\
\hline Defective & 1 & 5 & 1 & \\
\hline Dry Joints & 1 & 5 & 1 & 1 \\
\hline Lack of Continuity & 1 & 5 & 1 & 2 \\
\hline Damaged & 1 & 5 & 1 & 3 \\
\hline Incorrect Item & 1 & 5 & 2 & \\
\hline Incorrect Layout & 1 & 5 & 3 & \\
\hline Faulty Connections & 1 & 5 & 4 & \\
\hline Circuit Faults & 1 & 6 & & \\
\hline Assembly Faults & 1 & 7 & & \\
\hline Faults Causing Spurious Operation & 1 & 8 & & \\
\hline Control Systems and Instrumentation & 1 & 8 & 1 & \\
\hline Mains Interference & 1 & 8 & 1 & 1 \\
\hline Electrical Pickup & 1 & 8 & 1 & 2 \\
\hline Earth Faults & 1 & 8 & 1 & 3 \\
\hline Faulty Connections & 1 & 8 & 1 & 4 \\
\hline Others & $i$ & 8 & 2 & \\
\hline
\end{tabular}

*Code numbers left adjusted beginning with Column 32 . 
TABLE B. 6

EFFECTS*

\begin{tabular}{|lll|}
\hline Reactor Power Effects & 1 & 1 \\
Fluctuations & 1 & 1 \\
Overshoot & 1 & 3 \\
Reduction & 2 & \\
Fuel Handling Effects & 2 & 1 \\
Element Dropped & 2 & 2 \\
Element Lowered Inadvertently & 2 & 3 \\
Element Raised Inadvertently & 3 \\
Damage to Adjacent Plant or Equipment & 4 \\
Fuel Defects & 4 & 1 \\
Canning Failures & 4 & 2 \\
Fitment Failures & 4 & 3 \\
Element Bowing & 5 \\
Reactor Pressure Circuit Effects & 51 \\
Depressurization & 1 \\
\hline
\end{tabular}

*Code numbers left adjusted beginning with Column 37. 
TABLE B. 7

\section{MISCELLANEOUS COLUMN ALLOCATIONS*}

\section{Power}

M (Megawatts)

$\mathrm{K}$ (Kilowatts)

W (Watts)
Whether

Poisoned Out

P (Poisoned)
0

1

\section{Method of Trip}

Auto Trip

Manual Trip

Control Shutdown

No Shutdown

No Startup

Controlled Power, Reduction

Power Setback

Moderator Dumped

Moderator Partially Dumped

\begin{tabular}{l}
0 \\
1 \\
2 \\
3 \\
\hline
\end{tabular}

Reactor State

\begin{tabular}{|ll|}
\hline 0 & 0 \\
0 & 1 \\
0 & 2 \\
0 & 3 \\
0 & 4 \\
0 & 5 \\
0 & 6 \\
0 & 7 \\
0 & 8 \\
0 & 9 \\
\hline
\end{tabular}

Warning Only

Steady

Full Power

Raising Power

Startup

бK's (Low Power)

Subcritical

Experimental Run

Shutdown

Critical

Reducing Power

Being Shutdown

Partial Dumped

\begin{tabular}{|ll|}
\hline 0 & 0 \\
0 & 1 \\
0 & 2 \\
0 & 3 \\
0 & 4 \\
0 & 5 \\
0 & 6 \\
0 & 7 \\
0 & 8 \\
0 & 9 \\
1 & 0 \\
1 & 1 \\
1 & 2 \\
1 & 3 \\
\hline
\end{tabular}

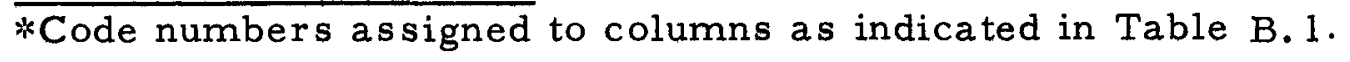


TABLE B.8

PM-1 MALFUNCTION PACKET CARD NO. 2

\begin{tabular}{|c|c|}
\hline Data & Column Allocation \\
\hline Packet Card Number & $1-2$ \\
\hline Malfunction Packet Identification & $3-4$ \\
\hline Reactor Identification & $5-9$ \\
\hline Code Number & $10-11$ \\
\hline Day & $12-13$ \\
\hline Month & $14-15$ \\
\hline Year & $16-17$ \\
\hline Subsystem Code & $18-20$ \\
\hline Component Location & $21-22$ \\
\hline Component Code & $23-27$ \\
\hline Time of Occurrence or Discovery & $28-31$ \\
\hline Repair Time, Hours & $32-35$ \\
\hline Time Generator Off, Hours & $36-39$ \\
\hline Time Reactor Not Critical, Hours & $40-43$ \\
\hline Malfunction Code & 44 \\
\hline Percent Power Cutback & $45-46$ \\
\hline Safety Code & 47 \\
\hline Shutdown Code & 48 \\
\hline Description of Shutdown & $49-78$ \\
\hline Packet Card Number & $79-80$ \\
\hline
\end{tabular}


TABLE B. 9

MALFUNCTION PACKET CARD NO. 3

\begin{tabular}{|l|r|}
\hline \multicolumn{1}{|c|}{ Data } & Column Allocation \\
\hline Packet Card Number & $1-2$ \\
Same as Card No. 2 & $3-17$ \\
Cost of Repair, Dollars & $18-23$ \\
Cost of Repair by Outside Contract, Dollars & $24-29$ \\
Failure Code & $30-31$ \\
Description of Failure Code & $32-78$ \\
Packet Card Number & $79-80$ \\
\hline
\end{tabular}


TABLE B. 10

PARTIAL LIST OF SUBSYSTEM AND COMPONENT CODES

\section{SUBSYSTEM CODES}

CA Coolant Chemical Addition

CC Coolant Charging System

CP Coolant Purification System

CW Cooling Water System

DB Decontamination Building and Equipment

DC Vital AC and DC Systems

DH Decay Heat Removal System

DV Coolant Discharge and Vent System

EP Emergency Power System

ES Extraction Steam System

FC Fuel Cask and Cooling System

FP Fire Protection and Alarm System

FW Feedwater System

HC Reactor Plant Heating and Cooling System

HV Heating, Air Conditioning, and Ventilating Systems

IA Instrument Air System

LS Lighting Distribution System

MC Main Condenser and Condensate System

MS Main and Auxiliary Steam System

COMPONENT CODES

$A B$ Auxiliary Boiler

AC Air Conditioner

AD Air Dryer

AE Air Ejector

AL Alarm

AM Amplifier

AN Annunciator

AS Air Sampler

AU Auctioneer

BA Battery

BAL Balance
DOR Door

DRY Dryer

DS Desuperheater

EPT Electropneumatic Transducer

EV Evaporator

EXC Exciter

EXT Extinguisher

FA Fan

FGB Blind Flange

FH Fume Hook

FI Filter 
TABLE B. 11

MALFUNCTION, SAFETY, AND SHUTDOWN CODES

\section{MALFUNCTION CODE}

$\begin{array}{ll}01 & \text { Normal Shutdown } \\ 02 & \text { Scram } \\ 03 & \text { Power Cutback } \\ 04 & \text { None of the Above }\end{array}$

SAFETY CODES

\begin{tabular}{|c|c|}
\hline 01 & Actual External Release of Radioactivity \\
\hline 02 & Potential External Release of Radioactivity \\
\hline 03 & Actual Internal Release of Radioactivity \\
\hline 04 & Potential Internal Release of Radioactivity \\
\hline 05 & Actual Inadvertent Increase in Reactor Reactivity \\
\hline 06 & Potential Increase in Reactor Reactivity \\
\hline 07 & No Effect \\
\hline
\end{tabular}

\section{SHUTDOWN CODES}

$\begin{array}{ll}01 & \text { Schedule for Maintenance } \\ 02 & \text { Schedule for Training or Demonstration } \\ 03 & \text { Schedule for Test } \\ 04 & \text { Refueling } \\ 05 & \text { Component Malfunction } \\ 06 & \text { Operator Error } \\ 07 & \text { Not Used } \\ 08 & \text { Other }\end{array}$




\section{TABLE B. 12}

\section{FAILURE CODES}

\begin{tabular}{|c|c|c|c|c|c|}
\hline & Mechanical & & Electrical & & Instrumentation \\
\hline 01 & Leak External & 21 & Short Circuit & 41 & Electronic Tube Failure \\
\hline 02 & Leak Internal & 22 & Open Circuit & 42 & Transistor Failure \\
\hline 03 & Rupture or Crack External & 23 & Ground & 43 & Geiger Mueller Tube \\
\hline 04 & Rupture or Crack Internal & 24 & Insulation Failure & 44 & BF3 Chamber Failure \\
\hline 05 & Bearing Failure & 25 & Brush Failure & 45 & Ion Chamber Failure \\
\hline 06 & $\mathrm{Clog}$ & 26 & Loss of Sensitivity & 46 & Alignment or Calibration \\
\hline 07 & Jammed or Stuck & 27 & Diode Failure & 47 & Connectors--Terminal Fail \\
\hline 08 & Gasket Failure & 28 & Relay Failure & 48 & Resistor Failure \\
\hline 09 & Mechanical Linkage Failure & 29 & Overload Heater & 49 & Power Supply Failure \\
\hline 10 & Packing & 30 & Coil Failure & 50 & Cable-Radiation Damage \\
\hline 11 & Seal Failure & 31 & Contact Failure & 51 & Controller--M62 \\
\hline 12 & Corrosion & 32 & Switch Failure & 52 & Capacitor Failure \\
\hline 13 & Valve Seats & 33 & Overload & 53 & D-P Cell Failure \\
\hline 14 & Score--Stem or Shaft & 34 & Physical Damage & 54 & Transformer \\
\hline 15 & Tube Failure & 35 & Power Cable & 55 & Drive or Torque Motors \\
\hline 16 & Lagging & 36 & Control Cable & 56 & Clean and Adjusted \\
\hline 17 & Adjustment and Clean & 37 & & 57 & Synchro-Dr. or Rec. \\
\hline 18 & Piping Failure & 38 & & 58 & Control Cable \\
\hline 19 & & 39 & & 59 & \\
\hline \multirow[t]{7}{*}{20} & Mechanical--Other & 40 & Electrical--Other & 60 & Instrumentation--Other \\
\hline & & & & \multicolumn{2}{|c|}{ Others } \\
\hline & & & & \multicolumn{2}{|l|}{61} \\
\hline & & & & \multicolumn{2}{|l|}{62} \\
\hline & & & & \multicolumn{2}{|l|}{63} \\
\hline & & & & \multicolumn{2}{|l|}{64} \\
\hline & & & & \multicolumn{2}{|l|}{65} \\
\hline
\end{tabular}


TABLE B. 13

LEVELS OF MARAD CLASSIFICATION

\begin{tabular}{|c|c|c|c|c|}
\hline LEVEL & DEFINITION & \multicolumn{3}{|c|}{ EXAMPLES $\begin{array}{c}\text { (Showing functional rela- } \\
\text { tions in the hie rarchy) }\end{array}$} \\
\hline $\begin{array}{l}\text { 1. Identification/ } \\
\text { Design }\end{array}$ & $\begin{array}{l}\text { Identification of the ship and characte rization } \\
\text { of the design. }\end{array}$ & \multicolumn{3}{|c|}{ MARAD Design Number } \\
\hline 2. Environment & Characterization of the ship's environment. & \multicolumn{3}{|c|}{ Trade Route } \\
\hline 3. System & $\begin{array}{l}\text { Collection of subsystems and/or units which } \\
\text { together perform a major function of the ship. }\end{array}$ & \multicolumn{3}{|c|}{ Power Supply and Propulsion } \\
\hline 4. Subsystem & $\begin{array}{l}\text { Collection of related units and components } \\
\text { which perform a major subfunction of the } \\
\text { system. }\end{array}$ & \multicolumn{2}{|c|}{ Steam } & Drive \\
\hline 5. Unit & $\begin{array}{l}\text { Set of one or more highly related components } \\
\text { and assemblies which are a major portion of } \\
\text { a subsystem. }\end{array}$ & \multicolumn{2}{|c|}{ Steam Supply } & Steam Expansion \\
\hline 6. Component & $\begin{array}{l}\text { Piece of equipment which performs some } \\
\text { specific basic operation. }\end{array}$ & Boiler & $\begin{array}{c}\text { Main Steam } \\
\text { Piping }\end{array}$ & $\begin{array}{l}\text { High Pressure } \\
\text { Drive Turbine }\end{array}$ \\
\hline 7. Assembly & $\begin{array}{l}\text { Set of subassemblies and/or parts which is a } \\
\text { major part of a component and which is usually } \\
\text { replaceable. }\end{array}$ & $\begin{array}{l}\text { Steam } \\
\text { Drum }\end{array}$ & Valves & Rotor \\
\hline 8. Subassembly & $\begin{array}{l}\text { Connected set of parts which is a removable } \\
\text { portion of an as sembly. }\end{array}$ & & $\begin{array}{l}\text { Main Steam } \\
\text { Sțop Valve }\end{array}$ & \\
\hline 9. Part & $\begin{array}{l}\text { Basic element which ordinarily cannot be } \\
\text { further disassembled. }\end{array}$ & Drum & $\begin{array}{l}\text { Stop Valve } \\
\text { Body }\end{array}$ & Blade \\
\hline 10. Subpart & Specific characteristic portion of a part. & & $\begin{array}{l}\text { Inlet } \\
\text { Flange }\end{array}$ & Root \\
\hline
\end{tabular}


TABLE B. 14

SYSTEMS AND SUBSYSTEMS

\begin{tabular}{|c|c|}
\hline $\begin{array}{l}\text { System and } \\
\text { Subsystem } \\
\text { Letter }\end{array}$ & System and Subsystem \\
\hline A & Automatic Control System \\
\hline $\begin{array}{l}\mathrm{C} \\
\mathrm{CB} \\
\mathrm{CC}\end{array}$ & $\begin{array}{l}\text { Communications System } \\
\text { External Communications Subsystem } \\
\text { Internal Communications Subsystem }\end{array}$ \\
\hline $\begin{array}{l}E \\
\text { EB } \\
\text { EC }\end{array}$ & $\begin{array}{l}\text { Electrical System } \\
\text { Generation Subsystem } \\
\text { Distribution Subsystem }\end{array}$ \\
\hline $\mathbf{F}$ & Fuel Oil and Ballast System \\
\hline $\begin{array}{l}\because \\
\mathrm{KB} \\
\mathrm{KC}\end{array}$ & $\begin{array}{l}\text { Cargo System } \\
\text { Dry Cargo Subsystem } \\
\text { Liquid Cargo Subsystem }\end{array}$ \\
\hline $\begin{array}{l}L \\
L B \\
L C\end{array}$ & $\begin{array}{l}\text { Life Support System } \\
\text { Accommodation Subsystem } \\
\text { Life Saving Subsystem }\end{array}$ \\
\hline M & Mooring System \\
\hline $\begin{array}{l}\mathrm{N} \\
\mathrm{NB} \\
\mathrm{NC}\end{array}$ & $\begin{array}{l}\text { Navigation System } \\
\text { Position Subsystem } \\
\text { Safety Subsystem }\end{array}$ \\
\hline $\begin{array}{l}\text { P } \\
\text { PB } \\
\text { PC }\end{array}$ & $\begin{array}{l}\text { Power and Propulsion System } \\
\text { Steam Subsystem } \\
\text { Drive Subsystem }\end{array}$ \\
\hline $\begin{array}{l}\text { R } \\
\text { RB } \\
\text { RC } \\
\text { RD }\end{array}$ & $\begin{array}{l}\text { Preservation and Repair System } \\
\text { Damage Control Subsystem } \\
\text { Maintenance and Repair Subsystem } \\
\text { Protection Subsystem }\end{array}$ \\
\hline $\begin{array}{l}S \\
S B \\
\text { SC }\end{array}$ & $\begin{array}{l}\text { Steering System } \\
\text { Main Stee } r \text { ing Subsystem } \\
\text { Auxiliary Steering Subsystem }\end{array}$ \\
\hline $\mathrm{T}$ & Structural System \\
\hline $\begin{array}{l}\mathrm{V} \\
\mathrm{VP} \\
\mathrm{VC} \\
\mathrm{VD} \\
\mathrm{VE}\end{array}$ & $\begin{array}{c}\text { Environmental Conditioning System } \\
\text { Accommodations Subsystem } \\
\text { Machinery Spaces Subsystem } \\
\text { Cargo Spaces Subsystem } \\
\text { Refrigeration Spaces Subsystem }\end{array}$ \\
\hline $\begin{array}{l}\text { W } \\
\text { WB } \\
\text { WC }\end{array}$ & $\begin{array}{l}\text { Water System } \\
\text { Freshwater Subsystem } \\
\text { Saltwater Subsystem }\end{array}$ \\
\hline
\end{tabular}


TABLE B. 15

COMPONENT AND ASSEMBLY LIST

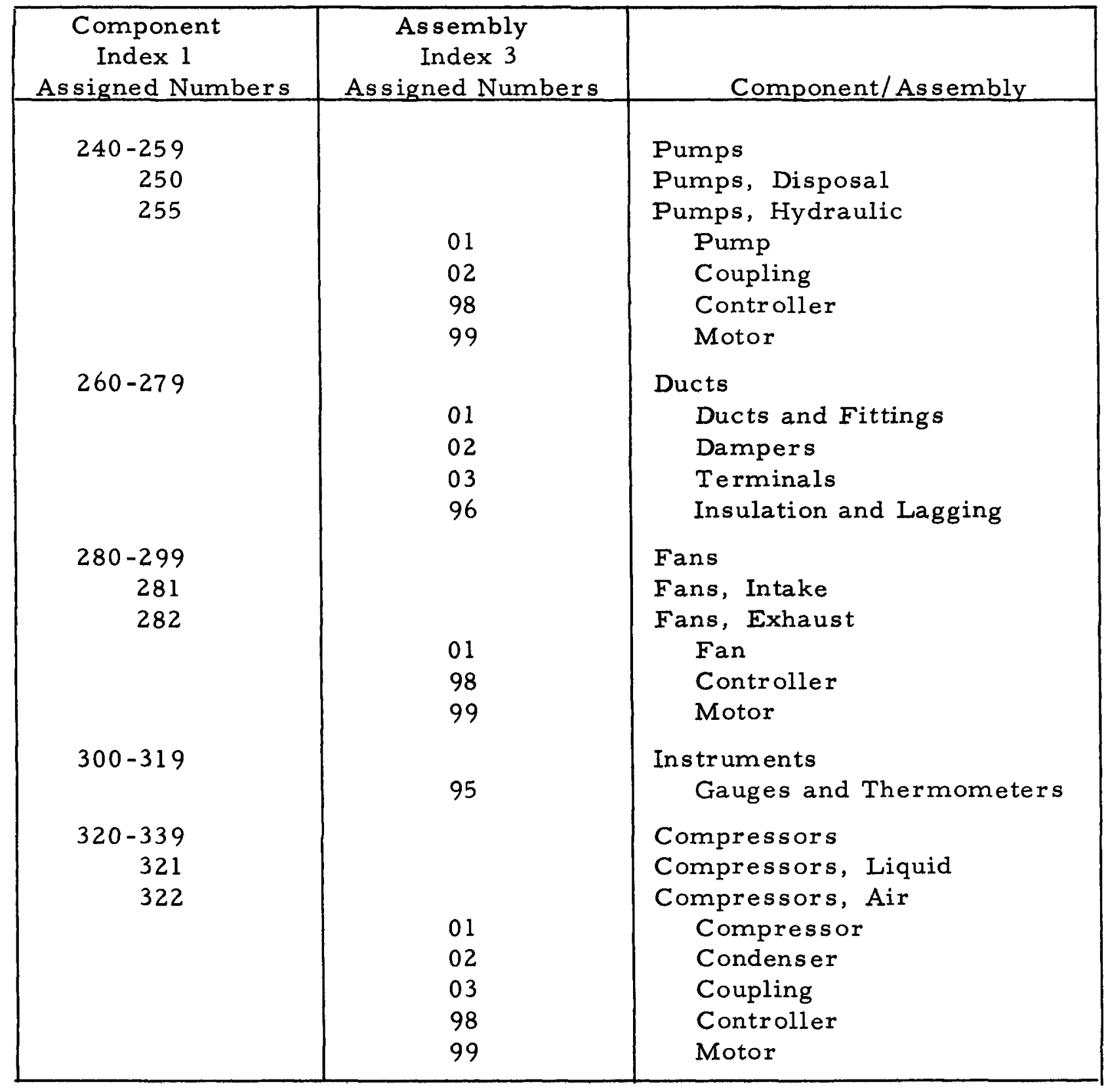


TABLE B. 16

COMPONENT TYPE LIST (INDEX 2)

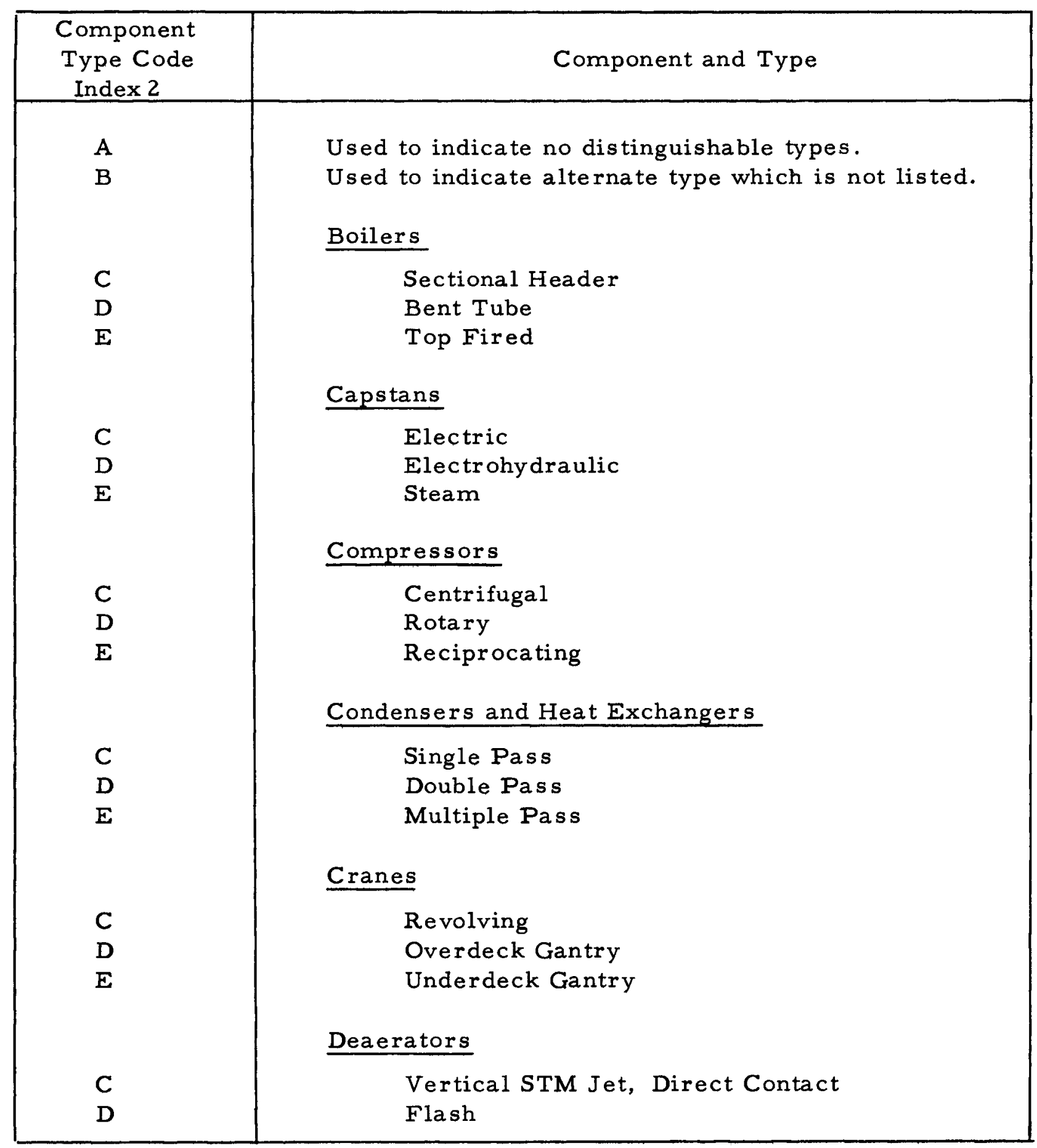


TABLE B. 17

EEI - TYPE OF OUTAGE

\begin{tabular}{|c|c|c|}
\hline $\begin{array}{c}\text { Code } \\
\text { No. }\end{array}$ & Type of Outage & Description \\
\hline 1 & Forced Outage & $\begin{array}{l}\text { An outage which requires a major piece } \\
\text { of equipment be taken out of service. }\end{array}$ \\
\hline 2 & Scheduled Shutdown & $\begin{array}{l}\text { An outage which can be planned in } \\
\text { advance or for which the starting date } \\
\text { is controllable beyond the weekend of } \\
\text { the week during which component } \\
\text { trouble occurred. }\end{array}$ \\
\hline 3 & Reserve Shutdown & $\begin{array}{l}\text { Shutdown in which the complete unit } \\
\text { or its turbine, generator, or boiler } \\
\text { could be operated, but is placed on } \\
\text { reserve because of fuel economy or } \\
\text { any other reason. }\end{array}$ \\
\hline 4 & Not Used At Present & \\
\hline 5 & $\begin{array}{l}\text { Forced Partial Out- } \\
\text { age }\end{array}$ & $\begin{array}{l}\text { A reduction in maximum capacity } \\
\text { available to the system of } 30 \mathrm{Mw} \text { or } \\
\text { more lasting for } 1 \text { hour or more. }\end{array}$ \\
\hline 6 & $\begin{array}{l}\text { Scheduled Partial } \\
\text { Outage }\end{array}$ & $\begin{array}{l}\text { Partial outage which, by definition, } \\
\text { not a forced partial outage. }\end{array}$ \\
\hline
\end{tabular}


TABLE B. 18

FAILURE TYPE

\begin{tabular}{|c|c|c|c|}
\hline \multicolumn{4}{|c|}{ BOILERS } \\
\hline$\underline{\text { Code }}$ & & $\underline{\text { Code }}$ & \\
\hline 100 & Boiler & 125 & Acid Cleaning \\
\hline 101 & Water Walls & 126 & Boiler Casing, Breeching and Ducts \\
\hline 102 & Generating Tubes & 127 & Soot Blowers \\
\hline 103 & Supe rheater & 128 & Boiler Circulating Pumps \\
\hline 104 & Reheater - First & 129 & \\
\hline 105 & Reheater - Second & 130 & Precipitator - Electrical \\
\hline 106 & Economizer & 131 & Precipitator - Mechanical \\
\hline 107 & Air Preheater - Tubular & 132 & Burners (including cyclones) \\
\hline 108 & Air Preheater - Rn: erative & 133 & Demineralizer \\
\hline 109 & Induced Draft Fans & 134 & Boiler Control** \\
\hline 110 & Forced Draft Fans & 135 & Miscellaneous \\
\hline 111 & Recirculating Fans & 136 & Furnace Slagging \\
\hline 112 & Desuperheaters and Attemperators & 137 & Superheater Fouling \\
\hline 113 & Bypass Dampers & 138 & Reheater Fouling \\
\hline 114 & Furnace Refractory & 139 & Air Heate $r$ Fouling \\
\hline 115 & Safety Valves & 140 & Induced Draft Fan Fouling \\
\hline 116 & Steam Valves and Piping & 141 & Precipitator Fouling \\
\hline \multirow[t]{2}{*}{117} & Valves and Piping - Feedwater and & 142 & Wet Coal \\
\hline & Blowdown & 143 & Poor Quality Coal (low BTU) \\
\hline 118 & Gage Glasses & 144 & Boiler Water Condition (silica control) \\
\hline 119 & Slag and Fly Ash Disposal System & 145 & Pulverizer Capacity Limited (due to \\
\hline 120 & Stack & & wear or outages) \\
\hline 121 & Pulverizers & 146 & Ashpit Trouble \\
\hline 122 & Stokers & 147 & Fly Ash Disposal Trouble \\
\hline 123 & Fuel Handling Equipment (gas-oil-coal) & & \\
\hline 124 & Fireside Cleaning & & \\
\hline \multicolumn{4}{|c|}{ TURBINES } \\
\hline 200 & Turbines & 209 & Wheels or Spindles \\
\hline \multirow[t]{2}{*}{201} & Bolting or Casing Dismantle and & 210 & Buckets or Blades \\
\hline & Reassembly & 211 & Vibration of Turbine Generator Unit* \\
\hline 202 & Governors & 212 & Lube Oil System and Bearings \\
\hline \multirow[t]{2}{*}{203} & Control, Turbine, and Reheat & & (except bearing vibration) \\
\hline & Stop Valves & 213 & Turbine Control $* *$ \\
\hline 204 & Shaft Packing & 214 & Miscellane ous \\
\hline 205 & Nozzles and Nozzle Blocks & 215 & Blade Fouling \\
\hline 206 & Nozzle Bolting (lst Stage) & 216 & Shell Leaks \\
\hline 207 & Diaphragms & 217 & Seal Leaks \\
\hline 208 & Shaft & & \\
\hline
\end{tabular}


TABLE B. 18 (continued)

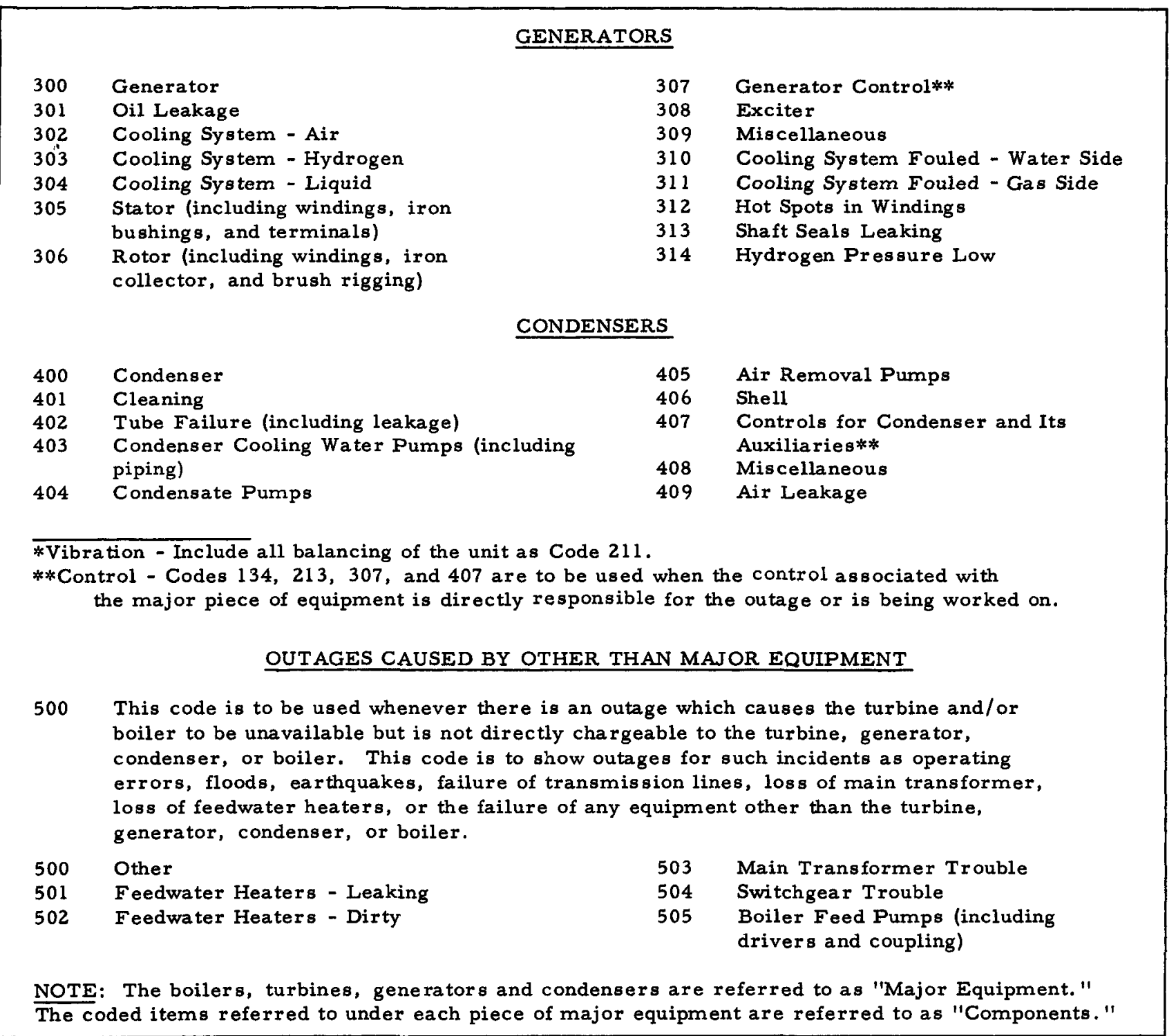


TABLE B. 19

EXAMPLE OF AEC-FPC ACCOUNT NUMBER CORRELATION

\begin{tabular}{|l|c|c|}
\hline \multicolumn{1}{|c|}{ Description } & $\begin{array}{c}\text { AEC } \\
\text { Account } \\
\text { Number }\end{array}$ & $\begin{array}{c}\text { FPC } \\
\text { Account } \\
\text { Number }\end{array}$ \\
\hline Nuclear Production Plant & & \\
\hline Land and Land Rights & 20 & 320 \\
Structures and Improvements & 21 & 321 \\
Reactor Plant Equipment & 22 & 322 \\
Turbine-Generator Units & 23 & 323 \\
Accessory Electric Equipment & 24 & 324 \\
Miscellaneous Power Plant Equipment & 25 & 325 \\
Transmission Plant & & \\
\hline Land and Land Rights & 50 & 350 \\
Clearing Land and Rights-of-Way & 51 & 351 \\
Structures and Improvements & 52 & 352 \\
Station Equipment & 53 & 353 \\
Towers and Fixtures & 54 & 354 \\
Poles and Fixtures & 55 & 355 \\
Overhead Conductors and Devices & 56 & 356 \\
Underground Conduit & 57 & 357 \\
Underground Conductors and Devices & 58 & 358 \\
Roads and Trails & 59 & 359 \\
General Plant & & \\
Communication Equipment & 97 & 397 \\
\hline
\end{tabular}


TABLE B. 20

AEC CLASSIFICATION OF CONSTRUCTION ACCOUNTS NUCLEAR POWER PLANTS

\begin{tabular}{|c|c|c|}
\hline \multicolumn{3}{|c|}{$\begin{array}{l}\text { Account } \\
\text { Number }\end{array}$} \\
\hline & \multicolumn{2}{|c|}{ NUCLEAR PRODUCTION PLANT } \\
\hline \multirow[t]{2}{*}{20} & \multicolumn{2}{|c|}{ Land and Land Rights } \\
\hline & $\begin{array}{l}201 \\
202 \\
203\end{array}$ & $\begin{array}{l}\text { Land and privilege acquisition } \\
\text { Relocating highways and railroads } \\
\text { Relocating telephone and power lines }\end{array}$ \\
\hline \multirow[t]{2}{*}{21} & \multicolumn{2}{|c|}{ Structures and Improvements } \\
\hline & $\begin{array}{l}211 \\
212 \\
218 \\
219\end{array}$ & $\begin{array}{l}\text { Ground improvements } \\
\text { Buildings } \\
\text { Stacks } \\
\text { Reactor containment structure }\end{array}$ \\
\hline \multirow[t]{9}{*}{22} & \multicolumn{2}{|c|}{ Reactor Plant Equipment } \\
\hline & 221 & Reactor equipment \\
\hline & 222 & Heat transfer systems \\
\hline & 223 & Fuel handling and storage equipment \\
\hline & $\begin{array}{l}224 \\
225\end{array}$ & $\begin{array}{l}\text { Fuel processing and fabricating equipment } \\
\text { Radioactive waste treatment and disposal }\end{array}$ \\
\hline & 226 & Instrumentation and control \\
\hline & 227 & Feedwater supply and treatment \\
\hline & 228 & Steam, condensate and feedwater piping \\
\hline & 229 & Other reactor plant equipment \\
\hline
\end{tabular}

23 Turbine-Generator Units

231 Turbine-generators

232 Circulating water systems

233 Condensers

234 Central lubricating system

235 Turbine plant boards, instruments and controls

236 Turbine plant piping

237 Auxiliary equipment for generators

238 Other turbine plant equipment 
TABLE B. 20 (continued)

Account

Number

24 Accessory Electric Equipment

241 Switchgear

242 Switchboards

243 Protective equipment

244 Electrical structures

245 Conduit

246 Power and control wiring

247 Station service equipment

25 Miscellaneous Power Plant Equipment

251 Cranes and hoisting equipment

252 Compressed air and vacuum cleaning systems

253 Other power plant equipment

TRANSMISSION PLANT

$50 \quad$ Land and Land Rights

51 Clearing Land and Rights-of-Way

$52 \quad$ Structures and Improvements

521 General yard improvements

522 Substation buildings

523 Outdoor substation structures

$53 \quad$ Station Equipment

531 Switchgear

532 Protective equipment

533 Main conversion equipment

534 Conduit

535 Power and control wiring

536 Station service equipment 
TABLE B. 20 (continued)

Account

Number

$54 \quad$ Towers and Fixtures

$55 \quad$ Poles and Fixtures

56 Overhead Conductors and Devices

$57 \quad$ Underground Conduit

$58 \quad$ Underground Conductors and Devices

$59 \quad$ Roads and Trails

GENERAL PLANT

97 Communication Equipment

DISTRIBUTIVES

98 Indirect Construction Costs

981 Engineering, design, and inspection

982 General and administrative

983 Other indirect costs

984 Earnings and expenses during construction

985 Interest during construction

99 Miscellaneous Construction Costs

991 Construction inventories

992 Temporary construction facilities

993 Construction equipment and tools

994 Construction clearing accounts

CONTINGENCY 


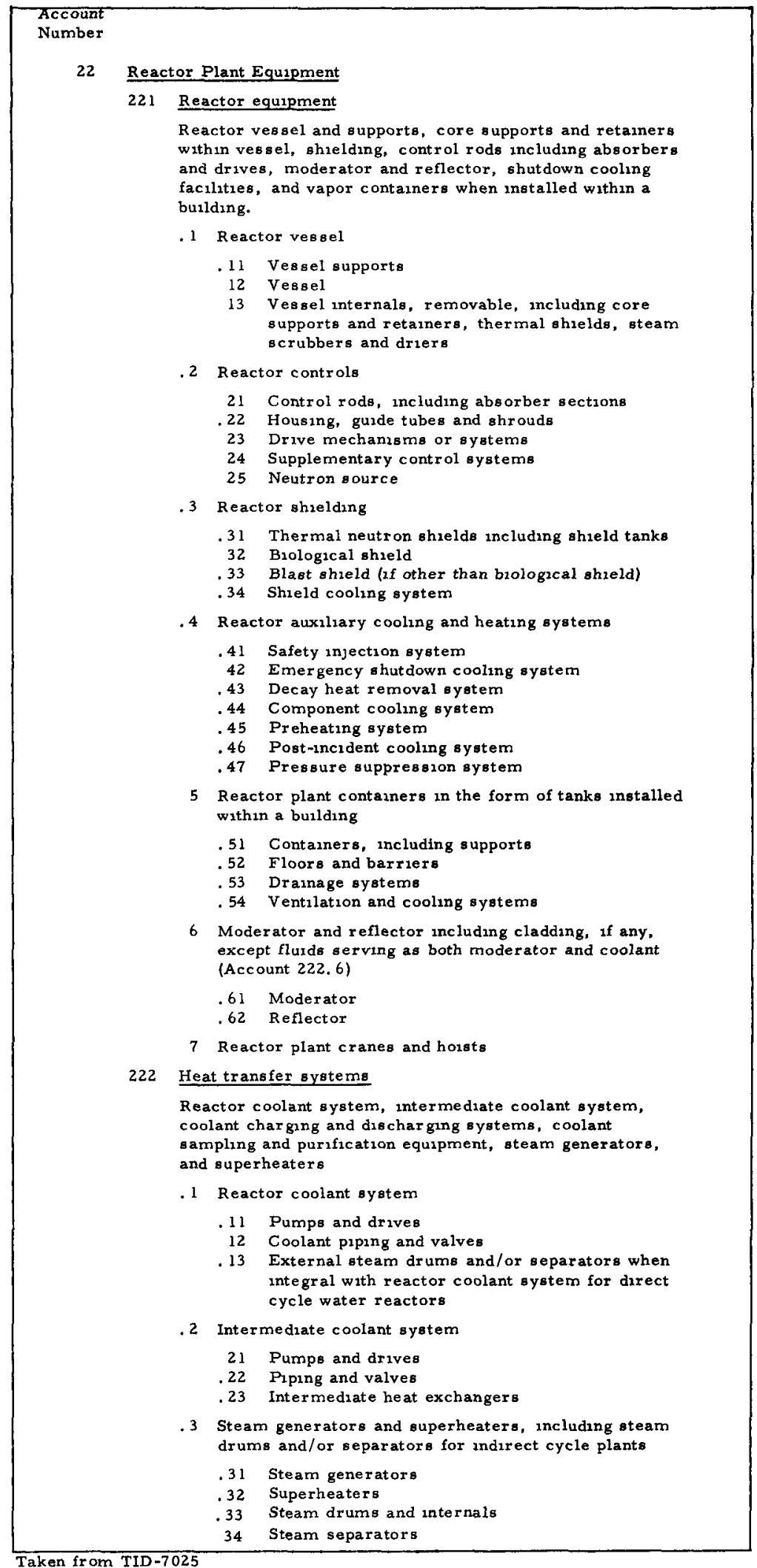




\begin{tabular}{|c|c|c|c|c|c|}
\hline \multicolumn{6}{|c|}{ SAFETY REPORT } \\
\hline \multicolumn{6}{|c|}{ COVERS PERIOD FROM 41163 to 311064} \\
\hline Plant & Date & Component & Fail Type & Shutdown & Safety Hazard \\
\hline PM 1 & 41163 & SUS & 17 & No & 7 \\
\hline PMl & 41163 & SC & 18 & No & 7 \\
\hline PMl & 51163 & MJ & 12 & Yes & 2 \\
\hline PM 1 & 51163 & PP & 11 & No & 7 \\
\hline PMI & 51163 & PP & 11 & No & 7 \\
\hline PM 1 & 51163 & MJ & 12 & Yes & 2 \\
\hline PM 1 & 51163 & MJ & 12 & Yes & 2 \\
\hline PM 1 & 51163 & MJ & 12 & Yes & 2 \\
\hline PMl & 51163 & MJ & 12 & Yes & 2 \\
\hline PM1 & 51163 & $\mathrm{MJ}$ & 12 & Yes & 2 \\
\hline PMl & 61163 & IM & 41 & No & 7 \\
\hline PM 1 & 61163 & LA & 60 & No & 7 \\
\hline PMl & 61163 & LA & 60 & No & 7 \\
\hline PMl & 61163 & SC & 18 & No & 7 \\
\hline PMI & 71163 & $\mathrm{IE}$ & 10 & No & 7 \\
\hline PMl & 81163 & IM & 46 & No & 7 \\
\hline PMl & 91163 & PS & 42 & No & 7 \\
\hline PMl & 91163 & PS & 42 & No & 7 \\
\hline PMl & 91163 & PS & 42 & No & 7 \\
\hline PM 1 & 111163 & VL & 10 & No & 7 \\
\hline PMI & 111163 & VL & 10 & No & 7 \\
\hline PM1 & 111163 & VG & 10 & No & 7 \\
\hline PM1 & 111163 & VG & 10 & No & 7 \\
\hline PMl & 121163 & $\mathrm{AM}$ & 42 & No & 7 \\
\hline PMl & 121163 & $\mathrm{RF}$ & 2 & No & 7 \\
\hline PM 1 & 131163 & IM & 47 & No & 7 \\
\hline PMl & 161163 & $\mathrm{CH}$ & 48 & No & 7 \\
\hline PMl & 181163 & WD & 22 & No & 7 \\
\hline PM1 & 181163 & IM & 49 & No & 7 \\
\hline PMl & 191163 & PHM & 41 & No & 7 \\
\hline PM 1 & 191163 & ND & 50 & No & 7 \\
\hline PM 1 & 201163 & VL & 13 & Yes & 5 \\
\hline PMl & 201163 & VL & 13 & Yes & 5 \\
\hline
\end{tabular}

FIGURE B. 1

SAMPLE SAFETY REPORT 
(ALL TIME IN HOURS)

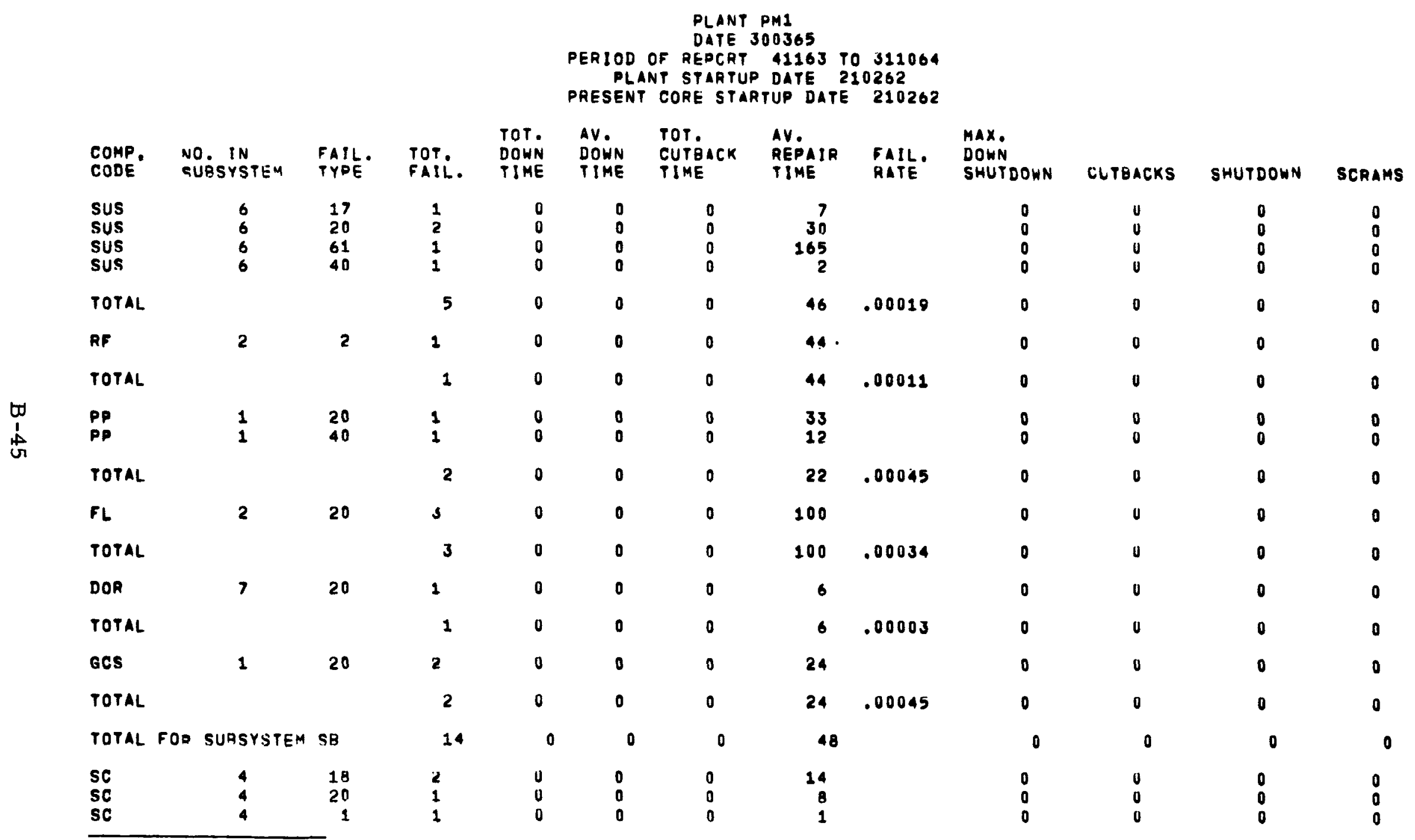

FIGURE B. 2

SAMPLE FAILURE, SHUTDOWN, AND AVAILABILITY REPORT 


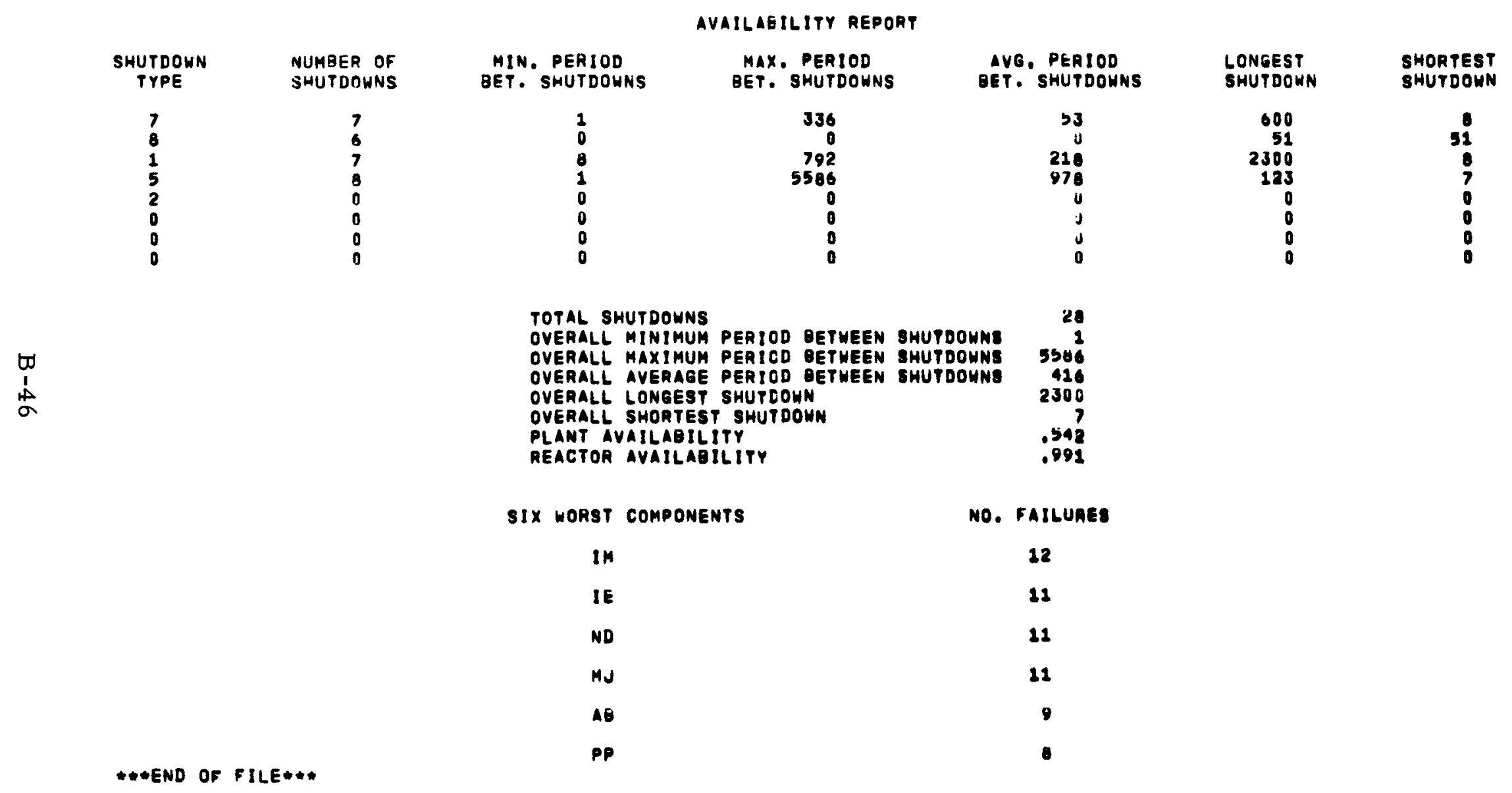

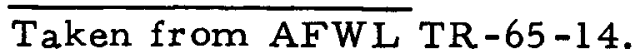


A. SHIP IDENTIFICATION

MAINTENANCE/REPAIM/INSPECTION REPONT

$$
\text { (1) Ship Name. }
$$

(3) Regzentered Holl No.

(O) Vorage No.

Propered by

(4) Job No.

8. COMPONENT/ASSEMBLY IDENTIFICATION

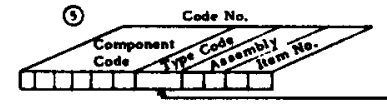

O Componome/anenembly deac ription

(7) Memulecturer_— O Mamelacturer' ' Model or Trpe No.

(9) In Tre code $A$ or B, dencribe tren

C. MANTENANCE, REPAIR, AND RUSPECTON ACTIVTYY DATA

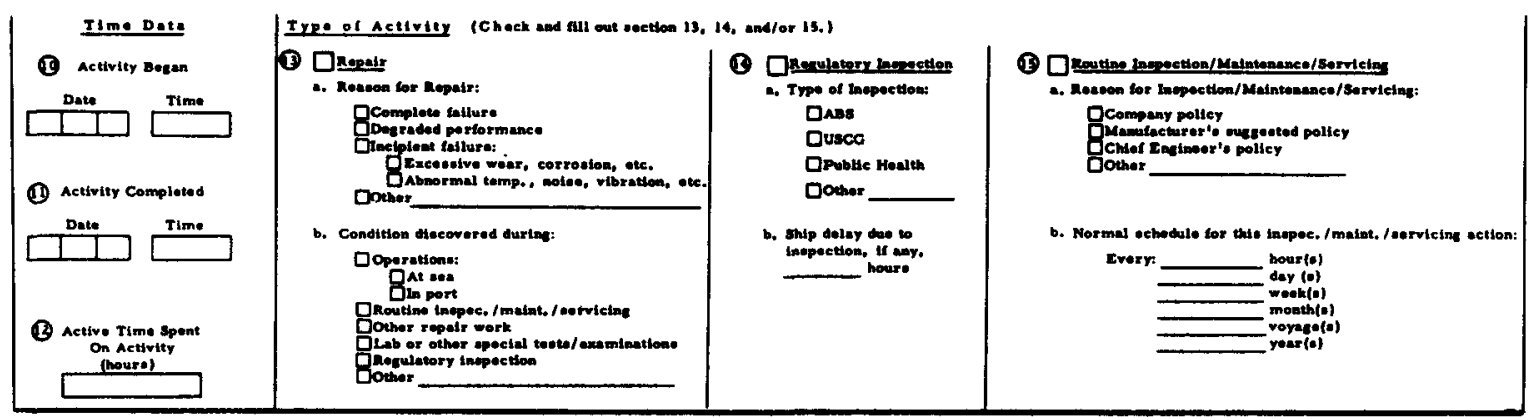

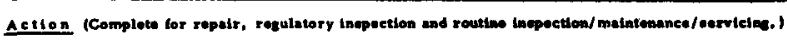
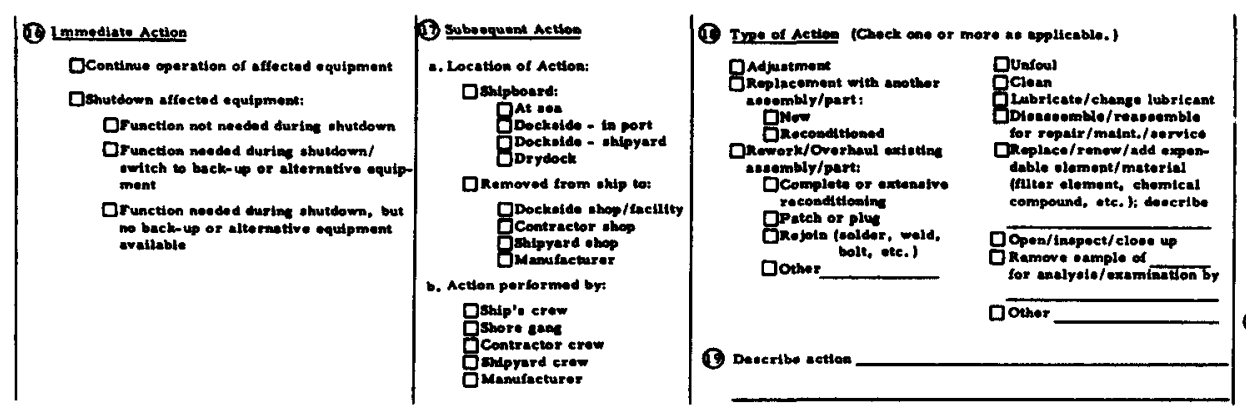

89 Reoult of Actios

No effoct of operation of

T Tomporary totarruption of

sormal optrentoms

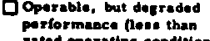
rated operating condition!

Ounsopairable/imoparable

(Mold for arrivel in port
or ehipyerd)

QTomporary ropeis

Opormenent repair

(3) What unie setion a follow-up on a 口No

Q Ye: (Job No

(20) what if the related preventive maintenance policy, Uf eny'

(3) What is the related inopection schedule?

D. FAlLURE DATA (Complote for repair aetivitin, only.)

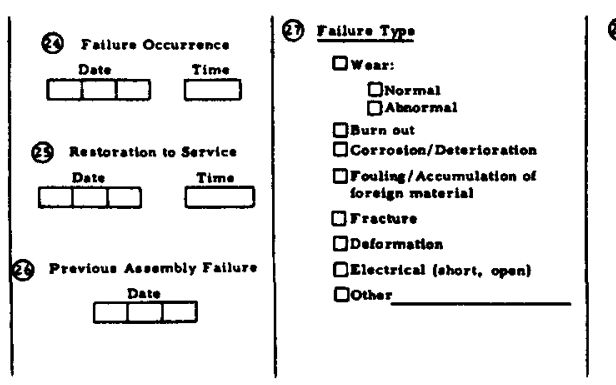

(1) retlure Canes

ONormal ue.

Qimproper

Oprosture busldeup

DEnrironmental oxponuse

Droresen sbrantve material

Dinsdequate proventive malinOLack of lube

$\square$ improper previouse repaly!

DDeresen

DDarmage by

Manulacturer'e defect

QPoor dectigs
(3) Effoct on Syaterm

D None

D Reduced to hell

DRudued to quarter

口Totel loso of function

(2)

(3) Describe tellure

(2) Deecribe fallure cause
(3) Effoct on Ship'e Proyrant:

$\square$ None

Delayed (in port

Q Roduced epeed (at oen)

How lone?

0 Dead-herema-water

E. COMMENTS/RECOMMENDATIONS

FIGURE B. 3

SAMPLE SHIPBOARD DATA COLLECTION FORM

(First of Two Sheets) 
F. COST DATA

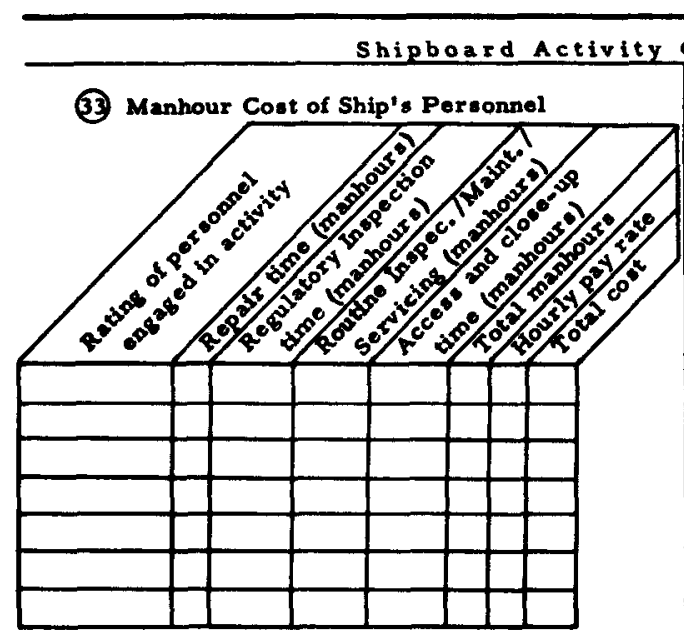

(35) Additional Conts or Fees (not covered above)
Description
Cost

$\overline{ }$
Shoreside Activity Cost

\section{(26) Labor Cost}

Cost due to access, close-up Cost for actual repair

Total of above, if not

broken down

37 Material Cost

Description of Part

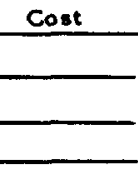

38 Ressen for using shoreside facility:

FIGURE B. 4

SAMPLE SHIPBOARD DATA COLLECTION FORM

(Second of Two Sheets) 


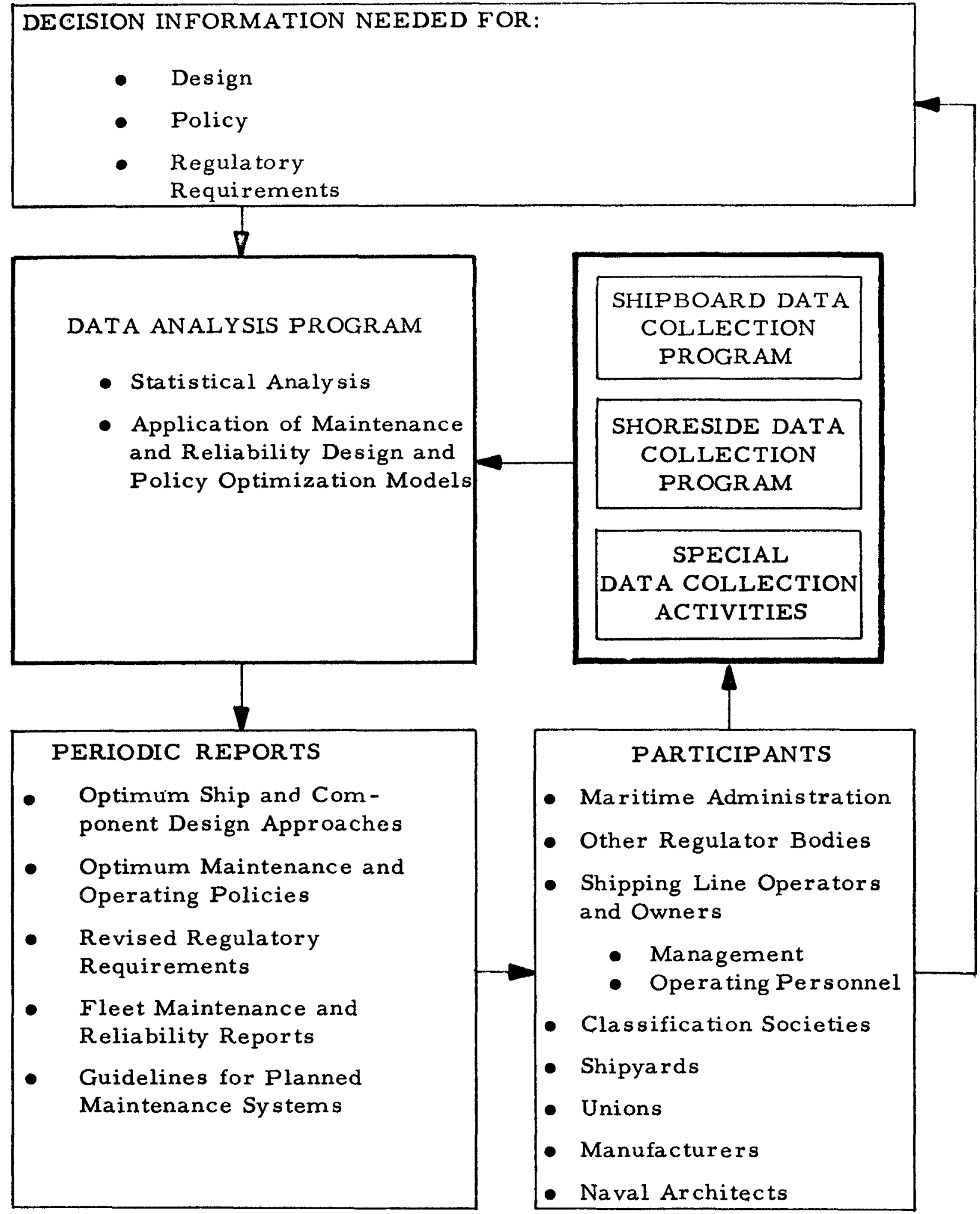

FIGURE B. 5

MARAD MAINTENANCE AND RELIABILITY PROGRAM 
A. FORCED OUTAGES

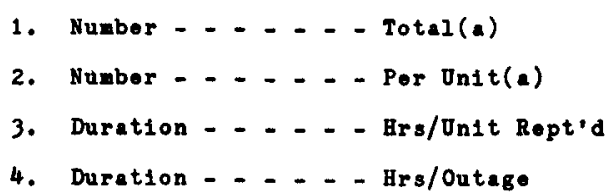

$\begin{array}{rrr}\text { Boiler } & \text { Turbine } & \text { Genorator } \\ 1,415 & 229 & 75 \\ 3.2 & 0.5 & 0.2 \\ 156 & 36 & 19 \\ 48 & 69 & 111\end{array}$

B. MAINTENANCE
5. Manhours (Total)
Por Onit(b)
6. Manhours (Forced)
Per Onft(b)

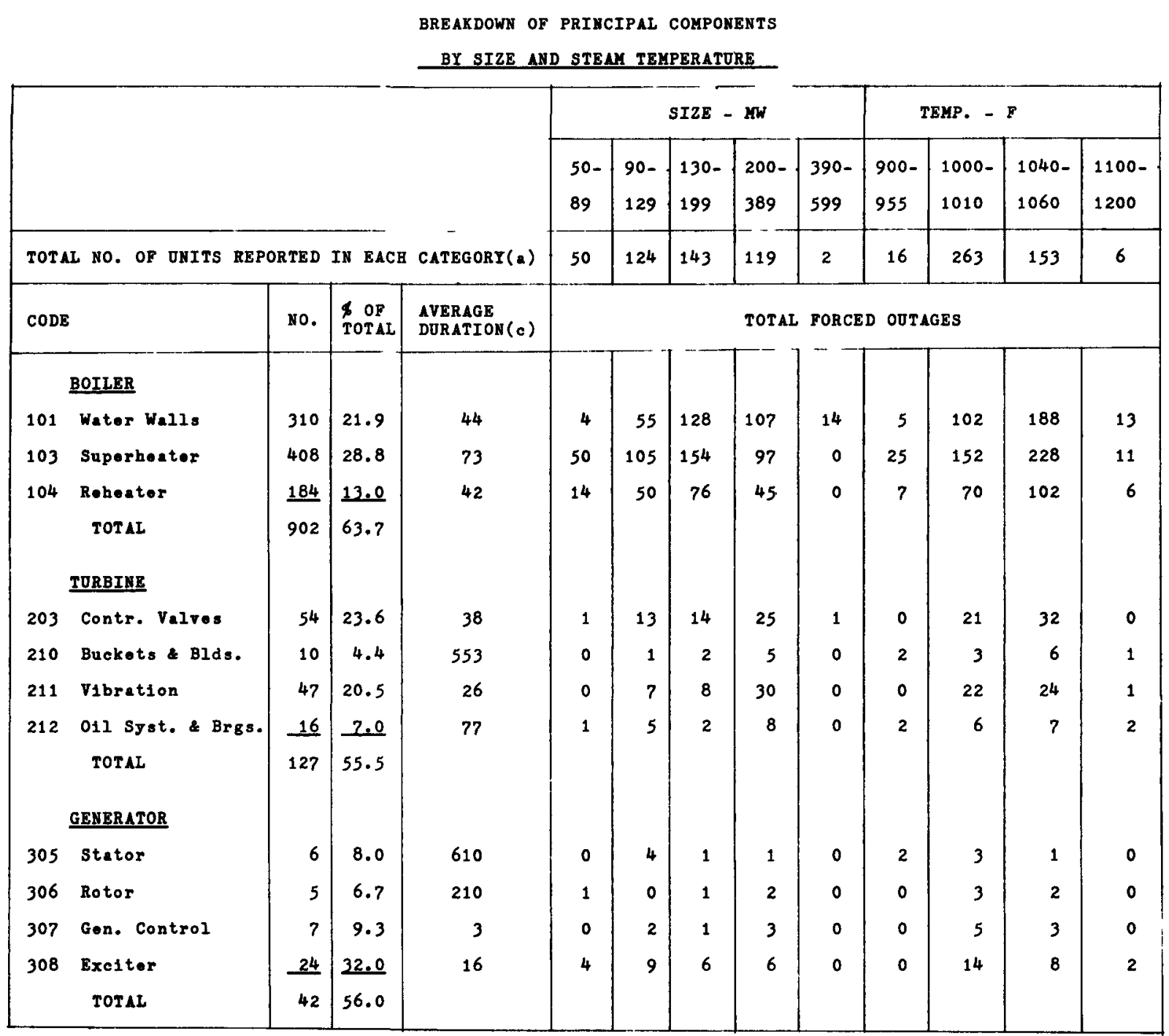

NoTES:

(a) Based on total of 438 units reported.

(b) Based on totel of 371 units for which manhours wore reported.

(c) Total foreed outago hours for eech conponent divided by the number of outeges.

Taken from EEI No. 65-35 "Report on Equipment Availability for the Five-Year Period 1960-1964," Edison Electric Institute, May 1965.

FIGURE B. 6

ANALYSIS OF COMPONENT FORCED OUTAGES FOR THE YEAR 1964 CONVENTIONAL DRUM TYPE UNITS ONLY 


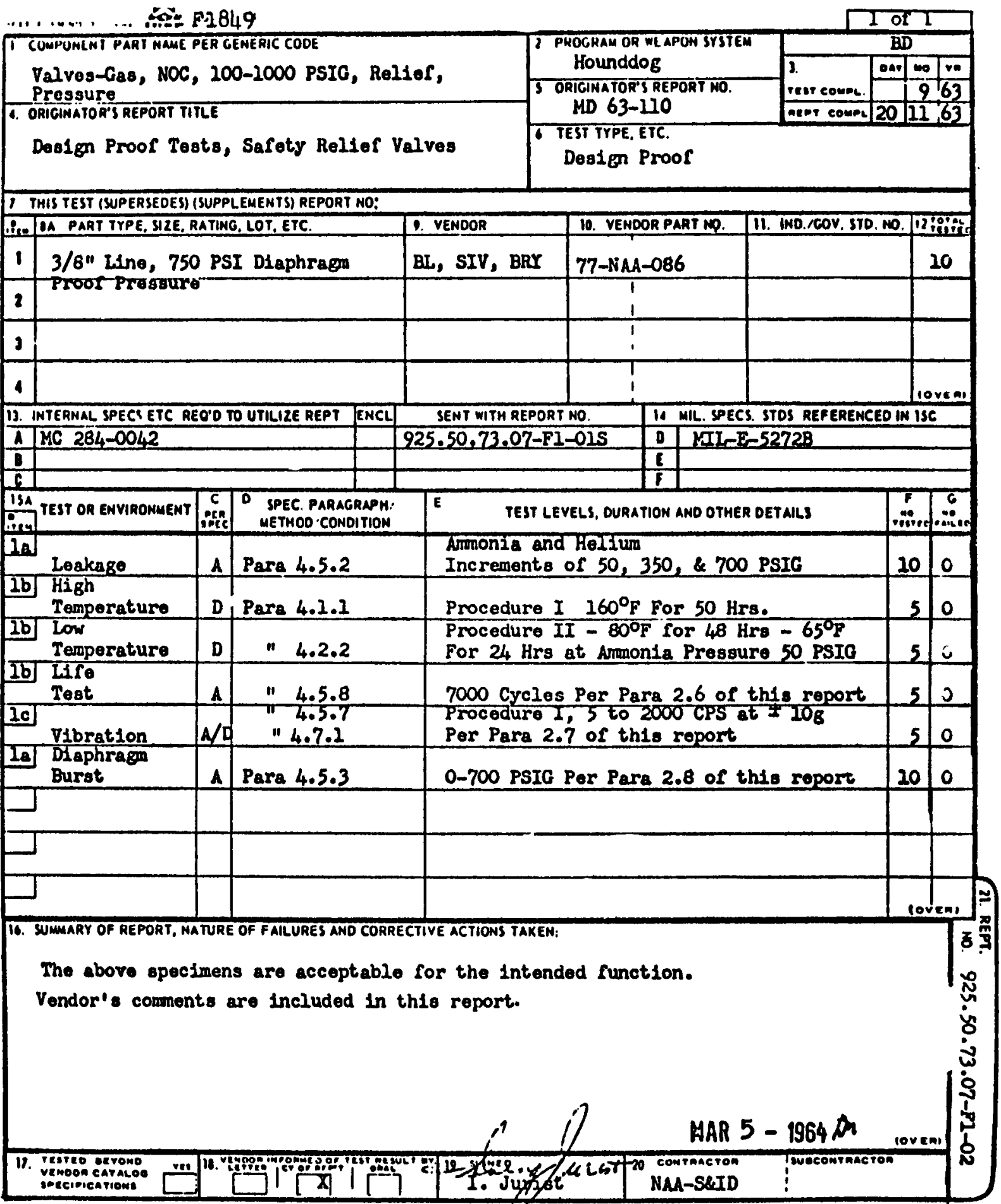

REPRODUCTION OR DISPLAY'OF THIS MATERIAL FOR SALES OR PUBLICITY PURPOSES IS PROHIBITEO.

FIGURE B. 7

REPOR T SUMMARY SHEET 
Primary Sources and Types of Failure Rate Data

Sponsored by Army, Navy, Air Force, and NASA

\begin{tabular}{|c|c|c|}
\hline \begin{tabular}{c|c} 
Government \\
Activities
\end{tabular} & $\begin{array}{c}\text { Prime Contractors and Major } \\
\text { Subcontractors on Military } \\
\text { Weapons and Space Programs }\end{array}$ & $\begin{array}{c}\text { Testing } \\
\text { Laboratories }\end{array}$ \\
\hline
\end{tabular}

Data Collection, Screening, Analysis

Summarization, Compilation, Distribution

FARADA Information Center Corono, California

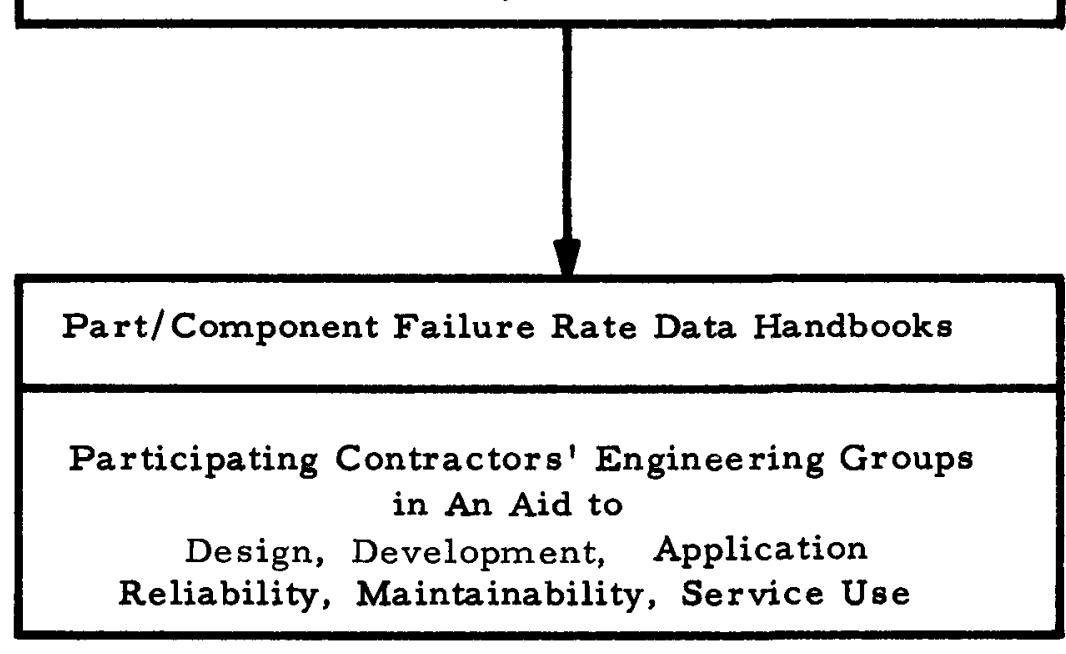

FIGURE B. 8

FARADA INFORMATION FLOW CHART 
MACKGROUND INFORMATION ON FAILURE RATE DATA - FARADA PROCAAM IIND FMSAEG $1200,(10.64)$

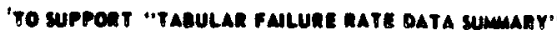

साप्ता

ACME Rompany

1. DESCRIPTION OF EQUIPMENTIS) TESTED OR UNDER SURVEILLANCE

AN/FSQ-7-Large high speed digital computer for use in real-time air defense problems

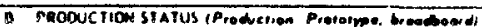

Prototype

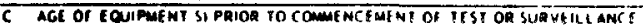

2 years

1962

II CONDITION OF TEST ror aurvelliance)

A DATL ANODOUATION

15 atober 1961 through 31 December 1963

Equipment housed in special envirommentally controlled building at a classified location.

some preventative maintenance in addition to montine replace and repair iil. SYSTEM STATISTICS

A TCTAL SYSTEMS inumber of averoment

1 Computer

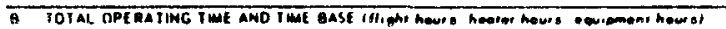

8500 nours of equipment operation

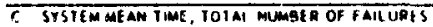

842 Catastrophic failures

Approximately 10 hours MTBF

IV FAILURE REPORTING SYSTEM

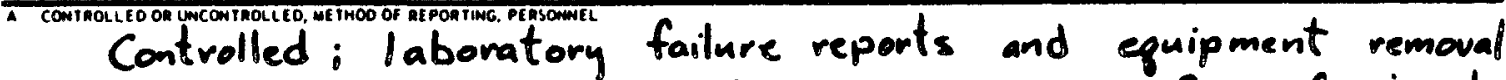
tags under the immediate surveillance of professional persomnel 3. ortmitionor raturef
Significant removal rate - defined as the total removals
minus accidental damage removals, removals due to failure of another component overload, and "no-defect-found" removals

95 to $100 \%$

FIGURE B. 9

SAMPLE OF COMPLETED

"BACKGROUND INF ORMATION ON FAILURE RATE DATA" FORM 


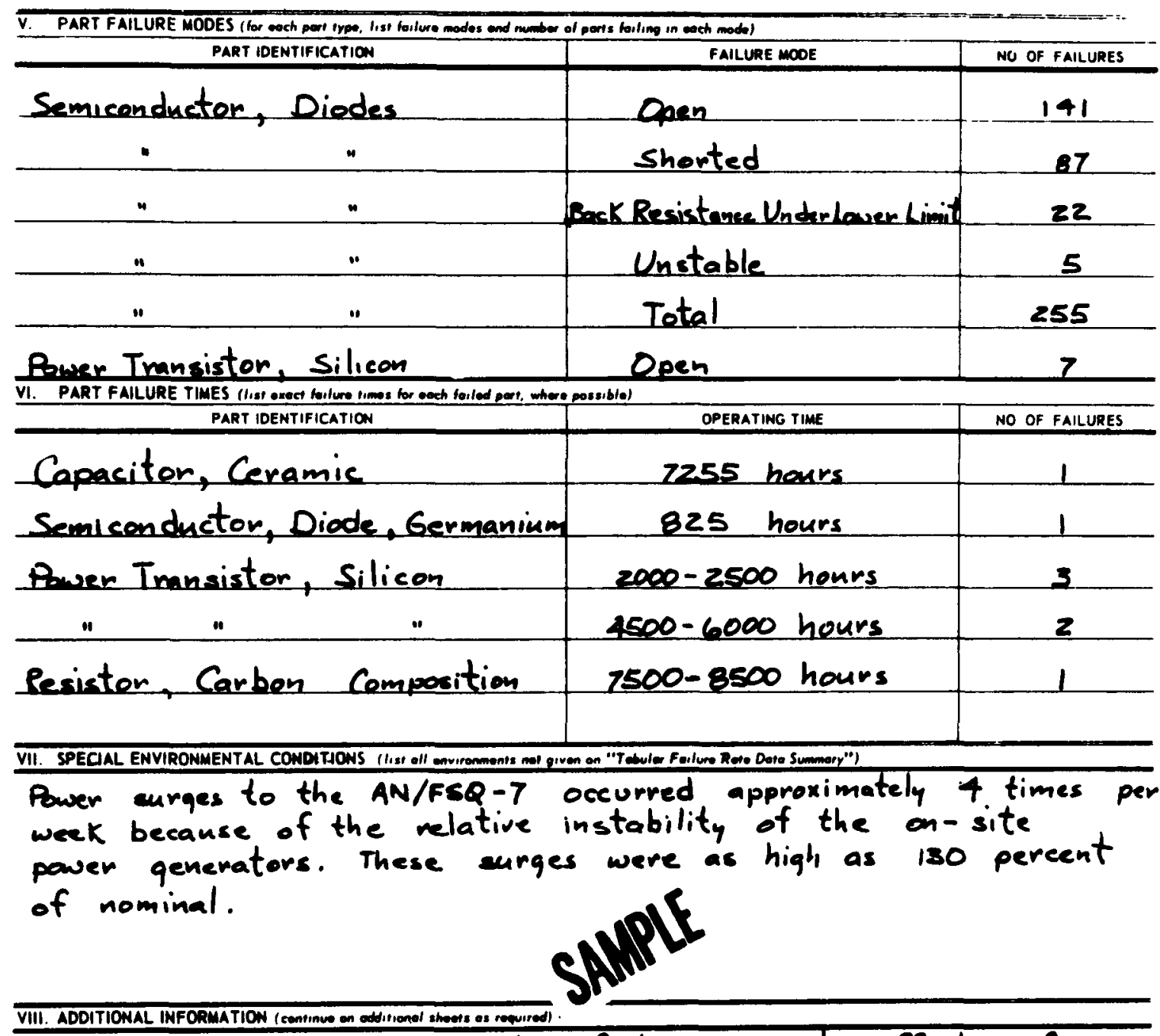

These data are a composite of two separate efforts of succeeding time periods (References $a$ and $b$, respectively). The two groups of data were integrated into this single report since they describe the same equipment and the component failure rates were very similar.

1X. REFERENCES

a) Doe, J.C.," A Preliminary Reliability Analysis of the MVFSQ-7 Computer," Proceedings of the $12^{\text {th }}$ National Symposium on Reliability and Quality Control, PP 372-380

(b) Mann, I.J., "The ANVTSQ-7, A Reliability Progress Report;" Proceedings of the 17 th RETMA Symposium of Applied Reliability, pp 75-82 
TAGULAR FALURE RATE DATA SUMMARY - FARADA PROGRAM TIMD-FMSAEG-2000/10 (nEV. 2-S6)

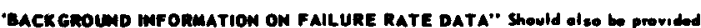

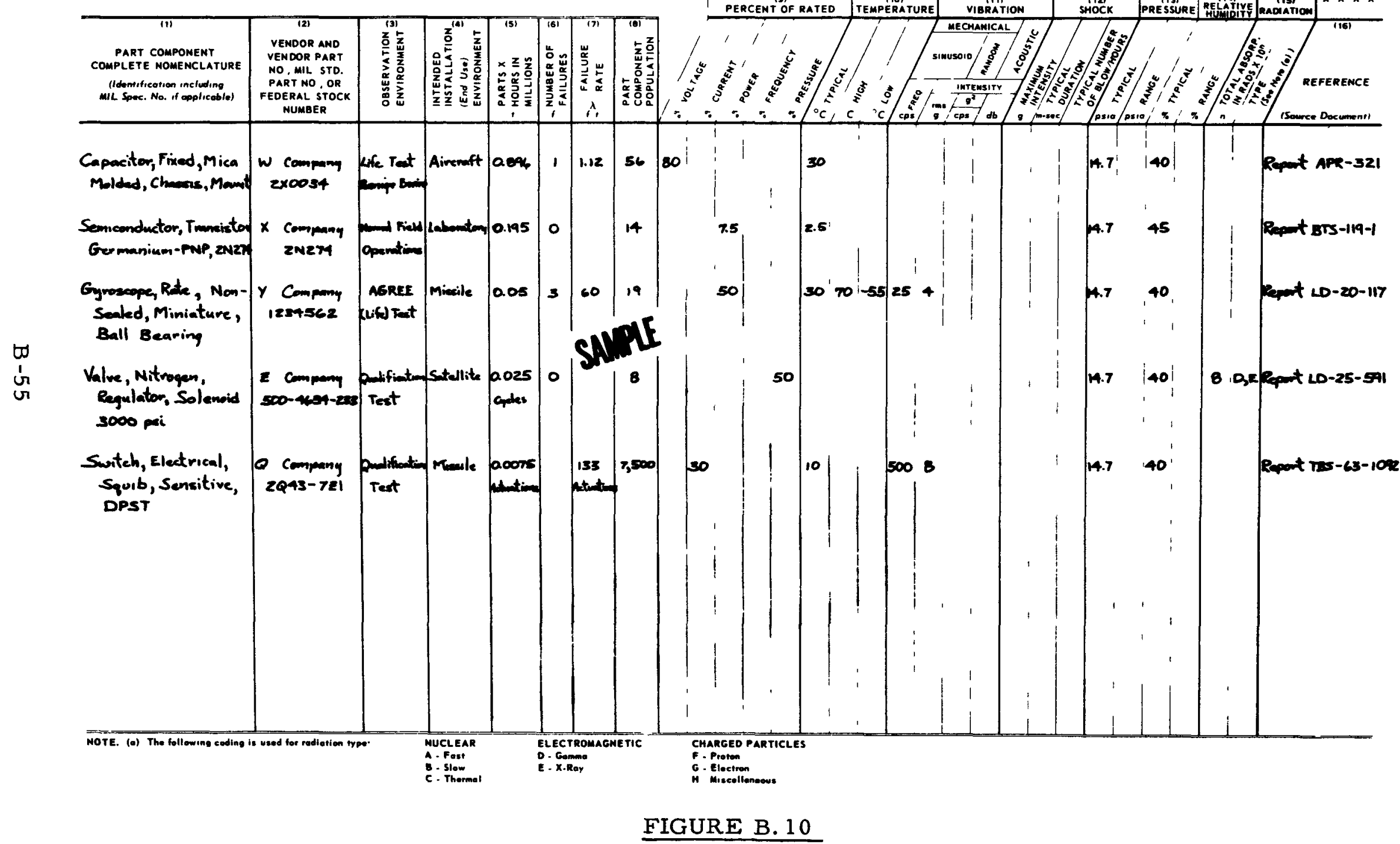

SAMPLE OF COMPLETED

"TABULAR FAILURE RATE DATA SUMMARY" FORM 


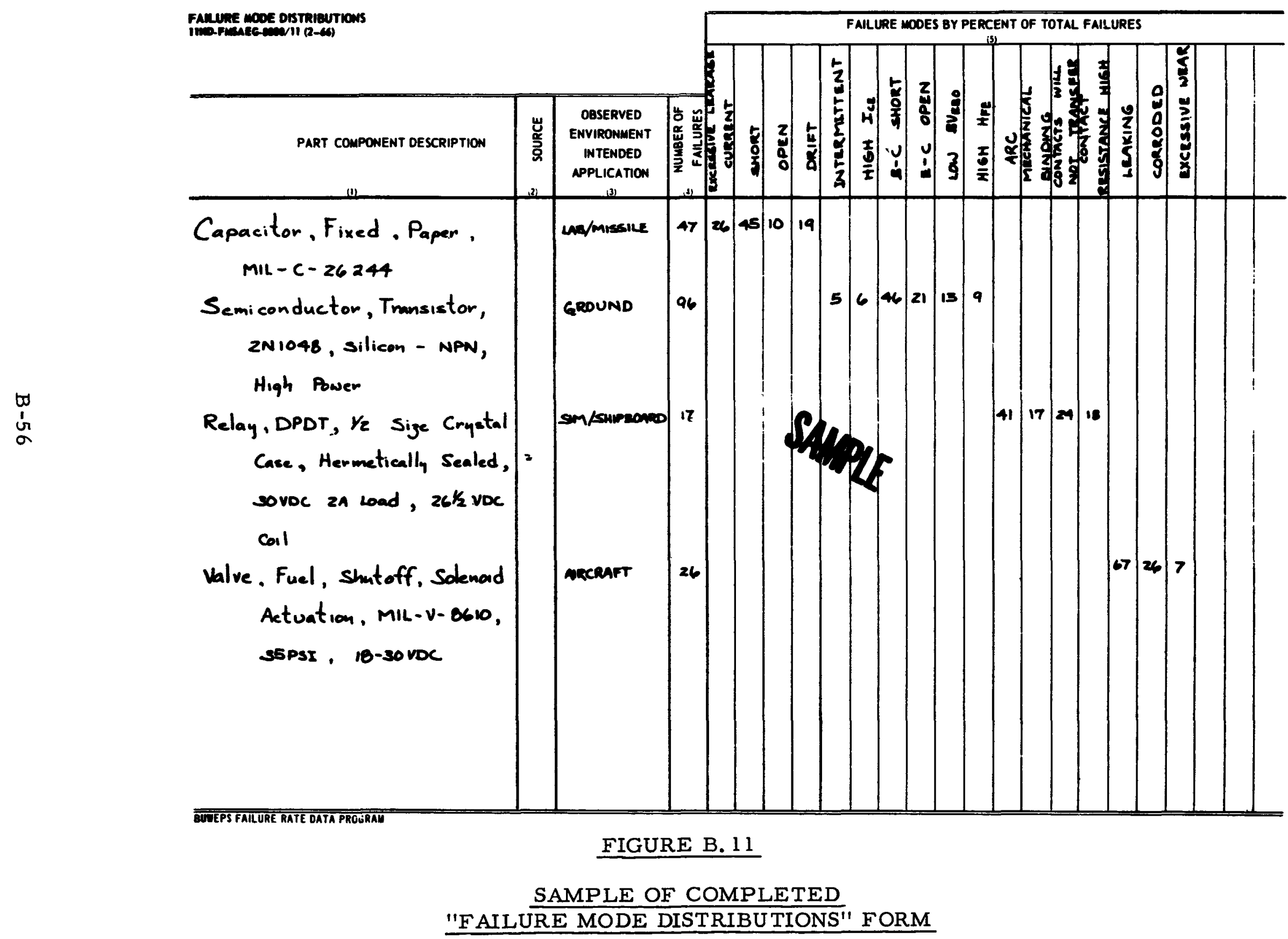




\begin{tabular}{|c|c|c|c|c|}
\hline $\begin{array}{l}\text { TE ISF } \\
\text { PORT } \\
-Y R\end{array}$ & $\begin{array}{l}\text { FALLURE RATE- } \\
\text { FAILURES PER MILLION } \\
\text { OPERAT ING HOURS }\end{array}$ & $\begin{array}{l}\text { Parts- } \\
\text { mouns } \\
\text { IN } \\
\text { mitl- } \\
\text { lous }\end{array}$ & $\begin{array}{l}\text { MUM- } \\
\text { DER } \\
\text { OF } \\
\text { FAIL- } \\
\text { URES }\end{array}$ & $\begin{array}{l}\text { COMP- } \\
\text { CNEKT } \\
\text { PART } \\
\text { POPU- } \\
\text { LATION }\end{array}$ \\
\hline $\begin{array}{l}-65 \\
-65 \\
-65 \\
-65 \\
-65 \\
-65 \\
-64 \\
-65 \\
-65 \\
-65 \\
-65 \\
-64 \\
-64 \\
-64 \\
-64 \\
-65 \\
-64 \\
-64 \\
-65 \\
-65 \\
-65 \\
-64 \\
-65 \\
-65 \\
-65 \\
-65 \\
-64 \\
-65 \\
-64 \\
-64 \\
-65 \\
-65 \\
-65 \\
-65 \\
-65 \\
-65 \\
-65 \\
-65 \\
-65 \\
-65 \\
-65 \\
-64 \\
\end{array}$ & 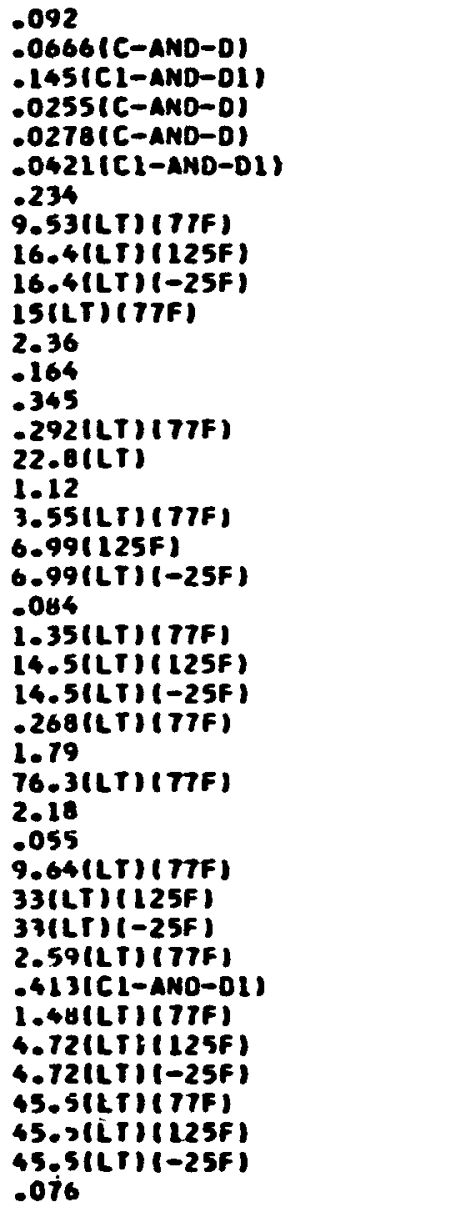 & $\begin{array}{l}43.609 \\
209.99 \\
124.13 \\
117.19 \\
1469.6 \\
1068.2 \\
5463.1 \\
.1049 \\
.0609 \\
.0609 \\
.0667 \\
1.6949 \\
170.92 \\
14.51 \\
3.424 \\
.04387 \\
39.311 \\
-2816 \\
.143 \\
.143 \\
344.07 \\
.7392 \\
.069 \\
.069 \\
3.7263 \\
3.9041 \\
.0131 \\
52.333 \\
274.5 \\
.1037 \\
.0303 \\
.0303 \\
.3864 \\
9.6801 \\
.6746 \\
.2119 \\
.2119 \\
.022 \\
.022 \\
0.022 \\
145.52\end{array}$ & $\begin{array}{l}1 \\
14 \\
18 \\
3 \\
41 \\
45 \\
1280 \\
0 \\
0 \\
0 \\
0 \\
4 \\
28 \\
5 \\
0 \\
0 \\
44 \\
0 \\
1 \\
0 \\
29 \\
0 \\
0 \\
0 \\
0 \\
7 \\
0 \\
114 \\
15 \\
0 \\
0 \\
0 \\
0 \\
4 \\
0 \\
0 \\
0 \\
0 \\
0 \\
0 \\
11\end{array}$ & $\begin{array}{l}5640 \\
40446 \\
23544 \\
22572 \\
283068 \\
202608 \\
689724 \\
27 \\
15 \\
15 \\
20 \\
212 \\
20793 \\
1855 \\
056 \\
5 \\
4705 \\
78 \\
30 \\
30 \\
47321 \\
184 \\
6 \\
6 \\
1028 \\
566 \\
6 \\
7580 \\
34532 \\
27 \\
5 \\
5 \\
99 \\
1830 \\
186 \\
50 \\
50 \\
5 \\
5 \\
5 \\
5 \\
1963\end{array}$ \\
\hline
\end{tabular}

OSSERVEO ENVISOUPARTS/COMPONENTS

SEMICONDUCTORS, DIUDES.IN2508, SILICON, PONER SEMICONDUCTORS, DIOUES. IN251, SILICON SEMICONDUCTORS, DIODES. IN25L, SILICON SEMICONDUCTORS, DIODES IN270, GERMANIUA SEMICONDUCTORS, DIUDES. IN276, GERMAMIUM SEMICONDUC TORS. DIODES.1N276, GERMANIUM SEAICONDUC TORS, DIODES. IN277. GERMANIUM, GOLD-BOND SEMICDNOUCTORS, DIODES. IN2T7 (JAN), GERMANIUA SERI CONDUCTORS, DIODES. IN2T7 (JAN), GERMANIUA SEMICONDUCTORS DIODES IM2T77 JANI GERMANIUM SERICONOUCTORS, DIODES. IN429, SILICON SEMICONDUCTORS, DIODES.1M429, SILICON, REFERENCE SEMICONDUC TORS, DIDDES. IN457, SILICON, DIFFUSED SEMICONDUC TORS, DIODES. IN458, SILICON, DIFFUSED SEAICONDUCTORS, DIODES. IN459, SILICDN

SEMICONOUCTORS, DIODES IN459, SII ICON.DIFEUSED SLMICONDUCTORS, DIODES. IN474A, SILICON, REF ERENCE SEMICOMDUCTORS, OIODES. IN4838, SILICON

SERICONDUCTORS, DIODES.

to SEICONDUCTORS, DIODES. IN4838, SILICON

SEMICANDUCTORS, DIODES.IN4838, SILICON

SEMICONDUCTORS, DIODES. IN538, SILICON, RECTIFIER

SEMICONDUCTORS, DIODES. INS38 (JAN), SILICON SEMICONDUC TORS. DIODES. INS38 (JAN), SILICON SEAICOMOUCTORS, DIODES.IN538(JAM), SILICUN SEMICONOUCTORS,DIDDES. INS40, SILICON SEMI CONDUCTORS, DIDOES I IN540, SILICON, REC TIFIER SEMI CONDUC TORS, DIODES - INS4T (JAN), SILICON SEMICONDUCTORS, OIUDES - IN629, SILICON, SWITCHING SEMICONDUCTORS, OIODES. IN645, SILICON, RECTIFIER SEMICONDUCTORS, DIODES. INGASIUSAFI,SILICON SEAI CONOUC TORS, DIODES. ING4SIUSAFI, SILICOM SEMICONDUC TORS DIODES ING45(USAF) SILICON

SËRICONDUCTORS, DIODES. IN647, SILICON

SEMICONDUC TORS, DIODES. IN658, SILICON

SEMICONDUC TORS, DIODES.IN658, SILICDM

SEMICONOUCTORS, DIODES.1N658, SILICON SEMICONUUC TORS, DIODES. IN658, SIEICON

SERICONUUCTORS, DIOOES. INGSB (USA), SILICON SEAICONDUCTORS, DIODES. IN658IUSAI SILICON SEAICOMOUCTORS, DIODES ING5BIUSAI SILICON

SERICONDUCTORS. DIUDES. ING9L, SILICON. SWITCHING

(1)

App

SRTUND

LAB,GND, SUB/SUB

LAB,GNO, SUB/SUA

LAB, GND, SUB/SUB

LAB, GND SUB/SUB

LAB, GND, SUB/SUE

GROUND

LAB/GRUUND

LAB IALI/GRUUNO

LAB (AL) / GROUND

LAB/GROUNO

GROUND

GROUND

LAB/GRIIUND

GRDUND

GRDUND

217

217

217

210

217

217

217

210

210
217
210
210

210
217

217
217
217
217

217

209

217

217

217

217
217
210

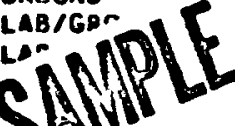

.ND

-iali/ground LAB (AL) / GRUUND GROUND

LAB/GRDUND

GRDUNO

LAE/GROUMD

LAB (AL)/GRUUND

LAB (AL) / GRUUND

LAB/GROUMD

LAB, GNO, SUB/ SUB

LAB/GRUUNO

LAB IALI / GRUUND

LAB IALI / GROUNO

LAB/GRUUNO

LAB I AL I / GRUUND

GAOTALI/GR

GROUND LAB / GRDUNO

FIGURE B. 12

TYPICAL PAGE OF FARADA COMPUTER PRINT-OUT FROM VOLUME 1 A 


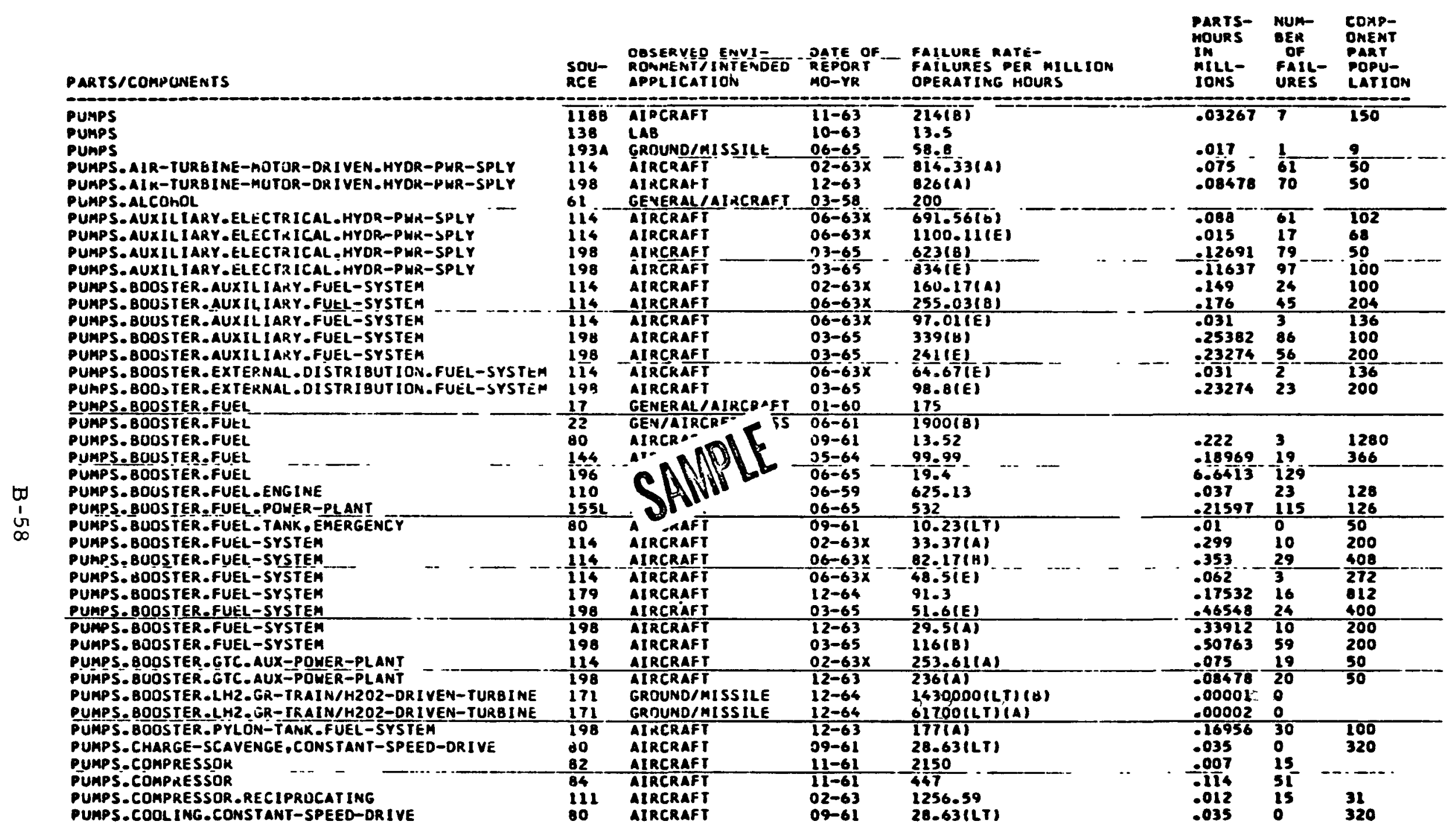




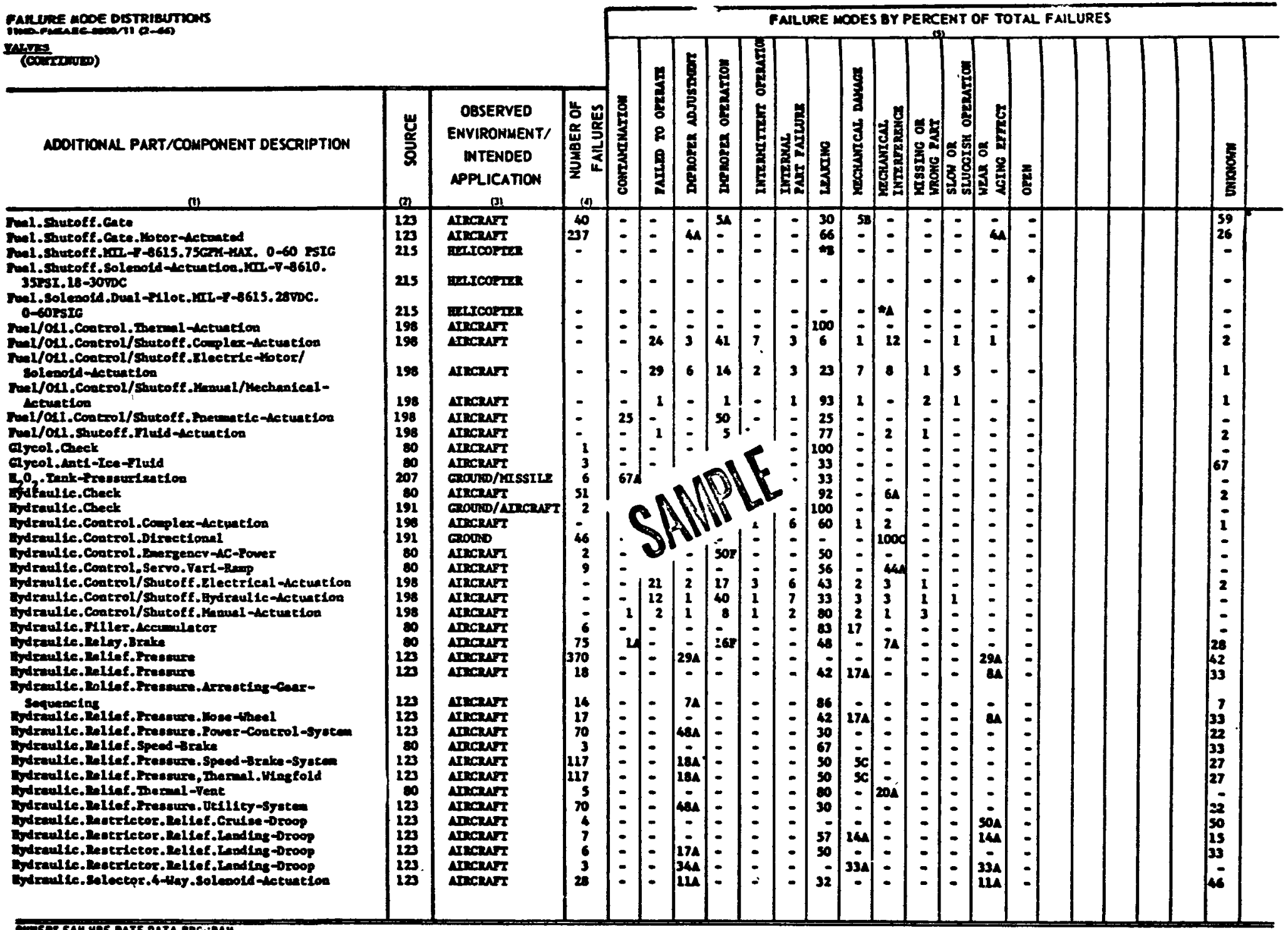

FIGURE B. 14

TYPICAL PAGE OF FARADA FAILURE MODE DISTRIBUTIONS 


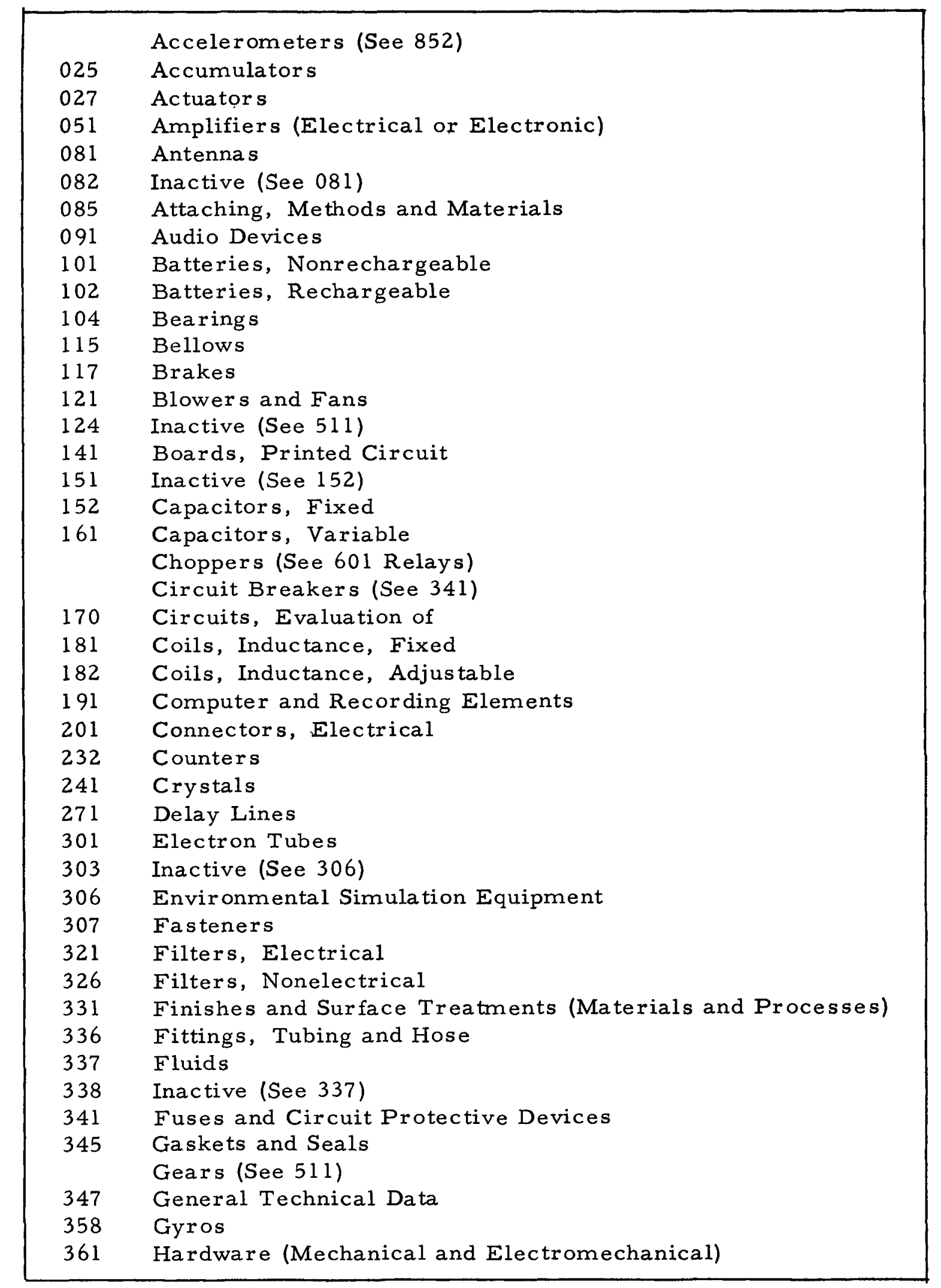

FIGURE B. 15

PAR TIAL LIST OF MAJOR CLASSIFICATIONS OF THE FARADA PART/COMPONENT GENERIC CODE 
SERVICE, MEDIA HANDLED

.10 Cryogenic Fluide

.20 Fuel, Exot1c

.30 Fue d, liydrocarbon

$.40 \mathrm{Cas}, \mathrm{llot}$

$.50 \mathrm{Cas}, \mathrm{NOC}$

.60 Hydraulic Fluld

.70 Oxidizers, NOC

.80 Pneumat1c

.90011

PRESSURE RANGE

$.100-25 \mathrm{mmHg}_{8}$

.20 25-35 umitg

$.3035-225$ mm 8 8 $\left(70,000^{\prime}-30,000^{\prime}\right)$

$.40225-760$ morks $\left(30,000^{\prime}-80\right.$ Ievel)

.50 S. L. only

$.600-100$ ps 18

.70 100-1000 peis

.80 1000-5000 pe18

.90 Over 5000 pele

POLER RATING

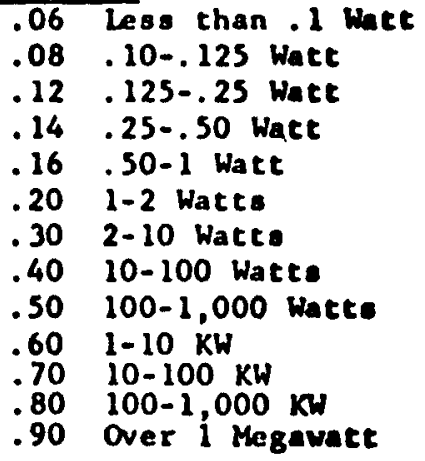

CONTACT ARRANGEMENT

.05 SPST (NC)

.10 SPST (NO)

.12 SPDT

.20 DPST (NC)

.25 DPST (NO)

.30 DPDT

.40 3PST (NC)

.45 3PST (NO)

.30 3PDT

.604 PST (NC)

.65 4PST (NO)

$.70 \quad 4 \mathrm{PDT}$

.80 6PST (NC)

.85 6PST (NO)

.90 6PDT

.95 Miltiple Pole
VOLTAGE RATING

.10 Under I MV

$.201-10 \mathrm{mV}$

$.3010 \mathrm{~m}-1 \mathrm{Volt}$

$.40 \quad 1-30$ Volta

$.5030-109$ Volts

.60 109-240 Volte

.70 240-1,000 Volte

$.80 \quad 1-10 \mathrm{kV}$

.90 Over $10 \mathrm{KV}$

CORE MATERTAL \& CONSTRUCTION

- I A1r, Encapsulated

. 2 " Hermetlcally Sealed

- 3 ". NOC

- 4 DLamagnet Ic, Encepoulated

- 5 " "Hermetically Sealed

- 6 " NOC

- 7 Ferromagnet1c, Encapoulated

- 8 " . Hermeticelly seled

. 9 , NOC

FREQUENCX RANGE

.02 D. C.

.0560 Cycles only

.06400 Cycles Only

$.07 \quad 0-3 \mathrm{Kc}$

.10 3-30 Kc

$.2030-300 \mathrm{Kc}$

$.30300-3,000 \mathrm{Kc}$

.40 3-30 Mc

$.50 \quad 30-300 \mathrm{Mc}$

$.60300-3,000 \mathrm{Mc}$

Aud 10

Low

Mediun

High

vlere

.70 3,000-30,000 He Super

.80 Orer $30,000 \mathrm{kc}$

Extrene 1y

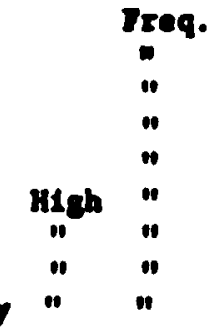

Note:

1. NOC - Not Otherwlee Claselfled

2. Range: The Range 10 defined to be greater than the lower value, up to and including the larger value. Samples Voltage Rating 1-10 w Any voltage greater than $1 \mathrm{w}$, up to and Including $10 \mathrm{mv}$.

FIGURE B. 16

FARADA COMMON SUBCLASSIFICATION 


\section{VALVES}

925. 00 Service, Media Handled (Common)

925.00.00 Pressure Range (Common)

$$
\begin{aligned}
& \text { 92.5.00 Function } \\
& \text { - } 1 \text { Check } \\
& \text { - } 2 \text { Multifunction } \\
& \text { - } 3 \text { Relief } \\
& \text { - } 4 \text { Servo } \\
& \text { - } 5 \text { Shutoff } \\
& \text { - } 6 \text { 3-way Selector } \\
& \text { - } 7 \text { 4-way Selector }
\end{aligned}
$$

$$
\begin{aligned}
925.00 .00 .00 & \text { Principle of Operation } \\
.10 & \text { Ball } \\
.20 & \text { Butterfly } \\
.30 & \text { Flapper } \\
.40 & \text { Poppet } \\
.50 & \text { Sleeve } \\
.60 & \text { Slide or Gate } \\
.70 & \text { Spool } \\
.80 & \text { Globe } \\
.90 & \text { Needle }
\end{aligned}
$$

$925.00 .00 .00 \quad$ Actuation or Control

. $\overline{4}$ Manual

- 5 Motor

. 6 Pilot

- 7 Pressure

- 8 Pyrotechnic or Explosive

- 9 Solenoid

FIGURE B. 17

EXAMPLE OF FARADA

PART/COMPONENT CLASSIFICATION CODE 


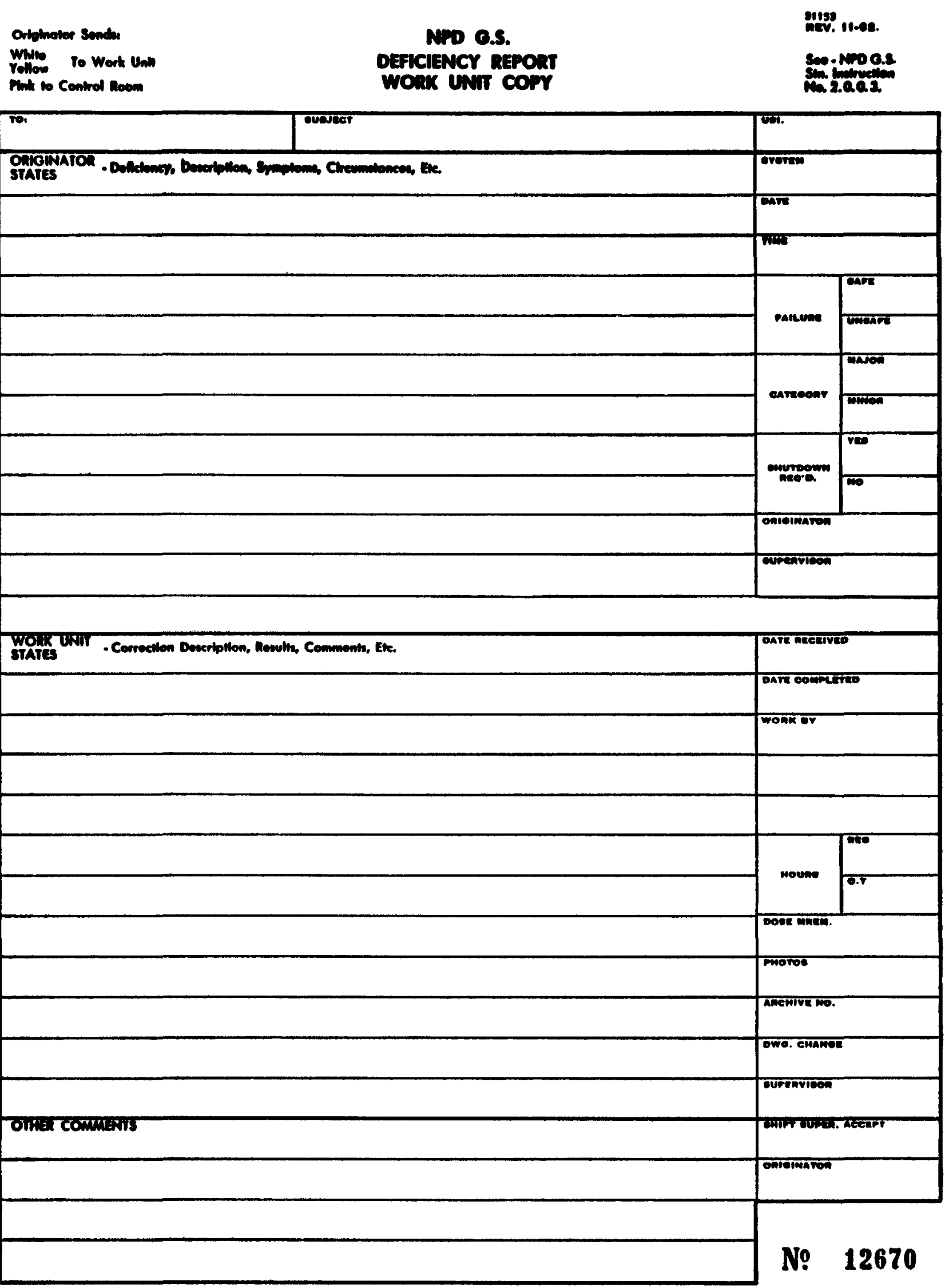

FIGURE B. 18

SAMPLE DEFICIENCY REPORT

B -63 


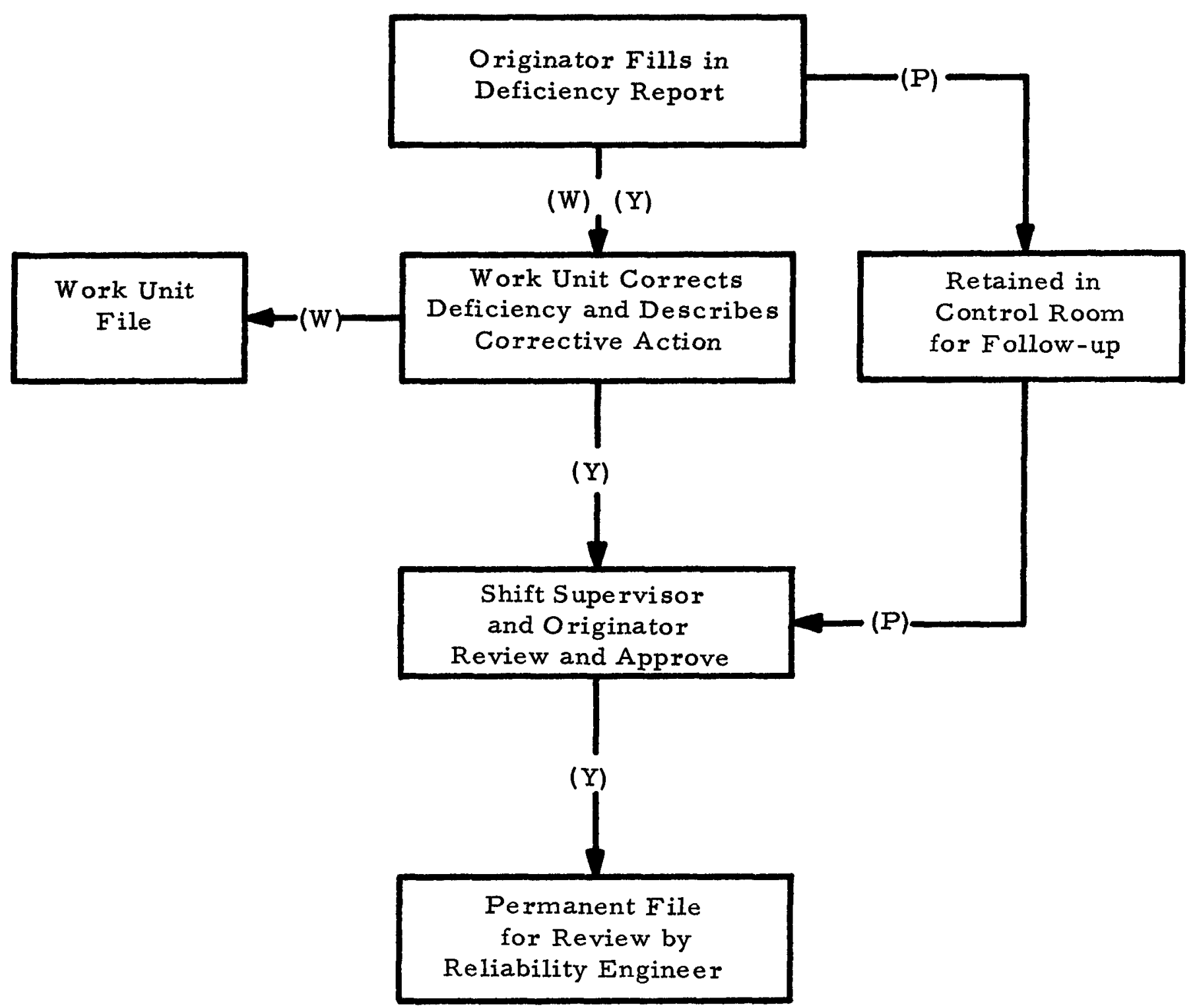

(W) - white original

(Y) - yellow copy

(P) - pink copy

FIGURE B. 19

DEFICIENCY REPORT FLOW CHART

B- 64 
APPENDIX C

EXAMPLE OF LOADSHEET PREPARATION AND DATA REDUCTION 


\section{APPENDIX C}

\section{EXAMPLE OF LOADSHEET PREPARATION AND DATA REDUCTION}

Loadsheet preparation and data reduction for failure rate analys is will be demonstrated by the use of a simple pumping system as shown schematically in Figure C. 1.

\section{SYSTEM DESCRIPTION}

The pumping system (system code 21.341 ) belongs in the reactor portion (system code 20) of a hypothetical power plant (I. D. No. 0123). It consists of one $50 \mathrm{gpm}$ pump, one flow switch ${ }^{*}$, and three loops each containing one motor operated control valve all as listed in Table C. 1. During plant operation it is assumed that the pump is operated continuously and the water flow in each loop is automatically adjusted by a motor operated valve. Automatic operation of the valves is controlled by a sensor measuring temperature or pressure. The sensor is not shown for simplicity.

Failure of any one valve in the closed position will not result in a system failure. Failure of any two valves in the closed position will resull in a system failure; and failure of the pump or all three valves will result in the actuation of the low flow switch which in turn will scram the reactor plant. The flow switch is tested daily during operation.

System History

It is assumed that the pumping system has previously completed approximately five years or 43,800 hours of operation with several component failures. During the next reporting period, which is on a three-month interval shown schematically in Figure C. 2 , the reactor plant was operating at the beginning of January 1966, and continued operation through the month of February until a scram occurred on March 20, 1966. It was started up again on March 24, 1966, and continued operation through the end of the reporting period, March 31, 1966. During this period

\footnotetext{
Flow switch belongs in System Code 26, 121 , Instrumentation and Control
} 
there were two concurrent valve failures resulting in total loss of system performance (effect code 3 ) and one valve failure causing no loss of performance (effect code 1). A pump failure, which resulted in system loss (effect code 3) and plant scram, alsowas reported.

Although operation of the example pumping system is correlated to the reactor plant (system code 20) in Figure C. 2, the operation of the turbo-generator ( $T / G$ ) plant (system code 30 ) is also shown to demonstrate how it is treated separately. The use data of a system whose operation is related to the $T / G$ plant would use $T / G$ data to more accurately derive "time to failure" estimates.

\section{LOADSHEET PREPAR ATION}

Loadsheets are prepared in order to provide information on individual failure events as well as to enable data reduction later by either manual computation or by computer programming. The manner in which a group of line entries within each reporting period are made is as follows:

Step 1: First make a separate line entry for reactor and $\mathrm{T} / \mathrm{G}$ operating status at the beginning of the reporting period (lines 1 and 2 of Figure C. 3 ).

Step 2: Enter all failure events in chronological order until plant shutdown or scram occurs (lines $3,4,5$, and 6 of Figure C.3).

Step 3: Make a separate line entry for reactor and $T / G$ shutdown or scram (lines 9 and 10 of Figure C. 3 ).

Step 4: Make a separate line entry for reactor and $T / G$ startup (lines 9 and 10 of Figure C. 3).

Step 5: Repeat line entries starting with Step 2.

Step 6: Finally make a separate line entry for reactor and $T / G$ operating status at the end of the reporting period (lines 11 and 12 of Figure C. 3).

Figure C. 3 illustrates the completion of input data loading for a quarterly reporting period on the example system. The entries in Figure C. 3 were made as follows:

Line 1: This is the status (use code 2) of the reactor plant (system code 20) on January 1, 1966. 
Line 2: This is the status (use code 2) of the $T / G$ plant (system code 30 ) on January 1.

Line 3: Two valve failures occurred on February 4 resulting in loss of system performance (effect code 3) but no plant shutdown (use code 2). Failure mode was that of premature operation (mode code 6) caused by maintenance error (cause code 34 ). Recovery of the system operation was made on the same day with six hours of total repair time. This entry is for one of the valve failures.

Line 4: This entry is for other valve failures indicated in Line 3 discussion.

Line 5: Another valve failure occurred on February 20 with no loss of system performance (effect code 1 and use code 2); however, repair was not performed until two days later.

Line 6: On March 20 a pump failure occurred which resulted in system loss (effect code 3 ) and reactor scram (use code 4). Failure mode was that of erratic operation (mode code 8 ) caused by high operating temperature (cause code 31 ). A new pump (new I. D. No. 5KY558AAl) from a different manufacturer (code 543) was installed and repair completed on March 21 with a total repair time of 20 hours.

Line 7: Reactor scram occurred following the pump failure.

Line 8: $T / G$ shutdown occurred following the pump failure.

Line 9: After remaining shut down for 3 more days another reactor startup was made on March 24.

Line 10: T/G startup followed 4 days later on March 28.

Line 11: The reactor plant was operating at the end of reporting period March 31.

Line 12: The $\mathrm{T} / \mathrm{G}$ plant was operating at the end of reporting period.

\section{DATA REDUCTION}

Using data presented in the input loadsheet, data reduction is possible by either manual computation or by computer programming. Two types of information that are provided in the output data sheet are as follows : 
Type 1: Listing of particular components that have failed during the reporting period. This information will provide the basis for deriving failure rates and distribution functions for components of similar kind.

Type 2: Summary of failure rate data of all components of interest as of the end date of the reporting period. These data are carried over to the next reporting period.

A summary of failure rate data from the previous reporting periods is shown in Figure C. 4. The listing of component failures and summary of failure rate data for the current reporting period are shown in Figures C. 5 and C. 6. The output data sheet listing component failures for the reporting period is produced from information on the current input loadsheet and the previous report periods. Subsequent to this, a summary of failure rate data is generated. Components are grouped by their generic code and also by the system code in which they belong. This entire process can be handled by machine processing with component failures and failure rate information being stored on tape for data update and for special searches such as examination of failure distribution functions. 


\begin{tabular}{|c|c|c|c|c|}
\hline Component & Type & $\begin{array}{c}\text { Manufacturer's } \\
\text { Code }\end{array}$ & $\begin{array}{l}\text { Plant } \\
\text { I. D. No. }\end{array}$ & $\begin{array}{l}\text { Generic } \\
\text { Code }\end{array}$ \\
\hline Pump & $\begin{array}{l}50 \mathrm{gpm} \text {, stainless } \\
\text { steel, centrifugal }\end{array}$ & 345 & $5 \mathrm{C} 50 \mathrm{~A}$ & $221: 56.22$ \\
\hline $\begin{array}{l}\text { Flow Control } \\
\text { Valve }\end{array}$ & $\begin{array}{l}1 \text { inch, stainless } \\
\text { steel, motor - } \\
\text { operated gate }\end{array}$ & 567 & $871 \mathrm{~A} 01 \mathrm{~T} 42$ & $202: 56.24 .22$ \\
\hline $\begin{array}{l}\text { Flow Control } \\
\text { Valve }\end{array}$ & $\begin{array}{l}1 \text { inch, stainless } \\
\text { steel, motor - } \\
\text { operated gate }\end{array}$ & 567 & $871 \mathrm{~B} 01 \mathrm{~T} 42$ & $202: 56.24 .22$ \\
\hline $\begin{array}{l}\text { Flow Control } \\
\text { Valve }\end{array}$ & $\begin{array}{l}1 \text { inch, stainless } \\
\text { steel, motor - } \\
\text { operated gate }\end{array}$ & 567 & $871 \mathrm{C} 01 \mathrm{~T} 42$ & $202: 56.24 .22$ \\
\hline Flow Switch & $\begin{array}{l}\text { Industrial instru- } \\
\text { ment }\end{array}$ & 789 & FS 971 & $500: 01.40$ \\
\hline
\end{tabular}

TABLE C. 1 


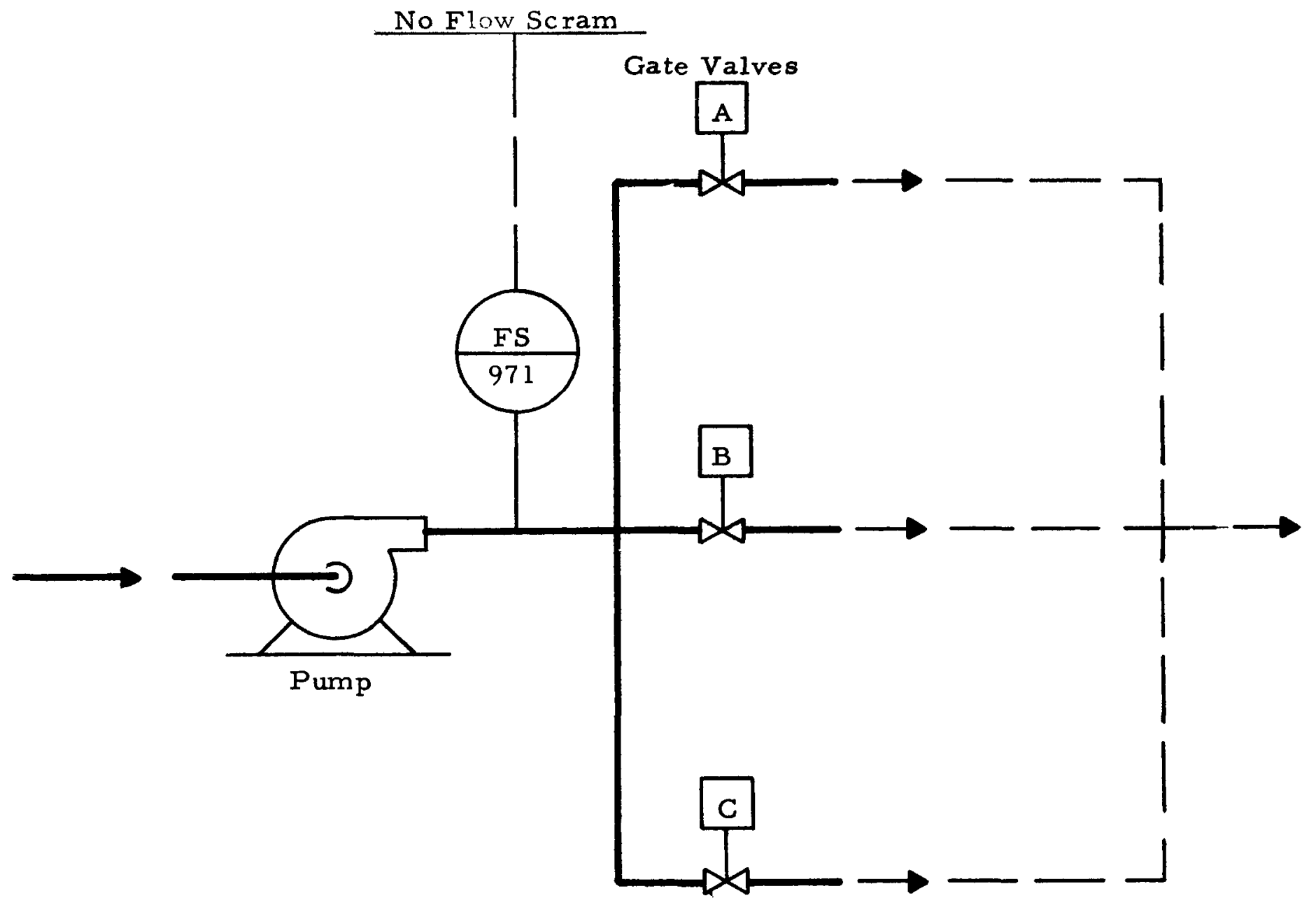

FIGURE C. 1

SIMPLE PUMPING STATION

$$
\text { C }-6
$$


System/Component

Reactor Plant

Pumping System

Pump

Valve (A)

Valve (B)

Valve (C)

Flow Switch

T/G Plant
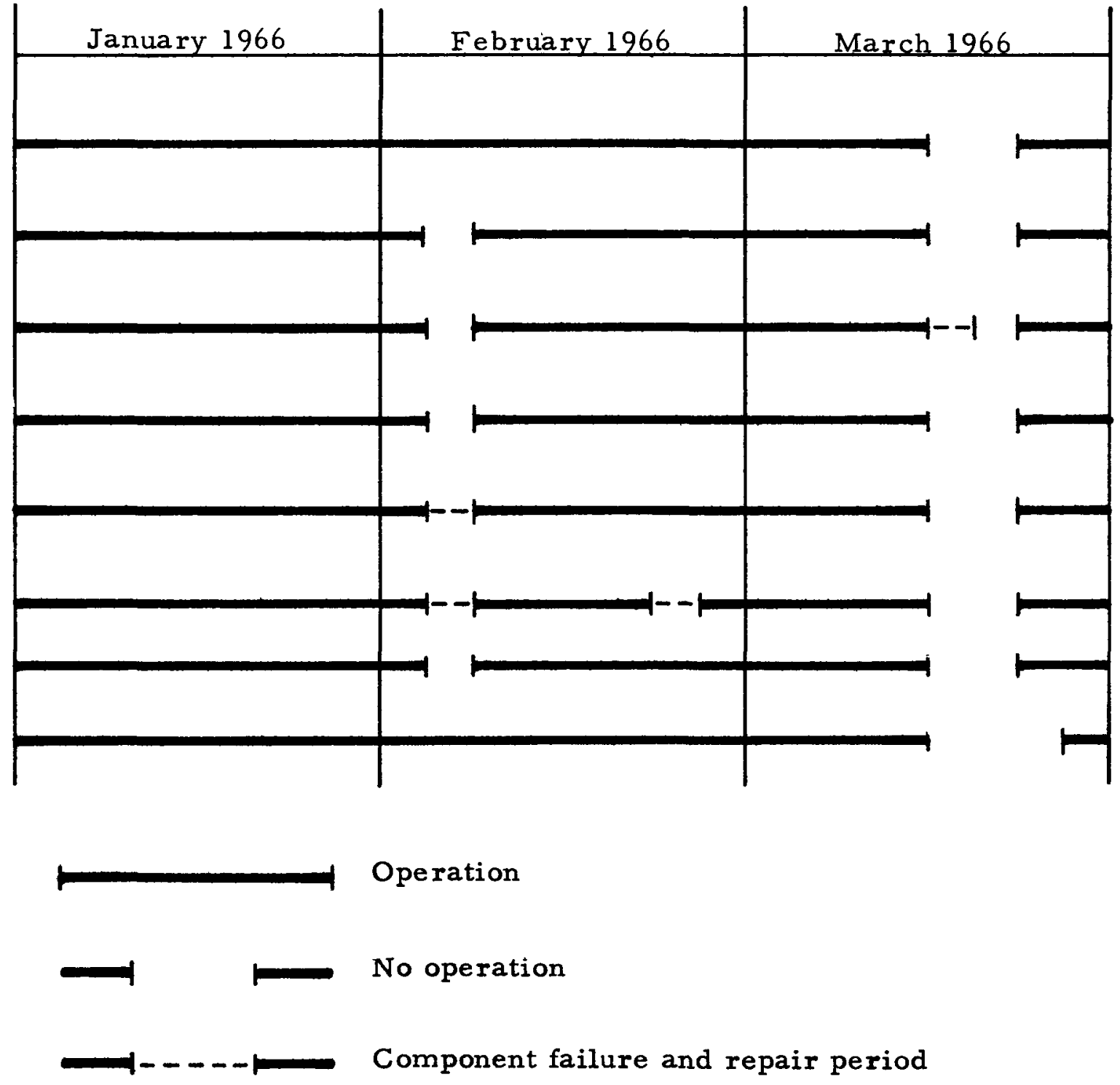

FIGURE C. 2

OPERATIONAL HISTORY OF A SIMPLE PUMPING SYSTEM 


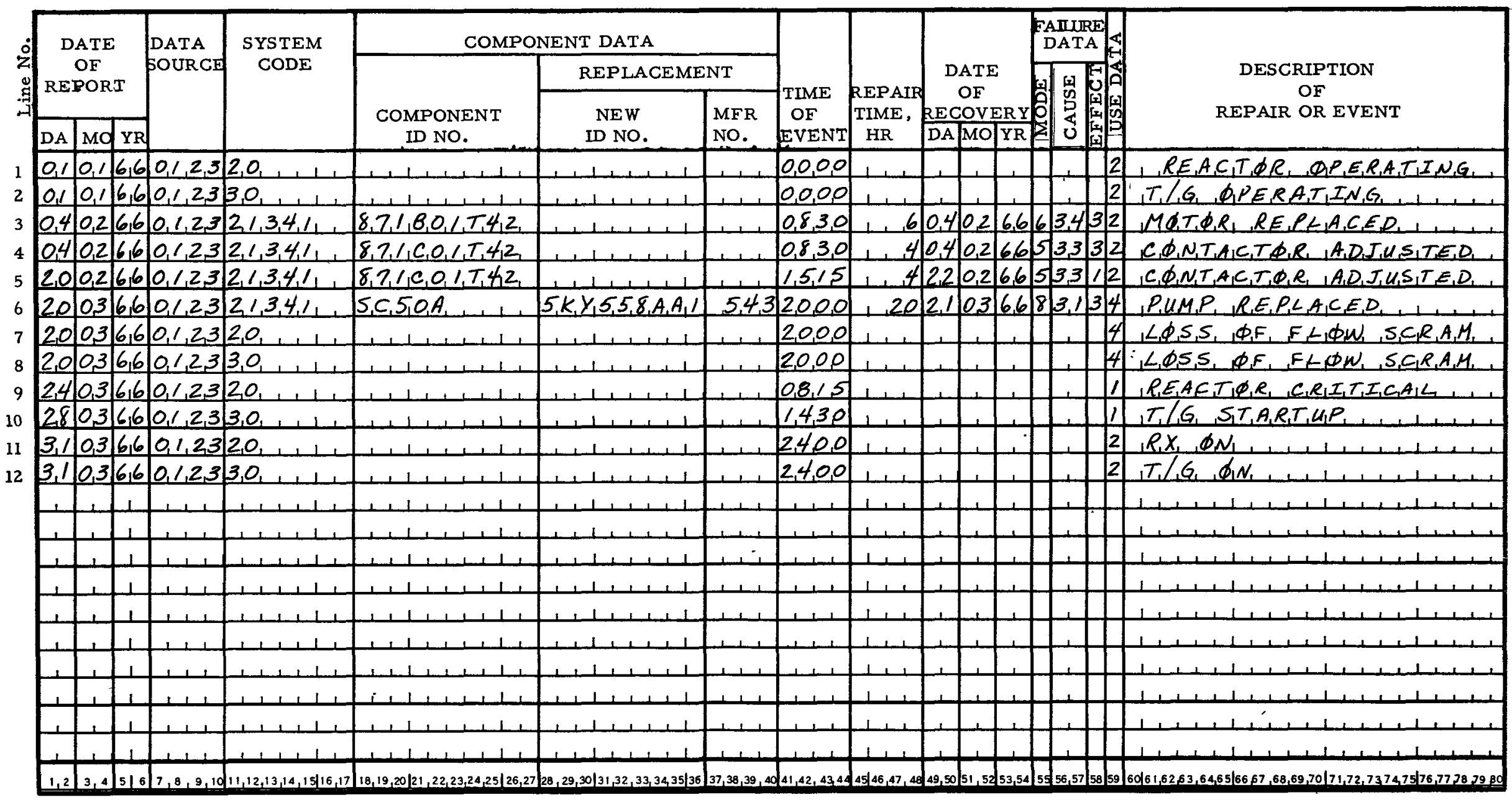

FIGURE C. 3

INPUT LOADSHEET 
ABC NUCLEAR POWER PLANT

PLANT I.D. NO. 0123

REPORTING PERIOD

311065 TO 311265

SUMMARY OF FAILURE RATE DATA

COM -

NO. OF

PONENT

I. D. NO.

PONENTS

$\begin{array}{cc}\text { FAILURE } & \text { NO. OF } \\ \text { MODE } & \text { FAILURE }\end{array}$

FAILURES

ACCUMU -

LATIVE FAILURE ACCUMU -

TIME IN PER STANDARD LATIVE MEAN

HOUR MILION , MILIION DEVIATION, REPAIR

OR HOUR OR HOUR OR HOUR OR TIME, TIME,

CYCLE CYCLE CYCLE CYCLE HOUR

TIME,

ST ANDARD

COMPONENTS IN SYSTEM CODE 21341

$\begin{array}{lcl}871 \mathrm{AO} 1 \mathrm{~T} 42 & 1 & 1 \\ 871 \mathrm{~B} 01 \mathrm{~T} 42 & 1 & 3 \\ 871 \mathrm{C} 01 \mathrm{~T} 42 & 1 & 3 \\ \text { COMPONENT CODE } & 202562555 \\ \text { SC50A } & 1 & \\ \text { COMPONENT CODE } & 2215622\end{array}$

$\begin{array}{ll}\text { Hour } & .043780 \\ \text { Hour } & .043800 \\ \text { Hour } & .043800 \\ \text { Hour } & .131380 \\ \text { Hour } & .043775 \\ \text { Hour } & .043775\end{array}$

2.3

10

COMPONENTS IN SYSTEM CODE 26121

FS971 1

COMPONENT CODE 5000140

52

2

$\begin{array}{ll}\text { Cycle } & .001828 \\ \text { Cycle } & .001828\end{array}$

$$
7.6
$$

22.8

22.8

\section{9}

109
DEVIATION, HOUR

FIGURE C. 4

TYPE 2 OUTPUT DATA SHEET 
ABC NUCLEAR POWER PLANT

PLANT I. D. NO. 0123

REPORTING PERIOD

010166 TO 310366

LISTING OF COMPONENT FAILURES

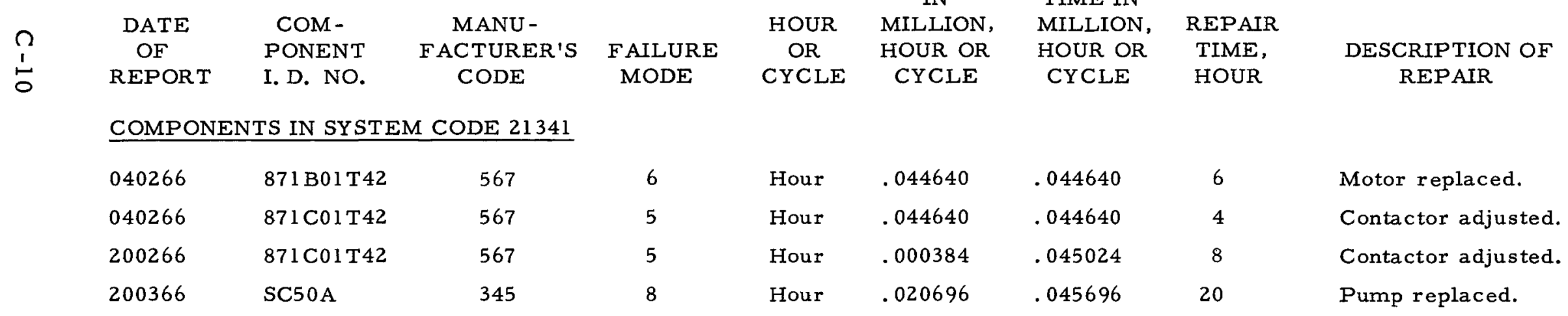

\section{FIGURE C. 5}

TYPE 1 OUTPUT DATA SHEET CURRENT LISTING OF COMPONENT FAILURES 
ABC NUCLEAR POWER PLANT

PLANT I. D. NO. 0123

REPORTING PERIOD

010166 TO 310366

SUMMARY OF FAILURE RATE DATA

\begin{tabular}{|c|c|c|c|c|c|c|c|c|c|c|}
\hline & & & & & $\begin{array}{l}\text { ACCUMU- } \\
\text { LATIVE } \\
\text { TIMEIN }\end{array}$ & $\begin{array}{l}\text { FAILURE } \\
\text { PER }\end{array}$ & STANDARD & $\begin{array}{l}\text { ACCUMU - } \\
\text { LATIVE }\end{array}$ & MEAN & \\
\hline $\begin{array}{l}\text { COM- } \\
\text { PONENT } \\
\text { I. D. NO. }\end{array}$ & $\begin{array}{l}\text { NO. OF } \\
\text { COM- } \\
\text { PONENTS }\end{array}$ & $\begin{array}{l}\text { FAILURE } \\
\text { MODE }\end{array}$ & $\begin{array}{l}\text { NO. OF } \\
\text { FAILURES }\end{array}$ & $\begin{array}{l}\text { HOUR } \\
\text { OR } \\
\text { CYCLE }\end{array}$ & $\begin{array}{l}\text { MILLION, } \\
\text { HOUR OR } \\
\text { CYCLE }\end{array}$ & $\begin{array}{l}\text { MILLION, } \\
\text { HOUR OR } \\
\text { CYCLE }\end{array}$ & $\begin{array}{l}\text { DEVIATION, } \\
\text { HOUR OR } \\
\text { CYCLE }\end{array}$ & $\begin{array}{l}\text { REPAIR } \\
\text { TIME, } \\
\text { HOUR }\end{array}$ & $\begin{array}{l}\text { REPAIR } \\
\text { TIME, } \\
\text { HOUR }\end{array}$ & $\begin{array}{l}\text { STANDARD } \\
\text { DEVIATION, } \\
\text { HOUR }\end{array}$ \\
\hline
\end{tabular}

COMPONENTS IN SYSTEM CODE 21341

$\begin{array}{lcc}871 \mathrm{~A} 01 \mathrm{~T} 42 & 1 & 1 \\ 871 \mathrm{~B} 01 \mathrm{~T} 42 & 1 & 6 \\ 871 \mathrm{C} 01 \mathrm{~T} 42 & 1 & 5 \\ \text { COMPONENT } & \mathrm{CODE} & 202562555 \\ \text { SC50A } & 1 & 8 \\ \text { 5KY558AAl } & 1 & \\ \text { COMPONENT } & \text { CODE } 2215622 \\ \text { COMPONENTS IN SYSTEM CODE 26121 }\end{array}$

FS971

COMPONENT CODE 5000140

$\begin{array}{lll}\text { Hour } & .045838 & 21.8 \\ \text { Hour } & .045858 & 21.8 \\ \text { Hour } & .045810 & 43.6 \\ \text { Hour } & .177506 & 22.6 \\ \text { Hour } & .045676 & 21.4 \\ \text { Hour } & .000168 & \\ \text { Hour } & .045844 & 20.7\end{array}$

10

10

6

6

7

28

22.5

25

25

FIGURE C. 6

TYPE 2 OUTPUT DATA SHEET CURRENT SUMMARY OF FAILURE RATE DATA 


\section{APPENDIX D}

SELECTED FAILURE RATE DATA 
APPENDIX D

SELECTED FAILURE RATE DATA

This appendix presents a list of failure rate data in Table $D .1$ for selected electrical, electronic, and mechanical parts or components. In Table D. 2 a list of references is given for additional information on values given in Table D. 1 as well as for additonal failure rate data, particularly on electrical and electronic parts.

The values given in Table D. I are provided to indicate the general level for failure rates on the parts listed. They are not necessarily the best values for a particular application since the rates must be adjusted according to the operational and environmental stresses imposed on the parts. Factors accounting for these stress factors may be found in most of the references listed in Table D. 2.

As a final comment, it should be noted that the data in Table D. 1 and the references cited in Table D. 2 are primarily derived from equipment associated with electronic and specialized electrical systems found in military, aerospace, and nuclear reactor instrumentation systems. Data for heavy electrical and mechanical equipment found in nuclear power plants is sparse to nonexistent. 


\section{REFERENCES}

1. Green, A. E. and A. J. Bourne, "Safety Assessment with Reference to Automatic Protective Systems for Nuclear Reactors, Part 3, " AHSB(S)R117, UKAEA, 1966.

2. Earles, D. R., "Reliability Application and Analysis Guide," MI-60-54 (Rev. 1), The Martin Company, July 1961.

3. Military Standardization Handbook "Reliability Stress and Failure Rate Data for Electronic Equipment, "MIL-HDBK-217A, Department of Defense, December 1, 1965.

4. Schmudde, A. A., "Engine-Generator Sets Meet Strict Reliability Limits, " Power, April 1967.

5. McGraw-Hill Book Company, "RADC Reliability Notebook," RADC-TR-58-111, October 30, 1959. 
TABLE D-1

FAILURE RATE DATA ON SELECTED

ELECTRONIC, ELECTRICAL, AND MECHANICAL EQUIPMENT

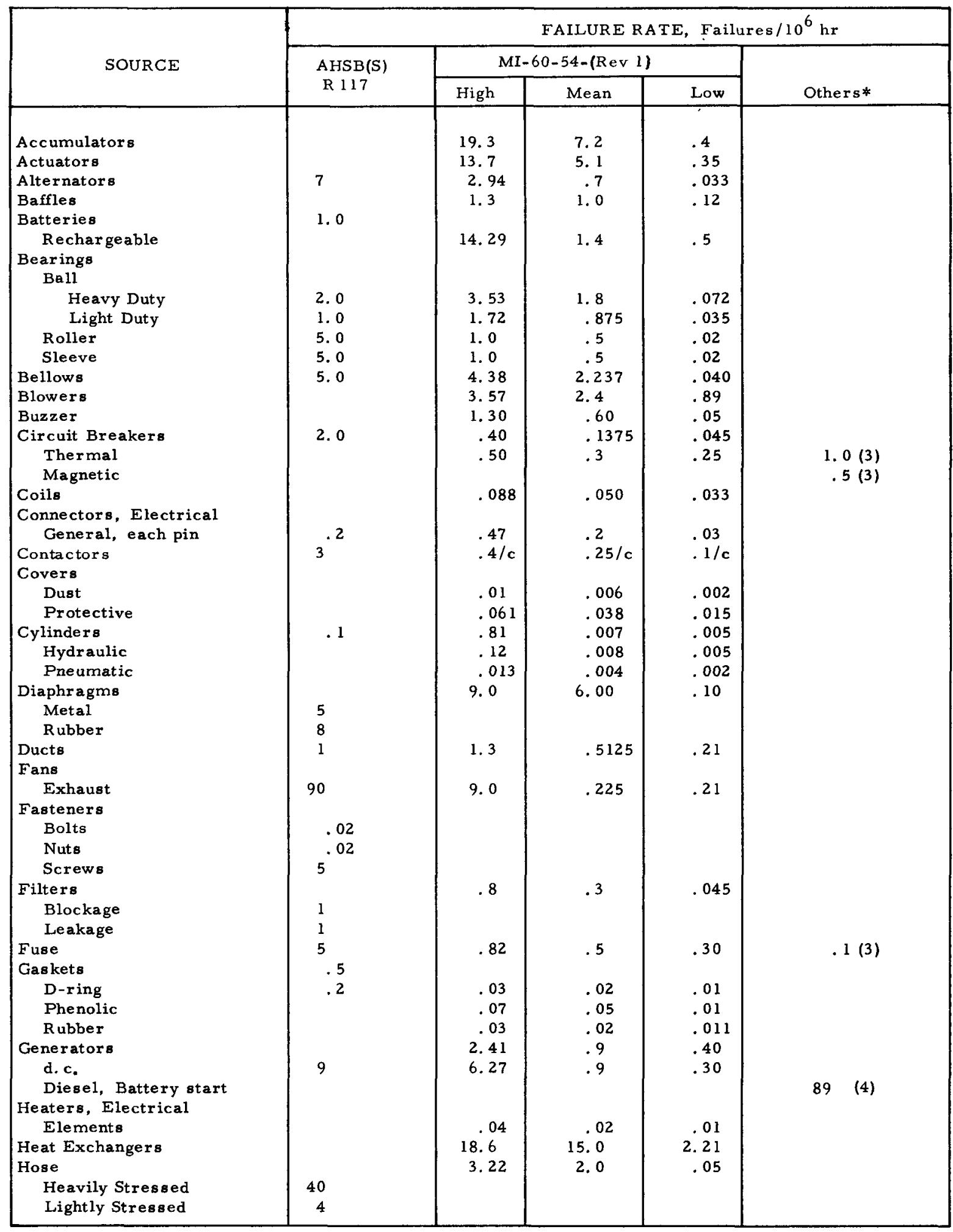

* See Table D-2 for reference sources 
TABLE D. 1 (continued)

\begin{tabular}{|c|c|c|c|c|c|}
\hline \multirow{3}{*}{ SOURCE } & & \multicolumn{4}{|c|}{ FAILURE RATE, Failures $/ 10^{6} \mathrm{hr}$} \\
\hline & \multirow{2}{*}{$\begin{array}{c}\text { AHSB(S) } \\
\text { R } 117\end{array}$} & \multicolumn{3}{|c|}{ MI-60-54-(Rev 1$)$} & \multirow[b]{2}{*}{ Others } \\
\hline & & High & Mean & Low & \\
\hline \multicolumn{6}{|l|}{ Instruments } \\
\hline Electrical & & 5.77 & 1.375 & 1.35 & \\
\hline Pressure & & & & & \\
\hline Gage & 10 & 7.8 & 4.0 & 0.135 & \\
\hline $\begin{array}{c}\text { Sensor } \\
\text { Temperature }\end{array}$ & & 6.6 & 3.5 & 1.7 & \\
\hline Bulb & & 3.30 & 1.0 & 0.05 & \\
\hline Sensor & & 6.4 & 3.3 & 1.5 & \\
\hline Metera (moving coil) & 3 & & & & \\
\hline Recorders & 25 & & & & \\
\hline Lamps & & 35.0 & 8.625 & 3.45 & \\
\hline Fiuorescent & 10 & & & & \\
\hline Incandes cent & & 32.0 & 8.0 & 5.20 & $1.0(3)$ \\
\hline Indicator & 5 & & & & \\
\hline Neon & 2 & 18.8 & 10.25 & 4.50 & $.2(3)$ \\
\hline Motor 8 & 10 & 7.5 & .625 & .15 & \\
\hline Blower & & 5.5 & .2 & .05 & \\
\hline Electrical & & .58 & .3 & .11 & \\
\hline Hydraulic & & 7.15 & 4.3 & 1.45 & \\
\hline Servo & & .35 & .23 & .11 & \\
\hline Stepper & 5 & .71 & .3 .7 & .22 & \\
\hline \multicolumn{6}{|l|}{ Mechanism, Power Transmittal } \\
\hline Belts & 40 & 15.0 & 3.875 & .142 & \\
\hline Clutches & & 1.1 & .4 & .06 & \\
\hline Friction & 3 & & & & \\
\hline Magnetic & 6 & .93 & .6 & .45 & \\
\hline Slip & & .94 & .3 & .07 & \\
\hline Coupling & 5 & & & & \\
\hline Flexible & & 1.348 & .6875 & .027 & \\
\hline Rigid & & .049 & .025 & .001 & \\
\hline Gear & & .20 & .12 & .0118 & \\
\hline Helical & 10 & .098 & .05 & .002 & \\
\hline Spur & 1 & 4.3 & 2.175 & .087 & \\
\hline Shafts & & .62 & .35 & .15 & \\
\hline Heavily Stressed & .2 & & & & \\
\hline Lightly Stressed & .02 & & & & \\
\hline Rack and Pinion & 2 & & & & \\
\hline Mounts, Resilient & 9 & 1.60 & .875 & .20 & \\
\hline \multicolumn{6}{|l|}{ Orifices } \\
\hline Fixed & & 2. 11 & .15 & .01 & \\
\hline Variable & & 3.71 & .55 & .045 & \\
\hline Pumps & & 24.3 & 13.5 & 2.7 & \\
\hline \multirow{2}{*}{\multicolumn{6}{|c|}{ Piping }} \\
\hline & & & & & \\
\hline Pipes & .2 & & & & \\
\hline Pipe joints & .5 & & & & \\
\hline Union and Junctions & .4 & & & & \\
\hline \multicolumn{6}{|l|}{ Pressure Vessels } \\
\hline General & 3 & & & & \\
\hline High Standard & 0.3 & & & & \\
\hline Regulators & & 5.54 & 2.14 & .70 & \\
\hline Flow and Pressure & & 5.54 & 2.14 & .70 & \\
\hline \multirow{2}{*}{\multicolumn{6}{|c|}{ Relays }} \\
\hline & & & & & \\
\hline General & & $.48 / \mathrm{c}$ & $.25 / \mathrm{c}$ & $.10 / \mathrm{c}$ & \\
\hline Each Coil & .3 & & & & \\
\hline Each Contact Pair & .2 & & & & \\
\hline High Speed & 5 & & & & \\
\hline Heavy Duty & 5 & $.81 / \mathrm{c}$ & $.5 / \mathrm{c}$ & $.30 / \mathrm{c}$ & \\
\hline Hermatically Sealed & .5 & $.19 / \mathrm{c}$ & $.04 / \mathrm{c}$ & $.02 / \mathrm{c}$ & \\
\hline Miniature & & $.25 / \mathrm{c}$ & $.06 / c$ & $.03 / \mathrm{c}$ & \\
\hline High Speed & & 1. $13 / \mathrm{c}$ & $.7 / \mathrm{c}$ & $.42 / \mathrm{c}$ & \\
\hline Power & & $4.10 / \mathrm{c}$ & $.3 / \mathrm{c}$ & $.15 / \mathrm{c}$ & \\
\hline P.O. Type & & & & & \\
\hline General & 2 & & & & \\
\hline Fully Tropicallized & 1 & & & & \\
\hline Restrictors & 5 & .983 & .59 & .197 & \\
\hline
\end{tabular}


TABLE D. 1 (continued)

\begin{tabular}{|c|c|c|c|c|c|}
\hline \multirow{3}{*}{ SOUR CE } & \multicolumn{5}{|c|}{ FAILURE RATE, Failures $/ 10^{6} \mathrm{hr}$} \\
\hline & \multirow{2}{*}{$\begin{array}{l}\text { AHSB(S) } \\
\text { R } 117\end{array}$} & \multicolumn{3}{|c|}{ MI-60-54-(Rev 1$)$} & \multirow[b]{2}{*}{ Others } \\
\hline & & High & Mean & Low & \\
\hline \multicolumn{6}{|l|}{ Seals } \\
\hline Rotating & 7 & 1.12 & .7 & .25 & \\
\hline Sliding & 3 & .92 & .3 & .11 & \\
\hline Solenoids & & & .05 & .036 & \\
\hline \multicolumn{6}{|l|}{$\begin{array}{l}\text { Springs } \\
\quad \text { Heavily tressed }\end{array}$} \\
\hline & ${ }^{1} .2$ & & & & \\
\hline Hair & 1 & & & & \\
\hline Calibration & & .42 & .22 & .009 & \\
\hline $\begin{array}{l}\text { Creep } \\
\text { Breakage }\end{array}$ & 2 & & & & \\
\hline \multirow{2}{*}{\multicolumn{6}{|c|}{$\begin{array}{l}\text { Switches } \\
\text { General, each contact }\end{array}$}} \\
\hline & .2 & - Sor & & & \\
\hline Micro & 2 & $.50 / c$ & $.25 / \mathrm{c}$ & $.09 / \mathrm{c}$ & \\
\hline Push Button & .5 & $.11 / c$ & $.063 / c$ & $.043 / \mathrm{c}$ & \\
\hline Rotary & 2 & $.660 / c$ & $.175 / c$ & $.118 / \mathrm{c}$ & \\
\hline Thermal & & $.261 / \mathrm{c}$ & $.161 / \mathrm{c}$ & $.114 / \mathrm{c}$ & \\
\hline Heater & 1 & & & & \\
\hline \multirow{2}{*}{\multicolumn{6}{|c|}{ Toggle }} \\
\hline & & & & & \\
\hline $\begin{array}{l}\text { General } \\
\text { Each Pair Contact }\end{array}$ & ${ }^{1} .2$ & & & & \\
\hline \multirow{2}{*}{\multicolumn{6}{|c|}{$\begin{array}{l}\text { Synchros } \\
\text { Tanks }\end{array}$}} \\
\hline & & .27 & .15 & .083 & \\
\hline Pressure, small & & .324 & .18 & .10 & \\
\hline High Pressure, small & & .144 & .08 & .044 & \\
\hline Tachometers & 5 & .55 & .3 & .25 & \\
\hline \multicolumn{6}{|l|}{$\begin{array}{l}\text { Transducers } \\
\text { Liquid Level }\end{array}$} \\
\hline Liquid Level & & 3.73 & 2.6 & 1.47 & \\
\hline \multicolumn{6}{|l|}{ Light } \\
\hline Photoelectric Cells & 15 & & & & \\
\hline \multirow{2}{*}{\multicolumn{6}{|c|}{ Radioactivity }} \\
\hline & & & & & \\
\hline $\begin{array}{l}\text { Beta Ray } \\
\text { Ion Chamber and Leads }\end{array}$ & 5 & 21.3 & 14.00 & 6.70 & \\
\hline \multicolumn{6}{|l|}{ Strain } \\
\hline Gage & 25 & 20.0 & 12.0 & 7.0 & \\
\hline Temperature & & 6.4 & 3.3 & 1.5 & \\
\hline Thermistors & & 28.00 & 15.0 & 10.0 & $.30(3)$ \\
\hline Thermocouples & 10 & & & & \\
\hline \multicolumn{6}{|l|}{$\begin{array}{l}\text { Thermocouples } \\
\text { Transformers }\end{array}$} \\
\hline \multirow{2}{*}{\multicolumn{6}{|c|}{$\begin{array}{l}\text { General, each winding } \\
\text { Mains }\end{array}$}} \\
\hline & & & & & \\
\hline Encapsulated & 5 & & & & \\
\hline \multirow{2}{*}{\multicolumn{6}{|c|}{ Power }} \\
\hline & & & & & \\
\hline Low Voltage & & .60 & .3 & .13 & \\
\hline High Voltage & & 1.88 & .94 & .407 & \\
\hline & 1.5 & & & & \\
\hline Low Voltage & & .235 & .15 & .065 & \\
\hline & & & & & \\
\hline Variable & 1 & .31 & .1 & .035 & \\
\hline A. F. & 0.3 & .04 & .02 & .01 & \\
\hline I. F. & 1 & .31 & .1 & .035 & \\
\hline AIEE Class & & & & & (3) \\
\hline 0 & & & & & $.21-10.00$ \\
\hline$A$ & & & & & $.20-19.50$ \\
\hline B & & & & & $\begin{array}{r}.22-12.00 \\
21-18.50\end{array}$ \\
\hline $\begin{array}{l}\mathrm{H} \\
\mathrm{C}\end{array}$ & & & & & $.21-18.500$ \\
\hline
\end{tabular}


TABLE D. 1 (continued)

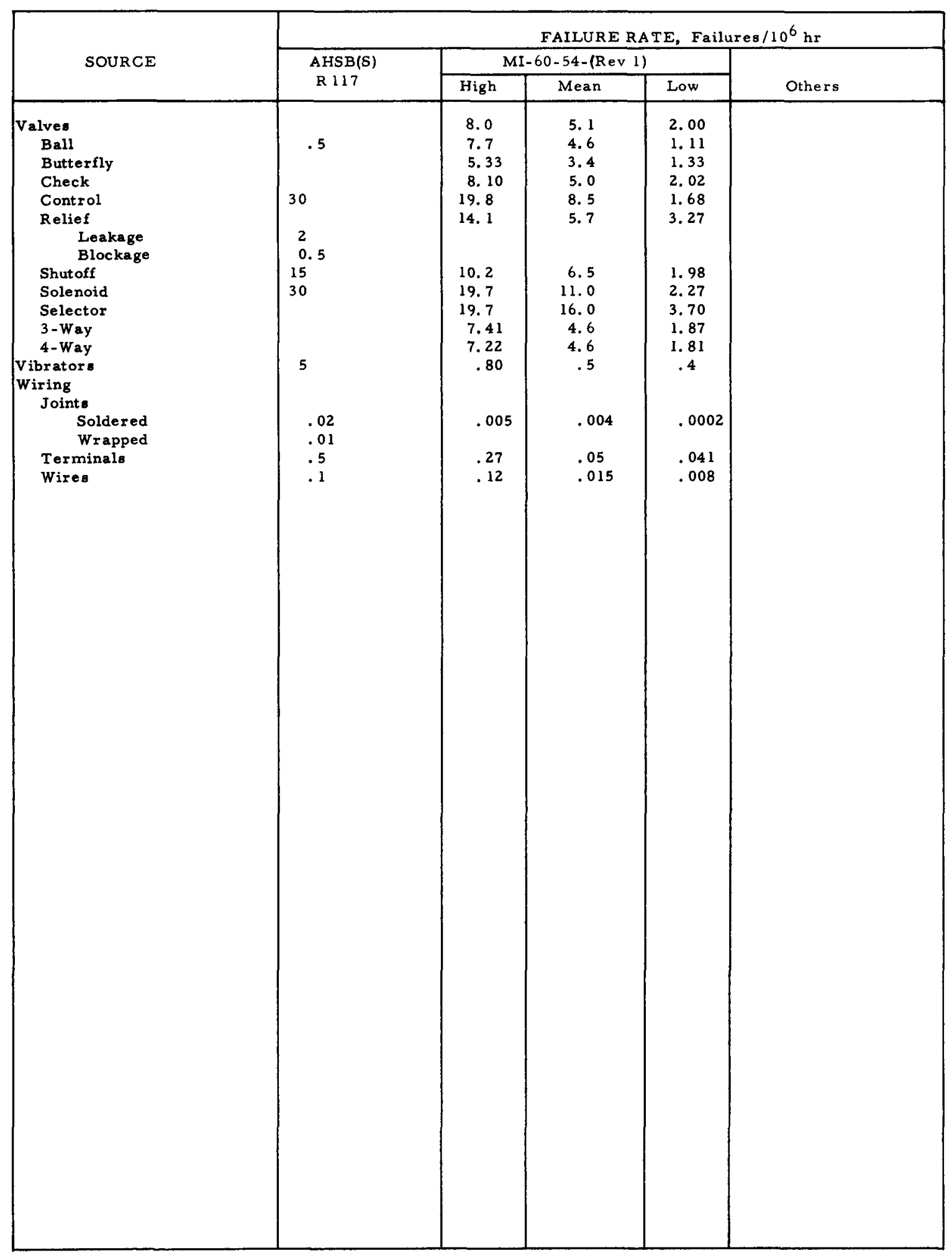


TABLE D. 2

\section{REFERENCE SOURCES FOR FAILURE RATE DATA}

\begin{tabular}{|c|c|c|c|c|c|c|c|c|c|}
\hline & Author & Title & & & & $\begin{array}{r}\text { Type } \\
\text { Electrical }\end{array}$ & $\begin{array}{l}\text { of Equipme } \\
\text { ETectrical }\end{array}$ & Tincluded & Mechanical \\
\hline $\begin{array}{l}\text { Reterence } \\
\text { No. }\end{array}$ & Author & $17 t 1 \mathrm{e}$ & or Publication & $\begin{array}{l}\text { Date or } \\
\text { Publication }\end{array}$ & Electronic & $\begin{array}{l}\text { Eiectrical } \\
\text { Light }\end{array}$ & $\begin{array}{l}\text { Electrical } \\
\text { Heavy }\end{array}$ & $\begin{array}{c}\text { Mechanical } \\
\text { Light }\end{array}$ & $\begin{array}{c}\text { Mechanica } \\
\text { Heavy }\end{array}$ \\
\hline 1 & A. E. Green and A. J Bourne & $\begin{array}{l}\text { Safety Assessment with Reference to } \\
\text { Automatic Protective Systems for Nuclear } \\
\text { Reactors, Part } 3\end{array}$ & AHSB (S) R 117 & 1966 & $\mathrm{x}$ & $\mathbf{x}$ & & $\mathbf{x}$ & \\
\hline 2 & D R. Earles & Reliabilaty Application and Analysis Gude & MI-60-54-(Rev 1$)$ & July 1961 & $\mathbf{x}$ & $\mathrm{x}$ & & $\mathbf{x}$ & \\
\hline 3 & Department of Defense & $\begin{array}{l}\text { Reliability Stress and Fallure Rate Data } \\
\text { for Electromic Equipment }\end{array}$ & MIL-HDBK-217A & Dec 1,1965 & $\mathbf{x}$ & $\mathbf{x}$ & & $\mathrm{x}$ & \\
\hline 4 & A. A. Schmudde & $\begin{array}{l}\text { Engine-Generator Sets Meet Strict } \\
\text { Relıabılity Limits }\end{array}$ & Power (Magazine) & April 1967 & & & $\mathrm{x}$ & & $\mathbf{x}$ \\
\hline 5 & McGraw-Hill Book Company & RADC Reliability Notebook & RADC-TR-58-111 & Oct 30,1959 & $\mathbf{x}$ & $\mathrm{x}$ & & $\mathbf{x}$ & \\
\hline 6 & Radio Corporation of America & $\begin{array}{l}\text { Rehability Stress Analysis for Electronic } \\
\text { Equipment }\end{array}$ & TR59-416-I & Jan. 15, 1959 & $\mathbf{x}$ & $\mathrm{x}$ & & $\mathrm{x}$ & \\
\hline 7 & $\begin{array}{l}\text { R. L. Harrington and } \\
\text { R. R. Riddick, Jr. }\end{array}$ & $\begin{array}{l}\text { Reliabuluty Engineering Applied to the } \\
\text { Marine Industry }\end{array}$ & $\begin{array}{l}\text { Marıne Technology } \\
\text { (Journal) }\end{array}$ & October 1964 & & & $\mathbf{x}$ & & $\mathbf{x}$ \\
\hline 8 & ARINC Research Corporation & $\begin{array}{l}\text { Prediction of Field Reliability for } \\
\text { Airborne Electronic Systems }\end{array}$ & $\begin{array}{l}\text { Publication } \\
\text { No. 203-1-344 }\end{array}$ & Dec 31,1962 & $\mathbf{x}$ & $\mathrm{x}$ & & $\mathrm{x}$ & \\
\hline 9 & D. R. Earles and M. F. Eddzns & Fanlure Rates & $\begin{array}{l}\text { Proceedings, Ninth } \\
\text { National Symposium on } \\
\text { Reliability and Quality } \\
\text { Control }\end{array}$ & January 1963 & $\mathbf{x}$ & $\mathbf{x}$ & & $\mathbf{x}$ & \\
\hline 10 & Vatro Corporation & $\begin{array}{l}\text { Reliability Prediction and Measurement } \\
\text { of Shipboard Electronic Equipments }\end{array}$ & Report No. 98 & Apral 15, 1957 & $\mathbf{x}$ & $\mathbf{x}$ & & $\mathbf{x}$ & \\
\hline 11 & $\begin{array}{l}\text { U.S. Naval Flset M1ssile Systems } \\
\text { Analys1s and Evaluation Group, } \\
\text { Corona, Calıfornaa }\end{array}$ & $\begin{array}{l}\text { Bureau of Naval Weapons Fallure Rate } \\
L \text { fandbook (Avallable only qualified } \\
\text { contractors and government agencies) }\end{array}$ & SP-63-470 & $\begin{array}{l}\text { Revised } \\
\text { Quarterly }\end{array}$ & $\mathrm{x}$ & $\mathrm{x}$ & $\mathrm{x}$ & $\mathrm{x}$ & $\mathrm{x}$ \\
\hline 12 & ARINC Research Corporation & Reliability Engineering (pp. 308-310) & Prentice-Hall, Inc. & 1964 & $\mathbf{x}$ & $\mathrm{x}$ & & $\mathrm{x}$ & \\
\hline
\end{tabular}


APPENDIX E

SAFTE-I SOURCE PROGRAM LISTING 
MAIN

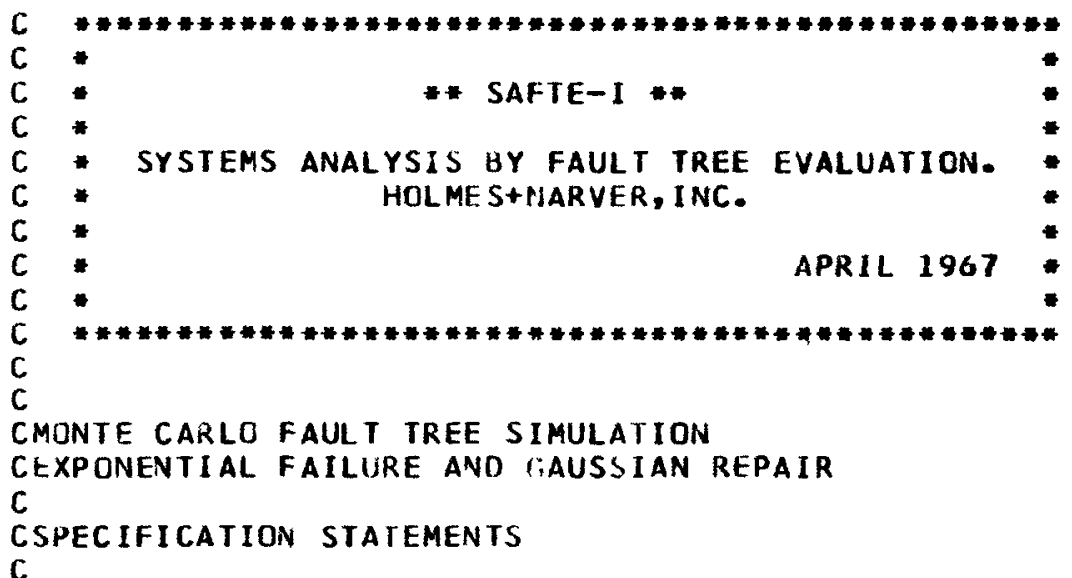

C

DIMENSION ARRAY $(90,90)$, XMTTF $(90), X M T T R(90), T T F(90), T T R(90), X I N D(90$ 1), DUMM(90), SIG (90), SIG $1(90)$, SIG2(90), CONS $(90)$, TF $(90)$, SYSPDF $(250)$, 2PTHCDF (20), PTHPDF $(20,250)$, PSEUD $(90), \operatorname{VARAY}(3,500), K 1(3), I S U M(3)$, 3BIN $(100)$

C 4.COMP (90)

C LOGICAL X(90),Y(90),A(90),B(90),G(90),TOP

C

COMMON NTRI AL, IMX, TMAX, NOINT, SIG, SIGI, SIGZ, AA, BB, CC, X, Y, A, B, G, TOP, 1 ARRAY, XMTTF, XMTTR, TTF, TTR,XIND, DUMM, CONS, TF, SYSPDF, PTHCDF, PTHPDF, 2SYSCDF, NCONS, COE, ARG, DELT, DUMMY, N,I JK, WHT

$3, I, J, I N D, K K, P S E U D, N P T H$

$4, K 1, I S U M$, VARAY, BIN

C

$5, \operatorname{COMP}$

203 READ $(5,1)$ NTRIAL, IMX,NOINT, NCONS, NP IH

READ $(5,2)$ TMAX, AA,BB,CC

$\operatorname{READ}(5,2)(X M T T F(I), I=1, I M X)$

READ (5.2) (XMTTR(I), I=1, IMX)

READ (5,2) (SIG(I), I =1,IMX)

$\operatorname{READ}(5,2)(\operatorname{CONS}(1), 1=1, N C O N S)$

$\mathrm{C}$

$\operatorname{READ}(5,1)(K 1(I), I=1,3)$

CNTR I AL $=$ NUMBER OF TRIALS

CIMX = NUMBER OF COMPONENTS (MAX. 90)

CNOINT=NUMBER OF TIME INTERVALSIMAX. 250 )

CNCONS=NUMBER OF SIDE CONSTRAINIS (MAX. 90)

CNPTH=ND. OF CRITICAL PATHS

CIMAX =LENGTH OF TIME BEYOND WHICH TRIAL IS TERMINATED(HOURS)

CAA $B$ IASING PARAMETER FO TIME TO FAILURE CALCULATIONS. VALUES OF AA GREAT C TER THAN 1.0 CAUSE SHOTR TIFS TO BE EMPHASIZED.

CBB=BIASING PARAMETER FOR TIME TO REPAIR CALCULATION. VALUES OF BB GREAT C ER THAN 1.0 CAUSE SHORT TTRS TO BE DE-EMPHASIZED.

CCC $=$ BIASING PARAMETER FDR TIME TO REPAIR CALCULATION.VALUES OF CC GREAT

C ER THAN 1.0 CAUSE LONG TTRS TO BE EMPHASIZED.

CXMTTF=MEAN TIME TO FAILURE (HOURS).

CXMITR=MEAN TIME TO REPAIR(HOURS). 
MAIN - EFN SOURCE STATEMENT - IFN(S) -

CSIG=STANDARD DEVIATION FOR TIME TO REPAIR DISTRIBUTIONS. CCONS= SIDE CINSTRAINTS.

CKI $I)=I N T E R V A$ NOS. FOR WHICH DETAILED ERROR ANALYSIS IS PERFORMED

C

WR I TE $(6,3)$

WR ITE $(6,4)$ NTRIAL

WR ITE $(6,5)$ I MX

WR ITE $(6,6)$ NOINT

WR I TE $(6,7)$ NCONS

WR I TE $(6,275)$ NP TH

WR ITE $(6,8)$ TMAX

WR I IE $(6,9)$ AA

WR I TE $(6,10) \mathrm{BB}$

WR ITE $(6,11) \mathrm{CC}$

WR ITE $(6,3)$

WR I T E $(6,12)$

DO $13 I=1,1 M X$

WR I IE $(6,14)$ XMTTF (I),XMTTR II)

13 CONT INUE

DO $15 I=1$, IMX

$S I G I(I)=S I G(I) / B B$

$S I G 2(I)=S I G(I) * C C$

15 CONT IIIUE

WR ITE $(6,3)$

WR ITE $(6,16)$

DO $18 I=1, I M X$

WR ITE (6,17) SIG(I),SIG1(I),SIG2(I)

18 CONT INUE

WR ITE $(6,3)$

WR I TE $(6,19)$

DD $211=1$, NCONS

WR ITE $(6,20)$ CONSI I)

21 CONT INIUE

C

WR ITE $(6,401)(K 1(1), I=1,3)$

1 FORMAT (5I5)

2 FORMAT(6E12.5)

3 FORMAT ( $1 H 1)$

4 FORMAT $(B H O N T R I A L=15 / 1$

5 FORMAT ( 5 HOI MX $=15 /$ )

6 FORMAT 17 HONOINT $=[5 / 1$

7 FORMAT 17 HONCONS $=15 / 1$

8 FORMAT (6HOTMAX $=E 12.5 /)$

9 FORMAT ( 4 HOAA $=E 12.5 /$ )

10 FORMAT $(4 \mathrm{HOBB}=\mathrm{E} 12.5 /)$

11 FORMAI $(4 \mathrm{HOCC}=\mathrm{E} 12.5 /)$

12 FORMATI $27 H O$ MTTF

14 FORMATIE 12.5, 2X,E 12.5)

16 FORMATI 4010 SIG

17 FORMATIE 12,5,2X,E12,5,2X,E12,5)

MTTR /

19 FORMATI 17 HOSIDE CONSTRAINTS/)

20 FORMAT (3X,E 12.5)

275 FORMAT 23 HOND. OF CRITICAL PATHS $=15 /$ )

C

401 FORMAT $(7$ HOK $1(1)=3[5 /)$

CSET SCORING ARRAYS EQUAL TO ZERO. 
C.

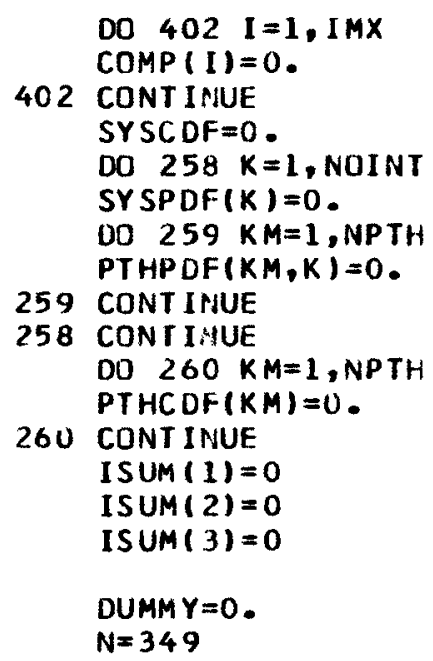

$96 \operatorname{TTR}(I)=0$.

99 CONT INUE 


$$
\text { MAIN - CFN SOURCE STATEMENT - IFN(S) - }
$$

C

C.

$$
\text { DO } 403 I=1, I M X
$$

CUETERMINE IF RIGHT OR LEFT GAUSSIAN WAS USED TO COMPUTE TTR(I). 


$$
\text { MAIN - EFN SOURCE STATEMENT - IFN(S) - }
$$

C

36 CALL LDG IC IF (TOP)GO TO 39

$J=J+1$

GO TO 40

39 CALL SUM

GO TO 100

C

CCURRENT FAILURE IS NOT FIRST OF SEQUENCE.

C

37 DO 28 IK $=1, I M X$

IK $=I K$

IF (ARRAY (IK, JK) ) $28,28,27$

28 CONT INIUE

27 IF $(X(I K)) G O$ TO 26

JK $=\mathbf{J K} \mathrm{K}-\mathbf{1}$

GO TO 38

26 IF (ARRAY (I, J)-TTF(IK)-TTR(IK) $) 25,25,202$

$25 \mathrm{JK}=J K-1$

$\mathrm{C}$

GO TO 38

CIT HAS BEEN DETERMINED THAT ONE

OF THE FIRST J FAILURES DO

CNUT OVERLAP. THE NON-DVERLAPPING COMPONENT $X(I K)$ IS

CKEPAIRED. A NEW TTF AND TTR ARE COMPUTED AND SEQUENCED INTO ARRAY(I,J). C

$202 R=E X P R N(D U M M Y)$

$\operatorname{TF}(I K)=X M T T F(I K) * R / \wedge A$

$\operatorname{TTF}(I K)=\operatorname{TTF}(I K)+\operatorname{TTR}(I K)+\operatorname{TF}(I K)$

$R=$ GAUS (N)

RI =FL IRN (DUMMY)

IF $(R 1-0.5) 22,23,23$

$23 \times I N D(I K)=1.0$

$\operatorname{TTR}(I K)=X M T T R(I K)+S I G Z(I K) * R$

COEI $=S I G 2(I K) /(A A * S I G(I K))$

ARGI $=+(-1$ 1.-AA) TF $(I K) / X M T T F(I K)-0.5 *(T T R(I K)-X M T T R(I K)) *(T T R(I K)-$

IXMTTK(IK))*(1./(SIG(IK)*SIG(IK))-1./(SIG2(IK)*SIGZ(IK)))

WHT $=$ WHT $*$ COE $1 * E X P(A R G I)$

GO TO 200

$22 \times I N D(I K)=-1.0$

$\operatorname{TTR}(I K)=X M T T R(I K)-S I G I(I K) * K$

IF(TTR(IK) $400,410,410$

$400 \operatorname{TTR}(I K)=0$.

410 CDE $I=S I G I(I K) /(A A * S I G(I K))$

$A R G 1=+(-(1 .-A A) * \operatorname{TF}(I K) / X M T T H(I K)-0.5 *(T T R(I K)-X M T T R(I K)) *(T T R(I K)-$

IXMTTR(IK)) *(1./(SIG(IK)*SIG(IK))-1./(SIGI(IK)*SIGI(IK)) )

WHT $=W H T *$ COE $1 * E X P(A R G 1)$

200 CALL SETLOG

GO TO 101

100 CONT INUE

CALL EDI I

GO TU 203

END 


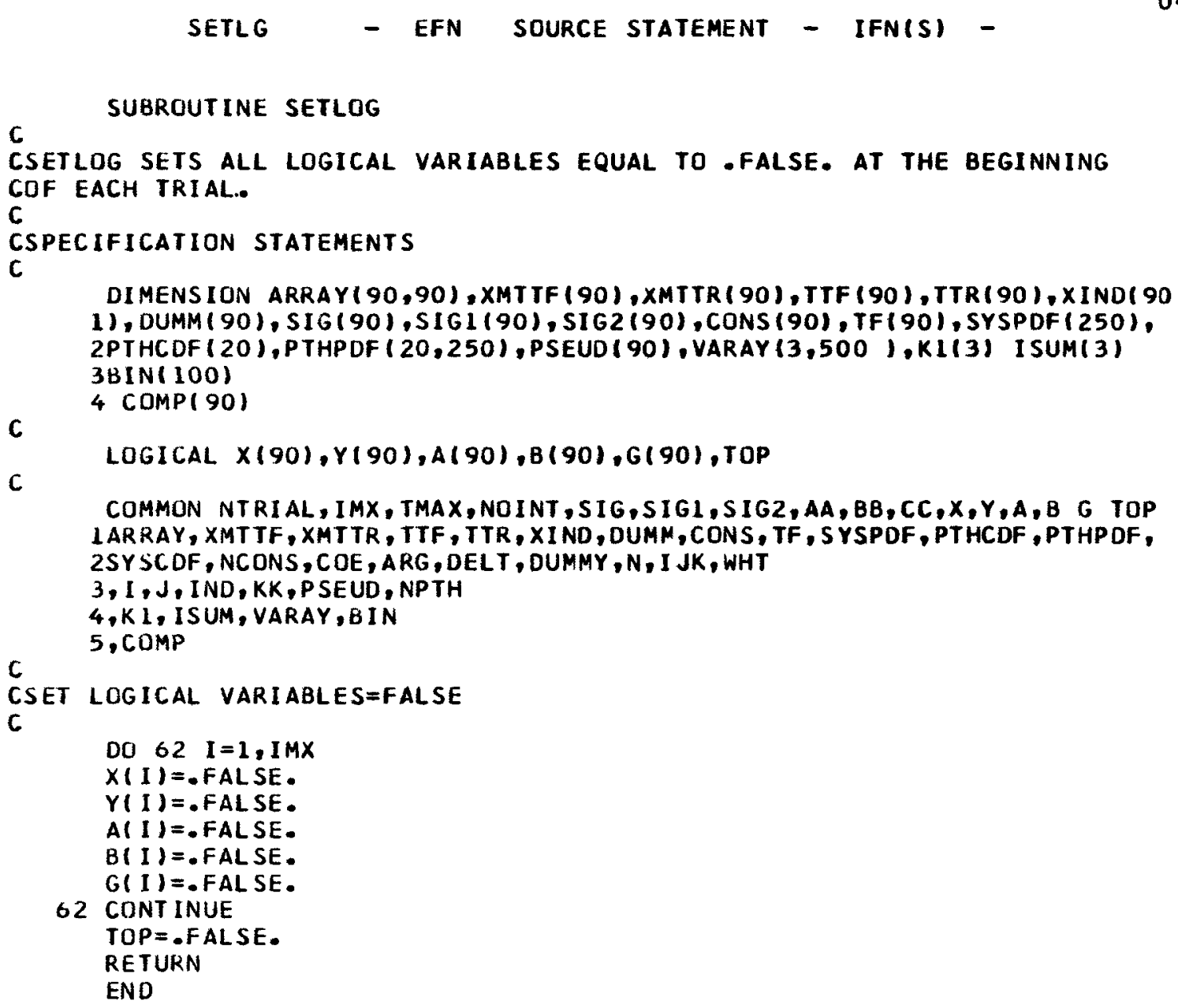




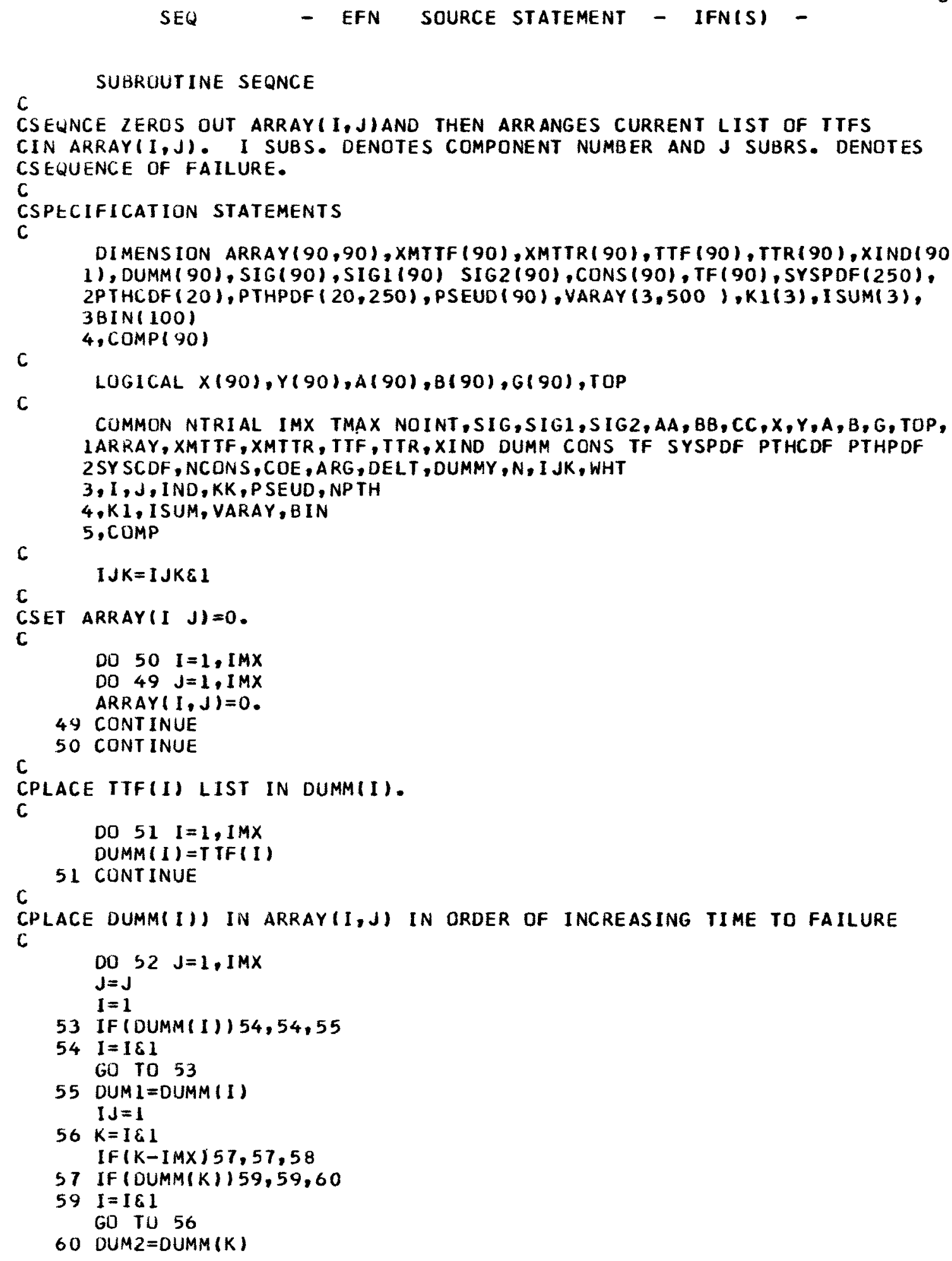




\section{SEQ - EFN SOURCE STATEMENT - IFN(S) -}

IF (DUM1-DUM2) 59,59,61

61 DUM1 = DUM2

I $J=K$

GO TO 59

58 ARRAY $(I J, J)=D U M 1$

$\operatorname{DUMM}(I J)=0$.

52 CONTINUE

RETURN

ENO 
C

SUBROUT INE LOGIC

CLHESDEN EMERGENCY POWER SYSTEM

C

CTHE FUNCTIUN OF LOGIC IS TO EXAMINE CURRENT COMPONENT FAILURES AND CUETERMINE IF THE UNDESIRED EVENT HAS OCCURED.

C

CSPECIFICATION STATEMENTS

C

DIMENSION ARRAY $(90,90), X M T T F(90), X M T T R(90), T T F(90), \operatorname{TTR}(90), X I N D(90$ 1). DUMM (90), SIG(90), 51G1 (90), S1G2(90), CONS $(90)$, TF $(90)$, SYSPDF $(250)$, 2PTHCDF( 20$)$, P THPDF $(20,250)$, PSEUD $(90)$, VARAY $(3,500), K 1(3), 1 S U M(3)$, $381 N(100)$

C $4, C O M P(90)$

C

LOGICAL $X(90), Y(90), A(90), B(90), G(90)$, TOP

COMMON NTRIAL, IMX, TMAX, NOINT, SIG, SIGI,SIG2, AA, BB, CC, X, Y, A, B, G, TOP, 1 ARRAY,XMTTF, XMTTR, TTF, TTR,XIND, DUMM, CONS, TF, SYSPDF, PTHCDF,PTHPDF, 2SYSC DF, NCONS, COE, ARG, DELT, DUMMY, $N, I$ JK, WHT

$3, I, J, I N D, K K$, PSEUD, NPTH

$4, K I, I S U M, V A R A Y, B I N$

C

5.COMP

$A(1)=X(1), 0 R \cdot X(2)$

$A(2)=X(3) \cdot O R \cdot X(4)$

$A(3)=A(1) \cdot D R \cdot A(2)$

$A(4)=X(5) \cdot 0 R \cdot X(6)$

$A(5)=A(3) \cdot O R \cdot A(4)$

$A(12)=X(13) \cdot O R \cdot A(5)$

$A(13)=X(14) \cdot O R \cdot X(15)$

$A(14)=A(12) \cdot O R \cdot A(13)$

$A(15)=X(16) \cdot O R \cdot A(14)$

c

$A(16)=X(17) \cdot O R \cdot A(15)$

$A(6)=X(2) \cdot O R \cdot X(7)$

$A(7)=X(8) \cdot O R \cdot X(9)$

$A(B)=A(6) \cdot O R \cdot A(7)$

$A(9)=X(10) \cdot O R \cdot A(8)$

$A(10)=X(11) \cdot O R \cdot A(9)$

c

$A(11)=X(12) \cdot O R \cdot A(10)$

$B(1)=\wedge(11) \cdot A N D \cdot A(16)$

$A(18)=X(19) \cdot O R \cdot X(20)$

C

$A(19)=A(18) \cdot O R \cdot B(1)$

$A(20)=x(23) \cdot O R \cdot X(24)$

$A(21)=x(21) \cdot 0 R \cdot x(22)$

$A(22)=A(20) \cdot O R \cdot A(21)$

C

$A(23)=X(25) \cdot 0 R \cdot X(26)$

$A(24)=x(27) \cdot$ OR $\cdot x(28)$

$A(35)=X(38) \cdot O R \cdot X(39)$

$A(25)=X(29), O R . A(35) \cdot O R . A(24)$

$A(26)=A(23) \cdot O R \cdot A(25)$ 


\title{
LOGIC1 - EFN SOURCE STATEMENT - IFN(S) -
}

C

$B(2)=A(19) \cdot A N D \cdot A(22) \cdot A N D \cdot A(26)$

\begin{abstract}
$A(31)=X(34) \cdot D R \cdot X(35)$
$A(29)=X(32), 0 R \cdot X(33)$

$A(30)=A(9) \cdot O R \cdot A(29)$

$A(17)=X(18) \cdot O R \cdot A(15)$

$A(27)=X(30), O R \cdot X(31)$

$A(28)=A(17) \cdot O R \cdot A(27)$

$B(3)=\wedge(28)$. AND $A(30)$

$A(32)=A(31) \cdot O R \cdot B(3)$
\end{abstract}

C

C

$A(33)=X(36) \cdot 0 R \cdot X(37)$

$A(34)=A(20) \cdot O R \cdot A(33)$

$A(36)=X(40) \cdot O R \cdot X(41)$

$A(37)=A(25) \cdot O R \cdot A(36)$

$c$

$B(4)=A(32)$. AND.A $(34) \cdot A N D \cdot A(37)$

C

$B(5)=B(2) \cdot A N D \cdot B(4)$

$A(38)=X(42) \cdot 0 R \cdot X(43)$

$A(39)=A(38) \cdot O R \cdot B(2)$

$A(40)=X(44), O R \cdot A(39)$

$A(41)=X(45), O R \cdot X(46)$

$A(42)=A(40) \cdot O R \cdot A(41)$

$A(43)=x(47) \cdot O R \cdot A(42)$

$A(44)=x(48) \cdot O R \cdot X(49)$

C

$A(45)=A(43) \cdot O R \cdot A(44)$

$A(52)=x(56) .0 R \cdot X(57)$

$A(46)=X(50), O R \cdot X(51)$

$A(47)=A(46) \cdot Q R \cdot B(4)$

$A(48)=X(52) \cdot O R \cdot A(47)$

$A(49)=X(53) \cdot D R \cdot X(54)$

$A(50)=A(48) \cdot O R \cdot A(49)$

$A(51)=A(50) \cdot O R \cdot A(55)$

6

$A(53)=A(51) \cdot O R \cdot A(52)$

c

$B(6)=A(45) \cdot A N D \cdot A(53)$

C

$B(7)=B(5) \cdot A N D \cdot B(6)$

$\mathrm{TOP}=\mathrm{B}(7)$

IND $=1$

RE TURN

END 
SUM 1

- EFN SOURCE STATEMENT - IFN(S) -

SUBROUTINE SUM

C

CTHE PURPOSE OF SUM IS TO TO RECORD TRIAL WEIGHT IN THE APPROPIATE CARAYS AT THE END OF EACH TRIAL.

C

CSPECIFICATION STATEMENTS

DI ME ISSION ARRAY $(90,90)$, XMTTF (90),XMTTR(90),TTF(90),TTR(90),XIND(90 1), DUMM (90), SIG (90), SIG1 (90), SIG2(90), CONS $(90)$, TF $(90)$, SYSPDF $(250)$, 2PTHCDF(20), PTHPOF $(20,250)$, PSEUD (90), VARAY $(3,5001, K 1(3)$, ISUM(3), 38 IN $(100)$

C 4. COMP $(90)$

C

LOGICAL X(90),Y(90),A(90),B(90),G(90),TOP

COMMON NTRI AL, I MX, TMAX, NOINT, SIG, SIGL, SIG2, AA, BB, CC, X, Y, A, B, G, TDP, 1ARRAY, XMTTF, XMTTR, TTF, TIR, XI ND, DUMM, CONS, TF, SYSPDF, PTHCDF, PTHPDF, 2SY SCOF, NCONS, COE, ARG, DELT, OUMMY, N, I JK, WHT

$3, I, J, I N O, K K, P$ SEUD,NPTH

$4, K I, I$ SUM, VARAY, BIN

C $5, C O M P$

TIME $=A R R A Y(I, J)$

CHECK $=D E L T$

DO $255 K=1$, NOINT

$K=K$

IF (T IME-CHECK) $256,256,257$

257 CHECK $=$ CHECK+DEL

255 CONT INIUE

256 SYSPDF $(K)=S Y S P D F(K)+W H T$

$S Y S C D F=S Y S C D F+W H T$

PTHPDF $(I N D, K)=P T H P O F(I N O, K)+W H T$

PTHC DF (IND) $=$ PTHCDF $(I N D)+W H T$

DO $260 \quad I=1,3$

IF $(K-K I(I)) 253,253,260$

253 I SUM $(I)=I \operatorname{SUM}(I)+1$

IF (I SUM( I) $-500 \quad 1254,254,260$

254 IND $=$ ISUM (I)

VARAY $(I, I N D)=$ WHT

260 CONT INUE

CCOMPONENT I IS RESPONS IBLE FOR CATASTROPHIC FAILURE $\mathrm{C}$

$1 \operatorname{COMP}(I)=\operatorname{COMP}(I)+W H T$

RE TURN

END 


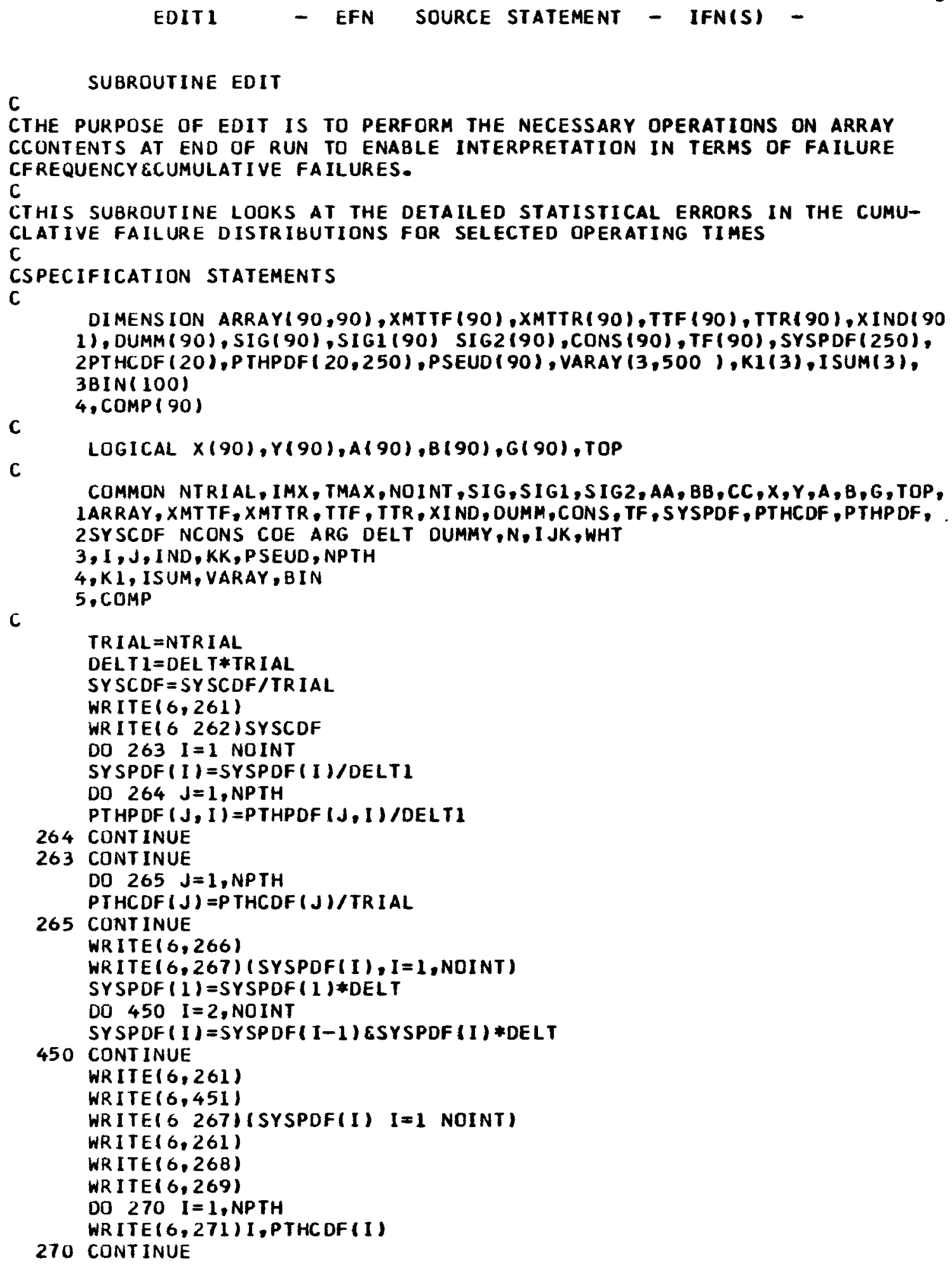


EDIT 1

211 CONTINUE

SUMI $=$ SUM $1 / T R I A L$

SUM2 $=$ SUM $2 / T R I A L$

VAR $2=S U M 1-S U M 2 *$ SUM 2

IF (VAR2) 111,111,212

111 DEV $2=0$.

GO TO 213

212 DEV $2=V A R 2 * 0.5$

213 WRITE $(6,214)$ VAR2

WRITE(6 215) DEV2

IF ( DEV 1$) 223,223,224$

223 WRITE $(6,225)$

RETURN

224 DO $218 \quad K I=1,48$

$B I N(K I)=0$.

218 CONT INUE

DO $219 J=1$, INO

VARAY $(I, J)=\operatorname{VARAY}(I, J) /(T R I A L * X M E A N * 0.25 * D E V I)$

WRITE $(6,229)$ VARAY $(I, J)$

CHECK $=$ XMEAN-5.75*DEV 1

DO $220 \mathrm{KI}=1,48$

$K I=K I$

IF(CHECK-VARAY(I J)) 221222222

$222 B I N(K I)=B I N(K I)$ EVARAY (I J)

GO TO 219

221 CHECK $=$ CHECK \&0.25*DEVI

220 CONT INUE

219 CONT INUE

WR ITE $(6,226) \mathrm{K} 1(1)$

CHECK $1=X M E A N-5.75 *$ DEV 1

CHECK2 $=X M E A N-6.0 * D E V I$

CHECK $3=$ CHECK $2 \& 0.125 *$ DEV1

WR ITE $(6,227$ !

DO $228 \mathrm{KI}=1,40$

EXACT $=1.0 /(D E V 1 * 2.508)$

EXACT $=$ EXACT $* E X P(-C H E C K 3 * C H E C K 3 /(2,0 * V A R 1))$

WRITE $(6,229)$ CHECK2,CHECK1,BINIKI), EXACT

CHECK $1=$ CHECK $1 \& 0.25 *$ DEV 1

CHECK2 $=$ CHECK2\&0.25*DEV1

CHECK $3=$ CHECK $3 \& 0.25 *$ DEV 1

228 CONT INUE

C

200 CONTINUE

261 FORMAT (1HI)

262 FORMAT (43HOPROBABILITY OF SYSTEM FAILURE BEFORE TMAX=E12.5/)

266 FORMAT (25HOSYSTEM FAILURE FREQUENCY/)

267 FORMAT (5E 12.5)

268 FORMAT (5OHOPROBABILITY OF DISCRETE PATH FAILURES BEFORE TMAX/)

269 FORMAT 24 HO PATH NO. PROBABILITY/)

271 FORMAT (16,6X,E12.5)

273 FORMAT (26HOFAILURE FREQUENCY OF PATH 14/)

281 FORMAT ( THOASTEP=E 12.5)

451 FORMAT ( 25 HOCUMULATIVE FAILURE DIST. 1 )

C

453 FORMAT (33HOCUMULATIVE FAILURE DIST.OF PATH I4/)

225 FORMAT 1 14HOEFF.ST.DEV.$=0 / 1$ 


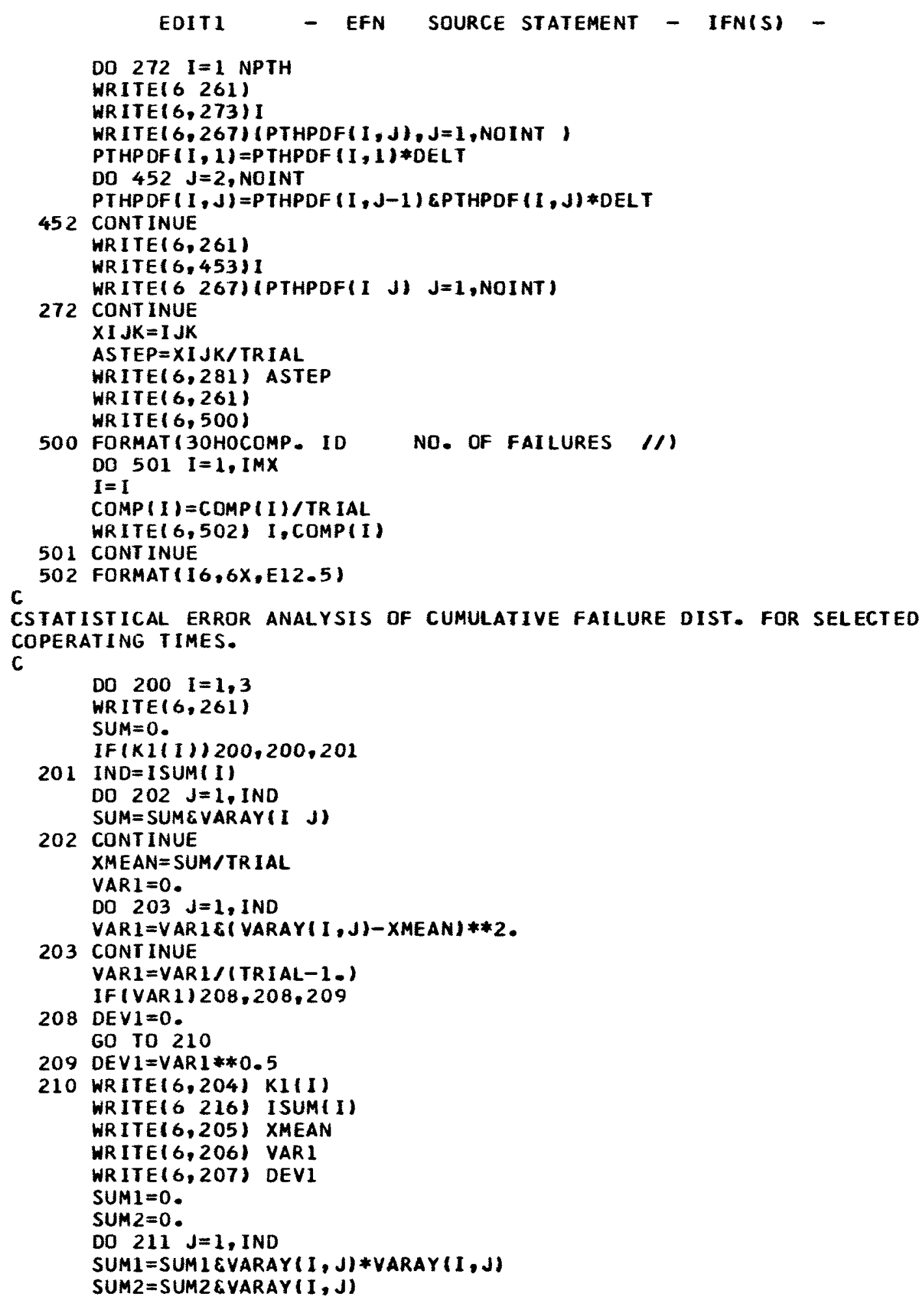

EOIT1 - EFN SOURCE STATEMENT - IFN(S) - 
204 FURMAT (3OHOERROR ANALYSIS FOR INTERVAL I5//)

205 FORMAT $(16$ HOEFF. MLAN VALUE $=E 12.5 /)$

206 FORMAT $(14$ HOEFF.VARIANCE $=E 12.5 /)$

207 FORMAT (24HOEFF.STANUARD DEVIATION=E12.5/)

214 FORMAT $(18$ BOMON.CAR . VAR IANCE $=E 12.5 /)$

215 FORMAT $(17$ HOMON.CAR.ST . DEV. $=E 12.5 /)$

216 FORMAT (24HONO.OF NON-ZERO SAMPLES $=$ I5/)

226 FORMAT ( 37 HONCRMALIZED SAMPLE DIST.FOR INTERVAL I5/)

227 FORMAT $(57$ HO INTERVAL SAMPLE 1 (/)

229 FORMAT $12 \mathrm{E} 13.53 \times 2 \mathrm{E} 12.51$

RETURN

END 


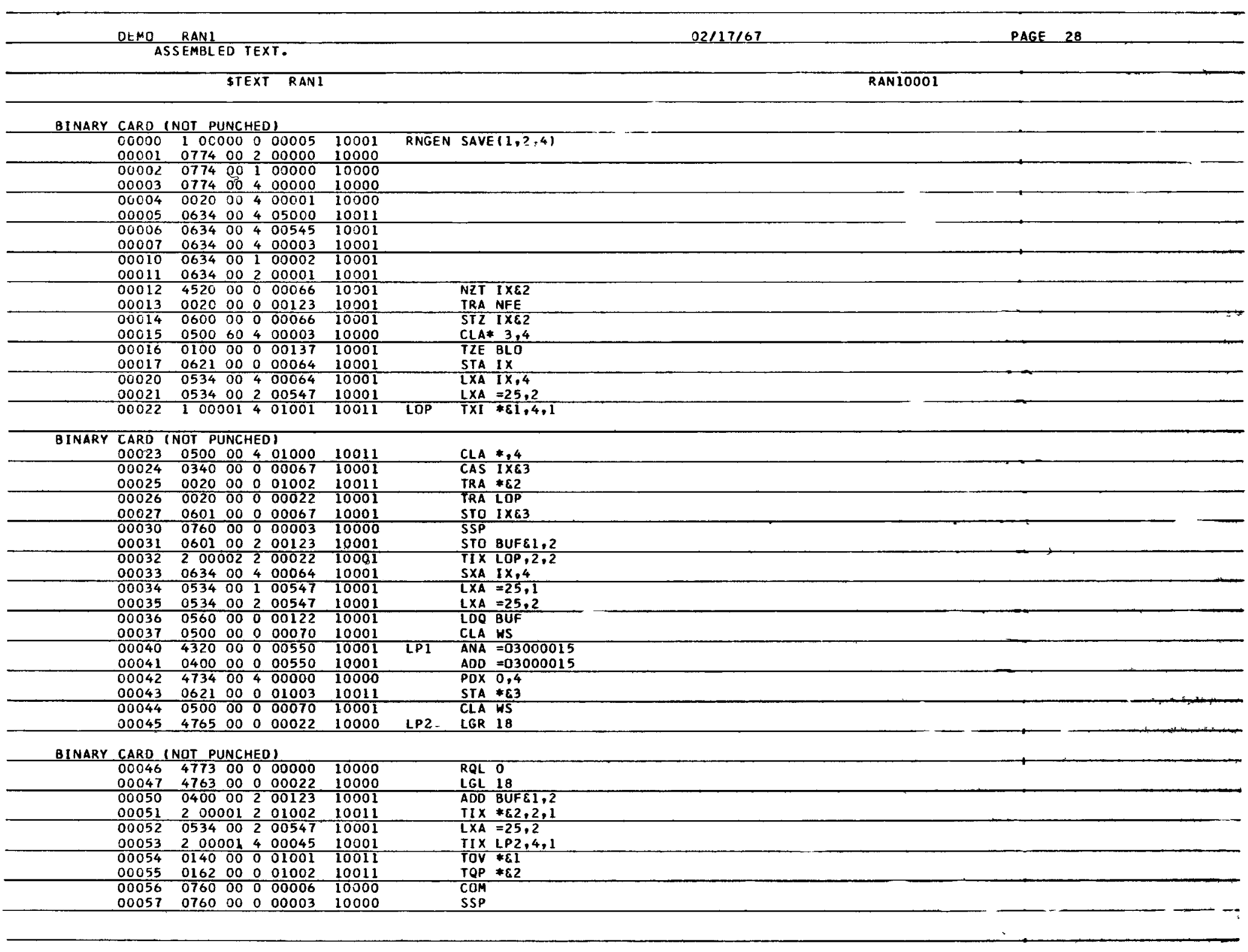




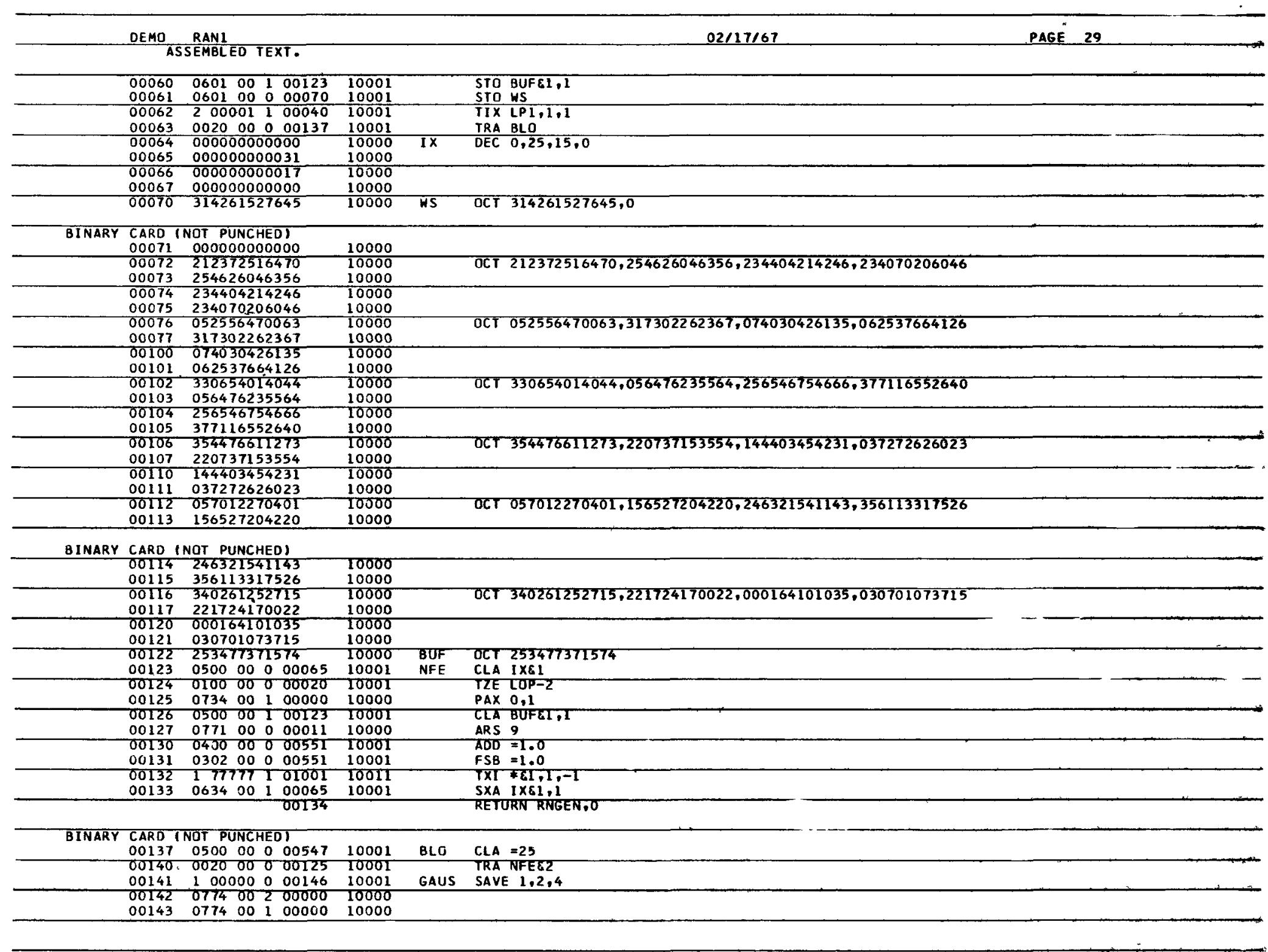




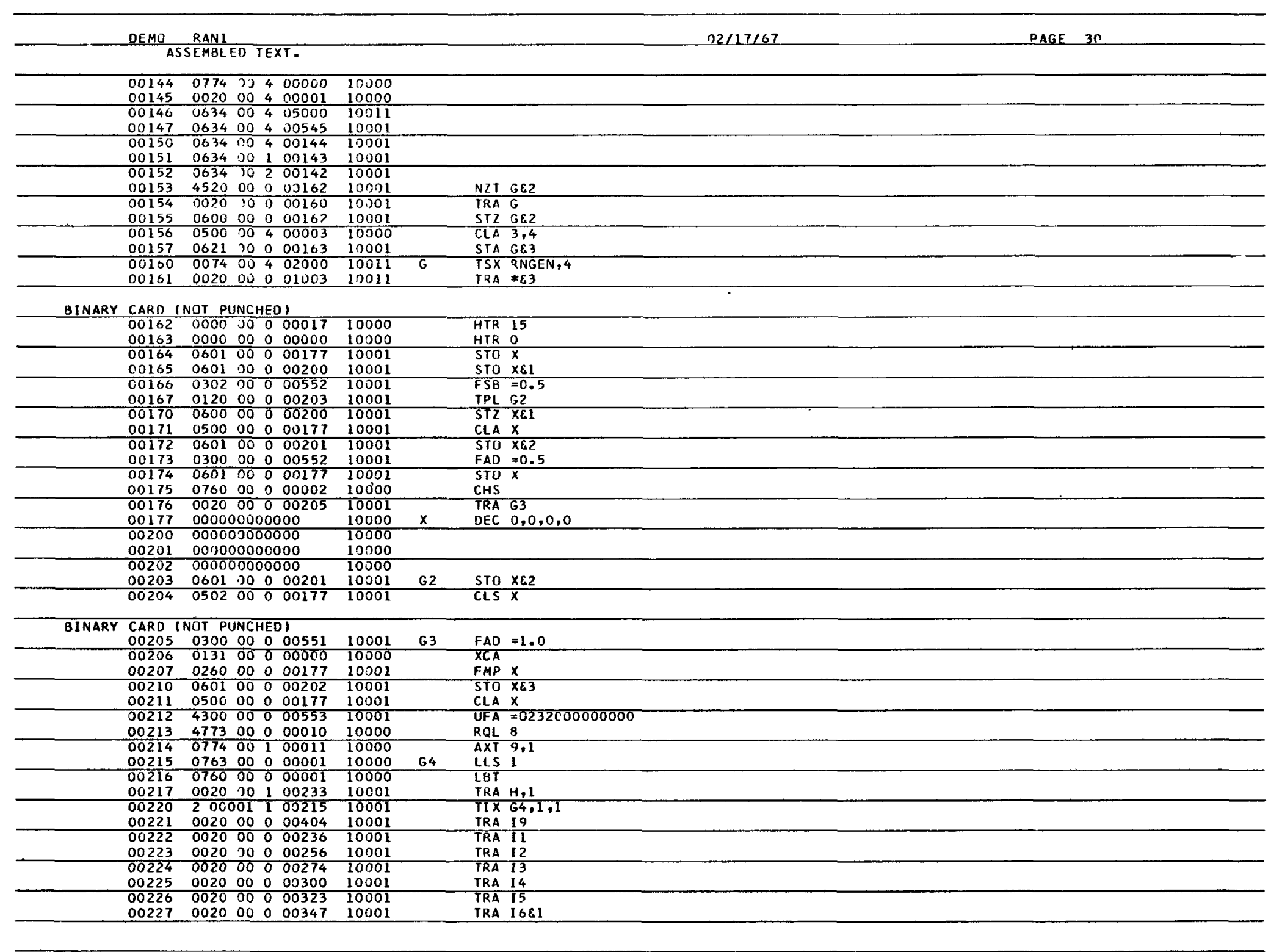




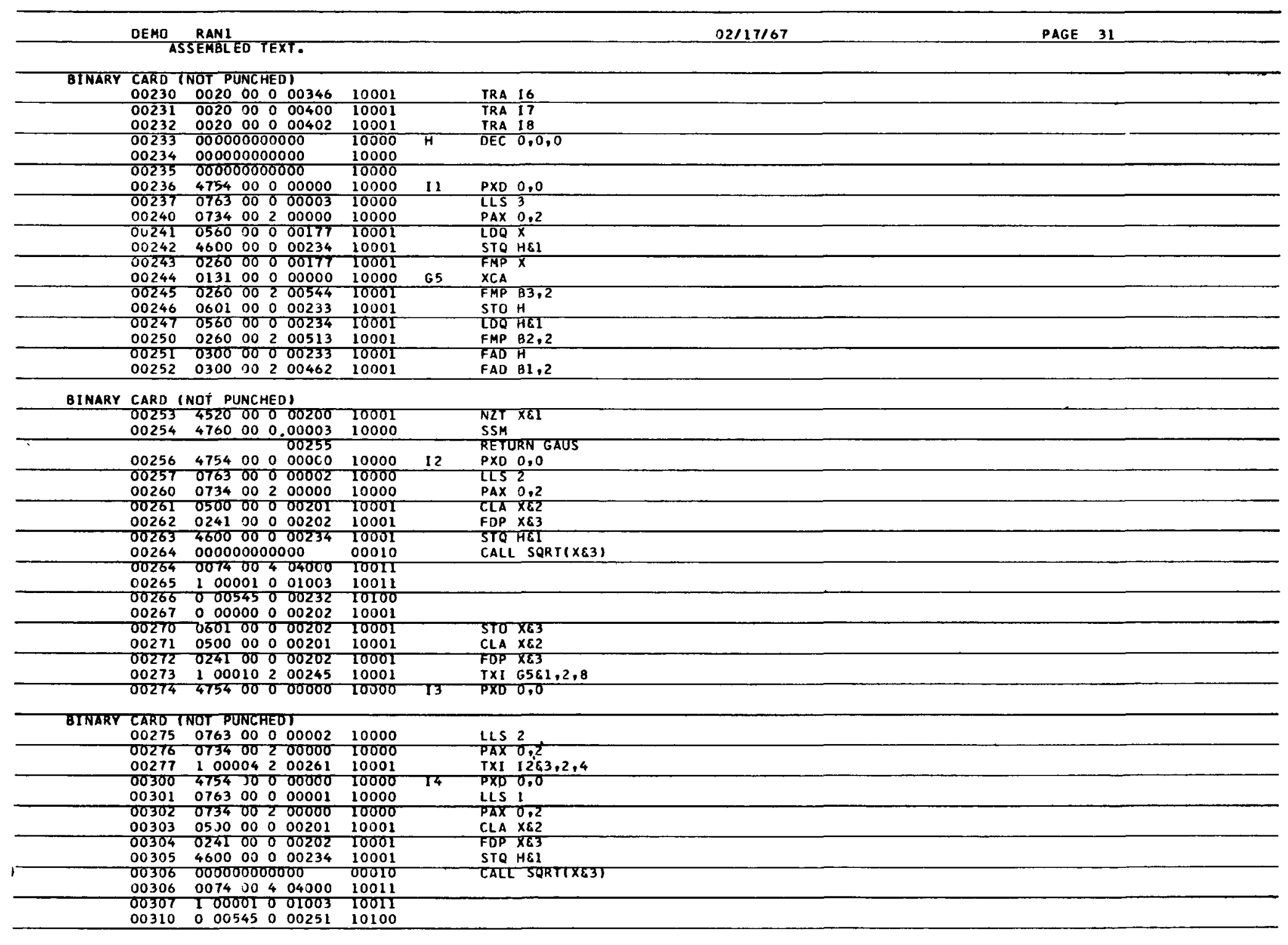




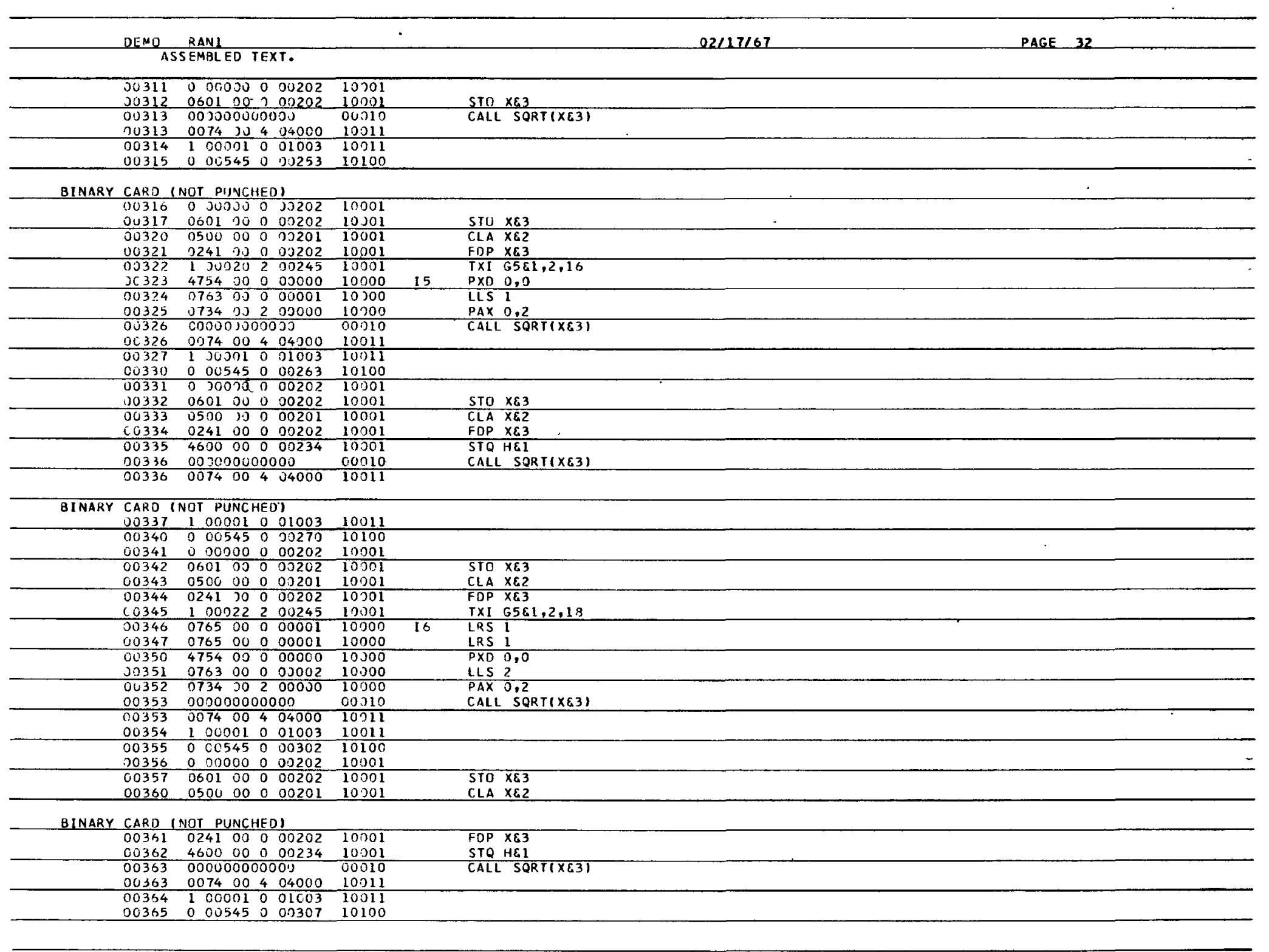




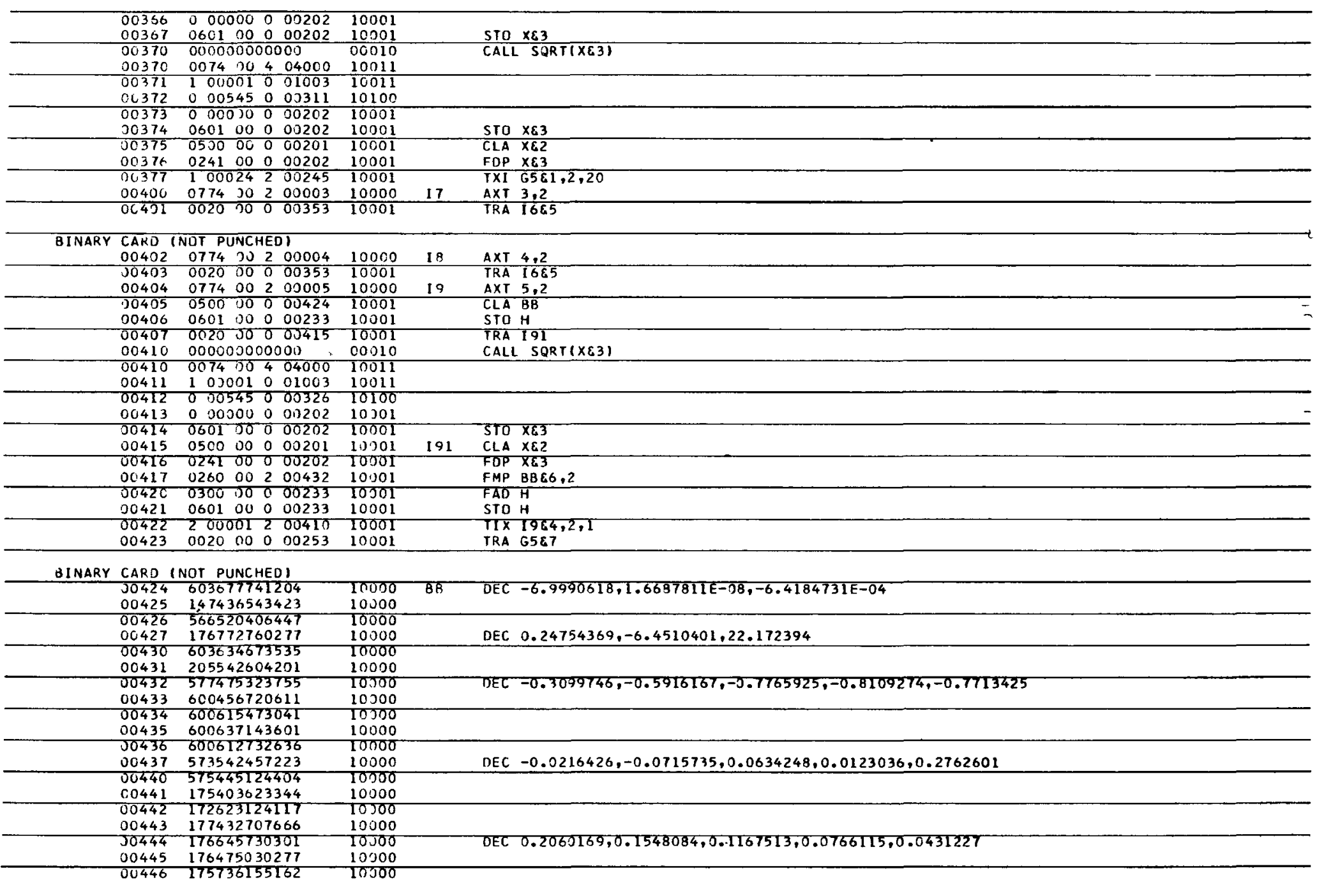




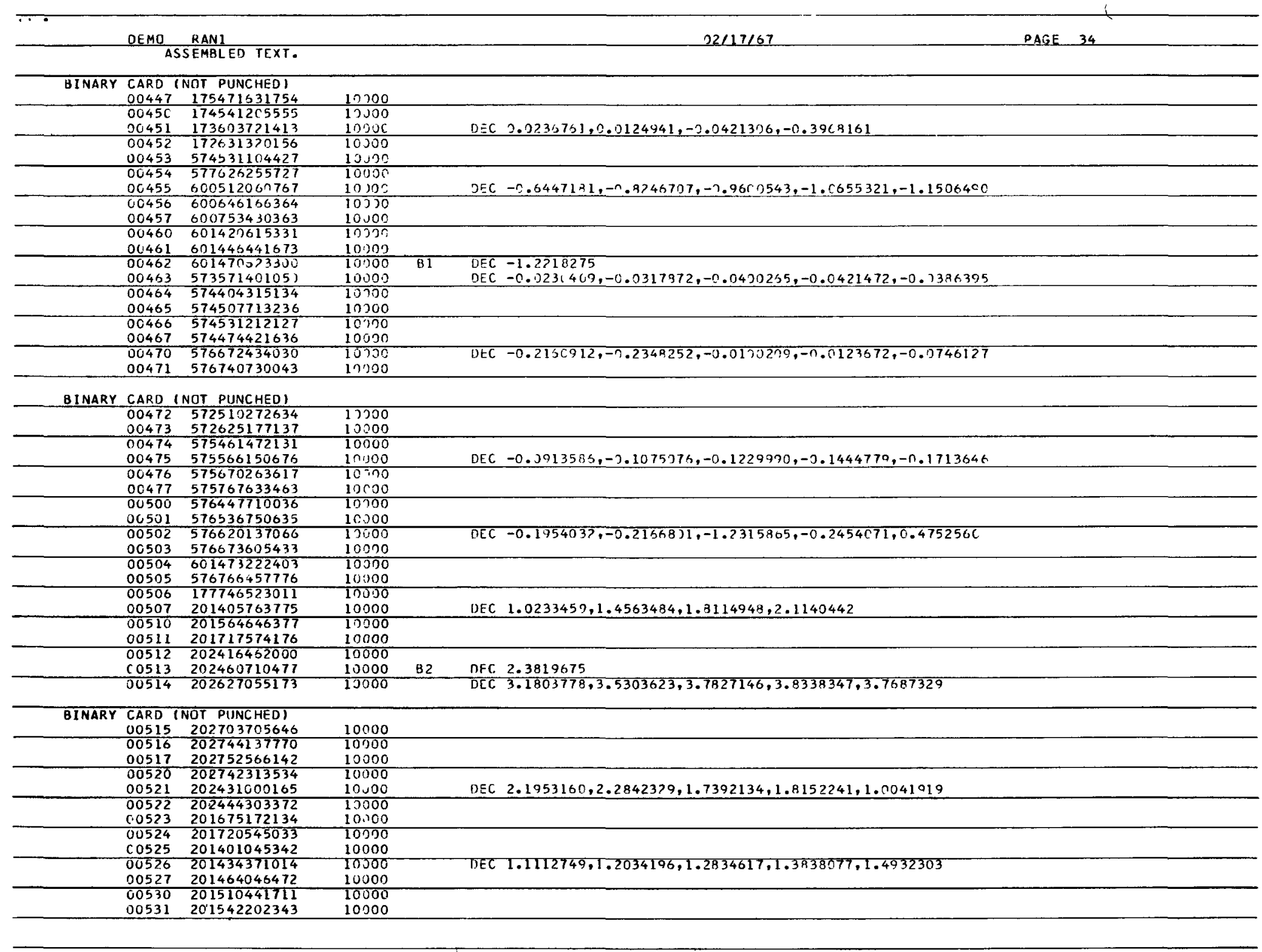


OEC $1.5791 C 7 C, 1.6470113,2 .+161072,2.2306061,1.7068558$

$4751002 \quad 10000$

BINARY CARD (NOT PUNCHED)

$00541 \quad 200742757773 \quad 10000$

$00542 \quad 200511721005 \quad 10000$

$00543 \quad 177600404773 \quad 1000$

$00545000000000000 \quad 10000$

0054651214501606010000

$\begin{array}{lll}00547 & 000001300031 & 10000 \\ 00550 & 00000300015 & 10000\end{array}$

$\begin{array}{lll}00550 & 000603500015 & 10000 \\ 00551 & 201400000000 & 10000\end{array}$

$\begin{array}{lll}00551 & 201400000000 & 10000 \\ 00552 & 200400000000 & 10300\end{array}$

005532320000000001000

\section{B3 DEC 0.1233749}

DEC. $1.2895203,0.9432983,0.5443520,0.3754978$

ए0000 0III

*LOIR

*LORG

00000 0IIII

END 


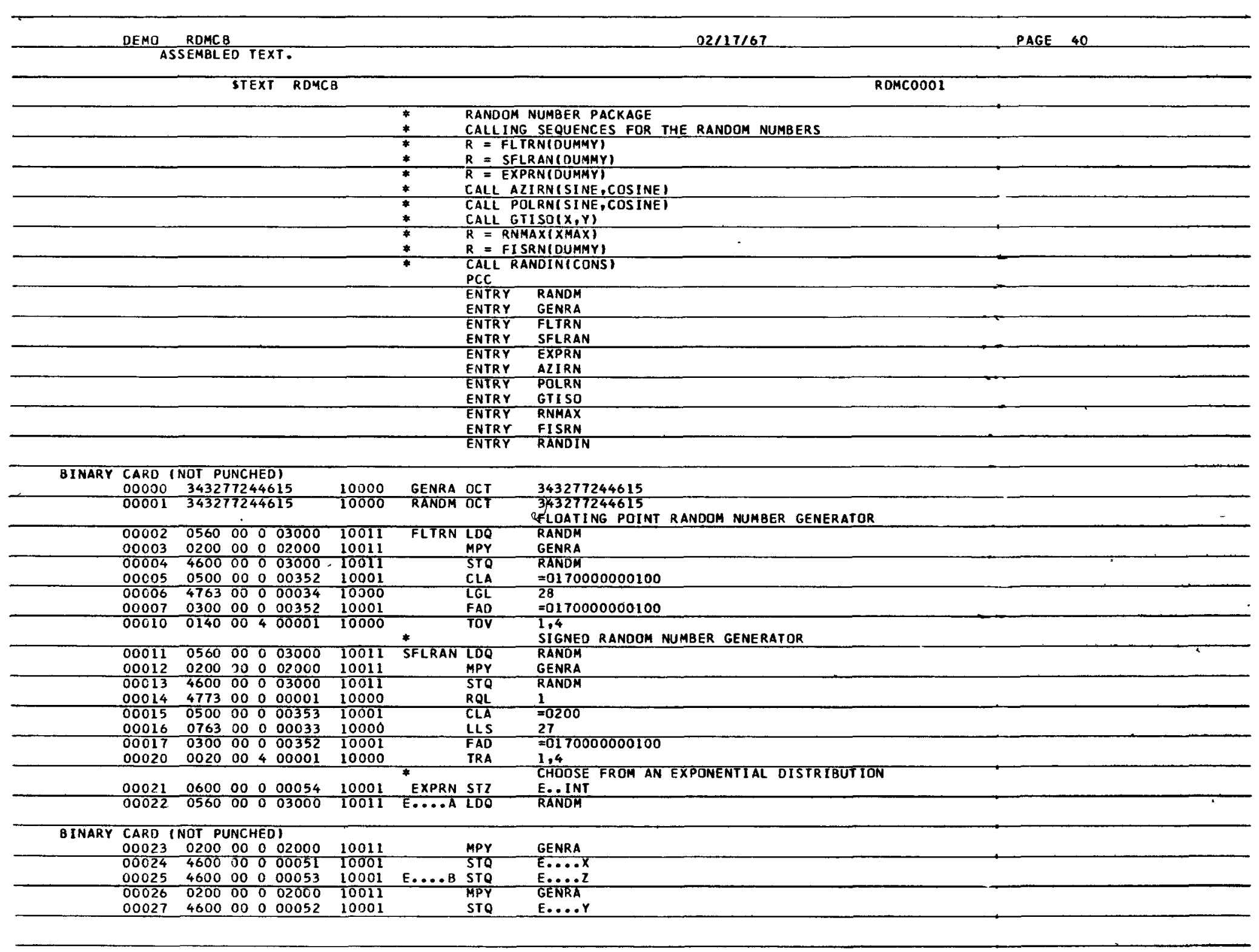


PAGE 41

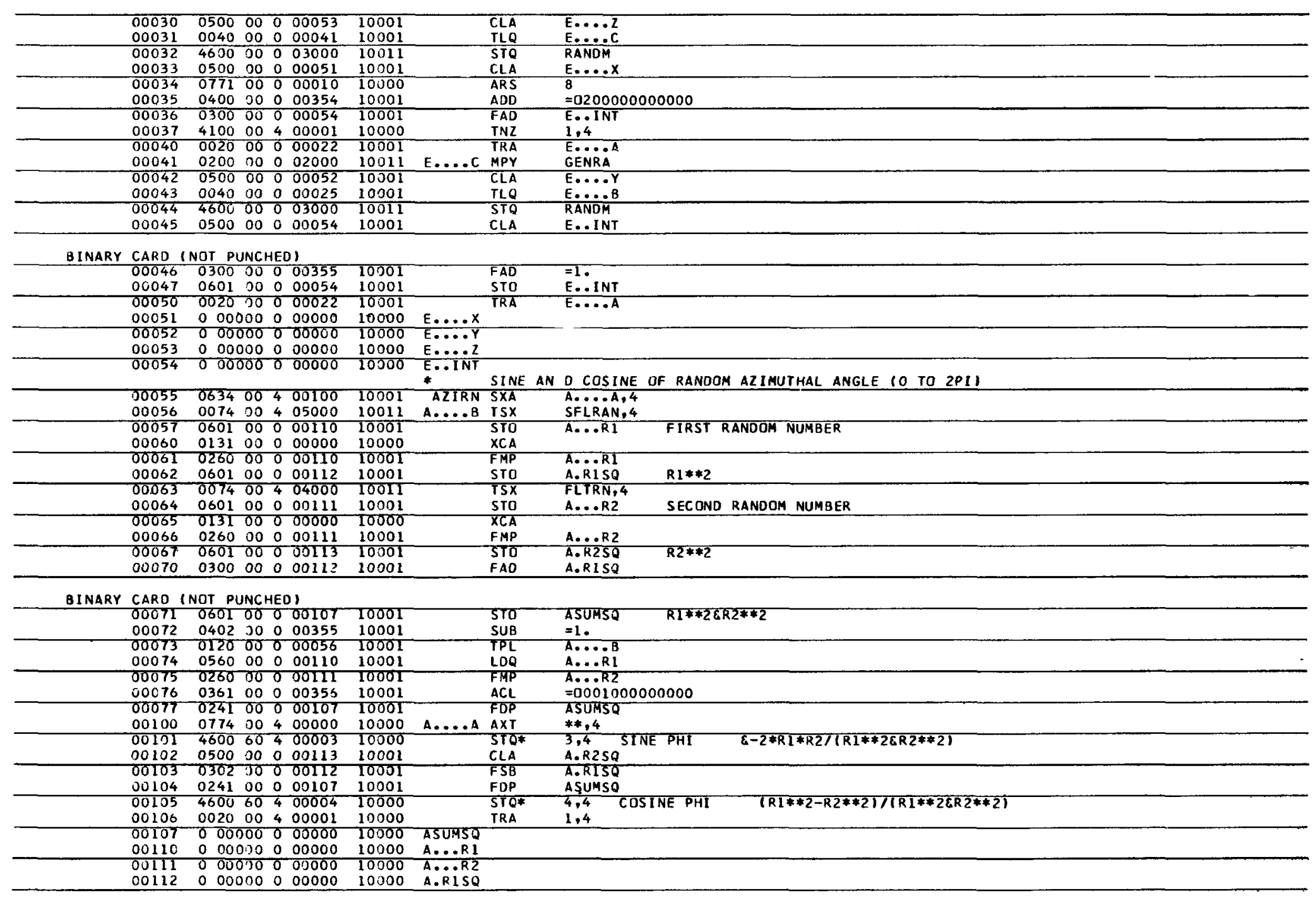


00113000000 U 0000010000 A.R2SQ

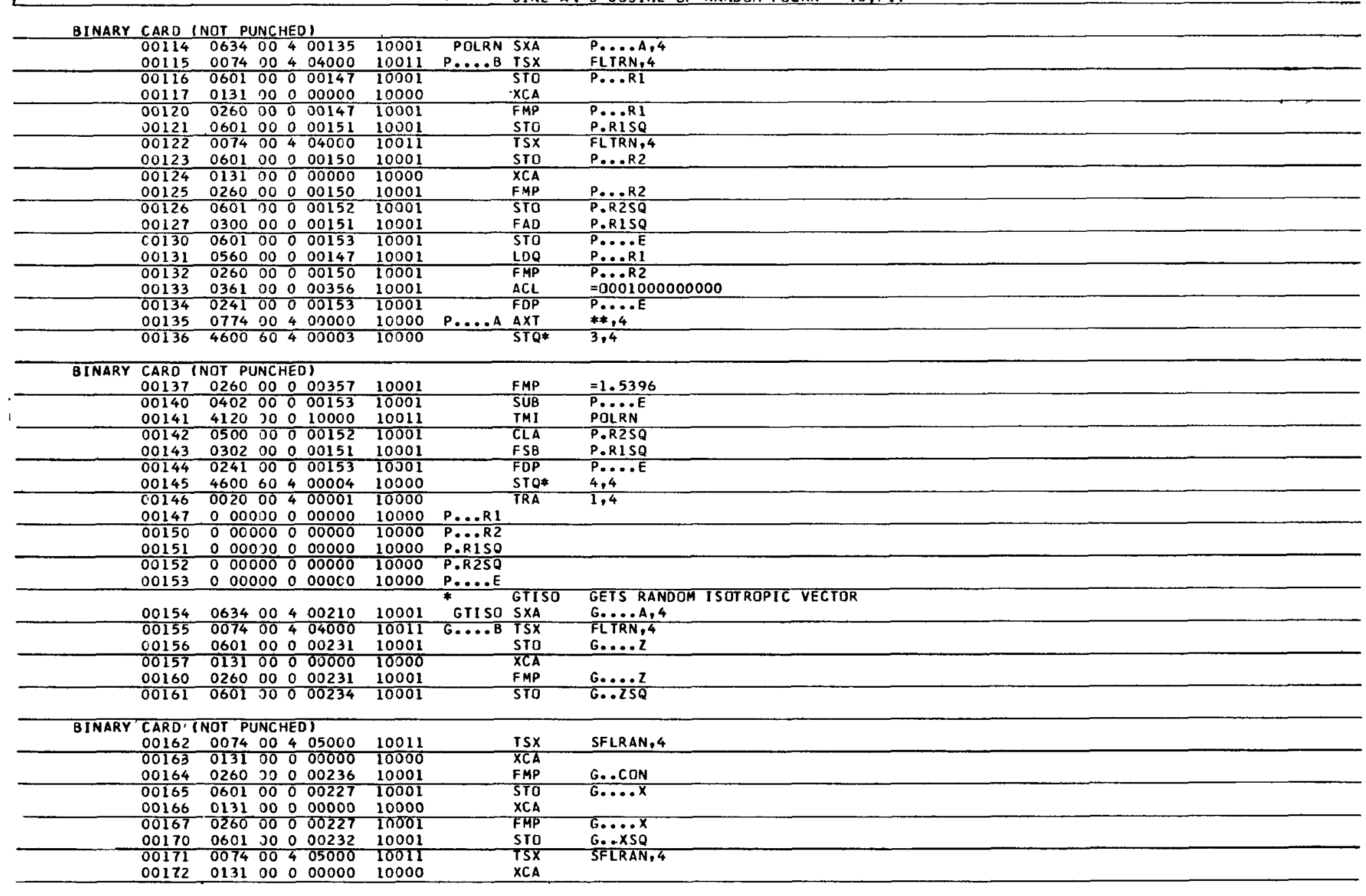




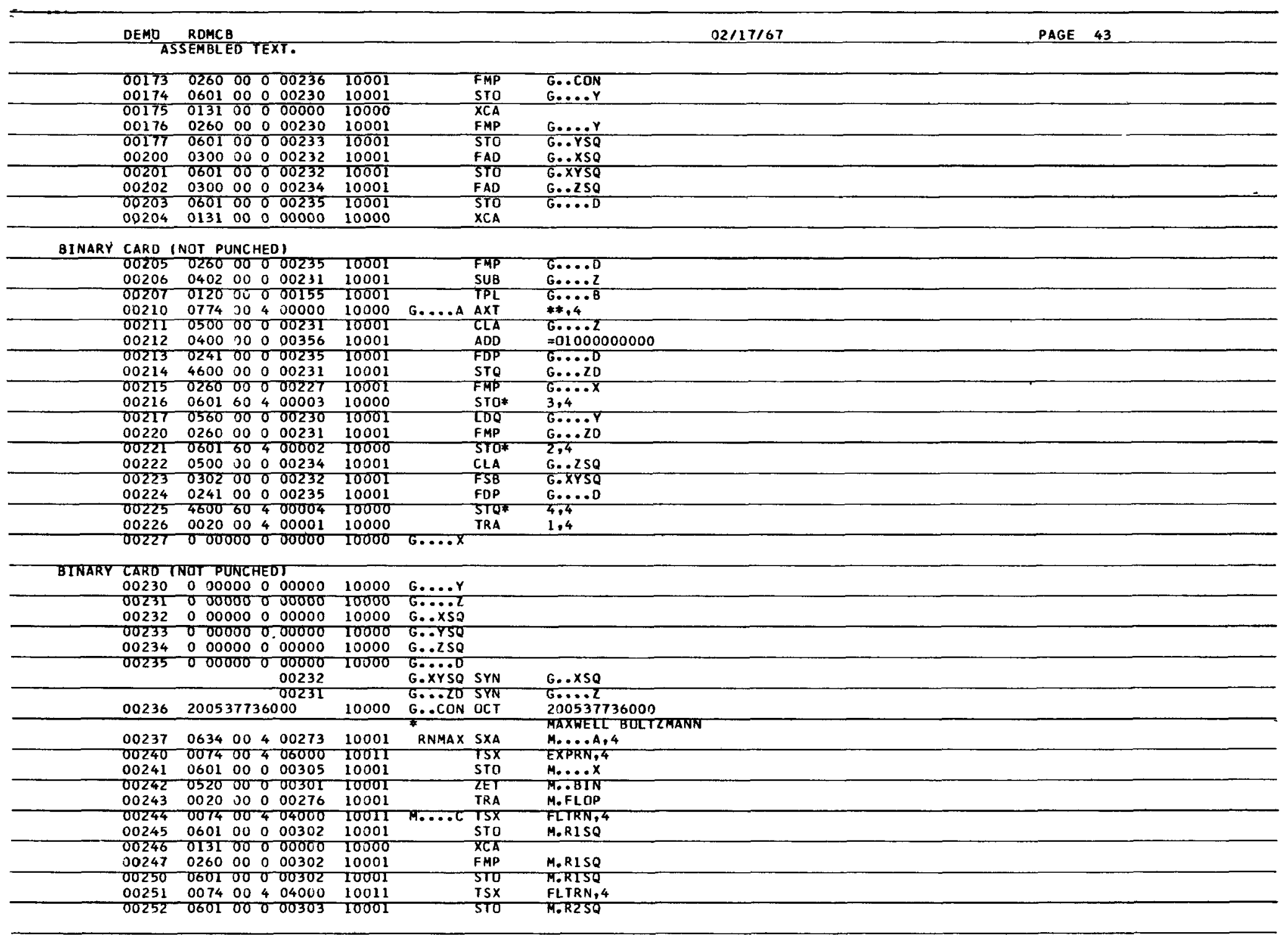




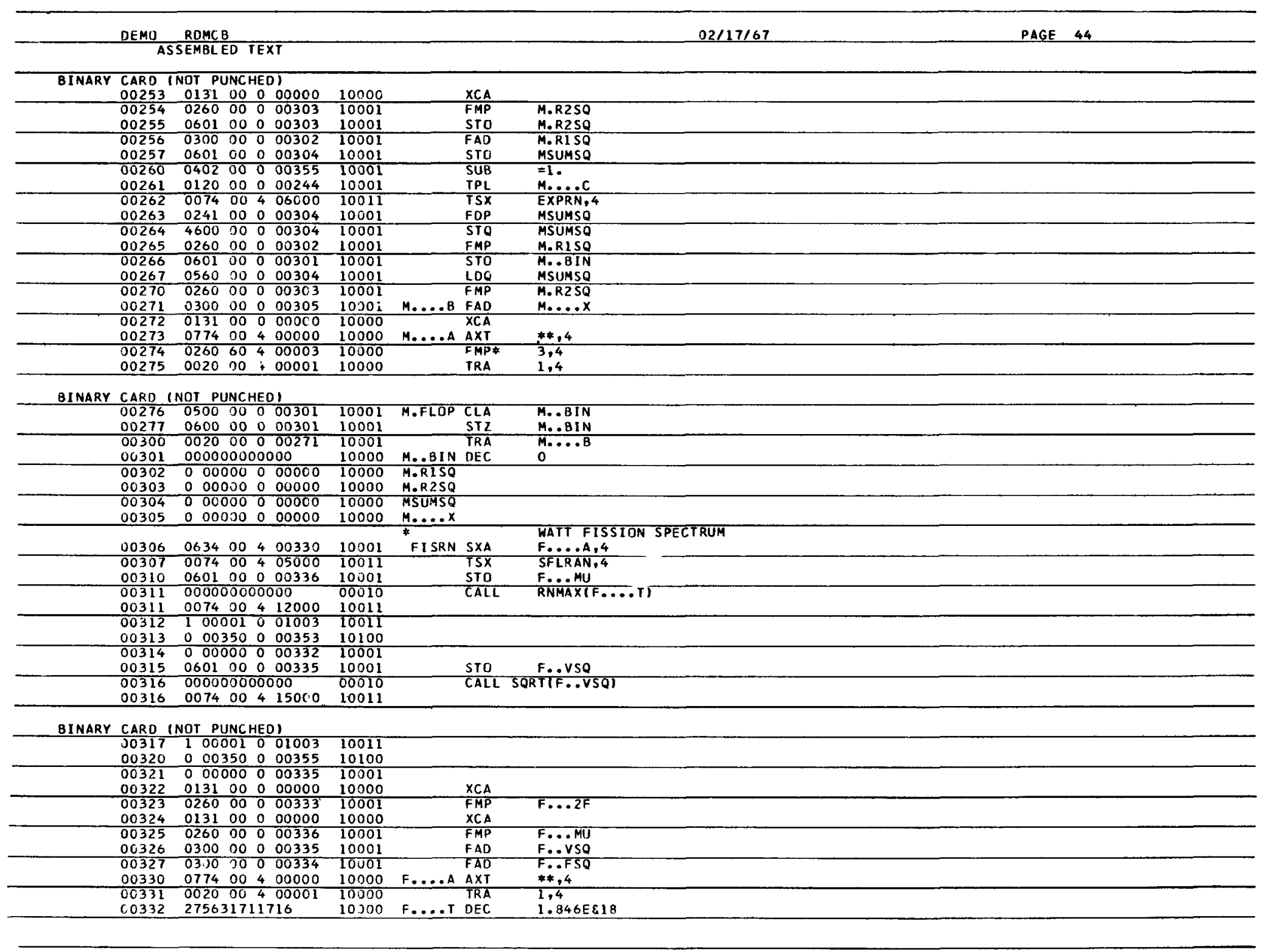


$\begin{array}{lllll}00333 & 237741405150 & 10000 & \text { F...2F DEC } & 2.0196 \mathrm{FEg}\end{array}$

$00334274704761114 \quad 10000$ F..FSQ DEC $1.020 E$ E 18

0033500000000000010000 F...VSO

\begin{tabular}{lllllll}
00336 & 000000 & 0 & 00000 & 10000 & F....MU & \\
\hline 0337 & 050060 & 400003 & 10000 & RANOIN CLA* & 3,4
\end{tabular}

\begin{tabular}{cccccc}
00340 & 01,00 & 00 & 0 & 00345 & 10001 \\
\hline 00341 & 0766 & 00 & 0 & 00003 & 10000
\end{tabular}

TZE

R.....A

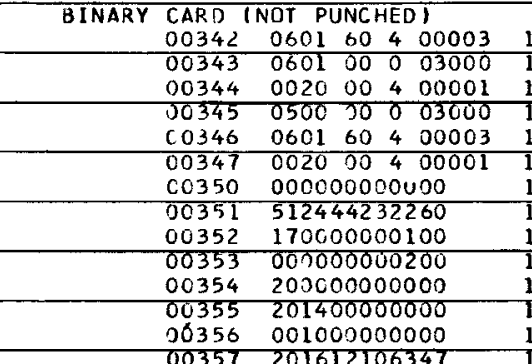

00357201612106347

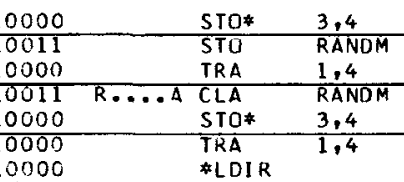

*LORG

*LORG


APPENDIX F

TENTATIVE GUIDELINES FOR RELIABILITY ANALYSIS OF CONT AINMENT ISOLATION VALVE SYSTEMS 


\section{APPENDIX F}

\section{TENT ATIVE GUIDELINES FOR RELIABILITY \\ ANALYSIS OF CONTAINMENT \\ ISOLATION VALVE SYSTEMS}

Assessment of isolation valve system reliability can be based on the assumption that at least one isolation valve must close or remain closed on each piping penetration; and, subsequently, these valves must maintain a certain degree of leak tightness. The question then arises, "should one consider all penetrations or only particularly critical penetrations?" This consideration is especially important for containments with a large number of penetrations. For example, the Dresden-3 drywell or primary containment system is penetrated by approximately 488 pipes or tubes and 61 electrical or instrumentation cartridges. Of the piping penetrations, 370 are control rod-drive hydraulic lines which terminate outside the drywell in the control rod hydraulic system.

To attack the problem of assessing isolation valve reliability, the penetrations and the associated valves have been classified according to importance by the degree of separation they afford between potentially radioactive systems and the atmosphere or the secondary containment. These classes in order of decreasing importance are:

1. Penetrations open to containment and terminating in systems not sealed to atmos phere.

2. Penetrations open to primary coolant system and terminating in systems not sealed to atmosphere.

3. Penetrations originating in systems separated from primary coolant or containment atmosphere by one or more impermeable membranes and terminating in systems not sealed to atmosphere.

4. Penetrations open to containment and terminating in sealed systems.

5. Penetrations open to primary coolant system and terminating in sealed systems.

6. Penetrations originating and terminating in sealed systems.

These penetration classes are summarized in Table F. 1 along with isolation valve classifications based on normal valve status, i.e., normally open and normally closed valves. 
With the preceding breakdown the most critical isolation valves are normally open valves on Class 1 penetrations; e.g. , containment ventilation lines which communicate with the atmosphere during normal operation. Such lines are used in some early power reactor designs (e.g., Shippingport and Dresden-1) but are not included in recent facilities. As developed, the classification system says that isolation valve reliability is most important on lines which do not require a second order failure (i.e., rupture of a sealed system) to result in leakage to the atmosphere, and that lines which require a third order failure, i.e., rupture of two sealed systems, are the least important. Using this ranking, the isolation valve reliability model can be simplified to focus on the more important lines with knowledge that other lines have even less probability of becoming a leakage path, since they will require second or third order failures to become significant. 
TABLE F. 1

PENETRATION CLASSIFICATION

\begin{tabular}{|c|c|c|}
\hline $\begin{array}{l}\text { Class } \\
\text { Designation }\end{array}$ & $\begin{array}{l}\text { Terminates Within } \\
\text { Containment In }\end{array}$ & $\begin{array}{l}\text { Terminates Outside } \\
\text { Containment In }\end{array}$ \\
\hline 1 & $\begin{array}{l}\text { Line open to containment } \\
\text { atmos phere }\end{array}$ & $\begin{array}{l}\text { Line or apparatus not } \\
\text { sealed to atmosphere }\end{array}$ \\
\hline 2 & $\begin{array}{l}\text { Line open to primary } \\
\text { coolant system }\end{array}$ & $\begin{array}{l}\text { Line or apparatus not } \\
\text { sealed to atmosphere }\end{array}$ \\
\hline 3 & $\begin{array}{l}\text { Equipment separated from } \\
\text { primary coolant or contain- } \\
\text { ment atmosphere by one } \\
\text { impermeable membrane }\end{array}$ & $\begin{array}{l}\text { Line or apparatus not: } \\
\text { sealed from atmo- } \\
\text { sphere }\end{array}$ \\
\hline 4 & $\begin{array}{l}\text { Line open to containment } \\
\text { atmosphere }\end{array}$ & $\begin{array}{l}\text { Equipment separated } \\
\text { from atmosphere by } \\
\text { one impermeable } \\
\text { membrane }\end{array}$ \\
\hline 5 & $\begin{array}{l}\text { Line open to primary } \\
\text { coolant system }\end{array}$ & $\begin{array}{l}\text { Equipment separated } \\
\text { from atmosphere by } \\
\text { one impermeable } \\
\text { membrane }\end{array}$ \\
\hline 6 & $\begin{array}{l}\text { Equipment separated } \\
\text { from primary coolant } \\
\text { or containment atmos- } \\
\text { phere by one imper- } \\
\text { meable membrane }\end{array}$ & $\begin{array}{l}\text { Equipment separated } \\
\text { from atmos phere by } \\
\text { one impermeable } \\
\text { membrane }\end{array}$ \\
\hline & $\frac{\text { Valve Classification }}{\underline{\text { Description }}}$ & \\
\hline NO & \multicolumn{2}{|c|}{$\begin{array}{l}\text { Valves open during normal plant operation } \\
\text { includes check valves on in-flowing lines. }\end{array}$} \\
\hline $\mathrm{NC}$ & \multicolumn{2}{|c|}{$\begin{array}{l}\text { Valve closed during normal plant operation } \\
\text { includes check valves on stagnant lines. }\end{array}$} \\
\hline
\end{tabular}


APPENDIX G

GLOSSARY 


\section{APPENDIX G}

\section{GLOSSARY}

This glossary is a reference for definitions of reliability terms and phrases which are used throughout this report. For broader coverage of reliability nomenclature, the reader is referred to handbooks, reports, and texts such as those listed at the end of this appendix.

Progress is closely associated with the ability to communicate especially in technical fields. At the beginning new concepts and ideas are expressed in many different ways, but in time they are amalgamated into a single word or phrase which greatly increases the understanding between different groups of people. As concepts, ideas, and philosophies change, old words take on new meanings. So it is with the words included in this glossary; they reflect present concepts and ideas, but as the field progresses, these words will acquire new meanings.

Automatic Reliability Mathematical Model (ARMM) - A general purpose computer program for deriving and solving a mathematical model of the reliability of complex systems based on a sequential application of the conditional probability the orem (Bayes' Theorem) to the probability of system failure. The program can handle up to a maximum of 300 functions and 500 components and has a built-in capability to handle dependent components, standby components, and mutually exclusive failure modes.

Component - A self-contained unit which performs a distinctive function in the overall operation of a system and which can be disassembled, reassembled, or replaced. For example, count rate amplifier, high voltage power supply, centrifugal pump, and solenoid operated valve.

Confidence Level - The probability that a given statement is correct or the chance that a given value lies within a stated numerical range. For example, a stated reliability of 99 percent at a confidence level of 75 percent means there is a 75 percent probability that the actual reliability is at least 99 percent (lies between 99 and 100 percent).

Derating - The process, in design or test, of employing a component which will carry a greater load, or is more accurate, or will provide a larger output than is necessary for the application. 
Derating Factor - A numerical measure of the design margin provided by derating. If a pump with a design head of 500 feet is used where only a 250 -foot head is required, the derating factor becomes 2 .

Degradation - A gradual deterioration in performance, strength, resistance, etc.

Environmental Stress - The stress or load imposed on a component by a given set of environmental conditions. Conditions which determine environmental stress include humidity, power, pressure, temperature, and voltage. Variations in these conditions tend to affect the failure rate of equipment.

Exponential Failure Density Function - A failure density function which represents the probability of failure in a population composed of units experiencing a constant failure rate. It is of the form

$$
f(t)=\lambda e^{-\lambda t}
$$

Exponential Failure Distribution - The distribution describing the probability of failure before time $t$ in a population with a constant failure rate. It is of the form

$$
F(t)=1-e^{-\lambda t}
$$

Failure - The occurrence of any condition which renders an item incapable of operating within its specified performance parameters.

Failure Cause - That which made the component fail the way it did. Cause description usually involves several levels which are difficult to identify. If an amplifier fails, the immediate cause may be a malfunctioning diode. However, the basic cause may be conditions or events such as a misapplication of the diode in the circuitry or inadequate inspection of the diodes employed in assembling the amplifier.

Failure Data - A collection of discrepancy and malfunction data derived from field and test experience.

Failure Density Function, $f(t)$ - The probability that a unit will fail per unit time at time $t$. Thus

$$
f(t)=\frac{d F(t)}{d t}
$$

sometimes called the failure frequency function. 
Failure Distribution Function, $F(t)$ - The probability that a unit will fail by time t. Thus

$$
F(t)=\int_{0}^{t} f(t) d t
$$

This is sometimes called the cumulative distribution function and is also recognized as the unreliability function.

Failure Effect - What happened to the system when a component failed the way it did.

Failure Event - Occurrence of failure as related to point in time.

Failure Mode - The physical description of the manner in which a component failed. For example, a valve failed to open; a pump failed to continue operation; and a bistable amplifier trip unit operated prematurely.

Failure Rate, $\lambda$ - The probability of failure per unit time in a given time interval. For an exponential failure distribution (constant failure rate), the failure $r$ ate is the reciprocal of the mean time between failures for reparable components and the mean time to failure for irreparable components. Failure rate is normally expressed in number of failures per million hours.

Fault Tree - A graphical presentation of a fault tree analysis which displays the sequential arrangement of events interconnected by logic operators (or gates) that ultimately lead to an undesired event.

Fault Tree Analysis - The analys is of a functional system to establish the logical sequence of events leading to an undesired event. The analysis includes a detailed understanding of system design and operation, the construction of a fault tree, and the mathematical simulation of fault tree logic to establish the likelihood of and individual failure contributions to an undesired event.

Malfunction - Any occurrence of unsatisfactory performance. It need not constitute a failure if readjustment of operator controls can restore an acceptable operating condition. 
Mean Life, $\theta$ - The arithmetic average of the life times of all items considered. It is equivalent to

$$
\theta=\int_{0}^{\infty} t f(t) d t
$$

Mean Time Between Failure, MTBF - The average time between failures which can be expected for a reparable component during the portion of its life cycle in which the exponential failure distribution applies.

Mean Time to Failure, MTTF - The average or mean life of an irreparable component.

Mean Time to Repair, MTTR - The average time to repair a failed component including detection of failure, definition of corrective action, performance of corrective action, and testing before return to service.

Normal Distribution - Same as Gaussian distribution. A distribution defined by

$$
f(t)=\frac{1}{\sigma \sqrt{2 \pi}} e^{-\frac{(t-\theta)^{2}}{2 \sigma^{2}}},-\infty<t<+\infty
$$

where

$$
\sigma \quad=\text { standard deviation }
$$

Operating Error - An error that is traceable principally to some human action or inaction occurring in any activity subsequent to design and fabrication.

Operating Time - The time period between turn-on and turn-off of a system, subsystem, or component during which operation is as specified. Total operating time is the summation of all operating time periods.

Performance - Operation with some degree of effectiveness.

Population - The total collection of items from a common source.

Probability of Failure, $P(\bar{A})$ - The likelihood that $A$ will fail during a specified period of time under a given environment. 
Probability of Success, $P(A)$ - The complement of the probability of failure. Thus

$$
P(A)=1-P(\bar{A})
$$

Random Failure - Any failure which occurs by chance, in an accidental, casual, or haphazard manner. Sometimes called chance failure.

Redundancy - The existence of more than one means of performing a function in order to prevent an overall failure in the event that all but one of the means fail. Parallel redundancy is the application of two or more means of performing a function, all cf which are functioning at the same time but each of which is capable of performing the function in the event of a failure to the other means. Standby redundancy applies where there is an alternate means of performing a function which is held in abeyance until a failure of the primary means is sensed and the alternate means is actuated to perform the same function.

Reliability, $R(t)$ - The probability of performing a required function under specified conditions for at least a given length of time t. For exponential failure distribution

$$
R(t)=e^{-\lambda t}
$$

System - A combination of components joined together to perform a specific operational function or functions. For example, reactor coolant system, reactor control system, and safety injection system.

Systems Analysis by Fault Tree Evaluation (SAFTE-1) - A Monte Carlo fault tree simulation program capable of handling exponential distribution for component failure and normal distribution for component repair.

Unreliability - The complement of reliability, namely, l-R(t).

Use Data - Data which describe the accumulated use by which successful operation of a plant or system is measured.

Wearout - The point at which the continued operation and repair of a component becomes uneconomical due to the increasing frequency of failures. 
Weibull Failure Density Function - Failure density function of the form

$$
f(t)=\beta \alpha t^{\beta-1} e^{-\alpha t^{\beta}}
$$

where

$$
\begin{aligned}
\alpha= & \text { Scale parameter } \\
\beta= & \text { Shape parameter } \\
& \text { If } \beta=1, f(t) \text { becomes exponential function } \\
& \text { and } \alpha \text { is equivalent to failure rate, } \lambda .
\end{aligned}
$$




\section{REFERENCES}

1. Ireson, W. G., et al., Reliability Handbook, McGraw-Hill, New York, 1966.

2. Von Alven, W. H., et al., Reliability Engineering, Prentice Hall, New Jersey, 1964.

3. Definitions of Reliability Terms, AD-480164, Defense Document Center, Virginia, August 1962.

4. Reliability Glossary, North American Aviation, Autonetics Division, Fourth Edition, April 1967. 
APPENDIX H

RELIABILITY ESTIMATES WITH CONFIDENCE LEVELS 
$\underline{\text { APPENDIX H }}$

RELIABILITY ESTIMATES WITH CONFIDENCE LEVELS

In estimating component and system reliabilities the question arises, "what is the potential error associated with reliability estimates made from available reliability data?" That is, one wishes to know the probability that the probability estimate is correct. In safety applications confidence level estimates are generally one-sided and state the probability that the reliability is at least the level estimated, or the failure $r$ ate is equal to or lower than the value used. Confidence levels can be readily estimated for failure rates or reliabilities of either single components or simple systems. For complex systems, the problem of assigning a confidence level to a system reliability estimate, based on component reliabilities and confidence levels, is not as straightforward. The problem here is that the failure distribution function for the system is not necessarily the same as that for the components nor is it easily obtainable.

This appendix presents analytical methods for determining component failure rates or reliabilities at specified confidence levels. In addition, two techniques are discussed for using component data in estimating system reliabilities with certain confidence levels.

\section{COMPONENT LIFETIMES}

An infinitely large component sample size would allow one to predict the true value of a measured parameter with 100 percent confidence. For the finite or particularly small sample size, an estimate of the mean life with a certain confidence level is all that can be obtained. The methods outlined in the following discussion can be used to correlate the accuracy of an estimate with the size of the sample from which it is obtained. These methods are separated, for clarity, into two classes - standby and operating.

\section{Standby Components}

Following Roberts ${ }^{(1)}$, let us consider a population whose individual units have an exponential failure distribution with mean life of $\theta_{0}$. Then the reliability (probability of successful operation) to time $t$ for each unit is

$$
R(t)=e^{-t / \theta} 0
$$


Hence the failure probability is

$$
F(t)=1-R(t)=1-e^{-t / \theta_{0}} \text {. }
$$

If $n$ such units are tested for a certain time $t$, the probability that $c$ or fewer units fail is

$$
Q=\sum_{i=0}^{c} \frac{n !}{i !(n-i) !}[F(t)]^{i}[R(t)]^{n-i}
$$

The confidence level $P$ then can be determined from

$$
P \leqq 1-Q
$$

For the case of no failures, which is of importance in this study, Equation (4) yields

$$
P \leqq 1-[R(t)]^{n} .
$$

Equation (5) is readily solved for $P$, given $t$ and $n$. However, for $c \geqq 1$, Equation (4) does not have a simple representation, and the relation of $c, t, n$, and $P$ must be obtained numerically.

Numerical Computation - If $P$ is specified, $c$ and $t$ can be chosen and an iterative approach used to obtain $\mathrm{n}$ from the equality given by Equation (4). Hence for some $n$ we write

$$
Q_{n}=\sum_{i=0}^{c} \frac{n !}{i !(n-i) !}[F(t)]^{i}[R(t)]^{n-i} .
$$

Now assuming a truncated Taylor series of the form

$$
l-P \approx Q_{n}+\frac{d Q_{n}}{d n} \Delta n
$$

this yields as a correction for $n$

$$
\Delta \mathrm{n}=\frac{(1-\mathrm{P})-\mathrm{Q}_{\mathrm{n}}}{\frac{\mathrm{dQ}}{\mathrm{dn}}} .
$$


Thus if $n$ is used for the $j^{\text {th }}$ iterate, then $(n+\Delta n)$ should be used in the $(j+1)^{\text {st }}$ iterate.

Using Stirling's formula for $n$ ! and neglecting terms in $\frac{1}{n}$

$$
\begin{aligned}
\frac{d Q_{n}}{d n}= & Q_{n}\left[\ln R(t)-\frac{F(t)}{R(t)}\right] \\
& -\left[\frac{F(t)}{R(t)}\right]^{c+1} \frac{n^{c}}{c !}[R(t)]^{n}
\end{aligned}
$$

This expression is used in calculating the denominator of Equation (6). Using this method, Figure H. 1 was obtained for the 75 percent confidence level case.

Example - As an example of the use of Figure H. 1 in obtaining lifetime estimates, consider the following problem. All components in an emergency cooling system are tested periodically. The most critical components are tested by conducting functional tests prior to each reactor startup. Other components are tested annually. These tests are assumed to show that the component can or cannot perform adequately under accident conditions.

Functional tests were conducted 24 times with an average testing interval of 38. 4 days. An estimate will be made for the mean time to an unsafe failure of a certain component $w$ ith an associated confidence level $P$ of 75 percent. For a component with no reported failures during the entire operating period, $c=0$ and $n=24-1=23$. We find from Figure $H .1$ $\frac{t}{\theta_{0}} \leqq 0.060$ and $\theta_{0}$ is, therefore,

$$
\theta_{0} \geq \frac{38.4}{0.060}=640 \text { days }
$$

which is equivalent to a failure rate $\lambda$ of

$$
\lambda \leqq \frac{1}{640 \times 24}=65 \times 10^{-6} \mathrm{hr}^{-1}
$$


Since there are several components generically and operationally similar, the test data from these components may be pooled together. For two similar components $w$ ith no reported failure, $c=0$ and $n=23 \times 2=46$. At a confidence level of $P=0.75, \theta_{0}$ becomes

$$
\theta_{0} \geqq \frac{38.4}{0.030}=1,280 \text { days }
$$

or

$$
\lambda \leqq 32.6 \times 10^{-6} \mathrm{hr}^{-1} \text {. }
$$

Thus doubling the number of components has doubled the lifetime estimate at the same confidence level.

Suppose the second component failed once during the entire period. Then for $c=1$ and $n=46$

$$
\theta_{0} \geqq \frac{38.4}{0.060}=640 \text { days }
$$

or

$$
\lambda \leqq 65 \times 10^{-6} \mathrm{hr}^{-1} \text {. }
$$

Similar estimates can be made for annually tested components. In this example, annual tests were conducted five times with an averaged testing interval of 324.5 days. For a component tested annually with no failure reported during the entire period, $c=0, n=5-1=4$, and

$$
\theta_{0} \geqq \frac{324.5}{0.34}=954 \text { days }
$$

or

$$
\lambda \leqq 44 \times 10^{-6} \mathrm{hr}^{-1} \text {. }
$$

\section{Operating Components}

Consider $n$ similar components which operate for a time $t$. The point estimate of the mean life of these components, $\theta_{0}$, when $c$ failures occur, is

$$
\theta_{0}=\frac{n t}{c} .
$$


Confidence Level - An alternative estimate ${ }^{(2)}$ of $\theta_{0}$ can be made based on the chi-square $\left(X^{2}\right) *$ distribution. In this case, it can be specified with a certain confidence that $\theta_{0}$ will lie between two numbers, the upper and lower confidence level estimates. For a given confidence level $P$

$$
\frac{2 n t}{x_{\frac{Q}{2}, 2 c+2}^{2}} \leqq \theta_{0} \leqq \frac{2 n t}{x_{1}^{2}-\frac{Q}{2}, 2 c}
$$

where

$$
Q=1-P \text {. }
$$

Equation (8) is useful provided $c \geq 1$.

In reliability analysis, a one-sided estimate of the mean lifetime is often of particular interest. That is, the minimum value of $\theta_{0}$ can be specified at a given confidence level. Thus

$$
\theta_{0} \geqq \frac{2 n t}{X_{Q, 2 c+2}^{2}}
$$

This expression holds for $c \geqq 0$.

A family of curves for operating time nt versus $\frac{1}{\theta_{0}}$ is plotted in

Figure H. 2 for 0 to 6 failures with 75 percent confidence level. Note that $\frac{1}{\theta_{0}}$ is equivalent to failure rate $\lambda$.

Example - Consider a system which has been operated 17,604 hours and which contains six similar pumps with no reported failures. Entering Figure H. 2 with $c=0$ and $n t=6 \times 17,604=105,624$ hours, the failure rate is estimated to be

$$
\frac{1}{\theta_{0}}=\lambda \leqq 13.3 \times 10^{-6} \mathrm{hr}^{-1} \text {. }
$$

\section{DISCUSSION}

It can be shown that the method used for the lifetime estimates of standby components is equivalent to that used for continuously operating *The values of $x^{2}$ are obtained from probability tables. 
components. In fact, many continuously operating components can be considered as standby in that they are cyclically monitored by an operator or operate cyclically and therefore can be treated in the manner for standby components.

Example

Consider the previous example in which six pumps operated for 17,604 hours with no failure and yielded

$$
\lambda=13.3 \times 10^{-6} \mathrm{hr}^{-1}
$$

according to the continuous method.

Treating each hour as a successful test, i. e., pump operation is monitored once each hour

$$
\begin{aligned}
& \mathrm{n}=6 \times 17,604=105,624 \text { tests } \\
& \mathrm{c}=0 \\
& \mathrm{t}=1 \text { hour. }
\end{aligned}
$$

Extrapolating from Figure H. 1

$$
\frac{t}{\theta_{0}}=13.3 \times 10^{-6}
$$

or

$$
\lambda=\frac{1}{\theta_{0}}=13.3 \times 10^{-6} \mathrm{hr}^{-1}
$$

Similarly if each day is treated as a successful test

$$
\begin{aligned}
& \mathrm{n}=105,624 / 24=4,401 \text { tests } \\
& \mathbf{c}=0 \\
& \mathrm{t}=24 \text { hours. }
\end{aligned}
$$

We find from Figure H. 1

$$
\frac{t}{\theta_{0}}=0.000316
$$


or

$$
\lambda=\frac{1}{\theta_{0}}=\frac{0.000316}{24}=13.2 \times 10^{-6} \mathrm{hr}^{-1} .
$$

The difference is due to round-off.

\section{SYSTEM LIFETIMES}

Confidence level estimates for individual components are quite simply obtained for either operating or nonoperating components. Similar estimates of confidence are difficult for the reliability of a system when based on the probabilistic behavior of components. In fact, such analytical estimates are presently limited to a narrow class of system models and probability distributions. This difficulty results from the fact that system reliability is strongly dependent on the structure of the system functional logic as well as component reliability.

Takenaga ${ }^{(3)}$ has analyzed systems composed of 10 components in series with all components assumed to have exponential failure distributions. He has found, for exampie, for a set of components all having failure rate estimates at the same confidence level, that the system confidence level behaves as follows:

Component Confidence Level System Confidence Level

$\begin{array}{ll}40 \% & 13.5 \% \\ 50 \% & 31.3 \% \\ 60 \% & 62.35 \% \\ 70 \% & 90.0 \% \\ 80 \% & 99.9 \%\end{array}$

Failure data are given in Reference (3). Another method for estimating the confidence level on system reliability with serially arranged components having exponential failure distributions is given in Reference (4).

Kniss ${ }^{(5)}$ has investigated simple systems which contain components in parallel as well as in series. He obtains system confidence levels by generating random values of the component confidence levels and using these to generate random values of the system reliability. His results agree with those obtained by alternative analytical methods in three simple cases. 
Of course, if a system were constructed, then data on its reliability characteristics could be easily obtained through testing the system. However, it should be possible to predict system reliability based on a knowledge of its components and structure. The two approaches mentioned above are attempts to solve this problem. The latter approach appears promising since one is not restricted to analyzing serial systems. However, this technique may become costly for complex systems. Further work in this area is needed. 


\section{REFERENCES}

1. Roberts, N. H. , "Mathematical Methods in Reliability Engineering," McGraw-Hill, New York, 1964.

2. Bazovsky, I., "Reliability Theory and Practice," Prentice Hall, Inc., Englewood Cliffs, New Jersey, 1961.

3. Takenaga, R., "Predicting System Reliability With Associated Confidence Level From Component Test Data," AD 459713.

4. Von Alven, W. H., "Reliability Engineering," Arinc Research Corporation, p. 320, Prentice Hall, Inc., Englewood Cliffs, New Jersey, 1964.

5. Kniss, J, R., "Reliability Estimation for Multi-Component Systems, "AD 633163 . 


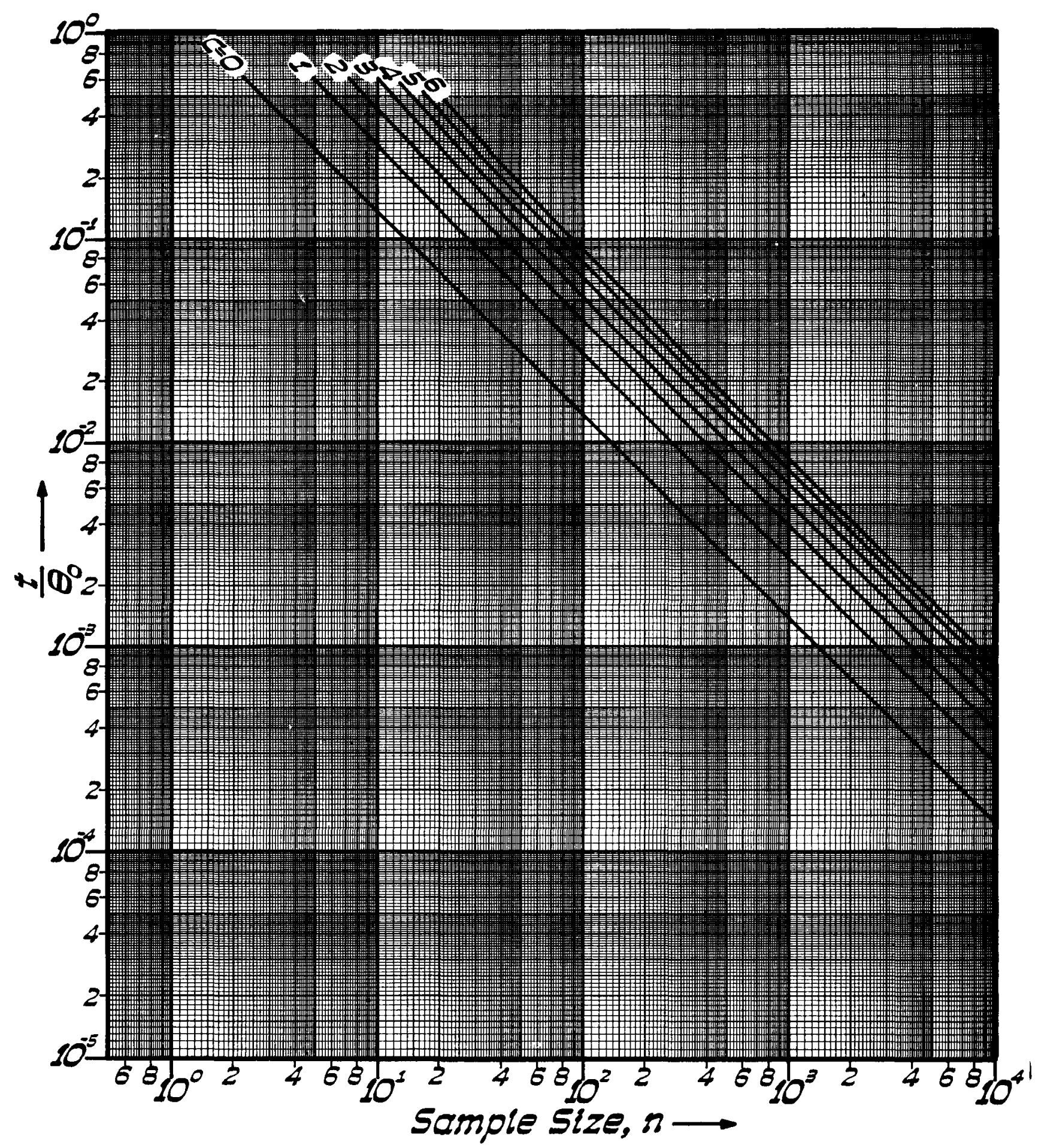

FIGURE H. 1

LIFETIME ESTIMATE FOR STANDBY COMPONENTS AT 75 PERCENT CONFIDENCE LEVEL 


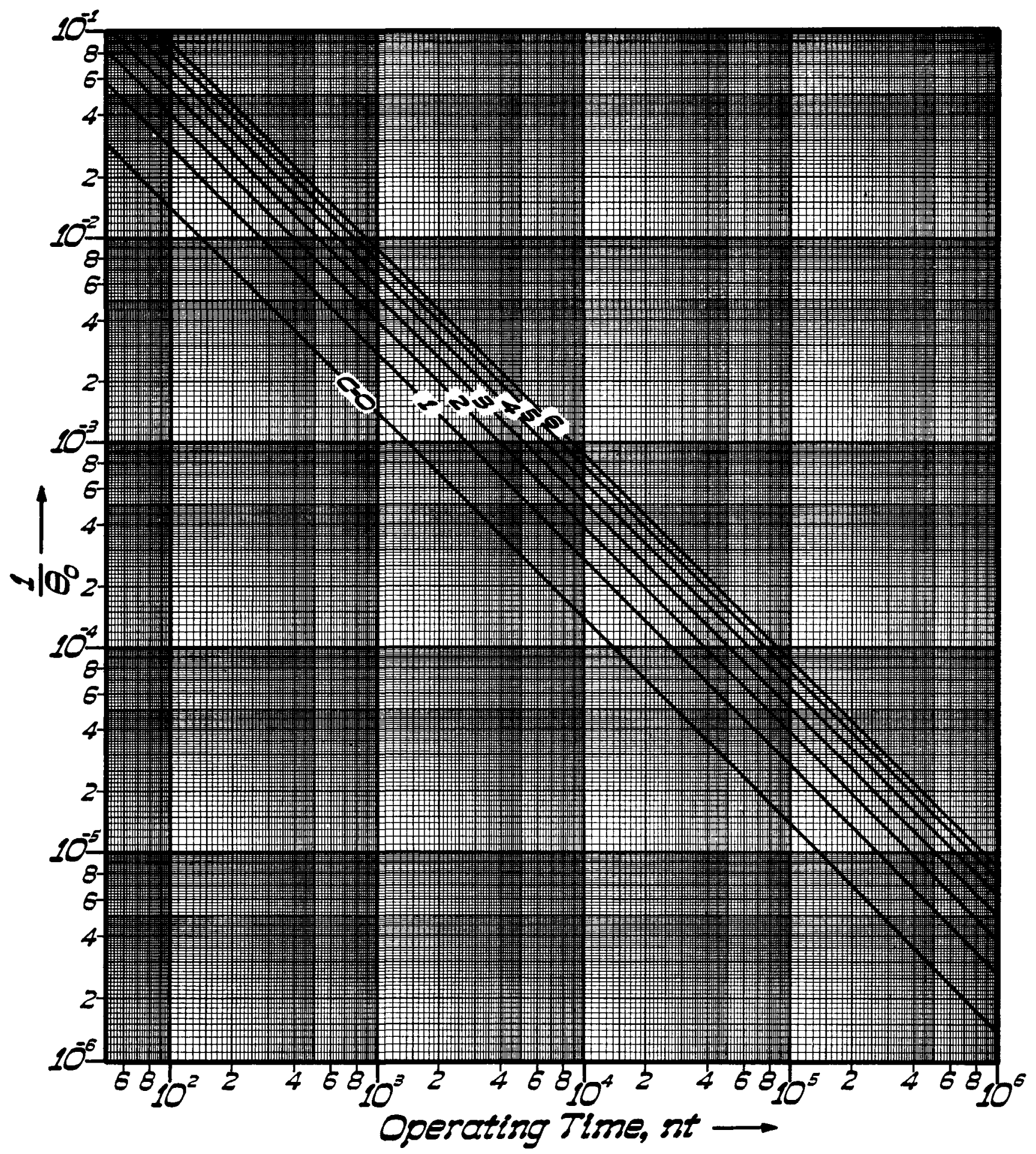

FIGURE H. 2

LIFETIME ESTIMATE FOR OPERATING COMPONENTS AT 75 PERCENT CONFIDENCE LEVEL 